\section{Indonesian Day}

\author{
1A-S01-01

\section{GMP VS ISO IN A BLOOD ESTABLISHMENT} \\ VA Armstrong \\ Vee Armstrong Quality and Regulatory Consultant, Perth, Australia
}

Blood Establishments have undergone a significant cultural change over the past 25 years, moving from a predominantly scientifically focussed culture to that of a manufacturer of therapeutic goods.

Part of this transition has been driven by the emergence of, initially, quality management systems (QMS), and subsequently, principles of good manufacturing practice (GMP) as requirements for blood establishments. The manufacture of fresh blood and blood components has also become highly regulated in many countries, with Codes of Good Manufacturing Practice written specifically for the industry.

Certification to the international Standard ISO 9001: Quality management systems Requirements has been undertaken by a number of Blood Establishments in countries where regulation of the Blood Establishments has not yet been introduced. The Standard sets out the framework for a QMS that could be used by any industry that produces a product for a customer and as such, the requirements are general. ISO implementation has the advantage of auditing by a third party to determine the level of compliance, and formal certification once the level is acceptable.

Not only do Codes of GMP for Blood require a QMS to be implemented, but they also set out principles for the manufacture of therapeutic goods, focussing on safety through risk management and error minimisation. Additionally, the Codes include requirements specifically for each part of the manufacturing chain. Successful GMP implementation in regulated countries is formally acknowledged by documented accreditation or the award of a licence to operate, however in non-regulated countries, there is often no formal acknowledgement of successful compliance or third party auditing. Additionally, the concept of GMP and particularly the differences between it and practices already in place can be difficult to grasp.

As a result, some Blood Establishments in non-regulated countries that wish to improve practices have opted to use ISO implementation as a driver for quality improvement. However, while the ISO Standard and Code of GMP both set out the requirements for a QMS, ISO does not include the critical requirement that manufacture of blood and blood components must follow principles of GMP to ensure optimisation of safety through managing risks and minimising errors. Blood Establishments will therefore ultimately need to implement GMP regardless of ISO certification to be assured of safe, consistent and efficacious components.

The starting point for a Blood Establishment's implementation of GMP will depend on its ISO status if any. If it has already been ISO certified, a gap analysis should be conducted between ISO and GMP and a plan developed to close the gaps. If it is currently implementing ISO, the gap analysis should include the systems still to be developed so that GMP is built into those systems at the same time. If it has not implemented ISO, it is recommended that the focus be on GMP implementation.

The similarities and differences between ISO and GMP, and the implementation of GMP into an ISO QMS will be considered in more detail.

\section{A-S01-02}

\section{THE ROLE OF THE NATIONAL BLOOD CENTRE IN A COUNTRY WITH A MIXED SYSTEM OF BLOOD SERVICES \\ U Charoonruangrit}

National Blood Centre, the Thai Red Cross Society, Bangkok, Thailand

Background: National Blood Centre (NBC), the Thai Red Cross Society is a charitable organization under principles of the International Federation of Red Cross and Red Crescent Societies (IFRC). It is designated by the Government to implement the national blood transfusion service with safe and adequate supply. Apart from NBC headquarters in Bangkok, there are 12 Regional Blood Centres (RBCs) in the provincial regions running blood collection and testing services for the network provinces in each particular region. Blood donation at NBC and RBCs are from voluntary nonremunerated donors (VNRD). Six government hospitals in Bangkok (under universities, militaries and Bangkok Metropolitans) and also approximately 160 government provincial hospitals operate blood collections for their own uses. Clinical blood transfusion practices run by the hospitals.

National blood policy: The Government adopted the current National Blood Policy 3rd version in 2010. It consists of 9 policy objectives including effective management, adequacy of blood supply from VNRD, safety of blood in accordance with the principles of the World Health Organization, promoting quality assurance systems, appropriate blood utilization, implementing related laws and regulations, conducting the researches and developments, producing plasma derived medicinal products and providing the stem cell from unrelated donor volunteers.

Blood donation: Coordinating with National Blood Centre, each Provincial Red Cross Chapter led by Red Cross Chapter President, shall conduct blood recruitment program. Red Cross Chapters together with provincial hospitals, which are assigned as Blood Centre Branches, shall arrange blood donation schedules to meet their need and also supply to neighboring districts. Due to effective public relations campaign during the past 4 years, NBC, RBCs and also hospital blood centre branches all over the country attained blood collections beyond the setting goal. By the year 2014, overall whole blood collections were 2,274,788 units which was equal to $3.5 \%$ of the number of the whole country populations.

Blood testing: Centralization of donor blood testing is one of the national policy strategies for safe and standard blood supply. All 12 RBCs are the centralized labs for their regions and 10 of them are Nucleic Acid Testing (NAT) lab centres. At present, nearly $95 \%$ of donated blood in the country is NAT tested.

Standards and guidelines: NBC and experts on Transfusion Medicine had revised and published the 5th edition of Donor Selection Guideline, the 1st edition of Physician Handbook on The Appropriate use of Blood and Blood Components, the 3rd edition of the Standard Blood Banks and Transfusion Services and the 1st edition of Hemovigilance Handbook. They are the references for national blood service practices.

Quality systems: NBC provides training and accreditation on Blood Collection Technique and Blood Donation Administration course for hospital staff who operate blood donations. All hospitals shall follow quality assurance programs such as Hospital Accreditation (HA), ISO etc. and shall be regularly assessed by the professional council committees.

\section{A-S01-03}

\section{CONSOLIDATION OF LABORATORIES FOR BLOOD TESTING}

$\mathrm{J}$ Chen

Taiwan Blood Services Foundation, Taipei, Taiwan

Background: Annually we collect 1.8 million of donor bloods, either whole blood or apheresis platelets, through six regional centers. Before the release of blood and blood components, samples of each donation are taken to test for infectious diseases and blood grouping. Since the population of the service area of certain center is low and the number of specimens to be tested by the center is few, they are in a difficult position in recruitment of qualified staff and in use of advanced technology for donor blood screening. Aims: We report a laboratory consolidation program that, with minimal cost, improve the quality of laboratory screening for donor blood collected by each centers. Methods: Before laboratory consolidation, donor bloods were tested in the laboratories of each center. The laboratory of the smallest center performed screening for around 200 donors per day. In 2001, we initiated a laboratory consolidation program. The fundamentals of the program were (1) centralization of donor blood screening at two laboratories, (2) each equipped with advanced screening analyzers, (3) blood specimens arrived at laboratory before 09:00 on the next morning, (4) result report before 17:00 on the next day, (5) uniformity in testing kits and equipment between laboratories, (6) back-up plan in place. As a part of risk management, the program was carried out with two phases that began with the transition from 6 to 5 laboratories, and after 6 months, to 2 laboratories. Results: After consolidation, the two laboratories each performs an average of 2,500 donor blood screening and the subsequent confirmatory testing per day in the work hours from 08:00 to 17:00. These include blood group serology (ABO, RhD, antibody screening), chemistry, viral immunoassay and nucleic acid testing (NAT). Before 17:00 on the second day of collection, test results are available for regional center to release blood and blood components except that donations are subject to test for bacteria contamination. Since that culture-based bacteria detection requires specimens for analysis being taken during $24-36 \mathrm{~h}$ of platelet storage, for this reason, we detect bacteria contamination for platelets in the laboratory of each regional center rather than centralization. In addition, it is essential that contingency back-up plans are in place for donor blood screening. Up to the present time, we had experienced a number of events that switched the usual procedure for donor blood screening to the back-up procedure.

Summary/conclusion: Through laboratory consolidation, we are able to provide high-quality and cost-effective donor blood screening and, most importantly, to ensure the safety of donor blood collected by each individual center. At present the two laboratories, together with a head laboratory that performs reference testing service, ensure blood safety and provide specialized transfusion support in Taiwan. 
1A-S02-01

\section{BLOOD APHAERESIS IN BEIJING}

Y Qiu

Beijing Red Cross Blood Center, Beijing, China

In July 1978, Beijing Red Cross Blood Center (BRCBC) collected the first aphaeresis platelet with Haemonetics M30. Since then BRCBC has imported Haemonetics PCS+, MCS3P, MCS+, Baxter AMICUS and Caridian BCT Trima successively into blood collection and components preparation. We collect platelet and plasma with aphaeresis machine only for clinical usage. The number of plateletpheresis has been increased annually. In 1994 we only collected 2249 therapeutic unit plateletpheresis and reached to 65,088 units in 2014. At present the SDP supply had replaced of the RDP totally in Beijing.

We follow the national regulation 'whole blood and component donor selection requirements GB 18467-2011' t o screen and select the aphaeresis donors and set up a efficient SDP donation workflow including donor identification, pre-donation testing and health evaluation, donor acceptance, aphaeresis collection preparation, collection process and post-donation treatment, etc. The rate of plateletpheresis donors deferred is 17.56 in 2014. The choice of aphaeresis procedures can be collecting platelet only, including single, double platelet or both plasma and platelet component is collected at the same time. We program the aphaeresis procedure depending on the donor intention and health examination results. The rate of double platelet collection is $62.7 \%$ and the number of plasma for transfusion units derived from aphaeresis collections is 4950 units in 2014.

In order to meet the increasing progressively clinical consumption of platelets annually, we take advantage of any accessible Medias to promote the blood donation and try to recruit and retain the blood donors in Beijing. Plateletpheresis donation frequency per donor in Beijing is about 3 times per year. Both the new guidelines of shortened the plateletpheresis donation interval and the increasing ratio of repeated aphaeresis donor help to overcome the shortage of platelet supply in Beijing. The platelet issues per 1000 population in Beijing are 5.69 in 2014

PCs product random test monthly is a method of quality surveillance and also a part work of the established blood quality management system. 'Standards for whole blood and blood components quality: GB $18469-2012$ ' is a statutory national guideline for blood services to implement quality control and supervise the Labeling, appearance, volume, $\mathrm{pH}$ value, platelets count, residual of WBC, residual of RBC and the sterility of all labile blood products.

Immunohaemotology lab is responsible for investigating frequencies of HLA and HPA antibodies among patients who transfusion the platelet. Patient's characteristics and the positive $\%$ of platelet-specific antibody push us to screen the platelet-specific and HLA antibodies screening routinely. Platelet cross-match is the mainly way to prevent the platelet transfusion refractory in Beijing.

By now we have not organized an integrated heamovigilance system in Beijing. Optimization the blood aphaeresis system deeply, fulfilling the clinical demand, expanding the HLA and HPA typed platelet donors pool and traceability the aphaeresis blood product will be the goal in the near future.

\section{A-S02-02}

\section{HEPATITIS B UPDATE ON DIAGNOSTIC AND SCREENING TEST} MW Thedja

Eijkman Institute for Molecular Biology, Jakarta, Indonesia

Hepatitis B remains a major health concern worldwide with impact on various clinical manifestations including chronic infection, cirrhosis, fulminant hepatitis, and hepatocellular carcinoma. Further, hepatitis B virus (HBV) accounts as the most common transfusion-transmitted viral infection that might give significance impact on morbidity and mortality. Thus, diagnostic and screening tests for HBV infection are the entry point to reduce the burden of clinical and socio-economic consequences of HBV complication. Hepatitis B surface Antigen (HBsAg) serology test is still used as the first-line diagnostic marker for hepatitis B, and also the first-line blood-donor screening test for HBV infection. However, in some situations HBsAg might not be detected in serum either in chronic HBV-patient or in blood donation despite the presence of HBV DNA. This condition might be due to window period, HBV mutant particularly in surface gene, and occult carriage of HBV infection (OBI) that usually corresponds with low titer of HBV DNA. In addition to HBsAg, anti-HBc test has been widely used for blood donation screening in some countries; however, the implementation of anti-HBc screening is inefficient for those countries with HBV endemic in term of blood donation loss. Mutations in HBsAg that is associated with conformational changes may account solely or in conjunction with the failure of HBsAg detection by immunoassay. In the last few years, NAT (nucleic acid testing) multiplex assay that combines simultaneous HBV, hepatitis C virus (HCV), and human immunodeficiency virus (HIV) detection has been introduced and

\section{(C) 2015 The Author}

Vox Sanguinis (C) 2015 International Society of Blood Transfusion

Vox Sanguinis (2015) 109 (Suppl. 2), 1-96 implemented either in minipools or more efficiently in individual samples. NAT discriminatory assay for HBV/HCV/HIV can be applied for samples with positive NAT multiplex. In comparison with serological tests, HBV NAT has the ability to reduce the window period and to detect OBI cases. However, for countries with high endemicity of HBV infection, the implementation of NAT multiplex/discriminatory may not be affordable due to the high operational cost. Alternatives to simplify methods and to reduce NAT multiplex/discriminatory operational cost should be challenge for scientific community, and also develop more sensitive serological tests for HBsAg mutants.

1A-S02-03

PLATELET ANTIBODY TESTING AS PART OF PRETRANSFUSION TESTING FOR MULTI-TRANSFUSION PATIENTS WHO HAD PLATELET REFRACTORINESS

NK Ritchie

Jakarta Blood Transfusion Unit, DKI Jakarta, Indonesia

Platelets as part of blood cells, which are multi-antigenic, have human platelet antigen (HPA), human leukocyte antigen (HLA) class I beside ABO antigen on its surface. The polymorphism of those antigens are very high, especially HLA antigen. The patients could develop alloantibody to antigen which not exists on his platelets. At next transfusion, that alloantibody could react with donor's platelet, resulting in platelet refractoriness. Beside alloantibody, there were other factors contributed to platelet refractoriness. The clinicians could use corrected count increment at $1 \mathrm{~h}$ and $24 \mathrm{~h}$ after platelet transfusion to predict the cause of platelet refractoriness. Then, they could ask the reference laboratory to screen and identify the platelet antibody. In Indonesia, according to Chunaeni study, 20-70\% of hemato-oncology patients in Dharmais Hospital had platelet refractoriness due to platelet transfusion. Most of them had platelet transfusion from random donors. Another study by Lubis et al has shown 39\% of hemato-oncology patients who received repeatedly platelets transfusion, developed anti-HLA class I antibody and only one anti-gpIIb/IIIIa (3\%). In other cases, we might find anti-HLA class I antibody in patients with no history of platelet transfusion. In such cases, the source of HLA class I antigens were leukocytes which contaminated the PRC. If the alloantibodies detected, there is a probability, patients could get a compatible platelet so the transfusion would be effective.

Platelet antibody testing consists of antibody screening and identification test. The principle of this test is testing the patient's serum with intact/whole platelets. There are many methods for this test, e.g. enzyme immunoassay, monoclonal antibody immobilization platelet antigen (MAIPA), solid-phase enzyme immunoassay (SPEIA), platelet immunofluorescence tests, and radioactive-monoclonal anti-IgG assay. Each method has its advantages and disadvantages. When we detect antibodies in patients, we should do platelet antigen typing either with molecular testing or serologic testing. If the patient needs blood then we select the antigen-negative platelet or do platelet cross-matching to get the most compatible platelet for the patient. So, if we implemented platelet antibody testing in Indonesia, it would help multitransfusion patients who have developed allo-antibodies to get the effective platelet transfusion.

\section{Plenary Session: Overcoming Resource Limitations}

\author{
1B-PL1-01 \\ OVERCOMING RESOURCE LIMITATIONS OF BLOOD SERVICES \\ IN INDONESIA \\ Y Soedarmono \\ Ministry of Health of Indonesia, Jakarta, Indonesia
}

Background: Indonesia with 250 million population is the fourth most populated country in the world. Blood service is an important part of health services especially for Indonesia with a high maternal mortality rate which one of the cause is inadequate and limited access to blood supply. In the last ten years, strong government commitment and good strategies have made blood services improved significantly in its supply, quality and access.Aims. To describe strategies and steps of improvement of blood services in Indonesia.

Methods: Indonesia with 539 district has various conditions of geography and infrastructure. Since 1950 the blood services in 210 districts were run by the Indone- 
sian Red Cross Blood Transfusion Services (IRC BTSs). In 1997-2002, Indonesia received a project from Japan Bank for International Cooperation (JBIC) on Strengthening of Safe Blood Supply System in Sulawesi. This project was a milestone to start government attention on the need for improving of blood services in the country. There were three main problems of blood services identified: (1) inadequate organization of blood services; (2) limited access and inadequate blood supply; and (3) varying and poor control of quality of blood product. To cope with these problems, the Ministry of Health (MOH) using the WHO guidelines, has made some strategies and actions.Results: To improve the organization of blood services, in 2005 a National Committee of Blood Services has been established to formulate policy and design strategies for MOH's approval. After the committee has been established, the government regulation on blood services and MOH decree on BTS, Hospital Blood Bank (HBB) and blood supply network have also been issued. These regulations emphasized that blood should 100\% come from voluntary donors, and their quality should be ensured. Under these regulations, the BTSs were classified based on their level of services; every hospital should establish a HBB to maintain cold chain of blood; and National Agency for Food and Drugs Control (NAFDC) was appointed to control the quality of blood products. Moreover, MOH decree on Functional Standard of BTS; plasma fractionation policy; and personnel resource and development will be issued. The functional Standard of BTS guides implementation of quality system and GMP of all aspects of BTS operational. To increase access and achieve adequate blood supply, mixed organization of BTS has been chosen by building 164 Hospital Based Blood Transfusion Services (HBTS) in the districts with no IRC BTSs. And nonetheless, the NAFDC in cooperation with the WHO, MOH and relevant stakeholders has set up the GMP audit tools of blood establishment. In the future the national system on GMP accreditation is expected to be established.

Conclusions: Blood services is a complex activity that should be well organized, maintained and controlled. A mixed organization of blood services run by IRC and hospitals might be the best choice for Indonesia. However, clear and precise government regulations with strong commitment of government and IRC is key to achieve adequate and qualified blood services in Indonesia.

\section{B-PL1-02}

\section{OVERCOMING RESOURCE LIMITATION IN MYANMAR}

T Aung ${ }^{1}$ and I Nozaki ${ }^{2}$

${ }^{1}$ Government, Yangon, Myanmar, Burma ${ }^{2}$ NCGM, Tokyo, Japan

Background: As many of other developing countries, Myanmar has been straggled with improving the blood transfusion services in resource limited setting. It was started in 1935 with paid donor and Central National Blood Bank committee, established in 1962, introduced voluntary blood donation. The frequent repeated donors who donate more than 50 times started to be awarded by Ministry of Health, and the ceremony for awarding has been conducted 38 times since then. Transfusion transmissible infections (TTIs) screening was started with HIV in 1995 by the support of National AIDS Program and extended to HBs Antigen and HCV Antibody screening in 2002 with cost sharing basis. Major challenges in those days were (1) lack of awareness on blood transfusion among both administrators and Public, (2) relatively high prevalence of TTIs in general (3) weakness in human, technical and financial resources.

Aim: To raise quality status of Blood transfusion services with available resources. Method: Retrospective review of system.

Result: To overcome those challenges, Blood and Blood Product Law were enacted in 2003, and National Blood Center (NBC) was assigned to strengthen the nationwide service.

Firstly, NBC has focused on awareness rising on blood transfusion service including importance of voluntary donation. HIV serology screening reactive rate was $1.08 \%$ in 2000 and was decreasing as increasing voluntary donors. Donor deferral and systematic donor registration were introduced to strengthen the donor selection since down trend was obtained in 2004. After that, it has been decreasing to $0.18 \%$ in 2013. Hepatitis B vaccine program was also introduced as motivation and retention program for safer donors. As a result, voluntary donations were increased from 8035 $(34.9 \%)$ in 2000 to $38814(88.5 \%)$ in 2013.

Secondly, NBC has tackled quality improvement of the services. NBC participated in International External Quality Assessment Scheme (EQAS) for TTI testing since 2002. NBC has a chance to change HBs Antigen testing method which used locally produced reagents, in 2003 because of false negative results from that program. National EQAS Program for HIV testing by National Health Laboratory covered other hospital blood banks. Monitoring for those hospital blood banks has been conducted since 2008. Finally, the guidelines on blood transfusion services, which adapted to countryss situation, was published in 2010 and started to be utilized in the training as reference.
Summary/Conclusions: Looking back the journey of improving blood transfusion service in Myanmar, we would like to emphasize three key points. First, social mobilization was the most important fundamental for developing blood transfusion services. Without the support from community, private sectors, and publics, we cannot do anything. Second, technical supports from expert groups were crucial for quality improvement in various areas and various ways. Third, the political commitment from the government was the most essential for long time sustainability. We are still in the middle of the journey and continue to work harder to improve quality of BTS for our patients, remembering those three keys.

\section{B-PL1-03}

OVERCOMING RESOURCE LIMITATIONS IN PAPUA NEW

GUINEA

ML Mathias

National Blood Service, National Capital District, Papua New Guinea

Background: Papua New Guinea is the larget island in the South Pacific with a population of 7.8 milliion. It is made up of 600 islands and 50\% of the land is mountainous with many areas inaccessible by road. Communication is very difficult in some places and $87 \%$ of the population is rural.

Current situation: The National Blood Service faces many challenges in making safe blood available. There are 35 blood banks scattered throughout the country. Annually, 30,000 units of blood is collected, 50\% of these from VNRBD whilst many small centres rely entirely on FR donors. A quater of these bloods colected is discarded due to TTIs.

Due to the isolation of these centres, blood banks are set up to be self sufficient in recruiting, bleeding of donors and screening blood for transfusion.

The NBS has recently been taken over by the DoH after being managed by NGOs in the las 50 years. It continues to struggle with lack of a management team, a central office, and the relevant logistical support. Funding has always been a main issue.

The issues that NBS faces are in effectively coordinating the activities of all the 35 blood centres, improve and maintaining quality systems, improving and maintaing an adequate blood supply, reduce the rate of TTIs and improve VNRBD to $100 \%$. Achievements: NBS has completed the Blood Policy that has been endosed by the National Health Board awaiting Government approval for funding. The Quality Management Checklist and revised Donor Management SOP has been approved for implementation. 120 persons have been trained in various activities of blood banking. NBS is gradually establishing regular contact with the blood centres. Way forward:

1. National Blood Policy and Strategic Plan 2015-2019 to be approved and funded by the Government

2. Establish a fully functional office

3. Finalise Clinical Blood Guidelines to be implemented

Acronyms: FR, family replacement donors; NBS, National Blood Service; PNG, Papua New Guinea; SOP, Standard Operating Procedures; VNRBD, Voluntary Nonremunerated Blood Donor; WHO, World Health Organization.

\section{Academy Session: The What and How of Immunohaematology}

\author{
1C-S03-01 \\ MOLECULAR APPLICATIONS IN ROUTINE TRANSFUSION \\ TESTING \\ CE van der Schoot, B Veldhuisen, L Haer-Wigman and M de Haas \\ Sanquin Research, Amsterdam, The Netherlands
}

For safe transfusion practices it is important to transfuse compatible donor red blood cells to an immunized patient. Blood group antigen status in donors and recipients is most often determined via serological typing. Nevertheless, serological typing is not always possible, for instance, in patients who recently received a red blood cell transfusion or in individuals with auto-antibodies. Also for fetal typing, serology is impossible and genotyping is the method of first choice. Furthermore, serological typing can be cumbersome when a large set of antigens needs to be determined or 
when an antigen has variable expression levels. Blood group antigen prediction via genotyping can overcome situations in which serology is impossible or impractical. At the moment some blood centers have implemented genotyping assays to accurately predict the blood group status of blood donors and recipients. More frequently blood group genotyping has also been used to screen donors for rare blood types in a high-throughput fashion. Nevertheless, most blood group genotyping assays are still used as a tool to facilitate serological typing. In this presentation an overview will be given on the presently available genotyping methods, and the potential advantages as well as drawbacks of genotyping in routine transfusion testing will be discussed.

\section{C-S03-02}

HOW DO WE APPROACH DILEMMAS IN RED CELL TESTING?

VS Nadarajan

University Malaya, Kuala Lumpur, Malaysia

Despite the large number of serologically identified red cell antigens to date, it is reassuring to note that in the common clinical scenario, the transfusion laboratory would only likely deal with a narrow range of clinically significant antibodies. Accurate $\mathrm{ABO}$ and $\mathrm{RhD}$ typing still remains the most crucial step in pre-transfusion testing followed by the antibody screen. The correct identification of single antibodies or multiple antibodies and whether they are allo- or autoantibodies is necessary so that corresponding antigen-negative red cells can be supplied. Clinically insignificant antibodies, or what some would denote as 'nuisance' antibodies would need to be differentiated from clinically significant antibodies. Conversely, the laboratory should also be able to rapidly recognize situations where an antibody to a high-incidence antigen developing within null phenotype individuals may occur.

When discrepancies occur between forward and reverse $\mathrm{ABO}$ typing, it is crucial that laboratories resolve the discrepancy before a specific $\mathrm{ABO}$ blood group is assigned. The inclusion of A2 cells and 0 cells in reverse grouping, anti-H and -A1 lectins in the forward, performing the typing on a range of temperatures using washed red cells and careful interpretation of agglutination patterns would usually provide an answer. On occasions, a saliva test may be necessary and rarely molecular typing. With regards to $\mathrm{RhD}$ typing, test systems should be in place to identify $\mathrm{RhD}$-negative and D-variant individuals, so that D-negative units can be issued to them. Testing with anti-sera from different clones, use of adsorption-elution techniques and RhD.CcEe typing aids in coming to a conclusion, before molecular testing becomes necessary. Performing an extended red cell phenotype is indicated especially if the patient harbours or is at risk for developing a red cell antibody. Using good quality validated anti-sera together with appropriate controls is always necessary. Washed red cells should always be used for phenotyping as substances in the plasma may either interfere with the anti-sera or cause false positive reactions. It is also important that both antithetical antigens are tested in order not to miss a null-phenotype. In a DAT-positive patient, phenotyping may be complicated especially when performed using an AHG phase. In such situations, we choose to use acid-eluted cells although other methods of stripping antibodies from the red cell surface may be employed as long as one is cognizant of the effect of the technique on antigen expression. Another issue that often arises with red cell phenotyping is when patients have been recently transfused. Careful interpretations of mixed field populations using column agglutinations are often helpful in assigning a preliminary phenotype without having to resort to molecular genotyping. Techniques for harvesting reticulocytes, which are more likely of patient origin in a post transfused sample are available but are often cumbersome and has largely been abandoned in favour of molecular typing. On occasions, atypical phenotyping patterns may be observed. Common red cell membrane abnormalities among Asians such as South East Asian ovalocytosis may down regulate red cell antigen expressions as would some acquired disorders in particular leukemia and myelodysplastic syndromes.

\section{C-S03-03}

\section{HOW DO WE MANAGE PATIENTS WITH RARE BLOOD TYPES}

Z Zhu

Shanghai Blood Center, Shanghai, China

In many countries, rare blood type is defined as red blood cell antigen phenotype rate below one of thousand. As their rare rate among population, clinical blood transfusion is always a major challenge for all countries, especially with a high titer antigen-specific antibody in the circulation.

As for blood services that supply blood components for clinical usage, selecting and determining rare blood type among donors is the most effective way of obtaining Although there are different strategy and methods of rare blood screening, foundation of a unified rare blood donor information system is a common pattern. From

\section{(C) 2015 The Author}

Vox Sanguinis (C) 2015 International Society of Blood Transfusion

Vox Sanguinis (2015) 109 (Suppl. 2), 1-96 80s in last century, ISBT set up a working party for rare blood donors. This party connects several major rare blood banks in the world, so as to form an international rare blood exchange system.

Currently, more and more laboratories are applying molecular engineering methods for blood type gene test. As technique evolves, more new blood type is found in genetic level. By means of high-flux genetic screening technology, such as genechip, mass diagnosis, etc, we conduct overall screening for all populations. This greatly remedies the lack of antiserum in rare blood screening.

According to the regional rate of blood type antigen and alloantibody if occur, it is needed to predict regional rare blood type and their possible demanding. If national blood center or regional blood center can estimate rare blood type demanding and therefore screen and prepare in advance, this will greatly cut the clinical waiting time and meet most clinical blood transfusion requirement. Meanwhile, as for scarce and unique blood type, national-wide registration and allocation system is required as well as international exchange system.

of course, not all rare blood types can determine its specificity in a short period. Sometimes even specificity is determined; you cannot obtain antiserum right away. Therefore, emergency blood transfusion, including monocyte monolayer assay (MMA), Chemiluminescence Test(CLT) and different blood substitute transfusion is needed.

Rare blood type is not only restricted to red blood cell or platelet. In plasma, rare blood type also matters. For instance, IgA deficiency patients usually suggest IgA negative blood transfusion. Therefore, setting up IgA negative donor bank is anothe emphasis in clinical blood transfusion.

\section{Scientific Advances in Blood Donation and Components}

\section{C-S04-01 APPLYING BEHAVIOUR CHANGE THEORY TO BLOOD DONOR RECRUITMENT AND RETENTION \\ C Tan}

Singapore Red Cross, Singapore, Singapore

A successful system, based on voluntary, non-remunerated blood donation (VNRBD) to ensure a safe and sustainable blood supply for the country, requires acceptance and support from all stakeholders. In order for this to happen, communications and engagement of stakeholders, especially existing blood donors and potential blood donors are key tenets of success. In this digital age, where content is king, blood services that have embarked on social media, in addition to traditional media, to communicate and engage potential donors have seen many successes in the recruitment of new blood donors. The challenge, however, is in bringing about more repeat donations. Studies and researches have been conducted to better understand donor behaviours; with the aim of enhancing communications with target audiences. In Singapore, we incorporated the 5W's and $1 \mathrm{H}$ in examining the 5 steps of behavioral change, from sharing the idea to bringing about comprehension and conviction; to taking action and repeating the action of voluntary blood donation; using both traditional and digital media to communicate our ideas and to heighten interest and acceptance of blood donation as an integral part of life, especially among the youth.

\section{C-S04-03}

\section{NEW DEVELOPMENTS IN PLASMA DERIVATIVES \\ PRODUCTION}

T Burnouf

Taipei Medical University, Taipei, Taiwan

Human plasma derivatives obtained by large-scale fractionation represent a unique range of approximately 20 therapeutic protein bioproducts. They are used to treat patients affected with life threatening conditions due to trauma, rare bleeding or metabolic disorders, immunodeficiency, auto-immune diseases, or bacterial or viral infections. Some plasma derivatives obtained by genetic engineering technologies are used mostly in developed economies due to limited supply and high cost. Plasma fractionation is thus required to prepare many protein products and to contribute to the worldwide supply.

The production of plasma derivatives is an integral manufacturing process starting from donors screening and blood/plasma collection. Plasmas obtained from whole blood ('recovered plasma') or collected by apheresis are both suitable for the produc- 
tion of fractionated plasma derivatives, as long as they meet specific specifications defined by the fractionator and approved by regulatory authorities overlooking plasma collection and plasma product marketing authorization.

In line with the clinical needs and considerations for cost-effectiveness, plasma fractionation involves integrated manufacturing steps to extract multiple products from plasma pool of typically $3000-6000 \mathrm{l}$. The current fractionation process remains largely influenced by technological choices made some 70 years ago when a technology (known as the Cohn method) using sequential ethanol precipitations under defined conditions of $\mathrm{pH}$, negative temperature, and conductivity was developed to prepare albumin and immunoglobulins. Combined with cryoprecipitation to isolate Factor VIII, it still remains the core methodology in use to date. Multiple products are obtained through the incorporation of chromatographic procedures to extract coagulation factors, protease inhibitors, anticoagulants from the cryoprecipitate-poor plasma or early fractions of the 'Cohn process'. As a means to increase immunoglobulin recovery without affecting other products, current plasma fractionators have successfully engaged into and achieved process modifications that replace dedicated ethanol precipitation steps of IgG by chromatographic purifications. The current fractionation process of plasma combining refined purification tools and robust viral reduction treatments allow preparing a range of plasma products with established quality and safety profile, albeit sometimes at sub-optimal yield.

Several attempts are on going to bring to the market new extraction processes of plasma derivatives. Most are intending to change the economics of plasma fractionation, mostly by allowing (a) higher recovery of the leading protein (currently Immunoglobulin) or that of key value proteins (such as alpha 1-antitrypsin) that suffer from very low yield during the current ethanol fractionation process or (b) extraction of novel plasma proteins. Such methodologies are based on core processes using integrated affinity chromatography, expanded bed chromatography, membrane electrophoresis, aqueous two-phase system, or salt precipitation and diafiltration. Regardless the technology used to improve product yield, the needed implementation of robust viral reduction treatments does require extra manufacturing steps with a toll on protein yield, therefore leveling off the initial recovery claims. Finally, a mini-pool fractionation/viral inactivation approach using disposable equipment is under development to facilitate access to the plasma fractionation technology in developing economies with minimal capital investment.

This presentation will summarize the current technology in place for industrial plasma fractionation and present an update on the methodologies in development.

\section{C-S04-04 \\ THERAPEUTIC APHERESIS}

T Triyono

Faculty of Medicine, Gadjah Mada University/ Sardjito Hospital, Yogyakarta,

Indonesia

Implementation of therapeutic apheresis varies in any areas around the world. It is a routine procedure in developed countries, but still a special procedure in many developing countries.

Apheresis may be performed to collect a therapeutic dose of a particular component, therapeutically reduce the circulating amount of a particularly harmful component, or collect a particular blood cell/ precursor from a patient for re-infusion. Many therapeutic apheresis procedures can be applied i.e. Therapeutic plasma exchange (TPE), Leukocytapheresis, Thrombocytapheresis, Erythrocytapheresis, RBC exchange, LDL apheresis, Adsorptive cytapheresis, Lymphocytapheresis, ECP, and Rheopheresis. Therapeutic plasma exchange is the most common procedure performed among therapeutic apheresis procedures. It is a therapeutic procedure in which blood of the patient is passed through a medical device which separates out plasma from other components of blood, the plasma is removed and replaced with a replacement solution such as colloid solution (e.g., albumin and/or plasma) or combination of crystalloid/colloid solution. In general,TPE is used for the treatment of autoimmune or immune mediated diseases or disorders to remove monoclonal antibodies, paraproteins, autoimmune antibodies, or antigen-antibody complexes.Some aspects should be considered i.e. indication, venous access, anticoagulant used, doses of exchange, number of procedures and its frequency. Other important aspects were replacement fluid and the complications may be happened.

Red cell exchange is usually done in case of sickle cell disease, and other cytapheresis is aimed to reduced pathologic or excess leukocytes/ platelets.

Technical aspects of the procedure, socialization, and economical coverage should be considered to improve implementation of therapeutic apheresis.

\section{C-S04-05}

THE EFFECTS OF $22^{\circ} \mathrm{C}$ AND $4^{\circ} \mathrm{C}$ STORAGE OF PLATELETS ON VASCULAR ENDOTHELIAL INTEGRITY IN VITRO AND IN VIVO

G Baimukanova and S Pati

BSRI, San Francisco, United States of America

Background: With the ever increasing demand for blood components in transfusion, methods of extending the short platelet shelf life have been examined recently, with a particular emphasis on cold storage $\left(4^{\circ} \mathrm{C}\right)$. While most studies are focused on platelet hemostatic function, the effect of $4^{\circ} \mathrm{C}$ platelets on endothelial vascular integrity is still not known. Maintenance of endothelial barrier stability is a critical goal of especially in prophylactic transfusion and the context of an injury or inflammatory response.

Aims: In this study, we compared the effects of platelets stored 1 up to 7 days at $22^{\circ} \mathrm{C}$ or $4^{\circ} \mathrm{C}$ on the vascular endothelial barrier function, in vitro and in vivo in mice.

Methods: Leukoreduced apheresis platelets were used fresh (whiting 1 day) or aged 5,7 days in plasma at $22^{\circ} \mathrm{C}$ or $4^{\circ} \mathrm{C}$ with a horizontal agitation. In vitro, platelet effects on HUVEC monolayers was assessed by analyzing transendothelial electrical resistance (TEER), adhesion, adherens junctions assembly revealed by VE-cadherin staining and impedance aggregometry. Cytokines in plasma have been detected by using Milliplex cytokine/chemokine panels. In vivo, platelets were investigated in a vascular permeability leakage model in NSG mice, and platelet circulation was measured by flow cytometry.

Results: Treatment of HUVEC monolayers with 5-day stored $22^{\circ} \mathrm{C}$ and $4{ }^{\circ} \mathrm{C}$ platelets resulted in a similar enhance resistance via TEER, while at 7 days of storage, some donors of $4^{\circ} \mathrm{C}$ stored platelets showed increased barrier function over $22^{\circ} \mathrm{C}$ platelets. In response to VEGF-A, $4^{\circ} \mathrm{C}$ platelets demonstrated stronger inhibition of endothelia permeability over their $22^{\circ} \mathrm{C}$ counterparts. Cytokine measurements revealed a significant decline in pro-inflammatory mediators in plasma during cold storage. $4^{\circ} \mathrm{C}$ platelets demonstrated diminished non-specific platelet-endothelial adhesion and loss of aggregation over time to relevant agonists: ADP (adenosine diphosphate), collagen, TRAP-6 (thrombin receptor activated peptide-6), and ASPI (arachidonic acid). In mice, while $22^{\circ} \mathrm{C}$ and $4^{\circ} \mathrm{C}$ platelets both revealed protection against VEGF-challenged vascular leak, $22^{\circ} \mathrm{C}$ platelets demonstrated stronger protection after 5 and 7 days of storage compared to $4^{\circ} \mathrm{C}$ platelets. Systemic circulating levels of $4^{\circ} \mathrm{C}$ platelets were diminished compared to $22^{\circ} \mathrm{C}$ platelets.

Summary/Conclusions: Treatment of endothelial cells with platelets results in increased endothelial barrier stability. In vitro, $4^{\circ} \mathrm{C}$-stored platelets exhibit greater capacity to maintain vascular stability than $22^{\circ} \mathrm{C}$-stored platelets. In vivo, $22^{\circ} \mathrm{C}$ platelets provide stronger control of VEGF-induced vascular leak. This discrepancy could possibly be due earlier clearance of $4^{\circ} \mathrm{C}$ platelets from the systemic circulation.

\section{Basic Science in Transfusion Medicine}

\author{
1D-S05-01 \\ A MURINE MODEL OF FOETAL/NEONATAL ALLOIMMUNE \\ THROMBOCYTOPENIA CAUSED BY CD36 ANTIBODIES \\ $\mathrm{X} \mathrm{Xu}{ }^{1}, \mathrm{X} \mathrm{Ye}^{2}, \mathrm{~W} \mathrm{Xia}{ }^{3}$, D Chen ${ }^{3}$ and S Santoso \\ ${ }^{1}$ Guangzhou Blood Center, Guangzhou, China ${ }^{2}$ Guangzhou Blood Centre,Institute of \\ Blood Transfusion, Guangzhou, China ${ }^{3}$ Guangzhou Blood Centre, Institute of Blood \\ Transfusion, Guangzhou, China ${ }^{4}$ Institute for Clinical Immunology and Transfusion \\ Medicine, Giessen, Germany
}

Background: Foetal/neonatal alloimmune thrombocytopenia (FNAIT) is bleeding disorder of foetus/neonates caused by maternal antibodies which recognize paternalderived antigen on foetal platelets leading to platelet destruction. The majority of FNAIT cases in Caucasians are caused by anti-HPA-1a antibodies reacted with the B3 integrin subunit (CD 61). Recent data indicate that anti-Nak ${ }^{\mathrm{a}}$ isoantibodies against CD36 seem to be the most frequent antibodies responsible for FNAIT in Asian populations. However, little is known about the pathomechanism of anti-CD36 mediated FNAIT. Whereas CD36 is found on different cells including platelets, erythrocytes, monocytes and endothelial cells, CD61 expression is only found on platelets and endothelial cells.

Aim: In this study, we developed a murine model of FNAIT to investigate the role of maternal anti-CD36 on foetal platelets and bleeding tendency of the foetus.

(C) 2015 The Author Vox Sanguinis (C) 2015 International Society of Blood Transfusion Vox Sanguinis (2015) 109 (Suppl. 2), 1-96 
Methods: CD36-1- deficient female mice were immunized two or three times with $1 \times 10^{8}$ wild-type (WT) platelets at weekly intervals. After immunization, anti-CD36 antibodies in serum of immunized mice were detected by flow cytometry using wild-type platelets as targets. Subsequently, the immunized CD36-/- female mice were bred with WT male mice. During pregnancy and following delivery, miscarriage, platelet counts and bleeding disorders were analyzed.

Results: After breeding with WT mouse, we found that anti-CD36 antibodies can only be measured in some CD36- - - female mice $(1 / 5)$ in the third and subsequent deliveries. In contrast, immunization CD36-/ - female mice with WT platelets led to anti-CD36 antibodies in the majority of immunized mice (12/12). In the control experiment, CD36- $/+$ heterozygous mice did not produce anti-CD36 antibodies after immunization (0/4). When the immunized female CD36-1- mice were bred with WT male mice, miscarriages, head bleeding and massive skin bleeding in some delivered pups were observed. In addition, the number of delivered pups was lower than control mice (immunized CD36- $/+$ female mice), and the mortality rate was $21.57 \%$ (Table 1). Interestingly, the platelet counts of the pups delivered from immunized CD36-/ - female mice were significantly lower when compared to control mice $\left(335.9 \pm 50.90 \times 10^{9} / 1\right.$ vs $\left.594.8 \pm 64.05 \times 10^{9} / 1 ; P<0.05\right)$. In comparison to antiCD61 mediated thrombocytopenia $\left(132.2 \pm 10.5 \times 10^{9} / 1\right.$ vs $618.3 \pm 42.5 \times 10^{9} / 1$; $\mathrm{P}<.001$ ), the level of the platelet counts in these pups are higher.

Conclusions: In this study, we established an animal model of FNAIT using CD36 knockout mice (CD36-/- mice) and observed that anti-CD36 antibodies can induce severe bleeding and miscarriage although only mild thrombocytopenia was observed in the foetus. This result indicates that other mechanism, besides platelet clearance, may participate on the mechanism of severe bleeding and miscarriage induced by anti-CD36 antibodies.

\begin{tabular}{|c|c|c|c|c|c|c|}
\hline Nr. & Breeding & $\begin{array}{c}\text { Breeding } \\
\text { cages }\end{array}$ & Anti-CD36 & Total pups & Dead pups & Miscarriage: \\
\hline 1 & WT(f) $\times W T(m)$ & 5 & $0 / 5$ & $37(7.40 \%)$ & $1(2.70 \%)$ & 0 \\
\hline 2 & $\operatorname{CD} 36-\mu(f) \times W T(m)$ & 4 & $0 / 4$ & $25(6.25 \%)$ & $1(4.00 \%)$ & 0 \\
\hline 3 & 1.CD36-l+(f) $\times$ WT $(\mathrm{m})$ & 4 & $0 / 4$ & $27(6.75 \%)$ & 0 & 0 \\
\hline 4 & $\mathrm{i}-\mathrm{CD} 36-f(\mathrm{f}) \times \mathrm{WT}(\mathrm{m})$ & 12 & $12 / 12$ & $51(4.25 \%)$ & $11(21.57 \%)^{\prime}$ & 2 \\
\hline
\end{tabular}

Table 1: An alysis of pups delivered by breedingwild-type or $\mathrm{CD} 36$ deficientfernale mice with wildtype male mice in the absence or presence of matem al anti-CD 36 antibodies ") miscariage was determined as signific
weight of mothers during pregnancy. 1) pe0.05 compared with experimental settings 1.2 and 3 .

Caption 1: Bleeding disorders caused by anti-CD36 antibodies.

1D-S05-02

DEGLYCOSYLATED HUMAN MONOCLONAL ANTIBODY AGAINST HPA-1A PREVENTS PLATELET CLEARANCE AND ENDOTHELIAL DYSFUNCTION MEDIATED BY ANTI-HPA-1A ALLOANTIBODIES

H Wihadmadyatami ${ }^{1}$, T Bakchoul ${ }^{2}$, B Bayat ${ }^{3}$, M Burg-Roderfeld $^{3}$, S Werth ${ }^{3}$, C Ruan ${ }^{4}$, W Mingwang ${ }^{5}$, G Bein ${ }^{3}$, UJ Sachs ${ }^{3}$ and S Santoso ${ }^{3}$

${ }^{1}$ Faculty of Veterinary Medicine Universitas Gadjah Mada, Yogyakarta, Indonesia ${ }^{2}$ Institute for Immunology and Transfusion Medicine Ernst Moritz Arndt University, Greifswald, Germany ${ }^{3}$ Institut for Clinical Immunology \& Transfusion Medicine Justus Liebig University, Giessen, Germany ${ }^{4}$ Jiangsu Institut of Hematology, TheFirst Affiliated Hospitalof Soochow University, Suzhou, China ${ }^{5}$ Dongying Lida Pharmaceutical, Suzhou, China

Background: Foetal/neonatal alloimmune thrombocytopenia (FNAIT) is caused by the destruction of fetal platelets by maternal platelet alloantibodies which crossed through the placenta during pregnancy. Alloantibodies (aabs) against Human Platelet Antigen-1a (HPA-1a) residing on the b-subunit of the platelet fibrinogen receptor (aIIbb3 integrin) are responsible for intracranial hemorrhage (ICH) in severe FNAIT cases. Recently, we could demonstrate that the deglycosylated mouse mab SZ21 against HPA-1a is able to cross through placenta, and prevents the destruction of platelets in mouse model (Bakchoul et al, Blood 2013). Meanwhile, this mab is humanized as chimeric antibody (mab 813). It is known that the b3 is abundantly expressed as vitronectin receptor ( $\alpha \mathrm{v} \beta 3$ integrin) on endothelial cells.

Aim: In this study, we sought to investigate the effect of deglycosylated mab 813 (deg-813) towards endothelial function.

Methods: Deg-813 was prepared and characterized as previously described (Bakchoul et al, Blood 2013). Binding of antibody binding to platelets, endothelial cells and purified $\alpha \mathrm{v} \beta 3$ integrin was performed by flow cytometry and surface plasmon

\section{(C) 2015 The Author}

Vox Sanguinis (C) 2015 International Society of Blood Transfusion

Vox Sanguinis (2015) 109 (Suppl. 2), 1-96 resonance technology, respectively. Apoptosis and tube formation assays were conducted to analyze the function of antibody binding on endothelial function. Results: Flow cytometry analysis showed similar binding activity of intact 813 and deg-813 with platelets and endothelial cells. This result could be confirmed by surface plasmon resonance technology; native and deg-813 interacted equally with purified $\alpha \mathrm{v} \beta 3$ immobilized on the sensor chip (Kd $7.4 \times 10^{-11}$ and $6.7 \times 10^{-11}$, respectively). To study the influence of mab 813 on endothelial function, apoptosis and angiogenesis experiment with HUVEC was performed using Caspase-3/7 and tube formation assays. In Caspase-3/7 assay, endothelial apoptosis was not observed with intact 813 and deg-813. In contrast, inhibitory mabs against $\alpha v \beta 3$ integrin (clone 23C6) caused significant apoptosis of these cells. Moreover, in contrast to anti-avb3, both intact and deg-813 did not alter angiogenesis as shown by the tube formation assay. Interestingly, treatment of endothelial cells with deg-813 prevents apoptosis and anti-angiogenesis effect of anti-HPA-1a antibodies. Furthermore, in comparison to native 813 , d-813 was able to inhibit platelet clearance mediated by anti-HPA-1a antibodies in NOD-Scid mouse model.

Conclusions: Taking together, our observations demonstrate that deg-813 can protect not only anti-HPA-1a mediated platelet destruction but also prevent endothelial dysfunction caused by anti-HPA-1a antibodies. These results suggest that deg-813 may represent a novel drug to prevent ICH in severe FNAIT during pregnancy. This approach may also applicable for the treatment of FNAIT caused by other platelet antibody specificities.

\section{D-S05-03 \\ MICRORNA EXPRESSION PROFILES AND TARGET GENE ANALYSIS IDENTIFIED A NOVEL MIRNA REGULATOR OF OK/ BSG \\ LY Ye, SS Han, FY Zhao, QX Yang and ZY Zhu \\ Shanghai Blood Center, Shanghai, China}

Background: Recently, the PfRh5-BSG interaction was proved to be essential for erythrocyte invasion by $P$. falciparum. Furthermore, this interaction was found to be species specific, and emerged as a promising malaria vaccine candidate target. In our previous work, the expression level of OK antigen/BSG protein was found to correlate with the recombinant PfRh5 binding. We also found that post-transcriptional regulation might be important for OK/BSG expression. However, the involved mechanisms still remain unclear.

Aims: We aim to find out microRNA regulators of OK/BSG and validate computationally predicted microRNA target sites.

Methods: The blood samples were collected from Shanghai Blood Center. All the exons of $B S G$ gene, as well as the promoter, $5^{\prime}$-UTR and $3^{\prime}$-UTR were sequenced. The total RNA was extracted and reversely transcripted into cDNA to analyze the relative level of $B S G$ mRNA expression. The expression levels of different domains of BSG protein and $\mathrm{Ok}^{\mathrm{a}}$ antigen were detected by flow cytometry analysis. MicroRNA sequencing and bioinformatics analysis were performed. For the candidate microRNAs, luciferase reporter plasmids were constructed by cloning the BSG $3^{\prime \prime}$ untranslated regions containing respective potential miRNA binding sites into the psiCHECK-2 vector. The negative control constructs were generated by site-directed mutagenesis, containing the mutated bases on the predicted sites. Cell transfection and dual luciferase reporter assay were performed in K562 cells. Cells were transiently transfected with either luciferase reporter plasmids or mutated negative control constructs, and various concentrations of corresponding miRNA mimics or negative controls. The data were obtained from three independent experiments performed in triplicate. Quantitative statistical analyses were carried out using the twotailed t-test.

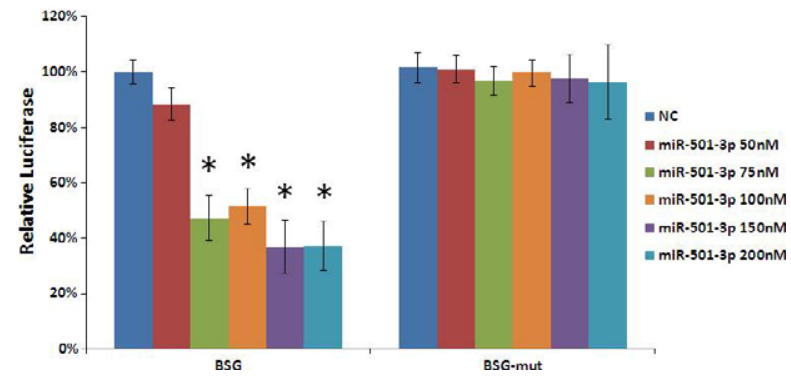

Figure 1: BSG is a target of miR-501-3p in K562 cells. 
Results: A significantly high level of $B S G$ mRNA was found in one sample with lower OK/BSG expression. MicroRNA sequencing was performed in this sample and another one bearing similar BSG polymorphisms but with higher OK/BSG expression and lower BSG mRNA level, used as a control. Among all miRNAs predicted to be the potential miRNAs to target $B S G$, miR-15b-5p, miR-99b-5p, and miR-501-3p were up-regulated in the experimental sample compared with the control. The respective dual luciferase reporter and control plasmids were constructed for luciferase assays, confirmed by sequencing. Overexpression of miR-501-3p mimic rather than other mimics significantly suppressed the luciferase activity, and the inhibition was rescued when the binding sites were mutated (Fig. 1). These results suggest that miR-501-3p targets the 3'-UTR of BSG in K562 cells, by directly binding to the predicted target sites.

Conclusions: For the first time, our results suggest that miR-501-3p might be a novel negative regulator of $B S G$ gene, which enriches the knowledge of the regulation of $\mathrm{OK} / \mathrm{BSG}$

1D-S05-04

ANTIGEN-DECORATED ENGINEERED RED BLOOD CELLS AS A NOVEL IMMUNE TOLERANCE-INDUCING AGENT

N Pishesha ${ }^{1}$, A Bilate ${ }^{2}$, MC Wibowo ${ }^{1}$, HL Ploegh ${ }^{1}$ and HF Lodish ${ }^{1}$

${ }^{1}$ Massachusetts Institute of Technology/Whitehead Institute for Biomedical Researc, Cambridge, United States of America ${ }^{2}$ Whitehead Institute for Biomedical Research, Cambridge, United States of America

Currently available therapies for autoimmune diseases are traditional immunosuppressive medications, exposing patients to higher risks of opportunistic infection, cancer, and other discomforts. Therefore, immunoregulatory modalities, which are able to prophylactically educate or therapeutically re-educate the immune system to induce antigen-specific tolerance are desirable. Here we employed engineered red blood cells carrying desired synthetic or natural antigens to induce long term, drugfree, specific immunotolerance while maintaining immune competence. Utilizing genetic engineering and Sortase A-mediated protein modification strategies, we generated both mouse and human red blood cells covalently carrying the desired antigens. Covalent conjugation allows precise quantification of antigens attached and sortagging method robustly attaches consistent amount of various antigens. More importantly, these modification strategies do not inflict any damage to the red blood

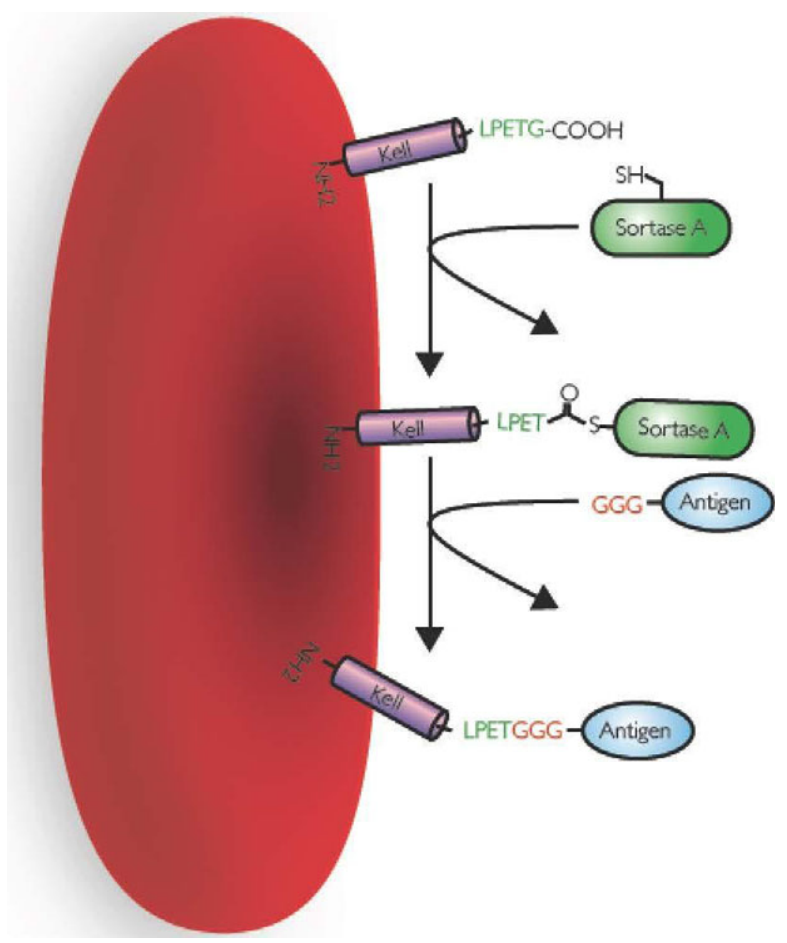

Figure 1: Sortagging of genetically engineered RBCs.

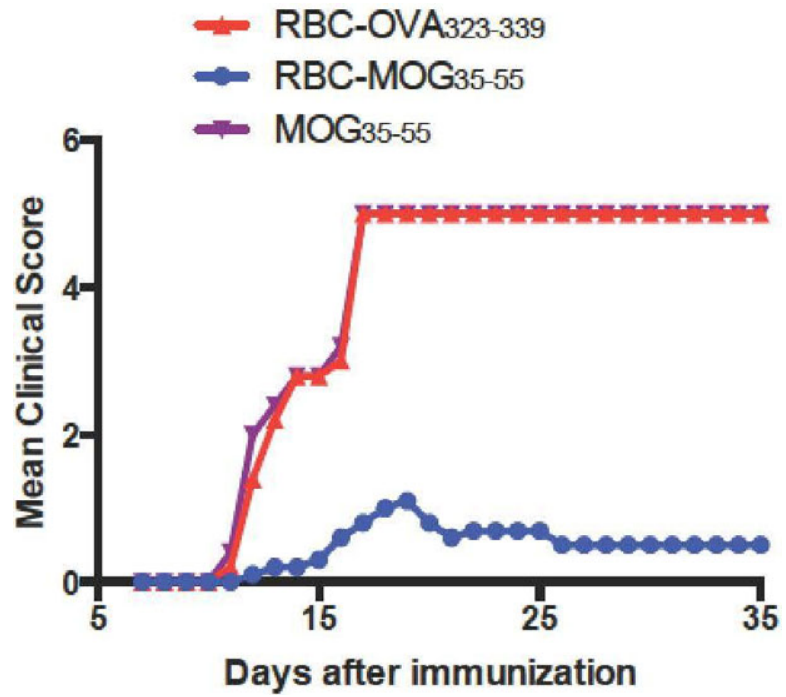

Figure 2: RBCs carrying MOG peptides protect against Experimenal Autoimmune Encephalomyelitis (EAE) - a disease model of multiple sclerosis.

cells as the engineered red blood cells can circulate in vivo with normal half-life. As a proof of concept using the ovalbumin-specific $\mathrm{T}$ cell receptor transgenic mouse models, we found that administration of red blood cells that carry the relevant peptide epitopes leads to induction of tolerance, as inferred from the drastic reduction in number of adoptively transferred CD4 + and CD8 $+\mathrm{T}$ cells. We further illustrate the tolerance-induction capacity of these engineered red blood cells to a model of autoimmune diseases, experimental autoimmune encephalitis (EAE), a mouse model for multiple sclerosis, we showed that myelin oligodendrocyte glycoprotein (MOG)decorated red blood cells could suppress, or at least delay the onset of, the disease. We have therefore highlighted the potential of hijacking the natural properties of red blood cells to curb autoimmune diseases.

\section{D-S05-05}

CRITICAL ROLE OF TGF- $\boldsymbol{\beta} 1$ IN EXPANDING HUMAN NATURAL REGULATORY T CELLS AND STABILIZING NTREGS UNDER INFLAMMATORY CONDITIONS

$\mathrm{J} \mathrm{Sun}^{1}$, BB Zhu ${ }^{2}$, YM Yang ${ }^{1}, \mathrm{~J} \mathrm{Yang}^{1}$, XY Jiang ${ }^{1}$, RF Xie ${ }^{1}, \mathrm{~L} \mathrm{Gao}^{1}$ and HH Fan ${ }^{1}$ Shanghai Blood Center, Shanghai, China ${ }^{2}$ East China Normal Univesity, Shanghai, China

Background: Recent studies have shown that thymus-derived naturally $\mathrm{CD} 4^{+} \mathrm{CD} 25^{+} \mathrm{Foxp}^{+}$regulatory $\mathrm{T}$ cells (nTregs) has an important role in suppressing autoimmune disease, preventing GVHD and maintaining immune tolerance and makes it a potent candidate for cell-therapy. However, it may be unstable and dysfunctional in the presence of pro-inflammatory cytokines (such as TGF- $\beta 1+\operatorname{IL} 1 \beta+$ IL6) and convert into Th1 and Th17 cells, loss Foxp3 expression and functionality in vitro and in vivo. Moreover, converted cells may become pathogenic and promote disease development. So maintenance of nTregs stability under inflammatory condition becomes critical toward sustaining their functionality for cell therapy. Transforming growth factor- $\beta 1$ (TGF- $\beta 1$ ) is a multifunctional cytokine which regulating T-cell growth and development, has been shown to convert peripheral $\mathrm{CD} 4^{+} \mathrm{CD} 25^{+}$ $\mathrm{T}$ cells into $\mathrm{CD}^{+} \mathrm{CD} 25^{+}$induced regulatory cells, suppress $\mathrm{T}$-cell cytotoxic activity and inhibit antibody production.

Aims: In this study, we hypothesize that added TGF- $\beta 1$ to human nTregs expansion culture in vitro stabilizes nTregs under inflammatory conditions.

Methods: Human $\mathrm{CD}^{+} \mathrm{CD} 25^{+}$nTregs were isolated from PBMCs, expanded under anti-CD3/28 beads, IL-2, rapamycin and with or without TGF- $\beta 1$ in vitro for 12 days. Harvest nTregs following encounter pro-inflammation with IL1 $\beta+$ IL6 + TGF- $\beta 1$ for 3 days. The percentage of Foxp3, IL17A and IFN- $\gamma$ and functionalities of expanded and pro-inflammation treated nTregs were investigated.

Results: 1 . Added TGF- $\beta 1$ expanded human nTregs become resistant to inflammatory-driven Th1 and Th17 conversion. Only $1.31 \%$ of TGF- $\beta 1$ expanded nTregs cells

(C) 2015 The Author

Vox Sanguinis (C) 2015 International Society of Blood Transfusion Vox Sanguinis (2015) 109 (Suppl. 2), 1-96 
(a)

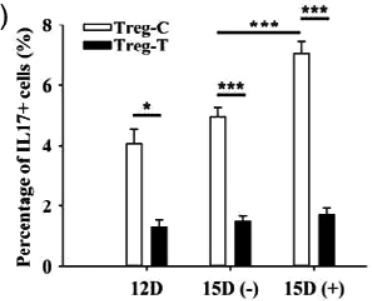

(b)
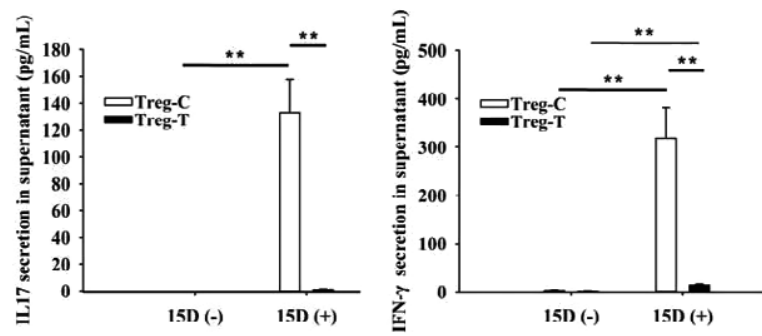

Fig1. IL17A and IFN- $\gamma$ expression in nTregs; A: \% of IL17A and IFN- $\gamma$ on day 12 (12D) or in inflammatory for 3 days (15D); B: secretion level of IL17A and IFN- $\gamma$ under inflammatory stimulation. Treg-C, Treg-T represent control or TGF- $\beta 1$ expanded nTregs; $(+),(-)$ stand for with or without inflammatory stimulation. ${ }^{*} \mathrm{P}<0.05,{ }^{* *} \mathrm{P}<0.01,{ }^{* * *} \mathrm{P}<0.001 ; \mathrm{n}=3$.

Figure 1: Foxp3 and Helios express in nTregs. (a)

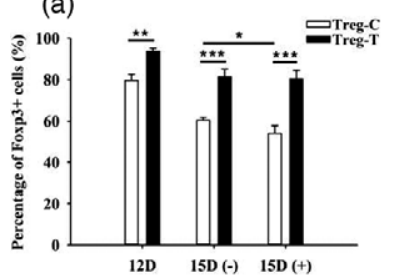

(b)

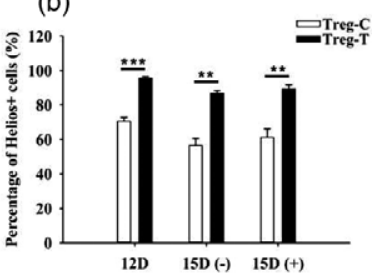

Fig2. Foxp3 and Helios expression in nTregs; A: \% of Foxp3on day 12 (12D) or in inflammatory for 3 days (15D); B: \% of Helios. Treg-C, Treg-T represent control or TGF- $\beta 1$ expanded $n$ Tregs; $(+)$, (-) stand for with or without inflammatory stimulation. * $\mathrm{P}<0.05, * * \mathrm{P}<0.01, * * * \mathrm{P}<0.001 ; n=3$

Figure 2: IL17A and IFN- $\gamma$ express in nTregs.

expressed IL17A, 1.44\% expressed IFN- $\gamma$ whereas the ratios were $4.07 \%$ and $12.42 \%$ respectively in controlled cells (without added TGF- $\beta 1$ expanded). When expanded nTregs encounter pro-inflammation cytokines for 3 days, the percentage of IL17 $\mathrm{A}^{+}$ cells significantly increased in controlled cells, however, TGF- $\beta 1$ expanded nTregs were stabilized. In addition, the secretion level of IL17A and IFN- $\gamma$ in supernatant was significantly increased under inflammatory condition in controlled nTregs but not in TGF- $\beta 1$ expanded nTregs. 2 . TGF- $\beta 1$ expanded nTregs expressed significantly higher Foxp3 and Helios and stabilized in inflammatory condition compared with controlled nTregs. 3. The suppression ability in vitro and anergy of TGF- $\beta 1$ expanded nTregs were stabilized when compared with control nTregs. 4. TGF- $\beta 1$ expanded nTregs express higher level of STAT1, STAT3, and STAT5 and increasing P- STAT1, P-STAT3, and P-STAT5 under stimulation of inflammatory cytokines. It was STAT3 but not STAT5 plays an important role in TGF- $\beta 1$ down-regulation of $\mathrm{IL}_{17 \mathrm{~A}^{+}}$cells conversion. Use STAT3 inhibition S3I-201 in TGF- $\beta 1$ expanded culture significantly increased IL $17 \mathrm{~A}^{+}$cells.

Conclusion: The results revealed that added TGF- $\beta 1$ in nTregs expansion systems in vitro could not only increased the inhibition ability, expression of Foxp3 and Helios but also decreased IL17A and IFN- $\gamma$ express in nTregs. In addition, what's even more important is TGF- $\beta 1$ expanded nTregs stabilized under inflammatory condition. Our researches contribute to maintain the stability of nTregs in vitro but further in vivo studies are needed for cell therapy. And as a component of blood, potential nTreg therapy can promote the development of component blood transfusion and blood utilization.

\section{Plenary Session: Immunohaematology}

\author{
2A-PL2-01 \\ WHY BLOOD GROUP O PROTECTS YOU AGAINST MALARIA \\ ML Olsson \\ Lund University, Lund, Sweden
}

It has been known for a long time, both from in vitro experiments and from clinical trials assessing malaria-caused mortality or morbidity, that individuals whose red blood cells (RBCs) lack A and B antigens do better when infected with Plasmodium falciparum. This is particularly evident in children with the most fatal form of the disease, cerebral malaria. Whilst other blood group molecules regulate entry of the parasite into RBCs by acting as involuntary receptors, $\mathrm{ABO}$ does not alter the degree of invasion, intracellular proliferation or egress. Cerebral malaria is largely due to rosetting, i.e. adhesion of malaria-infected RBCs (iRBCs) to other uninfected RBCs or to endothelial cells. Interestingly, the $\mathrm{ABO}$ blood groups were identified early as involved in rosetting (Carlson \& Wahlgren, J.Exp.Med. 1992;176:1311-7) but until now, rosetting has been attributed mainly to expression of $P$. falciparum erythrocyte membrane protein-1 (pfEMP1) on iRBCs. However, antibodies against pfEMP1 only cause disruption of rosettes with RBCs of group 0, not A. Furthermore, most strains including fresh clinical isolates prefer group A RBCs to form rosettes.

Based on the above, it was hypothesized that there is another parasite-encoded protein that binds A epitopes to cause rosetting. In a recently published study (Goel et al., Nat.Med. 2015;21:314-7 + a 15-page supplement), Prof. Wahlgren's group from Stockholm, Sweden, collaborated with other investigators including our group in Lund, Sweden, to elucidate the structure and function of the repetitive interspersed families of polypeptides (RIFINs) encoded by 150 rif genes in P. falciparum and divided into A-RIFINs and B-RIFINs. RIFINs are the largest family of antigenically variable molecules in P. falciparum but their function has remained unknown. Enzymatic removal of pfEMP1 on the iRBC surface reduced rosetting with group 0 RBCs but not group A. This strongly indicated that pfEMP1 is not the only rosettemediating molecule. RIFINs were found to be expressed on iRBCs and consequently candidates for mediating rosetting. In addition, RIFINs are resistant to enzymatic treatment. By using selected RBCs with common $\left(A_{1}, A_{2}, 0\right)$ and rare $\left(A_{\text {weak }}\right) A B O$ phenotypes or RBCs treated with bacterially-derived exoglycosidases clipping off the $\mathrm{A}$ / B-specific monosaccharides, it was proven that A-RIFINs bind A antigen to form large rosettes. Fewer A antigens on RBCs lead to smaller rosettes. Some remaining RIFIN-dependent rosetting was noted despite absence of A antigen. The target for RIFINs on group 0 RBCs was defined as sialic acid on glycophorin A (GPA) since rosetting could be inhibited by soluble sialic acid, by neuraminidase or trypsin (but not alpha-chymotrypsin) treatment of RBCs, and because En(a-) RBCs lacking GPA showed little rosetting compared to RBCs lacking GPB or GPC.

This study demonstrates that RIFINs are expressed on iRBCs and bind to uninfected RBCs, preferentially of group A, to form large rosettes and mediate adhesion to the microvasculature (shown in vivo in rats). We therefore suggest that RIFINs play an important role in the pathogenesis of severe malaria. Their preference for A antigen is likely to have contributed to the varying distribution of $\mathrm{ABO}$ groups among human populations globally.

\section{A-PL2-02}

RARE BLOOD TYPES IN THE ASIA PACIFIC REGION

\section{Y Tani}

Japanese Red Cross Kinki Block Blood Center, Ibaraki, Japan

Rare blood types are generally defined as those that occur at a frequency of 1:1,000 or less. However, some rare blood types have much different frequencies in global regions or specific ethnic groups. Here I present a categorization of rare blood types from the following points of view.

1. Rare blood types that lack high frequency antigens and how to find them in Japan: Most countries in the Asia Pacific Region screen for rare blood types using serological methods. The Japanese Red Cross (JRC) has established many kinds of monoclonal antibodies, with which numerous rare blood types can be identified. JRC has used them to find many rare donors with $\operatorname{Jr}(\mathrm{a}-)$, Di(b-), D-, Ko, McLeod and Lan- blood types among others.

2. Rare blood types that express low frequency antigens: The incidence of the low frequency antigen MiIII (Mur) is $<0.1 \%$ in most populations but has an incidence of $10 \%$ in Thai, $6 \%$ in Chinese, and 7\% in Taiwanese (with 88\% representation in the 
Ami Tribe). The incidence of $\mathrm{St}^{\mathrm{a}}$ is also $<0.1 \%$ in Caucasians but has an incidence of $6.3 \%$ in Japanese and $1.6 \%$ in Chinese.

3. Rare blood types with higher frequencies in the Asia Pacific Region: The frequency of $\mathrm{Di}(\mathrm{a}+)$ is $<0.01 \%$ in most global populations, but is $2-54 \%$ in South American Indians, 12\% in Japanese, 11\% in Chippewa Indians (Canada), and 5\% in Chinese. The incidence of Bombay is 1:1,000,000 in Europe, 1:100,000 in India, and $1: 10,000$ in Reunion Island. The frequency of Jk(a-b-) is $0.9-1.4 \%$ in Pacific Island populations but $<0.1 \%$ in Caucasians. Furthermore, $10-50 \%$ of Melanesian populations are Ge- (Yus type).

4. Rare blood types with lower frequencies in the Asia Pacific Region: Although $\mathrm{RhD}$ - is not typically regarded as a rare blood type, $\mathrm{RhD}$ - populations are very few (0.58\% in Japanese, $0.28 \%$ in Chinese, $0.33 \%$ in Taiwanese, and $0.23 \%$ in Thai) compared with $16.9 \%$ in Caucasians, and $8.4 \%$ in Blacks. The frequency of Fy(a-) is 34\% in Caucasians, and 22\% in Blacks but only $0.3 \%$ in Chinese, $0.9 \%$ in Japanese, and $3 \%$ in Thai. Fy (a-b-) is very rare but not in Central Africa because of a selection pressure resulting from widespread Plasmodium vivax.

5. Rare blood types that are difficult to find in the Asia Pacific Region and rare blood supplied from JRC to other Asia Pacific countries: Rhnull, En(a-), U-, Ge- and Emm- are very difficult to find. JRC has supplied units of Di(b-), Ko, D-, McLeod, p, Lan-, and Para-Bombay blood to other Asia Pacific countries.

\section{A-PL2-03}

\section{THE EVOLVING ROLE OF PLATELET ANTIGENS AND}

\section{ANTIBODIES}

\section{S Santoso}

Clinical Immunology and Transfusion Medicine, Giessen, Germany

Human platelet membrane glycoproteins (GPs) play a major part in platelet adhesion and aggregation; the key events in the thrombosis and hemostasis process. Some of these GPs have been found to be encoded by two or more allelic isoforms that differ by a single nucleotide polymorphism (SNP). This nucleotide exchange results in amino acid substitutions leading to conformational changes in the three-dimensional structure of the platelet GP, and form thereby new alloantigenic determinants which is recognizable by our immune system.

Immunization against human platelet antigen (HPA) can lead to the production of platelet alloantibodies responsible for the pathomechanism of immune mediated bleeding disorders including platelet transfusion refractoriness (PTR), post transfusion purpura (PTP) and fetal neonatal alloimmune thrombocytopenia (FNAIT).

In the last two decades, genotyping methods have been developed to determine the risk of immunization. However, the frequencies of these SNPs differ among different populations suggesting different clinical impact of HPAs between Caucasian and Asian populations. Furthermore, the identification of platelet antibodies as the cause for thrombocytopenia is mandatory for the accurate diagnosis and treatments of immune mediated thrombocytopenia. Several methods for platelet antibody detection are available. However, all these techniques have some limitations for the detection of certain HPAs which need urgently to be improved in the near future.

Several studies in the past indicated that the amount of platelet alloantibodies did not always correlate with the severity of bleedings; a phenomenon which complicates the clinicians to define good treatment strategy. Recently, several evidences indicated that the nature of antibodies (e.g. glycosylation) and antigens (e.g. heterogeneity of antigenic determinant, expression on other blood cells or endothelial cells) may also play important role on the pathomechanism of alloimmune mediated bleeding disorders.

In this presentation, the clinical important of different HPAs among Caucasian and Asian population will be underlined. In this context, the current problems and challenge of antigen/antibody detection will be discussed. Scientific results gained from recent in vitro as well as from in vivo studies will be presented to illustrate the possible translation from bench-to-bedside in the future.

\section{Academy Session: The What and How of Donors and Donation}

\author{
2B-S06-01 \\ PLANNING DONOR RECRUITMENT STRATEGIES WITH AN EYE \\ ON THE FUTURE \\ M Satake
}

Japanese Red Cross, Tokyo, Japan

National health authorities should have the ultimate responsibility for blood programs that encompass the entire process from blood collection to delivery. This has to be enshrined in laws governing blood in all countries and include a description of the responsibility of each relevant party, namely central or local government, blood facilities that execute blood collection, production, testing, and delivery and medical facilities. Two major policies underlying the law are voluntary non-remunerated blood donation (VNRBD) and self-sufficiency. Obviously, donated blood constitutes an essential element of medical materials/devices that are indispensable to modern medicine. Blood donation could thus be considered as an activity in which citizens should individually contribute to constructing the medical system. This is a simple and principled idea beyond service, goodwill or kindness. In this regard, for any country to foster the growth of future generations who will be actively engaged in blood donation is very important. The introduction of an educational program about the significance of blood donation into school curricula is one strategy with which to accomplish this.

In developing countries where the blood supply remains insufficient, the optimal size of the blood business, namely, the amount of needed blood, has not yet apparently been established. Considering the issue of 'blood management' that has been intensely debated and is leading to a shrinking blood business in advanced countries, defining the scale of blood programs in developing is essential. To achieve this, blood facilities should have a good relationship with medical facilities that practice blood transfusion. Through such collaboration, blood facilities could promote good transfusion practice and avoid unnecessary transfusion, which will help blood facilities estimate the annual optimal volume of blood to be collected and delivered. This would serve as a basis for constructing a concrete plan for establishing a blood program in less developed countries.

The declining birthrate and an increasing elderly population is an important issue in most developed and in some developing countries. Planning for annual blood collection and delivery requires prudent consideration of changing demographics. A declining birthrate means that fewer people will be eligible for future blood donation. The importance of educating young people about blood and blood programs is also stressed in this regard. To raise awareness about the importance of blood donation among general populations only through staff at blood facilities is difficult and insufficient. Recruiting, and then educating and training college students or volunteers at blood centers as local leaders before dispatching them to areas of interest might be very helpful to explore novel donor populations.

Finally, establishing repeat donor registries is essential to enable stable blood collection. Repeat donors are the greatest donor population in terms of stability, safety and donation frequency. Every effort should be focused on acquiring young repeat donors using IT communication methods.

\section{B-S06-02}

\section{CHOOSING THE RIGHT CRITERIA FOR DONOR HEALTH AND}

\section{SAFETY}

N Ishak

National Blood Centre Kuala Lumpur, Kuala Lumpur, Malaysia

Blood transfusion services (BTS) have the responsibility to collect and provide safe blood, and also to deliver sufficient quantity to their health systems. Among the key elements to ensure the safety and adequacy of the blood supply are the collection of blood from voluntary non-remunerated blood donors and availability of qualityassured testing for transfusion-transmissible infections on all donated blood. Each country BTS should establish a national system for blood donor selection criteria for all types of donations and a rigorous process to assess the suitability of prospective donors.

Blood donors are healthy volunteers who give either whole blood or blood components by apheresis for altruistic motives. Blood donation is a rather simple proce-

(C) 2015 The Author Vox Sanguinis (c) 2015 International Society of Blood Transfusion Vox Sanguinis (2015) 109 (Suppl. 2), 1-96 
dure, and most people donate blood because they want to help others. However choosing the right criteria for blood donors' health and safety are extremely important issues that could carry impact to both donors and blood donation

Criteria for blood donor selection includes

1. General factors involving age, donor appearance and inspection, weight, vital sign, donor iron status, fluid intake and food, gender, occupation and leisure activities. Special considerations for apheresis donor selection should also be considered.

2. Detailed medical and surgical history.

3. Detailed history related to transfusion transmitted disease.

4. Assessment of the suitability of individuals to donate.

Though blood donation is in general considered to be safe, it does carry some risk and adverse reactions that can occur during and after blood donation. Therefore, safe blood donation in the perspectives of donor safety and satisfactory experience is the two important targets to achieve.

Strategies for achievement of donor safety and satisfactory experience includes

1. Strengthening the public education programs for blood safety with effective public information and donor education, targeting blood donation from low-risk blood donors and employing rigorous blood donor selection process especially to those who belonged to the lapsed donor's category.

2. A donor questionnaire that able to elicit a prospective donor's demographic, medical and risk history should be used as the key tool in donor selection for assessing donor health and safety and for reducing the risk of transmission of infection.

3. The system of confidential unit exclusion (CUE) offers donors the opportunity to inform the BTS immediately after donation or subsequently if they consider that their blood may be unsafe for transfusion.

4. Donor adverse reactions and monitoring of donor hemovigilance should be managed in a way that ensures high standards of care towards donor as donors who have suffered an adverse reaction have been shown to be less likely to return to donate again.

Careful selection and evaluation of blood donors involved proactive and protective measures in assuring donor health and safety and indirectly towards an effective outcome in blood transfusion service.

\section{B-S06-03}

\section{ADVANCES IN BLOOD STORAGE BAGS AND PRESERVATIVE SOLUTIONS}

WC Tsoi

Hong Kong Red Cross Blood Transfusion Service, Hong Kong, China

Several factors determine the ability to provide quality blood components for clinical transfusion, which include the methods how the components are prepared, the storage conditions and donor related characteristics. The properties of the plastic material, of which blood storage containers are made, as well as their dimensions and the composition of the preservative solutions, exert influence on the quality parameters of the stored blood components and the development of blood cell storage lesions.

The ISO 3826 series of standards set out the physical and functional requirements for plastic collapsible containers for human blood and blood components. The essences of an ideal blood bag plastic are flexibility and deformability, resistance to extremes of temperature for heat sterilization and freeze storage, strength and sturdiness, safety and compatibility and manufacturing consideration such as allowing radiofrequency sealing, etc. Polyvinyl chloride (PVC) plasticized with di-ethylhexyl phthalate (DEHP) has been used as an ideal blood bag material for decades in virtue of possessing these properties as well as its red cell membrane stabilizing effect with lower hemolysis rate upon storage and better red cell recovery after transfusion. There are current scientific and public concerns about potential toxicities of DEHP in particular for male newborns exposed to high concentrations of the material. Newer plasticizers, such as n-butyryl-tri(n-hexyl)-citrate (BTHC)-PVC, have been researched into as potential alternatives to DEHP-PVC. Platelets are metabolically very active blood components, which consume much oxygen and produce considerable amount of lactic acid and carbon dioxide. Blood bag plastics with optimal gaseous permeability properties are of paramount importance in maintaining platelet viability upon storage through facilitating oxygen supply and carbon dioxide removal. Platelet containers made of newer materials with improved gaseous permeability, such as tris-octyl tri-mellitate (TOTM)-PVC, di-isononyl cyclohexane-1,2-dicarboxylic acid (DINCH)-PVC, BTHC-PVC and polyolefin, have been available in the commercial market for preparation of platelet concentrates for clinical application. Additive solutions for preserving red cells, such as SAG-M, AS-1, AS-5 and PAGGS$\mathrm{M}$, contain adequate dextrose and adenine which allow 6-week storage. All these licensed RBC additive solutions have an acidic pH (5.6-5.8). Moreover, when stored in these solutions, the buffering capacities are usually exhausted in about 4 weeks

\section{(C) 2015 The Author}

Vox Sanguinis (C) 2015 International Society of Blood Transfusion

Vox Sanguinis (2015) 109 (Suppl. 2), 1-96 due to accumulation of lactic acid generated from the Embden-Meyerhof pathway. The acidic intracellular environment interferes with the formation of ATP and 2,3DPG, which are essential for red cell survival. An alkaline, chloride-free, hypotonic additive solution (AS-7) has been marketed recently with promising in vitro quality parameter and in vivo recovery outcomes. The extended buffering range of AS-7 is achieved through higher initial intracellular $\mathrm{pH}$ based on the 'chloride-shift' mechanism.

Platelet Additive Solutions (PAS) were developed for preserving platelet concentrates with the purposes of directing more source plasma to fractionation into plasma products and minimizing the frequency of adverse reactions associated with transfusion of plasma contained in platelet concentrates. The current generation of PAS generally contains acetate, citrate, phosphate, potassium and magnesium as their effective compositions. Novel PAS may also contain glucose, calcium and bicarbonate for further reduction in residual plasma content.

\section{Advances in Immunohaematology}

2B-S07-01

\section{RED BLOOD CELL GENOTYPING IN CHINA}

YL Ji ${ }^{1}$, B Veldhuisen ${ }^{2}$, JZ Wen ${ }^{1}$, L Haer-Wigman ${ }^{2}$, TC Stegmann ${ }^{2}$, Z Wang ${ }^{1}$, M Lodén $^{3}, \mathrm{~L} \mathrm{Wei}^{1}, \mathrm{H} \mathrm{Luo}^{1}$, CY Mo ${ }^{1}$, GP Luo ${ }^{1}$, CE van der Schoot ${ }^{2}$ and YS Fu ${ }^{1}$ ${ }^{1}$ Guangzhou Blood Center, Guangzhou, China ${ }^{2}$ Sanquin Research, Amsterdam, The Netherlands ${ }^{3}$ MRC-Holland b.v, Amsterdam, The Netherlands

Background: Since the accuracy of a blood group genotyping assay is not only determined by the causative SNP, but also on the DNA-sequences encompassing this SNP, a genotyping platform has to be validated in different ethnic groups. Furthermore, the presence of variant alleles interfering with correct interpretation can greatly differ between populations. So far, high-throughput genotyping platforms for RBCs antigens have been tested in Caucasian and Blacks, but not yet in Chinese Previously, we developed a comprehensive genotyping assay based on multiplex ligation-dependent probe amplification (MLPA) technology, which can determine 41 blood group and variant alleles of 17 blood group systems, and 79 RHD and 17 RHCE variant alleles of Rh blood system qualitatively and quantitatively (Haer-Wigman L, Transfusion 2013).

Aims: To determine the accuracy of the MLPA assay in Chinese South Han donors. Methods: The MLPA assay was performed on 200 random selected donors. Among them, 91 donors were also serologically typed for 14 blood group antigens. Besides, 63 donors with D variants phenotype, and $200 \mathrm{D}$ - donors were tested in the $R H D$ RHCE MLPA. For donors with aberrant quantitative MLPA results, or with discrepant serology and genotyping results, the corresponding genes (DI, GYPB, LU, KLF1, RHD, or RHCE) were sequenced.

Results: The MLPA analysis of 17 blood group systems in 200 donors revealed that the MNS, Duffy, Kidd, Diego, Yt, Lewis and Dombrock, were polymorphic, and serological data were consistent with genotyping results except for one donor with Lu(ab-) phenotype due to a novel KLF1 gene mutation (Wang Z. Transfusion 2013). An increased frequency of the GYPA and GYPB hybrid alleles encoding Mur (9\%) and $\mathrm{St}^{\mathrm{a}}(2 \%)$ antigens was found. Besides, one donor with chimerism, and one carrying a novel $D I^{*} 02(A 845 V)$ allele, with decreased expression of $\mathrm{Di}^{\mathrm{b}}$, were identified. For the RHD-MLPA analysis in D variant donors, four novel RHD alleles, RHD*01(L27del), $R H D^{*} 01(S 230 N), R H D^{*} 01(A 237 V)$, and $R H D^{*} 01(A 244 P)$, and most common $R H D^{*} D V I .3$ and $R H D^{*}$ weak partial 15 alleles in Chinese population, and less common $R H D^{*} D V I .4, R H D^{*} D L O, R H D^{*} D V .5$, RHD*weak D type 72 , RHD*weak D type 25 , and $R H D^{*}$ weak $D$ type 33 alleles, described before, were identified, either alone or in combination. In 200 D- donors, six known RHD alleles, RHD*DEL1, RHD*01N.04, RHD*01N.16, RHD*DFR2, RHD-CE(3-7)-D, RHD*weak partial 15, and one novel $R H D^{*} 01(G 385 V)$ alleles, were identified in 36.5\% (73/200) D- donors. In $200 \mathrm{D}+$ donors, $D D(168 / 200,84 \%), D d(12 / 200,6 \%), D D D$ genotype $(1 / 200)$, and $R H D$ variant allele carriers $(19 / 200,9.5 \%)$, were found. For $R H C E$ genotyping, a novel $R H C E^{*} c e(P 103 L)$ variant allele were identified in D- donors (6/200). Expression study in vitro showed that the mutant $R H C E^{*} C$ result in the variant $C$ antigen expression. Conclusions: The Blood-MLPA assay is validated to correctly identify the common blood group in the Chinese population. However, the probes specific for the relatively high frequency of variant alleles of the MNS and Rh system in Chinese, such as Sta, RHD*weak D type 72 , and $R H D^{*}$ weak $D$ type 25 , should be added into the MLPA assay and also included in any high-throughput genotyping assay aimed for Chinese population in the future. 
2B-S07-02

\section{THE RELEVANCE OF PLATELET ANTIGEN/ANTIBODIES IN MULTI ETHNIC IN MALAYSIA}

MI Armawai ${ }^{1}$ and S Santoso ${ }^{2}$

${ }^{1}$ National Blood Center of Kuala Lumpur, Kuala Lumpur, Malaysia ${ }^{2}$ Institute for Clinical Immunology and Transfusion Medicine, Justus Liebig Univer, Giessen, Germany

Background: Immunization against polymorphic structure on Human platelet antigens (HPA) have been implicated in the pathogenesis of fetal/neonatal alloimmune thrombocytopenia (FNAIT), post-transfusion purpura (PTP) and platelet transfusion refractoriness (PTR). Malaysia is multi-ethic country; the majority of the inhabitants are Malays (54.4\%), Chinese (25.0\%) and Indians (7.5\%). Recently, the distribution of HPA gene frequencies and has been reported in Malaysia (Tan et al, 2012). However, little is currently known about the relevance of platelet alloantibodies in our heterogeneous population.

Aim: In this study, the frequency of platelet reactive alloantibodies in patients with suspected FNAIT and PTR should be analyzed to estimate their clinical relevance. Methods: Serum samples were analysed by antigen capture assay, MAIPA (Monoclonal Antibody Immobilized Platelet Antigen (MAIPA) using a panel of HPA genotyped platelets derived from blood group donors. Five monoclonal antibodies (mabs) against GPIIb/IIIa, Ib/IX, Ia/IIa, CD109 and HLA Class I were used as capture antibodies. Incompatibility on HPA systems was verified by genotyping of maternal/paternal or donor/recipient using comnmercial kits (GTI and BAGene).

Results: In our heterogeneous patient's cohort, we detected anti-HLA Class I as the major antibody specificity anticipated in FNAIT (20.7\%) and PTR (31.5\%) cases. Screening of anti-HPA antibodies indicated that anti-HPA-3a (20.5\%) represents the most frequent platelet alloantibody in all three ethnic groups (Malay, Chinese and Indians). Importantly, neonates in FNAIT cases associated with anti-HPA-3a alloantibodies presented severe thrombocytopenia; HPA compatible platelet transfusion(s) was necessary to manage the bleeding in these babies. Although, HPA-1a negative individual is extremely rare in our population, when compared to Caucasians, we found one FNAIT case caused by anti-HPA-1a alloantibody. In addition, antiHPA-5b and anti-HPA-15b alloantibodies (36.9\%) were also found in our cohort. However, only mild thrombocytopenia in FNAIT babies associated with these alloantibody specificities.

Summary: Our study indicates that anti-HPA-3a, -HPA-5b and -HPA-15b seem to be the most platelet specific antibodies involved in FNAIT and PTR cases in Malaysian population. Among them, anti-HPA-3a appears to be associated with severe thrombocytopenia. Similar HPA allelic distribution in Malaysian and Indonesian populations have been observed (Asmarinah et al, 2013), Thus, immunization against these three HPA systems are expected to be the most potential risk of alloimmune mediated platelet disorders in Indonesia and probably in other South East Asian Countries. Collective donor register of HPA and HLA typed platelets may improve the diagnosis and treatment of alloimmune mediated thrombocytopenic disorders in our region.

\section{B-S07-03 \\ GLYCOSYLATION OF ALLOANTIBODIES AGAINST BLOOD \\ CELL ANTIGENS}

CE van der Schoot ${ }^{1}$, E Sonneveld ${ }^{1}$, R Kapur ${ }^{1}$, J Koelewijn ${ }^{1}$, M de Haas ${ }^{1}$, M Wuhrer ${ }^{2}$ and G Vidarsson ${ }^{1}$

${ }^{1}$ Sanquin Research, Amsterdam, The Netherlands ${ }^{2}$ Leiden University Medical Centre, Leiden, The Netherlands

Background: IgG-alloantibodies against red blood cell(RBC) and platelet antigens can lead to clearance of the blood cells through $\mathrm{Fc}$-gamma receptor $(\mathrm{Fc} \gamma \mathrm{R})$ - mediated uptake by phagocytes in the foetal spleen and liver. Classically, these antibodies are detected in the case of RBCs by agglutination assays and in the case of platelets via immune fluorescence tests or Monoclonal antibody-specific immobilization of platelet antigens(MAIPA)-assays. The titre of the alloantibody or the level of the antibody in arbitrary units are used to estimate the risk of cytopenia. However, although the antibody level is correlated with the severity of disease, this correlation is not strict, whereas there is an important need for risk assignment. For example, of children of mothers with anti-fetal-platelet IHPA-1a IgG, only 31\% develop fetal neonatal alloimmune thrombocytopenia(FNAIT) and only $2.3 \%$ suffer from severe hemorrhagic disease. For haemolytic disease of the foetus and newborn(HDFN) a functional assay (e.g. the antibody dependent cytotoxicity test (ADCC) can be used to identify cases at high risk of disease. One of the factors determining the functional activity of alloantibodies is the glycosylation pattern of the Fc-tail of the antibodies.
IgG antibodies are glycoproteins containing a variable branched sugar moiety attached to asparagine (Asn)297 on each chain in the Fc-domain. The carbohydrates have a direct influence on the effector functions of the antibodies. Galactose and sialic acid are believed to affect the binding affinity to $F c \gamma R$ in general and might enable binding to DC-SIGN. Notably, the absence of fucose causes markedly enhanced binding to Fc $\gamma$ RIIIa and Fc $\gamma$ RIIIb, increasing the antibody mediated cytotoxicity. Likewise, the addition of bisecting GlcNAc has been shown to increase the antibody-dependent cellular cytotoxicity of recombinant IgG.

Aim: To investigate the glycosylation pattern of alloantibodies and relate this pattern with clinical outcome

Methods: Antigen specific alloantibodies were purified of serum from pregnant women with alloantbodies against HPA-1a, RhD or Rhc, of which the outcome of disease was known (Kapur et al. Br.J.Haematol. 2014 166: 936; Kapur et al Blood 2014;123:471; Koelewijn et al. Transfusion. 2008;48:1721; Koelewijn et al. Transfusion. 2008;48:941). The glycosylation pattern was investigated by Mass Spectrometry as previously described (Kapur, Blood 2014).

Results: Unique skewing in antigen-specific IgG-Fc-glycans after alloimmune responses against the $\mathrm{RhD}$ antigen on red blood cells and the HPA-1a antigen on platelets was found. Whereas $\sim 90 \%$ of IgG-Fc glycan species present in serum are core fucosylated, the fucosylation of the antigen specific IgG-Fc-glycans was lowered to a varying degree, in some patients even down to $\sim 10 \%$. The level of core fucosylation correlated significantly with the $\mathrm{Hb}$-level and platelet count of the new-born, respectively and clinical outcome (Kapur,Blood 2014; Kapur,Br.J.Haematol 2014). A less pronounced decreased fucosylation was found in anti-c antibodies, possibly explaining the lower risk of anaemia in these cases.

Conclusion: Why alloantibodies against blood cell antigens show such unique glycosylation patterns is unknown, and is presently investigated. But also without understanding the physiological explanation for this observation, IgG-glycosylation is proving to become an important biomarker in screening for severe cases of HDFN and FNAIT.

\section{B-S07-04}

\section{ADSORPTION OF AUTO- ANTIBODIES IN PATIENTS WITH AIHA HELPS TO DETECT MASKED ALLO- ANTIBODIES TO IMPROVE SAFETY OF BLOOD TRANSFUSION}

\section{A Abd Elrahman}

National Blood Transfusion Services, Cairo, Egyp

Background: Warm type auto-antibodies are directed against patients' own red blood cells that may interfere with and complicate investigations for the detection of RBC allo-antibodies. The patients' phenotype of RBCs can be difficult to be determined, if the patients were recently transfused.

Finding of compatible blood for patients with warm type AIHA may be impossible as the auto- antibodies in the patient's serum can react with all red blood cells Complicated adsorption procedures in a reference laboratory allow detection and identification of clinically significant allo- Abs that may be masked by the autoAbs.

Aim: To highlight the importance of implementation of adsorption techniques for detection of co-existing allo-Abs which are present in a high percentage of our Egyptian patients, to improve safety of blood transfusion for those patients.

Methods: The study done on 292 patients with warm type AIHA, presented to the Red Cell Reference Lab at Egyptian NBTC, in the period from January 2012 to December 2014 .

According to the Egyptian national testing strategy; routine lab investigations in the form of screening for red cell Abs and auto-control were done for all these patients and revealed positive auto-control with pan-positive reactivity with different phenotyped RBCs, using Column Agglutination Technique.

The patients were subjected to allo-adsorption; in this procedure adsorption of autoantibodies from the patient's serum is carried out using three samples of allogeneic red cells of varying phenotypes, these phenotyped samples already prepared monthly from selected blood units for this purpose. These samples should be complementary lacking the clinical significant red cell Ags, and are used to adsorb auto-antibodies from the patient's serum at $37^{\circ} \mathrm{C}$ on several phases to ensure complete successful adsorption of auto-antibodies. The adsorbed serum then tested for the presence alloantibodies.

Results: Of the 292 patients; 108(37\%) revealed co-existing allo-antibodies, of which $46.3 \%$ of the antibodies were against the (Rh) blood group antigens, 17.6\% against (Kell) blood group antigens, 13.9\% against (MNSs) antigens, 7.4\% against (Kidd) antigens, 3.7\% against (Duffy) antigens and 11.1\% against other blood group antigens. Of the 292 patients; 184(63\%) revealed only auto-Antibodies without no co-existing allo-antibodies. 
Clear identification of the masked coexisting allo-Abs directed us to transfuse the patients with $\mathrm{Ag} / \mathrm{s}$ free blood units for the relevant identified $\mathrm{Ab} / \mathrm{s}$, and hence we avoided the risk of hemolytic transfusion adverse effect of these allo-Abs

The previous wrong concept of transfusion of the least incompatible blood units to patients with AIHA was changed accordingly.

Summary/Conclusions: In countries with limited recourses; proper management of patients with AIHA requires the implementation of adsorption techniques that can differentiate between auto- $\mathrm{Ct}$ allo-antibodies and allow the identification of co-existing allo-antibodies; however being time consuming.

Genotyping of RBCs, especially for recently transfused patients and patients with AIHA can help in that, so we have to think about implementing this technique; however being expensive.

\section{B-S07-05}

TO STUDY THE EFFICACY OF CROSS-MATCH COMPATIBLE

PLATELETS IN MULTI-TRANSFUSED ALLO-IMMUNIZED HEMATOLOGY-ONCOLOGY PATIENTS REFRACTORY TO PLATELET TRANSFUSION

RR Sharma, A Chavan, P Malhotra, B Saikia and N Marwaha

Postgraduate Institute Medical Education \& Research, Chandigarh, India

Background: Platelet refractoriness, which leads to platelet transfusion failure resulting in morbidity and mortality, long hospital stay and the resultant patient cost, is not routinely investigated. Platelet Refractoriness occurs in about 20\% to $70 \%$ of multi-transfused thrombocytopenic patients as a result of immunological or non-immunological causes.

Aims: This study aimed to determine the efficacy of cross-match compatible platelets in multi-transfused alloimmunized haemato-oncological patients refractory to platelet transfusion.

Materials and method: A case control study in which 149 ABO compatible single donor platelet transfusions given to 38 patients with immune causes of refractoriness were studied. Patients were defined to be refractory if their Corrected Count Increment (CCI) was $<5000$ at $1 \mathrm{~h}$ following two consecutive platelet transfusions. A solid-phase red cell adherence method on automated immunohaematology analyzer was used to determine the alloimmunization status and platelet cross-matching was performed on the same platform. Post Transfusion Platelet Increment (PPI), CCI and the Percentage Platelet Recovery (PPR) were used to monitor the effectiveness of each platelet transfusion. ANOVA test followed by Post hoc test Tukey HSD to compare group means for data classified into three groups depending upon the crossmatching and compatibility status. Categorical data analyzed for various outcomes using Pearson's chi square test or Fischer exact test whichever was applicable. Result: Patients showed statistically significant and better platelet recovery in terms of PPI, CCI and PPR at $1 \mathrm{~h}$ post SDAP transfusions when they received crossmatched compatible platelets than on occasions receiving uncross-matched platele units and receiving cross-matched incompatible platelets. The 1-h CCI (mean \pm SD) was significantly higher for cross-match-compatible platelets $(19,173 \pm 2692)$ than for incompatible ones $(5888 \pm 1526)$ and for uncross-matched ones $(8140 \pm 1480)$. Forty four $(97.8 \%)$ of 45 cross-matched compatible platelet transfusion episodes showed a satisfactory response in terms of PPI and CCI values as compared to 50\% and 53.9\% in uncross-matched group respectively, $(\mathrm{P}<0.0001)$.

Conclusion: Platelet cross-matching is an effective intervention in the management of multitransfused alloimmunized Haemato-oncological patients, refractory to platelet transfusion and may be a beneficial intervention in such patients for better outcome of transfusion therapy and should be encouraged.

\section{Plenary Session: Donors, Donations and Components}

\author{
2C-PL3-01 \\ UPDATES ON DONOR RECRUITMENT AND MANAGEMENT IN \\ BLOOD SERVICE \\ CK Lee \\ Hong Kong Red Cross Blood Transfusion Service, Hong Kong, Hong Kong
}

Voluntary Non-Remunerated Blood Donation (VNRD) is the cornerstone of modern blood service and it relies heavily on the community support and participation. Over

(C) 2015 The Author

Vox Sanguinis (C) 2015 International Society of Blood Transfusion

Vox Sanguinis (2015) 109 (Suppl. 2), 1-96 the years most efforts have been made on the adequacy of blood donation and blood safety. Though significant improvement in blood safety profile has been seen and a decreasing blood demand in developed countries is noted, ongoing challenges in safety and adequacy still remain in every country.

Since blood transfusion (and blood donation) will never disappear, a balance in matching right blood supply and managing donors expectation are area that needs serious consideration. Besides, counteracting factors including ageing population donors, population migration, socio-economic factors, increasing stringent donor selection criteria could definitely add on the difficulties in maintaining this balance. At the same time, the traditional donors' mission for donation - 'Give Blood Save Lives' is no longer adequate to reflect their expectations nowadays. Therefore, transfusion services have been working on ways to refresh and revisit the current donor recruitment and management. The wide spread use of social media and 'big data' analysis in blood donation settings are typical examples which have enabled them a totally different approach in reaching and understanding donor behaviours. While those can either be passively originated from the donors or proactively disseminated from the blood service, all do create a dynamic and interactive culture among donors, blood services and the communities. With proper strategies, targeted donors (right age, sex, blood groups or others) are reached, donated blood and returned to subsequent donation at their expectation. As a result, the right balance can hopefully be maintained while donor expectation could be met.

In summary, challenges and possible solutions have been discussed to allow blood service operators to revisit their needs; adapt and revise for their own applications.

\section{C-PL3-02}

\section{BAA BAA BLOOD SHEEP}

YL Fung $^{1}$ and JP Tung ${ }^{2}$

${ }^{1}$ University of the Sunshine Coast, Caloundra, Australia ${ }^{2}$ Australian Red Cross Blood Service, Brisbane, Australia

Background: Animal models have been a valuable tool for research into blood products as well, as the effects of blood transfusions. Small animal models have been applied extensively and large animal model less frequently. This session describes the experience and details the findings from a recent series of in vivo sheep transfusion models.

Aims and method: To discuss the benefits of sheep as a large animal transfusion model and to review conclusions from a series of sheep transfusion models.

Results and discussion: Similarities in size, anatomy and physiology of sheep compared to humans have supported its use to model a wide range of human diseases. The large size of the sheep enables continuous physiological monitoring using standard human techniques. Its size also equates to a higher total blood volume which permits the collection of multiple and serial samples.

In this series the first sheep model involved transfusion of supernatant (SN) from human blood products. These studies confirmed the two-hit TRALI mechanism, as both host factors (lipopolysaccharide modelling bacterial infection) and blood product factors (storage duration of either platelet or packed red blood cell (PRBC) units) contributed to the development of TRALI. The ability to control interventions in an animal model also enabled demonstration that pathophysiological changes were more severe in TRALI induced by stored PRBC-SN than in TRALI induced by stored platelet-SN.

Subsequent sheep transfusion models were more sophisticated with the collection and use of homologous sheep blood products, and pre-transfusion cross-matching. In addition, similarities in the storage lesions of sheep and human PRBC units across 42 days storage, supported the use of these models to investigate the effects of stored PRBC transfusion. In a haemorrhage model, transfusion of stored sheep PRBC affected pulmonary function, even in the absence of overt tissue injury (first hit), highlighting the sensitivity and vulnerability of the pulmonary system to transfusions.

Like human PRBC, sheep PRBC have negligible levels of selenium (trace element involved with antioxidant function). The sheep haemorrhage model demonstrated that the transfusion of sheep PRBC diluted selenium levels and compromised glutathione peroxidase antioxidant activity, which in turn allowed lipid peroxidation. Their large size also enabled development of a sheep model of extracorporeal membrane oxygenation (ECMO). In these studies the insults of lung injury and ECMO reduced selenium levels but transfusion with two units PRBC did not alter the measured oxidative stress markers.

A recent study has delineated the similarities and differences between ovine and human haemostasis. The study revealed the sheep had similar primary haemostasis response, however differences were detected in its secondary haemostasis response. For examples sheep platelets had reduced ADP and collagen response.

Summary: These findings demonstrate the value of large animal models for transfusion research and reveal the sophistication of current sheep transfusion models. Con- 
versely these studies have also identified where sheep differ (e.g. platelet response), and this information is useful in the design of future sheep transfusion models.

\section{C-PL3-03 \\ CELLULAR THERAPY ACROSS ASIA: HARMONISATION AND CHALLANGES \\ M Koh \\ Health Sciences Authority, Singapore, Singapore}

Cell based therapy including the use of stem cells continue to generate considerable interest worldwide as a viable modality of treatment in cancer, immunologically mediated diseases and in regenerative medicine. The heterogenenity of economies, health care systems and regulatory frameworks across Asia present particular challanges and opportunities for the development of such therapies. Valuable lessons can be learnt from the development and progress in blood transfusion, stem cell transplantation and solid organ transplantation across this region. Japan continues to lead the field with its Nobel prize winning discoveries in induced pluripotent cell (iPS) technologies as well as its novel approaches with regulatory frameworks and market approvals for cell based therapy in contrast to standard pharmaceutical medicines. On the other hand, high cost of cell manufacture, requirements for specialised infrastructure and the need for manpower expertise have imposed challenges for other countries. This fast developing field has witnessed a profusion of unproven therapies for a variety of medical conditions; which coupled with the ease of global travel, has paradoxically seen a proliferation of "stem cell and cell therapy treatment hubs", many of which are concentrated in the Asian region. Other than cost and the need for continued emphasis on evidence based medicine, the other most pressing issue is the goal for greater harmonisation of regulatory frameworks and standardisation across the entire continent. Current national and international initiatives directed at these issues will also be discussed. A spectrum of emerging cell therapies and products across the continent will also be discussed

\section{Academy Session: The What and How of Blood Transfusion Safety}

\author{
2D-S08-01 \\ ASSESSING THE RISK OF TRANSFUSION-TRANSMITTED \\ EMERGING INFECTIOUS DISEASES \\ B Custer \\ Blood Systems Research Institute, San Francisco, United States of America
}

Background: Emerging infectious diseases (EID) continue to be a source of significant concern in blood safety, and are expected to result in continued morbidity and potential mortality for transfusion recipients.

Aims: The objectives of this presentation and article are to review EIDs of current concern. The particular issues that make EIDs more challenging for blood safety compared to other areas of public health will be demonstrated through the use of specific examples.

Methods: EIDs of known and theoretical concern in transfusion will be described. The methods used to conduct EID risk assessments will be reviewed, with a focus on the context of transfusion medicine. Dengue, Chikungunya, and Babesia microti will serve as the primary examples to demonstrate the challenges of understanding risk to blood recipients compared to vector- or community-acquired infection.

Results: Current EIDs include mosquito-borne arbovirus infections such as Dengue, Chikungunya, and Zika virus. In addition, tick-borne infections such as Babesia, Borrelia, Anaplasma and Severe Fever with Thrombocytopenia Syndrome Virus (SFTSV) are or have the potential to become significant EIDs in transfusion. Other zoonoses, such as HEV, are expected to emerge or re-emerge. Given the range of pathogens that are threats, understanding the actual risk to blood recipients that each pathogen poses is not straightforward. Often in risk assessment assumptions are made that transfusion-transmitted infection will have the same disease progression course as infection transmitted in other ways. This assumption can lead to inaccurate risk assessments which could substantially over- or under-estimate the impact of an EID in transfusion. A study conducted in Brazil demonstrates that substantial transfusion-transmission of Dengue virus can occur, but evidence of significant clinical outcome differences between recipients of Dengue RNA(+) and RNA(-) transfu- sions was not found. The policy implications are important; because clinical disease from Dengue may be obscured by the underlying reason the patient required transfusion, preventing transfusion-transmitted Dengue may not impact the burden of disease in endemic countries. Chikungunya poses a different set of concerns. Even though there have been explosive mosquito-borne epidemics of Chikungunya virus, no evidence of transfusion-transmission has been documented in the literature. In contrast, while transfusion-transmission of Babesia microti in the USA is a clear threat to recipients, there is no consensus on how to screen for or in which parts of the country donor screening should be implemented.

Summary/Conclusions: The three divergent examples demonstrate the need for risk assessment of the impact of transfusion-transmitted infection. The lesson from the assessment of the risk of transfusion-transmitted Dengue virus is that generalizations from one route of infection acquisition to another and from one pathogen to another may be appropriate for precautionary reasons, but may lead to ineffective and costineffective policy decisions. Each pathogen is unique and so is the route of transfusion-transmission. In order for risk assessment and blood safety policy to be evidence-based, studies of the risk and consequences to blood recipients need to be conducted for each EID.

\section{D-S08-02}

\section{ROLE OF A PERFUSIONIST IN PATIENT BLOOD \\ MANAGEMENT}

SW Tara

National University Hospital, Singapore, Singapore

Perfusionists are essential members of the cardiac surgery team. They provide cardiopulmonary support for patients undergoing various cardiac surgeries with the use of the cardiopulmonary bypass (CPB) machine. This takes over the functions of a patient's native heart and lungs. Blood from the patient is drained (typically from the right atrium) into the СРB circuit where it gets oxygenated and then pumped back to the patient's aorta. It is important that the extracorporeal circulation of the $\mathrm{CPB}$ meets the oxygen demands of the patient. Central in this oxygen demand-supply relationship between the patient and CPB is blood and its components. Cardiac surgery involves many situations that may necessitate transfusion of blood products. $\mathrm{CPB}$ and its circuit components, albeit vital, are one of the major contributors to transfusion. It represents the single largest acute source of exogenous non-hemic volume infused into a patient at the commencement of CPB. Anticoagulation is also fundamental due to the foreign surfaces of CPB. Systemic cooling of the patient on CPB is another contributor of transfusion. At the National University Hospital, Singapore, measures, both in terms of practices and devices, have been put in place in order to minimize transfusion. Switching to albumin prime and maintaining mild hypothermia may help reduce prime- and hypothermia-induced coagulopathy. Additionally, devices such as the hemofilter and cell-saver can aid in concentrating and recycling patient's own shed blood. Despite each having its own limitations, together, they help to minimize patients receiving homologous blood in the course of their surgery.

\section{D-S08-03}

\section{GETTING THE RIGHT UNIT TO THE RIGHT PATIENT - HOW} CAN WE FIX THE PROBLEM

Y Ayob

National Heart Institute, Kajang, Selangor, Malaysia

Blood transfusion is a complex process involving many departments, multiple staff, and several steps. At every stage of the blood transfusion process, error may occur but most incidents of wrong blood transfusion are caused by identification errors at the bedside.

In the laboratory, wrong sample, technical and clerical errors account for less than ward errors but, error in the laboratory is more likely to result in wrong blood transfusion.

Administration of incorrect blood to the wrong patient may result in fatality due to ABO incompatibility. Thus it is crucial that all staff understand the importance of appropriate match of the patient and blood.

Quality system should be implemented in all hospitals that include error management. Tracking transfusion-related events can be part of an overall facility quality improvement program. Identification of errors and analysis can assist the hospital to take appropriate corrective actions, including correction of latent system flaws and develop its own local policy and standard operating procedures (SOPs) in accordance with national guidelines. The Hospital Transfusion Committee plays an important role in enforcing and implementing these standard procedures.

(C) 2015 The Author Vox Sanguinis (C 2015 International Society of Blood Transfusion Vox Sanguinis (2015) 109 (Suppl. 2), 1-96 
Each hospital should make sure that identification procedures are appropriate for the facility and understood by staff. Special procedures may be needed in areas, such as the operating room or the emergency department that pose special challenges. There should be SOPs to ensure accurate labeling at the time of phlebotomy. Labeling of specimens at the bedside, either manually or using an automated system or on-demand labeling is more reliable than preprinted labels. Before administering the blood, identity check between patient and the blood unit at the bedside is important as it is the final step to avoid potentially fatality. This identification must be performed by the person administering the blood. Wristband systems, blood bag locking device, and barcode systems to confirm identity between wristband and blood that include handheld printers and bar code readers are available to assist identification at the bedside.

In case of any ambiguity, a second sample must be requested by to verify blood group or to verify patient identity.

There must be procedures should be in place so that the correct units could be retrieved from the blood fridge in the operating room.

Standard operating procedures must be available at all times in the immediate work area. All staff should be trained in the procedure they perform and be informed of the potential consequences if SOPs are not adhered to. They should be also be trained in identifying and handling transfusion reactions so that appropriate intervention can be instituted. In-service training in hospitals should be regularly done and kept up-to-date.

National haemovigilance programme that includes collection of near misses provide information on the frequency of error in the transfusion process but hospitals play a major role in patient safety relating to transfusion which includes appropriate transfusion and management of adverse events.

\section{New Perspectives in Blood Systems and Organisations}

\author{
2D-S09-01 \\ FIRST STEPS INTO LEAN AT THE AUSTRALIAN RED CROSS \\ BLOOD SERVICE \\ G Wilkie
}

Australian Red Cross Blood Service, Sydney, Australia

Background: The Blood Service collects, processes and tests blood throughout Australia, supplying fresh product to public and private pathology services as well as plasma for fractionation to CSL Behring. There are four main processing centres where blood is tested and processed, approximately eighty static collection sites and thirty mobile collection facilities. Blood products are distributed from ten distribution depots nationally.

Aim: The drivers for introducing lean thinking to the Blood Service were:

1. Pressure on the health dollar and a commitment to providing value for taxpayer funding;

2. sustained growth in demand for plasma for fractionation;

3. significant decline in demand for red blood cells;

4. competitive pressure from cheaper imported fractionated plasma products; and

5. Recognition of process inefficiencies including comparative international bench marking.

Method: In early 2013, the Manufacturing Division of the Blood Service launched a series of kaizen or rapid improvement events. Around thirty front line staff and supervisors were trained in the tools \& techniques and encouraged to address some long-standing improvement opportunities. The aim was to achieve some quick wins in order to build interest and enthusiasm for a broader lean approach. Despite some initial success, it quickly became clear that what was needed was a whole of organisation approach, as manufacturing processes interlinked with so many other areas of the Blood Service.

Results: As part of a planned culture change programme in the Manufacturing Division, the Blood Service invested in a number of dedicated roles to drive lean thinking. Lean awareness programmes were developed and rolled out to all manufacturing employees and this was built into the induction programme for new employees. Visual management, short interval production meetings and gemba walks were introduced and quickly drew attention from other parts of the Blood Service. A real breakthrough came when Lean Leadership programmes were run for the senior teams of all Divisions. This coincided with a refreshed strategic plan which is explicit in embracing continuous improvement, lean processes and customer value. Waste reduction, pull, flow, level production and lean production systems all feature in the new plan as key enablers to the achievement of strategic goals.

\section{(C) 2015 The Author}

Vox Sanguinis (C) 2015 International Society of Blood Transfusion

Vox Sanguinis (2015) 109 (Suppl. 2), 1-96
Conclusion: The Blood Service is already seeing great benefits from the pursuit of a lean continuous improvement culture. Whilst most of the gains have been within the Manufacturing Division to date, momentum is building in other parts of the business, supported by a small central team of business process improvement specialists.

The changes in Manufacturing have resulted in improvements in meeting customer demand; strong improvements in productivity in both processing and testing; a flatter organisation structure; greater levels of collaboration between different departments; and improving employee engagement.

The key to successful implementation of lean thinking is to invest time and effort at the beginning in educating people on the concepts and reassuring them that this is about making processes easier for them and better for customers, rather than cost cutting.

Acknowledgement: Australian governments fund the Australian Red Cross Blood Service for the provision of blood, blood products and services to the Australian community.

\section{D-S09-02 \\ HEMOVIGILANCE AND PATIENT BLOOD MANAGEMENT: A FRESH PERSPECTIVE}

C Bianco

ISBT, Bethesda, United States of America

Background: Efforts to prevent another AIDS tragedy drove the development of approaches to mitigate risk and adverse reactions associated with blood transfusions. Hemovigilance (HV) was created in France and SHOT (Serious Hazards of Transfusion) in the United Kingdom in 1996. Their concepts spread around the world, similar programs appeared in other countries and earlier this year the World Health Organization posted an Aide-Mémoire on national HV systems (www.who.int/bloodsafety/haemovigilance/en/). ISBT contributed to this work through its Hemovigilance Working Party.

Benefits: HV stretched our focus from prevention of transfusion-transmitted diseases (serious but rare events) to other frequent and preventable clinical consequences of transfusion. Successes of HV included the reduction of TRALI events by the preferential use of male plasma and the decline in bacterial contamination by introduction of bags with diversion pouches. Ultimately, clinical trials informed evidence-based guidelines and generated the new vision of Patient Blood Management (PBM), a program focused on evidence-based practice of transfusion.

Concerns: HV is a one-sided assessment of bad outcomes; it does not quantify successes or lives saved. HV and PBM, by their nature, promote reduction of the number of components transfused and consequently, reduction of costs. PBM led to a substantial reduction in blood component collection and transfusion in many high HDI countries including the United States, Canada and European countries, supported by clinical trials showing that patients subjected to restrictive transfusion strategies do as well as patients under liberal transfusion strategies. There are fewer studies of the benefits of transfusion. Trial results have valorized red blood cell transfusions in sickle cell and thalassemia patients, in trauma and surgical oncology, and in reduction of mortality of cardiac surgery patients.

Critique: Tragically, the most common measures of success of PBM are not increases in appropriate transfusions and improved patient outcomes (difficult to measure); they are simply the percentage reduction of transfusions and costs (easy to measure). This allowed a perverse convergence of HV and PBM with the financial interests of health provider organizations. In the United States, cost became the focus and austerity became the rule. HV and PBM mutated into programs aiming at reduction of transfusions, instead of aiming at the increase in appropriate transfusions. We need to restore a balanced vision of evidence-based transfusion therapy in the same way we approach pharmaceuticals, by prescribing transfusions when benefits exceed risks. We need to balance HV and PBM with data about clinical improvements and lives saved because of appropriate use of blood components. Conclusion and Recommendations

Reduction of the number of components transfused and diminished costs are not sufficient to define success of HV and PBM. These programs need data to quantify increases in the proportion of appropriate transfusions and improvement of clinical outcomes in addition to data about adverse reactions, reduction of transfusions and cost savings. Our patients deserve well-indicated transfusions with improved clinical outcomes, not compulsory avoidance of transfusions because of fear of adverse reactions. 
2D-S09-03

GOVERNANCE AND CLINICAL TRANSFUSION

R Benjamin

Cerus Corporation, Concord, United States of America

Background: The concept of clinical governance in medical practice was first introduced in the U.K. in response to local catastrophic failures in a health care system focused on efficiency and cost containment. Lessons learned regarding the central importance of, and management responsibility for, patient outcomes and optimum care can be applied to transfusion medicine in developing countries, as well as in high development countries where blood products are becoming increasingly commoditized.

Aims: To review the elements of clinical governance as it relates to transfusion, highlighting the responsibility of all members in the transfusion chain, up to the highest level of management, to ensure the optimal outcome for patients.

Methods: The concept of clinical governance will be explored in the in the context of changing transfusion medicine environment.

Result: Blood collectors are generally regulated using current good manufacturing principles that ensure product quality and the concepts of organizational governance is well established. In contrast, the process of transfusion is considered in the realm of medical practice. Clinical governance is a system through which organizations are accountable for continuously improving the quality of their patient services and safeguarding high standards of care by creating an environment in which excellence in clinical care will flourish. For transfusion, this incorporates ensuring staff prescribing and dispensing blood products, as well as those performing actual transfusion, have the appropriate education and training, and their performance is audited regularly. Certification in transfusion principles provides one means of ensuring high quality care, but only if continuous education and maintenance of skills is required. The clinical effectiveness of transfusion should be monitored, and research and development focused on ensuring appropriate transfusion indications. Hemovigilance systems focused on adverse outcomes provide one measure that may highlight areas for potential improvement, but only if data are routinely analyzed and interventions are implemented based on the available data. An environment is necessary that fosters the open reporting and discussion of problems and concerns, with formalized corrective action plans leads to continuous clinical quality improvement, supported by risk management principles and information management systems.

Summary: Clinical governance principles should be applied to transfusion medicine in order to ensure optimum patient outcomes in an environment of limited resources, with responsibility firmly ensconced at the highest level of the organization.

\section{D-S09-04}

\section{A SUCCESSFUL AND SUSTAINED MULTI-SITE PLATELET WASTAGE MINIMISATION PROGRAM}

R Tocchetti ${ }^{1}$, R Sinha ${ }^{1}$, S Ireland ${ }^{2}$ and D Roxby ${ }^{3}$

${ }^{1}$ SA Health / SA Pathology, Collinswood, Australia ${ }^{2}$ SA Health, Adelaide, Australia ${ }^{3} S A$ Pathology, Adelaide, Australia

Background: Blood product wastage minimisation is a stewardship obligation for all transfusion laboratories and hospitals and requires conscientious efforts to achieve this goal. Platelet inventory management and wastage minimisation forms part of this stewardship and poses significant challenges due to the limited product shelf life.

In Australia, government health services purchase blood products from the sole Australian blood supplier, the Australian Red Cross Blood Service (ARCBS) which is a non-government organisation.

Aim: For the financial year 2012/13, the South Australian platelet wastage rate was $17 \%$ which equated to an approximate cost of AUD 900,000. The South Australian BloodMove Platelets Project aim was to develop a sustainable city-wide multi-site process to significantly decrease platelet outdating and associated costs.

Method: The Project commenced in August 2014 after initial planning, preparation and education and was followed by a staged implementation, consisting of the: Routine transfer of near expiry platelets from low use hospital sites to high use sites: Establishment of a metropolitan-wide common Day 5 platelet inventory to be used by all sites prior to using available fresher platelets or ordering from the ARCBS

Results: Within the first month of operation, the Project achieved a significant drop in the platelet wastage rate for metropolitan public hospitals from a typical $21.5 \%$ in July 2014 to 10.7\% in August 2014.This decrease has been sustained for 10 months, post Project with an average discard rate of 7.7\% [95\% Confidence Interval (CI) 7.18.3]. The average number of platelets discarded per month post Project is $57 \mathrm{com}-$ pared to 130 prior to the project representing a decrease in discard of $56 \%$. The overall reduction in discarded platelets is shown in the graph.

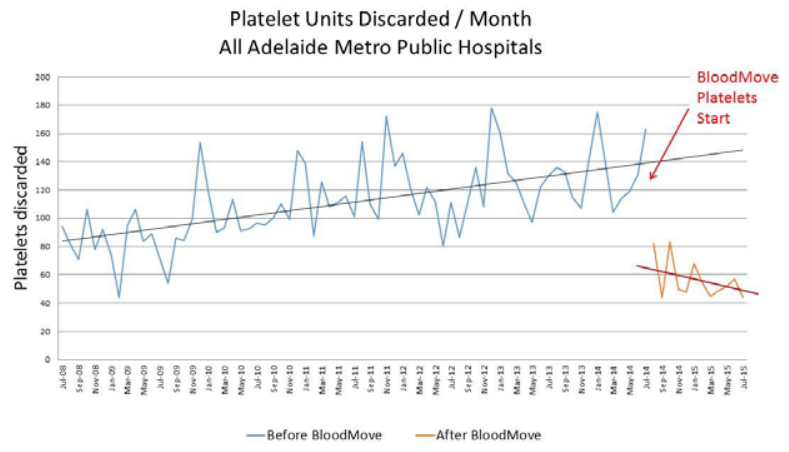

Figure 1: WBDD 2013 Global event in Paris.

Other key performance indicators of the Project were the median number of days with zero discards per month which increased to from pre Project of 3 [Interquartile range (IQR) 1-4] to post Project of 10.5 (IQR) 9-13 and the median number of units discarded per day deceased from 4 (2-7) pre Project to 1 (0-3) post Project.

Summary and conclusions: The transfer of near expiry platelets from low use sites to high use sites proved beneficial. This practice whilst maintaining standby stocks at the low use sites for emergency situations also resulted in wastage rates approaching zero for those sites.

The sourcing of platelets for elective transfusion from the multi-site Day 5 inventory prior to using available fresher platelets or ordering from the ARCBS resulted in significant wastage reduction. This practice was also extended to $\mathrm{ABO}$ cross group issues ie. pooled or low titre apheresis Day 5 group 0 platelets were issued to nongroup 0 patients instead of using fresher ABO specific platelets.

Through detailed planning, education, collaboration and support, the BloodMove Platelets Project has been extremely successful, resulting in significant and sustained reduction of platelet wastage and financial savings across all public metropolitan Adelaide hospitals. Projected ongoing annual saving being approximately AUD 519,000.

\section{Plenary Session: All About Transfusion Transmitted Infectious Diseases}

\section{A-PL4-01}

\section{THE PROBLEM WITH HEPATITIS}

\section{Satake}

Japanese Red Cross, Tokyo, Japan

The transmission of hepatitis C (HCV) and B (HBV) has been a widespread and serious side effect of blood transfusion. Transfusion-transmitted HCV (TT-HCV) infection was mostly eliminated by introducing anti-HCV screening for donated blood during the 1990s. Persistent TT-HCV was attributed to blood collected during the window period before anti-HCV seroconversion. Nucleic acid testing (NAT) for HCV and HIV have been introduced into blood facilities in most developed countries. The doubling time of HCV in peripheral blood is very short, which enables NAT with a pool format to be very efficient. Very little TT-HCV is currently being identified where NAT screening is implemented.

Screening donated blood for hepatitis B surface antigen (HBsAg) started during the 1970s. Some transfusion-transmitted HBV (TT-HBV) infection that occurred despite improvements in the sensitivity and specificity of HBsAg testing were mostly attributed to window-period donations before $\mathrm{HBsAg}$ conversion during the early phase of acute infection. Because the doubling time of HBV in peripheral blood is relatively long at that time, unlike HCV, NAT yield and sensitivity correlate. The efficiency of HBV NAT, particularly with the large sample pool format, has been questioned in countries with a low HBV prevalence, and HBV NAT was initially introduced in countries where HBV prevalence is intermediate-to-high.

A proportion of HBsAg-negative/ $\mathrm{HBCAb}$-positive blood was verified as being infectious during the late 1970s. This status is now defined as occult HBV infection (OBI) being HBsAg-negative and HBV NAT-positive and it is generally represented as being $\mathrm{HBCAb}$-positive. The status of OBI mostly depends on resolved prior acute

(C) 2015 The Author

Vox Sanguinis (C) 2015 International Society of Blood Transfusion Vox Sanguinis (2015) 109 (Suppl. 2), 1-96 
HBV infection and in a small fraction, the late acute phase before anti-HBs conversion and the late stage of HBV carriage losing HBsAg. The extremely low viral load of OBI-derived blood and the very small fraction of infectious OBI pose problems from the perspective of screening. Even individual donation NAT (ID-NAT) could miss infectious OBI blood. The strategy of disqualifying all HBcAb-positive blood would be a final solution to this issue, but a considerable number of blood donors would be lost. This could be serious in areas that have $>10 \% \mathrm{HBcAb}$ positivity rates among donors. In such areas, ID-NAT or pool format NAT with equivalent sensitivity would be a second-best strategy to eliminate most infectious donations although some infectious blood with a viral load below ID-NAT sensitivity might be missed. Debate is beginning to focus on whether or not hepatitis E virus (HEV) screening should be implemented in blood facilities because of TT-HEV infection. Despite an unusually high prevalence of HEV, HEV-hepatitis occurs only occasionally among the general population. However, HEV-related chronic liver damage is not uncommon among immunocompromised patients. These issues complicate decisions about which strategy to be implemented, no screening at all, selectively screening for patients at high risk, or universal screening. Nucleic acid testing for HEV would also further burden blood facilities from a cost viewpoint.

\section{A-PL4-02}

\section{PATHOGEN REDUCTION TECHNOLOGY - IS IT TIME?}

SY Kwon

Seoul Nambu Blood Center, Korean Red Cross, Seoul, South-Korea

With the application of stringent donor selection criteria and sophisticated screening tests, including the introduction of nucleic acid testing (NAT), the risk of the socalled 'traditional' transfusion-transmitted infections (TTI), HBV, HCV and HIV, has dramatically decreased. However, as no system is $100 \%$ safe, breakthrough cases are still being reported. Known pathogens that are not screened for and new emerging ones represent a constant threat. Moreover, bacterial contamination still remains the major infectious risk in blood transfusion. As opposed to our current safety measures which are reactive, the development of pathogen reduction technologies has presented a proactive approach to our blood safety paradigm. Pathogen reduction technology for platelets and plasma has been approved for use in Europe for more than 10 years. Although pathogen reduction efficiency against non-enveloped viruses and spore forming bacteria is less satisfactory, these technologies show a robust pathogen reduction capability for a broad spectrum of pathogens. There are concerns regarding the toxicity of the photochemical agent used to treat the platelets but current data indicate that transfusion reactions after transfusion of treated platelets are comparable to that of non-treated platelets. Another concern is that the treatment might affect platelet function and increase risk of bleeding. Clinical data have shown that recovery and survival of treated platelets are decreased compared to non-treated platelets; therefore an increase in platelet use can be anticipated after the implementation of pathogen reduction technology. According to current data, transfusion of treated platelets does not increase bleeding. As most pathogen reduction technologies target nucleic acids, they are not only effective in reducing infectious risks but also in inactivating leukocytes, thereby preventing non-infectious risks like transfusion-associated graft-versus-host disease (TA-GVHD) and alloimmunization. Despite the multiple risks prevented through pathogen reduction, the cost to implement these technologies remains a major issue, especially in developing countries. On the other hand, pathogen reduction could be an alternative to bacterial screening and gamma irradiation. Furthermore, cessation of some of the current screening items and modification of some donor exclusion criteria might be possible, especially when pathogen reduction technologies for red blood cells or whole blood become available. Therefore, if we consider all correlated efforts involved to make blood transfusion safe, the introduction of the pathogen reduction technology might prove to be beneficial.

\section{A-PL4-03}

THE CURRENT STATUS OF NUCLEIC ACID AMPLIFICATION TECHNOLOGY IN TRANSFUSION TRANSMITTED INFECTIOUS DISEASE TESTING

$\mathrm{M}$ Vermeulen $^{1}$ and $\mathrm{N}$ Lelie $^{2}$

${ }^{1}$ South African National Blood Service, Johannesburg, South Africa ${ }^{2}$ Lelie Research, Paris, France

Fully automated multiplex (MPX) Nucleic Acid Amplification Technology (NAT) enabling individual-donation (ID) or minipool of 6 (MP6) screening for HIV, HCV and HBV has been available for approximately 10 years. These systems are provided by two major commercial players in the NAT industry (Grifols/Hologic and Roche).

\section{(C) 2015 The Author}

Vox Sanguinis (C) 2015 International Society of Blood Transfusion

Vox Sanguinis (2015) 109 (Suppl. 2), 1-96
In addition a few manufacturers provide MP24-96 NAT systems for the local market. The pool size is indirectly proportional to the sensitivity of the assay. Prior to August 2014 Grifols was the main supplier of assays for ID-NAT as the Roche s201 platform is not conducive to ID-NAT screening for high numbers. However in August 2014 Roche released the new MPX assay performed on the high through-put Cobas 8800 and 6800 instruments (which are also ID-NAT analysers, the former able to process 1000 donations in an $8 \mathrm{~h}$ shift). Grifols also recently released their Ultrio Elite new assay which includes detection of HIV-2 and is performed on the Panther platform. This system has a smaller footprint than the former Tigris but allows random access of samples and loading of reagents during processing, which streamlines functionality and improves turn-around time. It is better suited for smaller laboratories than the previous Tigris instrument. At the end of June 2015, 61 countries perform NAT to screen the blood supply. Of these 16 and 18 countries screen entirely with either NAT systems from Roche or Grifols respectively and the remaining 27 countries use a combination of both methods in their country. Twenty countries screen their entire blood supply using ID-NAT and the remaining 41 countries use either MP or a combination of MP and ID-NAT. The MPX Roche assay has a 95\% limit of detection (LOD) of $25.7 \mathrm{IU} / \mathrm{ml}$ (CI 21.1-32.8), $7.0 \mathrm{IU} / \mathrm{ml}$ (CI 5.9-8.6) and $1.4 \mathrm{IU} / \mathrm{ml}$ (CI 1.2-1.7) for HIV-1, HCV and HBV respectively. The Ultrio Elite Grifols assay has a LOD of $18 \mathrm{IU} / \mathrm{ml}(15-23.5), 3.0 \mathrm{IU} / \mathrm{ml}(2.5-3.9)$ and $4.3 \mathrm{IU} / \mathrm{ml}$ (CI $3.8-$ 5.0) for HIV, HCV and HBV respectively according to the manufacturers' package insert claims. A number of publications most notably in South Africa, Europe, Australia and Japan have shown, using modelling or lookback analysis, that a small risk still remains of transmitting HIV and HBV even when using highly sensitive IDNAT. These models have in part been validated by 4 anecdotal cases of proven IDNAT breakthrough transmission cases of both window period (WP) and occult HBV infection (OBI) by red blood cell or fresh frozen plasma (FFP transfusions). In addition MP-NAT HIV and HBV WP and OBI breakthrough infections have been found in Brazil, Japan and Thailand, which also could have been missed by ID-NAT. These latter cases highlight the importance of selective donor recruitment in addition to state of the art screening.

\section{Academy Session: The What and How of Blood Components}

\section{B-S10-01 AUTOMATING THE PRODUCTION OF BLOOD COMPONENTS M Lozano and J Cid}

University Clinic Hospital, Barcelona, Spain

For many years the preparation of blood components from whole blood (WB) donations was totally manual, from centrifugation of the WB bag to the transfer of the blood components to the connected bags or the seal of the plastic tubing. Since the introduction of the buffy coat $(\mathrm{BC})$ method for preparing platelet concentrates from WB donations, automation started to enter the blood components preparation laboratory of the blood banks. Thus, in the late 1980 several devices were developed that allow the automation of the first step of blood processing, i.e. the separation of the centrifuged WB bag into red blood cells (RBC), plasma and BC. The introduction of a device that performed the sterile connection of the plastic tubing had a tremendous impact in the blood component preparation laboratory, since it opened the possibility of pre-storage pooling of the components and allowed the discovery that the processing of pooled $\mathrm{BC}$ instead of individual $\mathrm{BC}$ increased significantly platelet recovery in the platelet concentrates (PC). In 2003, a device that combined the centrifugation of one pooled $\mathrm{BC}$ with the expression of the supernatant plasma rich plasma was introduced in the market (OrbiSac, Terumo BCT). Later a new device was developed that allowed the processing of up to six pooled BC in each centrifugation run (TACSI, Terumo BCT). More recently a new generation of devices, which allow the automation of almost all the necessary steps for preparing blood components from WB donations has been introduced. The first one was Atreus system (Terumo $\mathrm{BCT}$ ) that allowed the processing of one whole blood donation into $\mathrm{RBC}$ and plasma (protocol 2C) or RBC, plasma and $\mathrm{BC}$ (protocol 2C+). The system was not refrigerated and due to high temperatures generated during the centrifugation process in routine use, RBC vesiculation occurred leading to the appearance of reddish PC. The recently introduced Reveos system (Terumo BCT) could process simultaneously 4 units of WB and is refrigerated. The Reveos $2 \mathrm{C}$ protocol produces from one WB donation $\mathrm{RBC}$ and plasma while the $3 \mathrm{C}$ protocol produces $\mathrm{RBC}$, plasma and one intermediate plate- 
let unit (IPU). The IPUs can be manually pooled with other IPUs using a pooling kit with integrated leukoreduction filter to form a transfusable adult-size PC without the need of an additional centrifugation step for RBC removal. As the Atreus 3C, the Reveos $3 \mathrm{C}$ system also includes a platelet yield index, which provides an estimation of the platelet content in the IPU, making it possible to increase and/or standardize platelet content of the final PC by selecting the optimal IPUs. Several in vitro and in vivo studies have shown that the blood components produced using semi-automated and automated devices met the European quality standards and provide a similar transfusion response in the recipients compared to those prepared using manual methods

\section{B-S10-02 \\ INTRODUCING NOVEL BLOOD COMPONENTS - PRINCIPLES AND PRACTICE \\ SM Kirwan}

New Zealand Blood Service, Auckland, New Zealand

The development of novel blood components requires input from a number of functional areas within the Blood Service and must be managed in close consultation with the Regulator. Prior to commencing discussions with the Regulator an evaluation is performed and presented to the Executive management team for discussion and inclusion into the business strategic plan.

Before approval to implement a new product can be granted, the Regulator needs to understand what the benefits to the patient and organisation are, what other international Blood Services are doing in regard to this component and whether the Blood Service has the capability to implement the change. The information submitted to the Regulator for approval will inevitably be comprehensive and likely require several detailed submissions.

Initial input is required from the clinical team to provide comment on international practice and evidence of clinical efficacy of the new component. The technical /operational team will develop the process and procedures to manufacture the product providing confidence that the organisation has the required expertise and capability. Validations of new equipment and processes must be completed, reported and approved including any changes to the Blood Management System. The Quality system within the organisation will provide assurance that the development is robust and as per defined change management procedures. Training plans specific to the new process must be implemented and delivered to manufacturing staff. Post implementation plans are developed to review and verify the effectiveness of the process. Any change requires timely and relevant communications with clinical end users and all others with a vested interest. Not only is it important to communicate the benefits of the change, it is unavoidable that costs will rise when creating a new product so revised pricing and its impact must be announced.

Recognition that the evolution of a de novo product, within the Blood Service, is a complex and multifaceted process is essential for success, together with effective communication and careful planning.

\section{B-S10-03 \\ GMP SUPERVISION ON BLOOD ESTABLISHMENT \\ T Hutadjulu \\ Badan POM/NADFC, Jakarta Pusat, Indonesia}

Blood products are life saving medicine that often used in life threatening situations of severely ill patients. Therefore, blood safety has become an important issue where high standards of quality and safety of these products have to be assured prior to use in human. These high standards can be achieved by implementing the principles of Good Manufacturing Practice (GMP) in blood establishment, which is providing a systematic approach for incorporating quality into the entire manufacturing process. This approach ensuring that quality system are in place for the collection, preparation, storage, testing and distribution of blood products and consistently produce product that appropriate to their intended use and meet the required specifications. GMP in blood establishments also increasing availability of plasma and is one of the key issues for a successful plasma contract fractionation program. Plasma for fractionation is needed to generate essential plasma-derived medicinal products (e.g. clotting factors and immune globulins). Implementation of GMP standards are mandatory for collection of plasma as starting material for plasma-derived products. By assuring that standards are met, blood regulation serves to protect donors and to promote and maintain quality and safety of both blood components for transfusion and plasma for fractionation.

GMP implementation in Blood Establishment is relatively new, especially in developing countries. Consistent compliance in GMP implementation depends upon a coordinated service with an appropriate national blood policy. Blood regulation within a legal framework is an essential element of any national blood system. The competent national regulatory authority (NRA) is responsible for assessing and ensuring the compliance with GMP in blood establishments in order to ensure quality and safety of the blood products.

Badan Pengawas Obat dan Makanan (Badan POM) or National Agency of Drug and Food Control (NADFC) is a regulatory body which is responsible for ensuring the safety, efficacy and quality of drug and food marketed in Indonesia. Blood products along with other pharmaceuticals are included as commodities under the supervision of Badan POM/NADFC. As a developing country which become the pilot project on WHO's blood safety program, Indonesia has strong commitment to strenghten the national blood regulation and enforcing the GMP aspect on blood establishment in order to provides high quality plasma and plasma derived product.

\section{Advances in Blood Safety}

\section{B-S11-01 \\ A PRIMER ON MALARIA IN THE ASIA PACIFIC \\ $\mathrm{J}$ Baird \\ Eijkman-Oxford Clinical Research Unit, Jakarta, Indonesia}

Five species of protozoan parasites in the genus Plasmodium cause acute malaria in humans and other primates. These parasites follow complex life cycles in the blood and liver of humans, and are transmitted between humans by the bite of mosquitoes in the genus Anopheles - the only means of acquiring malaria except for blood transfusion or tissue and organ transplantation. Malaria remains highly endemic across much of the Asia Pacific. Among all nations of the region, only Japan, Russia, Australia and Singapore are free of endemic malaria transmission. All of the five species of plasmodia occur in the Asia Pacific, putting almost 3 billion people at risk of infection and typically causing tens of millions of attacks of illness. Each year many thousands in the region do not survive their malaria attacks, mostly due to delayed or improper diagnosis and treatment. This lecture will review the plasmodia species causing malaria, the clinical course and consequences of them, and their geographic distribution in the Asia Pacific. Diagnostic methods for malaria will be reviewed, including ultra-sensitive molecular techniques needed to detect the very often microscopically sub-patent infections. The lecture will also describe the relationship between glucose-6-phosphate dehydrogenase deficiency and malaria, especially as regards sensitivity to acute hemolytic anemia caused by the antimalarial drug primaquine. All of these issues may be considered relevant to the practices of blood banking and transfusion in the Asia Pacific.

\section{B-S11-02}

\section{NEW STRATEGIES FOR BACTERIAL CONTAMINATIONS}

M Schmidt and E Seifried

German Red Cross Institute, Frankfurt, Germany

Background: The detection of bacterial contaminations in blood components can be classified in culture methods, rapid detection methods and experimental new technologies.

Culture methods: Many countries have currently implemented culture methods into blood donor screening. Culture methods convinced with easy handling and high analytical sensitivity. The challenge with culture methods, with the negative-to-date release concept, is a risk for false negative screening results due to sample failures and a reduced clinical efficiency. To reduce this risk, the time for sample collection was extended and the input volume improved to a maximum of $10 \mathrm{ml}$. An additional advantage of culture systems is the option to screen all blood components including stem cells. Nevertheless, due to storage conditions at room temperature, most countries have implemented bacterial screening only for platelet concentrates. Rapid detection methods: Within the last decade, rapid bacterial detection systems (RBDS) were developed as an alternative option to culture systems. In RBDS testing time is reduced to $2-3 \mathrm{~h}$. Therefore a negative-to-date release concept is not necessary by using these systems. Three of them are currently CE or FDA certified (bactiflow system NAT and Verax PGD test). The clinical efficiency is high for the rapid detection methods but the analytical sensitivity is a little lower than for culture methods

New technologies: In addition, new technologies are currently in development to detect bacterial strains in blood components. One option could be the measurement of the bacterial esterase activity by an automated bio-imaging system. First data demonstrated an analytical sensitivity of app. $20 \mathrm{CFU} / \mathrm{ml}$ with a detection time of $45 \mathrm{~min}$. Another approach focuses on the detection of the bacterial cell wall by an ELISA technology. A third new technology uses ATP luminometry. Finally, electrochemical biosensors were developed for many different analytes, which range in size 
from individual ions and small molecules to nucleic acids and proteins up to whole viruses and bacteria. This 'lab-on-a-chip' method of patient or donor diagnosis and monitoring provides a more rapid diagnosis, which allows for faster and more effective therapeutic intervention, thereby preventing full-blown infection and mortality and also decreasing the spread of disease.

Conclusions: Bacterial detection in blood components, especially in platelets, is implemented in many blood donor services. Timing of all steps (sample collection, detection method) is very important to achieve the highest safety level. Although pathogen reduction methods are available for some blood components like platelets or packed red cell concentrates, testing methods offer in addition options to screen also stem cell concentrates. New approaches are promising to come to a next level to be implemented into routine screening, but the analytical sensitivity has to be improved for some of the new test systems in the coming years. Due to the fact that a risk for bacterial contamination still occurs in all blood components, the focus in transfusion medicine in the next decade will be the development of general principles and strategies to detect or inactivate bacteria in all blood components. Therefore, new developments are gladly welcome.

\section{B-S11-03}

\section{DEVELOPMENT OF A HIGHLY SENSITIVE DETECTION} METHOD FOR BACTERIAL DNA IN BLOOD FOR TRANSFUSION

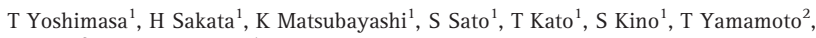
H Ikeda $^{2}$ and S Takamoto ${ }^{1}$

${ }^{1}$ Japanese Red Cross Hokkaido Block Blood Center, Sapporo, Japan ${ }^{2}$ Hokkaido Red Cross Blood Center, Sapporo, Japan

Background: Bacterial contaminated blood product for transfusion is known to result in severe side effects in patients. Although the Japanese Red Cross Blood Centers introduced the diversion of the initial blood collection and leukoreduction in addition to a short shelf-life of blood products, bacterial contamination of blood products has not been completely excluded. Therefore additional preventive measures such as bacterial screening are required. Culture-based bacterial detection methods are highly sensitive but take much time to obtain the results, whereas PCRbased methods such as broad-range real-time PCR of 16S rRNA gene (rDNA) are rapid but not so sensitive because of contamination of the exogenous bacterial DNA in the PCR reagents.

Aims: We aimed to develop a rapid and highly sensitive method for broad-range bacterial $16 \mathrm{~S}$ rDNA detection using real-time PCR.

Methods: A primers/probes targeting the highly conserved region of the bacterial $16 \mathrm{~S}$ rDNA was used to amplify a 160-bp fragment by real-time PCR (Yang S, et al, 2002). A plasmid including the target region of $16 \mathrm{~S}$ rDNA was synthesized and used as a standard 16S rDNA. Three methods for decontamination or inactivation of the exogenous bacterial DNA in the two reagents, namely an amplification reagent (FastStart Universal Probe Master, Roche Applied Science, AMP) and a primers/probes mix (PP), were explored. Method 1, a mixture of AMP and PP was digested with the Alu I restriction enzyme. Method 2, only AMP was digested with DNase I, and PP was filtered through a centrifugal ultrafiltration device (Amicon Ultra-0.5 50K, Merck Millipore). Method 3, the same as the method 2 but AMP was light-inactivated with ethidium monoazide instead of enzymatic digestion. A serial diluted standard DNA at $0,1,2,3$ and $4 \log$ copies/reaction and no template controls (NTCs) were tested by real-time PCR with reagents treated by the methods described above. For evaluation of the sensitivity for practical bacterial detection, peripheral blood spiked with Staphylococcus aureus or Escherichia coli at 0, 1, 2, 3 and $4 \log$ bacteria/reaction was prepared and tested along with NTCs. DNA was extracted from blood spiked with bacteria using QIAamp MinElute Virus Spin kit (QIAGEN) with bead beating and subjected to real-time PCR using a bacterial DNA-reduced reagents.

Results: The amount of exogenous bacterial DNA contaminated in the PCR reagents was estimated approximately 100 copies/reaction. The assay sensitivity using standard DNA was approximately 100, 1 and 10 copies/reaction for the method 1,2 and 3 , respectively. No amplification was also observed for NTC in the method 2 . In the bacterial spiking experiments, the sensitivity of the real-time PCR assay in which the reagents were treated by the method 2 was approximately 10 bacteria/reaction both for S. aureus and E. coli.

Conclusions: In the broad-range bacterial detection by real-time PCR, a combination of DNase I digestion and ultrafiltration was proven to be very effective for reducing contaminated bacterial DNA in the PCR reagents. Broad-range bacteria 16S rDNA real-time PCR assay using bacterial DNA-reduced reagents was considered to be feasible for a bacterial screening of blood for transfusion.

(C) 2015 The Author

Vox Sanguinis (C) 2015 International Society of Blood Transfusion

Vox Sanguinis (2015) 109 (Suppl. 2), 1-96
3B-S11-04

TRANSFUSION RISK FROM EMERGING PATHOGENS IN THE ASIA-PACIFIC REGION

HM Faddy $^{1}$, E Viennet ${ }^{2}$ and R Flower ${ }^{1}$

${ }^{1}$ Australian Red Cross Blood Service, Brisbane, Australia ${ }^{2}$ Research School of

Population Health, Australian National University, Canberra, Australia

Background: An emerging infectious disease (EID) refers to a disease that has recently appeared in a population or one that has rapidly increased in incidence or geographic range. Examples of EIDs include Ebola, HIV/AIDS, hepatitis E and the vector-borne diseases caused by Zika and dengue virus. EIDs pose a risk to transfusion safety, which can be direct, if the agent can be transmitted through blood transfusion, or indirect, where outbreaks reduce the pool of available donors. Although examples of both have been observed, the former will be the focus of this review. Many transfusion risks have become global due to globalisation and increased international travel; however, unique region-specific concerns exist. The Asia-Pacific region varies in size depending on context, but for the purposes of this review will be defined as Southeast Asia and Oceania. This region is a hotspot for genetic diversity, as well as the emergence of a number of infectious diseases. The Asia-Pacific region is also geographically, culturally, socioeconomically, and climatically diverse. Moreover, the level of sophistication in blood operators within this region varies, from a national supplier based on voluntary blood donors as is seen in Australia, to hospital-based services relying on replacement donors, which is seen in many nations across the pacific.

Aims: To review current EID risks to blood transfusion safety in the Asia-Pacific region. Methods: Review of available public health reports and literature for the occurrence of EIDs in the Asia-Pacific region that pose a transfusion risk.

Results: EIDs that pose a transfusion risk occur across the Asia-Pacific region, with differing levels of endemicity between and within countries. Reports of hepatitis $\mathrm{E}$ virus (HEV) seropositivity vary from $2.2 \%$ in Fiji to $15.2 \%$ in Papua New Guinea. As a result of high HEV prevelance in the Hokkaido region of Japan, HEV nucleic acid testing (NAT) has been implemented for screening blood donations. Dengue infection is widespread across the Asia-Pacific, with major outbreaks occurring in 2014 in Fiji, Malaysia, the Philippines, and Thailand. In Australia, dengue is episodic in the North-east, resulting in donation restrictions in affected areas. Zika virus, the firs arbovirus demonstrated to also be sexually transmitted, emerged in the Asia-Pacific in early 2007 and outbreaks have spread across the pacific, with epidemics in French Polynesia, the Cook Islands, Easter Island and New Caledonia. Due to the theoretical transfusion risk several preventative procedures were implemented in French Polynesia during an outbreak in 2013, including NAT.

Summary/Conclusions: EIDs pose a transfusion risk globally, and in the Asia-Pacific region. A number of EIDs that can be transmitted through blood transfusion are endemic to the Asia-Pacific, with countries managing the transfusion risk depending on the level of endemicity of the agent in question, and the maturity of blood operations in each county. Climate change is predicted to increase the distribution and density of a range of disease vectors, which is likely to increase the transmission of a number of vector-borne diseases, representing a significant threat to the maintenance of a safe blood supply in the future.

\section{B-S11-05}

\section{MIDDLE EAST RESPIRATORY SYNDROME CORONAVIRUS} (MERS-COV) OUTBREAK IN SOUTH KOREA: RISK MANAGEMENT AT THE KOREAN RED CROSS SEOUL NAMBU

\section{BLOOD CENTER}

SY Kwon, EH Lee, HS Kim, JH Kim, YO Lim and GR Choi

Seoul Nambu Blood Center, Korean Red Cross, Seoul, South-Korea

Background: On 20 May 2015 the first confirmed case of MERS was identified in South Korea. Unexpectedly this case triggered an outbreak with a total of 186 cases of which 36 have died. Nearly half of all confirmed cases have been traced to Samsung Medical Center (SMC), which is in the service area of the Seoul Nambu Blood Center (SNBC) of the Korean Red Cross (KRC).

Aims: To review measures taken both at a national and SNBC level to deal with the impact of the MERS crisis on blood availability.

Methods: National and SNBC blood collection and supply data were analyzed using the Blood Information Management System (BIMS) of the KRC. Measures taken to deal with the decrease in collections were reviewed.

Results: To ensure the safety of the national blood supply, the KRC applied enhanced donor criteria and guidelines on post-donation information. Donors with overseas travel history were already deferred for 1 month since 2008. Although there was no evidence of community transmission, public anxiety caused by the out- 
break led to decreased outdoor activities, temporary closure of schools and cancellation of various events. This resulted in a decrease of donors at fixed donation sites and cancellation of mobile blood drives. From 21 May to 20 June, this includes the period with a peak in case confirmation, national and SNBC collection at fixed sites decreased by $2.5 \%$ and $2.3 \%$, respectively compared to 2014 . Decrease of collection at mobile blood drives was more pronounced, with a $9.6 \%$ and $22.2 \%$ decrease at a national and SNBC level. Overall national and SNBC total collection decreased by $4.9 \%$ and $8.1 \%$, respectively. While blood supply still met the demand, to ensure adequate blood supply level, the KRC made public appeals to participate in blood donation. Opening time of fixed donation sites was also extended. At SNBC level, regular blood donation campaigns were held to raise awareness about the decrease in donation in our area. With these efforts, national and SNBC collection increased during 21 to 30 June by $9.0 \%$ and $7.1 \%$, respectively. National blood demand decreased by only $0.5 \%$ compared to 2014 . However, at SNBC the demand dropped by $9.1 \%$. This was mainly due to the shutdown of SMC after the increase in confirmed MERS cases, because about 35\% of SNBC's supply is issued to SMC.

Conclusion: Emerging infections that have not been confirmed to be transmitted by transfusion can also threaten the safety of the blood supply. Timely measures were effective in ensuring an adequate blood supply at a national level. Although decrease in collection at SNBC was more pronounced, adequate supply level could be maintained because the demand declined remarkably compared to other regions. In cases of an emergency, blood collection target should be adjusted according to local needs as the incident may have different impact on the blood service at a local level. Experience gained through this crisis will add to our preparedness planning for future incidents.

\section{Academy Session: The What and How of Organisation and Blood Supply Management}

\author{
3C-S12-01

\section{WHAT'S THE RIGHT MODEL FOR A NATIONAL BLOOD} \\ PROGRAMME? \\ P Flanagan \\ New Zealand Blood Service, Auckland, New Zealand
}

The World Health Organisation advocates for a nationally co-ordinated blood system in order to promote uniform standards, economies of scale, consistency in the quality and safety of blood and blood products and best transfusion practices. The structure of the national blood system will however depend on a range of factors including the size and population of the country, geographical and transport constraints, the level of development of the overall health-care system and the level of funding available to develop and maintain the system. The Ministry of Health will play a pivotal role in defining the strategy and policy of the overall blood transfusion system and for ensuring that it is fully integrated into the wider health system of the country.

Internationally a number of different models exist for delivery of blood transfusion services. There is no one model that fits all circumstances. Each country must determine its own optimal model and in doing so ensure effective oversight and monitoring against pre-agreed standards. Governance of the blood system must extend beyond the activities of the blood transfusion services and include consideration of regulation, access to plasma fractionation and clinical transfusion systems within hospitals.

A range of options should be considered when defining the optimal organisation structure within a country. This includes the number of organisations that will provide the service, the types of organisations that will be involved (government provision or non-governmental organisation) and the level of integration between the collection, manufacturing and hospital blood banking activities. Inevitably the type and effectiveness of pre-existing structures will also need to be considered.

A key consideration is the level of centralization of the national blood transfusion service. Centralization of the collection and manufacturing aspects of BTS provision will reduce duplication, enable better national management of inventory and enable introduction of consistent national standards. Conversely a decentralized approach whereby individual hospitals undertake broad range of activities will enable closer and better integration with clinical services and links to University and Teaching hospitals with improved opportunities for education and research. Either approach can work. Many countries have adopted a hybrid model.

Three types of organisation may be involved in the delivery of the BTS. These are government managed providers, non-government organisation providers (Red Cross/
Red Crescent) and hospital/ university providers. In some countries commercial provides are also involved. Many countries adopt a mixed model. The Red Crescent/Red Cross is a major provider of BTSs. This avoids a direct link to government and might provide a useful source of finance for the system. It does however result in a more complex governance model. Overall direct provision of a centrally co-ordinated BTS by government is probably the most efficient model. Nonetheless an NGO model does provide other benefits and might be more appropriate in some circumstances. An effective model for delivery of a national co-ordinated BTS requires government commitment and support, clarity of the roles and functions of individual providers, clear lines of authority, responsibility \& accountability and adequate and sustainable resourcing.

\section{C-S12-02}

\section{PUTTING IN PLACE A RISK-BASED DECISION FRAMEWORK} FOR BLOOD SAFETY

PC McDonald and ABO Alliance of Blood Operators

Alliance of Blood Operators, Melbourne, Australia

Background: Decisions about blood safety need to take numerous factors into account, such as risks, benefits, costs, ethical issues, and stakeholder perspectives. Since the 1980s, numerous measures aimed at maximising blood safety have been implemented based on varying interpretations of the precautionary principle and an unsustainable pursuit of 'zero risk', without evident consideration of cost effectiveness and opportunity cost. In 2010, the international Alliance of Blood Operators (ABO) held an International Consensus Conference on Risk-Based Decision-Making for Blood Safety to address the issue. Among the consensus panel's conclusions were the following principles: that risk is inherent 'vein to vein'; that zero risk is unattainable; that the well-being of transfusion recipients must be central to blood safety decision-making.

Based on these conclusions, ABO has developed an integrated, internationally applicable, risk-based decision-making framework to guide major policy and operational change. The Framework is: evidence-based and takes advantage of quantitative and qualitative information; provides measurable outcomes; improves consistency in decision making and proportional responses to risk; improves confidence and trust in investment decisions; allows redirection of resources thus improving efficiency and effectiveness; increases transparency and communication; and enables defensibility and commitment across the system.

Summary: The ABO Risk-based Decision-making (RBDM) Framework is a structured approach to blood safety risk management. It lays out a step by step methodology for setting the best course of action under uncertain conditions by identifying, assessing, acting on and communicating risk. The RBDM Framework has been tailored for the specific needs of blood operators. The Framework provides a set of approaches to help users identify and prioritise risks to blood safety and to evaluate the effectiveness of potential risk management options, such as introducing a new intervention or withdrawing an existing one. The approaches included in the Framework help the user analyse and account for a series of contextual, often qualitative, factors that affect decision making in the management of blood risks. The Framework takes a societal perspective, enabling consideration of social, economic, and ethical perspectives that go beyond quantitative calculations of risk and can alter risk tolerability. The RBDM Framework is intended to be embedded in an organisation's risk management program. As such, it has two distinct components: policy foundations; and the decisionmaking process. Risk management policy foundations are background elements that express the values, priorities, and policies of a blood service in relation to risk management. They guide the decision-making process and must be in place before the process begins. The policy foundations include: risk management principles; risk communication and stakeholder participation; assessment principles; and risk tolerability.

The stages of the decision-making process are common to many decision frameworks. The RBDM Framework steps the user through each of the stages: preparation; problem formulation; participation strategy; assessments (including health economics \&t outcomes assessments); evaluation; and decision.

\section{C-S12-03}

\section{HAEMOVIGILANCE-SETTING UP ONE FROM NONE}

N Marwaha

Postgraduate Institute of Medical Education and Research, Chandigarh, India

Haemovigilance is a surveillance system which aims to detect and analyse adverse events and reactions in the blood transfusion chain to improve blood safety by instituting corrective and preventive actions. Haemovigilance activities may be organized at an institutional level to improve clinical transfusion practices, but this does not contribute to risk assessments of transfusion at a national level. Hence, there is a Vox Sanguinis (2015) 109 (Suppl. 2), 1-96 
need to establish a nationally co-ordinated system of haemovigilance. Setting up such a system is a complex task, it involves both organizational and operational aspects, which may vary between countries depending upon the structure and regulation of blood transfusion services.

First and foremost it should be a part of the National Blood Policy. The organizational structure should ideally have governmental oversight to the program through its Ministry of Health or equivalent authority. Haemovigilance advisory committees are required to give direction to the program. All stakeholders need to be identified and systems worked out for their collaboration and coordination. During the phase of planning the following issues need to be addressed: (i) whether reporting of all adverse events in the transfusion chain is required (donors, processes and recipientrelated events) or restricted to recipients, that is, reporting only transfusion reactions to begin with, whether to include near miss events and/or rapid alerts, (ii) whether reporting will be voluntary or mandatory, or a combination of both, that is mandatory reporting of serious adverse reactions only, rest being voluntary, (iii) what would be the flow of information - who reports to whom, (iv) adoption of definitions of adverse events and reactions (AEs, ARs) as per international consensus, (v) uniform reporting formats and (vi) requisite resources both financial and technical to implement the program. The initial focus of haemovigilance activities could be on the transfusion recipients, that is, reporting of transfusion reactions.

At the reporting end, awareness and training of all staff who would be involved in haemovigilance activities needs to be conducted. This would include clinicians and other bedside transfusion staff, personnel in blood centers and hospital based departments of transfusion medicine, members of hospital transfusion committees and donor organizations. A very important aspect to be dealt with during awareness sessions is to allay fears of any punitive action for the reporting staff. The reporting units initially could be regional blood transfusion centers and their associated hospitals or the tertiary care hospitals and gradually when the system gains ground the scope could be extended to include other hospitals and blood centers and subsequently also the donors.

The haemovigilance reports generate a large amount of data, hence a validated system for collection, collation and analysis of data is required. Mechanisms for regular publications of haemovigilance reports with recommendations for better transfusion practices should be put in place. This would help in directing policy decisions to improve blood safety

It should also be aimed to establish linkage with the international haemovigilance network and database for exchange of information between member countries.

\section{Advances in Clinical Transfusion}

\section{C-S13-01 \\ HAEMOGLOBINOPATHIES IN INDONESIA \\ I Setianingsih \\ Eijkman Institute for Molecular Biology, Jl. Diponegoro 69, Jakarta}

Around 6000 patients (mostly are beta-thalassaemia) are currently registered for regular blood transfusion, where 5000 out of these patients are living in Java island. The management of thalassaemia in Indonesia is far from the standard required. Most patients were late diagnosed and received inadequate blood transfusion, Only a very small number of patients received adequate blood transfusion and iron chelating agent. However, since 2011, Government: has subsidized the optimal management of all patients registered.

The management and prevention program for thalassemia in Indonesia is complicated by the diversity of the carrier frequency, the molecular defects and the phenotype expression of the disease in this country. The carrier frequency ranged from 5 to $10 \%$, and that of $\mathrm{HbE}$ which is $1-33 \%$, in the various ethnic populations. Likewise, the spectrum of the underlying $\beta$-globin mutations varies widely between regions. Twenty four mutations accounted for more than $90 \%$ alleles in Indonesia. $\mathrm{HbE}$ and IVS1-nt5 are found in all geographical areas, albeit with different frequencies, whilst other mutations are specific for particular ethnic groups such $\mathrm{as} \mathrm{Hb}$ Malay and $\mathrm{Cd} 26$ (GAG $\rightarrow$ TAG) in the Malay populations of Sumatera, IVS1-nt1 in the Sundanese and Cd-35 in the Javanese (both of the Java island), and large - and -globin gene deletions in Buginese and Makasarese of South Sulawesi.

The relationship between these mutations, their overall genetic background and their clinical expression is also of major interests. Clinically the patients showed a wide range of phenotypic expression, from thalassemia major to the mild type of thalassemia intermedia. All patients with $\beta^{\circ} / \beta^{\circ}-, \beta^{\circ} / \beta+-$ and $\beta+/ \beta+-$-mutations presented at early of age and were clinically $\beta$-thalassaemia major. In contrast, the age at first

\section{(C) 2015 The Author}

Vox Sanguinis (C) 2015 International Society of Blood Transfusion

Vox Sanguinis (2015) 109 (Suppl. 2), 1-96 presentation in patients compound heterozygous for $\mathrm{HbE}$ with either $\beta^{\circ}$ or $\beta+$-thalassemia mutations varied from 6 months to 20 years and showed a broad spectrum of clinical severity, ranging from thalassemia major to mild thalassemia intermedia. The findings have major implications in the management of thalassaemia in Indonesia. The variation in the distribution of carriers in the various ethnic populations and the size of the populations resulted in unequal anticipated carrier screening and prenatal diagnosis workload. The diagnostic protocol for carrier screening and for prenatal diagnosis could be specifically designed on a regional basis.

\section{C-S13-02 \\ OPTIMISING TRANSFUSION THERAPY IN THE CHRONICALLY TRANSFUSED PATIENTS}

AL Ang

Health Sciences Authority, Singapore, Singapore

Patients may require regular transfusion due to congenital or acquired causes of ineffective blood cell production or bone marrow failure. In Asia, the most common congenital cause is thalassaemia while acquired causes include myelodysplastic syndrome (MDS) and severe aplastic anaemia. The major goal of transfusion in these patients is to minimise the complications from their condition with the lowest possible amount and risks of transfusion.

Due to the frequent transfusions, their cumulative risks from transfusion are higher than other patients. These include mild adverse reactions such as febrile non-haemolytic transfusion reactions and urticaria, as well as severe risks such as haemolytic transfusion reactions, anaphylaxis, transfusion transmitted infections, transfusion associated circulatory overload and TRALI. Leucodepleted blood products not only help to reduce the risks of febrile non-haemolytic transfusion, it may also reduce the risk of HLA-alloimmunisation and hence platelet refractoriness in patients with significant thrombocytopaenia requiring platelet transfusion. Washed blood products may also be needed in those with recurrent severe anaphylactic reactions to transfusion. These chronically transfused patients have a higher frequency of red cell alloimmunization, which in turn increases their risks of haemolytic transfusion reactions. It is therefore important to regularly monitor for the development of new clinically significant red cell alloantibodies before each transfusion. Patients with clinically significant red cell alloantibodies need to receive compatible antigen-negative red cells. An extended red cell phenotyping before commencement of red cel transfusion may be useful for subsequent antibody identification in case of alloimmunisation later. If the patient is already transfused regularly, molecular typing should be carried out instead. Prophylactic matching of blood groups (other than $\mathrm{ABO}$ and $\mathrm{RhD}$ ) which are associated with high frequencies of alloimmunization helps to reduce the incidence of this, and is particularly useful for patients with high risks of red cell alloimmunization. To minimise the risk of transfusion transmitted infections, the blood should be collected from carefully screened non-remunerated voluntary blood donors, and each donation should be screened for HIV, Hepatitis B/C syphilis and where relevant, other infections that pose a risk to blood safety. Patients should also be vaccinated against hepatitis $B$.

In addition, patients who need regular red cell transfusion such as those with transfusion dependent thalassaemia, are at risk of transfusional haemosiderosis. Transfusing red cell concentrate close to its collection may result in better in vivo recovery and longer half-life, and this may decrease the red cell transfusion requirements of these patients. This led to the recommendation of transfusing red cells in additive solution within 2 weeks from collection. For thalassaemia major patients, maintaining pre-transfusion $\mathrm{Hb}$ at 9 to $10.5 \mathrm{~g} / \mathrm{dl}$ had been shown to provide the most appropriate balance between adequate bone marrow suppression and transfusional iron loading. Thalassaemia patients should also be assessed carefully on the indications and benefits of regular transfusion before being commenced on this.

\section{C-S13-03 \\ INTRODUCTION OF POINT-OF-CARE TESTING AND INTERVENTION IN THE TRANSFUSION UNIT DECREASES PERIOPERATIVE BLEEDING, SHORTENS OPERATIVE TIME AND FACILITATES APPROPRIATE DOSAGE OF BLOOD TRANSFUSIONS}

C Yamada, H Furumaki, H Fujihara, H Watanabe, M Tsuzuki, T Shinba, M Kaneko, H Shibata, K Ishizuka, Y Hosaka and A Takeshita

Hamamatsu University School of Medicine, Hamamatsu, Shizuoka, Japan

Background and aims: Perioperative blood components should be supplied on the basis of the latest clinical symptoms and laboratory data, while many transfusion 
units have not been always and completely attainable. We introduced a blood cell and coagulation analyzer into the operating room, which members from the transfusion unit use to analyze samples. We previously reported this system significantly decreased the total incidence of perioperative bleeding (Yamada et al, AABB, 2014). In this study, we had deeper analyses on the advantage of adopting this system in each operative procedure and each blood component.

Methods: Samples were analyzed on the spot by members of the transfusion unit using the analyzer placed in the operating room, and shared the data with anesthesiologists and surgeons. We analyzed the amount of total bleeding, operative time and dosage of blood components transfused during each operating procedure, and compared these findings before and after introducing the system. The data was compared by Chi-square test and Fisher's exact test (SAS, Tokyo), and P-values of less than 0.05 were considered statistically significant.

Results: The total number of operations performed before and after introducing the system was 1757 and 2044, respectively. After initiating the new system, the amount of perioperative bleeding significantly decreased during endovascular aortic repair, aortic aneurysmectomy, colectomy, valve replacement, cervical and uterine cancer, spinal fusion, and scoliosis correction (Table 1). Operative time was significantly shortened in bypass graft, hepatectomy, colectomy, valve replacement, cervical and uterine cancer surgery and spinal fusion $(\mathrm{P}<0.001,0.007,0.042,<0.001,<0.001$ and $<0.001$, respectively) after introduction of point-of-care testing. In addition, dosage of RBC and FFP were significantly decreased in bypass graft $(\mathrm{P}=0.028$ and 0.002 , respectively) and valve replacement $(\mathrm{P}<0.001$ and 0.015 , respectively) upon adoption of the new system. The dosage of RBC was significantly decreased in hepatectomy $(\mathrm{P}=0.016)$. The dosage of FFP, which was adversely increased in scoliosis correction $(\mathrm{P}=0.011)$ compared to those before introduction of the system, improved postoperative bleeding.

Conclusion: Perioperative interventions include monitoring the amount of blood loss, management of coagulopathy, and treatment of adverse effects of transfusion. According to the Perioperative Blood Management Guidelines (Jonathan et al, AABB, 2014), point-of-care testing has a positive effect on perioperative care and improves patient outcome. In this study, we moreover clarified the advantage of the system not only with regard to each operative procedure but also with each blood component. Perioperative bleeding and dosage of blood components varies depending on the operative procedure (Thompson et al, Transfusion, 2014). Our results suggest it is important to promptly and continuously provide latest perioperative data, which can then be disseminated to other members of the team in the operating room.

Table 1 . The amount of total bleeding and perioperative time during each operating procedure.

\begin{tabular}{|l|c|c|c|c|c|c|}
\hline \multicolumn{1}{|c|}{ Operative procedures } & \multicolumn{2}{|c|}{ Amount of Total Bleeding (mean \pm SD) } & \multicolumn{3}{c|}{ Perioperative Time (mean \pm SD) } \\
\cline { 2 - 7 } & Before (dil) & After (dil) & p-value & Before (hours) & After (hours) & p-value \\
\hline Endovascular aortic repair & $6.1 \pm 27.7$ & $3.6 \pm 7.9$ & $<0.001$ & $3.2 \pm 0.1$ & $3.1 \pm 0.1$ & 0.475 \\
\hline Bypass graft & $7.3 \pm 6.3$ & $7.2 \pm 5.8$ & 0.218 & $8.1 \pm 0.2$ & $7.1 \pm 0.2$ & $<0.001$ \\
\hline Hepatectomy & $10.6 \pm 10.6$ & $9.0 \pm 22.2$ & 0.182 & $9.1 \pm 0.1$ & $8.0 \pm 0.1$ & 0.007 \\
\hline Aortic aneurysmectomy & $23.3 \pm 23.3$ & $20.0 \pm 22.8$ & 0.020 & $7.1 \pm 0.1$ & $6.5 \pm 0.1$ & 0.319 \\
\hline Colectomy & $4.9 \pm 6.6$ & $5.4 \pm 17.6$ & 0.004 & $3.1 \pm 0.0$ & $2.4 \pm 0.0$ & 0.042 \\
\hline Valvereplacement & $11.9 \pm \pm 11.3$ & $6.3 \pm 5.1$ & 0.004 & $6.5 \pm 0.1$ & $5.2 \pm 0.1$ & $<0.001$ \\
\hline Cervical and uterine cancer & $8.0 \pm 16.7$ & $6.0 \pm 12.4$ & 0.004 & $2.4 \pm 0.1$ & $2.2 \pm 0.1$ & $<0.001$ \\
\hline Spinal fusion & $4.8 \pm 5.2$ & $3.5 \pm 4.5$ & 0.001 & $3.5 \pm 0.1$ & $3.2 \pm 0.1$ & $<0.001$ \\
\hline Scoliosis correction & $15.0 \pm 11.9$ & $12.7 \pm 11.2$ & 0.007 & $5.5 \pm 0.1$ & $5.5 \pm 0.1$ & 0.381 \\
\hline
\end{tabular}

\section{C-S13-04}

\section{RISKS AND BENEFITS OF ANTIFIBRINOLYTIC THERAPY WITH TRANEXAMIC ACID VARY BY CARDIOVASCULAR SURGERY \\ TYPE}

Y Kanaumi, T Maeda, S Seguchi, S Kawamura, M Kodama, T Kawai and S Miyata National Cerebral and Cardiovascular Center, Suita, Japan

Background: Massive bleeding is associated with increased morbidity and mortality in cardiovascular surgery patients. Since surgical intervention on the cardiovascular system, use of cardiopulmonary bypass (CPB), and hypothermia can potentially activate the fibrinolytic system, hyperfibrinolysis is a major cause of massive bleeding. Indeed, recent meta-analyses have indicated that antifibrinolytic agents such as tranexamic acid (TXA) are effective in reducing blood loss in cardiovascular surgery patients when used prophylactically. However, several concerns remain regarding appropriate indications and adverse events, such as an increased incidence of postoperative seizures that may be associated with increased mortality. Recent studies suggest that the use of higher doses of TXA can be associated with a higher incidence of seizures. Since relatively high doses of TXA are required in cardiovascular surgery, we discontinued TXA use in April 2013, which permitted us to conduct a before-and-after study to clarify the balance of risks and benefits of TXA for different types of cardiovascular surgery.

Aims: The aim of the study was to investigate the risks and benefits of TXA therapy for different types of cardiovascular surgery.
Methods: We retrospectively collected the data including surgery type, perioperative adverse events, and surgical blood loss after the ethical committee of our institution approved the study. Patients who underwent surgery requiring CPB at our hospital were included and divided into two groups: cardiac surgery (CS) and aortic surgery (AS). The groups included patients who underwent coronary artery bypass grafting, valve replacement, or removal of intracardiac tumors and those who underwent aortic replacement or thoracic aorta aneurysm repair, respectively. Patients with congenital heart disease were excluded from the study. We compared the incidence of postoperative seizures up to postoperative day 28 and total surgical blood loss volume before (April 2008-March 2013) and after (April 2013-July 2014) April 2013, when we discontinued TXA use, utilizing Student's t-test or Fisher's exact test as appropriate.

Results: A total of 2387 patients were enrolled ( $n=1312$ in the CS group and $\mathrm{n}=1075$ in the AS group). Overall, the incidence of postoperative seizures significantly decreased after April 2013 (before: 4.3\% vs after: 2.4\%; P = 0.03). However, mean blood loss was similar (before: $1844 \mathrm{ml}$ vs after: $1993 \mathrm{ml} ; \mathrm{P}=0.17$ ). In the AS group, the incidence of postoperative seizures decreased significantly (before: 6.1\% vs after: $2.1 \% ; \mathrm{P}=0.01$ ) and mean blood loss increased significantly (before: $2394 \mathrm{ml}$ vs after: $3012 \mathrm{ml}$; $\mathrm{P}=0.003$ ) after April 2013. In contrast, significant alterations were not observed in the CS group.

Summary/conclusions: Antifibrinolytic therapy with TXA may reduce surgical blood loss only in patients who undergo aortic replacement or thoracic aorta aneurysm repair, probably due to more surgical invasiveness and prolonged CPB time. However, we must take into account the increased risk of postoperative seizures associated with TXA use.

\section{C-S13-05 \\ A COMPARISON OF PLATELETS TRANSFUSION PRACTICE IN DENGUE HOSPITALIZED PATIENTS DURING SEPARATE OUTBREAK YEARS - 2005 VS 2013}

KP Myint and J Chay

Health Sciences Authority, Singapore, Singapore

Background/Introduction: Dengue outbreaks occurred in Singapore in 2005 and 2013. In 2005, there were a total of 14,209 dengue fever cases and 25 people died. 2013 saw 22,318 dengue fever cases, with 8 deaths reported.

Clinical management of dengue in-patients in Singapore has changed significantly in recent years, with regards to transfusion support. Prior to 2009, the majority of hospital doctors, including infectious disease physicians, subscribed to the practice of prophylactic platelets transfusion for non-bleeding dengue patients when platelets count below $20,000 / \mathrm{mm}^{3}$. Clinical practices have undergone a paradigm shift in the early 2010s, led by infectious disease physicians; findings from a series of small studies, as well as the ongoing ADEPT.

Adult Dengue Platelet Study suggest that prophylactic platelet transfusion in patients with thrombocytopenia (platelet count $<20,000 / \mathrm{mm}^{3}$ ) may not be effective in preventing complications of dengue, including bleeding. On basis of this emerging evidence, prophylactic platelet transfusion triggered by platelet count $<20,000 / \mathrm{mm}^{3}$ alone is no longer current standard practice among most hospital departments. Anecdotally this has led to a dramatic drop in platelet demand for dengue cases. Aim/Objective: This retrospective audit study compares the platelet usage for dengue cases in the two outbreak years 2005 and 2013 to analyze for any significant drop in platelet demand in dengue cases with the change in transfusion support practices in dengue management.

Methodology: We analyzed the usage of platelets for Dengue per 100,000 populations for the Dengue outbreak years of 2005 \&t 2013, to observe if there is any statistically significant difference. We also studied the trend of overall platelet usage and platelet used for Dengue, as well as the case mortality.

Result: Out of 18,254 whole-blood derived platelet units used in 2005, 3857 units were used for Dengue cases (representing 21.13\% of overall platelets usage for 2005). This translates to 271.45 platelet units transfused per 1000 Dengue cases. In 2013, total platelets usage was 23,592 whole blood derived units, but only 776 platelets units were used for Dengue cases, representing only $3.29 \%$ of total platelets usage. There were only 35 whole blood derived platelet units transfused per 1000 Dengue cases.

Dengue mortality rates in 2005 and 2013 were $0.19 \%$ and $0.04 \%$ respectively. Conclusion: This study shows there was a very significant drop in platelet transfusions to dengue patients with the decrease in prophylactic platelet transfusions given in the absence of significant bleeding. The decline in dengue mortality rates also suggest that this change in practice has not resulted in harm to dengue patients. 


\section{Plenary Session: Clinical Transfusion Updates}

\author{
3D-PL5-01 \\ UPDATE ON MANAGEMENT OF HEMOGLOBINOPATHIES AND \\ IRON OVERLOAD: BEYOND BLOOD TRANSFUSION \\ V Viprakasit \\ Siriraj Hospital, Mahidol University, Bangkok, Thailand
}

Background: Chronic anemia, ineffective erythropoiesis (IE) and secondary iron overload due to iron hyperabsorption from reduced hepcidin levels and transfusional iron influx are three main pathological changes underlie complications in thalassemia patients; both Transfusion Dependent Thalassemia (TDT) and Non-Transfusion Dependent Thalassemia (NTDT). Although stem cell transplantation (SCT) can offer a curative measure, as it could abolish completely anemia and abnormal erythrocyte production. However there is a limitation due to unavailable donors. Aims: To review alternative treatment options for thalassemia patients.

Methods: Literatures on thalassemia management in the last two decades were reviewed and evaluated.

Results: Recent success on SCT from haploidentical donors has provided an alternative source of stem cells, however this approach require further clinical trials before this could be a mainstream management. Therefore, blood transfusion remain a standard of care and this requires appropriate red cell typing to avoid potential alloimmunization and adequate hemovigilance. One unit of blood contains at least 100$120 \mathrm{mg}$ of iron that is much higher than usual iron intake through duodenal absorption (2-3 mg/day). TDT patients on regular red cell transfusion will soon develop iron overload. At present, three iron chelators; desferioxamine (DF0), deferiprone (DFP) and deferasirox (DFX) are available. Together with a better iron monitoring by MRI with appropriate usage of these iron chelators, optimal iron chelation treatment could be achieved with acceptable iron status and minimizing risks of iron related end-organs' complications and improving a long term survival of these patients.

In recent years, several modalities for thalassemia have been studied and reported. These include somatic gene therapy using lentivirus. The aim is to transfer $\beta$-globin into the patients'stem cells to reduce the $\alpha-\beta$ imbalance in erythroid cells, eventually leading to transfusion independence. In an exploratory clinical trial, an adult patien with severe $\mathrm{HbE} / \beta$-thalassemia dependent on monthly transfusions since early childhood has become transfusin independent for over 5 years. At least 10 additional patients are currently under clinical trails. Several new molecules that could ameliorate anemia in TDT and NTDT are under investigated including: (1) Janus kinase 2 inhibitors: this may limit the overproduction of immature erythroid cells in thalassemia, potentially reversing extramedullary hematopoiesis and preventing splenectomy, (2) ACE-011 (sotatercept), (3) ACE-536 (luspatercept); these are modified activin type IIa and -b receptor fusion protein respectively that inhibit signaling induced by other members of the transforming growth factor $\beta$ super family in thalassemia and (4) Hepcidin agonist; it replaces endogenous hepcidin and improve regulation over hepcidin-feroportin axis enhancing iron utilization and reducing ineffective erythropoiesis. These molecules could result in a promotion of normal maturation of terminally differentiating erythroblasts and enhancing hemoglobin production. These compounds are under-investigation at Phase II-III studies on both TDT and NTDT patients and they were expected from preclinical trials to be effective and possibly reduce transfusion requirement in some, but not all thalassemia patients.

Summary: It remains to be seen whether these new modality as a mono or combo therapy could provide a new pathway for future management of thalassemia patients when blood transfusion might become a history.

\section{D-PL5-02}

\section{UPDATE ON INDICATIONS FOR RED CELL TRANSFUSION}

AL Ang

Health Sciences Authority, Singapore, Singapore

The first attempt of blood transfusion dates back to as early as the 17th century, and the first successful blood transfusion in human took place in the early 19th century. Blood transfusion became an established clinical practice in the 20th century after World War I which propelled the improvement in knowledge and technological advancements that made blood transfusion safer and more effective.

The benefits of blood (or red cell) transfusion were first recognised and demonstrated in patients with massive haemorrhage and haemorrhagic shock. In such patients, red cell transfusion helps to quickly restore oxygen delivery to the tissues by replacing

\section{(C) 2015 The Author}

Vox Sanguinis (C) 2015 International Society of Blood Transfusion

Vox Sanguinis (2015) 109 (Suppl. 2), 1-96 the rapidly lost red cells. For patients with chronic isovolaemic anaemia, there may be differences in their tolerance of anaemia due to variations in their ability to recruit physiological reserves (eg. increase cardiac output), depending on their age and comorbidities. Therefore in patients with chronic anaemia, decision to transfuse red cells should not be based purely on the Hb level, but should also depend on their clinical assessment.

Unlike massive haemorrhage, the benefits of red cell transfusion in chronic anaemia are less evident. This has been subsequently confirmed in a few randomised controlled trials. Since the randomized controlled trial on Transfusion Requirements in Critical Care was first published in the late 1990s, there have been more randomized controlled trials conducted which showed that a restrictive transfusion strategy is not inferior to a liberal strategy in other patient groups despite a significantly lower transfusion requirement. These patient groups include post-operative patients with history of ischaemic heart disease or risk factors for this, patients with stable upper gastrointestinal tract bleed, septic and obstetric patients. These studies have generally led to the consensus that in patients with chronic anaemia, transfusion is no appropriate when $\mathrm{Hb}$ is at least $10 \mathrm{~g} / \mathrm{dl}$, transfusion is likely beneficial when the $\mathrm{Hb}$ is less than $7 \mathrm{~g} / \mathrm{dl}$, and those with $\mathrm{Hb}$ of 7 to $10 \mathrm{~g} / \mathrm{dl}$ requires individual assessment of their comorbidities and clinical condition. However, a safe red cell transfusion trigger for patients with acute coronary syndrome is unknown, and the pilot study seems to suggest that a liberal transfusion strategy may be better.

\section{D-PL5-03}

AN UPDATE ON INDICATIONS FOR PLATELET TRANSFUSION

EM Wood ${ }^{1}$, GL Crighton ${ }^{1}$, LJ Estcourt ${ }^{2}$, ZK McQuilten ${ }^{1}$ and SJ Stanworth ${ }^{2}$

${ }^{1}$ Monash University, Melbourne, Australia ${ }^{2}$ NHS Blood \& Transplant/Oxford

University Hospitals NHS Trust, Oxford, United Kingdom

Platelet concentrates are the most commonly prescribed blood component after red cells, and demand for platelet concentrates appears to be increasing. Numerous clinical factors likely influence a patient's need for platelet support; and patient factors, donor variables, and product characteristics (such as methods of collection, preparation and storage) all affect the outcomes from platelet transfusion.

Most platelet transfusions are administered to haemato-oncology patients, where these serve as an important adjunctive therapy in treatment of hypoproliferative thrombocytopenia secondary to chemotherapy or haematopoietic stem cell transplantation (HSCT). International guidelines recommend prophylactic platelet transfusions for hypoproliferative thrombocytopenia to reduce the risk of spontaneous bleeding when platelet count is $<10 \times 10^{9} / 1$. A recent Cochrane systematic review in this patient cohort found that, overall, prophylactic platelet transfusions appeared to reduce bleeding events and days with significant bleeding. However, the benefit from prophylactic platelet transfusions differed between treatment groups, with autologous HSCT patients deriving least benefit. Bleeding frequently occurs in these patients despite prophylactic platelet transfusions, and other risk factors such as fever, duration of thrombocytopenia, underlying disease and treatment regimen should be considered in transfusion decision-making.

In thrombocytopenic patients, platelet concentrates may also be given prophylactically prior to surgery or other invasive procedures. However, the causes of thrombocytopenia are different between haemato-oncology and other clinical contexts (e.g. cardiac surgery), and relationship between platelet count and risk of bleeding in many of these settings is not strong. Evidence to guide transfusion practice remains limited. There are no randomised controlled trials (RCTs) to guide platelet transfusion prior to invasive procedures. National guidelines vary. AABB recommends patients are transfused at a platelet count of $<20 \times 10^{9} / 1$ prior to elective CVC insertion, or $<50 \times 10^{9} / 1$ prior to elective lumbar puncture and major non-neuroaxial surgery Higher thresholds may be needed for surgery in critical sites (eye, brain).

Neonates in the neonatal intensive care unit frequently become thrombocytopenic and intracranial haemorrhage (ICH) is a major concern. However, many platelet transfusions are prescribed to treat neonatal thrombocytopenia with little evidence to support this practice. Guidelines directing neonatal platelet transfusion practice vary considerably, and are generally consensus rather than evidence-based. There has only been one RCT in preterm neonates; this found no difference in the incidence of ICH between a liberal regimen (platelet transfusion when platelet count $<150 \times 10^{9} / 1$ ) and a more restrictive regimen (platelet count $<50 \times 10^{9} / 1$ or clinical concern about bleeding). The Planet-2 RCT is comparing a platelet transfusion threshold of 25 vs $50 \times 10^{9} / 1$ in neonates $<34$ weeks in whom ICH has been excluded. Therapeutic platelet transfusions are usually indicated in the setting of active bleeding when thrombocytopenia or platelet dysfunction are contributing factors, or in critical bleeding necessitating large-volume transfusion and with a falling platelet count. However, platelet transfusions are not indicated in all cases of thrombocytopenia. Importantly, conditions where platelet transfusions are generally avoided unless critical bleeding is present include immune-mediated thrombocytopenia 
thrombotic microangiopathies and congenital thrombocytopenias such as Glanzmann's thrombasthenia.

\section{POSTERS}

\section{Management and Organisation 1.1 Organisational Issues}

P-001

This abstract has been withdrawn.

\section{$\mathrm{P}-002$}

INFRASTRUCTURE OF BLOOD TRANSFUSION ROOMS AT INPATIENT FACILITIES IN KAZAKHSTAN

M Ospanova, S Abdrakhmanova, R Magzumova and Z Burkitbayev

Scientific and Production Center of Transfusiology, Astana, Kazakhstan

Background: Currently, the provision of highly specialized hospital care requires using transfusion therapy. New national transfusion care approaches are therefore developed to improveequipment in order to modernize the blood transfusionfacilities. Aim: is to assess the organizational aspects of clinical transfusion practices in Kazakhstan.

Materials and methods: Statisticalanalysis conducted on the results of monitoring the data obtained from all transfusion rooms at in-patient facilities in Kazakhstan Results: In Kazakhstan, there are currently 473 health care organizations (HCO) that provide blood transfusion services, of which 427 include a specific department (room) for blood transfusion, which is on average 91.9\% $\pm 11.1,100 \%$ median throughout the country.

At the national HCO where they have high number of transfusion therapies, full-time rate is allocated to $70 \%$ of transfusion experts. Whereas, in regional, municipal and district-level HCOs, full-time rate is allocated to less than $27 \%$ of transfusion experts. In 275 HCOs (representing 58\%), 0.25-0.5 of the full-time rate is allocated for transfusionexperts, in combination with other physicians.

In $102 \mathrm{HCO}$ no full-time transfusion expert rates are present, accounting for $22 \%$, which requires further strengthening of the organizational and methods in order toprovision of good quality transfusion services.

Analyzing the absolute number of transfusion-certified physicians, we can assume that there is a lack of transfusionexperts in the national-level HCOs in Almaty. It is necessary to train transfusionexperts for further employment at regional, municipal and district-level medical organizations.

In 427 departments (rooms) certified transfusionexperts staffing ratio is 0.71 . In some regions, this figure does not exceed 0.3 .

The table below gives a brief description on specialized equipment availability of blood transfusion rooms at in-patient health care organizations of different level: national, regional, municipal and district-level.

Performing immune-haematological studies on surface does not allow to correctly diagnose complex cases (rare phenotype, chimerism), and the specialized equipment for agglutination microcolon method are available only in $9 \%$ of hospitals

There is a tendency to develop a policy of blood conservation, particularly reinfusion of autologous blood components, which reduces the risk of transfusion of allogeneic haemo-components. 26 health care organizations are equipped with the device for intraoperative reinfusion of autologous red blood cells, which is 5\%

Table 1. Equipment availability of blood transfusion rooms at in-patient health care organizations throughout Kazakhstan.

\begin{tabular}{|l|l|c|c|c|c|}
\hline № & Basic equipmenttype & $\begin{array}{c}\text { National } \\
\mathrm{HCO}(\%)\end{array}$ & $\begin{array}{c}\text { Regional } \\
\mathrm{HCO}(\%)\end{array}$ & $\begin{array}{c}\text { Municipal } \\
\mathrm{HCO}(\%)\end{array}$ & $\begin{array}{c}\text { District- } \\
\text { level HCO } \\
(\%)\end{array}$ \\
\hline 1 & $\begin{array}{l}\text { Specialized refrigeration equipment for } \\
\text { storage of erythrocyte-containing blood } \\
\text { components and frozen blood } \\
\text { components }\end{array}$ & 100 & 51.7 & 52.2 & 51.9 \\
\hline 2 & $\begin{array}{l}\text { Platelets mixers } \\
3\end{array}$ & 23 & 3 & 6 & 0.5 \\
\hline 4 & Plasma defrosters & 95 & 79 & 44 & 20 \\
\hline 5 & Machines for heating of red blood cells & 23 & 16 & 13 & 0 \\
\hline 6 & $\begin{array}{l}\text { Water bath } \\
\text { hel serology systems for immune- } \\
\text { haematological tests }\end{array}$ & 64 & 43 & 55 & 35 \\
\hline 7 & Thermal containers & 80 & 100 & 1 & 15 \\
\hline & $\begin{array}{l}\text { Average overall equipment } \\
\text { avallability }\end{array}$ & $\begin{array}{l}81,3 \pm 49,9, \\
\text { median } 80 \%\end{array}$ & $\begin{array}{l}41.8 \pm 31.0, \\
\text { median 43\% }\end{array}$ & $\begin{array}{l}40.3 \pm 22.0, \\
\text { median 44\% }\end{array}$ & $\begin{array}{l}28.1 \pm 27.5 \\
\text { median 20\% }\end{array}$ \\
\hline
\end{tabular}

Conclusions: Overall, $71 \%$ of in-patient facilities are staffed with transfusionexperts. Overall, 43\%of transfusiondepartments (rooms) are equipped with basic equipment throughout the whole country $(37.2 \% \pm 25.2,43 \%$ median$)$.

\section{P-003 \\ A BRIEF LOOK, WORK LOAD AND SUPERVISION OF A DAY CARE CENTER IN A TERTIARY LEVEL HOSPITAL BSMMU, BANGLADESH A FOUR YEAR STUDY \\ SS Miah \\ Bangabandhu Sheikh Mujib Medical University (BSMMU), Dhaka, Bangladesh}

Background: Transfusion of blood component is now widely used to maintain of the glowing survivility of the patients. Transfusion and its safety invariably depends on the physicians and its being a good choice in using the rationale use of components. The day care now being a new door for the patients as it is a key intervention in decreasing morbidity and mortality of patients and for those patients who are totally dependent on transfusion. In esteem of remote area, still there is working of whole blood. As a transfusion specialist, it is now important to demonstrate the actual picture of good practice of component, which makes the entire transfusion more effectual for the patients.

Aims: To determine the out patient treatment that is increasing in the day care in year to year. The effectiveness of component therapy that have been proven in making the clinical practice more accepted. Day care transfusion therapy permits less hospital stay and can be more proficient for the patients who are only dependent on transfusion. It could guide the others to provide these poor patients in maintaining their transfusion with the presence of a skilled team and also the balanced use of components.

Methods: It is a retrospective type of study and total data of the patients were taken from the records of patient registration who had taken transfusion in the day care. Study period started from July 2011 to June 2015. The recorded data also provide which type of component is transfused for patients. Before transfusion therapy a prescribed data like name, age, sex, pre and post transfusion status and clinical diagnosis were recorded. The required components were selected carefully for transfusion of each patient. and were monitored carefully by the doctors.

Results: It is an analysis of four years (from July 2011 to June 2015) and total attending patients were 7203 to 8362 . In the overall objervation male patients were $62.9-63.6 \%$ and female patients were $36.4-38.3 \%$, Male: Female ratio is about 1.6 1.7:1. Among them RCC were transfused in patients with thalassemia were 88.43$90.14 \%$, anaemia due to chronic disorder $8.45-11.54 \%$, anaemia due to malignancy $0.10-0.49 \%$, anaemia due to chemotherapy $0.75-1.95 \%$, IDA $0.04-0.23 \%$, and others $0.02-0.05 \%$. RCC with Platelet concentrate were transfused in patients with Leukaemia 53.0-64.2\%, aplastic anaemia 35.1\%- 46.3\% and others $0.5-0.7 \%$. Total FFP and Cryoprecipitate were supported to haemophillic patients were 98.1-99.5\%, VWD $0.5-1.7 \%$ and others were about $0.1-0.2 \%$

Conclusion: The clinical transfusion of blood and components is required for expanded indications. The benefits of transfusion must be balanced with the inherent risks. Transfusions remain as an crucial part for the management of patients like thalassemia, haemophillia, aplastic anaemia, leukaemia etc. where there is no alternative for transfusion. By making more day care, also in remote areas we can minimize the costs. Therefore, donor availability with donor panel will also ensure through this placing and for those who are critically ill and dependent in the day care.

\subsection{Information Technology}

P-004

STANDARDIZED 2D LABEL DESIGN FOR BLOOD PRODUCTS

E Cabana ${ }^{1}$, P Ashford ${ }^{1}$, A Brazier ${ }^{2}$, B Kirkpatrick ${ }^{3}$, B Wray ${ }^{4}$, J Georgsen ${ }^{5}$, L Lodge ${ }^{6}$, N Kok Quan ${ }^{7}$, R Skagestad $^{8}, \mathrm{~S}$ Grabowski ${ }^{9}$, T Campbell $^{10}$ and W Bolton ${ }^{11}$ ${ }^{1}$ ICCBBA, San Bernardino, United States of America ${ }^{2}$ NHS Blood and Transplant, Newcastle upon Tyne and Wear, United Kingdom ${ }^{3}$ Digi-Trax, Lincolnshire, United States of America ${ }^{4}$ Computype, St. Paul, United States of America ${ }^{5}$ Odense University Hospital, Odense, Denmark ${ }^{6}$ Scottish National Blood Transfusion Service, Edinburgh, United Kingdom ${ }^{7}$ Health Sciences Authority, Singapore, Singapore ${ }^{8}$ Labcraft, Oslo, Norway ${ }^{9}$ Gulf Coast Regional Blood Center, Houston, United States of America ${ }^{10}$ One Blood, Tampa, United States of America ${ }^{11}$ Australian Red Cross, Adelaide, Australia

Background: The ISBT 128 Standard was designed to ensure the highest levels of accuracy, safety, and efficiency for donors and patients. Blood establishments using ISBT 128 reported increasing challenges of including more information on blood component labels. Additionally, facilities seek to increase efficiency in processing

(C) 2015 The Author

Vox Sanguinis (C) 2015 International Society of Blood Transfusion Vox Sanguinis (2015) 109 (Suppl. 2), 1-96 
hundreds of thousands of units each year without compromising safety. The current international ISBT 128 blood label was designed over 15 years ago and has four, sometimes five, standardized linear barcodes. It was proposed that a new label design, using a single 2D barcode (i.e., Data Matrix), be developed to satisfy these demands.

Aims: Moving towards a single 2D barcode on the label will:

1. Increase efficiency by reducing the number of barcode scans;

2. Allow for better organization of information;

3. Decrease training and confusion on which barcode to scan.

Methods: An international committee was formed by ICCBBA with experts from various regions.

They began with published survey results (Transfusion Med. 2014 Apr;24 (2): 89-98) from the UK which determined hospital views on the optimal placement of critical information on a label. Two of the authors participated on this committee. Then label information was categorized as either necessary or unnecessary. The Committee then surveyed blood facilities and hospitals in various regions to identify elements of the label that could be internationally standardized.

Different scanning mechanisms (e.g., hand held scanners and flatbed scanners) were taken into consideration when determining the best placement of the $2 \mathrm{D}$ barcode. Several draft labels were distributed for comment and a best-fit design was created based on feedback.

Results: A new label design was created. Information that is important to the enduser is in the upper half of the label. This includes the Donation Identification Number, Product Code, product name, $\mathrm{ABO} / \mathrm{RhD}$, expiry date, volume, and the Data Matrix symbol.

The lower right of the label includes test results and the processing facility (if different than the collection facility).

The lower left of the label contains the collection date, storage conditions, regulatory text, static information, and collection facility. Component specifications may be provided via a second $2 \mathrm{D}$ barcode (using $\mathrm{QR}$ symbology) that takes the user to a website that displays this information.

An ISBT 128 compound message will be used to encode the information into the Data Matrix symbol. Minimally it will include the Donation Identification Number, Product Code, $\mathrm{ABO} / \mathrm{RhD}$, and expiry date. Additional information may optionally be encoded

Summary/Conclusions: The ISBT 128 standardized blood label will move towards the use of a single Data Matrix barcode and eventually eliminate linear barcodes on the label. This will improve efficiency at the blood center and hospital by requiring a single scan to capture all the information currently encoded within 4 or 5 linear barcodes. It will group key information for the transfusionist in the upper half of the label, while static information will appear in the lower half of the label. A transition period of ten years is anticipated. Facility software must be updated before the new label can be implemented. Design feedback can be submitted to ICCBBA.

\section{P-005}

\section{SOLVENT DETERGENT PLASMA STANDARDIZED LABEL}

E Cabana ${ }^{1}$, P Ashford ${ }^{1}$, A Brazier ${ }^{2}$, S Greenwell ${ }^{3}$, T Laakso ${ }^{4}$, W LeFresne ${ }^{5}$, B Quirke $^{6}$, $\mathrm{M}$ Rechsteiner $^{7}$, A Riezebos $^{8}$ and R Skagestad ${ }^{9}$

${ }^{1}$ ICCBBA, San Bernardino, United States of America ${ }^{2}$ NHS Blood and Transplant, Newcastle upon Tyne and Wear, United Kingdom ${ }^{3}$ American Red Cross, Washington DC, United States of America ${ }^{4}$ Finnish Red Cross Blood Service, Helsinki, Finland ${ }^{5}$ Canadian Blood Services, Nova Scotia, Canada ${ }^{6}$ Irish Blood Transfusion Service, Dublin, Ireland ${ }^{7}$ Octapharma AG, Lachen, Switzerland ${ }^{8}$ Sanquin, Amsterdam, The Netherlands ${ }^{9}$ Labcraft, Oslo, Norway

Background: Unlike most products that are made from plasma from a large number of donors, $\mathrm{ABO}$ blood group is relevant to solvent detergent-treated plasma (SDP). Therefore, the blood group must be on the affixed label, preferably in both eye- and electronically-readable format. The relevancy of blood group, and the fact the product is handled in a similar way to Fresh Frozen Plasma, mean it is typically managed by blood banks rather than pharmacies. Because blood banks use ISBT 128, and because ISBT 128 supports the encoding of blood groups and other key information, both ICCBBA and GS1 recommend labeling SDP using the ISBT 128 standard on the affixed label. Aims: The aim of the work was to design a globally-standardized affixed label with key information presented in both eye- and electronically-readable format. Such a label would improve patient safety, as well as improve the accuracy and efficiency of recording information required for traceability.

Methods: ICCBBA, the organisation that manages the ISBT 128 Standard, established a team of experts to design an internationally-standardized affixed label for SDP. The team, which met by conference call, comprised professionals responsible for managing SDP in blood centres and hospitals, ICCBBA staff, and a representative of Octapharma, a major manufacturer of SDP. A proposed label design was released for public comment and the comments received were incorporated into a final label design.

Results: The standardized affixed label includes critical information in the upper half of the label encoded into linear bar codes following the ISBT 128 standard. Corresponding text information is placed near each bar code. This critical information includes:

1. Donation Identification Number (DIN) to uniquely identify each plasma product

2. ABO blood group

3. Product code

4. Expiration date

The relative position of the bar codes is the same as on an ISBT 128 blood label DIN and blood group bar codes are at the top of the label placed in a way to support concatenation, while product code and expiration date bar codes are beneath the DIN and blood group, also placed to support concatenation.

Regulatory and other country-specific information is just beneath the horizontal centre of the label and may be bar coded. Manufacturer information, lot number and peel-off labels are on the bottom of the label.

Text may be in any language and two languages on a label are possible.

Other electronic information may appear on outer packaging of an SDP unit. Twodimensional (2-D) symbols may be used to encode this information.

Summary/Conclusions: An internationally-standardized affixed label for SDP utilizing ISBT 128 has been designed and approved. ISBT 128 is commonly used in blood banks around the world and its use on SDP supports automated capture of critical information by computer systems in these facilities. Accurate entry of key information improves patient safety and as well as the accuracy and efficiency of recordkeeping. Organisations are encouraged to request that their supplier of SDP provide products labelled with ISBT 128.

\section{P-006 \\ METRICS FOR ACCEPTANCE TESTING FOR EGYPTIAN BLOOD MANAGEMENT SYSTEM (BMS)}

A Elgohary, L Borhan and M Labib

National Blood Transfusion Services, Cairo, Egypt

Background: The implementation of the BMS in Egyptian blood transfusion services is a necessary step towards monitoring, saving time and decreasing human errors. Acceptance testing (AT) is a vital prerequisite for the implementation of BMS needed to fulfill the business rules of any blood transfusion centre.

(AT) is a means to measure quality by challenging the efficiency of the utilized software. This can be achieved by choosing reflective in-process metrics which validate and quantify the ability of the system in place.

Aims: To quantify the accuracy of (AT) performed on the BMS software using inprocess metrics as useful indicators.

To utilize these metrics to highlight problems, encountered in (AT) to ensure the quality of all BMS application components and their successful module integration from donation to patient care

Methods: Test data was collected from January to July 2015

A review of 'Software requirement specifications'(SRS) was done to:enable the detection of missing requirements, detect the need for more information, and identify mistakes in SRS as 'comments' which are analyzed in terms of severity.

The ability of the system to meet these comments was evaluated; defects were recorded and assigned the metric 'requirement review'.

Test cases (TCs) were developed based on the SRS, subsequently The degree of requirements coverage by (TCs) was calculated using an equation and named the metric 'requirement coverage'

Requirement Coverage $=$ Total no. of requirements supported by TCs/Total no. of requirements

The third important metric is number of defects vs fixed defects. These defects are weighted in terms of severity and this evaluation process must be reflected in the acceptance criteria

Finally TCs were executed, recorded and analyzed, then execution results were categorized as: pass/ fail. The 'fail' status generated a defect record and indicated that the targeted function hadn't been achieved. This metric was named 'Number of defects/executed TC'.

Result: Regarding the metric 'Requirements Review', Our study found 128 missing requirements, in the SRS which otherwise wouldn't be included in the final applied software.

The percentage of requirement coverage found by (TCs) was $81 \%$. However the remaining 19\% lacking coverage need to be addressed by increasing number of TCs (effectiveness) or enhancing the efficiency of the existing TCs. 


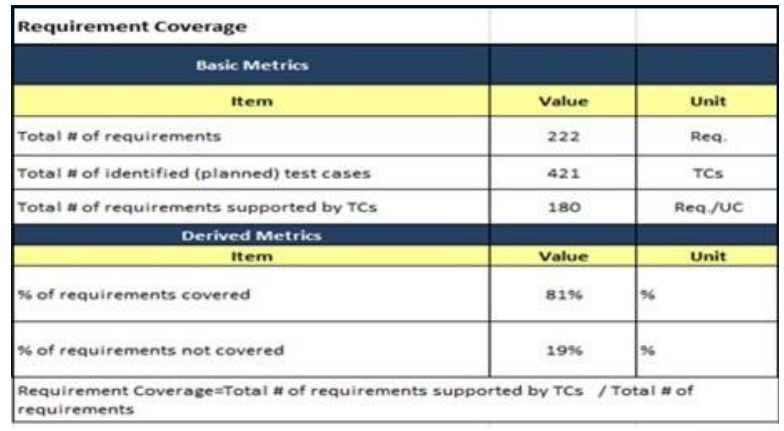

Requirement coverage by test cases

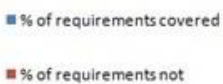
covered

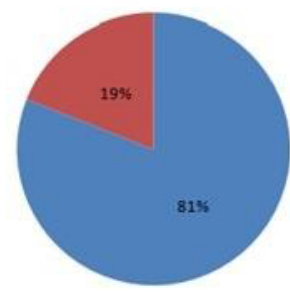

Metrics related to Requirements Review

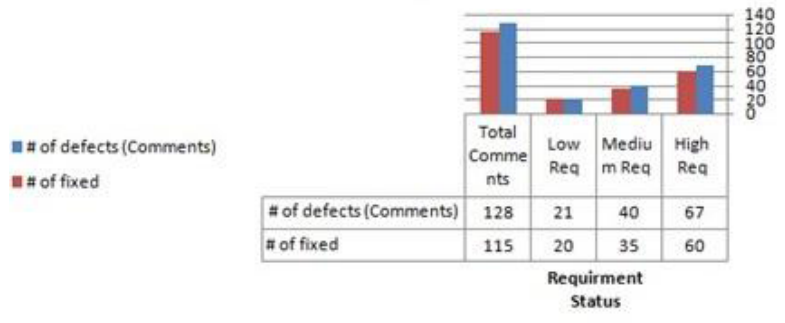

Number of defects found and fixed per executed test cases

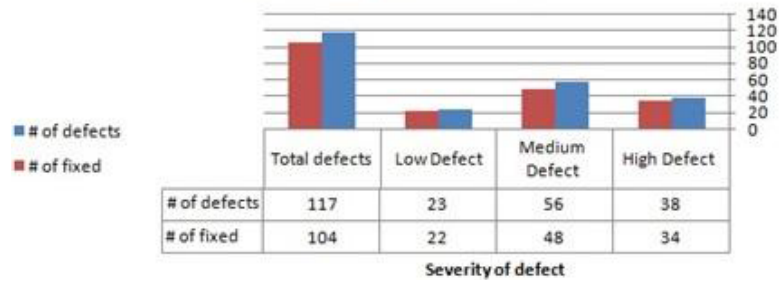

The percentage of fixed defects in relation to total defects was calculated at $88 \%$ (104 fixed / 117 Total defects), it is noted that $88 \%$ defects fixed, is a positive indication of software integrity and

Conclusion: These metrics are vital to (AT) in isolating missing requirements, evaluating requirement coverage through test case analysis and achieving quality assurance by assessing number of fixed defects. Our study illustrated how the development of these metrics was achieved through the customization of already existing data. Such metrics can be utilized by similar blood banks, in the process of (AT) provided that they follow the same steps and adapt these metrics to conform to their own business rules.

In conclusion it's clear that implementation of these metrics merits serious consideration for efficient running of Blood management projects.
P-007

TESTING SECURITY OF THE BLOOD MANAGEMENT SYSTEM, THROUGH THE USAGE OF NON FUNCTIONAL TESTING, IN THE NATIONAL BLOOD TRANSFUSION CENTRE (NBTC), CAIRO, EGYPT

S Abdullah ${ }^{1}$, A Ahmed $^{2}$, M Labib ${ }^{2}$, A Ibrahim ${ }^{1}$, M Shokri ${ }^{2}$ and A Abady ${ }^{2}$ ${ }^{1}$ NBTC, Cairo, Egypt ${ }^{2}$ National Blood Transfusion Center, Cairo, Egypt

Background: The Blood Management System (BMS) is a project which will automate manual operations in 17 sites of the Egyptian National Blood Transfusion Services (ENBTS). Protecting data is a vital issue to guarantee business continuity. Security is an essential requirement that is fulfilled by having the suitable hardware and network architecture. Security functions are granted to each user according to the degree of their involvement in the blood transfusion system. These user privileges limit the transfer and sharing of data, and other functionalities amongst users in real time.

Non Functional Security Testing is a method used to measure whether the BMS application and its infrastructure is secure or not.

Aims: To examine how Non Functional Security Testing can be used as a tool to measure how far the BMS software, hardware and network are secured.

Methods: The IT team at ENBTS arranged a series of meetings to study the BMS security requirements, namely 'Test Basis' (the network, hardware and application design) in detail. In order to extract any points of weakness in the design and provide useful feedback.

A database of Non Functional Security Test cases was generated by using the check list testing technique, which is a technique that creates a catalog of requirements that need to be tracked within the BMS, concerning security.

The generated test cases were classified by site ('headquarters only' or 'all sites') and by severity, and their impact on the flow of work. The outcome from these test cases is embedded into the execution plan database.

The execution plan database, challenges the various security steps, detects and also categorizes defects according to severity. These findings are then relayed and solutions are found, taking security requirements into consideration.

Finally the execution plan in all its revised stages will be run, to achieve user acceptance with regards security of the BMS.

Results: The ENBTS IT team created a repository of about 100 test cases extracted from the security requirements, which highlighted several points:

The importance of firewall and optimal antivirus software installment.

Security lists should be applied on switches.

To protect data, it was found that full identification of each user must be monitored during login; our system is expected to dictate password changes at a pre-determined period (30 days).

Locking of the session if users don't use the system within a set period of time, to protect confidentiality.

Control of access authorization to: read, update, create and delete data; according to the user's personal functionality definition. Especially concerning data defined by the blood center's management as sensitive.

Conclusion: Non functional security testing insures the integrity, and safety of any system, especially one as the BMS. It predicts problems, protects businesses from data theft and saves money. Such considerations are crucial in low income settings and an institution as sensitive as a blood transfusion centre.

Achieving strong security is mandatory to achieve confidentiality, build trust, and strengthen the donor and patient relationship with the ENBTS.

P-008

BENEFITS OF IMPLEMENTATION OF BLOOD DONORS DATA

BASE MANAGEMENT SYSTEM VS MANUAL RECORDING

SYSTEMS IN DAR EL SALAM REGIONAL BLOOD TRANSFUSION CENTER

A Selim Sayed, R Farouk, R Farouk Badr and S Mohamed

Dar El Salam Regional Blood Transfusion Center, Cairo, Egypt

Background: Blood bank management system is a browser based system that is designed to store, retrieve and analyze information concerned with all aspects, starting from recruiting blood donors to issuing of blood units. Prerequisites for implementation of such technology include: adequate infrastructure, quality system, appropriate staffing, and sufficient funding. Blood donor module plays an important role to build secured donor database, recruiting regular donors, encouraging recall for aphaeresis and rare blood group donors, exclude disqualified donors from further donations and postpone temporary deferred donors.

(C) 2015 The Author Vox Sanguinis (C) 2015 International Society of Blood Transfusion Vox Sanguinis (2015) 109 (Suppl. 2), 1-96 
Aims: To highlight the impact of implementation of electronic Blood Management System over the current manual documentation system used in the Egyptian National Blood Transfusion Services (NBTS).

Methods: This study was conducted by studying the workflow in Dar El-Salam Regional Blood Transfusion Center, using the current manual documentation system versus the predicted results for Blood Management System software (E-Delphyn) which will be implemented soon in Egyptian National Blood Transfusion Services (NBTS).

Results: The database will include information of each donor visit: personal information, donation location, date, and type, serological tests and any additional tests (e.g. phenotyping). Users use the stored data to:

Provide a pool of regular blood donors.

Avoid wasting time/effort searching for rare blood group donors especially in emergencies.

Follow up temporarily deferred donors, with suitable notification system, preventing loss of healthy potential donors.

Follow up and prevent further donation for donors with reactive serological results. (In the year 2014, $144(0.68 \%), 470(2.23 \%), 43(0.20 \%)$ and $28(0.13 \%)$ units were reactive to $\mathrm{HBsAg}$, Anti-HCV, HIV, and Syphilis respectively of total blood collected and discarded. Those number could be drastically decreased by recognizing repeated donors with TTIs using the BMS)

Previous adverse reaction from donation can be managed correctly either with appropriate follow up or permanent deferral. (e.g. 1.87\% of Donors from JuneDecember 2014 suffered from Vasovagal Reactions, using a solid database would make their next donation process safer)

Help to monitor trends and progress of donation collection and donor pool through precise and effective information retrieval system and statistical analysis.

On the other hand, maintenance and updating of blood bank records, currently, are done manually. Listed below are some of the problems of using the present system: Records are subjected to loss and/or damage.

Difficulties in retrieving data.

Securities of records are minimal.

Difficulties in statistical analysis and making reports.

Summary/Conclusions: Computerization leads to rapid performance, accurate transcription, large volume storage of information, and fast retrieval of records improving performance and quality of blood banks operations.

\section{P-009}

IMPACT OF INFORMATION TECHNOLOGY IMPLEMENTATION IN MOBILE UNIT TO TRANSMITTED TRANSFUSION INFECTION RATE M Marwiyah, NK Ritchie, S Salim and SE Herny Paskah

Jakarta Blood Transfusion Unit, DKI Jakarta, Indonesia

Background: In this modern era, information technology (IT) is needed in almost every institution. Blood Transfusion Units (BTUs) also need IT to manage the blood donor's data, the production's data and many other data. In 2010, Jakarta BTU implemented an IT system from the $3^{\text {rd }}$ party, called 'Sistem Informasi Transfusi Darah' (SITD). This system linked all the data and we could trace any blood components from donor to patients. This traceability system is the most important things for blood safety. We can trace the history of donations so we could prevent them to donate again if their previous result was reactive in transfusion transmitted infection (TTI) screening. The great about this system was not just used inside our building but we also carried them to mobile unit and mobile bus. Years before SITD implemented, we used manual system to know the donation's history. It was not effective and time-consuming. So, we hope by implementing SITD, it will reduce the frequency of TTI screening because we could prevent the 'reactive' donor donating their blood.

Aims: To know the impact of SITD implementation in the frequency of TTI screening. Methods: Every day, mobile unit team must synchronize the data into their laptops. In every mobile unit team, there was a laptop, functioning as a server, and minimum a laptop, as a client. The server contained the database and the client used for the data-input. Server and client were connected with wi-fi. There was a unique code for any 'reactive' donors, so the staff could directly refer or counsel them to defer the donation. In the afternoon, when they arrived back at office, they synchronize the 'new' data into the main server. Results: After 10 year of SITD implementation, we found there were decreasing trend in the frequency of TTI screening results. From $1.32 \%$ in 2009 , now only $0.58 \%$ for HBsAg-results; from $0.69 \%$ to $0.36 \%$ in anti-HCV results, $0.28 \%$ to $0.19 \%$ in HIV and $1.1 \%$ to $0.49 \%$ in syphilis testing-results. We could the effective impact of SITD implementation in TTI screening results. The staff also feel satisfy and helped by SITD implementation. Summary/conclusions: IT implementation in mobile unit was effective to reduce the TTI screening results.

(C) 2015 The Author

Vox Sanguinis (C) 2015 International Society of Blood Transfusion

Vox Sanguinis (2015) 109 (Suppl. 2), 1-96
P-010

INCREASED PRODUCTIVITY WITH A PAPERLESS WORKING ENVIRONMENT AT APHERESIS

SC Khoo, NR Rahamat and CHW Sim

Health Sciences Authority, Singapore, Singapore

Background: Manual documentation of donation procedures at Apheresis requires effort and space. Relevant data such as donor's full blood count and other blood results in relation to each procedure are written on paper records, which in turns, require the manual retrieval of these records for future references, especially when donor returns for a donation.

Limited space for record-keeping proves to be perpetual challenge as the Bloodbank currently has an estimate of 9000 procedure manual records on a yearly basis. There is a need to explore, with the use of current technology, a more efficient method on how the Apheresis records are stored and retrieved.

Aims: A recording system with the use of the latest available technology enables the department to reduce time spent on documentation. Easier retrieval of records would eventually help to improve work processes, thus cutting down manpower and turnaround time. With the elimination of manual records, space concerns would be resolved as all records would be kept electronically.

Methods: In September 2014, a customized software programme, with the use of hand-held portable devices (PDA), was implemented. The system is designed to manage Apheresis donations in a paperless working environment and provides real -time update to allow Apheresis staff to easily access vital information in a mobile fashion. With this, the process of donor monitoring during a procedure also enables staff to record directly the procedure status to the system.

The connection of the system to the hematology analyzer also allows instant update of the donors' full blood count and calculated platelet QC results for easy reference. Results: Immediate access to donor information resulted in a smoother work process as less time is spent tracking for records as staff only need to enter donor's identification number to the system upon retrieval. In general, staff satisfaction is improved as time savings permits them to deliver better quality care to Apheresis donors. As all Apheresis records are now kept electronically, space-savings too, is one of the many benefits the department has gained.

Summary/Conclusions: The world around us is constantly developing and life has dramatically changed in the last few decades. Using advanced technology has transformed traditional system into intelligent system to enhance work efficiency and productivity. The conversion to electronic records is initially costly, but over time, easy access to information proves to be a determinant in overall improved productivity and strengthening key aspects of work. As technology has been integrated into our lives, staff is also able to adapt naturally to the relevant changes in order to achieve a positive outcome.

\subsection{Cost/Effectiveness}

P-011

\section{RESULTS OF ESTABLISHMENT OF WALK-IN BLOOD DONOR PANELS ON ISLAND AND REMOTE AREAS IN VIETNAM} MQ Ngo, TH Le, TMA Bui, AT Nguyen and TD Pham

National Institute of Hematology and Blood Transfusion, Ha Noi, Vietnam

Background: In every region and in every situation, even in the most remote and island areas, ensuring safe and adequate supply of blood and blood components has to be met. In the remote regions, blood supply is based on three main sources: emergency supply from large blood transfusion centers, storage of blood components and maintenance of a pool of walk-in blood donors.

Vietnam is a geographically diverse country with a coastline of 3,444 kilometers, more than 4,000 different islands and 287 remote districts part of 48 provinces, which significantly affects the quality of health care service in general and blood safety in detail. Ensuring a sufficient blood supply in that situation is challenging; however, so far, this was not studied before. Therefore, a pilot study titled 'Establishing substantial and stable walk-in blood donor panels for island and remote areas has been implemented in Vietnam during 2011-2014.

Aims: This paper aims (i) to describe the result of establishing of walking blood bank in some island and remote districts and (ii) to describe the result of collection and usage of whole blood from walk-in blood donors in emergencies.

Methods: We selected two largest islands (Cat Hai and Phu Quoc district) and two typical rural and remote districts in the Northern (Dien Bien Dong district) and the Southern part (Tinh Bien district) of Vietnam to conduct a prospective study from 2011-2014. Results: National Institute of Hematology and Blood Transfusion cooperated with the four described districts to establish the walk-in donor panels with 321 walk-in blood donors. The average age of blood donors was $33.9 \pm 8.1$ years old, 65.7\% 
was male and $65.4 \%$ had type 0 blood. Blood was screened for Hepatitus B (HBsAg), Hepatitus C (anti-HCV), anti-HIV by using ELISA techniques and these tests were performed annually. Among 617 screening tests, we found that 24 tests were positive (3.9\%) including 17 positive for $\mathrm{HBsAg}$ and 7 positive for anti-HCV.

Four rehearsals were carried out in 4 districts to access the suitability of the procedure, the ability of applying as well as the effectiveness of walk-in blood donor panels. 25 walk-in blood donors were called, of who 13 came to donate blood; the average time for presence of blood donors to give blood was $16.46 \pm 8.9 \mathrm{~min}$. In emergency situations, 12 patients required 30 units of whole blood; therefore, we called 43 walk-in blood donors, of who 30 walk-in donors donated blood, the percentage of people coming was 68.3\%. 30 units of whole blood were collected, $60 \%$ of them were blood group 0; donation rate was $100 \%$. Additionally, the results of both rapid tests and ELISA for HBsAg, anti-HCV and anti-HIV were found negative. Conclusions: Four districts in remote and island areas of Vietnam established a substantial, effective and stable walk-in donor panels comprised of 321 donors. 12 activations and 30 safe units of whole blood were collected, which ensured a good blood supply in emergencies.

Table 1: The result of annual blood screening tests.

\begin{tabular}{|l|c|c|c|c|c}
\hline & $\begin{array}{c}\text { Cat } \\
\text { Hai }\end{array}$ & $\begin{array}{c}\text { Phu } \\
\text { Quoc }\end{array}$ & $\begin{array}{c}\text { Dien Bien } \\
\text { Dong }\end{array}$ & $\begin{array}{c}\text { Tinh } \\
\text { Bien }\end{array}$ & Total \\
\hline Number of donors & 72 & 86 & 83 & 106 & 347 \\
\hline $\begin{array}{l}\text { Registration to join in walk-in } \\
\text { blood donor panel }\end{array}$ & 5 & 5 & 7 & 7 & 24 \\
\hline Being rejected & 1 & 1 & 0 & 0 & 2 \\
\hline Leaving & 66 & 80 & 76 & 99 & 321 \\
\hline Walk-in donors & & & & & 24
\end{tabular}

Table 2: The recruitment of walk-in blood donors.

\begin{tabular}{|l|c|c|c|c|c}
\hline & Cat Hai & $\begin{array}{c}\text { Phu } \\
\text { Quoc }\end{array}$ & $\begin{array}{c}\text { Dien } \\
\text { Bien } \\
\text { Dong }\end{array}$ & $\begin{array}{c}\text { Tinh } \\
\text { Bien }\end{array}$ & Total \\
\hline $\begin{array}{l}\text { Number of } \\
\text { screening tests }\end{array}$ & 115 & 154 & 131 & 217 & 617 \\
\hline HBsAg (+) & 3 & 2 & 6 & 6 & 17 \\
\hline Anti-HCV (+) & 2 & 3 & 1 & 1 & 7 \\
\hline Anti-HIV & 0 & 0 & 0 & 0 & 0 \\
\hline $\begin{array}{l}\text { Number of positive } \\
\text { screening tests }\end{array}$ & 5 & 5 & 7 & 7 & 24 \\
\hline $\begin{array}{l}\text { The rate of positive } \\
\text { screening tests }\end{array}$ & 4.3 & $3, .2$ & 5.3 & 3.2 & 3.9 \\
\hline
\end{tabular}

$\mathrm{P}-012$

\section{A REVIEW OF SEGMENT TESTING ON OLYMPUS PK AND THE DESIGN OF AN AUTOMATED SEGMENT CUTTER \\ B Mitchell and S Valensky}

Western Province Blood Transfusion Service, Cape Town, South Africa

Background: In South Africa, it is legislated that the ABO/Rh be confirmed on the segment from the pack. When crossmatches were performed manually, this took place at the crossmatch stage.

When WPBTS changed to an automated crossmatch method, segment testing was still performed manually at WPBTS blood banks because of the cost of automated grouping. This resulted in an unacceptable increase in workload, was tedious and not practical.

Intervention: Costing was done comparing manual testing, Autovue and testing on the Olympus PK. Routine segment testing on the Olympus PK was implemented in December 2006 after adapting a test method. Approximately 650 segments from the initial packs are tested daily and 13\% of these packs are modified (e.g. paediatric or filtered units) and retested.
Outcome: The cost of testing the ABO/Rh on the Olympus PK is significantly lower than other methods.

From Dec 2006-July 2015, segment testing on the Olympus PK has cost R167,219 compared to manual grouping at R2,246,050 and using the Autovue at R26,094,842 for the same period. Workload in the Blood Banks was reduced and no extra staff had to be employed in Blood Grouping laboratory where the Olympus PK was situated. While there were financial benefits other problems surfaced i.e. the tediousness of manually cutting segments for testing on the Olympus PK and manual verification of serial numbers.

Further changes: In 2010, WPBTS contracted a S.A. company LTS to design an automated segment cutter. A prototype was designed and tested in 2013 but a decision was taken to scrap the prototype due to many problems experienced. The automation was re-engineered. This was made easier by the fact that SANBS (South Africa's other transfusion service) had decided to use the 'Beval' automation to cut segments and also perform segment testing on the Olympus PK. There was now a demand for 6 segment cutters in South Africa.

Design of Automated 'Beval': The barcoded segments are placed in a jig to keep them rigid. The jig is then placed in an input rack on the 'Beval' automation. The jig containing the segments pass a scanner which reads the segments barcode, replicates it and places the barcode on a tube which is situated in an Olympus PK input rack. The jig then proceeds to a station which cuts the top and bottom of the segment and allows the cells to run into the labelled tube. Saline is added and the Olympus rack is then ready to load on the Olympus PK.

Late July 2015 the 'Beval' was placed at WPBTS after batches being validated at the manufacturer.

Conclusion: Making the decision to test segments on the Olympus has proved successful for WPBTS. It has been cost effective and time saving. The design of an automated segment cutter has eliminated the manual cutting of segments and streamlined the process in the laboratory.

P-013

\section{EFECTIVITY AND COMPARATION CHECKING HAEMOGLOBIN}

USING CUPRIC SULFATE WITH HB CHECKER

D Devitri, F Femi Luwita, DDH Sidabutar and I Irsan Sirajudin

Indonesian Red Cross, Tangerang City Blood Centre, Tangerang, Indonesia

Background: Hemoglobin is a protein molecule in red blood cells, that function as media transport oxygen from the lungs to all body tissues and carrying carbon dioxide from the body's tissues to the lungs. Checking of $\mathrm{Hb}$ is one part to selection process before blood tapping and the absolute thing to do because the results is relates to the health of donors and the quality of the blood to be transfused to patients. The normal values of $\mathrm{Hb}$; Male 14-16 g/dl, Female $12-14 \mathrm{~g} / \mathrm{dl}$.

\section{$\mathrm{Hb}$ Checker}

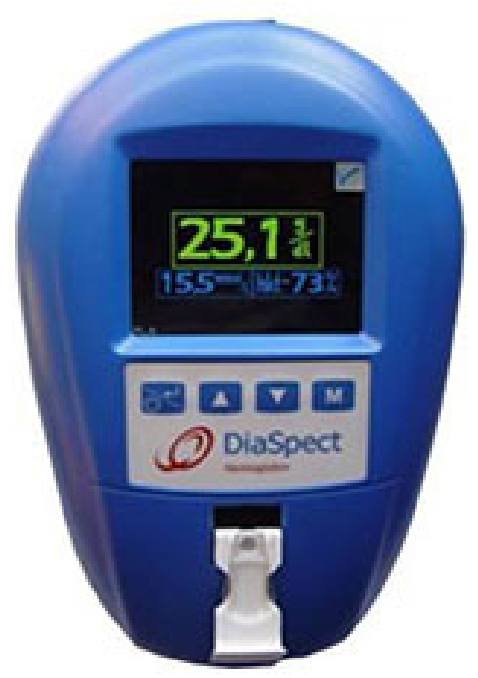

Figure 1: Hb Checker.

(C) 2015 The Author

Vox Sanguinis (C) 2015 International Society of Blood Transfusion Vox Sanguinis (2015) 109 (Suppl. 2), 1-96 
Aims: To determine the effectiveness of inspection hemoglobin levels using cupri sulfate with $\mathrm{Hb}$ Checker.

Methods: This research methods used a solution of cupric sulfate (CuSO4) and $\mathrm{Hb}$ Cheker, where CuSO4 measuring Hb levels is based on differences in the density of blood with a specific gravity of a solution CuSO4. Checking used BJ 1053 and BJ 1062, For donor requirements with BJ 1053 may be taken if the blood sinks which means normal $\mathrm{Hb}$. The interpretation is go down is mean $\mathrm{Hb}>12.5 \mathrm{~g} / \mathrm{dl}$, floating means $\mathrm{Hb}=12.5 \mathrm{~g} / \mathrm{dl}$ and cut in half means $\mathrm{Hb}<12.5 \mathrm{~g} / \mathrm{dl}$. Using $\mathrm{BJ} 1062$ to ensure that $\mathrm{Hb}$ is still in the normal range, which is less than $17 \mathrm{~g} / \mathrm{dl}$ with blood interpretations that can be taken to be floated $\mathrm{Hb}<17 \mathrm{~g} / \mathrm{dl}$. Hb Cheker: represents the development of the method of determination of $\mathrm{Hb}$ by means by spectrophotometer. This method is based on optical density in the cuvette which has a capacity of 10 microliters by light rays coming from within 0.133 millimeters to the wall parallel optical slits are a cuvette, which will then display the number of $\mathrm{Hb}$ values measured on the screen

Results: The effectiveness of using a solution cupric sulfate BJ 1.053 and 1.062 with $\mathrm{Hb}$ Checker if reviewed from service cost then cupric sulfat from BJ 1.053 and $1.062 \times$ Rp. $100,000=$ Rp. 200,000 , the volume each $500 \mathrm{ml}$ were used together Once usage in $1 \mathrm{ml}$ beaker glass $30-40$ to \pm 20 drops of inspection. then: $500 \mathrm{ml} /$ $30 \mathrm{ml}=16$ beaker. 1 beaker glass to \pm 20 drops/person. Beaker $16 \times 20$ people $=320$ people. So, cupric sulfate with BJ $1053(\mathrm{Hb}<17 \mathrm{~g} / \mathrm{dl})$ and BJ $1062(\mathrm{Hb}>$ $17 \mathrm{~g} / \mathrm{dl}$ ) for 320 donors only need Rp. 200,000. Hb Cheker method using a cuvette with price Rp.5000 per cuvette. 1 box cuvette $50 \times$ Rp $5000=R p$ 250,000. Eg, 320 donors ( 320 cuvette) $\times \operatorname{Rp} 5000=\operatorname{Rp} 1.600 .000$

Conclusion: Based on comparation data, using cupric sulfate was more effective, we can saving Rp. 1,400,000 with an equally quality already established by the WHO

Table 1: Comparation Using $\mathrm{Hb}$ Checker with Cupric sulfate.

\section{Comparation using $\mathrm{Hb}$ checker with Cupric sulfate}

\begin{tabular}{|c|l|l|l|}
\hline No & Method & Checking & Price \\
\hline 1 & Cupric Sulfate & $\begin{array}{l}\text { 320 donors } \times 2 \text { botle } \\
\text { (@1 botle Rp. 100.000) }\end{array}$ & Rp. 200.000; \\
\hline 2 & Hb Checker & 320 cuvette $\times$ Rp. 5000; & Rp. 1.600.000; \\
\hline
\end{tabular}

\subsection{Training and Education}

P-014

\section{RED CELL SEROLOGY WORKSHOP}

KK Leou ${ }^{1}$, KS Kang ${ }^{1}$ and Y Chen ${ }^{2}$

${ }^{1}$ Health Sciences Authority, Singapore, Singapore ${ }^{2}$ Singapore International

Foundation, Singapore, Singapore

Background: A Blood Group Serology practical workshop was organised in partnership with the National Blood Centre (NBC) in Yangon, in conjunction with a Blood Transfusion Symposium, co-organised by the International Society of Blood Transfusion and the NBC in March 2015.

The 41 participants who attended the workshop were mainly from the peripheral hospitals in Yangon and Mandalay. Although they had been trained to perform tests using tube method, it was not common practice for hospitals in Myanmar to perform red cell antibody screen for pre-transfusion testing except for the haematological cases in NBC.

Aims: To assess the practical abilities of laboratory staff in blood group serology in performing $\mathrm{ABORh}$ typing, resolving $\mathrm{ABO}$ discrepancy, compatibility tests and antibody screen using tube method.

Methods: The workshop was prepared and organised by the Singapore International Foundation Volunteers (SIVs) in NBC. As the laboratory space was only able to accommodate 21 workstations, 2 workshop sessions were conducted with participants sharing table-top centrifuges, microscopes and water baths.

Participants were briefed on the purpose of the workshop before its commencement. They were instructed to test 3 samples for $\mathrm{ABO}$ and $\mathrm{RH}$ typing with one $\mathrm{ABO}$ discrepancy, 2 samples for antibody screening, 2 samples for compatibility testing and one sample for DAT, including multiple choice questions, followed by a demonstration on $\mathrm{ABO}$ blood group typing and antibody screen using tube method.

There were also discussions on the problems faced in performing the test procedures and how to resolve blood group discrepancies.

\section{(C) 2015 The Author}

Vox Sanguinis (C) 2015 International Society of Blood Transfusion

Vox Sanguinis (2015) 109 (Suppl. 2), 1-96
Results: Participants were asked to self-assess their knowledge before and after attending the workshop based on a scale of 1-5; 1 indicating 'Very Poor' and 5 being 'Very Good'. 20.0\% reported an improvement from 'Average' to 'Very Good', 73.3\% from 'Average' to 'Good', 6.7\% from 'Poor' to 'Average' after attending the workshop. Practical results showed that the participants performed fairly well in antibody screen, compatibility tests and the DAT, despite the fact that hospitals in Myanmar did not perform these tests using tube method. However, resolving ABO discrepancies remained a challenge.

The discussions at the end of the workshop provided further understanding and emphasised the important of accurate serological testing, reagent control and quality assurance on the tests performed.

Conclusions: The post workshop evaluation questionnaire was encouraging, with requests for longer workshop duration.

Table 1: Practical Results for Antibody Screen \& XM.

\begin{tabular}{|c|c|c|c|c|}
\hline Table 1 shows the results for ABO Rh typing \\
\hline \multirow{2}{*}{$\begin{array}{c}\text { Test } \\
\text { Samples }\end{array}$} & $\begin{array}{c}\text { ABO Typing } \\
\text { Expected Results }\end{array}$ & $\begin{array}{c}\text { No. of } \\
\text { Correct } \\
\text { Result }\end{array}$ & $\begin{array}{c}\text { No. of } \\
\text { Incorrect } \\
\text { Result }\end{array}$ & $\begin{array}{c}\text { Wrong } \\
\text { interpretation }\end{array}$ \\
\hline Sample A & B Positive & 41 & 0 & 0 \\
\hline Sample B & A Positive & 41 & 0 & 0 \\
\hline & $\begin{array}{c}\text { O Positive } \\
\text { Sample C } \\
\text { with unexpected cold } \\
\text { antibodies present. }\end{array}$ & 17 & 4 & 20 \\
\hline
\end{tabular}

Table 2: Practical Results for ABO \& DAT.

\begin{tabular}{|c|c|c|c|}
\hline \multicolumn{2}{|c|}{ Table 2 shows the results for Direct Anti-globulin Test (DAT) } \\
\hline \multirow{2}{*}{ Test Sample } & $\begin{array}{c}|c| \\
\text { DAT }\end{array}$ & \multicolumn{2}{|c|}{ Participants results } \\
\cline { 3 - 4 } & Expected Results & Positive & Negative \\
\hline Sample F & Positive & 40 & 1 \\
\hline
\end{tabular}

\begin{tabular}{|c|c|c|c|c|c|c|}
\hline $\begin{array}{c}\text { Test } \\
\text { Samples }\end{array}$ & $\begin{array}{l}\text { Antibody } \\
\text { Screen } \\
\text { Expected } \\
\text { Results }\end{array}$ & $\begin{array}{l}\text { No. of } \\
\text { Correct } \\
\text { Results }\end{array}$ & $\begin{array}{c}\text { No. of } \\
\text { Incorrect } \\
\text { Results }\end{array}$ & $\begin{array}{c}\text { No } \\
\text { conclusions } \\
\text { given }\end{array}$ & $\begin{array}{l}\text { Errors in } \\
\text { coombs } \\
\text { neg ctrl }\end{array}$ & Total \\
\hline Sample D & Negative & 31 & 1 & 4 & 5 & 41 \\
\hline Sample E & $\begin{array}{l}\text { Positive with } \\
\text { abnormal } \\
\text { antibodies } \\
\text { present }\end{array}$ & 33 & 2 & 4 & 2 & 41 \\
\hline
\end{tabular}

\begin{tabular}{|c|c|c|c|c|c|c|}
\hline \multirow{3}{*}{$\begin{array}{c}\text { Test } \\
\text { Samples }\end{array}$} & \multirow{3}{*}{$\begin{array}{l}\text { Expected } \\
\text { Results }\end{array}$} & \multicolumn{5}{|c|}{ Participants results } \\
\hline & & \multirow[b]{2}{*}{ Compatible } & \multirow[b]{2}{*}{$\begin{array}{c}\text { In- } \\
\text { compatible }\end{array}$} & \multirow[b]{2}{*}{$\begin{array}{l}\text { Unable to } \\
\text { interpret }\end{array}$} & \multicolumn{2}{|c|}{ Coombs QC } \\
\hline & & & & & $\begin{array}{l}\text { Not } \\
\text { Done }\end{array}$ & $\begin{array}{l}\text { Invalid } \\
\text { results }\end{array}$ \\
\hline $\begin{array}{l}\text { Sample } \\
\text { D vs C }\end{array}$ & Compatible & 39 & 0 & 2 & 4 & 2 \\
\hline $\begin{array}{l}\text { Sample } \\
\text { E vs H }\end{array}$ & $\begin{array}{c}\text { In- } \\
\text { compatible }\end{array}$ & 2 & 37 & 2 & & \\
\hline
\end{tabular}

The workshop had introduced performing blood group serology using the tube method which is still accepted as the 'gold standard' for testing. However, most of the equipment and reagents were not easily available in Myanmar and this needed to be resolved first before antibody screening could be implemented. Antibody screening is important as it is a basic requirement to minimize transfusion reactions and difficulties in getting compatible blood for patients. While SIVs had provided training in preparing screening cell panels in NBC, it had been a challenge for them to supply to hospitals in wide scale.

Participants in the workshop were keen to adopt the enhanced testing method, retrain staff and improve the quality control procedures in providing safer blood supply in Myanmar. 
P-015

ACHIEVING STANDARDISATION OF EGYPTIAN BLOOD

TRANSFUSION PRACTICES, THROUGH A NATIONWIDE TRAINING PROGRAMME ON THE IMPLEMENTATION OF THE EGYPTIAN NATIONAL BLOOD TRANSFUSION STANDARDS

L Samir Borhan Ragheb, S Shabayek and M Fag El Nour

NBTC, National Blood Transfusion Centre, Cairo, Egypt., Cairo, Egypt

Background: The Ministry of Health and Population, in recognition of the magnitude of the HCV burden in Egypt, collaborated with multiple stakeholders and developed, the 'Plan of Action for the Prevention, Care and Treatment of Viral Hepatitis, Egypt'20142018, the PoA, encompassing seven components:surveillance, infection control, blood safety, HBV vaccination, patient care and treatment, communication and research. To address blood safety, the National Blood Transfusion Services in Egypt with WHO support, launched the nationwide implementation of the Egyptian National Standards, $3^{\text {rd }}$-Edition. Training on these standards was vital to achieve uniform dissemination of, approved blood transfusion practices to all transfusion service providers in Egypt.

Aims: A qualitative research that aims to: illustrate how implementation of Egyptian National Standards can consolidate all blood bank services, under one common vision and practice.

To examine how the inclusion of practical recommendations from this training can improve transfusion education.

Methods: Focus groups were held in the form of training sessions, which were standardised in terms of content and trainers, and focussed on the 12 sections and annexes of the Egyptian National Blood Transfusion Standards.

Trainees were from hospital blood banks within 7 health-sectors: Ministry of Health, university, teaching, medical insurance, the Red Crescent, private sector, and Armed Forces. Trainees included: physicians, nurses, lab-technicians and transfusion practitioners from all disciplines, especially those involved in other staff members' education.

Table 1: Training Schedule for the implementation of the National Blood Transfusion Standards, 3rd Edition, July to August 2015.

Iraining Schedule for the implementation of the National Bloo
Iransfusion Standards. 3rd Edition. July to August 2015

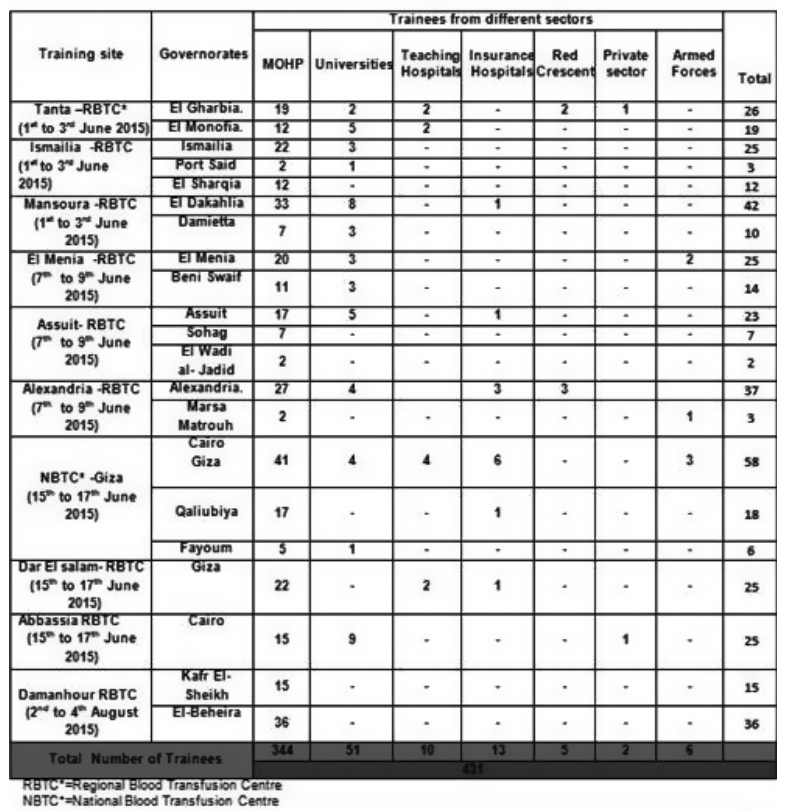

Trainee contributions were transcribed and categorised into two themes:

1. Standardising transfusion practices.

2. Improving training strategies.

Results: 431participants from 20 governorates were trained from June -August 2015, and formed 10 focus groups with approximately 35-40 participants /group. Recurrent themes from focus groups highlighted the following recommendations:

1. The importance of achieving standardisation through the establishment of a nationally recognised regulatory body to ensure consolidation of all blood transfusion practices in all sectors across Egypt.
2. The establishment of hospital transfusion committees whose duities include: overseeing standards implementation in transfusion practices from 'vein to vein', revising Appropriate-Clinical- Use-of-blood protocols, adverse events accounting.

3. Training should: meet needs of blood bank staff and be executed through case scenarios rather than cascade learning, have CME credit points assigned to all training activities, be a continuous process, encourage constructive feedback by staff that is incorporated in the standard's biannual revision.

4. Training should follow a defined curriculum in Arabic and English, implemented in 'protected time', and assessed by yearly competency tests in three levels: Basic, Intermediate and Advanced.

5. The Egyptian fellowship of Blood transfusion, (a nationally recognised multi-disciplinary course) should be a mandatory qualification for all blood bank service providers. 6. Accommodating staff from distant governorates through incorporating e- learning in training regimens.

Conclusion: The inclusion of recommendations from these focus groups will address issues that are raised by the staff themselves, and re-structure blood transfusion services to the needs of those involved in service delivery.

Such restructuring ultimately aims at standardising transfusion practices in Egypt which is a mandatory step towards achieving blood safety, preventing blood related infections and controlling the viral hepatitis epidemic, and realising the 2018 goal of HCV control in Egypt.

P-016

WHO WORKSHOP 2014: THE BLOOD TESTING, RISK ASSESSMENT, ENFORCEMENT AND IMPLEMENTATION AS PART OF GOOD MANUFACTURING PRACTICES (GMP) IN BLOOD ESTABLISHMENT IN INDONESIA

Blood Transfution Centre, Tangerang City Red Cross, Tangerang City, Indonesia

Background: The blood testing, risk assessment, enforcement and implementation as part of Good Manufacturing Practices (GMP) in blood establishment is considered a priority as a tool to minimize the risk of transmission. GMP is a system for ensuring that products are consistently produced and controlled according to quality standards appropriate to their intended use and as required by the product specification. This priority has been consistently highlighted by medicines regulatory authorities at International Conferences of Drug Regulatory Authorities (ICDRA). Blood centre In Indonesia still don't have reference of quality standard, such as ISO, AABB, and National Standard, Many Blood centre in Indonesia still can't fulfilled natural quality standard of Indonesia. there are no blood centre using GMP standard. A number of educational programs and training Workshops are being jointly developed by WHO Headquarters and its Regional Offices, to respond to the needs it. including this workshop.

Aim: The purpose of this workshop was to strengthen and upgrade the technical expertise and provide information of Medicines Regulatory Authorities and National Blood Programmes on GMP to assure the quality and safety of blood components, and to evaluate available information and summarise what conclusions can be made and what research is still needed to fill gaps in knowledge about enforcement and implementation of GMP in blood establishment in Indonesia.

\section{Participants}

The Blood Testing, Risk Assessment, Enforcement and Implementation as Part of Good Manufacturing Practices (GMP) in Blood Establishment In Indonesia

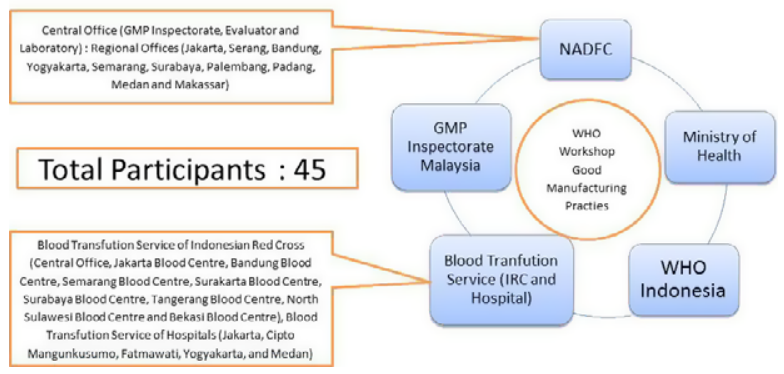

Figure 1: Participant.

(C) 2015 The Author

Vox Sanguinis (C) 2015 International Society of Blood Transfusion Vox Sanguinis (2015) 109 (Suppl. 2), 1-96 


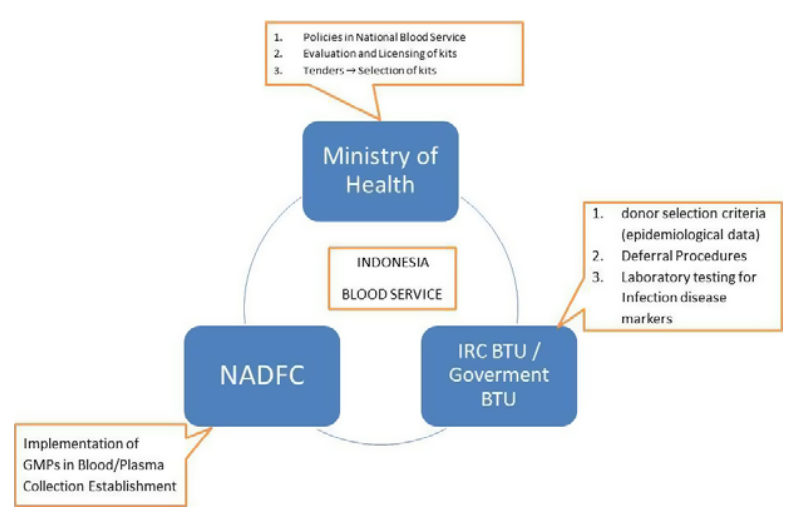

Figure 2: Indonesian Blood Service.

Methods: The WHO workshop on enforcement and implementation of gmp in blood establishment do with two open sessions (9-12 June 2004 and 24-27 June 2014) which is the first session invited speakers presented on specialized topics on Blood Testing and Risk Assessment as Part of GMP in Blood Establishment and other related topics. There was a closed Working Group meeting to finalise a report for WHO on Thurday 12 June 2014. And in the second session invited speakers presented on specialized topics on Enforcement and Implementation of GMP in Blood Establishment and do the Mock Inspection in working Groups in Blood Centres, among other Jakara Blood Centre, Tangerang City Blood Centre, and Bekasi City Blood Centre. There was a closed Working Group meeting to summing up and presentation result of the Mock Inspection, Conclusion and Recommendations on Friday 27 June 2014

Result: The WHO workshop 2014 enforcement and implementation of gmp in blood establishment followed by National Agency of Drag and Food Control-Indonesia, Ministry Of Health, WHO Indonesia, Blood Transfusion Service (Indonesian Red Cross and Hospital), and total participants is 45 Participants. Participants can practice enforcement and implementation of GMP in blood centre fit in section. Participants also knowing about blood testing and risk assessment as part of gmp in blood establishment

Conclusion: The GMP in blood establisment can be as Part quality standards In Indonesia. Enforcement and implementation of GMP in blood establishment and the WHO workshop for GMP system must be consistently each years in Indonesia.

P-017

This abstract has been withdrawn.

\section{P-018}

\section{INDONESIAN APHERESIS EDUCATION EXPERIENCE}

$\mathrm{R}_{\text {juwita Sembiring }}{ }^{1}$ and $\mathrm{H}$ Vrielink $^{2}$

${ }^{1}$ Columbia Asia Hospital, Medan, Indonesia ${ }^{2}$ Sanquin Blood Supply, Amsterdam, Netherlands Antilles

Background: Workers in medical health, especially for Apheresis care need to be qualified and competent for their job. Indonesian Society on Thrombosis Hemostasis collaboration with Joint Task Force for Apheresis Education a group of experienced apheresis physicians and nurses from Europe and the USA was given certification course for physician and nurses/operators at the end of March 2015 in Medan Indonesia.

Aims: To do a certification course of apheresis in physicians and nurses/operatorsMethods: The topics of certification course consist of basics and instrumentation apheresis, blood a versatile, collection of platelets, adverse events of platelet collections, prevention and treatment of apheresis complications, therapeutic apheresis introduction, therapeutic plasma exchange procedure, blood cell reduction and pediatric apheresis was given in March 2015. The participant made a test prior and posttest. The test could be made in Bahasa Indonesia.

Results: The participants of certification course consisted of 38 clinical phatologists doctor, 2 general doctor and 21 nurses/operators. There is significant increase of the post test of doctors from $56.6 \pm 8.1$ to $77.4 \pm 6.9$ ( $\mathrm{P}<0.0001)$ and nurses/operators from $49 \pm 6.6$ to $72.8 \pm 4.3(\mathrm{P}<0.0001)$. There are a significant different of pre(C) 2015 The Author

Vox Sanguinis (C) 2015 International Society of Blood Transfusion

Vox Sanguinis (2015) 109 (Suppl. 2), 1-96 test and post- test value of physicians compare nurses/operators $56.6 \pm 8.1$ and $49.0 \pm 6.6(\mathrm{P}<0.001), 77.4 \pm 6.9$ and $72.8 \pm 4.3(\mathrm{P}<0.008)$.

Conclusions: The course in apheresis as given in Medan in March 2015 has been shown of value for physician as well as for nurses/operators. All participants score > 54 points in the post-course test and could be certified. There is increase of knowledge on apheresis and all participants certified.

\section{P-019}

DEVELOPING HUMAN CAPITAL IN GLOBAL TRANSFUSION SAFETY RESEARCH: A MID-COURSE ASSESSMENT OF THE PROGRAM

E Murphy ${ }^{1}$, E Bloch ${ }^{2}$, B Custer ${ }^{2}$, C Shiboski ${ }^{3}$, E Sabino $^{4}$, AB Proietti ${ }^{5}$, W McFarland ${ }^{6}$, $\mathrm{M} \mathrm{Busch}^{2}$ and S Laperche ${ }^{7}$

${ }^{1}$ UCSF/BSRI, San Francisco, United States of America ${ }^{2}$ BSRI/UCSF, San Francisco, United States of America ${ }^{3}$ UCSF, San Francisco, United States of America ${ }^{4}$ University of Sao Paulo, Sao Paulo, Brazil ${ }^{5}$ Hemominas, Belo Horizonte, Brazil ${ }^{6}$ San Francisco Department of Public Health (SFDPH), San Francisco, United States of America ${ }^{7}$ Institut National de la Transfusion Sanguine (INTS), Paris, France

Background: Clinical research in viral infections, donor selection and quality assessment is an important contributor to blood safety. However in low and middle income countries, such research has been limited by a lack of trained clinical research personnel. Long-term training in Europe or the USA is impractical due to high cost and long absence from primary job responsibilities.

Aims: Assess an existing program of clinical research training and develop new approaches to build a new generation of transfusion medicine researchers in lowand middle-income countries.

Methods: The existing program recruits blood transfusion professionals in low and middle income countries who wish to pursue clinical research. In 2-week short courses, experts in transfusion medicine and epidemiology teach the principles of clinical research with readings from a textbook and illustrated with examples from the experts' own research. Each trainee brings a research question relevant to his/her local blood bank and develops it into a 6-page research protocol during workshop sessions. After the course, many trainees accomplish their projects with assistance from a seed grant program and ongoing mentorship by course professors. The most promising trainees are later invited for 6-week internships at UCSF and BSRI in San Francisco to analyze data, learn laboratory techniques and write manuscripts. A

\section{Table 1. Short Course} History and Trainees

\begin{tabular}{|l|l|l|}
\hline Date & Place & Trainees \\
\hline May 2004 & Sao Paulo, Brazil & 9 \\
\hline Sep 2005 & Buenos Aires, Argentina & 13 \\
\hline Oct 2006 & Tegucigalpa, Honduras & 7 \\
\hline May 2007 & Paris, France* & 7 \\
\hline Oct 2007 & Johannesburg, South Africa & 12 \\
\hline May 2008 & Paris, France* & 10 \\
\hline Sept 2008 & Belo Horizonte, Brazil & 16 \\
\hline Oct 2008 & Durban, South Africa & 10 \\
\hline May 2009 & Paris, France* & 11 \\
\hline Aug 2009 & Recife, Brazil & 17 \\
\hline Oct 2009 & Cape Town, South Africa & 12 \\
\hline May 2010 & Paris, France* & 13 \\
\hline Aug 2010 & Riode Janeiro, Brazil & 14 \\
\hline Nov 2010 & Kruger Park, SouthAfrica & 8 \\
\hline May 2011 & Paris, France* & 11 \\
\hline May 2012 & Paris, France* & 8 \\
\hline June 2012 & Manaus, Brazil & 15 \\
\hline Nov 2012 & Nairobi, Kenya & 13 \\
\hline May 2013 & Paris, France* & 11 \\
\hline May 2014 & Paris, France* & 9 \\
\hline May 2015 & Paris, France* & 10 \\
\hline & TOTAL & 236 \\
\hline
\end{tabular}

comprehensive review of the program was conducted by the first author during a sabbatical leave in 2014-2015.

Table 1.

Results: The Table summarizes locations and number of trainees for research training courses conducted from 2004-2015. A total of 236 trainees from Latin America, Africa and other regions have participated in 21 courses. Fifteen trainees have participated in subsequent 6-week internships at UCSF and BSRI in San Francisco. Transfusion research networks have been developed in both Brazil and South Africa (funded by the NHLBI REDS-III International Program) and francophone Africa (coordinated by the French INTS). The sabbatical assessment included visits to training alumni and potential trainees at blood centers in 7 African countries (Zimbabwe, South Africa, Tanzania, Malawi, Cameroon, Cote d'Ivoire and Burkina Faso. There 
was great enthusiasm for the training courses but alumni requested: more mentoring; skills development in data analysis, biostatistics and manuscript preparation; and the possibility of Master's degree training in disciplines relevant to clinical research. Following the sabbatical, an application for a NIH Fogarty HIV Research Training Grant has been submitted.

Conclusions: After eleven years, a new curriculum in clinical and epidemiological research has produced new human capital in clinical research but better mentoring of past trainees during their early research careers is needed. We are now focused on obtaining stable funding to allow advanced training for the network of trainees using a hybrid approach of in-country short courses, medium term internships in the USA and Master's degree training in South Africa. (Supported by an ISBT Foundation Grant, Blood Systems Research Institute, French ART and GEMHEP grants, UCSF Center for AIDS Research, an educational grant from Novartis and NIH grants K24-HL-075036, D43-TW000003 and D43-TW05799).

\section{$\mathrm{P}-020$}

This abstract has been withdrawn

\subsection{Blood Supply Management and Utilization \\ P-021 \\ RETROSPECTVE AUDIT ON THE APPROPRIATENESS OF} PLATELETS USAGE IN A TERTIARY HOSPITAL IN SINGAPORE N Yacob Marican ${ }^{1}$, W Chang ${ }^{1}$, PLK Lim ${ }^{1}$, TTS Lim ${ }^{1}$, SY Lee ${ }^{2}$, ES Yap ${ }^{2}$ and LK Tan ${ }^{2}$ ${ }^{1}$ National University Hospital, Singapore, Singapore ${ }^{2}$ Department of HaematologyOncology, National University Cancer Institute, Singapore, Singapore

Background: The decision to transfuse platelets should be based on assessment of risks vs benefits to patients. Several established clinical guidelines including American Association of Blood Banks and British Committee for Standards in Haematology, exist to serve as guides to appropriate platelet transfusions. However, in spite of these guidelines, inappropriate platelet transfusions still persist.

Aims: We conducted a retrospective audit on platelet usage in our institution to assess the appropriateness of platelet transfusion in different medical disciplines. Methods: Data was collected over a six month period from August 2014 to Jan 2015. As part of our transfusion criteria, a platelet count must be determined prior to any platelet transfusion. In addition, the patient's clinical situation and other risk factors for bleeding were also considered for the appropriateness of transfusion.The decision whether the platelet transfusion was deemed appropriate or not, was based on the criteria set by our haematologists in concordance to available guidelines.Requests were deemed appropriate if transfused for: (1) active bleeding in the presence of platelet dysfunction, regardless of platelet count.(2) platelet count $<20 \times 10^{9} / \mu \mathrm{l}$, (3) platelet count $\left(20-50 \times 10^{9} / \mu \mathrm{l}\right)$ with active bleeding, undergoing invasive procedure in next $24 \mathrm{~h}$, major surgery in the last $48 \mathrm{~h}$, massive transfusion, epidural catheter insertion in next $24 \mathrm{~h}$ and undergoing surgery to CNS or eye in next $24 \mathrm{~h}$, (4) platelet count $\left(50-80 \times 10^{9} / \mu \mathrm{l}\right)$ with massive transfusion, epidural catheter insertion in next $24 \mathrm{~h}$, CNS or intraocular bleeding, undergoing surgery to CNS or eye in next $24 \mathrm{~h}$, bleeding from multiple trauma, active bleeding with platelet dysfunction, (5) platelet count $\left(80-100 \times 10^{9} / \mu \mathrm{l}\right)$ with CNS or intraocular bleeding, undergoing surgery to CNS or eye in next $24 \mathrm{~h}$, bleeding from multiple trauma, active bleeding with platelet dysfunction. Requests were deemed inappropriate if transfused for: (1) platelet count $>20 \times 10^{9} / \mu$ l, without active bleeding and any risk factors (2) platelet count $>20 \times 10^{9} / \mu \mathrm{l}$, without any of the situations above. Risk factors for bleeding include sepsis, rapid fall of platelet count, presence of other associated coagulopathies, co-existing medical conditions such as liver disease and renal failure. Results: A total of 2918 requests for platelet transfusions were received and reviewed. A total of 2729 requests were deemed appropriate with 189 inappropriate requests, an overall incidence rate of 6.5\%. Orthopaedic, opthamology and geriatric medicine had 100\% inappropriate platelet transfusion request. The main reason is for prevention of bleeding rather than treating a bleeding episode as all these patients have platelet count $>200 \times 10^{9} / \mu$ l. The most common inappropriate requests identified were (1) transfusion when platelet count $>100 \times 10^{9} / \mu$ l without any bleeding or undergoing any invasive procedures (2) platelet count $>100 \times 10^{9} \%$ $\mu \mathrm{l}$ without any indication of platelet transfusion.

Summary/Conclusions: The rate of inappropriate platelet transfusion was found to be $6.5 \%$ in our facility. Physicians need to be educated and clinical guidelines to be adhered strictly prior to any platelets requests, to minimize unnecessary platelet transfusion.
$\mathrm{P}-022$

ANALYSIS OF TRANSFUSION REQUIREMENTS AND FACTORS PREDICTING PERI-OPERATIVE BLOOD AND BLOOD COMPONENT TRANSFUSION IN PATIENTS UNDERGOING RENAL TRANSPLANT

RN Makroo, B Kakkar, A Bhatia, M Chowdhry and S Naik

Indraprastha Apollo Hospitals, New Delhi, India

Background: Transfusion therapy plays an important role in the success of solid organ transplant programmes. Given the complexity of these surgeries auditing the transfusion requirements and identifying risk factors of excessive blood consumption is a tool that aids in better inventory management and improved preparedness for such procedures.

Aim: To audit transfusion of blood and blood components and to identify factors predicting transfusion requirements in patients undergoing renal transplant at our centre.

Methods: The records of all renal transplant surgeries performed between 1st May 2014 to 31st December 2014 were retrieved and reviewed. Patient demographics, medical and surgical history, duration of dialysis, medications along with pre-operative laboratory parameters and PLOS (post-operative length of stay) were recorded. The blood and blood component usage including packed red cells (PRC), fresh frozen plasma (FFP), cryoprecipitate (cryo) and platelet concentrate (PC), during the intraoperative as well as postoperative phase was obtained from the blood bank records. Pediatric renal transplants (less than 18 years) were excluded from the study.

Results: A total of 510 patients (398 males; 78\% and 112 females; 22\%) ranging from 18 to 77 years in age underwent renal transplant during the study period. Of these, 269 (52.7\%) patients did not receive any transfusion, while 241 (47.3\%) patients received a total of 845 units of blood and blood components. Out of these, 263 units were utilised in intra-operative period $(\mathrm{PRC}=184 ; \mathrm{FFP}=79)$ and 582 units $(\mathrm{PRC}=340, \mathrm{FFP}=219 ;$ Cryo $=6 ; \mathrm{PC}=17)$ in post-operative period. The mean pre-operative haemoglobin in the transfused group was $8.7 \mathrm{~g} / \mathrm{dl}$ while in nontransfused group it was $10.3 \mathrm{~g} / \mathrm{dl}$. Average consumption of blood and blood components per patient per transplant was 1.03 units PRC, 0.6 units of FFP, 0.03 units of platelet concentrate and 0.01 units of cryoprecipitate. Intraoperative blood consumption was largely limited to 1-2 units of PRC and FFP each, with only 8 patients receiving 3 or more PRC and 6 patients receiving 3 or more FFP units during the surgery. Multivariate regression analysis revealed that pre-operative haemoglobin was a major predictor of intra-operative PRC transfusion $(\mathrm{P}=0.000)$ but did not affect the post-operative blood consumption. Average PLOS was $10 \pm 6$ days. There was no significant difference in the PLOS between the transfused and non-transfused groups of patients, however, a statistical significant increase in utilization for both PRC $(P=0.044)$ and FFP $(P=0.002)$ was observed with increased PLOS. Among the other parameters assessed as predictors of blood consumption none reached statistical significance. Mortality was seen in 2 patients while post-operative graft dysfunction was noted in 25 patients, out of which 6 were re-explored.

Conclusion: Nearly $47.3 \%$ of patients undergoing renal transplant received transfusion and PRC was the most common product transfused. Pre-operative haemoglobin and PLOS were identified as strong predictors of blood consumption.

P-023

This abstract has been withdrawn

$\mathrm{P}-024$

A STUDY OF BLOOD UTILIZATION TREND IN A TERTIARY CARE HOSPITAL LOCATED IN THE FOOTHILLS OF HIMALAYAS IN NORTHERN INDIA

DK Bains and MK Kandwa

Max Superspeciality Hospital, Dehradun, India

Background: Blood being a biologic agent, is associated with both infectious and noninfectious complications. As blood components are a scarce and expensive resource, there is a continuous need for monitoring of blood utilization and auditing of transfusion practices which serves in identifying key areas in the blood component usage and inappropriate blood component request or use, wherein corrective actions can be planned. This study was designed to assess and review the blood component requisitions and utilization as per each speciality in a tertiary care corporate hospital. Aim: To determine the quality indicators of blood utilization and analyze the trend of utilization of different blood components by various medical and surgical specialties at our hospital.

(C) 2015 The Author

Vox Sanguinis (C) 2015 International Society of Blood Transfusion Vox Sanguinis (2015) 109 (Suppl. 2), 1-96 
Method: The transfusion data was reviewed for all the patients requiring blood component transfusion at our hospital for a period of six months from January 2015 to June 2015 using patient transfusion request forms, hospital information system, crossmatch register, and issue register.The information obtained from the documents included age, gender, blood group, consultant incharge, speciality, diagnosis and indication of transfusion, pre transfusion hemoglobin/platelet count/coagulation profile The data pertaining to number of whole blood (WB)/packed red blood cells (PRBC)/fresh frozen plasma (FFP) /random donor platelet concentrates (PC)/single donor apheresis (SDAP) platelet concentrates requested as well as crossmatched and issued was collected. The quality indicators for blood utilization were calculated as crossmatch to transfusion ratio (CT ratio), Transfusion Probability (\%T) and Transfusion index (TI). Result: A total of 1210 (795 male and 415 female patients) blood component requisitions were received by Department of Transfusion Medicine during the study period. Of total of 3711 blood components requested, PRBC, FFP, PC, SDAP and WB requisitions contributed 49.63\%( $\mathrm{n}=1842), 28.59 \%(\mathrm{n}=1061), 21.34 \%(\mathrm{n}=792), 0.30 \%(\mathrm{n}=11)$ and $0.13 \%(n=5)$ respectively. The requisitions and issue for surgical specialties $[62.97 \%(n=762), 67.81 \%]$ exceeded the demand from medical specialties [37.02\% ( $\mathrm{n}=448$ ), 32.19\%].The maximum blood component requisitions were received for the patients of cardiothoracic surgical unit followed by neurosurgery.The transfusion requests were observed to be maximum for the age group of 51-60 years (19.67\%, $\mathrm{n}=238)$ followed by $61-70$ years $(18.26 \%, \mathrm{n}=221) . \mathrm{BRh} \mathrm{D}$ positive $(38.01 \%)$ blood components were maximally requested followed by A Rh Dpositive (25.70\%), O Rh Dpositive (22.72\%) and AB Rh Dpositive (10.33\%) blood group. The overall mean pretransfusion hemoglobin trigger for packed red cell transfusion was $7.42 \mathrm{~g} / \mathrm{dl}$.The overall average CT Ratio was 1.57, highest being in cardiothoracic surgery department (1.92), followed by general surgery (1.84) and neurosurgery (1.79) departments. The Transfusion probability (\%T) and Transfusion Index were $79.91 \%$ was 0.97 respectively. Of the total blood component utilization, PRBC constituted 44.47\%, FFP utilization was 33.83\% and platelet concentrates as $21.21 \%$.

Summary: The retrospective audit helped in assessment of the transfusion practice at our centre which is found to be heartening. The CT ratio, Transfusion probability and Transfusion index demonstrated significant blood utilization at our hospital. However, excessive blood ordering for elective surgeries so observed leads to wastage of testing reagent resources, further adding extra cost to the patient .Therefore, the transfusion auditing should be made a common feature with hospital transfusion committee meetings where maximum surgical blood ordering schedule (MSBOS) can be modified and implemented progressively.

\section{P-025}

\section{SUPERVISION AND COMMUNICATION WITH THE BLOOD BANKS EFFECTIVE FOR IMPROVING BLOOD SAFETY IN JAKARTA PROVINCE}

E Pratiwi, P Rini, NK Ritchie and S Salim

Jakarta Blood Transfusion Unit, DKI Jakarta, Indonesia

Background: Jakarta Blood Transfusion Unit (BTU) is the biggest BTU in Indonesia, served 51.084 blood components per month to 173 Jakarta and 300 other hospitals and clinics in around Jakarta. According to Ministry of Health Regulation in 2007, every hospitals should have a blood bank in their hospitals. In 1991, there were only 14 blood banks. The number is increasing and in 2014, there were 42 blood banks. The responsibility of blood banks were maintaining blood stock in their hospitals and preparing blood for the patients in their hospitals. Jakarta BTU as the blood provider should monitor the blood usage in blood banks. The blood banks usually give reports to BTU and BTU should evaluate it. In 2009, we realize that many blood banks request whole blood and they produce PRC by open system in not proper procedure. Those blood components were not safe for the patients and that practice must be stopped. There should be a more efficient monitoring program for blood usage in blood bank. Aims: To reduce the production of open system PRC in blood bank.

Methods: We analyze and evaluate annual reports. We set up 2 programs for blood bank, first is impromptu visit and second is an 'audit' visit. We prepared audit checklist and reports. In internal of Jakarta BTU, we coordinate with logistic teams to order double bags instead single bags. The blood banks always request whole blood for their stock because the expiry date was longer than packed red cell (PRC) in Jakarta BTU. In this case, we already did some research to extend the expiry date but it seemed difficult unless we used additive solutions. Therefore, if we provide only whole blood in unprocessed-double bag, they could produce PRC in closed system which was safer than the previously. We also held routine meeting, seminars and workshop to gain their knowledge and skill in blood banking.

Results: After 5 years of supervision, we had a decreasing number of whole-blood request from $6.2 \%$ to $1.6 \%$. Now, opened-system already left behind. We hope the transfusion reactions in patients would also be reduced although the hemovigilance system not yet well-established. Since the communication between BTU and blood banks are more frequent and intense especially for the 'worst' blood bank so the hospital managements now gave their attention to the blood banks and supported them more than previously. So, we got another value from the supervision programs that more than $80 \%$ of blood banks in Jakarta were in the scale of good and very good.

Summary/conclusions: Supervision and communication were effective to improve the quality of blood bank services.

\section{P-026}

KOREAN RED CROSS BLOOD SERVICE HEADQUARTERS' RISK MANAGEMENT OF MERS-COV OUTBREAK

SJ Shin, NS Cho, JN Ko, JY Eom and JW Kim

Korean Red Cross, Wonju-si, South-Korea

Background: On May 20, 2015, Korea confirmed the first Middle East Respiratory Syndrome (MERS) patient. The unexpected MERS outbreak resulted in 36 deaths among 186 confirmed cases, and 16,693 people quarantined. The increased anxiety of the emerging outbreak had people limit outdoor and group activities, which led to the decreasing number of blood donations.

Aims: To assess the risk management of the potential crisis related to blood collection and supply due to the MERS outbreak.

Methods: The Korean Red Cross Blood Service headquarters (KRC) compared the number of collected blood and supplied blood with the same period of time (May 20-July 10) in 2014 and assessed if the activities implemented by the KRC were effective.

Results: The KRC conducted various activities to protect blood donors and KRC employees from the outbreak of MERS. The KRC strengthened strict blood donor interview, medical examination, and hygiene control at blood collection sites. Because of temporary closure of schools, cancellation of mobile blood drives, and the lower number of blood donors, the KRC identified the decreasing number of blood donations. On June 2, 2015, the KRC recognized that the inventory days of red blood cells were 7.3 days (the optimal days are 5 days) and predicted that the inventory days would be less than the optimal days after June 19 (20-30\% and 10\% of reduction in the number of blood donations and blood supply respectively). Therefore, the KRC implemented the risk management including securing blood fo transfusion as a priority at all blood centers, strengthening blood donation promotion, following the risk response manual, and extending blood donation center opening hours. Additionally, the KRC put an effort in protecting the health of employees and ensuring the safety of blood collection sites by providing the guideline for the prevention of MERS proliferation. As a result, compared with the same period of time (May 20-July 10) in 2014, there was no significant difference in blood collection and supply. While the total number of blood donations decreased by $0.5 \%$, the number of whole blood and apheresis platelet donations increased by $4.0 \%$ and $19.7 \%$ respectively. The total number of blood supply decreased by 3.8\% because of the closure of medical institutions, and the blood centers located in the center of MERS outbreak showed the reduction in blood supply, more than twice the national average reduction, resulting in significant variances among regions. Contrary to the prediction, the inventory days of blood products recorded the lowest number of 6.1 days on June 13 and increased to 7.4 days on July 10. Overall, the blood products were maintained over the optimal inventory days.

Summary/Conclusions: Disease outbreak that has high mortality or infection is one of the causes that threaten blood collection and supply. However, the prediction an preparation of the risk in advance contributed to overcoming the potential crisis of blood collection and supply as the KRC responded to the case of MERS outbreak.

\subsection{Quality Management}

\section{P-027}

THE RESULTS OF EXTERNAL PROFICIENCY TESTS FOR HBSAG AND ANTI-HCV DONOR TESTING IN BLOOD CENTERS IN KOREA (2012-2014)

MN Lee ${ }^{1}$, JR Kwon ${ }^{1}$, YS Choi ${ }^{1}$, QE Park ${ }^{2}$, YJ Cha ${ }^{3}$ and JN Kim

${ }^{1}$ Korea Centers for Disease Control \& Prevention, Cheongju, South-Korea ${ }^{2}$ Blood Center, Chung-Ang University Hospital, Seoul, South-Korea ${ }^{3}$ Department of Laboratory Medicine, Chung-Ang University College of Medicine, Seoul, South-Korea

Background: Blood donor screening tests for infectious diseases are one of the most important processes of blood safety management. In Korea, these tests include 
HBsAg (CLIA), HBV DNA (ID-NAT), Anti-HCV (EIA), HCV RNA (ID-NAT), Anti-HIV1/2 (EIA), HIV RNA (ID-NAT), Anti-HTLV-1/2 (CLIA) and Syphilis qualitative assay (TPPA). The Korean Centers for Disease Control and Prevention (KCDC) has been doing an annual proficiency test program which includes the distribution of the specially manufactured panels for HBsAg and anti-HCV to blood centers.

Aim: We report here the results of these proficiency tests during 2012 2014 for donor screening tests of HBsAg and anti-HCV performed in all of the licensed blood centers in Korea.

Methods: The materials for the proficiency tests were manufactured with well-proven reactive sera and healthy donors' sera which were acquired from Human Serum Bank of Chung-Ang University Hospital. The panels were designed to evaluate the ability of each blood center to detect very low concentrations of HBsAg and AntiHCV. The panels consisted of four reactive panels of various concentration for each of HBsAg and Anti-HCV and two non-reactive panels.

Results: Most blood centers reported correct answers even with very low concentration panels. The average percentages of correct answers for HBsAg proficiency tests were 98.7\% (2012), 98.5\% (2013) and 99.1\% (2014). The percentages for Anti-HCV were $97.7 \%, 99.5 \%$ and $99.1 \%$, respectively.

Conclusion: KCDC will administering the proficiency test program continuously to maintain the quality of the donor screening tests of HBsAg and anti-HCV. The panels of low concentrations of HBsAg and Anti-HCV will be used to improve the ability of the donor screening tests. The blood centers which failed to report correct answers will have to be educated and report the causes. Finally, satisfaction research will be taken to improve the quality of the proficiency test program.

\section{P-028}

ENVIRONMENTAL MONITORING ABOUT BACTERIA TO BLOOD PRODUCTS STORAGE, INCLUDING RED BLOOD CELL, PLATELET AND PLASMA

YC Lan ${ }^{1}$, JS Chang ${ }^{2}$, YS Lin ${ }^{1}$ and YJ Peng ${ }^{3}$

${ }^{1}$ Department of Clinical Medicine, Peking University Health Science Center, Beijing, China ${ }^{2}$ National Taiwan University Hospital, Taipei, China Taiwan ${ }^{3}$ Department of Clinical Pathology, Taipei City Hospital, Ren Ai branch, Taipei, China Taiwan

Background: There is still not an international guideline for bacteria monitoring in environment of blood products storage, including the 4 interlayer-refrigerator to store red blood cells, the temperature of platelets conservation and the way of thawing fresh frozen plasma. In Hong Kong and Macao, it has proved that the pollution of pseudomonas spp. in water bath would contaminate the plasma package and cause the neonatal sepsis. The purpose of this article is to monitor the space of blood product storage, such as refrigerators, platelet oscillators, and the number of bacteria in water bath of plasma-thawing, in order to set a standard of regular disinfection. Method: Red blood cell and platelet storage environment: the quality control of all the equipments are carried out according to the relevant rules. We tested once at each period of time: 10:00 to 10:30 in the morning, 4:30 to 5:00 in the afternoon and 9:30 to 10:00 at night for five days.

Plate Position: due to the difference of $\mathrm{A}, \mathrm{B}, \mathrm{O}, \mathrm{AB}$ blood types, the frequency of opening the refrigerators are not the same. The positions are placed like Pic.1. Water Bath: Take the fresh R.O water each time before changing the old one and use the sterile container to load some as a blank of R.O water. Before pouring the water into the bath, take the fresh R.0 water as a blank of transfer container. After 5 min, we took the R.O water in the water bath as a $0 \mathrm{~h}$ of bacteria content, then, we took the sample after every $4 \mathrm{~h}$ and stop at the fourth time.

Result: The monitoring of refrigerators: we could find that the number of colony counting will be maximum at 4:00 in the afternoon. The amount of bacteria in different position distinguished by $\mathrm{A}, \mathrm{B}, \mathrm{O}, \mathrm{AB}$ blood type also comply with the proportion of blood group. On average, the amount of bacteria in 0 type was higher than A type, than $B$ type, than $A B$ type (Table 1 and Table 3). It also because of the storage environment are different between platelet and red blood cell, we could see that the number of colonies in $25^{\circ} \mathrm{C}$ is higher than $4^{\circ} \mathrm{C}$ (Table 2 and Table 3).

Table 3. Average counting to colony in different environment and position.

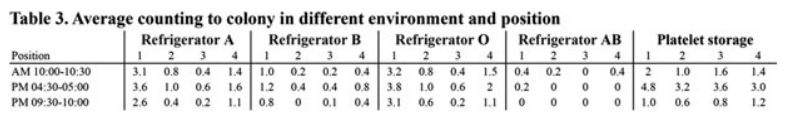

in the contrast, disinfection is a better way to inhibit the growth. In generally thinking, change the water is the best way to reduce the chance of being polluted, but in this study, we shows that if we do not go through sterile procedures, the initial amount of bacteria will be in a high concentration and affect the result. Poured out

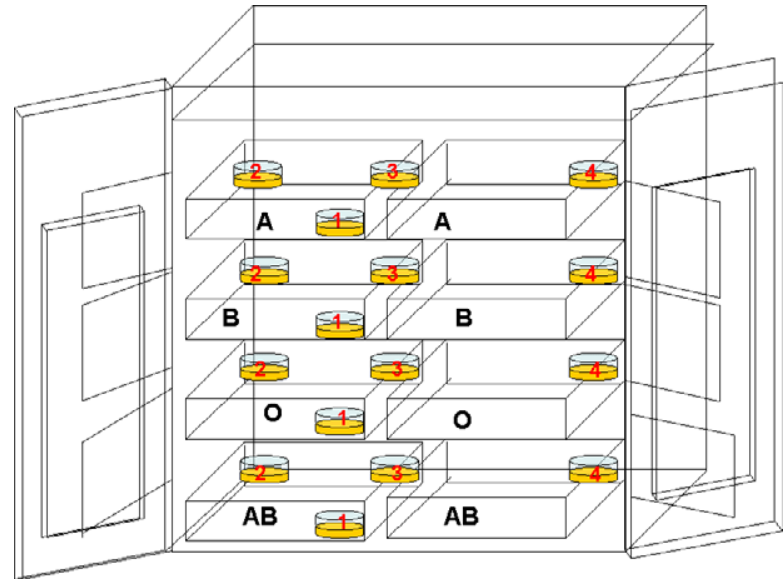

Figure 1: The plate positions in refrigerators.

the R.0 water after $24 \mathrm{~h}$ without sterilization, then, poured into 5 liters of sterile diluents and repeated three times, the amount of bacteria obtained as $5870 \mathrm{CFU} / \mathrm{ml}$, $3240 \mathrm{CFU} / \mathrm{ml}, 50 \mathrm{CFU} / \mathrm{ml}$. It can be seen that the steps required to do at least three times in order to effectively reduce the amount of residual bacteria in water bath.

P-029

STUDY OF DIFFERENT FACTORS OF BLOOD BANK REFRIGERATORS AND REFRIGERATED CENTRIFUGES USED IN IRANIAN BLOOD BANKS (IBTO) CENTERS

M Fallah Tafti, G Karimi, K Mousavi Hosseini and R Morshedlou

Iranian Blood Transfusion Organisation (IBTO), Tehran, Iran

Background: Blood safety mostly depends on the quality of medical devices used in the blood donors, productions, storage and distribution of a blood bank center.

Aim: The aim was study of different factors (body, door and glass doors insulation, door seals, door hinges, lock and doorknob, rotor, bucket, shield, leaner, plug, socket, cables, equipment's earthing, speed, shield field temperature, room temperature and humidity plus refrigerator's shelves temperatures, heat and direct sunlight, air circulation around the equipment, equipment level, distance between it and other equipment, alarm systems) belongs to blood bank refrigerators and refrigerator centrifuges of 10 Iranian blood bank centers

Methods: Iranian Blood Transfusion Organization (IBT0) was established in May 1974 and presently is functioning with 31 regional blood centers throughout the country to ensure a safe and sufficient blood supply. IBTO within a period of 12 months (from 21 Mar 2014 to 20 Mar 2015) had a total collection rate of $2,250,341$ blood units. In order to study the results of P.M. for blood bank refrigerator, and refrigerated centrifuges, the data of 36 important factors ( 3 factors pertaining body, door and glass doors insulation, 1 factors on door seals (gaskets), 3 factors on door hinges, lock and doorknob, 8 factors on shield's field, shield's door, rotor, bucket, bucket's hung, leaner, shaft, shaft's spring, 5 factors on fluorescent lighting, plug, socket, cables, earthing, 6 factors room temperature and humidity plus shelves temperatures, 4 factors pertaining on heat and direct sunlight, air circulation around the equipment, equipment is level, adequate distance between it and other equipment, 3 factors on alarm systems, speed of centrifuges with full load and the related data are collected for 10 centers analyzed by SPSS.

Results: The present abstract shown only the results belongs to some factors obtained for sixty tree blood bank refrigerators and forty eight refrigerated centrifuge from 10 blood bank regional centers. The averaged speed of refrigerated centrifuges were $5014 \mathrm{rpm}$, corrosion of bucket 13\%, corrosion of shaft's spring 9\%, corrosion of shield's field 17\%, temperatures from four shelves of blood bank refrigerators from the top shelve were $\left(2.5-8.4^{\circ} \mathrm{C}\right),\left(2.6-8.2^{\circ} \mathrm{C}\right),\left(2.4-7.4^{\circ} \mathrm{C}\right)$ and $(2.51-7.55$ ${ }^{\circ} \mathrm{C}$ ) respectively. The averaged amount of other factors like: room humidity $49.3 \%$, laboratories environment temperature $26.53{ }^{\circ} \mathrm{C}$ and differences temperature between the refrigerators' body and environment were $3.2-7.7^{\circ} \mathrm{C}$.

Conclusion: This study contributes to a better knowledge of parts states used in different part of blood bank refrigerators and refrigerated centrifuges with the working life time of 5-12 years.

(C) 2015 The Author Vox Sanguinis (C) 2015 International Society of Blood Transfusion Vox Sanguinis (2015) 109 (Suppl. 2), 1-96 
Key words: Blood bank refrigerator, refrigerated centrifuge. Speed, rotor, bucket, leaner, shaft, shield, humidity, laboratories environment temperature, humidity, body and glass insulator, ribbon and door lock of refrigerator.

\section{P-030}

\section{AN INSTITUTIONAL EFFORT AT MONITORING QUALITY OF PACKED RED CELLS AND PLATELET CONCENTRATES \\ RN Makroo, M Chowdhry, A Bhatia and UK Thakur \\ Indraprastha Apollo Hospitals, New Delhi, India}

Background: The Standards for Blood Banks \& Blood Transfusion Services, laid down by the Ministry of Health and Family Welfare, Government of India, outline recommendations for the quality of blood and blood components in India. Monitoring the quality of blood components prepared establishes the efficacy of procedures and protocols being followed, aids in identifying problem areas and determining corrective actions.

Aims: To analyze the quality of blood components processed at our blood bank, to test whether they meet the recommended national standards.

Design and methods: Our hospital based blood bank routinely collects blood in quintuple top and bottom bags with integral leucocyte filters. Blood is then processed for preparation of blood components as per departmental protocols and manufacturers' instructions using automated component separation systems. Pre-storage leucoreduction of Packed Red Cells (PRC) is performed using integral filter. Platelet Concentrates (PC) are prepared from the Buffy coat.

As per the national standards, a minimum of $1 \%$ of the blood components prepared undergo quality control testing. PRC and PC units, for which no incidents are reported during collection and processing, are randomly selected for quality control Weights are measured and volumes are calculated based on specific gravity. Cell counts, hemoglobin and hematocrit are performed in a automated cell counter (Beckman Coulter). In addition, a Nagoette's chamber is used for manually confirming WBC counts. The $\mathrm{pH}$ is recorded at the day of collection as well as on the 5th day of storage of PC. Swirling is examined visually.

We retrieved and reviewed the Quality Control data of the cellular blood components (PRC and PCs) for 2013 and 2014.

Results: The volume of PRC units (Mean \pm SD) was $286.8 \pm 12.6 \mathrm{ml}$. Hemoglobin levels ranged from $17.8-23.3 \mathrm{~g} / \mathrm{dl}$ and the mean hematocrit (Hct) was $59.6 \pm 4 \%$. None of the units had a Hct of less than 50\%, while 2 units had a Hct of over 70\%. All the units tested were adequately leucoreduced as per standards $\left(<5 \times 10^{6} \mathrm{WBC}\right)$ unit after use of leucocyte filter).

As regards to PCs tested, the mean volume of PC was $73.8 \pm 5 \mathrm{ml}$, and the platelet yield was $6.65 \pm 1.4 \times 10^{10} /$ unit. Platelet yield in $96.8 \%$ of the units tested were in compliance with the national standards of minimum $4.5 \times 10^{10}$ platelets/unit. The $\mathrm{pH}$ ranged from 6 to 7.5 on the last day of storage, in compliance with the recommended standards. Swirling was visible in all the units. The mean WBC counts of the units was $2.8 \pm 1.6 \times 10^{8}$ per unit and $91.4 \%$ of the PC tested were adequately leucoreduced as per the standards $\left(<5 \times 10^{8}\right)$. The red cell counts were $0.02 \pm 0.001 \times 10^{12} /$ unit

Conclusion: Both red cell as well as platelet concentrates prepared on the automated component separation systems at our centre comply with the recommended national standards. All PRC units were adequately leucoreduced (upto 3 log reduction), while $91.4 \%$ of the PC achieved over 1 log leucoreduction.

Table 1: Quality Parameters of packed red cells and platelet concentrates.

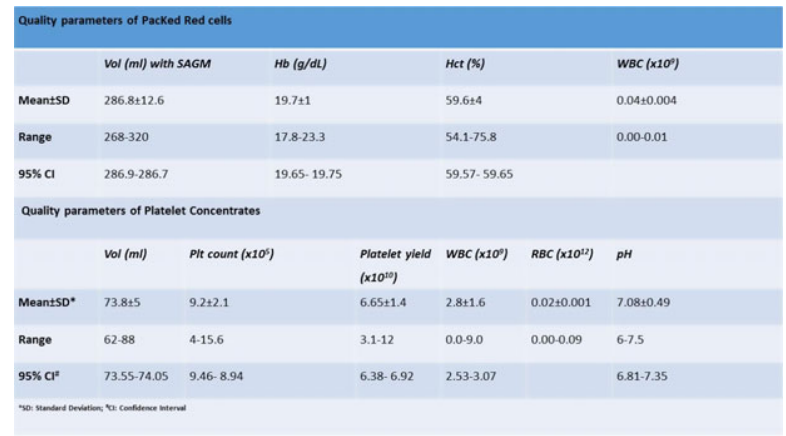

(C) 2015 The Author

Vox Sanguinis (C) 2015 International Society of Blood Transfusion

Vox Sanguinis (2015) 109 (Suppl. 2), 1-96

\section{P-031}

\section{ANALYSIS AND CLINICAL AUDIT OF UNUSED PLATELET CONCENTRATES IN H. ADAM MALIK HOSPITAL MEDAN INDONESIA}

I Adhayanti ${ }^{1}$, T Triyono ${ }^{2}$ and R Juwita ${ }^{1}$

${ }^{1}$ Adam Malik Hospital, Medan, Indonesia ${ }^{2}$ Sardjito Hospital, Yogjakarta, Indonesia

Background: Platelet concentrate (PC) is given to avoid or cure patients with bleeding disorders. Platelet transfusion must be performed with the right indication for patient safety reason. One of the quality indicators in H. Adam Malik Hospital Blood Transfusion Unit is the percentage of unused PC per-month. The data showed that the percentage of unused PC were $2.63 \%, 6.45 \%, 8.74 \%$, and $12.40 \%$ for January, February, March and April 2015 respectively. The analysis and clinical audit were needed for PC usage.

Aims: To reach the efficacy of PC usage and to fix the unused PC quality indicator in H. Adam Malik Blood Transfusion Unit.

Method: This research was held in H. Adam Malik Blood Transfusion Unit, Medan Indonesia using cross sectional design. The data were collected from the hospital information system in the period of January-June 2015. The data then analyzed and compared with the data from Hospital transfusion unit.

Result: There was an increased PC used (2604 units) from January to June 2015. The unused PC were declined but still above the standard $(>1 \%)$.The strategies that were used for increasing quality indicator in Blood Transfusion Unit started on May 2015 were spreading platelet transfusion indication brochures that were signed by the hospital main director, the responsible doctors must sign the platelet concentrate demand letters, requesting the platelet agitator with incubator device for patient safety reason. The result still high $4.12 \%$ in May and 5.50\% in June. The departments with the highest number of unused PC were Internal Medicine 28.4\%; Surgery 20.4\%; Pediatric 19.8\%, Obstetrics and Gynecology 17\%; Cardiology 10.8\%, others 3.4\%.

Conclusion: The number of unused PC in H. Adam Malik Hospital Blood Transfusion Unit has not reached the standard. There were still many constraints found, such as: different education background of the responsible doctors, ineffective communication, unsigned platelet concentrate order letter, broken platelet agitator with incubator device. By improving the communication, implementing the strategies that have been made and provisioning platelet agitator with incubator device, the decrease of unused platelet concentrates was expected.

\section{P-032 \\ QUALITY ANALYSIS OF PRE-OPERATIVE PREPARED PACKED RED CELLS STUDY OF INDEXES RBC, CULTURE AND IDENTIFICATION OF BACTERIA}

RA Muhiddin ${ }^{1}$, T Triyono ${ }^{2}$ and B Mulyono ${ }^{2}$

${ }^{1}$ Wahidin Sudirohusodo Center Hospital, Makassar, Indonesia ${ }^{2}$ Gadjah Mada University, Jogjakarta, Indonesia

Background: Pre-operative Packed Red Cell (PRC) is prepared to anticipate acute blood loss during surgery. In the absence of intraoperative bleeding complication, the PRC would not be transfused. The handling of pre-operative PRC performed under the cold chain system to ensure it was safe to be distributed to the patients.

Aims: To determine the differences of red blood cell (RBC) indexes, and the bacterial culture of the pre-operative prepared PRC before being transferred to the operating room (group I) compared to the unused PRC in operating room then returned to the blood bank and stored for 3 days (group II). Furthermore, to determine the percentage of hemolysis in plasma after 3 days storage at the Blood Bank, and its relationship with the day of PRC storage after donation.

Method: This study was conducted in the period of August to October 2014, in the Blood Bank and Clinical Pathology Laboratory of Dr. Wahidin Sudirohusodo General Hospital Makassar, Indonesia. The RBC indexes was examined by Sysmex XT1800 hematology analyzer. Bacterial culture was examined by Bact/ALERT, Bacterial identification was examined by VTEK2 Compact.

Results: The Mean Corpuscular Volume (MCV) of group I and group II were $85.95 \mathrm{fl}$ and $86.45 \mathrm{fl}$ respectively $(\mathrm{P}=0.109)$. The Mean Corpuscular Hemoglobin (MCH) group I was higher $(28.05 \mathrm{pg})$ than group II $(28.00 \mathrm{pg})$, but insignificant $(\mathrm{P}=0.386)$. The Mean corpuscular hemoglobin concentrate (MCHC) of Group I and group II were $32.70 \%$ and $32.20 \%$ respectively $(\mathrm{P}=0.001)$. Red Cell Distribution Width (RDW) group I was $14.90 \%$, RDW Group II was $15.47 \%(\mathrm{P}=0.006)$. There wasn't difference positivity bacterial culture in both group I and II. There was 6.67\% samples found percentage hemolysis $>1 \%$. There was weak relationship between percentage of hemolysis with day of PRC storage after donation $(r=0.128, \mathrm{P}=0.463)$. Conclusions: There was a significant difference for MCHC and RDW between group I and II. There wasn't difference positivity bacterial culture in both group I and II. Hemolysis can occur along the length of the storage period PRC. 
P-033

TRANSFUSION REACTIONS AS AN INDICATOR OF SERVICE QUALITY OF BLOOD TRANSFUSION IN SANGLAH HOSPITAL

\section{DENPASAR BALI}

NK Mulyantari, IW Putu Sutirta Yasa and AAN Subawa

Faculty of MedicineUdayana University, Denpasar-Bali, Indonesia

Background: Each blood transfusion should be based on a strong indication and always takes into consideration the amount of benefits compared to the risks to be borne. Transfusions done without an indication is a contraindication. One of the fatal side effects of transfusion is transfusion reactions. The incidence of transfusion reactions in Sanglah Hospital is quite high. In 2014 the average incidence of transfusion reactions was $0.52 \%$ per month. It was very far from the target set by the government, the target was $0.01 \%$. The high number of the incidence needs serious attention, so that the transfusion reaction was raised as one of the indicators of the quality of hospital services. To achieve the quality indicator target, it is necessary to set up an activity program so that the number of incidence of transfusion reactions can be decreased. The activity program is based on the analysis of cases of transfusion reactions that have occurred.

Aims: To analyze the effect of educational programs and the training of the implementation of closed system and cold chain in the distribution of blood products as well as improving service system on decrease of the incidence of transfusion reactions.

Methods: The study was an intervention study, pre and post test. The study population was all nurses in Sanglah Hospital. The research sample included all nurses who served in the wards with the largest amount of usage of blood products. There are Internal Medicine Department, Department of Surgery, Department of Obstetrics and Gynecology and Department of Pediatric. Interventions in this study was in the form of a program of education and training on the application of the closed system and cold chain in the distribution of blood products and improved service system. The program was run for 6 months and it evaluated the decrease in the incidence of transfusion reactions that occurred every month.

Results: The results showed a decline in the incidence of transfusion reactions every month during the study. From September 2014 until February 2015 the incidence of transfusion reactions every month was $0.77 \%, 0.56 \%, 0.5 \%, 0.49 \%, 0.45 \%$ and $0.38 \%$ respectively. The average reduction in the incidence of transfusion reactions was $0.08 \%$ every month. Type of transfusion reaction that occurs was 59\% urticaria, 29\% fever, 7\% shortness of breath, 4\% fever and chills, 1\% shock.

Conclusion: Education and training programs on the application of the cold chain system and closed system in the distribution of blood products as well as the improvement of service system helped reduce the number of the incidence of transfusion reactions in Sanglah Hospital Bali.

\section{$\mathrm{P}-034$}

This abstract has been withdrawn

P-035

THE IMPORTANCE-PERFORMANCE ANALYSIS APPROACH IN CUSTOMER SATISFACTION SURVEY TO IMPROVE DONOR

\section{SERVICES IN JAKARTA BLOOD TRANSFUSION UNIT}

S Sumiyanti, NK Ritchie and S Salim

Jakarta Blood Transfusion Unit, DKI Jakarta, Indonesia

Background: Blood Transfusion Units (BTUs) are responsible for producing quality products while delivering excellent customer service. It is important to know who and what the customer's need and expectations. A BTU should have systems in place that seek or encourage customer feedback and accommodate different modes of communication. This feedback loop is an important part of the continuous improvement process. Since 2009, Jakarta BTU has been certified for ISO 9001:2008 by SGS. To maintain the quality of our services, we held a customer satisfaction survey twice in a year. At the beginning, we use simple questionnaire with 4 level of satisfaction (very satisfy-satisfy-not satisfy- very not satisfy). During 2009-2011 we achieved $90 \%$ customer satisfaction with some notes of suggestions and complaints. First, we must know what are the important services needed for the customer and how we already achieved that. But at the mean time, we had no valid information about that, so we have to use another approach to get that.

Aims: To know what the customer most needed in donor and blood services.
Methods: In April 2012, we started using importance-analysis approach in the new questionnaire. The responders were 100 blood donors, organizing coordinator of blood donation in 100 institutions, and 300 customers from hospitals. The customers were asked to give points about how important is 'the aspect' in our services and what they felt about the performance of 'this aspect'. We calculate the importance average score and the performance average score to analyze the gap between realization with customer's expectation. We also reckon the importance index score (IIS) and the performance index score (PIS) and then plot the index score into a chart. The $\mathrm{x}$-line of the chart was IIS and the $\mathrm{y}$-line was PIS. The chart was divided into 4 areas to show which aspect should be maintained (high IIS, high PIS); de-emphasized (low IIS, high PIS); need high effort (high IIS, low PIS) and low effort (low IIS, low PIS). Results: All questionnaires come back to us and 99\% completely filled in and valid. During 2012-2015, we achieved 96\% customer satisfaction. The top 3 importance aspects that should be maintained with us in donor services were competency of phlebotomist doing the venepuncture, the hospitality and the supporting facilities are. The most important aspect for organizing coordinator of blood donation in institution is timeliness arrival of our mobile unit team. The 3 importance aspects that should be maintained in blood services for hospitals are turn-around-time, the hospitality and the accuracy of information given by our staff. Based on that, we can more focus on improving the competency and communication skill of our staff in donors and patients services. The aspects that needed high effort to improve are supporting facilities such the cleanliness of toilets and the availability of parking lot.

Summary/Conclusion: The importance-performance analysis approach is very useful to evaluate the customer satisfaction on the BTU services.

$\mathrm{P}-036$

\section{COMPARISON QUALITY WITH FINANCING IN TANGERANG CITY BLOOD CENTRE}

R Anggraini, K Kamaludin and D Hasudungan Sidabutar

Indonesian Red Cross Tangerang City Blood Centre, Tangerang, Indonesia

Background: Blood transfusion is no longer aimed for saving lives but also shifted toward medicine treatment. One of indicator increasing blood needed for transfusion services especially in Tangerang is increasing of blood supply in Tangerang City Blood Centre every year. So we realize is very importance to improving quality of blood product in our Blood Centre.

Aim: To improve quality of Tangerang City Blood Centre blood product with financing effectiveness.

Methods: We analized and evaluated the result of external Audit from Central Blood Transfusion Service of Indonesia and Australian Red Cross for blood product in Tangerang City Blood Centre. As a first step, we formed a QC department and hold Learning Carier Development for all employees, strengthen of Standart Operating Procedure and documentation, close system, monitoring temperature and cold chain. Cooperation and communication between department is also very necessary for quality improvement. At the phlebotomy department, Infection Disease Department, IT Department and administration cooperation in blood safety system with high effectiveness through Self deferral donors. At laboratories, administration and logistics are cooperate to maintain the cold chain and close system. We also do

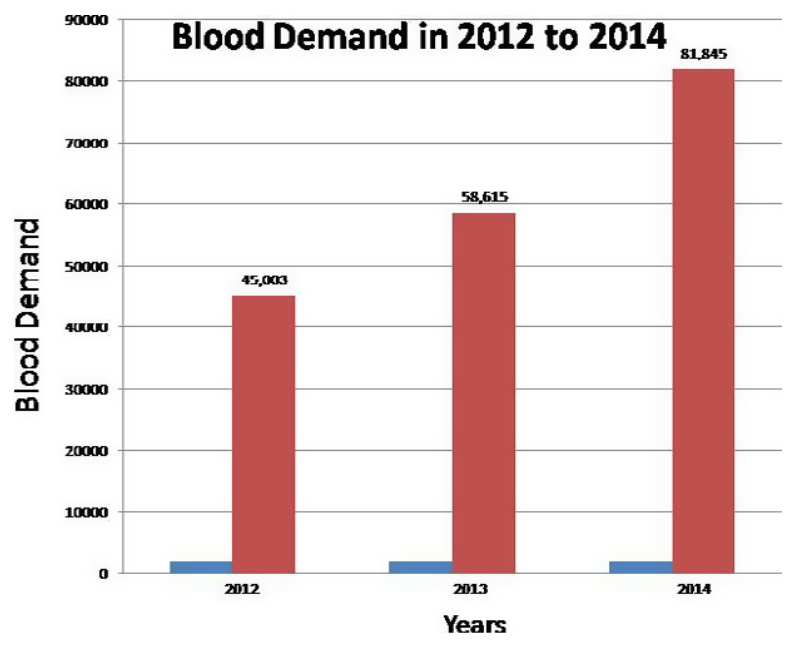

(C) 2015 The Author Vox Sanguinis (C) 2015 International Society of Blood Transfusion Vox Sanguinis (2015) 109 (Suppl. 2), 1-96 


\section{Comparison Between Quality, Cost and Risk of Blood Product}
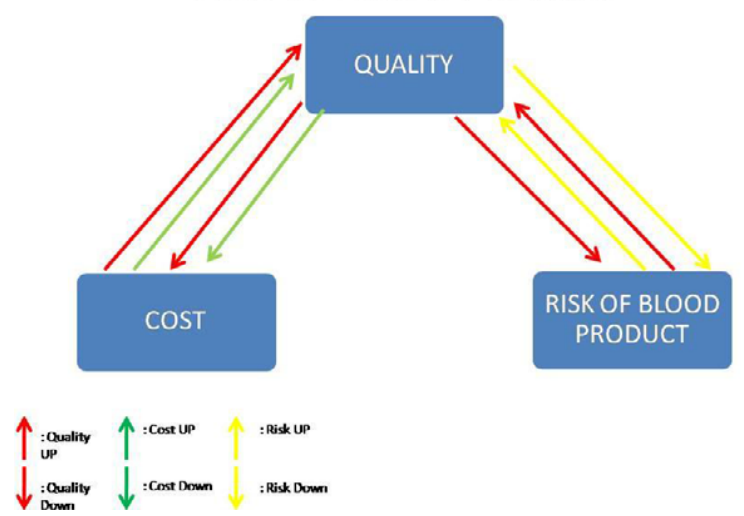

Figure 2: Blood Demand in 2012 to 2014.

external QC blood product with Central Blood Transfusion Service of Indonesia. The quality should not be interrupted just in our Blood Centre but also for Patient safety, therefore we also hold socializations to nurses, doctor and clinicians about blood transfusions safety and quality. At finance department had to think about the effect of improving the quality of the costing to effectiveness but it should not damaging the quality framework.

Result: Calculation that we arrange for improving the quality increased by $141.21 \%$ from previous year, with QC improving strategies the quality of our human resources increases, the percentage of blood taping failure decreased 12.5\%, and increasing of blood distribution roughly 39.63\%. Comparison of test results and quality product from Whole Blood and Packed Red Cell 3 times external QC with CBTS: not pass the standarts (87.5\%), passing standarts (100\%), passing standart (100\%) good morphology test, hematology and bacterial contamination. As a rough calculation of the ratio between the costing and quality is directly proportional, but the ratio is reserved when compared to the risks from products that are not qualified. For example, the cost of blood screening by using NAT or CLIA is $\$ 20.01$ and $\$ 11.56$ while for rapid test we using two kinds of reagen for comparison the cost is $\$ 23.13$, not including the cost would be the risks of the results obtained. From the comparison of two parameters it can see the cost to obtain good quality products is not too high could even be cheaper than the regular product.

Conclusion: Quality is expensive but the effects of unqualified product is more expensive. Quality is something that is not negotiable. So we should not be afraid to improve the quality of our products and our services.

\section{Blood Donation}

\subsection{Blood Donor Recruitment}

P-037

FREQUENT WHOLE BLOOD AND APHERESIS DONORS: UNDERSTANDING CHARACTERISTICS AND RETURN BEHAVIOUR

A Thijsen, CN Gemelli, D Waller and J Hayman

Australian Red Cross Blood Service, Alexandria, Australia

Background: Blood donor management is supported by examining segments in the donor population. An essential donor group is the frequent donor population who provide the largest portion of donations on a regular basis. A few studies have determined frequent donor characteristics but no analysis has included donation frequency, phlebotomy type and donor return behaviour. Thus, the Australian Red Cross Blood Service set out a large database research to understand trends in the frequent donor population to provide support for retention and reactivation strategies of this specific donor group.

Aims: The aim of this study was to identify segments within the frequent donor population based on phlebotomy type, medical history, and donation history.

(C) 2015 The Author

Vox Sanguinis (C) 2015 International Society of Blood Transfusion

Vox Sanguinis (2015) 109 (Suppl. 2), 1-96
Methods: Based on donor records two study populations were identified: (1) frequent whole blood donors who had donated successfully 3 times or more between December 2010 and November 2011, and (2) frequent apheresis donors who had donated 6 times or more between January and December 2012. Donors were further segregated by deferral status, those who started donating in the study period, and those who had lapsed from blood donation.

Descriptive analysis using means and proportions was conducted on both donor populations. Donor characteristics examined included age, gender, occupation, socioeconomic status, previous donation history, and deferral status. Survival analysis was used to determine the probability of lapsing following a temporary medical deferral. Results: Donor records identified 90,649 frequent whole blood donors who had donated 3 or more times. These donors were predominately male (51.36\%), with a mean age of 48.07 years (range 16-81). They had made a mean number of 22.67 donations prior to December 2010 (range 0-270). These donors were followed through to the end of 2013 to determine numbers of lapse with 13.59\% lapsing during the follow-up period $23.26 \%$ of these due to deferral.

Frequent apheresis donors were a smaller group of 17,231 donors. They were found to have a greater portion of males $(64.5 \%)$, with a mean age of 50.24 years (range 18-81). The mean number of total donations prior to January 2011 was 45.73 donations (range 1-334). The majority of these donors were apheresis only donors (77.7\%), with a mean number of 12.15 apheresis donations made in 2012 (range 629). These donors were followed through to the end of 2014 to determine numbers of lapse with 6.15\% lapsing during follow-up period, $13.6 \%$ of these due to a deferral. Conclusions: Whilst previous studies have examined donor characteristics of the entire frequent donor population, this is the first study to examine segments within this donor population. Compared to frequent apheresis donors, frequent whole blood donors were younger, consisted of more female donors, and a greater number of lapsed donors. Survival analysis showed that being younger, female, having a shorter donation career, donating whole blood only, and receiving a medical deferral were associated with an increased probability of lapsing. Segmenting the donor population in this way can inform retention and reactivation strategies designed to maintain a stable blood supply.

\section{P-038}

\section{SOME CHARACTERISTIC FACTORS ASSOCIATED WITH DONOR RECRUITMENT}

IP Rupasena

National Blood Center, Colombo 05, Sri Lanka

Background: Blood supply has always been scarce. Therefore blood donation programs face challenges in recruiting of blood donors and maintaining their blood supplies without any shortages throughout the year. These challenges make urgent the exploration of factors affecting blood donation in order to identify effective strategies for increasing the donor base and fulfilling future blood needs.

The main strategies of any blood donor programmes are donor motivation, donor recruitment and donor retention. Donor recruitment is an essential strategy in any blood donor programme because it aids in maintaining a sustainable and safe blood donor pool.

Aim: Descriptive study of donor factors such as donor intention, 1st motivation and advertizing method of donor recruitment.

Methods: The prospective descriptive study was conducted a period of six months from 01/10/2015 to 30/06/2015 in blood collection campaigns conducted by Blood Bank, District General Hospital, Negombo, Sri Lanka.

Results: Total of 2759 voluntary blood donors, $81.1 \%(n=2237)$ males and $18.9 \%$ $(\mathrm{n}=522)$ females were donated blood as voluntary non remunerated blood donor during relevant period. 29.6\% $(\mathrm{n}=816)$ was first time donors and $70.4 \%(\mathrm{n}=1943)$ was regular donors.

Out of 2759 donors, randomly selected 879 donors were participated for this study. $40.9 \%(\mathrm{n}=360)$ was first time donors and $59.1 \%(\mathrm{n}=519)$ was regular donors. Male donors accounted for $73.3 \%$ while the female counterpart was responsible for $26.7 \%$. Considering the educational level of donors, $79.1 \%$ had primary educational level (school education) and 20.9\% had secondary educational level (diploma or higher). In first time donors, the highest number of donors $12.7 \%(\mathrm{n}=177)$ were motivated for blood donation in their 1st blood donation by printed media (poster/news paper etc.) while $14.7 \%(n=129)$ in regular donors who were motivated by friends. The least number of donors motivated by electronic media (TV/Radio) which was $0.6 \%$ $(\mathrm{n}=6)$ in both 1 st time and regular donors. 26.9\% and 36.2\% donors donated blood for self satisfaction in 1st time and regular donors respectively. $12.9 \% 1$ st time donors and 18.8\% regular donors donated blood as a responsibility. 25.7\% 1st time donors and 30\% regular donors thought that most effective ways for advertizing the blood donation campaigns was displaying the poster or banner. Also 7.2\% 1st time and 11.3\% regular donors thought it was Facebook/Internet. 
When considering the educational level, $12.7 \%$ of donors who had primary educational level were motivated their blood donation by printed media (poster/news paper etc.) and $14.7 \%$ of donors who had secondary educational level were motivated by friends. $26.9 \%$ of primary educated donors and $36.2 \%$ of secondary educated donors donated their blood for self satisfaction. Both type of donors believed that the most effective ways for advertizing the blood donation campaigns was displaying Posters or Banners. (25.6\% and 30.\% respectively)

Summary: Donor population of Sri Lanka is 100\% voluntary non-remunerated donors. The information about donor's opinion and feelings towards donor recruitment is more important to make future decisions and improvements.

Donors are found to vary in their behaviors, attitudes and beliefs but not enormously. These data may enable National blood center to optimize recruitment by tailoring a more effective strategy.

P-039

RECRUITMENT STRATEGIES FOR INCREASING NONREMUNERATED VOLUNTARY DONOR AT FASTING MONTH IN JAKARTA BLOOD TRANSFUSION UNIT

S Juwariyah, U Khasanah, D Winarti, NK Ritchie and S Salim

Jakarta Blood Transfusion Unit, DKI Jakarta, Indonesia

Background: The blood stock depends on the availability of blood donors. In Jakarta Blood Transfusion Unit (BTU), 95\% was non-remunerated voluntary donor, and 5\% was replacement donor which increasing in fasting month because $88.1 \%$ of Indonesian population were moslem. At fasting month, the blood collection from voluntary donor was dropped to $60 \%$ compared to other months, voluntary donors were $99.5 \%$. Usually, we have 10-15 Mobile Unit teams, went to various institution and got $800-1200$ blood donors every day. At fasting month, only 1-2 mobile unit teams per day, except Saturday and Sunday. This condition affected the blood stock and delayed the medical treatment of patients. So, we have to make a breakthrough program to increase the blood collection. In 2013, we have new recruitment teams and new mobile bus, a facility for collecting blood inside the bus. We have socialization program in social media such as radio to socialize fatwa MUI which allow Muslims donated blood in fasting month. We also placed our mobile bus unit in some mosques and malls almost every day to get more donors. By those programs, the number of voluntary donors increased to 65\%, but then in 2014 it dropped to $59 \%$ because less anticipated programs were conducted before fasting month.

Aims: To increase the blood collection at fasting month in Jakarta BTU.

Methods: We always ask the active institutions and non Muslims organizations to donate in fasting month. Few weeks before fasting month, we went to some institutions, door to door to explain the situation of unbalancing between blood stock and demands in fasting month and encouraged them to engage blood donations in fasting month. Other routine programs such as socialization programs still conducted. Results: During fasting month in 2015, we achieved the highest number of voluntary donors ever, $71.3 \%$ of total donations or $21.2 \%$ higher than 2014 . The increasing voluntary donors were seen in our buildings (25.4\%) and in mobile unit teams $(16.8 \%)$ compared to 2014 . The responds from the coordinator of blood donations in all institutions were excited to help. They also gave us time to campaign about 'blood donations in the fasting month' for the next fasting months.

Summary/conclusions: Pro-active communication and good personal skill were very helpful increasing the number of blood collection in the fasting month.

P-040

This abstract has been withdrawn

\section{P-041}

\section{PUBLIC AWARENESS, ATTITUDE AND PRACTICES OF BLOOD DONATION AMONG THE GENERAL POPULATION LIVING IN RIYADH, SAUDI ARABIA}

AW Al-Johar ${ }^{1}$, MA Abolfotouh ${ }^{2}$, MH Al-Assiri ${ }^{3}$, MA Al-Omani ${ }^{2}$, AM Al-Hakbani and AS Alaskar ${ }^{3}$

${ }^{1}$ King Saud University, Riyadh, Saudi Arabia ${ }^{2}$ King Saud bin-Abdulaziz University for Health Sciences, Riyadh, Saudi Arabia ${ }^{3}$ King Abdullah International Medical Research Center, Riyadh, Saudi Arabia

Background: Voluntary donors are the only source of blood donation in Saudi Arabia. Despite frequent blood donation campaigns and encouragement by the media,

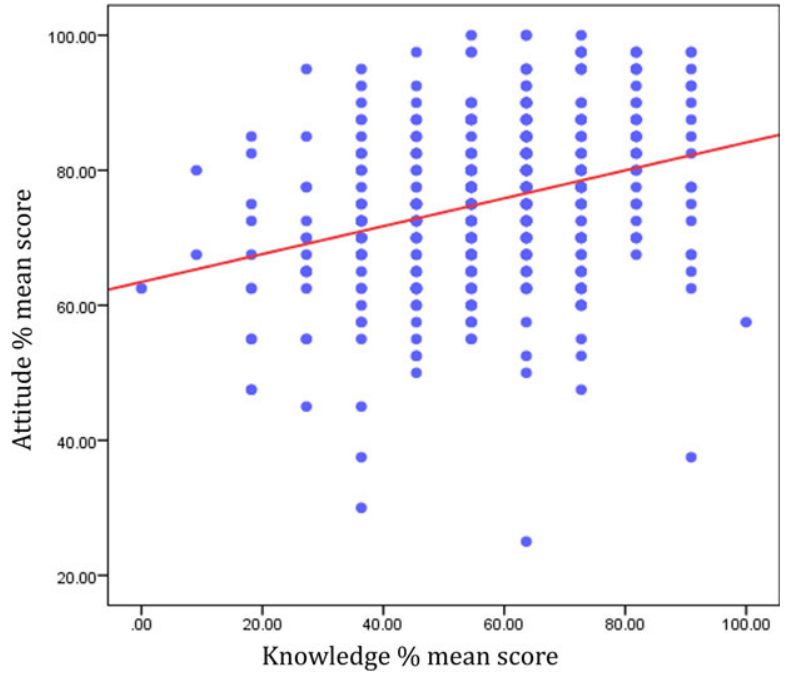

Figure 1: Correlation between knowledge and attitude mean scores.

lack of blood resources remains significant and problematic in advanced healthcare centers.

Aim: The aim of this study was to assess the level of public knowledge and attitude toward blood dona-tion. In addition, to describe the donating and non-donating populations with identification of motivating and restraining factors in each.

Methods: Using a previously validated questionnaire that comprises 38 questions to assess the level of knowledge, attitude, and practices towards blood donation, 469 Saudi adults who attended different shopping malls in Riyadh, Saudi Arabia were interviewed. Based on calculated scores, level of knowledge and attitude of our study cohort were categorized into different groups. Multiple regression analyses were used to identify significant predictors of blood donation.

Results: Approximately, half of all subjects (53.3\%) reported previous history of donation, 39\% of whom had multiple donations, with predominance of males (66\% vs $13.3 \% ; \mathrm{P}<0.001)$. Knowledge percentage mean score was $58.0 \%$, denoting poor level of knowledge. Attitude percentage mean score towards donation was $75.4 \%$, reflecting neutral attitude. After adjustment of confounders, multiple linear regression showed that higher knowledge score, higher attitude score, and male gender were significant predictors of blood donation $(\mathrm{P}=0.01, \mathrm{P}=0.001, \mathrm{P}<0.001$, respectively). Correlation between knowledge and attitude mean scores showed significant association between the variants $(\mathrm{P}<0.001)$. Notable reasons that refrained females from donation were inability to reach the blood donation centers and fear of anemia (49.9\% and 35.7\%, respectively), whereas lack of time was the only significant reason amongst males (59.5\%).

Conclusion/Summary: 0verall, level of knowledge about blood donation was poor, and attitude towards blood donation was generally neutral. Higher level of knowledge was associated with more positive attitude for blood donation. After adjustment, significant predictors of blood donation were higher knowledge and attitude scores, and male gender.

P-042

\section{A STUDY ON THE EFFECTIVENESS OF ADVERTISEMENTS AT THE WOODLANDS SATELLITE BLOOD BANK}

R Vamadevan ${ }^{1}$, LPB ms Tan ${ }^{2}$ and R ms Othman ${ }^{2}$

${ }^{1}$ HSA, Singapore, Singapore ${ }^{2}$ Health Sciences Authority, Singapore, Singapore

Background: Singapore's first satellite blood collection centre, Blood Bank at Woodlands, was opened in 2011. Situated within a shopping cum office building, with close proximity to a busy shopping mall and subway train station, it was hoped that this location would be more convenient to donors living in the Northern part of Singapore and would encourage more donations. Early this year, in order to increase public awareness regarding its location as well as to serve as reminders to donate blood, advertisements in the form of a lighted signboard mounted on the building's facade and advertorial stickers on the building's first floor escalator were installed. Yet blood collection at this satellite site still lags behind all other collection centres, including newer satellite sites. Faced with increasing blood demand, the staff at Woodlands were challenged to investigate the effectiveness of the advertisements.

(C) 2015 The Author Vox Sanguinis (C) 2015 International Society of Blood Transfusion Vox Sanguinis (2015) 109 (Suppl. 2), 1-96 
Aims: To determine the effectiveness of advertisements at the building where the Woodlands Satellite Blood Bank is located.

Methods: A survey was conducted using questionnaire forms given out to al potential donors who walked into the donation centre. The questionnaire was intentionally kept simple with only three questions so that donors could fill up the forms while waiting for their turn to donate blood. Sample size was not expected to be large as data collection was over two months, the intention was to have an overview of whether donors noticed the advertisements put up and therefore were informed of the availability of a donation centre in their neighbourhood.

Results: A total of 972 repeat donors and 88 first-time donors were surveyed. Almost no one rejected the questionnaire forms, because it was simple and quick to complete. The results showed that $62 \%$ of repeat donors and $49 \%$ of first-time donors did not notice the advertisements. There were more repeat donors than firsttime donors who did not notice the advertisements; this could be due to first-timer donors being keener on looking out for directions to the blood bank. On the question of accessibility, the results were encouraging. The vast majority of donors said the blood bank was accessible via lifts or stairs, even though it is situated on the fifth floor.

Summary/Conclusions: The role of satellite blood banks is central to overall blood collection in Singapore, and thus the location and visibility of the blood banks can have major implications on donor recruitment and retention. The results of this survey showed that advertisements placed at Woodlands served little to direct people to the blood bank or to get the blood bank noticed. It is therefore recommended that further measures should be taken to explore other possible avenues of advertisements as well as being more tactical and creative with the information conveyed.

\section{$\mathrm{P}-043$}

KNOWLEDGE, ATTITUDES AND BELIEFS TOWARDS

VOLUNTARY BLOOD DONATION AMONGST UNDERGRADUATE MEDICAL STUDENTS AT A TERTIARY HEALTHCARE INSTITUTION IN UTTARAKHAND, INDIA

A Doda, G Negi, DS Gaur and M Harsh

Himalayan Institute of Medical Sciences, Dehradun, India

Background: Blood donors form the backbone of blood transfusion services. Statistics show that there are 234 million major operations in India, 63 million trauma-induced surgeries, 31 million cancer-related procedures and 10 million pregnancy related complications which require blood transfusions. Apart from these there are also disorders like sickle cell anemia, thalassemia and haemophilia that require repeated blood transfusions. Blood donation is the only way of acquiring blood to meet the requirements. Globally, 80 million units of blood are donated each year. India, despite being a country with a population of 1.25 billion, faces a blood shortage of 3 million units every year.

Aims: The objective of this study was to determine the knowledge and attitude of medical students of a tertiary healthcare institution in Uttarakhand, India towards voluntary blood donation.

Methods: This descriptive cross-sectional study was conducted amongst second year MBBS students at Himalayan Institute of Medical Sciences, Dehradun, Uttarakhand (India). We assessed awareness, knowledge, attitude, perceptions and factors affecting voluntary blood donation in the students by a validated and pretested questionnaire. The consented information was analyzed using Microsoft Excel.

Results: More than half of the respondents had never donated blood. Of those that had donated, most were voluntary donors. Approximately two-thirds of total respondents had good knowledge of blood donation. Most students showed a positive attitude towards blood donation. Among those that had donated, males were more than females. Many donated for moral satisfaction in the regularly held blood donation camps at our institution. The reasons why many did not donate were lack of awareness, lack of opportunity due to tight lecture schedule, lack of knowledge and inadequate information about blood collection services along with fear of weakness and fear related to venepuncture. Some students agreed that IEC material and gift items such as T-shirts, stationery, mugs and wristbands would motivate them to donate blood. Use of print, electronic and social media was suggested for donor motivation. Students mentioned that a simple explanation of the procedure and reassurance would alleviate fear and apprehension about blood donation.

Conclusion: The role of youth in voluntary blood donation is crucial to meet the demand of safe blood. It was possible to understand the various factors that influence blood donation which is the basis for donor mobilization and retention strategies. A positive attitude and practice can be improved by inculcating knowledge about voluntary donation amongst medical students so as to recruit regular donors. Owing to ease of access to hospital services, the students organizations in the uni-

\section{(C) 2015 The Author}

Vox Sanguinis (C) 2015 International Society of Blood Transfusion

Vox Sanguinis (2015) 109 (Suppl. 2), 1-96 versity should include a blood donation drive in their activities. The university authorities along with the Department of Pathology \& Transfusion Medicine should collaborate in promoting voluntary blood donation among the students more actively.

\section{$\mathrm{P}-044$ \\ KNOWLEDGE, ATTITUDE AND PRACTICE TOWARDS WALK-IN BLOOD DONATION AMONG INHABITANTS OF SOME ISLANDS AND REMOTE DISTRICTS IN VIETNAM}

MQ Ngo, TH Le, VN Nguyen, TMA Bui and AT Nguyen

National Institute of Hematology and Blood Transfusion, Ha Noi, Vietnam

Background: One of the most important aspects of blood safety for inhabitants of remote and island areas is to ensure safe, adequate and timely supply of blood and blood components. In these geographical areas, it is important to ensure that a constant pool of donors is always available to donate, particularly in emergencies. Therefore, walk-in blood donor panels are set up as alternative and the donors mus commit to donate blood at any time and without the need for an appointment. During 2012-2013, the National Institute of Hematology and Blood Transfusion (Vie Nam) organized campaigns in some islands and remote districts in order to promote walk-in blood donation with a variety of activities such as broadcasting, leaflets, posters and delivery of the information to each family. In addition, 4 walk-in blood banks were established in 4 districts.

Aims: This paper aims (i) to assess the knowledge, attitude and practice towards walk-in blood donation of inhabitants of various islands and remote districts in 2013 and (ii) to identify the key factors affecting this.

Methods: A cross-sectional descriptive study was conducted among 810 inhabitants of the two largest islands (Cat Hai and the Phu Quoc district) and two typical rural and remote districts in the Northern (Dien Bien Dong district) and Southern part (Tinh Bien district) of Viet Nam. Responders were 18-60 years of age, lived in the central area of 4 different districts for more than 2 years and had to complete questionnaire.

Results: Among all 810 responders (211 in Cat Hai, 206 in Phu Quoc, 186 in Dien Bien Dong and 207 in Tinh Bien), 58.3\% were male and the average age was $37.4 \pm 10.47$.

$67.8 \%$ of responders were aware of the walk-in blood donation; $58.3 \%$ of respondents had the knowledge of the $\mathrm{ABO}$ blood group system, of which, $70.6 \%$ were aware that blood group 0 was the most needed blood group in recruiting walk-in blood donors.

96\% of the responders agreed that it was necessary to establish a walk-in donation panel and $64.9 \%$ agreed that joining such a blood bank was each person's own responsibility. $63.6 \%$ were willing to register themselves as donors in a walk-in donation panel, which was higher in males when compared with females $(66.9 \%$ and 58.9\% respectively). 6.2\% participated in walk-in blood donation before.

Factors that positively affected the general knowledge, attitude and practice towards walk-in blood donation were male gender, educational level of at least high schoo diploma, presence of relatives and friends who donated blood or received blood. The awareness of the walk-in blood donation enhanced the responder's willingness to participate in waking blood bank.

Conclusions: Inhabitants of various islands and remote districts were overall wel informed and had a positive attitude about walk-in blood donation; however, good practices of joining in the walking blood bank can still be improved.

\section{P-045}

\section{WHY WOMEN DON'T GIVE BLOOD? BACKGROUND}

\section{Kasraian and MH Karimi}

Blood Transfusion Research Centre, High institute for research and education in, Shiraz, Iran

In Iran, $100 \%$ of blood donations are collected from voluntary non-remunerated blood donors. The distribution of men and women donors is similar in many countries. However in previous studies in Iran, only 8\% of Blood collected from women blood donors. In shiraz, women account for only $3.2 \%$ of blood donors

Aims: The aim of this study was to assess donor motivations and barriers of donation of blood by women. methodsIn this cross-sectional study, 658 women were selected by cluster sampling method and asked to fill a specially formatted questionnaire. Data were analysed by chi squre, ANOVA and the t-test.ResultsThe mos important motivation for donation was altruism (98.6\%). The most important barriers for blood donation were fear of needle, fear of anemia, medical unsuitability for donation anemia, and lack of time..Conclusion Increase in the level of knowledge of 
women and correction of false beliefs could improve blood donation in women. Iron supplementation therapy can promote blood donation

P-046

DATA ANALYSIS OF BLOOD DONORS POST 2014 KAOHSIUNG GAS EXPLOSIONS

KT Lin, CH Yeh, SB Tseng and CM Hung

Kaohsiung Blood Center, Kaohsiung City, Taiwan

Background: A series of gas explosions occurred on July 31, 2014 in Kaohsiung, Taiwan. A large number of people came to donate blood after the incident as information of hundreds of wounded had been reported by the media.

Aims: It is worth noting that the number of first-time blood donors increased tremendously after the disaster. The aim of this study is to analyze the characteristics and demographics of the blood donors a week post disaster and compare these to that of the same period in the previous year. The findings of this study will help direct future publicity and recruitment efforts of blood donation.

Methods: All donors from August 1 to August 7, 2014, were subjects of the study group, while all donors during the same period in 2013 were subjects of the control group. The chi-square test and multiple variables logistic regression were used to analyze throughout the study.

Results: 3425 donors represent a 73.6\% increase in the same period from 2013 to 2014. 909 donors who are first time donors in the study group, compared to 171 donors in the control group, an increase of 738 donors and 431.6\%. Comparing from the study group to the control group, the average of age is younger and a positive correlation was found. The number of female donors was higher than males $(\mathrm{OR}=1.276)$. The number of $250 \mathrm{ml}$ donors was higher than that of $500 \mathrm{ml}$ donors $(O R=1.276)$. More donors came to the fixed sites than mobiles $(O R=3.311)$. As aforementioned, there is an increase of the number of first-time donors, as well as donors who have not donated blood over last three years $(O R=3.525)$. No difference was found on the rate of pre-donation deferral between the study and control groups. Conclusions: Since this disaster did not increase the demand of blood; we immediately mitigated the volume of donors arriving at various sites to prevent over donation. Nonetheless, reporting by unprofessional media causing chaos at donation sites. While both the number of first-time donors and repeat donors increased post disaster, the latter seemed better at gauging their donation effort based on the demand of the blood bank and understanding that donating regularly is the key to keep the blood bank working. Although the actual need of blood did not match the donors' expectation for donating blood in this gas explosion disaster, through this study, this experience can be utilized in establishing future regulations and recruitment efforts.

P-047

ANALYSIS ON THE CHARACTERISTICS OF BLOOD DONORS IN KOREA, 2011-2013

MJ Kim ${ }^{1}$, I Park ${ }^{2}$, YG Kim ${ }^{3}$, KI Na ${ }^{3}$, YS $\mathrm{Choi}^{3}$ and JN Kim ${ }^{3}$

${ }^{1}$ Seonam University College of Medicine, Goyang, South-Korea ${ }^{2}$ Pukyong

NatiolUnivrsity College of Natural Science, Busan, South-Korea ${ }^{3}$ Korean Centers for

Disease Control \& Preventon, Cheogju, South-Korea

Background: Transfusion comprises the essential parts of therapy in medical practice. Various products by many researchers have tried the clinical trials; no truly safe and effective artificial blood product is currently marketed. So, blood donation by healthy donors is required to fulfill the supply the adequate amount of blood component in time. To accomplish this goal, thorough understanding of the characteristics of blood donors is critical to recruit and maintain the donor pools.

Aims: Analysis on the blood donors was performed to identify the characteristics of majority of blood donors in Korea to be used as base line data to make a strategy to recruit and maintain the donors.

Methods: Age, gender, body weight, height, past donation history of donors, and the blood center, mobile/fixed site where the donation was performed from 2011 to 2013 were analyzed. BMI (body mass index) was calculated with donors' body weight and height. Descriptive statistics were used to analyze our data.

Results: 3,147,300 donors donated blood, including whole blood, plateletpheresis, and plasmapheresis, 7,699,232 from 2011 to 2013 in Korea. The average age of donors was 25.3 in male, and 21.9 in female. The average body weight was $70.4 \mathrm{~kg}$ in male and $56.6 \mathrm{~kg}$ in female, and the average height of donors was $174.2 \mathrm{~cm}$ in male and $161.7 \mathrm{~cm}$ in female. The average BMI was 23.2 in male and 21.6 in female. $65.8 \%$ was donated in fixed sites. $33.1 \%$ of male donors donated platelets or plasma through cell separators, and $98.5 \%$ of female donors donated whole blood. The med- ian number of past donation history was 7 in male and 5 in female, and 10 in fixed site and 4 in mobile drive.

Conclusions: Most blood donation is performed by young male with reference BMI in fixed site whose past donation history is seven times. Decreased donation rate in middle aged population, decreased participation rate in female, apheresis performed only in male, shows that the strategy customized to middle aged population and especially for female is needed to increase and maintain the stable donor pool in the long term.

\section{P-048}

\section{DEFERRED DONORS IN R. MACEDONIA IN THE YEAR 2014}

M Blagoevska, L Mitevska, E Velkova and T Makarovska Bojadzieva

National Institute of Transfusion Medicine, Skopje, Macedonia

Introduction: R. Macedonia is a small Balkan country with around 2 million citizens; $3 \%$ of population is registered as regular voluntary blood donors. In the last 5 years, 98\% of donations are from voluntary donors and only 2\% are family donors. Even though that at national level is reached self-sufficiency in red blood cells, albumin, immunoglobulin and coagulation factors are still imported. In the meantime the criteria for donor selection become more rigorous; the donor population is getting older which implicates on the number of collected blood units and reasons for deferrals.

Aim: To evaluate the number of deferred donors for the year 2014 and the reasons for deferral at national level; to compare number and reasons for deferral between National Institute of Transfusion Medicine-Skopje (NITM) and other 19 Blood Transfusion Services (BTSs) from other cities that are part of NITM since integration (year 2011).

Material and method: Data from monthly rapports from all BTSs and from NITMSkopje were used. With the method of comparative analyze, average and percentage were used to estimate the most frequent reasons for blood donors deferral.

Results: At national level for the year 2014 were collected 49.931 units. From total number of registered donors (55.962), deferred donors are 6.031 (10.8\%), out of which $46.1 \%$ are from BTSs and 53.9\% from NITM-Skopje. The most frequent reasons for deferral were: low hemoglobin level (women-30.4\%, men 24.9\%), hypotension (8.3\%), hypertension (7.3\%), infections (4.9\%, with antibiotic treatment), infections (3.6\%, without antibiotic treatment), cardiovascular diseases $(2.3 \%)$ and other (high risk behavior, travels in foreign countries, surgical treatment with received blood, recent tattoo and piercing, low weight) which represent $18.3 \%$.Permanent deferred donors are 3\% (first screen positive to TTI, severe chronic diseases etc.). There are significant variations in the reported data for deferred donors: two BTSs didn't report any case of deferral, which implicates on the average number.The number of deferred donors had variations from maximum $14.3 \%$, till minimum 1.6\%; at NITM- Skopje the number of deferred donors is high (14.3\%) which is questionable issue compared with previous years, when the referral rate was around 10\%. Conclusions: In the year 2014 for the first time there is evidence of deferred donorsat national level. It is obvious that the data are still incomplete, with big variations and differences between BTSs and NITM. Due to the lack of integrated informatics system for registration of blood donor deferrals, not all deferred donors are in the base. Other important factor is a new employed staff, which is in a process of education and has a lack of proper skills.

P-049

EVALUATION AND PATTERN OF DONOR DEFERRAL IN A TERTIARY LEVEL HOSPITAL, BANGABANDHU SHEIKH MUJIB MEDICAL UNIVERSITY, DHAKA

SS Miah

Bangabandhu Sheikh Mujib Medical University (BSMMU), Dhaka, Bangladesh

Background: Healthy donor recruitment and retention is a challenge that faces the health today. The most important steps in improving the safety of blood is donor selection. Hence proper assessment is essential to ensure the safety of the recipient and on entire transfusion process. By the evaluation of donor questionnaire and physical examination, the donors are deferred for many reasons which is allied to our patient and donor safety. Therefore it's very important to scrutinize the reasons for such deferral among the potential donors in order to categorize them into temporary and permanent deferrals.

Aims: To evaluate the causes of donor deferral pattern in the centre of Transfusion Medicine dept.in BSMMU, Dhaka. The main objective is to assess the current reasons for donor deferral so that temporarily deferred donors with corrective reasons can be

(C) 2015 The Author Vox Sanguinis (C) 2015 International Society of Blood Transfusion Vox Sanguinis (2015) 109 (Suppl. 2), 1-96 
identified, informed and guided to improve their quality and later on come back as a regular donor .Therefore continuous process of blood supply can be regulated. Methodology: Data was collected from the records maintained by the blood bank and it is a retrospective descriptive study based on evaluation of history and physical examination. Donors came were classified as physically fit or unfit for the comprehensive donation. Participants included between 1st January to 31st December on 2014.

Results: This study shows total 45,688 donors were found fit for donation over a period of one year and bloodletting was done from 22,167. Total 1239 donors were found unfit and among them 54.9\% were temporal and 45.1\% were permanently deferral. Male were $77.4 \%$ and Female were 22.5\%. Frequent causes were donor with hypertension and drug $22.9 \%$, asthma or severe allergy were found $12.5 \%$. Furthermore, most common reasons for temporal deferral were earlier donation within 3 months $18.0 \%$, anemic donor was found $14.7 \%$, weight $(<50 \mathrm{~kg}) 5.1 \%$, jaundice within 6 months were 4.4\%, H/0 taking antibiotic 4.2\%, enteric fever, dengue or chickenpox within 6 months were found 3.4\%, Age restriction like $>60$ years were $2.6 \%$, Vaccinated for $<1$ week $0.8 \%$, Tooth extraction or any dental procedure $<15$ days $0.6 \%$.

Remaining reasons for permanent deferral was Diabetes with drug or insulin 4.4\%, recent major surgery $<6$ months $2.0 \%$, TB or taking anti-TB drug $0.5 \%$, residing any endemic zone or malaria within 3 years were $0.2 \%$ and any allergic, fungal or viral infection, tattoo on donation site $0.2 \%$.

Conclusion: Healthy donor recruitment is the challenge that faces the health today. While, it is also important to ensure the sufficient collection process. The act of returning of donors must be shared, otherwise it could become a one way effort and meaningless because it gives the opportunity to save lives but paradoxically, it may put them in infection. The criteria for this selection and controls on donation as provided by law, are rigid and strict and we must not forget that donating blood is not just a way to save more lives, but also because the donor receives the result of analysis and can easily keep an eye on this health.

P-050

PROMOTION OF BLOOD DONATION PROGRAMS DURING FASTING MONTH BY PARTICIPATION OF GROUP DONATION IN TANGERANG BLOOD CENTRE

F Fitri, F Femi, I Ismi and D David

Tangerang Blood Centre, Tangerang, Indonesia

Background: The increasing need of blood demand for people who need it and blood stocks availability is very limited for patients, especially during fasting month in Indonesia, especially in Tangerang Blood Centre, so the massive campaign we do the marathon promotion for donor groups in Tangerang Blood Centre. One alternative by using the bus fleet S2MU that providing new facilities and services that easier in fullfilled blood stock availability required of the national community in Indonesia.

Aims: To reach and easier in provide facilities for donors in a blood donation action and provide the blood stock in according with the needs of the community during the fasting month in Indonesia.

Methods: Promotion of blood donation method using visual and electronic media and also short message via broadcast message in our management information sys-

\section{Blood Donation}

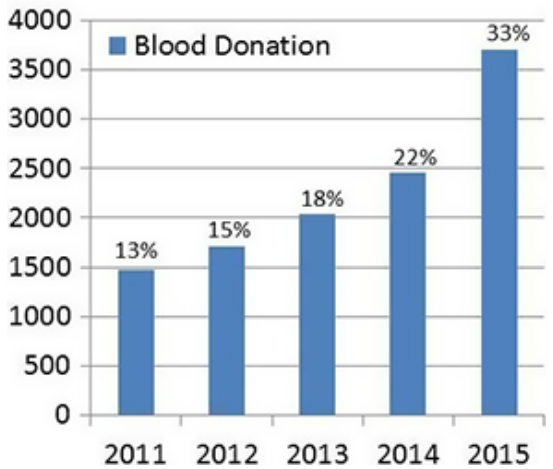

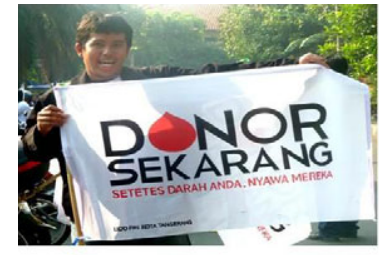

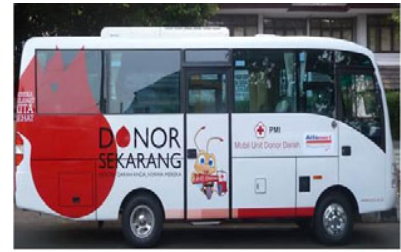

ACTIVITY OF BLOOD DONATION
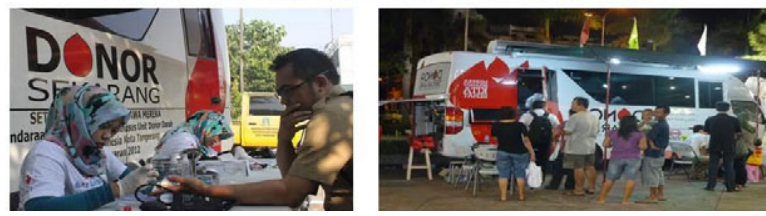

Figure 2: Chart Of Blood Donation.

tem SIMMUDA in order to obtain an agreement schedules with big donor groups during fasting month. Besides that, the promotional programs are considered effective and successful is use a fleet of bus (S2MU) that easier to reach society in downtown to keep doing blood action in fasting month.

Results: With blood donation activities method since 2011 to 2015, obtained the results such 1475 donors or 13\% (2011), 1715 donors or 15\% (2012), 2037 donors or $18 \%$ (2013), 2453 donors or 22\% (2014), 3700 donors or 33\% (2015). This method is effective in provide stocks of blood for people who need it during fasting month, not only patients in Tangerang City area, but also Jakarta, Bogor, Depok, Tangerang Regency, South of Tangerang, and Bekasi even Banten and West Java. Interestingly, these conditions can handled by UDD Tangerang Blood Centre, during the fasting month in 2015. The blood requests are always fulfill maximally and there is no taking blood from the patient's family like usually experienced all blood centre in Indonesia. Summary: Promotion of blood donation program during fasting month in Tangerang Blood Centre will developed continually and variety in order to fulfill the demand of blood for community, not least in the fasting month. Therefore, the promotion of blood donation program organized massively by Tangerang Blood Centre is proven effective in overcoming in the lack of blood stock during the fasting month in Indonesia.

P-051

This abstract has been withdrawn.

P-052

ANALYSIS OF RISK FACTORS LEADING TO NON COMMUNICABLE DISEASES AMONG A GROUP OF BLOOD DONORS AND ORGANIZERS OF BLOOD DONOSR SESSIONS

L Morawaka, HBNN Bandara, N Hettiarahchi and T Pathirana

National Blood Transfusion Service, Maharagama, Sri Lanka

Background: Annual Health Bulletin, Sri Lanka, enumerates ischemic heat disease as the leading cause of hospital deaths and cerebrovascular diseases as the fourth leading cause. Since there is an increasing tendency of being affected by non communicable diseases screening for risk factors has become a vital process in modern preventive medicine.

National Blood Transfusion Service (NBTS), Sri Lanka, collects more than 350 thousand blood units annually from voluntary non remunerated blood donors. While a minority of blood donors do their donations at hospital based blood banks majority of the donations take place at Mobile Blood Donor Sessions, organized regularly by motivated community groups.

Since blood donors are subjected to a brief medical examination at each donation and they have concerns over the other patients, it is assumed that such donors are healthy and motivated to maintain their physical well being.

Aims: This analytical study aims to detect the risk factors leading to non communicable diseases among a group of volunteer blood donors and organizers of Mobile Blood Donor Sessions. The results will also be used to encourage the group to main-

Figure 1: Picture of activity blood donation.

(C) 2015 The Author

Vox Sanguinis (C) 2015 International Society of Blood Transfusion

Vox Sanguinis (2015) 109 (Suppl. 2), 1-96 


\begin{tabular}{|c|c|c|}
\hline Cause of deferral & Male & Female \\
\hline 1. LOW HEMOGLOBIN & 7 & 167 \\
& $(4.0 \%)$ & $(96 \%)$ \\
\hline 2. INTAKE OF ANTIBIOTICS & 100 & \\
\hline 3. ALCOHOL INTAKE & $(96.2 \%)$ & 4 \\
& & $(3.8 \%)$ \\
\hline 4. UNDER WEIGHT & 29 & 0 \\
& $(100 \%)$ & $(0 \%)$ \\
\hline & 10 & 16 \\
\hline
\end{tabular}

tain their good health or guide them for counselling or treatments where the test results warrants to do so.

Method: A randomly selected group of blood donors, platelet donors and organizers of blood donor sessions were invited for the screening tests for non communicable diseases. It was done in parallel to World Blood Donor Day celebrations in order to motivate donor recruitment. Blood sample were taken from each individual for; Fasting Blood Sugar, Lipid Profile, Blood Urea, Serum creatinine, Full Blood Count and Serum Proteins. While a team of medical officers recorded their blood pressure Body Mass Index (BMI) was calculated by nursing officers.

Relevant test results were annexed to the consent form containing basic demographic data completed by each participant. Individuals with deviated results were summoned back on a subsequent date for counselling and referral. A reassessment was planned for them after one year.

Results: Two hundred people were invited for the screening and 120 were participated. Out of the participants 33 had normal results for tested parameters. Criteria considered at the assessment of remaining 87 participants needed either counselling or referral. While the age distribution of participants with deviated results ranged from 18 to 79 years $62 \%$ belonged to $40-59$ age group.

Details of the deviated test results are summarized in the given table. According to the results, blood pressure was out of the range only in 6 individuals and none of them were blood donors. Body mass index was more than 30 among 9 participants. Haemoglobin level and fasting blood sugar level ware out of range among 10 and 12 participants respectively. However the lipid profiles were abnormal in majority of the participants. Triglycerides, Total cholesterol and LDL were above the desirable levels among 46, 52 and 56 individuals respectively.

Conclusion: Out of the tested risk factors for non communicable diseases lipid profile was the most deranged parameter in the majority of participants.

P-053

This abstract has been withdrawn.

P-054

IMPLEMENTATION OF PRODUCTIVE APHERESIS IMPROVES PLATELET TRANSFUSION SAFETY OF POLYTRANSFUSED PEDIATRIC PATIENTS

C Napoli ${ }^{1}$, MR De Pascale ${ }^{1}$, DF Vitale ${ }^{2}$, A Belsito ${ }^{1}$, M Vasco $^{1}$, L Sommese ${ }^{1}$, M Di Mauro ${ }^{1}$ and B Nobili ${ }^{1}$

${ }^{1}$ Secon University of Naples, IT, Naples, Italy ${ }^{2} 3$ Fondazione Salvatore Maugeri, IRCCS Telese Terme 82037, Benevento, Italy

Background: The potential adverse effects of transfusion therapy is still debated and the search of the best transfusion practice is still going on. The focus of this study addresses the question regarding the benefit of apheresis (AP) donation in comparison to whole blood donations especially regardingthe safety profile of pediatric patients. In this context, we analyzed pediatric transfusion related events comparing the type and the frequency of adverse reactions both for standard and apheretic blood components during the follow-up period 2010-2013.
Methods: From 2010 to 2013, we performed a total of 17,190 donations with an annual increase of total APs and whole blood donations.

Results: The percentage of AP donations in comparison to total donations was $0.75 \%$ in 2010 until $15.8 \%$ in 2013 showing a significant test for trend $\left[\chi^{2}=493\right.$; $\mathrm{P}<0.001]$; at the same time, we registered a significant increase of percentage of AP in comparison to total blood donors $\left[\chi^{2}=432 ; \mathrm{P}<0.001\right]$. Reported transfusion related reactions to red blood cells were $0.49 \%$ for erithroapheresis transfusion related reactions, $0.08 \%$ for packed red blood cells without buffy coat transfusion related reactions as well as $0.32 \%$ for leucodeplete red blood cells transfusion related reactions. None adverse events for platelet aphaeresis, for plasma fresh frozen and for plasma aphaeresis were observed.

Conclusions: Our data highlighted that the implementation of AP donations were coupled with significant lower transfusion reaction rates for platelets. On the other hand, we observed a significant reduction of adverse events associated to erithroapheresis with respect to packed red blood cells without buffy coat and leucodeplete red blood cells.

\subsection{Blood Collection Including Apheresis}

$\mathrm{P}-055$

INCREASING THE NUMBER OF VOLUNTARY BLOOD DONORS

IN TANGERANG OF BLOOD CENTRE FROM 2011 UNTIL 2014

F Luwita, F Nugroho, I Martiyani, DH Sidabutar and M Kamaludin

Indonesian Red Cross, Tangerang City Blood Centre, Tangerang, Indonesia

Background: Since the establishment of blood centre tangerang in 2006, blood requests Need annually increasing every year, it encourages blood donation unit tangerang increasing the number of donations each year to fulfill the demand of blood

Aim: To determine the development of increasing voluntary blood donors at blood center Tangerang in the last four years and as a Tangerang blood center annual report

Method: Based on statistical data taken from the our management information system SIMUDDA (2011-2014), we are also compare and analyze the registration number the group of blood donors, and the level participation of individual blood donors during its period
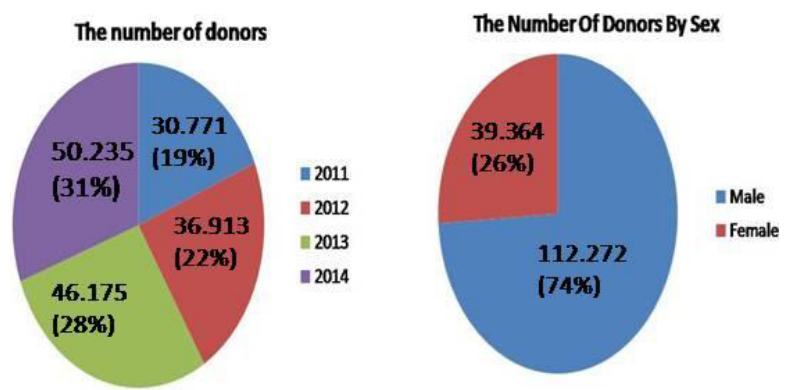

Figure 1: The Number of donors by Age and The Number of donors by blood type.
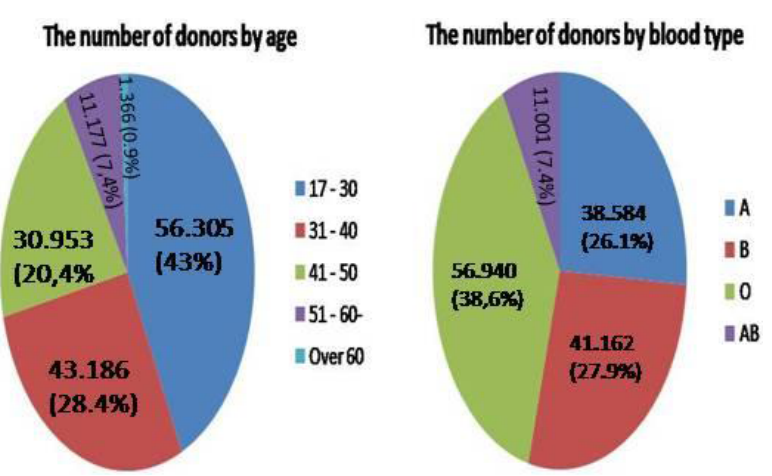

Figure 2: The Number of donors and The Number of donors by sex.

(C) 2015 The Author

Vox Sanguinis (C) 2015 International Society of Blood Transfusion Vox Sanguinis (2015) 109 (Suppl. 2), 1-96 
Result: There is increasing number of voluntary blood donors each year, such 30.771 people (2011), 36.913 people (2012), 46.175 people (2013), 36.390 people (2014), so total number of donors until now is 369.302 people. By sex; 112,272 male (74\%), 39.364 female (26\%). Based on the age of the donor; $65.305(43 \%)$ age $17-$ $30,43.186(28.4 \%)$ age $31-40,30.953(20.4 \%)$ age $41-50,11.177(7,4 \%)$ age $51-60$, $1.366(0.9 \%)$ age over 60 years. Based on blood group, such; blood group A 38.584 (26.1\%), blood group B 41.162 (27.9\%), blood group 056.940 (38.6\%), blood group AB $11.001(7.4 \%)$

Conclusion: The needs of underserved blood demand in blood center tangerang very influential to the participation of voluntary blood donors. To achieve enhancement number of voluntary blood donors every year, need to increase publicity about the importance of blood donation, comfortable place with good facilities, promotion of blood donation, etc.

\section{P-056}

\section{THE CAUSE OF THE BLOOD COLLECTION'S FAILURE ON} BLOOD DONOR BEGINNERS IN BLOOD DONOR SERVICES

R Aprianti, YM Djoechro, U Muktimanah, W Suryawidjaja, Y Mulyati, H Purwati, A Sriati and R Mulyani

Indonesian Red Cross, Bandung, Indonesia

Background: Until now the failure of the blood collection still occurs, primarily on blood donor beginners.

One of the main reasons is donor reaction occurs during donation, either mild reactions or moderate reactions, therefore will result in failure of blood collection.

Blood donor beginners are people who donate blood for the first time either voluntary or as a replacement donor with the age limit from 17 years old to 60 years old and weigh equal or more than $45 \mathrm{~kg}$.

Aims:

1. To determine the cause of failure in the blood donor beginners.

2. To identify the unknown factors that may affect the donor beginners.

Methods:

1. Descriptive research design with sample of 100 cases donor beginners.

2. Likert scale questionnaire data collectio, open questionnaire and structured interviews.

Result: From 100 donor beginners there are 20 donors failures of blood collection, caused by:

1. Sleep after 12.00 a.m.

2. Excessive activity before donor.

Conclusions: Blood collection's failure is have higher rates in donor beginners with sleep after 12.00 a.m, excessive activity before donor, and having high levels of anxiety.

Keywords: : failure, blood collection, blood donor beginner

\section{P-057}

\section{DONOR ARM PREPARATION TECHNIQUE EFFICACY EVALUATION}

\section{Toh, SC Khoo and HH Tan}

Health Sciences Authority, Singapore, Singapore

Background: Viral transmission via allogeneic blood is very low due to transfusion safety measures, bacteria contamination is still a great concern. Though the source of bacterial contamination in blood cannot always be easily identified, donors' arm nevertheless is a potential source of contamination. Type of antiseptic and cleaning technique have an effect on the efficacy of skin disinfection.Currently Blood Services Group, Singapore (BSG) is using a 1 step cleansing procedure with swab stick containing $2 \% \mathrm{w} / \mathrm{v}$ Chlorhexidine Gluconate in $70 \% \mathrm{v} / \mathrm{v}$ Isopropyl Alcohol in circular motion from within out from the intended venepuncture site for $30 \mathrm{~s}$, covering an area of about $8 \mathrm{~cm}$ by $8 \mathrm{~cm}$ and allowed to air dry for at least 30 s.FDA in its options for Arm Preparation (Method 4), approved scrubbing the intended venepuncture site with 2\% Chlorhexidine Gluconate in 70\% Isopropyl Alcohol with repeated back and forth strokes and paper published in Vox Sanguinis (2001) by C.P. McDonald on 'evaluation of donor arm disinfection techniques' found that the up and down motion of the disinfectant application was more superior to spiral.

Aims: In this evaluation, the efficacies of circular motion technique currently practise by BSG and the repeated back and forth strokes technique with the same type of

\section{(C) 2015 The Author}

Vox Sanguinis (C) 2015 International Society of Blood Transfusion

Vox Sanguinis (2015) 109 (Suppl. 2), 1-96 antiseptic (2\% w/v Chlorhexidine gluconate and 70\% v/v Isopropyl alcohol), were compared.

Methods: All phlebotomists in BSG were recruited as the subjects for this evaluation to minimise inconvenience to donors. Those who are sensitive to either Chlorhexidine or alcohol or pre-existing skin conditions on the antecubital fossa area were excluded.Direct swabbing on the antecubital fossa area of both arms of each subject was taken prior to skin disinfection as baseline.The antecubital foss area of the right arm of each subject was prepared with the antiseptic solution using circular motion from within out and that of the left arm was prepared using repeated back and forth strokes.Post disinfection swab on each arm was taken immediately after the required drying time and sent to Pathology Laboratory for plating, incubation and bacteria count.

Results: Post disinfection culture per plate for both methods had shown no growth in $93 \%$ of the cases. In both methods there were 3 cases each or $7 \%$ with colony growth of 1 to 4 colonies per plate post disinfection.

Summary and conclusion: There was no significant difference in efficacy with both methods. In view of the findings and FDA's option for arm preparation with $2 \%$ Chlorhexidine Gluconate in 70\% Isopropyl Alcohol, BSG has decided to adopt repeated back and forth strokes technique for donors' arm preparation.

\section{P-058}

VALIDATION OF THE HAEMOGLOBIN COLOUR SCALE IN BLOOD DONORS IN MALAWI

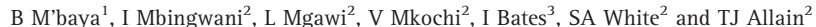

${ }^{1}$ Malawi Blood Transfusion Service, Blantyre, Malawi ${ }^{2}$ College of Medicine, Blantyre, Malawi ${ }^{3}$ Liverpool School of Tropical Medicine, Liverpool, United Kingdom

Background: The Malawi Blood Transfusion Service (MBTS) was established in 2004 as the nationally coordinated blood service based on voluntary non-remunerated blood donation. Malawi is a densely populated low Human Development Index (HDI) Southern African country (2009 GDP per capita of USD290) with a population of 15 million, $85 \%$ of whom live in rural areas. It cannot routinely use HemoCue for anaemia screening due to the high cost of cuvettes. Since 2009, the MBTS uses the WHO Haemoglobin Colour Scale (HCS) to screen all blood donors to determine their haemoglobin eligibility for blood donation and to detect those that need additional testing with HemoCue before their eligibility can be decided. Before 2009, clinical assessment was used in addition to this but it was removed because it was felt that this criteria was being abused due to its subjective nature. The 2009 HCS based protocol is used as follows: those with $\mathrm{Hb}<12 \mathrm{~g} / \mathrm{dl}$ are deferred for blood donation; those with $\mathrm{Hb}>12 \mathrm{~g} / \mathrm{dl}$ are accepted for blood donation, while those with $\mathrm{Hb}=12 \mathrm{~g} / \mathrm{dl}$ are referred for testing with HemoCue and are only accepted for donation if the $\mathrm{Hb}$ is $\geq 12.5 \mathrm{~g} / \mathrm{dl}$.However, both the pre and post 2009 protocols had not been validated. Published studies of the accuracy of the HCS to screen potential blood donors show varying levels of accuracy and opinion varies whether this is an appropriate screening test or not hence the need for this study.

Aims: The aim of the study was to assess the validity of the MBTS protocol of using the HCS and HemoCue in screening for anaemia in potential blood donors.

Methods: This was a blinded prospective study in potential blood donors aged over 18 years, at MBTS in Blantyre, Malawi. Capillary blood samples were analysed using the HCS and HemoCue, independent of each other. The sensitivity and specificity of correctly identifying eligible blood donors as per MBTS protocol were calculated. Results: From 242 participants 8 (3\%), were wrongly allocated on the basis of the HCS compared to HemoCue. All were subjects that were wrongly accepted as donors when their haemoglobin results were $\leq 12.0 \mathrm{~g} / \mathrm{dl}$. This gave a sensitivity of $65 \%$ and specificity of $100 \%$ to detect eligibility for donation. No subjects were wrongly excluded as donors.

Conclusions: Initial screening with the HCS correctly predicts eligibility for blood donation in the majority of potential blood donors at considerable cost saving compared with use of HemoCue as the first line anaemia screening test. 
P-059

PERIPHERAL BLOOD STEM AND PROGENITOR CELL COLLECTION BY TWO SEPARATORS: A PROSPECTIVE RANDOMIZED MULTICENTER STUDY

$\mathrm{H} \mathrm{Ohto}^{1}$, K Ikeda ${ }^{2}$, K Muroi ${ }^{3}$, SI Fujiwara ${ }^{4}$, K Minagawa ${ }^{2}$, M Yamada-Fujiwara ${ }^{5}$, Y Fujimori $^{6}$ and R Tanosaki ${ }^{7}$

${ }^{1}$ Fukushima Medical University, Fukushima, Japan ${ }^{2}$ Dept. Blood transfusion \& Transplantation Immunology, Fukushima Med University, Fukushima, Japan ${ }^{3}$ Division Cell Transplantation and Transfusion, Jichi Medical University, Tochigiken, Japan ${ }^{4}$ Division of Hematology, Department of Medicine, Jichi Medical University, Tochigi-ken, Japan ${ }^{5}$ Division of Blood Transfusion and Cell Therapy, Tohoku University Hospital, Sendai, Japan ${ }^{6}$ Department of Transfusion Medicine and Cellular Therapy, Hyogo College of Medicine, Nishinomiya, Japan ${ }^{7}$ The Japan Society of Transfusion Medicine and Cell Therapy, Tokyo, Japan

Background: Collection of peripheral blood stem and progenitor cell (PBSPC) imposes various apheresis-related risks including thrombocytopenia, hypocalcemia, and thromboembolism. Therefore, an efficient PBSPC collection is crucial with fewer risks and less contamination. Previously we have shown preventive measures against hypocalcemia and performances of apheresis devices and software (Transfusion, 2003, 2007 and 2014). A new automated apheresis machine, the Spectra-Optia, was recently introduced but this device was not yet established for safety and efficiency.Aims: To compare the novel Spectra-Optia device with the currently widely used automated apheresis machine, Spectra-Auto (Software version 6.1), we performed a prospective randomized multicenter study, for the first time.Methods: The apheresis device used for granulocyte-colony stimulating factor (G-CSF) given adults $(\geq 18$ years) was randomly assigned between November 2013 and May 2015. A total of 154 first-day apheresis collections, 79 with Spectra-Auto and 75 with Spectra-Optia, including 46 collections for autologous (auto-) patients and 108 for allogeneic (allo-) donors were analyzed. Apheresis was performed according to the manufacturer's protocols, with a 12:1 to 15:1 ratio of whole blood to acid citrate dextrose (ACD), without heparin. Pre-apheresis parameters including age, sex, body weight, circulating blood volume, and peripheral blood cell counts were similar between devices.Results: The Spectra-Auto processed larger volumes when compared with the Spectra-0ptia $(\mathrm{P}<0.05)$, although the run times were similar $(\mathrm{P}=0.6)$. Mild apheresis-related reactions occurred in some cases, but the frequencies of reactions and volumes of ACD used were not different between the machines. Harvested product volumes were greater in the Spectra-Optia when compared with the Spectra-Auto (158 vs $95 \mathrm{ml}, \mathrm{P}<0.001)$. Although mononuclear cell yields and CD34 + cell collection yields were similar, CD34+ cell collection efficiency (CE-1) using the Spectra-Optia device was higher $(\mathrm{P}<0.01)$ than the Spectra-Auto in allo-donors, rather than auto-patients $(P=0.3)$. However, regardless of the program used, pre-apheresis peripheral cell count strongly correlated with the number of CD34 + cells collected. The products collected using the Spectra-Optia contained more contaminating red blood cells ( 64 vs $36 \times 106$ cells, $\mathrm{P}<0.001$ ), but not platelets $(\mathrm{P}=0.1)$, without inducing anemia in patients/donors.Conclusion: Following apheresis collection of PBSPC, the performance of the Spectra-Optia machine is comparable in the CD34+ cell yield or adverse events, when compared with the currently used Spectra-Auto device.

Table 1: Apheresis yields: Spectra-Auto vs Spectra-Optia.

\begin{tabular}{|c|c|c|c|}
\hline & $\begin{array}{l}\text { Spectra-Auto } \\
(n=79)\end{array}$ & $\begin{array}{l}\text { Spectra-Optia } \\
(n=75)\end{array}$ & $P$ value \\
\hline Allo-donor/auto-patient & $54 / 25$ & $54 / 21$ & N.S. \\
\hline Gender M/F & $48 / 31$ & $39 / 36$ & N.S. \\
\hline Age (years) & $42[18-66]$ & $41[20-68]$ & N.S. \\
\hline Body weight $(\mathrm{Kg})$ & $61.2[36.8-94.0]$ & $61.9[41.1-91.1]$ & N.S. \\
\hline Pre-WBC $\left(10^{9} / \mathrm{L}, \pm \mathrm{SD}\right)$ & $37.3 \pm 19.0$ & $36.0 \pm 16.2$ & N.S. \\
\hline Pre-CD34+ cell $\left(10^{6} / \mathrm{L}, \pm S D\right)$ & $5.9 \pm 4.3$ & $5.7 \pm 6.7$ & N.S. \\
\hline Processed volume $(\mathrm{mL}, \pm \mathrm{SD})$ & $8803+2199$ & $8053 \pm 1598$ & $<0.05$ \\
\hline Run time (min., \pm SD ) & $185 \pm 30$ & $187 \pm 24$ & N.S. \\
\hline WBC yield $\left(10^{\%} / \mathrm{bag}, \pm S D\right)$ & $23.1 \pm 12.6$ & $24.7 \pm 12.0$ & N.S. \\
\hline CD $34+$ cell yield $\left(10^{6} / \mathrm{bag}, \pm\right.$ SD $)$ & $287.5 \pm 325.2$ & $215.0 \pm 193.1$ & N.S. \\
\hline $\begin{array}{l}\text { CD34+ cell Collection Efficiency-1 } \\
(\%,+ \text { SD })\end{array}$ & $66.3 \pm 31.3$ & $82.2 \pm 24.3$ & $<0.001$ \\
\hline
\end{tabular}

P-060

\section{THE ADVANTAGE OF PLATELET INCREMENT ON PEDIATRICS AFTER PLATELETPHERESIS' TRANSFUSION AT HERMINA HOSPITAL}

Y Djoechro and W. Suryawidjaja

Indonesian Red Cross, Bandung, Indonesia

Background: Patient in need for platelets, can be transfused with platelet blood components which derived from multi donors or single donor platelet.

Common blood components is one of the options due to it has lower cost than plateletpheresis, but the usage of plateletpheresis is more beneficial to the patients. The reason for plateletpheresis' transfusion is for therapeutic supportive.

Aims: To analyze the increment of platelets number in pediatric after plateletpheresis' transfusion.

Methods:

1. We performed an observational study with cross-sectional design to assess the increment of platelets number after plateletpheresis.

2. Data collection before and after plateletpheresis' transfusion.

3. We examined platelets before and after plateletpheresis' transfusion.

4. Thirteen pediatrics received plateletpheresis for observation.

Results: The average of platelets increment after plateletpheresis' transfusion was $81,212 / \mathrm{mm}^{3}$.

Ten tranfusions (77.5\%) had platelet increment: 60,000-90,000/ $\mathrm{mm}^{3}$. One transfusions $(7.5 \%)$ had platelet increment until $160,000 / \mathrm{mm}^{3}$.

Two transfusions (15\%) had platelet refractioness, which received repeated tranfusion of plateletpheresis.

Conclusion: The average of platelets increment was $81.212 / \mathrm{mm}^{3}$.

Good response was found in $77.5 \%(10 / 13)$ transfusions. Platelet refractioness was found in $15 \%(2 / 13)$. Some transfusions are with rash.

Keywords: platelets increment, plateletpheresis.

\section{P-061}

\section{SINGLE \& DOUBLE DOSE PLATELETPHERESIS DONATION AND PLETELETPHERESIS DEMAND IN TANGERANG CITY \\ BLOOD CENTRE}

B Supriyono, D.H. Sidabutar, F Nugroho and M Kamaludin

Indonesian Red Cross, Tangerang City Blood Centre, Tangerang, Indonesia

Background: Dharmais Cancer Hospital is the first hospital which doing plateletpheresis in Indonesia.Number of platelete needed for transfusion is increasing every years in indonesia. For those things, Tangerang city Blood Centre adding more service in apheresis technology, which adding more machine become 3 unit of apheresis machine for increasing blood supply services. Plateletpheresis donation have done at the first time in Tangerang city blood centre at 2013, until now 2015, Tangerang City Blood Centre still continues for increasing the services to fullfil the blood supply needed of Plateletpheresis which taking Double Dose Donation.

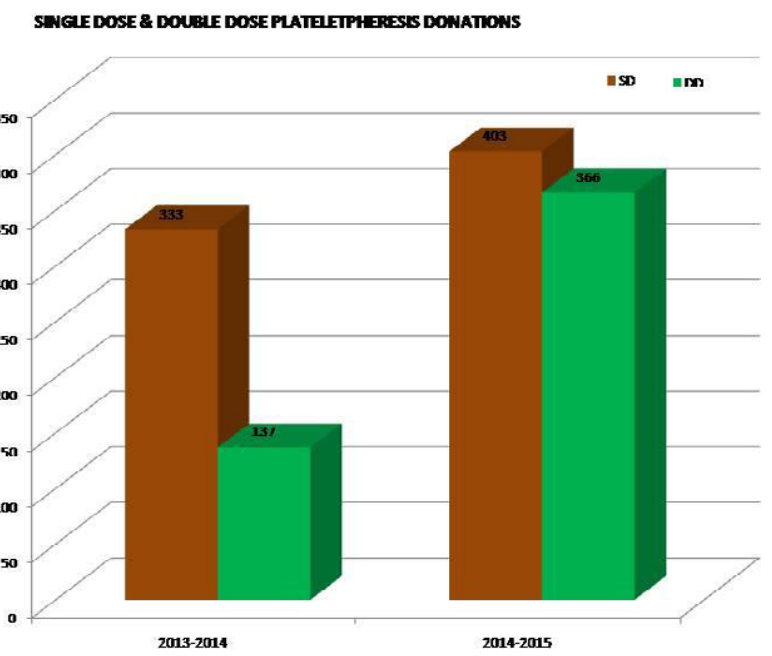

Figure 1: Single dose \&t double dose Plateletpheresis donation.

(C) 2015 The Author Vox Sanguinis (C) 2015 International Society of Blood Transfusion Vox Sanguinis (2015) 109 (Suppl. 2), 1-96 


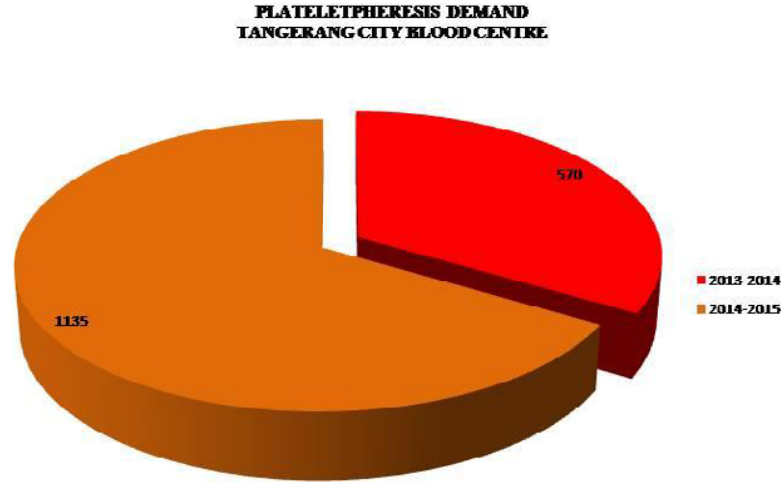

Figure 2: Plateletpheresis demand tangerang city blood centree.

Aim: Observing increasing of single and double dose plateletpheresis donation and blood supply demand of plateletpheresis In tangerang city blood centre

Method: collection of data retrieval single \& double dose Plateletpheresis from April 2013-April 2014 as many as 470 Donations. Single dose as much as 333 donations and double dose as much as 137 donations. The number of hospital demand from April 2013-April 2014 are 580 bags. data collection single and double dose in Apri 2014-April 2015 are739 donations. There are 403 single Dose plateletpheresis donations and 336 plateletpheresis double dose donations. Blood Supply demand of Plateletpheresis from hospital are 1135 bags.

Result: From those data, we have increasing number of donations and demand from hospital every year. From 2013 to 2015 there are increasing number to $21 \%$ of single dose plateletpheresis donation, and increasing $161.7 \%$ of double dose plateletpheresis donation and also blood demand of plateletpheresis product until 95.6\% Conclusion: From those data of plateletpheresis an increasinge number of double dose donations more than 100\% and also increasing blood demand of plateletpheresis from the hospital. This may indicate a progressive increase in the quality of blood services in Indonesia and in particular in Tangerang city blood centre. The number of donors needed more to be able to further improve service and fullfil the demand of plateletpheresis from hospitals in Tangerang City or Indonesia globally.

\subsection{Donor Adverse Events}

P-062

\section{DELAYED VASOVAGAL REACTIONS FOLLOWING BLOOD DONATION}

A Thijsen, D Waller, J O'Donovan and B Bell

Australian Red Cross Blood Service, Alexandria, Australia

Background: Delayed vasovagal reactions (VVR) are an important problem for the safety of donors; blood services are unable to provide onsite care and there is a high risk of injuries. Several studies have documented underreporting of delayed VVRs and much remains unknown as to the determinants and experiences of a delayed VVR. It is relevant for blood services to improve their understanding of delayed VVRs as it may aid in the development of strategies to reduce the incidence and severity of these events.

Aims: The aim of this study was to increase our understanding of delayed VVRs by examining the incidence rates of reported events, examine underreporting of delayed VVRs, and to describe the experience of delayed VVRs among the Australian blood donor population.

Methods: A mixed-method approach was used to understand the impact of a vasovagal reaction on the blood donor. First, descriptive analysis $(n=25,852)$ was conducted of reported vasovagal reactions in 2014-15 in the Australian blood donor population. Second, data was used from the Donor Safety Survey collected in 2015 ( $n=431)$ among donors with no registered adverse event to explore the rate of unreported events in this sample. Third, in-depth telephone interviews were conducted with donors who recently experienced a delayed VVR $(n=14)$. Descriptive analysis of the quantitative data was conducted using means and proportions. Qualitative data was thematically analysed using NVivo.

Results: Adverse event records from 2014-15 indicated that 12.2 percent of the total number of VVRs occurred offsite, of which 61.5 percent of the donors experienced a loss of consciousness and 5.0 percent sustained an injury. The group consisted primarily of females $(79.1 \%)$ who were aged between 20-29 years (22.8\%)

\section{(C) 2015 The Author}

Vox Sanguinis (C) 2015 International Society of Blood Transfusion

Vox Sanguinis (2015) 109 (Suppl. 2), 1-96
Survey data revealed a further number of additional unregistered adverse events including haematoma, and delayed VVRs without an associated loss of consciousness. Those with an unregistered delayed VVR were predominately female and were aged between 20-29 years. The interview data provided insight into the context of the delayed VVRs. Frequently mentioned causes were inadequate hydration after donation, strenuous physical exercise, and prolonged period of walking or standing post donation. Whilst donors reported receiving information regarding how to prevent VVRs, none of the participants were aware of how to manage vasovagal symptoms. Interestingly, instructions on how to manage VVRs are available to donors but this study finding illustrates the importance of improving communication strategies. Conclusions: Donors reporting a delayed VVR are at a high risk of a loss of consciousness. Unreported VVR without an associated loss of consciousness might contribute to the lower than expected donor return rate among female and younger donors. Interview data indicated strong donation preparation behaviours and a knowledge gap in post-donation behaviours which may be contributory to the onse of a delayed vasovagal response. Further work is required to improve donor adverse events reporting and to enhance donor education of post-donation prevention and management strategies.

\section{P-063}

THE RELATIONSHIP BETWEEN DONOR'S DIET SHORTLY BEFORE DONATION AND SPORT ACTIVITY WITH TOTAL CHOLESTEROL AND TRIGLYCERIDES LEVEL IN JAKARTA BLOOD TRANSFUSION UNIT

LC Sari, H Lestari, N Agung, NK Ritchie and S Salim

Jakarta Blood Transfusion Unit, DKI Jakarta, Indonesia

Background: Diet and lifestyle can affect donor's health. A healthy diet contains all the nutrients needed in appropriate amount. Unbalanced diet and less exercise were predisposed of dyslipidemia. High cholesterol in plasma could be seen as white and milky plasma. The milky plasma could not be used for transfusion and would be discarded. From 2012 until 2013, there was 10\% increasing of milky plasma discarded in Jakarta Blood Transfusion Unit. In our quality control laboratory, we found that most of the milky plasma has high cholesterols and high triglycerides. After a meal, triglycerides level can rise five to ten times higher. We always told donor to have meal $2-4 \mathrm{~h}$ before donation. As part of donor services, we have to educate dono with the right information but first we must know what the cause of the high cholesterols and triglycerides in blood donors.

Aims: To know the relationship between donor's diet shortly before donation and donor's lifestyle to lipid level in blood donor.

Methods: We use questionnaire and interview method to get information of donor's diet shortly before donation and donor's lifestyle. We take $3 \mathrm{ml}$ of donor's blood in a non-anticoagulant tube and check the cholesterol and triglyceride level using CHOD-PAP method. The data was processed with chi square test and regression test. Results: We interviewed 50 Donors. 21 of 50 have high cholesterol $(>200 \mathrm{mg} / \mathrm{dl}$ ), 33 of 50 have high triglyceride $(>160 \mathrm{mg} / \mathrm{dl})$. There was no significant correlation between donor's diet shortly before donation and the level of cholesterol and triglyceride $(\mathrm{P}>0.05)$. There was also no significant correlation between sport activity with cholesterol level $(\mathrm{P}>0.05)$ but there was significant correlation between sport activity with triglyceride level $(\mathrm{P}<0.05)$. We also found that more frequent the donor exercised, the level of triglyceride was lower.

Summary/conclusions: Donors could have meal $2-4 \mathrm{~h}$ before donation and we should encourage them to do sport activities more often.

\section{P-064}

\section{HAEMOVIGILANCE OF BLOOD DONORS: IMPACT OF PRE- DONATION COUNSELING ON DONOR REACTION}

\section{Abdul Quader ${ }^{1}$, MA Shakoor ${ }^{2}$ and MA Islam²}

${ }^{1}$ AD-DIN medical college hospital, Dhaka, Bangladesh ${ }^{2}$ Bangabandhu Sheikh Mujib Medical University (BSMMU), Dhaka, Bangladesh

Background: The safety of blood for transfusion depends on careful selection of donors, the backbone of a transfusion service. So donor's safety should be maintained throughout the whole blood collection process. During blood donation, various adverse events might occur, including sweating, weakness, dizziness, fainting convulsion and local injuries.AIMS: To study the influence of pre- donation counseling on blood donor reactions in order to maintain a steady donor stream.

Methods: In a medical college hospital, we performed a cross-sectional study on blood donors (both male and female) who donated blood from January 1until end of June 2014. Blood donors were selected randomly and categorized either into first- 
time or regular donors. Adverse donor reactions were documented in donors with or without counseling. In this study, only blood donors who donated within the last two years included into the regular donor group.

Results: 256 out of 4145 randomly selected donors were investigated in total. 111/ 256 selected donors were first-time and 145/256 were regular donors.54/ 111 firsttime donors were counseled, while 57 were not.76/145 of regular donors were counseled, 69 were not. The mean age of first-time donors was $30.27 \pm 2.34$, for regular donor's $31.57 \pm 1.95$ years. In $20.37 \%$ of first-time donors who were being counseled, donation reactions occurred, while this was $45.61 \%$ in the group who did not undergo counseling. Among regular donors who received counseling, 15.79\% experienced a donor reaction. In contrast, this was $31.88 \%$ who were not counseled. In total, $27.73 \%$ of studied donors experienced a donor reaction. In the counseling group, this was $8.98 \%$ while this was $18.75 \%$ in the without counseling group. A total of $185(72.27 \%)$ showed negative reaction: 107 (41.80\%) were part of the counseling group, and $78(30.47 \%)$ in non- counseled group.

Conclusions: We found that in the counseled group less adverse donor reactions occurred but the donors also responded more negatively towards counseling when compared with the non- counseled group. Although our null hypothesis was: no association between donor reaction and counseling, this study did show a strong association $(\mathrm{P}<0.001)$. In general, we found that counseling before blood donation has great impact on the number of adverse donor reactions. Therefore, in order to maintain a high quality donor chain, we should therefore implement counseling for all donors pre-donation.

\section{$\mathrm{P}-065$}

\section{SOME CHARACTERISTIC FACTORS ASSOCIATED WITH ADVERSE REACTIONS IN WHOLE BLOOD DONATION IP Rupasena \\ National Blood Center, Colombo 05, Sri Lanka}

Background: Blood donation programme in Sri Lanka is conducted by National Blood Transfusion Service. The National Blood Transfusion Service is responsible for the blood collection, processing, testing and distribution of blood to both public and private hospitals. All the requirements of blood are fulfilled by the voluntary nonremunerated blood donation.

Blood donors are the backbone of Blood Transfusion Service. Ensuring the safety of blood donors is of extremely importance. If the donors are ensured of a pleasant experience during blood donation they could be motivated to become regular blood donors. This can be accomplished by way of preventing adverse reactions in the donor.

Aims: This study was conducted to determine and estimate the frequency of occurrence of adverse reactions in whole blood donors and to identify associated factors like age, sex, and body weight in the causation of donor adverse reactions.

Methodology: The retrospective study was conducted a period of one year from 01/ 10/2013 to 30/09/2014 in National Blood Center, Colombo, Sri Lanka. The data was collected from four mobile blood collecting in relevant period.

Result: Total of 62,130 voluntary blood donors, $67.3 \%(n=41,814)$ males and $32.7 \%$ ( $n=20,316$ ) females were studied during relevant period for adverse reaction of blood donation.

$2.22 \%(n=1382)$ of voluntary blood donors reacted to blood donation. Male donors accounted for $58.18 \%(n=804)$ reactions while the female counterpart was responsible for $41.82 \%(n=578)$.First time donors were significantly more likely had adverse effect 60.85\% $(\mathrm{n}=841)$ than regular blood donors 39.15\% $(\mathrm{n}=541)$

The age distribution of donors were $24.6 \%(\mathrm{n}=15,311), 47.9 \%(\mathrm{n}=29,695), 16.7 \%$ $(\mathrm{n}=10,390)$ and $10.8 \%(\mathrm{n}=6734)$ for aged $18-25,26-35,36-45$ and $46-60$ years respectively. The rate of adverse effect was highest, 3.85\% among donors $18-25$ years compared to $1.2 \%, 3 \%$, and $1.8 \%$ respectively for other age groups $(\mathrm{P}<0.001)$.

The rate of adverse reactions was higher among donors who weighing between 50$59 \mathrm{~kg}$ than the higher weight group (weight $60 \mathrm{~kg}$ or above) $(\mathrm{P}<0.001)$. Table 01.

Table 01

\begin{tabular}{|c|c|c|c|c|c|c|c|}
\hline Age range & Total No & $\%$ & Non $\mathrm{Re}$ & $\%$ & Rea & $\%$ & $P$ value \\
\hline $18-25$ & 15311 & $24.6 \%$ & 14722 & $96.15 \%$ & 589 & $3.85 \%$ & \multirow{4}{*}{$p<0.001$} \\
\hline $26-35$ & 29695 & $47.9 \%$ & 29333 & $98.8 \%$ & 362 & $1.2 \%$ & \\
\hline $36-45$ & 10390 & $16.7 \%$ & 10081 & $97 \%$ & 309 & $3 \%$ & \\
\hline $46-60$ & 6734 & $10.8 \%$ & 6612 & $98.2 \%$ & 122 & $1.8 \%$ & \\
\hline
\end{tabular}

Conclusion: There was a predictable low rate of adverse reaction to blood donation among voluntary blood donors. First time donors were more prone to get adverse reactions than regular donors. When considering the age of donors, there was significant reactions among donors who were in between 18-25 years of age .Adverse reactions of blood donation was significantly occurred in donors who had 50-59 kg body weight than donors $60 \mathrm{~kg}$ body weight or more. Donor friendly environment, relieving of peer pressure, shorten the waiting time and good communication with donor may help to resolve the occurrence of adverse reactions.

Table 02.

Table 02
\begin{tabular}{|l|lc|l|l|lr|r|r|}
\hline Weight range (kg) & Total No. & $\%$ & \multicolumn{2}{|l|}{ Non Reactors } & $\%$ & Reactors & P value \\
\hline $\mathbf{5 0 - 5 9}$ & 24911 & $40.1 \%$ & 24472 & $98.2 \%$ & 439 & $1.8 \%$ & \multirow{2}{*}{ p $<0.001$} \\
\hline $\mathbf{6 0 - 6 9}$ & 15968 & $25.7 \%$ & 15586 & $97.6 \%$ & 382 & $2.4 \%$ & \\
\hline$>70$ & 21251 & $34.2 \%$ & 20690 & $97.3 \%$ & 561 & $2.7 \%$ & \\
\hline
\end{tabular}

P-066

OMAN EXPERIENCE AFTER IMPLEMENTING DONOR

HEMOVIGILANCE (DHV) AT CENTRAL BLOOD BANK, DEPARTMENT OF BLOOD SERVICES, MUSCAT

AH Sakr, T Ashraf and A Al-Balushi

Ministry of Health, Sultanate of Oman, Muscat, Oman

Background: Donor health and safety should be of equal concern to blood safety. Donor hemovigilance is the systemic monitoring of adverse reactions and incidents in the whole chain of blood donor care, with a view to improving quality and safety for blood donors.

Aim: The aim of this study is to monitor complications related to blood donation (adverse reactions), errors in donor care (adverse incidents) and to focus on aspects related to donor safety.

Methods: Donor hemovigilance reporting system (DHV Form) based on ISBT/IHN classification is adapted. Full training of all nurses conducted to ensure proper recognition, reporting and classification of adverse events. Donor reactions are stratified under age, sex and frequency of donation. Age was classified into three categories (18-24 years), (24-40 years) and (40-65 years). Frequency of donation was only divided into 1 st timers and repeated donors.

Results: This programme started in 20/9/2014 and data given till 31/5/2015. Through this period 18,878 donors males represented $83.47 \%$ while females $16.53 \%$. Repeated donors were 52.64\% against 47.32\%. Repeated donors had the upper hand in males by $53.5 \%$ while the 1 st timers were in females by $51.63 \%$. As total levels of reactions we had 440 males $(2.79 \%)$ and females $254(8.14 \%)$. 1st timers donors in both sexes were $400(57.80 \%)$ while repeated donors reactions were $292(42.20 \%)$. According to age classifications the mean of reactions percentage in relation to their total number in the same age group, males between 18-24 years had reactions percentage $(5.66 \%)$ while females showed (11.94\%). Age between $24-40$ years males had $(2.48 \%)$ while females had (4.97\%). Last age group between $40-65$ years males had $(1.49 \%)$ females had $(2.36 \%)$. Through the details of reactions types the programme of donor vigilance showed that we recorded (1213) form of reactions presented as hyperventilation (118), Sweating (252), Dizziness (539), loss of consciousness (72), vomiting (15), convulsions (46), hematomas and re-bleeding (52), nerve irritation (4), offsite reactions (112) and only one case of hypocalcaemia symptoms. Many cases of multiple forms of reaction occurred to same donors.

Conclusion: However, the current study covers short time period with limited number of donors but gives us a conclusive understanding that DHV is an important and valuable tool in monitoring the quality of blood donation services delivered. Most frequently recorded reaction remains dizziness. There is notified number of convulsions reaction (46) and off-site reactions (112) for further investigations. Upon analysis, reactions occurred more frequently in younger age, female and first time donors. The data is comparable to results reported from blood banks in other parts of the world. With comparable classification (ISBT/IHN), one could not only look into other countries, but also look into effectiveness of risk reduction strategies and follow-up trends. We started to implement pre-donation hydration as a method of intervention to reduce levels of complications.

\section{Blood Products \\ 3.1 Blood Processing, Storage and Release}

P-067 HEMOLYSIS POTENCY OF WASHED ERYTHROCYTE AND INLINE RED CELL LEUKODEPLETED COMPONENTS

TSA Akbar ${ }^{1}$, ASM Sofro ${ }^{2}$ and RSE Gantini ${ }^{3}$

${ }^{1}$ Indonesian Red Cross - Banda Aceh Blood Center, Banda Aceh, Indonesia ${ }^{2}$ Faculty of Medicine YARSI University, Jakarta, Indonesia ${ }^{3}$ Center Blood Transfusion Service, Jakarta, Indonesia

Background: Washed erythrocyte (WE) and red cell leukodepleted (RC-LD) are normally used in clinical transfusion to prevent transfusion reaction. However, in Indonesia there is no evidence on hemolysis due to erythrocyte washing and leukocyte filtration that may cause significant clinical impact.

(C) 2015 The Author

Vox Sanguinis (C) 2015 International Society of Blood Transfusion Vox Sanguinis (2015) 109 (Suppl. 2), 1-96 
Aims: To provide scientific evidence on the potency of hemolysis, hematology specification and total protein count of WE and RC-LD blood components

Methods: Hemolysis, hematology testing and total protein count were run on 26 blood samples before and after erythrocyte washing using normal saline open system and on another 26 blood samples before and after leukocyte filtration using in-line filter. The hemolysis and hematology specification was tested using the Osmotic Fragility Test (OFT) method and Automatic Sysmex Xn-2000 system, while the total protein count was tested using the ADVIA 1650/1800 system.

Results: A very low level of hemolysis $(<0.8 \%)$ was observed in all samples of WE and RC-LD. The decrease of hemoglobin and hematocrit level after washing on WE was not significant $(\mathrm{P}>0.05)$ and their average value still met the standard which were $39.83 \pm 5.59 \mathrm{~g} / \mathrm{unit}$ and $64.04 \pm 5.36 \%$ respectively. Meanwhile, the decrease of protein concentration and leukocyte count after washing on WE were significant $(\mathrm{P}<0.05)$ in which their average value were $0.19 \mathrm{~g} /$ unit and $3.27 \times 10^{8}$ per unit respectively. Moreover, for the RC-LD the decrease of hemoglobin and hematocrit level after leukocyte filtration was not significant $(P>0.05)$ and their average value still met the standard which were $45.34 \pm 6.44 \mathrm{~g} / \mathrm{unit}$ and $53.58 \pm 6.25 \%$, respectively. Meanwhile, the decrease of protein concentration and leukocyte count after washing on WE were significant $(\mathrm{P}<0.05)$ in which their average value were $6.52 \mathrm{~g} / \mathrm{unit}$ and $0.79 \times 10^{6}$ per unit, respectively.

Conclusion: Hemolysis during the preparation of both WE and RC-LD blood components could be considered negligible. The erythrocytes' washing decreased the plasma protein concentration significantly, and the leucodepletion by filtration decreased the leukocyte count significantly. Therefore the WE and RC-LD components are considered to be useful for preventing transfusion reaction such as allergy to plasma protein and reaction to human leukocyte antigen.

Keywords: Washed Erythrocyte, Red Cell Leukodepleted, Hemolysis, Transfusion Reaction.

\section{$\mathrm{P}-068$}

COMPARATIVE STUDY OF THE PERCENTAGE OF

HAEMOLYSIS OF RED BLOOD CELLS STORED IN TWO DIFFERENT ADDITIVE SOLUTIONS: SAGM AND AS-1

E Siomi, SS Chua, WC Tan and S Lam

Health Sciences Authority, Singapore, Singapore

Background: Red blood cell (RBC) transfusion is the most common therapeutic procedure performed in hospitals. Since the 1980's, RBC components have been prepared as concentrates suspended in nutrient additive solution to preserve and extend the shelf-life of the RBC components. Studies have shown that storage in different additive solutions may have some effects on the RBC membranes which could lead to varied level of haemolysis. In Blood Services Group (BSG) Singapore, two different additive solutions are regularly used for the preparation of the buffy-coat removed RBCs. The two additive solutions used are (i) Saline-Adenine-Glucose-Mannitol (SAGM) in the JMS T-BEX System and (ii) Adsol (AS-1) in Macopharma topand-bottom triple blood bag. Both additive solutions allow the RBC units to be stored up to 42 days at refrigerated temperature before transfusion. To monitor the post-storage haemolysis of red cells, measurement of the plasma haemoglobin in the supernatant of RBC units is performed at the end of shelf-life.

Aims: Our aim is to study the effect of the additive solutions SAGM and AS-1 on the storage of RBCs by measuring the extent of haemolysis after 42 days of refrigerated storage.

Methods: Routine Quality Control (QC) data were collected retrospectively. A total of 28 units of SAGM RBC and 40 units of AS-1 RBC were tested for the level of haemolysis on day 42 . The percentage (\%) haemolysis of the RBC units were calculated using the haematocrit obtained from a Full Blood Count from the Sysmex ${ }^{\circledR}$ XS-1000i and supernatant haemoglobin measured using the HemoCue ${ }^{\circledR}$ Plasma/Low $\mathrm{Hb}$ System. The Student's t-test was used to determine the statistical difference between the SAGM RBC and the AS-1 RBC groups.

Results: All RBC units met the Council of Europe guideline on the QC requirement for haemolysis of $<0.8 \%$ of the red cell mass. The average \% haemolysis for the SAGM RBC units is $0.31 \%$ (range: $0.11 \%$ to $0.59 \%$ ) while the AS- 1 RBC units have a mean value of $0.25 \%$ (range: $0.05 \%$ to $0.55 \%$ ). Graphical analysis shows that the AS-1-RBC units have comparatively lower \% haemolysis measurements than the SAGM RBC units. The Student's t-test yields P-value of 0.053 which is fairly close to statistical significance $(\mathrm{P}<0.05)$. Our results are consistent with other reported studies that AS-1 RBCs have significantly lower hemolysis compared to RBCs stored in SAGM although factors such as donor red cell variations and type of storage containers are not excluded in our study.

Conclusions: The extent of haemolysis for RBCs stored in AS-1 appears to be lower in comparison to storage in SAGM. Nonetheless, the level of haemolysis for both

\section{(C) 2015 The Author}

Vox Sanguinis (C) 2015 International Society of Blood Transfusion

Vox Sanguinis (2015) 109 (Suppl. 2), 1-96
RBCs stored in SAGM and AS-1 are still within the acceptance limit of $0.8 \%$ as stated by the Council of Europe guideline.

\section{P-069}

OSMOTIC FRAGILITY TEST OR HEMOLYSIS, WHICH ONE IS MORE WORTHY FOR BLOOD CENTRE?

N Nurhayati, HT Lestari, N.K. Ritchie and S Salim

Jakarta Blood Transfusion Unit, DKI Jakarta, Indonesia

Background: Blood is a viable organ. Red blood cells (RBCs) needs glucose for glycolysis to produce ATP for maintaining the flexibility of red cell membrane. Stored RBCs undergo biochemical, structural, and functional changes, so its shelf life was shortened. The membrane was more fragile and easily hemolysed. Seventy percent of RBCs were expected still viable in patients after it is being transfused. Osmotic Fragility Test (OFT) could be used to detect the viability of RBSs pre-transfusion. So this test was used in Jakarta Blood Transfusion Unit (BTU) to see the quality of RBCs. But, when we referred the parameters of RBCs quality in the European guidelines and AABB standards, it was stated less than $0.8 \%$ of red cell mass hemolysed OFT definitely is not the method used to meet the criteria in the standards. We've been introduced with photometer (HemoCue plasma/Low Hb Photometer) to check the $\mathrm{Hb}$ level in plasma which could be used to see the level of hemolysis in packed red cells (PRCs).

Aims: To know the impact of OFT method and photometer to the decision for discarding PRCs.

Methods: We have total 84 aliquot of PRCs from 14 PRCs stored in CPDA-SAGM for 35 days. At the first day and every week after, we did the oft method and check the level of hemolysis with HemoCue plasma/Low Hb Photometer and Sysmex KX21. The OFT method is a test to find out the fragility of RBCs by suspend the RBCs in various concentration of sodium chloride. In $0.54 \%$ or less than $0.54 \%$ of sodium chloride, the normal RBCs would not hemolysed. If it is hemolysed in concentration of more than $0.54 \%$ sodium chloride, the RBCs were interpreted already fragile and discarded. In the photometer, if the percentage of hemolysis was more than $0.8 \%$ then the PRC would be discarded. We also analyze the relationship between the OFT method with photometer with Fisher's exact test.

Results: At the first week of storage, no PRC discarded. At the second week of storage, only one PRC discarded due to both results. At the 3rd, 4th and 5th week of storage, more PRC discarded due to OFT method compared to photometer as follows 3 to 1,8 to 1 and 9 to 1 . We also found there were no significant relationship between the OFT method with photometer $(\mathrm{P}>0.05)$ because the OFT method was predicted the fragility of RBCs membrane or the possibility of RBC's viability in patient's circulation but it might be not hemolysed yet in PRC. In the other hand, the photometer method was detected the level of hemoglobin in plasma which means how many RBCs already hemolysed in PRC.

Summary/Conclusions: Less PRCs discarded if we referred to photometer result so the availability of PRCs stock could be maintained, but the adverse effect of transfusion should be monitored in patients.

\section{P-070}

BLOOD SEPARATION AND STORAGE WITH A NEW GRAVITY DRIVEN DEVICE

F Bonn $^{1}$, B Büttner ${ }^{2}$, D Krieter ${ }^{3}$, HD Lemke ${ }^{2}$ and C Sauvant ${ }^{2}$

${ }^{1}$ Membrana GmbH, Wuppertal, Germany ${ }^{2}$ EXcorLab GmbH, Obernburg, Germany

${ }^{3}$ University Hospital Wuerzburg, Department of Medicine I, Wuerzburg, Germany

Background: Disaster or lack of electrification in underdeveloped areas both are conditions, which make preparation of erythrocyte concentrates and plasma from whole blood donation difficult.

Aims: Aim of the present study was to assess the performance of PlasmaXpress ${ }^{\circledR}$, a novel CE-certified, hollow-fibre based device with integrated leucodepletion filter designated for gravity-driven red blood cell separation.

Methods: The blood components were investigated according to European guidelines. The weight of erythrocyte concentrates and plasma separated with the PlasmaXpress ${ }^{\circledR}$ system was determined. Red cells were stored for $42 \mathrm{~d}$ in red blood cell bags containing $100 \mathrm{ml}$ stabilizer solution SAG-M (saline-adenine-glucosemannitol, $376 \mathrm{m0sm} / \mathrm{l})$. Relative haemolysis was calculated as ratio of free haemoglobin to total haemoglobin after complete lysis of red cells. Sterility was assayed by standard broth tests (bioMérieux, Germany). Coagulation factor VIII was measured by a chromogenic activity assay (BIOPHEN Faktor VIII:C Kit, Coachrom, Austria) and platelet count in plasma was detected by FACS analysis (Plasma 
Count Kit BD, BD Biosciences, Germany). A total of $n=54$ experiments were performed.

Results: Mean weight of the blood units used for filtration was $474 \pm 10 \mathrm{~g}$, resulting in $319 \pm 13 \mathrm{~g}$ of red blood cell concentrate (including stabilizer solution) and $242 \pm 47$ g plasma (mean \pm SD of $n=54$ experiments). Duration of filtration was $57 \pm 15 \mathrm{~min}$. Haematocrit increased from $38 \pm 3 \%$ in blood before filtration to $53 \pm 3 \%$ in red cell concentrate. The red cell concentrate was free of leucocytes. Immediately after separation, no significant haemolysis was detected $(0.04 \pm 0.02 \%$ in plasma; $n=27$ ). Storage of red cells led to haemolysis of only $0.45 \pm 0.16 \%$. At the end of the storage period, sterility of the red cell concentrates could always be proven $(\mathrm{n}=23)$. The plasma generated contained $2.4 \pm 1.7 \times 10^{6}$ platelets $/ 1$ plasma $(\mathrm{n}=6)$. Taking into account a limit of detection of $0.6 \pm 0.3 \times 10^{6} / 1$ and a European quality limit for therapeutic plasma of $<50 \times 10^{9} / 1$, the plasma may be considered as virtually platelet free $(n=6)$. Activity of factor VIII in plasma was $91 \pm 19 \%(\mathrm{n}=29)$.

Summary/Conclusions: PlasmaXpress ${ }^{\circledR}$ is an easy to handle device which allows processing of high quality red cell concentrates and plasma from whole blood without centrifugation and automated separation. The results do not differ from blood products currently generated in blood banks and meet European Guidelines.

\section{P-071}

This abstract has been withdrawn.

\section{P-072}

EVALUATION OF THE TERUMO PENPOL TOP AND BOTTOM BLOOD BAGS USING A T-ACE II+ AUTOMATIC COMPONENT EXTRACTOR AT WPBTS IN SOUTH AFRICA

SL Sutton and DL Smith

Western Province Blood Transfusion Service, Cape Town, South Africa

BACKGROUND: Top top quad blood bags are currently used at Western Province Blood Transfusion Service (WPBTS) in South Africa. Blood separation is done using a refrigerated centrifuge and a component extractor. $1 \%$ of annual production is recommended for quality control testing. The leucocyte quality control of the red cell (RC) concentrates were not consistently meeting requirements of the Standards of Practice for Blood Transfusion in South Africa.

AIM: The objective was to determine if the components produced from whole blood donations using top and bottom (TAB) blood bags resulted in red cell concentrates with a lower leucocyte count and simultaneously obtaining buffy coats with a higher platelet yield than the top top bags currently in use at WPBTS.

METHOD: Predominantly group A donors were selected and bled into TAB bags. Initial separation of the whole blood was done by centrifugation and further separated on a T-ACE II+ into the various components viz. RC concentrate, buffy coat and plasma. $\mathrm{RC}$ concentrates produced were prepared for routine quality control i.e. weight, volume, leucocyte count and haematocrit. A Pentra XL80 was used for cell counts.

Six random donor platelets were made by pooling 5 buffy coats and adding $200 \mathrm{ml}$ of a corresponding plasma, which is our current method. A further twenty one random donor platelets were made using 4 buffy coats and an increased volume of plasma at the advice of a Component Processing Specialist. Platelet counts and pHs were done on random platelets. A second $\mathrm{pH}$ was tested on expiry of the random platelet.

RESULTS: Of the $171 \mathrm{RC}$ concentrates produced, the average haematocrit was $0.6 \mathrm{l} /$ 1 with no failures observed. The average volume per RC bag was $286.74 \mathrm{ml}$, sixteen were lower than the minimum requirement of $250 \mathrm{ml}$. Two RC concentrates had leucocyte counts exceeding the requirement of

$\leq 2.4 \times 10^{9} /$ unit with the average being $1.1 \times 10^{9} /$ unit.

There was a $94 \%$ total platelet recovery for fifteen platelet pools when pooling only 4 buffy coats.

One $\mathrm{pH}$ did not meet the requirement of $6.9 \pm 0.5$ on expiry, which could be attributed to a high platelet count.

CONCLUSION: The Standards of Practice for Blood Transfusion in SA require that $80 \%$ of monthly QC pass specifications. This requirement was met.

Leucocyte counts in the RC concentrates were significantly lower for blood donations bled into TAB bags with 99\% meeting requirements. Overall there was a greater than $80 \%$ pass rate for the various quality control requirements for the red cell concentrates.

The buffy coat was more stable and on comparing results of platelet pools of 5 with 4 buffy coats it would be suitable to rather pool 4 with the addition of 280-305 g of plasma.

\section{P-073}

\section{THE EFFECT OF PRE-STORAGE LEUKOREDUCTION FILTRATION ON SOLUBLE HUMAN LEUKOCYTE ANTIGEN-I LEVELS IN PLATELET CONCENTRATE}

\section{SA Herawati}

Udayana University, Denpasar, Indonesia

Background: Platelet concentrates (PC) are important blood components in order to manage bleeding in patients. In order to provide platelet concentrates with good therapeutic effect, preparation process and storage condition should be optimized and maintained properly. To ensure high quality, pre-storage leukoreduction filtration (PLF) can be performed during PC preparation. Human Leukocyte Antigen-I is a highly polymorphic glycoprotein which is expressed on all nucleated cells and also on platelets. The presence of HLA-I antigen in PC is considered a marker of immunological reactivity and cellular fragmentation; therefore it can be used as an indicator of the performance of PLF in PC preparation.

Aims: The purpose of this study was to determine the effect of PLF on soluble human leukocyte antigen-1 (sHLA-I) levels in PC stored for 1, 3 and 5 days.

Methods: In this study 34 PC were enrolled, which were randomly assigned into a PLF treated and control group. Samples from all PC were taken on day 1, day 3 and day 5 during storage. sHLA-I levels were determined using an Enzyme Linked Immunosorbent Assay (ELISA).

Results: In the PLF group, lower mean sHLA-I levels were found when compared with the control group. The mean sHLA-I level in PLF group at day 1 was $2.75 \pm 0.82 \mu \mathrm{g} /$ $\mathrm{ml}$ while this was $3.66 \pm 0.87 \mu \mathrm{g} / \mathrm{ml}$ in the control group $(\mathrm{P}<0.05)$. At day 3 , the mean sHLA-I level in the PLF group was $3.07 \pm 0.75 \mu \mathrm{g} / \mathrm{ml}$ and $4.05 \pm 1.29 \mu \mathrm{g} / \mathrm{ml}$ in the control group $(\mathrm{P}<0.05)$. The mean sHLA-I level in the PLF group at day 5 was $3.81 \pm 0.97 \mu \mathrm{g} / \mathrm{ml}$ vs $5.61 \pm 3.26 \mu \mathrm{g} / \mathrm{ml}(\mathrm{P}<0.05)$ in the controls.

Conclusion: It can be concluded that PLF significantly decreases sHLA-I levels in PC stored for up to five days, when compared with untreated controls. These findings suggest a positive effect of PLF treatment on the quality of PC, especially since it could potentially decrease the immunological reactivity and cellular fragmentation of platelets.

\section{P-074}

\section{VALIDATION OF RAD SOURCE RS3400 X-RAY IRRADIATOR} FOR THE IRRADIATION OF BLOOD AND BLOOD COMPONENTS TS Leong

Health Sciences Authority, Singapore, Singapore

Leong Tong Seng, Linda Corcoran: Blood Services Group, Health Sciences Authority, Singapore: Background: The rationale behind the irradiation of blood components prior to transfusion in immunocompromised patients is to prevent transfusionassociated graft-versus-host disease (TA-GvHD), a potential complication of blood transfusion whereby donor white cells in the blood product can replicate and mount an immune response against this vulnerable patient group. Irradiation of blood components can be performed using Gamma irradiation and more recently, X-ray irradiation. Irradiation of blood components is currently performed by Blood Services Group (BSG) using gamma irradiation. BSG will transit from the use of gamma irradiation to X-ray irradiation using the Rad Source RS3400 Blood Irradiator.

Aim: This study aims to validate the RS3400 X-Ray irradiator and compare its performance against current in use gamma irradiator Nordion Gammacell 1000 Elite (GC-1000E).

Methods: RAD-SURE XR15 Gy, XR 25 Gy and RadTAG RTX15 irradiation labels were used to verify the dosage delivered to blood components are within AABB/FDA guidelines. The equipment system functions, holding capacity and reliability (stress testing by running 24 continuous irradiation cycles) was tested by performing irradiation cycles using either dummy bags or blood components. The pre-set irradiation time was verified against a calibrated reference timer and total processing time was recorded.

Results: Dose mapping reports showed that the x-ray irradiator was able to deliver a central dose of $25 \mathrm{~Gy}$ (with minimum dose of $15 \mathrm{~Gy}$ and maximum dose of 50 Gy at any point) with uniformity maintained at 1.6, meeting the FDA and AABB requirements. The radiation emission level at $17 \mathrm{u} \mathrm{R} / \mathrm{hr}$ was within regulatory safety requirements $(<500 \mathrm{u} R / \mathrm{hr})$. All system controls/functions were working as per specifications. The RS3400 irradiator could accommodate up to 6 units of blood component per cycle run as compared to 1 unit per cycle for GC-1000E irradiator. Projected irradiation time per cycle for RS3400 and GC-1000E irradiators are 8.5 and 6.5 min respectively including setting up, documenting and verification of the irradiated unit's data. The turn-around time for irradiation cycles based on average 
daily requirement of 34 units of component was $0.9 \mathrm{hr}$ for RS3400 and $3.7 \mathrm{hr}$ for GC-1000E.

Summary/Conclusions: The RS3400 X-Ray irradiator is validated to be a safe and suitable replacement for the Gamma irradiator delivering the required dose and uniformity of exposure to blood components being processed.

Utilisation of this equipment will enhance the irradiation workflow due to the increased processing capacity. Based on the above, RS3400 is capable of meeting BSG operational requirements and needs.

\section{P-075}

\section{EFFECT OF LEUKODEPLETION AND PATHOGEN}

\section{INACTIVATION ON PLATELET CONCENTRATES}

T Burkitbayev $^{1}$, Z Baltabayeva ${ }^{1}$, S Abdrakhmanova $^{1}$, Z Bibekov $^{1}$ and E Zhiburt ${ }^{2}$

${ }^{1}$ Scientific and production center of transfusiology, Astana, Kazakhstan ${ }^{2}$ Pirogov National Medical Surgical Center, Moscow, Russian Federation

Introduction: The efficacy and safety of transfusion of donor platelets is increased by leukodepletion and pathogen inactivation. However, additional processing of blood components might change their morphological and functional characteristics. For example, in untreated erythrocyte suspensions, at least $45 \mathrm{~g} /$ dose of haemoglobin is present while following leukodepletion it needs to be at least $40 \mathrm{~g} /$ dose. In normal fresh frozen plasma, VIII factor activity should be at least $0.7 \mathrm{IU} / \mathrm{ml}$ while following pathogen reduction this should be at least $0.5 \mathrm{IU} / \mathrm{ml}$. To date, the effect additional processing might have on platelet counts in platelet concentrates (PC), are not taken into account.

Aims: Determine the effect of leukodepletion and pathogen inactivation on platele counts in PC

Materials and methods: We have prepared $21 \mathrm{PC}$, pooled from 4 buffy coats, isolated from whole blood (Makopharma, France). Additionally, 94 PC were prepared by apheresis (MCS +, Gemonetiks, USA). Leucocytes were removed from all PC byleuko-filtration (Pall, USA) and pathogen inactivation was carried out (Tserus, USA). At all stages, we determined PC volume and platelet counts (Sysmex XT2000i, Japan).

Results: PC volumes were not significantly different after additional processing. PC prepared by apheresis contained a $28.2 \%$ higher platelet count when compared with the pooled one $(\mathrm{P}<0.01)$. When $\mathrm{PC}$ were leukodepleted, platelets count in pooled PC did not significantly change while in apheresis PC this was reduced by $26.5 \%$ (P < 0.01). Pathogen inactivation does not influence platelet counts in PC (Table 1). Conclusion: Additional processing of PC, in particular leukodepletion might reduce platelet counts due to size of platelets during filtration. Therefore, in order to validate PC processing technologies, it is necessary to control the basic quality parameters for transfusion.

Table 1. PC characteristics during processing ( $\mathrm{M} \pm \mathrm{SD} *$ ).

\begin{tabular}{|l|c|c|}
\hline \multirow{2}{*}{} & \multicolumn{2}{|c|}{ PC type } \\
\cline { 2 - 3 } & Pooled & Apheresis \\
\hline Initial volume $(\mathrm{mL})$ & $277.0 \pm 26.2$ & $299.9 \pm 7.6$ \\
\hline After leukodepletion & $273.8 \pm 17.8$ & $289.0 \pm 9.0$ \\
\hline $\begin{array}{l}\text { After pathogen } \\
\text { inactivation }\end{array}$ & $253.3 \pm 15.3$ & $276.3 \pm 10.0$ \\
\hline Initial Platelet count, $\times 10^{9}$ & $273.7 \pm 84.7$ & $351.0 \pm 77.2$ \\
\hline After leukodepletion & $284.9 \pm 62.6$ & $258.1 \pm 62.1$ \\
\hline $\begin{array}{l}\text { After pathogen } \\
\text { inactivation }\end{array}$ & $266.0 \pm 52.4$ & $267.0 \pm 62.5$ \\
\hline
\end{tabular}

Note: ${ }^{*} \mathrm{M}$ - arithmetic mean, SD - Standard deviation

\section{P-076}

\section{NCI CAIRO UNIVERSITY EXPERIENCE IN MANUAL VS \\ AUTOMATED BLOOD COLLECTION}

\section{S Shalaby}

Cairo university, Cairo, Egypt

Background: For many years, the only available platelet component was a RPC obtained from whole blood donation by a two centrifugation steps process, the "platelet rich plasma' [PRP]. Since the beginning of the 1970s, APCs became available, using different techniques. Since the end of the 1980s, a new method of RPC preparation was developed, using the buffy-coat (BC-PC), providing a blood component with highly preserved platelet functions as compared to RPCs prepared by the PRP technique. Finally, the use of each of these components either native, leuco-reduced,

\section{(C) 2015 The Author}

Vox Sanguinis (C) 2015 International Society of Blood Transfusion

Vox Sanguinis (2015) 109 (Suppl. 2), 1-96

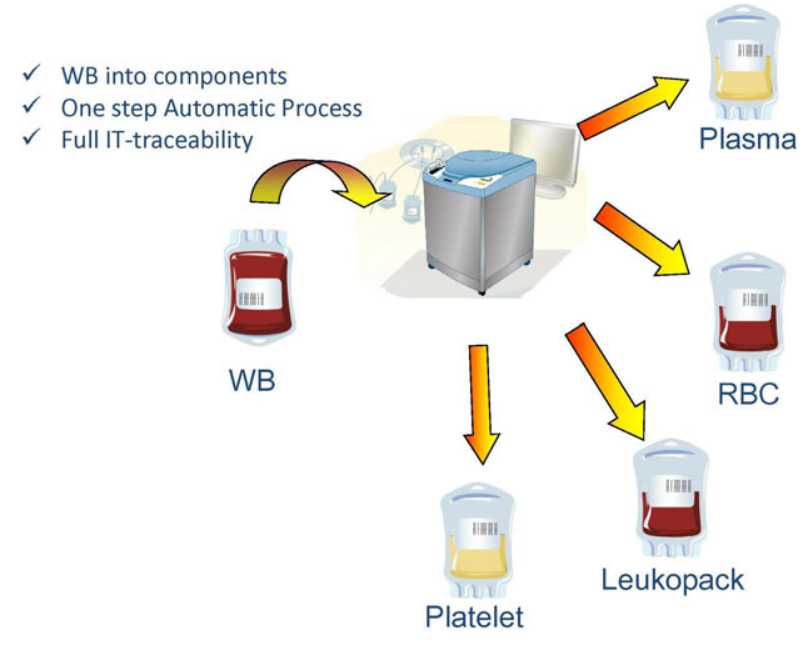

Figure 1: automated component quality.

\section{Atreus product quality}

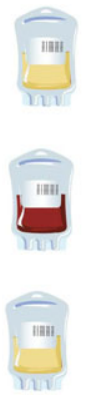

\begin{tabular}{|c|c|}
\hline Plasma & $\begin{array}{l}\text { - Volume } \sim 180 \mathrm{ml} \\
\text { - No RBC contamination } \\
\text { - No WBC contamination }\end{array}$ \\
\hline $\mathrm{RBC}$ & $\begin{array}{l}\text { - Volume } \sim 300 \mathrm{ml} \\
\text { - Hb content } \sim 50 \mathrm{~g} \\
\text { - Always LR }\end{array}$ \\
\hline Platelets & $\begin{array}{l}\text { - Platelet content } \sim 3.0 \times 10^{\wedge} 11 \text { (6-8 doses) } \\
\text { - No RBC contamination } \\
\text { - No WBC contamination }\end{array}$ \\
\hline
\end{tabular}

Figure 2: automated blood processing.

suspended in a storage solution, or processed with a pathogen inactivation technique adds new layers of complexity to compare them. Conversely, few studies have been published comparing clinical efficacy of RPC vs APC regarding CCI.The Atreus 3C system simplifies whole blood processing by making various manual process steps with the help of automation

that include: Balancing: Centrifugation: Expression: Sealing: Volume determination: Aim: The use of Random (RPC) and Apheresis (APC) platelet concentrates is highly heterogeneous among most countries. However some countries have changed their policy, switching from APC to RPC or vice versa.The goalof the study is to compare two whole blood processing methods manual and automated in terms of product quality and productivity. This presentation intends to analyze what factors may impact such decisions.

Methods: In our study we compared platelets processed using the Atreus $3 \mathrm{C}$ system, to those of the routine method either random (RPC) or apheresis (APC) platelet by assessing:

Time of the procedure.

RBCs: volume -hematocrite-WBCs contamination.

Plasma: volume -RBCs \& WBCs contamination.

Platelets: volume - PYI - WBCs contamination.

Transfusion reaction of the patients.

Result: We got more RBCs and plasma volumes which were leukodepleted, a platelet yield of $2.1 \times 10^{\wedge} 11$ (i.e. one therapeutic dose) from every 3-4 pooled bags which were leukoreduced and regarding transfusion reaction few patient showed minimal transfusion reaction as fever and rigors, similar to that shown in apheresis products. Conclusion: According to this study we will replace gradually all our needs using Atreus 3C system for its: Product quality.

Leukodepleted products. 
Cost effectiveness compared to conventional apheresis.

SAG M RBCs with longer life time.

Providing platelets without need for apheresis donors.

P-077

THE USE OF WASHED ERYTHROCYTE (WE) FOR CLINICAL

TRANSFUSION PRACTICE IN INDONESIA: SHOULD IT BE RETAINED OR SHIFT TO OTHER ALTERNATIVES?

ASM Sofro $^{1}$, TIS Akbar $^{2}$ and RSE Gantini ${ }^{3}$

${ }^{1}$ Universitas YARSI, Jakarta Pusat, Indonesia ${ }^{2}$ Faculty of Medicine Syiah Kuala University, Banda Aceh, Indonesia ${ }^{3}$ Central Blood Transfusion Service, Indonesian Red Cross, Jakarta, Indonesia

Background: Despite variation and limitation in resources and other facilities, safety issue is a priority in clinical blood transfusion practice in Indonesia archipelago. Increasing number of patients requiring multiple transfusion of blood component particularly Packed Red Cells (PRC) rendering Blood Transfusion Servive to provide blood components carefully matched in alloimmunized patients. Current leukoreduced praparation by using leukofilter is considered to be the best and savest blood component. However, the use of Washed Erythrocyte (WE) is a common practice in the majority hospitals in Indonesia.

Aims: The present work aims to justify the use of WE for patients with multiple transfusion is sufficient in countries like Indonesia where the WE preparation is as safe as leukoreduced blood prepared by leukofilter and still comply with the European standards, without spending extra cost for leukofilter.

Methods: Elecronic communication survey was carried out to map blood components service in six Blood Transfusion Service (BTS) in Java island providing aproximately 30\% of total blood supply in Indonesia. In addition, 26 PRC donors collected in $350 \mathrm{ml}$ blood bag for WE at Jakarta BTS were tested for their haematological parameters including haemoglobin levels, haematocrit volume, plasma protein concentration, leukocyte counts and possibility of haemolysis before and after WE preparation. Sysmex Xn-2000 was used to test the paramaters, whilst potential haemolysis was checked using Osmotic Fragility Test.

Results: A total of 584,661 donated blood were collected at six BTS, and prepared each 87.79\%, 3.54\%, 42.7\% for PRC, WE and TC respectively. In addition, 3798 unit of thrombocyte were prepared by apheresis at five BTS. Laboratory testing on 26 unit PRC donors for WE resulted in reduces blood parameters i.e. haemoglobin levels by $15.4 \%$, haematocrit by $8.55 \%$, protein concentration by $98.4 \%$ and leukocytes by $87.31 \%$ compared to those prior to washing. Practically no haemolysis was observed during Osmotic Fragility Test.ConclusionsDespite relatively low use of WE, it still meet the quality of blood components in terms of protein and leukocyte reduction approaching the European standard. Hemoglobin levels and haematocrit volume were both significantly reduced, but Osmotic Fragility test was practically normal. In general WE prepared applying good manufacturing practice can still be recommended for multiple transfusion patients. However, in certain circumstances, the use of alternative using leukofilter can also be encouraged.

\subsection{Blood Components}

P-078

\section{ANALYSIS OF ERYTHROCYTE FRAGILITY AND POTASSIUM LEVELS IN PACKED RED CELL COMPONENTS AFTER IRRADIATION WITH VARIOUS DOSES}

D Hasudungan Sidabutar ${ }^{1}$, Y Soedarmono ${ }^{2}$ and A Agus Kosasih ${ }^{3}$

${ }^{1}$ Indonesia Red Cross, Tangerang City Blood Centre, Tangerang, Indonesia

${ }^{2}$ Directorate of Basic Health Care, Ministry of Health, Jakarta, Indonesia

${ }^{3}$ Department of Clinical Pathology, Dharmais National Cancer Hospital, Jakarta, Indonesia

Background: One of the delayed transfusion reactions which is often fatal is Transfusion Associated Graft Versus Host Disease (TA GVHD). The incidence of TA GVHD in immunocompromised patients is estimated at 0.1 to $1.0 \%$ with a mortality rate of approximately $80-90 \%$. The irradiation of cellular blood components with appropriate dose is currently considered the most efficient and reliable way to prevent TA-GVHD. Aims: To determine the effect of irradiation on red blood cells fragility and its potassium level in different doses of irradiation and different length of storage.

Method: 72 bags of Packed Red Cell (PRC) component that were divided into 4 groups were evaluated for detection of red cell membrane fragility and their potassium level. The first group of PRC was a control group, and the other three groups

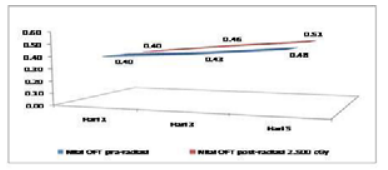

(1) Pre and Post Radiation OFT Value with $2500 \mathrm{cGy}$, in day 1 , day 2 and day 3
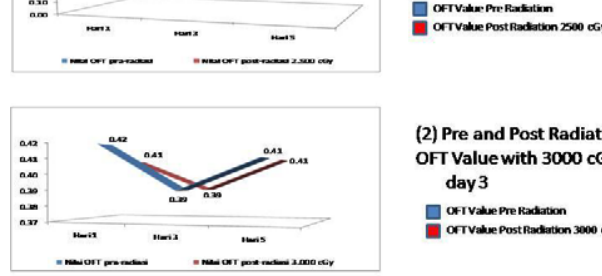

(2) Pre and Post Radiation

OFT Value with $3000 \mathrm{cGy}$, in day 1, day 2 and

day3
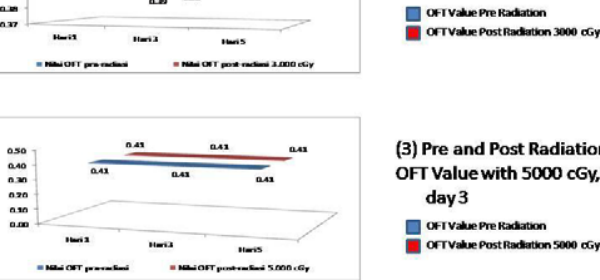

(3) Pre and Post Radiation

OFT Value with $5000 \mathrm{cGy}$, in day 1 , day 2 and

day 3

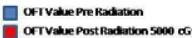

Figure 1: Pre and Post Pottasium Level with Various Dose Radiation in Day 1, Day 2 and Day 3.
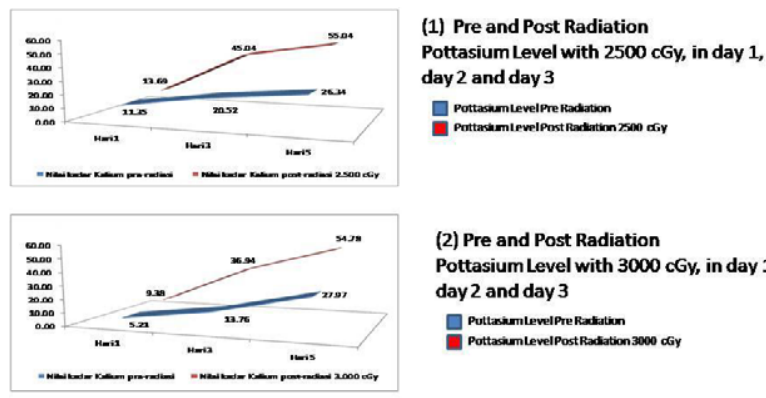

(2) Pre and Post Radiation Pottasium Level with $3000 \mathrm{cGy}$, in day 1 day 2 and day 3
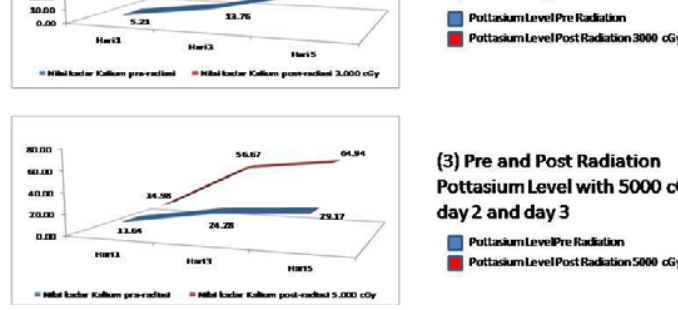

(3) Pre and Post Radiation Pottasium Level with $5000 \mathrm{cGy}$, in day 1 day 2 and day 3

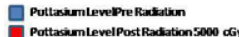

Figure 2: Pre and Post Pottasium Level with Various Dose Radiation in Day 1, Day 2 and Day 3.

of PRC received different irradiation doses of 2500, 3000, 5000 cGy respectively. The red cell membrane fragility and their potassium level were tested in the first, third and fifth days of storage, and the results were compared to those of the control group. The PRC irradiation was done using Biobeam gamma irradiation GSR C1 machine; the red cell membrane fragility was tested using the Osmotic Fragility Test (OFT) with normal saline; and the potassium level is measured using the automatic Easylite machine.

Results: The red cells in control group at three different times of storage started to hemolyse at the $\mathrm{NaCl}$ concentrate of $0.395-0.43 \%$, which met normal hemolysis status (normal hemolysis started at the $\mathrm{NaCl}$ concentration of $0.42 \% \pm 0.02 \%$ ). However, only red cells in 2500 cGy irradiated group and stored at only 1 day met normal hemolysis status. Compared to the control group, there was a significant increase of potassium level on the irradiated red cell at all different doses of irradiation $(\mathrm{P}<0.05)$. However, among them only a group of red cells stored at 1 day after irradiation had the normal value of potassium level $(<15 \mathrm{mmol} / \mathrm{l})$. The increase of potassium level was in parallel with the increase of irradiation dose and length of storage.

Conclusion: The irradiation dose of $2500 \mathrm{cGy}$ is recommended to be used and at the latest one day after irradiation, the red cell should be transfused to prevent the transfusion reaction due to hemolysis of red cell and high potassium level in PRC component caused by irradiation.

Keyword: : TA GVHD, PRC, Irradiation, cGy, OFT and Potassium. 
P-079

EFFECTIVENESS OF LEUCOCYTE AND CYTOKINE REDUCTION ON BUFFY COAT DEPLETED PACKED RED CELL AND MODIFIED BED SIDE LEUKOFILTERED PACKED RED CELL

$\mathrm{P}$ Agussalim $^{1}$, Y. Soedarmono ${ }^{2}$ and PAL Wahidiyat

${ }^{1}$ Central Blood Transfusion Service, Jakarta selatan, Indonesia ${ }^{2}$ Directorate of Basic Health Care, Ministry of Health, Jakarta, Indonesia ${ }^{3}$ Department of Pediatric, Cipto Mangunkusumo Hospital, Jakarta, Indonesia

Background. Data from Thalassemia Center of Cipto Mangunkusumo Hospital in Jakarta, showed that $65 \%$ of thalassemia patients who received regular Packed Red Cell (PRC) components had transfusion reaction, meanwhile only 15\% of those who received leukoreduced PRC components had transfusion reaction. Most of leukoreduced PRC components available in Jakarta were developed by buffy-coat depleted method because in-line leukocyte filtration method is considered expensive. Another method of leukoreduced is bed-side leukocyte filtration that has some weaknesses such as impractical operation and inability to eliminate cytokines because it is applied on post-storage PRC.

Aims. To evaluate the effectiveness of leukocyte and cytokine reduction on buffycoat depleted PRC compared to modified bed-side leukofiltered PRC.

Methods. The leukocyte count and pyrogenic cytokines of IL- 6 and TNF- $\alpha$ titer were analyzed on 30 samples of buffy-coat depleted PRC and 30 samples of modified bed-side leucofiltered PRC at $<48 \mathrm{~h}$ of storage using the automatic Sysmex-XT 1800-I cell blood counter and Quintikine HS sandwich enzyme immunoassay respectively. Buffy-coat depleted PRC was produced from whole blood in the JMS TopBottom blood bag using standard procedures. On the other hand, modified bed-side leukofiltered PRC was processed by connecting the Terumo bed side leukocyte filter with the blood bag tube of regular PRC using a sterile connecting device.

Result. Only one (3.33\%) sample of buffy-coat depleted PRC showed to be leucoreduced $\left(<5 \times 10^{8}\right.$ leukocyte/unit) and $29(96.7 \%)$ samples of modified bed-side leukofiltered PRC showed to be leukodepleted $\left(<5 \times 10^{6}\right.$ leukocyte/unit). The average titer of IL-6 of buffy-coat depleted PRC sample vs modified bed-side leukofiltered PRC sample was $0.08 \mathrm{pg} / \mathrm{ml}$ vs $0.53 \mathrm{pg} / \mathrm{ml}$ (normal titer of IL-6 is $0.16-10 \mathrm{pg} / \mathrm{ml}$ ), meanwhile the average titer of TNF- $\alpha$ on both kind of PRCs was $0.21 \mathrm{pg} / \mathrm{ml}$ vs $1.16 \mathrm{pg} / \mathrm{ml}$ (normal titer of TNF- $\alpha$ is $0.5-32 \mathrm{pg} / \mathrm{ml}$ ) with $\mathrm{P}>0.05$.

Conclusion. The leukocyte reduction of modified bed-side leukofiltered PRC product is more effective than that of the buffy-coat depleted PRC product. To prevent the accumulation of pyrogenic cytokines such as IL- 6 and TNF- $\alpha$, both of leukoreduced PRC products are recommended to be transfused $<48 \mathrm{~h}$ of storage.

Keywords: packed red cell, buffy-coat depleted, bed-side leucocyte filtration, transfusion reaction.

\section{P-080}

\section{FULLY AUTOMATED PREPARATION OF WASHED PLATELET CONCENTRATES IN BRS-A PLATELET ADDITIVE SOLUTION WITH A CLOSED-SYSTEM CELL PROCESSOR}

S Oikawa, M Minegishi, K Endo, T Hoshi, W Kawashima, K Suzuki and H Shimizu Japanese Red Cross Tohoku Block Blood Center, Sendai, Japan

Background: To reduce the adverse reactions following platelet (PLT) transfusion, the presence of plasma can be reduced in PLT products by concentrating or washing steps. In general, washing is more effective than concentrating. However, washing is time-consuming and significantly worsen PLT quality. Additionally, in Japan, platelet additive solutions (PAS) are not commercially available. We have previously reported that the automated processor, the ACP215 system is feasible for washing PLT concentrates (PC). More recently, we have developed a novel PAS: bicarbonated Ringer's solution (BRS) supplemented with acid-citrate-dextrose formula A (ACD-A), termed BRS-A, which can preserve the in vitro properties of PLTs for at least 7 days with less than 5\% plasma.

Aims: The aim of this study was to evaluate the in vitro qualities of PLTs washed with BRS-A in the automated closed-system cell processor ACP215, using two kinds of modes for washing: 'manually adding ACD-A to BRS' and 'automatically' adding ACD-A to BRS (during washing process).

Methods: Two apheresis PCs with identical ABO were mixed and divided. The PLT content was calculated by multiplying the PLT concentration with the PC volume (weight of unit/specific gravity, 1.03) or washed PC volume (weight of unit/specific gravity, 1.01). The PCs were stored on a flatbed agitator (60 cycles $/ \mathrm{min}$ ) at $20-24^{\circ} \mathrm{C}$ and washed within 2 days after collection. Platelet washing was automatically performed with the ACP215 system using BRS-A, which consists of $95.2 \mathrm{mmol} / \mathrm{l} \mathrm{NaCl}$, $3.8 \mathrm{mmol} / \mathrm{l} \mathrm{KCl}, \quad 0.9 \mathrm{mmol} / \mathrm{l} \mathrm{MgCl}_{2}, \quad 1.4 \mathrm{mmol} / \mathrm{l} \mathrm{CaCl}_{2}, 26.6 \mathrm{mmol} / \mathrm{l} \mathrm{NaHCO}_{3}$, $5.8 \mathrm{mmol} / \mathrm{l}$ glucose, $4.2 \mathrm{mmol} / \mathrm{l}$ trisodium citrate, and $2.4 \mathrm{mmol} / \mathrm{l}$ citric acid. Two (C) 2015 The Author Vox Sanguinis (C) 2015 International Society of Blood Transfusion Vox Sanguinis (2015) 109 (Suppl. 2), 1-96 washing procedures were compared: (1) manually added ACD-A to BRS premixing before washing ('control') and (2) automatically added ACD-A to BRS (mixing during washing process, 'test'). In both groups, the volume of washing solution was approximately $2000 \mathrm{ml}$ and the total time 25-26 min. Washed PC were stored on a flatbed agitator (60 cycles $/ \mathrm{min}$ ) at $20-24^{\circ} \mathrm{C}$. The platelet count, the volume, the residua plasma protein level, and the in vitro properties of washed PLTs were assessed (swirling, $\mathrm{pH}$, bicarbonate, glucose, lactate, hypotonic shock response, mean PLT volume, CD62P and CD42b expressions, PLT-derived microparticles) during the 7-day storage period $(\mathrm{n}=7)$.

Results: The characteristics of washed PLTs in both groups are summarized in Table 1 and 2. Swirling was well-maintained and $\mathrm{pH}$ values were above 7.0 in al units during 7-day storage. In vitro properties of washed PLT in the 'test' method were comparable to the 'control'. Glucose consumption and lactate production were comparable in both groups. On day 3, CD62P expression levels in both groups were approximately a 2-fold higher than pre-wash, while hypotonic shock response were comparable in both groups. CD42b expressions in both groups were decreased at day 7. No significant differences in PLT volumes and PLT-derived microparticles in both groups were found although those were time-dependently increased.

Conclusion: The automated closed-system cell processor ACP215 can successfully wash PLTs without manually premixing BRS and ACD-A and preserve the quality of washed PLTs stored in BRS-A.

\begin{tabular}{|c|c|c|c|}
\hline & Control & Test & $P$ value \\
\hline \multicolumn{4}{|l|}{ PLT count $\left(\times 10^{11} / \mathrm{bag}\right)$} \\
\hline pre-wash & $2.31 \pm 0.12$ & $2.30 \pm 0.11$ & 0.63498 \\
\hline post-wash & $2.00 \pm 0.12$ & $1.99 \pm 0.12$ & 0.35504 \\
\hline Platelet recovery (\%) & $86.2 \pm 1.7$ & $86.0 \pm 2.2$ & 0.80863 \\
\hline \multicolumn{4}{|l|}{ Volume $(\mathrm{mL})$} \\
\hline pre-wash & $203.0 \pm 2.1$ & $202.8 \pm 2.2$ & 0.66921 \\
\hline post-wash & $216.7 \pm 0.3$ & $215.9 \pm 0.9$ & 0.13919 \\
\hline \multicolumn{4}{|l|}{ Plasma protein (mg/bag) } \\
\hline pre-wash & $14,374 \pm 1,489$ & $14,246 \pm 1,281$ & 0.33742 \\
\hline post-wash & $177 \pm 50$ & $145 \pm 37$ & 0.14123 \\
\hline Plasma protein removal ratio (\%) & $98.8 \pm 0.3$ & $99.0 \pm 0.2$ & 0.15563 \\
\hline
\end{tabular}

\begin{tabular}{|c|c|c|c|c|c|}
\hline & \multicolumn{5}{|c|}{ Days after washing } \\
\hline & pre-wash & Day 1 & Day 3 & Day 5 & Day 7 \\
\hline \multicolumn{6}{|l|}{$\mathrm{pH}$ at $37^{\circ} \mathrm{C}$} \\
\hline Control & $7.12 \pm 0.03$ & $7.26 \pm 0.05$ & $7.15 \pm 0.08$ & $7.41 \pm 0.10$ & $7.53 \pm 0.05$ \\
\hline Test & & $7.26 \pm 0.06$ & $7.15 \pm 0.09$ & $7.41 \pm 0.09$ & $7.53 \pm 0.05$ \\
\hline \multicolumn{6}{|c|}{ Bicarbonate $(\mathrm{mmol} / \mathrm{L})$} \\
\hline Control & $12.6 \pm 1.1$ & $15.3 \pm 0.7$ & $10.3 \pm 1.0$ & $8.4 \pm 0.5$ & $8.9 \pm 0.6$ \\
\hline Test & - & $15.4 \pm 0.7$ & $10.3 \pm 1.3$ & $8.7 \pm 0.9$ & $9.7 \pm 0.5$ \\
\hline \multicolumn{6}{|c|}{ Glucose (mmol/L) } \\
\hline Control & $20.67 \pm 0.81$ & $4.59 \pm 0.35$ & $1.39 \pm 0.64$ & $0.09 \pm 0.13$ & $0.08 \pm 0.05$ \\
\hline Test & - & $4.50 \pm 0.41$ & $1.33 \pm 0.65$ & $0.07 \pm 0.07$ & $0.05 \pm 0.04$ \\
\hline \multicolumn{6}{|c|}{ Lactate $(\mathrm{mmol} / \mathrm{L})$} \\
\hline Control & $18.21 \pm 0.67$ & $2.87 \pm 0.59$ & $8.41 \pm 1.59$ & $10.37 \pm 0.75$ & $9.86 \pm 0.54$ \\
\hline \multirow{2}{*}{\multicolumn{6}{|c|}{ Hypotonic shock response (\%) }} \\
\hline & & & & & \\
\hline Control & $74.2 \pm 2.2$ & $73.7 \pm 3.0$ & $76.5 \pm 1.2$ & $63.5 \pm 4.7$ & $63.4 \pm 2.9$ \\
\hline Test & - & $73.1 \pm 3.4$ & $76.2 \pm 4.2$ & $65.3 \pm 7.0$ & $64.7 \pm 3.6$ \\
\hline \multicolumn{6}{|c|}{ Mean PLT volume (fL) } \\
\hline Control & $7.9 \pm 0.5$ & $7.8 \pm 0.5$ & $8.0 \pm 0.6$ & $8.4 \pm 0.7$ & $8.8 \pm 0.5$ \\
\hline Test & 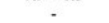 & $7.9 \pm 0.5$ & $8.0 \pm 0.5$ & $8.4 \pm 0.7$ & $8.8 \pm 0.6$ \\
\hline \multicolumn{6}{|l|}{ CD62P (\%) } \\
\hline Control & $8.2 \pm 2.4$ & $15.3 \pm 5.7$ & $15.7 \pm 5.7$ & $25.9 \pm 9.2$ & $25.9 \pm 9.2$ \\
\hline Test & 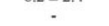 & $16.6 \pm 0.1$ & $15.9 \pm 0.9$ & $24.8 \pm 0.6$ & $26.2 \pm 0.7$ \\
\hline \multicolumn{6}{|l|}{$\mathrm{CD} 42 \mathrm{~b}(\%)$} \\
\hline Control & $99.5 \pm 0.3$ & $99.4 \pm 0.2$ & $99.3 \pm 0.3$ & $97.2 \pm 1.7$ & $92.1 \pm 5.6$ \\
\hline Test & - & $99.3 \pm 0.3$ & $99.1 \pm 0.4$ & $96.7 \pm 2.5$ & $92.5 \pm 5.9$ \\
\hline \multicolumn{6}{|l|}{ PMPs } \\
\hline Control & $2.0 \pm 1.0$ & $10.1 \pm 2.8$ & $11.6 \pm 4.0$ & $15.9 \pm 5.5$ & $18.2 \pm 6.1$ \\
\hline Test & - & $11.2 \pm 3.3$ & $12.4 \pm 2.6$ & $17.7 \pm 4.4$ & $18.7 \pm 3.5$ \\
\hline
\end{tabular}

P-081

EFFECT OF LIPID STATUS OF THE DONOR PLATELET APHERESIS TO CONTENT OF PLATELETS AND LEVELS OF MALONDIALDEHYDE WITH IN STORAGE

C Yanto ${ }^{1}$, M Sadikin ${ }^{2}$, NK Ritchie ${ }^{3}$, S Chunaeni ${ }^{3}$, A Rachman ${ }^{2}$ and SW Jusman ${ }^{2}$

${ }^{1}$ National Cancer Centre, Dharmais Hospital, Jakarta, Indonesia ${ }^{2}$ Medical Faculty University, Jakarta, Indonesia ${ }^{3}$ Indonesian Red Cross, Jakarta, Indonesia

Background: Platelet apheresis donors are encouraged to eat first before the proces begins. It can be effect of lipid status of the donors. Hyperlipidemia could increase platelet activation and lipid peroxidation which malaondialdehyde as end product. $\mathrm{AABB}$ recommends to dispose of all products from donors with plasma lipemia. 
Aims: This study aimed to determine the effect of lipid status of the donor platelet apheresis to content of platelets and levels of malondialdehyde (MDA) with in storage.

Methods: This study used descriptive analytic design in 31 platelet apheresis concentrates. We used apheresis machine from Haemonetic MCS $+{ }^{\circledR}$. Samples were divided into two groups, hyperlipidemia and normolipidemia. The assay for the content of platelets and levels of MDA was tested on the first day, second and fourth of platelet storage.

Results: The triglyceride concentration of hyperlipidemia and normolipidemia were $240.41 \pm 95.51$ and $89.50 \pm 30.51 \mathrm{mg} / \mathrm{dl}$. The results of platelet apheresis with in storage are shown in the table. An increase in the content of platelets during storage in both groups, which are associated with the process of apoptosis. In the first and second days of storage platelet counts in hyperlipidemia group was slightly lower than the normolipidemia group, whereas in the fourth day of storage obtained platelet counts in hyperlipidemia group that exceeded the platelet count in the normolipidemia group. This suggests that apoptosis occurs more frequently in hyperlipidemia group compared to the normolipidemia group. Increased platelet count is probably because platelets have an enlarged (swollen) and further fragments to form platelets derived procoagulant microparticles (PDMPs) in the apoptotic process but still count as platelets by hematology analyzer (false positive). PDMPs will increase the risk of thrombosis in patients who are sensitive. There was no significant correlation between levels of triglycerides and platelet content. On the fourth day found significant differences in the levels of MDA. There were positive and significant correlations between levels of triglyceride to the levels of MDA.

Conclusions: Improved of the lipid status of the donor platelet apheresis will increase platelet apoptosis and increase levels of MDA. The need to remind donors platelet apheresis to a low fat dietary before apheresis process implemented. Need for further research to determine the lipid levels that can still be tolerated. Table 2. Levels of MDA.

Table 1. Content of platelets

\begin{tabular}{lccc}
\hline & Hyperlipidemia & Normolipidemia & $p$ \\
& & & \\
\hline First day & $3,1 \times 10^{11} \pm 0,9 \times 10^{11}$ & $3,2 \times 10^{11} \pm 0,5 \times 10^{11}$ & 0,858 \\
Second day & $3,2 \times 10^{11} \pm 1,1 \times 10^{11}$ & $3,3 \times 10^{11} \pm 0,6 \times 10^{11}$ & 0,720 \\
Fourth day & $4,3 \times 10^{11} \pm 1,3 \times 10^{11}$ & $4,0 \times 10^{11} \pm 0,1 \times 10^{11}$ & 0,559
\end{tabular}

Table 2. Levels of MDA $(\mathrm{nmol} / \mathrm{mL})$

\begin{tabular}{lccc}
\hline & Hyperlipidemia & Normolipidemia & $p$ \\
\hline First day & $0,8797 \pm 0,5375$ & $0,6132+0,2152$ & 0,081 \\
Second day & $1,0215 \pm 0,5436$ & $0,6853 \pm 0,3412$ & 0,081 \\
Fourth day & $0,9847 \pm 0,3456$ & $0,7221+0,3934$ & 0,032 \\
\hline
\end{tabular}

$\mathrm{P}-082$

L-Carnitine effectively Improves the Metabolism and Quality of Platelet Concentrates During Storage

MR Deyhim ${ }^{1}$, SA Mesbah-Namin ${ }^{1}, \mathrm{~F} \mathrm{Yari}^{1}$, MR Taghikhani $^{2}$, A Naser $^{1}$ and F Khoshnaghsh

${ }^{1}$ Iranian blood transfusion research center, High institute for research and edu., Tehran, Iran ${ }^{2}$ Department of Clinical Biochemistry, Faculty of Medical Sciences, Tehran, Iran

Background: Human platelets undergo structural and biochemical alternations during storage which are collectively called platelet storage lesion (PSL). PSL is characterized metabolic and functionally changes causing decrease in platelet's recovery and survival.
Aims: Here, we evaluated the effect L-carnitine (LC) on the platelet metabolism, function and platelet mitochondrial enzyme activity during platelet storage.

Methods: Platelet rich plasma (PRP) was used to prepare platelet concentrate (PC) in Iranian blood transfusion organization. For this purpose, ten PC bags from healthy donors, stored at $22^{\circ} \mathrm{C}$ with gentle agitation in the presence or absence of LC. The effects of LC ( $15 \mathrm{mM})$ on the platelet quality were assessed by analyzing the levels of glucose, lactate, ATP and lactate dehydrogenase (LDH) activity. Platelet aggregation induced by arachidonate and ristocetin were analyzed by aggregometer. Platelet mitochondrial enzyme activity was measured by MTT assay and platelet count and mean platelet volume (MPV) were also determined by a hematology analyzer during 5 days of PC storage.

Results: The Results indicated that LC could significantly decrease lactate concentration and glucose consumption accompany with increasing oxygen consumption in stored PC. LDH activity was also significantly less increased in LC-treated PC on day 2 and 5 of storage. Platelet aggregation in response to the ristocetin and arachidonate was significantly higher in LC-treated PC than in untreated PC on day 5 of storage. Finally, platelet mitochondrial enzyme activity was significantly less decreased in LC-treated PC compared to the control group on day 2 and 5 of storage.

Conclusions: It seems that LC would be a good additive to reduce PSL and improve the platelet metabolism and quality of the stored PC for platelet-transfusion therapy. Keywords: L-carnitine; Platelet quality; stored platelet concentrates; Platelet storage lesion:

\section{$\mathrm{P}-083$}

\section{INFLUENCE OF DIFFERENT METHODS PREPARATION ON}

PLATELET ACTIVATION IN STORED PLATELET

\section{CONCENTRATES}

A Soleimany ferizhandy

Iranian Blood Transfusion Research Center, High Institute for Research and Educa, Tehran, Iran

Background: Platelet concentrates are routinely manufactured from whole blood by differential centrifugation. During this storage period under blood bank conditions, biochemical, structural and functional changes occur, a process that is also known as platelet storage lesion. Their quality was assessed using the following parameters: Platelets, leukocytes and erythrocytes counts, pH, CD63, lactate dehydrogenase and Annexin V. Materials and Methods: In this experimental study, 25 platelet concentrates prepared with platelet rich plasma-platelet concentrates, 25 units via buffy coat and apheresis-derived platelet methods. The percentages of Annexin V, CD63 expression, lactatedehydrogenase, platelet, leukocytes counts and $\mathrm{pH}$ were evaluated.Results: During storage for up to 5 days, no significant $\mathrm{pH}$, difference was observed among all three type of platelet concentrates $(\mathrm{P}>0.05)$. The mean leukocytes count buffy coat units, platelet rich plasma platelet concentrates and apheresis-derived units were comparable and statistically significant difference was observed $(\mathrm{P}<0.05)$. During storage for up to 5 days platelet concentrates units displayed significant an increase in lactatedehydrogenase, CD63 and Annexin V expressions, as compared with buffy coat units and apheresisderived units preparation on day $5(\mathrm{P}<0.05)$. Discussion: The kinetics of CD63 and annexin $\mathrm{V}$ levels are influenced by the method used to prepare platelets for storage. The different levels of $\mathrm{CD63}$, annexin $\mathrm{V}$ and lactatedehydrogenase in three types of units clearly demonstrating a progressive activation process of platelet concentrates units exceeds that of buffy coat and apheresis-derived units. Further clinical studies will be necessary to determine whether meet the quality criteria or is superior in predicting in vivo viability

$\mathrm{P}-084$

\section{CENTRIFUGATION SPEED AND TIME FOR PREPARATION OF PLATELET CONCENTRATE BY PRP METHOD. IS IT AFFECTED BY BLOOD BAG TYPE?}

CG Kohombange and KS Kuruppu

National Blood Center, Colombo, Sri Lanka

Background: Platelet count of a platelet concentrate is the main quality parameter which determines the efficacy of the product. Quality control laboratory of NBC monitors at least $1 \%$ of all the total production of platelet concentrate. According to AABB standards acceptable number of platelets per unit should be $5.5 \times 10^{10}$ and according to the EU guideline, $75 \%$ of the products tested should be at least having a platelet count of $6.0 \times 10^{10}$. Based on these guidelines,

(C) 2015 The Author

Vox Sanguinis @ 2015 International Society of Blood Transfusion Vox Sanguinis (2015) 109 (Suppl. 2), 1-96 
NBTS standards is set as 75\% from the tested products should contain at least $5.5 \times 10^{10}$ platelets per unit.

While monitoring the platelet counts in PRP derived platelets prepared at the blood component preparation laboratory of NBC, it was noted that from July 2014 onwards, the platelet count has not met the required pass rate of $75 \%$. This study was conducted as to find out the root cause for this trend.

Aim: Aim of this study was to find the root cause for the reduced platelet counts of the PRPD platelets.

Method: A root cause analysis was conducted to identify any deviations in all the quality system essentials involved in the process.

Results: Based on the above analysis it was evident that the properties of the blood bag matters in the final yield of the platelets prepared from PRPD method.

With further testing an optimum centrifugation programe was selected for platelet preparation by whole blood collected to Terumo Japanese triple blood bag system

Conclusion: Following aspects has to be taken into consideration while preparing PRPD platelets:

Centrifugation speeds and times of the initial centrifugation cycle of the whole blood collected to triple blood bag systems should be validated according to the blood bag type. When procuring blood bags it is recommended to get down supplier recommendations on centrifugation programs and obtain technical assistance of the suppliers in initial validation of the blood bag system. It is feasible to use a single brand of triple blood bag system in preparation of PRPD platelets in a single production cycle, as it may required to change the centrifugation programs when using different brands of blood bags.

Caption 1: Root cause analysis.

\section{$\mathrm{P}-085$}

COAGULATION FACTORS, THROMBIN FORMATION AND CLOT FORMATION OF THAWED FRESH FROZEN PLASMA

Y Naito ${ }^{1}$, Y Hayashi ${ }^{1}, \mathrm{~S}$ Wakamoto ${ }^{1}$, M Fujihara ${ }^{1}$, M Akino ${ }^{2}$, M Endo ${ }^{1}, \mathrm{C} \mathrm{Homma}^{1}$, T Yamamoto $^{3}, \mathrm{H} \mathrm{Ikeda}^{3}$, S Kino ${ }^{1}$ and S Takamoto ${ }^{1}$

${ }^{1}$ Japanese Red Cross Hokkaido Block Blood Center, Sapporo, Japan ${ }^{2}$ Japanese Red Cross Blood Service Headquarters, Tokyo, Japan ${ }^{3}$ Japanese Red Cross Hokkaido Blood Center, Sapporo, Japan

Background: In Japan, leukocytes reduced fresh frozen plasma (FFP-LR) must be used within $3 \mathrm{~h}$ after thawing. This is thought to be based on the decline of labile coagulation factors such as factor (F) V and FVIII activities during post-thaw storage. In 2012, on the other hand, the Food and Drug Administration (USA) approved extending the storage time of thawed FFP stored at 1 to $6^{\circ} \mathrm{C}$ up to $24 \mathrm{~h}$, instead of $6 \mathrm{~h}$. The longer shelf life of thawed FFP-LR makes it applicable for neonatal use or critical care settings.

Aims: We evaluated coagulation capacity of thawed FFP-LR up to $120 \mathrm{~h}$ with a thrombin generation assay, a viscoelastic assay and measurement of activities of coagulation factors.

Methods: 15 FFP-LRs (4 A-, 5 0-, 2 B-, 4 AB-type) were stored at $-30^{\circ} \mathrm{C}$ for approximately 12 months. FFP-LRs were thawed at $37^{\circ} \mathrm{C}$, divided into two equal volumes in plastic bags, and stored at either $4^{\circ} \mathrm{C}$ or $22^{\circ} \mathrm{C}$ for up to $120 \mathrm{~h}$. Samples were taken at $0,3,6,24,48$ and $120 \mathrm{~h}$ after thawing. Coagulation factors were assessed by CS-2000i automated coagulation analyzer (Sysmex Corporation). Thrombin generation was measured based on the use of a fluorogenic substrate in the presence of exogenous tissue factor, phospholipid, and calcium using the calibrated automated thrombogram system (CAT, Thrombinoscope BV). The thrombin generation curve was assessed by examining the lag time for thrombin generation to occur, peak amount of thrombin generated, the area under the thrombin generation curve (endogenous thrombin potential, ETP), time to peak and time to tail. Clot formation was assessed by rotational thromboelastometry (ROTEM, Pentapharm GmbH) using INTEM (ellagic acid/phospholipid activation) and EXTEM (tissue factor activation) The clotting time (CT, the lag time for clot formation to commence), alpha angle ( $\alpha$, rate of clot formation) and maximum clot firmness (MCF, a measure of clot strength) were assessed.

Results: The FVIII level decreased from $0.95 \pm 0.21$ to $0.50 \pm 0.11 \mathrm{IU} / \mathrm{ml}$ after $120 \mathrm{~h}$ of storage at both $4^{\circ} \mathrm{C}$ and $22^{\circ} \mathrm{C}$, and at the largest degree among other coagulation factors. The level of fibrinogen was almost unchanged after $120 \mathrm{~h}$ of storage. Levels of other coagulation factors in thawed FFP-LR after $120 \mathrm{~h}$ remained above $0.70 \mathrm{IU} / \mathrm{ml}$. In the thrombin generation assay, there was a significant increase in the lag time after $120 \mathrm{~h}$ of storage at $4^{\circ} \mathrm{C}$ and after $48 \mathrm{~h}$ and $120 \mathrm{~h}$ of storage at $22^{\circ} \mathrm{C}$ compared with $0 \mathrm{~h}$. However, there was no difference in the ability of thawed FFPLR to generate thrombin up to $120 \mathrm{~h}$ as assessed by the peak and ETP. There was no

(C) 2015 The Author

Vox Sanguinis (C) 2015 International Society of Blood Transfusion

Vox Sanguinis (2015) 109 (Suppl. 2), 1-96 difference in clot formation in thawed FFP-LR stored for $120 \mathrm{~h}$ as assessed by ROTEM in terms of $\alpha$ and MCF.

Conclusions: Despite the approximately 50\% loss of FVIII, the ability of thawed FFP-LR generating thrombin or forming a clot was unaltered. Therefore, we suggest that thawed FFP-LR can be used to treat coagulopathies except FVIII deficiency for up to $120 \mathrm{~h}$

\section{P-086}

\section{AGGREGATION AND RELEASE OF PRO-COAGULANT TISSUE FACTOR-EXPRESSING MICROPARTICLES BY THP- 1 MONOCYTIC CELLS ACTIVATED BY PLATELET-DERIVED MICROPARTICLES IN VITRO}

ML Chou ${ }^{1}$, HC Lin $^{2}$, HW Chang ${ }^{2}$, SH Hsiao ${ }^{1}$, JC Seghatchian ${ }^{3}$ and T Burnouf ${ }^{1}$

${ }^{1}$ Taipei Medical University, Taipei, Taiwan ${ }^{2}$ Taipei Medical University Hospital, Taipei, Taiwan ${ }^{3}$ Audit/Inspection and DDR Strategy, London, United Kingdom

Background: Microparticles (MPs) released by blood or endothelial cells are present in plasma for transfusion. They originate from the collected donor blood, or are triggered by the collection and production process of blood components. While MPs may contribute to hemostasis, their presence in transfused plasma may lead to uncontrolled thrombin generation when transfused to susceptible cancer or patients with hypercoagulable state. Understanding the biochemical and cellular triggers of MP-mediated thrombogenesis is therefore needed. We hypothesized that the prothrombotic role of PMPs could encompass interaction with and activation of immune cells leading to the release of pro-thrombotic microparticles.

Aim: In the current study, we have isolated and compared the characteristics of PMPs isolated from the human plasma compartment of platelet concentrates ( $N$ PMPs) or generated by thrombin activation of isolated platelets (T-PMPs) and evaluated their capacity to induce THP-1 leukemia monocytic cell aggregation and release of pro-coagulant tissue factor-bearing MPs.

Methods: We isolated platelet MPs (PMPs) present in platelet concentrate supernatant plasma (N-PMPs) or prepared by activation of isolated platelets using $0.1 \mathrm{IU} / \mathrm{ml}$ thrombin (T-PMPs). N-PMPs and T-PMPs were characterized dynamic light scattering and counted by tunable resistive pulse sensing to determine population size and number. $1 \times 10^{6}$ THP- 1 cells cultures were treated with $5 \mathrm{ml}$ of N-PMP or $5 \mathrm{ml}$ of T-PMP (corresponding to a 1 to 500 ratio of THP-1 cells to PMPs) at $37{ }^{\circ} \mathrm{C}$ for $4 \mathrm{~h}$. Supernatants from monocytes stimulated or not by N-PMPs or T-PMPs were collected by serial centrifugation at $300 \mathrm{xg}$ and $3000 \mathrm{xg}$ at $4^{\circ} \mathrm{C}$ for $10 \mathrm{~min}$ to remove monocytes and obtain the supernatant. The functional pro-coagulant activity of TF-MPs released by monocytes was determined by a TF-capture assay (Zymuphen MP-TF).

Results: THP-1 cells incubated for $5 \mathrm{~min}$ at $37^{\circ} \mathrm{C}$ in growth medium evidenced a typical regular round shape morphology. There was no immediate change in THP-1 cells morphology after 5 min of incubation following addition of N-PMPs and THP1 cells remained as a monodispersed suspension with round shape morphology. By contrast, T-PMP addition induced, within as little as $5 \mathrm{~min}$ of incubation, a strong cellular aggregation, also characterized by a change in THP-1 cell morphology from regular to irregular and rough appearance, with an apparent release of MPs budding from the cell surface. There was no detectable change in the morphology and shape of THP cells alone or co-cultured with N-PMP over $24 \mathrm{~h}$, whereas aggregation induced by T-PMPs remained strong. Release of pro-coagulant TF-MPs was significantly stimulated by the addition of both N-PMPs $(\mathrm{P}<0.05)$ and T-PMPs $(\mathrm{P}<0.001)$ compared to THP-1 cell alone. The control samples of growth medium spiked with N-PMPs and T-PMPs under the same conditions and at the same ratios did not contain detectable $(<1 \mathrm{pg} / \mathrm{ml})$ pro-coagulant TF-MPs, suggesting that the pro-coagulant TF-bearing MPs were released by THP-1 cells.

Conclusion: Our in vitro data indicate that, in addition to generating thrombin through protein activation, PMPs may trigger cell-mediated thrombogenicity leading to the generation of pro-coagulant TF-bearing MPs released by monocytes.

\section{P-087}

\section{EFFECT OF FREEZING TECHNIQUE ON THE QUALITY OF} FRESH FROZEN PLASMA

R Chaudhary, L Dhantole and A Sonker

Sanjay Gandhi Postgraduate Institute of Medical Sciences, Lucknow, India

Background: The quality of plasma depends on many factors, one of which is the technique used for freezing plasma.

Aim: In order to study effect of freezing method on quality of plasma, we conducted a study in which plasma components were frozen by contact shock freezer 
and by mechanical freezer, and studied the difference in quality parameters between the two study groups.

Methods: Two ABO identical plasma were pooled using sterile connecting device in a dedicated plasma container to make one pool. Fifty of such ABO identical pools were made for Fresh Frozen Plasma $(\mathrm{N}=100)$. This pooled plasma was divided into two aliquotes. One aliquote from each pool was frozen by contact shock freezer (Dometic MBF 21) which tool approximately $30 \mathrm{~min}$ to completely freeze the plasma. Another aliquote were frozen at $-40^{\circ} \mathrm{C}$ in a blood bank mechanical freezer which took nearly $24 \mathrm{~h}$ to completely freze the plasma. Samples from both aliquotes were tested for coagulation parameters after thawing at $37 \mathrm{oC}$ using automated coagulometer.

Results: There was a statistically significant difference $(\mathrm{P}<0.05)$ between mean values of PT, Factor V, Factor VII, Factor VIII and fibrinogen among the two study groups (Table 1).

Summary: The quality parameters were better in plasma frozen by contact shock freezer as compared to mechanical freezer.

Table 1: Effect of freezing technique on quality of plasma.

\begin{tabular}{|l|c|c|c|}
\hline Parameter & $\begin{array}{c}\text { Contact Shock } \\
\text { Freezing } \\
(\mathrm{N}=50)\end{array}$ & $\begin{array}{c}\text { Mechanical } \\
\text { Freezing } \\
(\mathrm{N}=50)\end{array}$ & P value \\
\hline PT ( seconds) & $13.58 \pm 0.59$ & $13.85 \pm 0.64$ & $0.03^{*}$ \\
\hline APTT ( seconds & $31.17 \pm 1.62$ & $31.08 \pm 3.0$ & 0.852 \\
\hline Factor V (IU/ml) & $1.01 \pm 0.10$ & $0.94 \pm 0.11$ & $0.03^{*}$ \\
\hline Factor VII (IU/ml) & $1.12 \pm 0.16$ & $0.96 \pm 0.12$ & $<0.05^{\star}$ \\
\hline Factor VIII $(\mathrm{IU} / \mathrm{ml})$ & $1.16 \pm 0.15$ & $1.02 \pm 0.15$ & $<0.05^{\star}$ \\
\hline Fibrinogen $(\mathrm{mg} / \mathrm{ml})$ & $4.22 \pm 0.57$ & $4.00 \pm 0.58$ & $0.05^{\star}$ \\
\hline
\end{tabular}

${ }^{*} P$ values are clinically significant

P-088

PROCESSING PRC LEUCOREDUCE PRODUCTION IN TANGERANG BLOOD CENTRE PERIOD JANUARY-JUNE 2015

A Agung, F Femy, I Ismi, K Fajar and D David

Tangerang Blood Centre, Tangerang, Indonesia

Background: Leucoreduce PRC has an important role in reduce the risk of posttransfuse.The methode in manufacture of PRC leucoreduce by reduce the leukocytes that containe in a blood bag is very helpful for patient blood recipients at hospital. One of the increasing quality service in Tangerang blood centre is provide products PRC leucoreduce. This produk is very welcomed by the hospitals, especially thalassemia patients whose lives depend on blood transfusions.

Aims: To determine the blood component production activities of PRC leucoreduce in Tangerang blood centre in the first half of 2015.

Methods: Collecting and processing data from the report leucoreduce PRC component process from the beginning of 2015 until mid-2015.

Results: The result of process PRC Leucoreduce production from January 2015 as 134 bags, February 2015 as 390 bags, March 2015 as 292 bags, April 2015 as 264

\section{PRC LEUCOREDUCE PRODUCTION IN MIDDLE 2015}

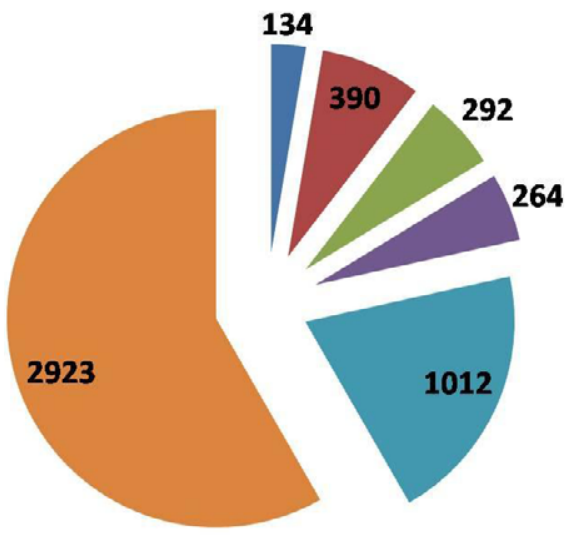

- Jan-15

- Feb-15

Mar-15

- Apr-15

May-15

Jun-15

Figure 1: Data analyze of production PRC Leucoreduction.

bags, May 2015 as 1012 bags, June 2015 as 2923 bags with total production in the first half 2015 as 5015 bags. The increasing production Leucoreduce PRC began from January until June 2015 amounted to 2081\%, with progress in each month from January to February 2015 191\%, February to March 2015 25\%, March to 9.5\% in april 2015, April to May 2015 283\%, may to June 2015 188\%.

Conclusions: PRC production leucoreduce certainly continue to rise because Tangerang Blood centre will improve continually the quality of blood products and services in accordance with the high demand of blood. One of the efforts made by Tangerang Blood centre in improving the quality of blood products and services is promotion the products to the hospital by having seminars between Tangerang Blood centre with the hospital. The increasing quality products and services that have done will make patients who received blood transfusions feel more secure.

Table 1: Production of PRC Leukoreduction in 2015.

\section{ANALYZE DATA OF PRODUCTION PRC LEUCOREDUCE}

\begin{tabular}{|c|c|c|c|}
\hline MONTH & PRODUCTION & $\begin{array}{c}\text { IMPROVE PRODUCTION } \\
\text { EVERY MONTH }\end{array}$ & PERCENTAGE \\
\hline JAN 2015 & 134 BAGS & 256 BAGS (JAN-FEB 2015) & $191 \%$ \\
\hline FEB 2015 & 390 BAGS & 98 BAGS (FEB-MAR 2015) & $25 \%$ \\
\hline $\begin{array}{c}\text { MAR } \\
2015\end{array}$ & 292 BAGS & 28BAGS (MAR-APR 2015) & $9.5 \%$ \\
\hline $\begin{array}{c}\text { APR } 2015 \\
\text { MAY }\end{array}$ & 264 BAGS & 748 BAGS (APR-MAY 2015) & $283 \%$ \\
\hline $\begin{array}{c}\text { JUN } 2015 \\
\text { 1015 }\end{array}$ & 2923 BAGS & 1911 BAGS (MAY-JUN 2015) & $188 \%$ \\
\hline
\end{tabular}

PETECTING YELLOWISH PLASMA IN BLOOD COMPONENTS FOR IMPROVING BLOOD DONOR SERVICE IN JAKARTA BLOOD TRANSFUSION UNIT

A Udyaningrum, U Bachrussani and HT Lestari

Jakarta Blood Transfusion Unit, DKI Jakarta, Indonesia

Background: Jakarta Blood Transfusion Unit (BTU) commits to give the best and quality service for donor. Every blood was processed and distributed in good condition. For blood safety, we also checked the visual parameter of blood components such as icteric, hemolysis, clots etc. For yellowish plasma or icteric, we concerned about the total bilirubin and SGOT/SGPT of the donor. This yellowish plasma indicated some metabolic problems in donor. If we told the donor about his condition, they could be treated if needed.

Aims: To know the level of total bilirubin, SGOT/SGPT in yellowish plasma.

Methods: We used DMSO method to detect total bilirubin, IFCC method for SGOT/ SGPT method.

Results: From 2013 there was 999 samples. Eight point three percent was at normal level and 91.7\% was at high level. In 2014, there was 1621 samples. Five point one percent at normal level and $94.9 \%$ at high level. This yellowish plasma didn't correlate with the TTI screening results. Considering this high level of bilirubin and SGOT/SGPT, so we thought that we should tell the donor's condition for their own good.

Summary/conclusions: Yellowish plasma indicates high total bilirubin and SGOT/ SGPT in donors.

P-090

TTHE USE OF BLOOD COMPONENT FOR TRANSFUSION BY BACKGROUND DISEASE/REASON TRANSFUSION IN BLOOD TRANSFUSION CENTER TANGERANG CITY

Btc tangerang City Red Cross, Tangerang city, Indonesia

Background: Blood transfusion is an action done on patients who need blood and/ or blood products by entering the blood through the veins by using a set transfusi.Aplikasi blood transfusion in clinical everyday can be as a replacement therapy

(C) 2015 The Author Vox Sanguinis (C) 2015 International Society of Blood Transfusion Vox Sanguinis (2015) 109 (Suppl. 2), 1-96 
and supportive. Appropriate consideration of the risks and benefits of transfusion of blood components such as thrombocyt concentrate (TC) packed red cells (PRC), fresh frozen plasma (FFP), more recommended than whole blood (WB).

Aims: To Know the amount of use of transfusions of blood components based on reasons and background of the disease.

Methods: This research study conducted in the tangerang blood center. Data obtained from the sum of blood request form in 2014. The background of the disease is determined by identifying the diagnosis/reason for transfusion. PRC blood components studied, TC, FFP. Utilization by counting the total number of blood components used per diagnosis of disease, and the average consumption per amount of blood product transfusion cases per tahun.analisa indicated using static test.

Results: Blood Component of the most widely used successively in 2014 was 25 503 units of PRC, TC 18526 units, 5143 units of FFP. Most patients with transfusion reason is 16098 anemia, thalassemia 13,568, dengue fever 7451, C-section 3434, heart surgery 2424, CKD 2398, another disease in 2118.

Conclusion: there is a coherency between disease background/rationale transfusion with blood components transfus use. Anemia, thalassemia, C-section and DHF is a disease background/rationale transfusion most widely used blood component transfusion in tangerang blood center, and blood components are most widely used is the PRC, platelet concentrate (TC), fresh frozen plasma (FFP),

TABLE1. DISTRIBUTION BY NUMBER OF BLOOD COMPONENTS IN 2014

\begin{tabular}{|l|c|c|c|}
\hline \multirow{2}{*}{ MOON } & \multicolumn{3}{|c|}{ BLOOD PRODUCTS } \\
\cline { 2 - 4 } & PRC & TC & FFP \\
\hline JANUARY & 2400 & 1588 & 390 \\
\hline FEBRUARY & 1766 & 1477 & 504 \\
\hline MARCH & 2479 & 1903 & 434 \\
\hline APRIL & 2992 & 2330 & 540 \\
\hline MEI & 1409 & 897 & 222 \\
\hline JUNE & 1138 & 765 & 344 \\
\hline JULY & 1875 & 1203 & 379 \\
\hline AUGUST & 1879 & 1195 & 400 \\
\hline SEPTEMBER & 2104 & 1598 & 613 \\
\hline OCTOBER & 2091 & 1585 & 167 \\
\hline NOVEMBER & 2574 & 1747 & 491 \\
\hline DECEMBER & 2793 & 2238 & 359 \\
\hline SUM & \multicolumn{3}{|c}{} \\
\hline
\end{tabular}

\subsection{Plasma Products}

\section{P-09}

\section{CERULOPLASMIN ISOLATION FROM CRYOSUPERNATANT BY ION EXCHANGE CHROMATOGRAPHY}

K Mousavi Hosseini, M Shahabi and M Fallah Tafti

Blood Transfusion Research Center, High Institute for Research and Education, Tehran, Iran

Background: Structure of ceruloplasmin enzyme is a multicopper oxidase. Ceruloplasmin is an enzyme in human blood which is essential for normal iron homeostasis. This research tries to separate the enzyme ceruloplasmin from the cryosupernatant from fresh frozen plasma.

Aims: This investigation attempts to separate the enzyme ceruloplasmin from the cryosupernatantfrom fresh frozen plasma by ion exchange chromatography.

Methods: Cryosupernatant from fresh frozen plasma was used as starting material. For the purpose of separation of ceruloplasmin from cryosupernatant, first the cryosupernatant was treated with polyethylene glycol. Then, ion exchange chromatography by DEAE-Sephacel was carried out on obtained enriched-ceruloplasmin to get the ceroluplasmin.

Results: The achieved ceroluplasmin enzyme from cryosupernatant by treatment of cryosupernatant with polyethylene glycol and ion exchange chromatography DEAESephacel showe purity of $90-92 \%$.

Summary/Conclusion: By treatment of fresh frozen plasma cryosupernatantwith polyethylene glycol followed by ion exchange chromatography a purity of $90-92 \%$ for ceroluplasmin was obtained. Further ion exchange chromatography by DEAESephadex may result in higher purity.

P-092

This abstract has been withdrawn.

(C) 2015 The Author

Vox Sanguinis (C) 2015 International Society of Blood Transfusion

Vox Sanguinis (2015) 109 (Suppl. 2), 1-96

\subsection{Pathogen Inactivation}

P-093

This abstract has been withdrawn.

P-094

EVALUATION OF SINGLE AND DOUBLE DOSE, INTERCEPTTREATED, POOLED RANDOM DONOR PLATELETS SUSPENDED IN 100\% PLASMA

M Green ${ }^{1}$, H Vermeij $^{1}$, L Amorim ${ }^{2}$, H Patel $^{3}$, S Graminske ${ }^{4}$, M Lopes ${ }^{2}$, T Berry ${ }^{1}$, E Weiner $^{1}, \mathrm{~J} \mathrm{Crane}^{3}$ and D Triulzi ${ }^{5}$

${ }^{1}$ Cerus Corporation, Concord, United States of America ${ }^{2}$ Hemorio, Rio De Janeiro, Brazil ${ }^{3}$ LifeSource, Chicago, United States of America ${ }^{4}$ Blood Center of Wisconsin, Milwaukee, United States of America ${ }^{5}$ ITxM, Pittsburgh, United States of America

Background: A photochemical treatment process utilizing amotosalen and UVA light (INTERCEPT[TRADEMARK] Blood System) has been developed for the inactivation of viruses, bacteria, parasites, and leukocytes that can contaminate blood components intended for transfusion.

Aims: The objective of this study was to determine the processing feasibility and in vitro platelet quality over 7 day storage of INTERCEPT-treated double dose pooled whole blood-derived random donor platelets (RDPs) in 100\% plasma.

Methods: To determine processing feasibility, 8 ABO-identical RDPs were produced from $\sim 450 \mathrm{ml}$ whole blood using an optimized method, pooled into a standard $600 \mathrm{ml}$ transfer container $(\mathrm{n}=12$ pools), and leukofiltered. The double dose RDP pools were then immediately INTERCEPT-treated, split in two platelet doses, and stored for 7 days. A second study was performed where 16 ABO-identical RDPs were pooled into a 1.31 transfer pack $(n=5$ pools), separated into two identical 8 unit pools, and then each pool was leukoreduced. One pool was INTERCEPT-treated (Test), while the remaining pool was untreated (Control). In vitro assays to assess platelet quality were performed on all pools on days 1 (pre-treatment), 2, 5 and 7. Results: In the first study, platelet quality was well preserved during 7 days of storage, and met EU guidelines for blood component specifications. The mean platele dose was $3.5 \pm 0.3 \times 10^{11}$ per product. The $\mathrm{pH}$ of the pools remained stable over 7 days of storage (mean $6.8 \pm 0.1$ ) with a platelet swirl rating of 2-3 for all pools Additionally, creating double-dose RDP pools resulted in a 20\% reduction in needed whole blood collections to produce the same number of platelet doses, a 50\% reduction in the use of filters and pooling systems, and a 30\% reduction in the use of sterile connections. Since the double dose INTERCEPT processing set was utilized, the time for producing 2 pathogen inactivated platelet doses was cut in half. In the second study, additional in vitro parameters for Test and Control pools were evaluated (Table 1). After 7 days of storage, the $\mathrm{pH}$ of the Test remained above 7.0 (mean $7.05 \pm 0.05$ ), and was similar to the Control (mean $7.17 \pm 0.08$ ). Additionally, Tes and Control showed a similar active metabolism $\left(\mathrm{pO}_{2}\right.$ levels), residual energy source (glucose), and buffering capacity (bicarbonate). Importantly, extent of shape change, hypotonic shock response, morphology, and CD62 expression were comparable for Test and Control.

Conclusions: INTERCEPT-treatment of double dose, whole-blood derived RDP pools in $100 \%$ plasma is feasible, cost effective, and the quality of the INTERCEPT-treated platelets is maintained throughout 7 days of storage.

Table 1: In Vitro Platelet Parameters Comparing INTERCEPT-Treated Platelets with Conventional Platelets.

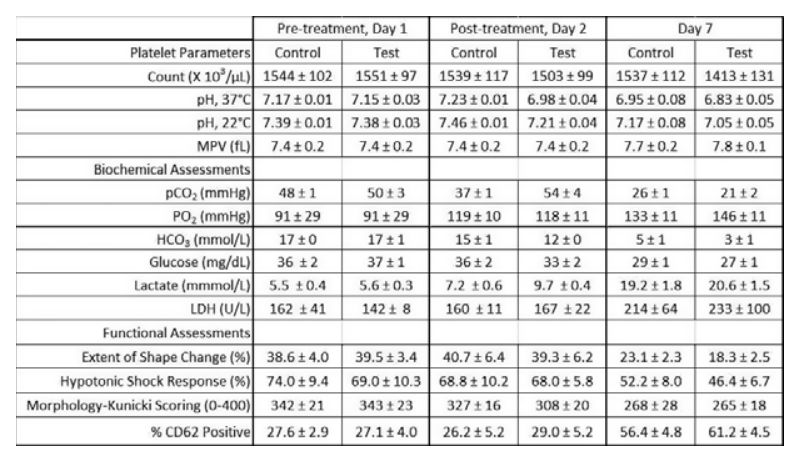


P-095

AN OBSERVATIONAL STUDY TO EVALUATE THE

THERAPEUTIC EFFICACY AND SAFETY OF PLATELET COMPONENTS PREPARED WITH THE INTERCEPT BLOOD

SYSTEM

JPY Sim ${ }^{1}$, C Lam ${ }^{2}$, CW Tsoi ${ }^{3}$, CK Lee ${ }^{3}$, CK Lin ${ }^{3}$, A Stassinopoulos ${ }^{4}$ and AKW Lie ${ }^{1}$

${ }^{1}$ University of Hong Kong, Hong Kong, China ${ }^{2}$ Department of Pathology and Clinical Biochemistry, University of Hong Kong, Hong Kong, China ${ }^{3}$ Hong Kong Blood

Transfusion Service, Hong Kong, China ${ }^{4}$ Cerus Corporation, Concord, California,

United States of America

Introduction: Transfusion support is an essential part of haematopoietic stem cell transplant (HSCT). Platelets components (PC) for transfusion are stored at room temperature as cold temperatures induce aggregation of von Willebrand factor receptors and subsequent platelet elimination by liver macrophages. However, storage at room temperature increases the risk of bacterial contamination (estimated to be $1 / 2000-1 /$ 3000 platelet units). The amotosalen/UVA-based INTERCEPT ${ }^{\mathrm{TM}}$ Blood System (Cerus Corporation, Concord CA, USA) is one pathogen inactivationtechnology which inactivates pathogens through a photochemical reaction preventing DNA replication and RNA transcription. I-PLT (INTERCEPT treated platelets) has the further advantage of lymphocyte inactivation, obviating the need for gamma-irradiation for transfusion associated graft-versus-host disease prophylaxis in HSCT recipients. The INTERCEPT Blood System has received national registration in France (Afssaps), Germany (Paul Ehrlich Institute), and Switzerland (Swissmedic). Here we conduct a prospective study to evaluate the therapeutic efficacy and safety of I-PLT in Hong Kong Chinese HSCT recipients.

Methods: Patients undergoing autologous HSCT or identical ABO blood group allogeneic HSCT were recruited. I-PLTS were prepared from pools of 5 ABO-matched non-leukofiltered buffy coat PC in platelet additive solution treated with the INTERCEPT system. Another 31 patients undergoing autologous HSCT, or ABO-matched allogeneic HSCT received conventional platelet rich plasma (PRP) PC in the run-in period were used as control. Each patient may receive up to 5 I-PLTs or PRP PC in this study. The primary efficacy endpoint is the 1-h corrected count increment (CCI) and the primary safety endpoint is the proportion of patients who experience transfusion reaction following transfusion of I-PLT. Patients with splenomegaly and/or history of refractoriness to platelet transfusion were excluded.

Results: At the time of this interim analysis, 28 patients had received 60 I-PLTS units and 31 patients 88 PRP PC. The mean 1-h CCI achieved after I-PLT transfusion was significantly higher than that achieved after conventional PRP $(n=0.037)$. One patient in the I-PLT group had fever post transfusion. This patient underwent haploidentical transplant but was complicated by graft failure and neutropenic sep-

\section{Table 1. Patients (Pts) and transplant characteristics}

\begin{tabular}{|c|c|c|}
\hline & PRP Platelets & $\begin{array}{c}\text { I-PLT } \\
\text { Interim analysis }\end{array}$ \\
\hline No. of patients & 31 & 28 \\
\hline No. of platelet transfusions & 88 & 60 \\
\hline \multicolumn{3}{|l|}{ Patients character istics } \\
\hline - Male:Female & $11: 20$ & $15: 13$ \\
\hline \multicolumn{2}{|l|}{ Diagnosis } & $43.5(23-66)$ \\
\hline - Acute Myeloid Leukaemía & 13 & 13 \\
\hline - Acute Lymphoblastic Leukaemia & 7 & 6 \\
\hline - Chronic Myeloid Leukaemia & 1 & 1 \\
\hline - Chronic Lymphocytic Leukaemia & - & 1 \\
\hline - Myelodysplastic syndrome & 2 & 2 \\
\hline - Myeloproliferative neoplasm & 2 & - \\
\hline - Multiple Myeloma & 1 & 2 \\
\hline - Non-Hodgkin lymphoma & 5 & 1 \\
\hline - Hodgkin lymphoma & - & 1 \\
\hline - Severe Aplastic Anemia & & 1 \\
\hline \multicolumn{3}{|l|}{ Graft type } \\
\hline - BM & 15 & 15 \\
\hline - PBSC & 16 & 12 \\
\hline - $B M+P B S C$ & & 1 \\
\hline \multicolumn{3}{|l|}{ ABO blood group } \\
\hline$-A$ & 6 & 7 \\
\hline$-B$ & 13 & 6 \\
\hline-0 & 11 & 14 \\
\hline$-A B$ & 1 & 1 \\
\hline \multicolumn{3}{|l|}{ Transplant characteristics } \\
\hline - Autologous & 5 & 5 \\
\hline \multicolumn{3}{|l|}{ - Allogeneic } \\
\hline ○ Myeloablative conditioning & 17 & 21 \\
\hline$\circ$ Reduced Intensity conditioning & 9 & 2 \\
\hline \multicolumn{3}{|l|}{ - Allo-donors } \\
\hline$\circ$ Related & 17 & 16 \\
\hline - Unrelated & 9 & 7 \\
\hline
\end{tabular}

Table 2. Post transfusion platelet (PLT) counts $\left(\times 10^{9} / L\right)$ and 1-hour corrected count increment (CCI)

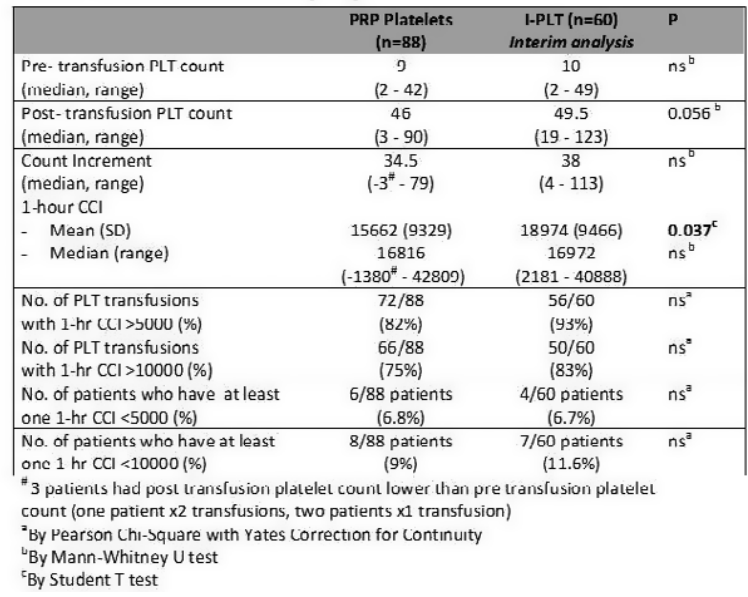

sis. The fever was likely due to underlying sepsis rather than transfusion related. Another one patient developed self-limiting urticarial rash after I-PLT which resolved with antihistamine.

Conclusion: The transfusion of I-PLT was associated with adequate platelet count increments and a good safety profile.

\section{P-096}

\section{COMPARATIVE EFFECTIVENESS OF PLASMA PREPARED WITH AMOTOSALEN-UVA (INTERCEPT) PATHOGEN INACTIVATION FOR THERAPEUTIC PLASMA EXCHANGE AND LIVER TRANSPLANT}

LM Corash

Cerus Corporation, Concord, United States of America

Plasma transfusion is a critical therapy for patients with thrombotic thrombocytopenic purpura (TTP) and acquired coagulopathy of liver transplant (LT). Plasma transfusion for these disorders requires large volumes of donor plasma with exposure to multiple donors. Exposure to multiple donors increases the risk of transfusiontransmitted infection (TTI) due to undetected low burden pathogens (HIV, HCV, and HBV); and to emerging pathogens which may be endemic or epidemic, and for which no tests are available (chikungunya, dengue, SFTS, and zika virus) to detect asymptomatic infections. In contrast to donor testing which is a reactive strategy with a limited spectrum, pathogen inactivation (PI) treatment of donor plasma using robust PI technology is a prospective and broad spectrum strategy to reduce TTI. PI treatment of plasma with amotosalen-UVA (INTERCEPT Blood System for PlasmaIBSP, Concord, CA) is a CE Mark Class III drug-device, and has been licensed by the US FDA for treatment of apheresis and whole blood plasma transfusion of patients with acquired coagulopathy of LT and patients with TTP requiring therapeutic plasma exchange (TPE). Randomized, controlled clinical trials were conducted for each of these indications as part of the European and US registrations. The 2007 international Consensus Conference on Pathogen Inactivation Technologies (Transfusion 2007; 47: 2338-47) recommended that post-marketing surveillance studies be conducted as these novel technologies were introduced into practice. In accordance with this recommendation post marketing studies of comparative effectiveness for INTERCEPT plasma used in treatment of patients with acquired coagulopathy of LT and TPE for TTP were conducted. A retrospective sequential cohort design was utilized for both studies. The LT study compared the therapeutic efficacy and key safety outcomes for liver transplants supported with quarantine plasma (QP), or amotosalen-UVA -plasma (IBSP). Records for 212 QP and 215 IBSP LT were reviewed. Among the LT requiring plasma, median pre-transplant values were worse among the IBSP cohort including: delay to LT $(\mathrm{P}=0.002)$, MELD score $(\mathrm{P}<0.001)$, hematocrit $(P=0.006)$, and graft cold perfusion time $(P=0.033)$. However, the median volumes of plasma transfused were not different for QP and IBSP $(2.1601$ vs $1.969 \mathrm{l}, \mathrm{P}=0.292$ ). No significant differences were observed for the frequency of acute hepatic artery thrombosis or mortality. The TTP study reviewed sequential TPE cohorts using QP and IBSP. 31 patients (18 IBSP and 13 QP) met inclusion criteria for auto-immune TTP. The proportions (\%) in remission at $30 \mathrm{~d}$ (IBSP $=61.1$,

(C) 2015 The Author Vox Sanguinis (C) 2015 International Society of Blood Transfusion Vox Sanguinis (2015) 109 (Suppl. 2), 1-96 
$\mathrm{QP}=46.2, \mathrm{P}=0.570)$ and $60 \mathrm{~d}(\mathrm{IBSP}=77.8, \mathrm{QP}=76.9, \mathrm{P}=1.00)$ were not different. Median days to remission were less for IBSP (15.0 vs $24.0, P=0.003$ ). Relapse rates (\%) 60d after remission were not different (IBSP $=7.1, \mathrm{QP}=40.0, \mathrm{P}=0.150$ ) ECG abnormalities before and during TPE were frequent; but cardiac AE and mortality were not different between treatment cohorts. Post market surveillance using retrospective, controlled comparative effectiveness studies with conventional plasma confirmed that IBSP was effective and safe for transfusion support of LT and TTP.

\section{P-097}

\section{INACTIVATION OF DENGUE AND CHIKUNGUNYA VIRUSES} WITH THE THERAFLEX MB-PLASMA SYSTEM

HM Faddy ${ }^{1}$, JJ Fryk ${ }^{1}$, PR Young ${ }^{2}$, D Watterson ${ }^{2}$, RA Hall ${ }^{2}$, NA Prow ${ }^{2}$, J HobsonPeters $^{2}$, S Reichenberg $^{3}$ and DC Marks ${ }^{1}$

${ }^{1}$ Australian Red Cross Blood Service, Brisbane, Australia ${ }^{2}$ Chemistry and Molecular Biosciences, University of Queensland, Brisbane, Australia ${ }^{3}$ MacoPharma

International GmbH, Langen, Germany

Background: Arboviruses are a group of arthropod-borne viruses that have the potential to cause disease in humans, with many considered emerging or re-emerging. Given that arboviral infection results in asymptomatic viraemia there exists a risk of transfusion-transmission. Two such arboviruses investigated in this project are dengue viruses (DENV), which have been reported to be transmitted through blood transfusion, and chikungunya virus (CHIKV), which could theoretically be transfusion transmissible. An approach for managing this risk is pathogen inactivation, such as the THERAFLEX MB-Plasma system.

Aims: To examine the effectiveness of the THERAFLEX MB-Plasma system at different visible-light doses to inactivate DENV or CHIKV in plasma.

Methods: DENV (types 1 or 2) or CHIKV were spiked into pooled plasma, and then treated with the THERAFLEX MB-Plasma system at different doses of visible light. Pre- and post-treatment samples were taken at each dose and viral infectivity determined by a modified version of a plaque assay. The reduction in viral infectivity was calculated.

Results: We observed dose-dependency in viral inactivation with increasing dose of visible light for DENV-1 and DENV-2. At $60 \mathrm{~J} / \mathrm{cm}^{2}$ (half the standard dose), we observed $>5.77 \log _{10},>5.17 \log _{10}$, inactivation for DENV-1 and DENV-2, respectively. Inactivation of CHIKV resulted in the residual viral infectivity being at the detection limit of the assays used after the first treatment dose, resulting in $>6.58$ $\log _{10}$ inactivation at $60 \mathrm{~J} / \mathrm{cm}^{2}$.

Summary/Conclusions: Arboviruses pose a risk to transfusion safety globally. This study has shown that the residual infectivity of both DENV and CHIKV at up to half the standard visible light dose was at the detection limit of the assays used. The level of inactivation that would prevent transmission of DENV or CHIKV disease through blood transfusion, or the viral load to elicit disease through this route, is currently unknown. Indeed this knowledge would be valuable for determining the minimum level of inactivation required to prevent transmission through transfusion. Nevertheless, this study has shown that DENV-1, DENV-2 or CHIKV spiked into clinical plasma units were effectively inactivated by the THERAFLEX MB-Plasma system, and these viruses can be added to the range of agents that are inactivated by this system.

\subsection{Novel Blood Products}

P-098

EVALUATION OF TWO HUMAN PLATELET LYSATES

SUPPLEMENTS TO SUPPORT EX VIVO EXPANSION OF BOVINE CORNEAL ENDOTHELIAL CELLS AND HUMAN UMBILICAL CORD BLOOD WHARTON'S JELLY MESENCHYMAL STEM CELLS

M.S. Chen ${ }^{1}$, TJ Wang ${ }^{2}$ and T Burnouf ${ }^{3}$

${ }^{1}$ Taipei Medical University, Taipei, Taiwan ${ }^{2}$ Department of Ophthalmology, Taipei Medical University Hospital,, Taipei, Taiwan ${ }^{3}$ Graduate Institute of Biomedical Materials and Tissue Engineering, TMU, Taipei, Taiwan

Background: Ex vivo expansion of stem cells and primary cells is needed to achieve a sufficient number of cells for some transplant and cell therapy procedures. Human platelet-lysates (HPLs), which contain numerous growth factors and nutrients, have generally been shown to be an efficient substitute for fetal bovine serum (FBS) as a supplement for ex vivo cell expansion. HPL is typically obtained by freeze and thaw (FT) cycles or calcium chloride/thrombin activation of platelet concentrates (PC). There is a need to improve HPL standardization and virus safety for optimal outcomes and safety in the ex vivo expansion of different cell types for therapeutic use.

\section{(C) 2015 The Author}

Vox Sanguinis (C) 2015 International Society of Blood Transfusion

Vox Sanguinis (2015) 109 (Suppl. 2), 1-96
Aims: To evaluate, and to compare to FBS, the capacity of HPL prepared by FT cycles or subjected to solvent/detergent (S/D) viral inactivation, as supplement of growth media for ex vivo expansion of bovine corneal endothelial cells (BCECs) and human Wharton's jelly mesenchymal stem cells (WJMSCs).

Methods: BCECs (BCE C/D-1b) were supplied from Pf. Tsung-Jen Wang. WJMSC (H-WJ001) were purchased from BCRC (Hinschu, Taiwan). HPL fractions were prepared from the same expired PCs $(\mathrm{N}=3)$ that were collected from whole blood using the platelet-rich-plasma preparation procedure. PCs were frozen at $-20^{\circ} \mathrm{C}$. The frozen PCs were thawed at $37^{\circ} \mathrm{C}$, separated into aliquots and subjected to three freeze and thaw cycles $\left(-20 /+37^{\circ} \mathrm{C}\right.$; FTPL), or virus inactived by S/D treatment using $1 \%$ TnBP and 1\% Triton X-45 at room temperature for $1 \mathrm{hr}$ followed by $10 \%$ soybean oil extraction and C18 chromatography (SDPL). Protein profile was determined by sodium dodecyl sulfate-polyacrylamide gel electrophoresis (SDS-PAGE) and growth factors by ELISA. HPL fractions were used at the same protein content as 10\% FBS as supplement of Dulbecco's Modified Eagle's medium (DMEM) for ex vivo expansion of BCECs and as the same protein content as 20\% FBS as supplement of Minimum Essential Medium Alpha Medium (MEM- $\alpha$ medium) for $e x$ vivo expansion of WJMSCs. The viability of WJMSCs and BCECs was evaluated by 3-(4,5-cimethylthiazol-2-yl)-2,5-diphenyl tetrazolium bromide assay (MTT), cell growth curve by counting cell number under microscopy for 7 days, and cell morphology was observed by microscopy.

Results: Protein profile of each PL fractions determined by SDS-PAGE was no visually different. The content of growth factor of SDPL and FT was within expected ranges. BCECs grown in medium supplemented with SDPL or FTPL exhibited a viability in the MTT assay similar to that found using FBS. BCECs cells showed higher expansion when grown until 7 days in a medium supplemented using SDPL or FTPL compared to FBS. WJMSCs growth in medium supplemented with SDPL or FTPL was similar with that achieved in the medium containing FBS. Therefore, the preliminary data support that both FTPL and SDPL fractions can be used as supplement to expand BCECs and WJMSCs ex vivo.

Conclusions: HPL obtained by FT or S/D lysis can be used to substitute for FBS as supplement of growth media for expanding BCECs and WJMSCs ex vivo.

\section{P-099}

\section{PLATELETS AS DRUG DELIVERY SYSTEM OF DOXORUBICIN} FOR BREAST CANCER THERAPY

\section{YW $\mathrm{Wu}^{1}$, CA Changou ${ }^{2}$, H.A. Goubran ${ }^{3}$ and T Burnouf}

${ }^{1}$ Graduate Institute of Biomedical Materials and Tissue Engineering, Taipei, Taiwan ${ }^{2}$ Graduate Institute of Translational Medicine, Taipei Medical University, Taipei, Taiwan ${ }^{3}$ Division of Oncology, College of Medicine, University of Saskatchewan, Saskatoon, Canada

Background: Platelets (PLTs) are known to interact closely with cancer cells in the tumor microenvironment, where a reciprocal amplification loop is ignited. Due to their human origin, biocompatibility, biodegradability, inherent non-toxicity, and tropism for tumors, platelets may emerge as a potential novel targeted drug delivery system of anti-cancer agents. Doxorubicin hydrochloride (DOX) is a widely used potent anti-cancer drug that has the drawbacks of inducing major toxic effects in patients. Encapsulation into PEG-liposomes has not eliminated all side effects of DOX. Therefore, safer and personal therapeutic strategies to deliver DOX to the tumor site, using immunologically 'transparent' blood cells, like autologous platelets, should be considered.

Aims: The aim of this in vitro study was to evaluate the capacity of human platelets to (a) entrap DOX and (b) exert a toxic effect in a MCF7 breast cancer cells model. Methods: Platelet concentrates were prepared from whole blood and centrifuged to pellet platelets. PLTs were incubated with $100 \mu \mathrm{M}$ doxorubicin for $1 \mathrm{~h}$ at $37^{\circ} \mathrm{C}$ to prepare platelet-entrapped DOX (PLT-DOX). Entrapment and localization of DOX within PLTs were observed by fluorescence microscopy. Size and morphology of PLT and PLT-DOX were determined by scanning electron microscopy (SEM) and compared to that of thrombin-activated PLT. The quantity of DOX entrapped by PLTs was quantified by spectrophotometry at $585 \mathrm{~nm}$. The capacity of PLT-DOX to reduce breast cancer cells viability was determined in vitro using MCF7 cells. Cells were treated with PLT, PLT-DOX and free DOX (control) for $48 \mathrm{~h}$ and viability was compared by trypan blue exclusion assay.

Results: DOX was efficiently entrapped by PLTs, as evidenced by fluorescence microscopy. 0.8-1 $\times 10^{6}$ PLTs were found to entrap 18-20 $\mu \mathrm{M}$ of DOX within $1 \mathrm{~h}$ of incubation at $37^{\circ} \mathrm{C}$. The size and morphology of PLT-DOX was similar to that of untreated PLT, and different from thrombin-treated PLT that exhibited speudopodes typical of an activation. In vitro cellular assays revealed that the viability of MCF-7 breast cancer cells treated with PLT-DOX was decreased to values similar to that obtained with DOX only (positive control). 
Conclusions: In conclusion, these in vitro data show that PLTs can entrap DOX without obvious impact on their microscopic morphology. PLT-DOX can exert a toxic effect on breast cancer cells in vitro suggesting release of DOX to targeted cells. Further studies on other cancer cells models and using animal experiments are needed to further evaluate the use of PLT-DOX as a potentially novel cell-based Trojan Horse therapeutic strategy against cancers.

P-100

This abstract has been withdrawn.

\section{Transfusion Transmitted Infections 4.1 Screening Strategies for TTI}

P-101 AUTOMATED SYSTEM FOR NUCLEIC ACID TESTING IN BLOOD DONORS

ZR Huang, SS Chua, KS Ng and S Lam

Health Sciences Authority, Singapore, Singapore

Background: Nucleic acid testing (NAT) is one of the mandatory tests performed to screen blood donations in Blood Service Group (BSG) Singapore since October 2000. For the past decade, a few fully automated, high-throughput molecular testing systems have been developed for NAT in blood donations. One of the latest addition to these testing platforms is the Roche cobas ${ }^{\circledR} 6800$ system. The cobas ${ }^{\circledast}$ MPX Assay is used on the cobas ${ }^{\circledR} 6800$ system to detect for HIV-1, HIV-2, HBV and HCV in blood donors through real-time PCR test.

Aims: Our aim is to evaluate the performance of the cobas ${ }^{\circledR} 6800$ system and cobas $^{\boxplus}$ MPX Assay and study if the system is suitable for the current laboratory workflow for blood donation testing.

Methods: Individual donor nucleic acid testing was performed on the cobas ${ }^{\circledR} 6800$ system for approximately 10,000 routine donations. The performance of cobas ${ }^{(1)}$ MPX6800 system was assessed by studying the following: (i) sample throughput and daily maintenance time; (ii) system failure rate; (iii) cross-contamination rate; (iv) Good Laboratory Practice (GLP) compliance features; (v) analytical sensitivity; (vi) assay specificity and (vii) assay reproducibility.

Results: 9619 donations were tested during the evaluation study in a month. For sample throughput time, the average time from sample loading onto instrument to result reporting for a processing plate of 92 samples plus cobas ${ }^{\oplus}$ MPX controls was $3 \mathrm{~h}$. There was no major unrecoverable system failure encountered but $0.8 \%$ invalid test results was reported during the study period due to sample aspiration and assay related errors. No cross contamination was observed. The cobas ${ }^{\circledR} 6800$ system was able to comply all the GLP features that our laboratory requires. The Limits of Detection (LODs) of the cobas $^{\circledR}$ MPX assay were estimated at $13.4 \mathrm{IU} / \mathrm{ml}$ for HIV-1, $5.7 \mathrm{IU} / \mathrm{ml}$ for $\mathrm{HIV}-2,8.7 \mathrm{IU} / \mathrm{ml}$ for $\mathrm{HCV}$ and $1.5 \mathrm{IU} / \mathrm{ml}$ for $\mathrm{HBV}$ and the overall specificity for cobas ${ }^{\circledR}$ MPX assay is established at $99.95 \%$. The reproducibility of the assay is at $100 \%$ for the 3 viral targets when tested over 3 days. For this study, the HBV NAT yield rate was estimated at 1 in 2405. No NAT yield cases were found for HIV and HCV.

Conclusions: The cobas ${ }^{\circledR}$ MPX 6800 system is suitable to be implemented for routine NAT as it has met the requirements on the analytical sensitivity and assay specificity needed for blood donation screening. The cobas ${ }^{\circledR}$ MPX 6800 system has the added advantage of a short throughput time with maximum sample loading capacity of 225 samples and our laboratory staff find the system easy to operate.

\section{$\mathrm{P}-102$ \\ SPECIFICITY STUDY OF THE COBAS MPX TEST ON THE COBAS 6800 SYSTEM AT NATIONAL BLOOD CENTER, THE THAI RED CROSS SOCIETY}

P Suriya, S Oota, K Chaiwong, P Dejhutsadin, W Saekram, T Sawangsub, P Wongkittikul, S Nanta, S Siriwicthayakul and T Sakuldamrongpanich

National Blood Center, Bangkok, Thailand

Background: Nucleic acid testing (NAT) of blood donations can detect infections that are not detected by serology (HBsAg, anti-HIV/HIV Ag, anti-HCV). We evaluated a new assay on a new highly automated NAT, $\operatorname{cobas}^{\circledR}$ MPX on the cobas ${ }^{\circledR} 6800$ system, and compared it to our current NAT, the cobas ${ }^{\circledR}$ TaqScreen MPX Test, v2.0 on the cobas s 201 system (MPX2). Both are multiplex real time PCR assays that incorporate fluorescent probes specific for HIV, HCV, and HBV, enabling simultaneous detection and viral target discrimination.

Aims: To evaluate the specificity performance of the cobas ${ }^{\circledR}$ MPX Test on the cobas $^{\circledR} 6800$ system for blood donor screening

Methods: 12,298 non-reactive blood donations on $\operatorname{cobas}^{\circledR}$ MPX2 were tested with cobas ${ }^{\circledR}$ MPX Test on the $\operatorname{cobas}^{\circledR} 6800$ system into 2 studies. Study 1, 8064 blood donations were screened in MP6 by cobas $^{\circledR}$ MPX. Study 2, 4234 blood donations were screened by cobas ${ }^{\circledR}$ MPX in MP6 and IDT. Reactive MP6 were individually resolved. The status of reactive samples was confirmed by 5 replicate testing of samples from the plasma bag by both cobas $^{\circledR}$ MPX and MPX2 and also viral load testing to calculate specificity.

Results: Study 1: 8064 blood donations were tested in MP6 on $\operatorname{cobas}^{\circledR}$ MPX, 3 minipools were reactive by cobas ${ }^{\circledR}$ MPX, each of which resolved to an individual sample reactive for HBV. Results of the replicate testing are shown in Table 1.

Study 2: 4234 blood donations were screened on $\operatorname{cobas}^{\circledR}$ MPX; 1 was reactive for HBV on $\operatorname{cobas}^{\circledR}$ MPX by MP6. 14 were reactive for HBV on $\operatorname{cobas}^{\circledR}$ MPX by IDT, including the donation identified by MP6. 6/14 were confirmed reactive by cobas ${ }^{(1)}$ MPX on replicate bag testing; 4 of these were also confirmed by MPX2 (Table 2). 8 donations were nonreactive on replicate bag testing. The difference in yield of cobas $^{\circledR}$ MPX in MP6 vs IDT was of borderline significance $(P=0.06)$. Among the 4228 donations not confirmed to contain HBV, 4220 were nonreactive on cobas ${ }^{\circledR}$ MPX by IDT, for a specificity of 99.81\% (95\% CI: 99.63-99.92\%).

Altogether 12,298 blood donations were screened in MP6 and only 4234 blood donations in IDT on cobas ${ }^{\circledR}$ MPX. The cobas ${ }^{\circledR}$ MPX detected 4 HBV reactive donations by MP6 and $14 \mathrm{HBV}$ reactive donations by IDT. The difference in confirmed yield of cobas ${ }^{\circledR}$ MPX vs MPX2 in MP6 was not statistically significant.

Summary/conclusions: The cobas ${ }^{\circledR}$ MPX on the highly automated cobas ${ }^{\circledR} 6800$ system shows excellent performance in both IDT and MP6. This small evaluation suggests cobas ${ }^{\circledR}$ MPX has shown a comparable specificity to the cobas MPX2.

Table 1: Additional testing of $14 \mathrm{HBV}$ reactive samples detected by cobas MPX by MP6 testing and/or IDT.

Table 1: Results of replicate testing of aliquots taken from plasma bags of 3 donations reactive for HBV detected by cobas MPX in MP6

\begin{tabular}{|c|c|c|c|c|c|c|c|}
\hline \multirow[b]{2}{*}{ Donor } & \multicolumn{2}{|c|}{ Screen Result } & \multicolumn{2}{|c|}{$\begin{array}{l}\text { Bag retest by } \\
\text { IDT* }\end{array}$} & \multirow{2}{*}{$\begin{array}{l}\text { Anti- } \\
\mathrm{HBC}\end{array}$} & \multirow{2}{*}{$\begin{array}{l}\text { Anti- } \\
\text { HBs }\end{array}$} & \multirow{2}{*}{$\begin{array}{l}\text { HBV Viral } \\
\text { load (IU/mL) }\end{array}$} \\
\hline & $\begin{array}{c}\text { MPX2 } \\
\text { by MP6 }\end{array}$ & $\begin{array}{c}\text { cobas } \\
\text { MPX } \\
\text { by MP6 }\end{array}$ & MPX2 & $\begin{array}{l}\text { cobas } \\
\text { MPX }\end{array}$ & & & \\
\hline 1 & NR & $\mathrm{R}$ & $5 / 5$ & $5 / 5$ & Pos & Pos & 22 \\
\hline 2 & NR & $\mathrm{R}$ & $0 / 5$ & $3 / 5$ & Pos & Pos & $\begin{array}{l}\text { Undetectable } \\
\text { (UD) }\end{array}$ \\
\hline 3 & NR & $\mathrm{R}$ & $0 / 5$ & $0 / 5$ & Neg & $\mathrm{Neg}$ & $\begin{array}{c}\text { Undetectable } \\
\text { (UD) }\end{array}$ \\
\hline
\end{tabular}

* Number of replicates reactive for $\mathrm{HBV} /$ /number replicates tested

Table 2: Results of replicate testing of aliquots taken from plasma bags of 3 donations reactive for HBV detected by cobas MPX in MP6.

\begin{tabular}{|c|c|c|c|c|c|c|c|c|}
\hline \multirow[b]{2}{*}{ Donor } & \multicolumn{3}{|c|}{ Screen Result } & \multicolumn{2}{|c|}{$\begin{array}{l}\text { Bag retest by } \\
\text { IDT* }\end{array}$} & \multirow{2}{*}{$\begin{array}{l}\text { Anti- } \\
\text { HBc }\end{array}$} & \multirow[b]{2}{*}{$\begin{array}{l}\text { Anti- } \\
\text { HBs }\end{array}$} & \multirow{2}{*}{\begin{tabular}{|c|} 
HBV \\
Viral \\
load \\
$\left(\begin{array}{c}\mathrm{IU} / \mathrm{mL}) \\
\star \star\end{array}\right.$
\end{tabular}} \\
\hline & $\begin{array}{c}\text { MPX2 } \\
\text { MP6 }\end{array}$ & $\begin{array}{c}\text { cobas } \\
\text { MPX } \\
\text { MP6 }\end{array}$ & $\begin{array}{c}\text { cobas } \\
\text { MPX } \\
\text { IDT }\end{array}$ & MPX2 & $\begin{array}{c}\text { cobas } \\
\text { MPX }\end{array}$ & & & \\
\hline 1 & NR & $\mathrm{R}$ & $\mathrm{R}$ & $5 / 5$ & $5 / 5$ & Pos & Neg & $<20$ \\
\hline 2 & NR & NR & $R$ & $2 / 5$ & $2 / 5$ & Pos & Pos & UD \\
\hline 3 & NR & NR & $\mathrm{R}$ & $1 / 5$ & $1 / 5$ & Pos & $\mathrm{Neg}$ & UD \\
\hline 4 & NR & NR & $\mathrm{R}$ & $5 / 5$ & $1 / 5$ & Pos & $\mathrm{Neg}$ & UD \\
\hline 5 & NR & NR & $\mathrm{R}$ & $0 / 5$ & $1 / 5$ & Neg & Pos & UD \\
\hline 6 & NR & NR & $\mathrm{R}$ & $0 / 5$ & $1 / 5$ & $\mathrm{Neg}$ & Pos & UD \\
\hline 7 & NR & NR & $\mathrm{R}$ & $0 / 5$ & $0 / 5$ & Pos & Neg & UD \\
\hline 8 & NR & NR & $\mathrm{R}$ & $0 / 5$ & $0 / 5$ & Pos & Pos & UD \\
\hline 9 & NR & NR & $\mathrm{R}$ & $0 / 5$ & $0 / 5$ & Pos & Pos & UD \\
\hline 10 & NR & NR & $\mathrm{R}$ & $0 / 5$ & $0 / 5$ & Pos & Pos & UD \\
\hline 11 & NR & NR & $\mathrm{R}$ & $0 / 5$ & $0 / 5$ & Pos & Pos & UD \\
\hline 12 & NR & NR & $\mathrm{R}$ & $0 / 5$ & $0 / 5$ & Pos & Pos & UD \\
\hline 13 & NR & NR & $\mathrm{R}$ & $0 / 5$ & $0 / 5$ & $\mathrm{Neg}$ & Pos & UD \\
\hline 14 & NR & NR & $\mathrm{R}$ & $0 / 5$ & $0 / 5$ & $\mathrm{Neg}$ & Pos & UD \\
\hline
\end{tabular}

(C) 2015 The Author Vox Sanguinis (C) 2015 International Society of Blood Transfusion Vox Sanguinis (2015) 109 (Suppl. 2), 1-96 
P-103

\section{HEAD-TO-HEAD COMPARISON OF THE NEW ROCHE COBAS 8800 AND THE GERMAN RED CROSS NAT-SYSTEM ZELOS $\mathrm{X} 100$}

M Schmidt, K Hourfar and EH Seifried

German Red Cross, Frankfurt, Germany

Background: The German Red Cross, Frankfurt, implemented NAT-testing for HIV, $\mathrm{HCV}$ and HBV as early as in 1997. At that time there were no commercially available methods suitable for NAT-screening in a large blood donor service. Therefore our institute developed an inhouse NAT-system, which in the meanwhile obtained the CE-mark. Our current system combines high sensitivities with economic testing in pools of 96. Although our system is tailor-made for our blood-donor service, we are periodically reviewing the commercially available systems. The introduction of the new Roche Cobas 6800/8800-system was the reason for initiating a head-tohead-comparison between our GRC NAT-system and the new Roche instrument.

Methods: The Roche Cobas 8800 system has been installed in our laboratory in 2014. Technicians as well as unskilled employees have been trained to operate the NAT-systems. The head-to-head comparison was performed for HIV, HBV, HCV using the Roche MPX-test, for HAV and B19 using the DPX-test, and for HEV and the GRC NAT-assays, respectively. All systems were analyzed with respect to specificity, genotype inclusivity and analytical sensitivity by probit analysis. Additionally work-flow analyses were performed for all systems.

Results: The results of the study confirm that the MPX-test on the Roche Cobas $6800 / 8800$ platform and the GRC system are very robust and specific. Sensitivity and specificity in pools of 96 is largely comparable between all three test systems (Table 1) and did not influence the diagnostic window period significantly. Additionally the commercial systems could be operated by unskilled workers after 3 days of training, whereas for the GRC system a significantly higher training level is required.

Conclusions: In the past sensitivity of commercially available NAT-systems was inferior compared to the GRC-system, when testing was performed in pools of 96 . However, with the Roche Cobas $6800 / 8800$ system a technology is available, which provide comparable sensitivities, even when used of pools of 96 . Additionally the system offers a very high throughput, which allows a reduction of pool-size, or even a change to individual donation NAT. The major benefit of these systems, however, is in our point of view, that they can be operated by unskilled personnel. This enables new concepts of laboratory-reorganization.

\section{Table 1:}

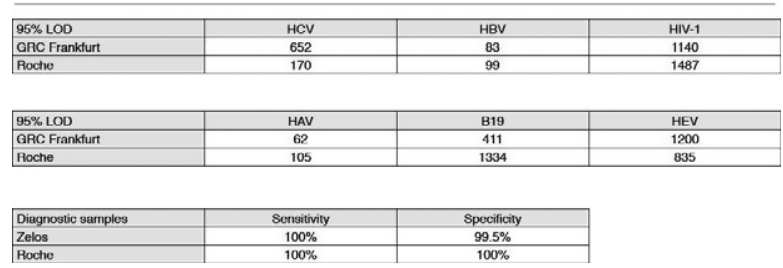

P-104

\section{NRL BLOOD SCREENING EQAS: UPDATE 2014}

G.A. Vincini, LM Cabuang, K Smeh, WJ Dimech and SJ Best NRL, Fitzroy, Australia

Background: In 2013 NRL introduced the Multimarker Blood Screening Serology (MMBS4320) EQA Scheme, specifically designed for the donor screening laboratory. Data from this inaugural year were presented in 2014 and, now in its second year, an update on observations sheds some interesting information in the performance of donor screening laboratories and the testing algorithm and tests used. In 2014, the early HIV infection scenario was expanded upon with inclusion of both manufactured HIV-1 p24 and natural early infection specimens.

Complementary to this serology scheme is the existing Multimarker Blood Screening NAT Scheme (NATA4315), already into its 7th year in this current format. For NATA4314, observations will be presented from the 2014 distributions, a most interesting year in this scheme's history due to an unexpected reactivity result submission pattern phenomenon.

Aims: To assess the performance of donor screening laboratories using serology and NAT tests for TTI viruses.

\section{(C) 2015 The Author}

Vox Sanguinis (C) 2015 International Society of Blood Transfusion

Vox Sanguinis (2015) 109 (Suppl. 2), 1-96
Methods: MMBS4320 2013 and 2014 consisted of three 20-member panels per year. Each panel contained samples positive for one or more of anti-HIV, anti-HCV, anti$\mathrm{HBc}$ total and HBsAg, as well as samples negative for all these markers, sourced from blood donors. In two samples for 2013 distributions, recombinant HIV-1 p24 antigen was spiked into normal human plasma to simulate early HIV infection. In 2014 distributions, both recombinant HIV-1 p24 antigen and a natural early infection (HIV-1 RNA and HIV-p24 antigen positive, but HIV antibody negative) samples were included to simulate early HIV infection. NATA4315 2014 consisted of plasma pooled samples spiked with nucleic acids for HIV-1 and HIV-2, HBV and HCV a various concentrations above the lower limit of detection for most donor screening tests used. Participants were requested to test the samples in the same manner as their donor samples. Results were submitted to NRL using OASYS, an internet-based proficiency test application. The results were then compared with internally-generated reference results and with those obtained by the peer group.

Results: A total of 129 serology and 110 NAT donor screening laboratories from up to 28 countries participated in MMBS4320 and NATA4315 generating over 59,000 serology and over 14,000 NAT results from 155 assays. In the analysis of testing strategies, there were many instances where the testing strategy did not allow fo adequate detection of HIV-1 p24 antigen positive samples. Many examples of aberrant test results were observed based on poor assay sensitivity, particularly where rapid tests were used or where third-generation tests were used in isolation. Furthe analysis of aberrant results revealed some of the errors identified were due to data entry, lot variation and/or peer group false reactivity. In addition, there was a large proportion of unexpected reactivity reported for HBV DNA and HIV-1 RNA for NATA4315 2014 across all distributions.

Conclusions: Overall, the performance of all assays used was of a high standard. NRL EQAS provided participants with valuable information regarding their performance relative to their peers and created many opportunities to educate and facilitate continuous improvement in institutions delivering donor screening at a local, regional or national level.

\section{P-105}

IMPACT OF NUCLEIC ACID AMPLIFICATION TEST ON SCREENING OF BLOOD DONORS IN NORTHERN PAKISTAN

SK Niazi ${ }^{1}$, FA Bhatti ${ }^{1}$, N Salamat ${ }^{1}$, E Ghani ${ }^{2}$ and M Tayyab

${ }^{1}$ Armed Forces Institute of Transfusion (AFIT), Rawalpindi, Pakistan ${ }^{2}$ AFIP, Rawalpindi, Pakistan

Background: Safety of blood products is a great challenge for a developing country like Pakistan with limited resources and a high prevalence of hepatitis B and hepatitis C. Armed Forces Institute of Transfusion (AFIT) is located in Rawalpindi, Northern Pakistan, and acts as a regional blood centre with more than 50,000 donations collected annually. To enhance safety of the blood products at our institute, Nucleic acid amplification test (NAT) was introduced in September 2012, for screening all seronegative blood donors.

Aims: To study the impact of NAT implementation on screening seronegative blood donors in northern Pakistan and to highlight the importance of additional testing for confirmation of reactive NAT results.

Methods: The study was conducted from 21st September 2012 to 20th September 2013. Serological screening was performed on Abbott ARCHITECT i2000 for HBsAg. anti-HCV and HIV antigen-antibody combination assay. Samples of the seronegative donors were run on Roche Cobas s201 platform in pools of six. Initially resolved reactive donors were followed up with additional tests including Individual donation-NAT (ID-NAT) as well as serological tests to confirm positive NAT results. If the donors could not be contacted or failed to re-visit to give fresh sample, fresh frozen plasma bag of the NAT reactive donors was retrieved and additional tests were performed. Viral load estimation was done for all NAT reactive donors.

Results: After serological screening of 56,772 blood donors, 2334 were found reactive; $719(1.27 \%)$ were reactive for HBsAg, 1046 (1.84\%) for anti-HCV, $12(0.02 \%)$ for anti-HIV and $557(0.98 \%)$ for anti-syphilis antibodies. NAT testing of the 54,438 seronegative donors led to 27 NAT reactive donors, with an overall NAT yield of in 2016 donors. 23 donors were found reactive for HBV-DNA (HBV NAT yield: $1: 2367)$ and 4 donors reactive for HCV-RNA (HCV NAT yield: 1:13,609). No case of human immunodeficiency virus (HIV) was detected by NAT during the study period On the basis of HBV serology of the index as well as follow-up samples of $23 \mathrm{HBV}$ NAT reactive donors, 3 had (13\%) window period infection (WP), $1(4.3 \%)$ acute, late stage infection, $14(61 \%)$ occult hepatitis B infection-Tail end (OBI-TE), 1 (4.3\%) chronic hepatitis B infection and $4(17.4 \%)$ seromarker-negative HBV infection. All four HCV-NAT reactive donors seroconverted to anti-HCV during follow-up. The Residual Risk (RR) after NAT implementation, calculated for the first time blood donors, was 62.5 and 4.4 per million donors for HBV and HCV, respectively. 

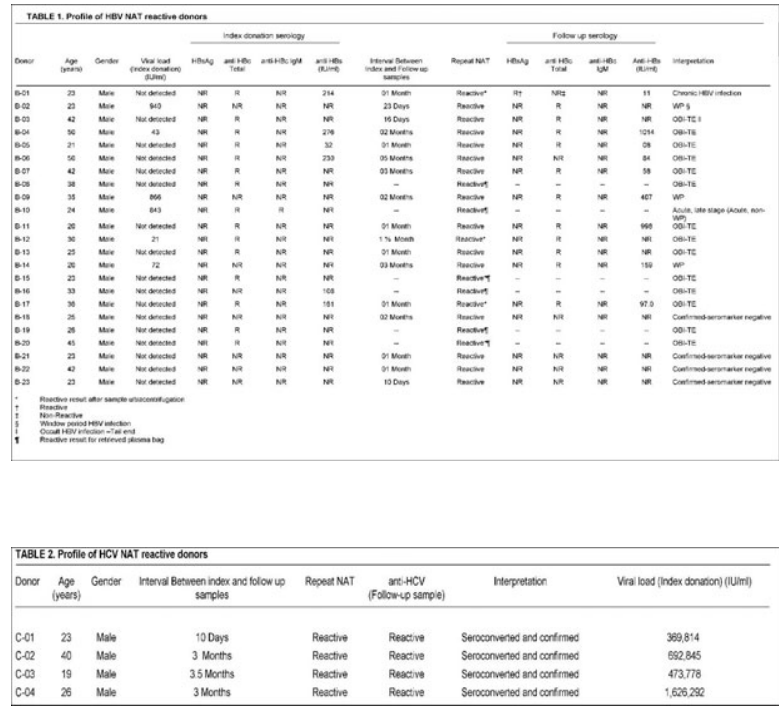

Summary/Conclusions: NAT has significantly improved the safety of blood products at our transfusion institute. Implementation of NAT is highly recommended in other transfusion centers in Pakistan to upgrade safety of blood products. Confirmation of NAT results must always be done either on follow-up samples or samples from retrieved frozen plasma bags.

P-106

\section{NUCLEIC ACID TESTING FOR SCREENING BLOOD DONATION} AT REGIONAL BLOOD CENTRE X CHIANG MAI, THAILAND

W Prasing ${ }^{1}$, W Tongsang ${ }^{1}$, R. Khuenkaew ${ }^{1}$, R Karong ${ }^{1}$, P Srisai ${ }^{1}$, P Kiertpornsakda ${ }^{1}$, T Sakuldamrongpanich ${ }^{2}$ and S Pikulsod ${ }^{2}$

${ }^{1}$ Regional blood Centre X Chiang Mai, Chiang Mai, Thailand ${ }^{2}$ National Blood Centre, Thai Red Cross Society, Bangkok, Thailand

Background: Transfusion-transmitted infections (TTIs) are a major problem associated with blood transfusion. Nucleic acid testing (NAT) in blood donor screening has been implemented in many countries to reduce the risk of TTIs and increase the safety for blood transfusion.

Aims: To assess the prevalence of hepatitis B virus (HBV), hepatitis C virus (HCV) and human immunodeficiency virus (HIV) NAT yield in blood donation and impact of NAT in improving the safety of blood supply.

Method: This study was performed in blood donation from April 2009 to June 2015 at Regional Blood Centre X Chiang Mai. The sero-negative blood donor samples were screened for HIV RNA, HCV RNA and HBV DNA by NAT (mini-pool 6) using $\operatorname{cobas}^{\circledR}$ Taqscreen MPX test on the Roche cobas ${ }^{\circledR}$ s 201 system. Repeated reactive pool for individual donor NAT reactive samples was resolved.

Results: A total of 429,534 sero-negative blood donations were screened. During 6 year period, total $356(1: 1206)$ NAT yields rate were detected which were 9 HIV NAT yield cases $(1: 47,726), 3$ HCV NAT yield cases $(1: 143,178)$ and 344 HBV NAT yield cases (1: 1248) respectively. The NAT yield rate for HBV was higher than HCV and HIV in every year. These results reflected the high prevalence of HBV infections in donor population. Moreover, this study found the majority of NAT reactive samples were male (74.16\%) with repeat donation (49.44\%).

Conclusions: NAT contributed to efficiency improvement of blood donor screening by significantly reduction of the transfusion-transmitted risk of window period donations and also improving the safety of blood supply. Therefore, NAT should be implemented in blood centers in countries with a high incidence of TTIs like Thailand that required a government support for NAT setting.

\section{$\mathrm{P}-107$}

\section{IMPLEMENTATION OF MULTI-DYE NUCLEIC ACID TESTING} FOR BLOOD SCREENING IN DELHI, INDIA

P Sangeeta, S. Singh, T Chakraborty and R Dubey

Max Super Speciality Hospital, New Delhi, India

Background: Nucleic Acid Testing (NAT) was first introduced at Max Super Specialty blood bank as part of the blood screening system in July 2010. Upon initial NAT implementation, blood donations were screened in pools of six using the Roche cobasTaqScreen MPXv1.0 test (MPX v1 test) which requires further discrimination of a reactive donation, in order to identify the reactive viral target. In September 2014, NAT was upgraded to the newly launched Roche cobasTaqScreen MPX v2.0 test (MPX v2 test) in India, which employs real-time detection and identification of 3 viruses in a single test by using multi-dye PCR technology. This technology enables the direct detection of HIV, HBV, HCV and the internal control in separate channels thus removing the need for any additional viral discriminatory testing. Aim: Evaluation of the MPX v2 test performance for routine blood screening. Methods: A total of 90,396 seronegative donations were screened byNAT in minipools of six from July 2010 till Feb 2015 for HIV-1 \& -2 RNA, HCV RNA and HBV DNA. Among the donations tested, a total of 77,614donations were tested on the MPX v1 test from July 2010 till August 2014 and thereafter, 12,782donations were tested on the MPX v2 test from September 2014 till February 2015.

Results: During the initial4 years and 2 months of NAT implementation usingthe MPX v1 test, there were a total of 17 HBV NAT yield donations detected (NAT reactive but seronegative cases) and 3 HCV NAT yield and 2 HIV-1 NAT yield cases detected. This would generate a NAT yield rate of 1:4566 for HBV, 1:25871 for HCV and 1:38,807 for HIV-1. With the advent of MPX v2 test with increased HBV analytical sensitivity, in the last 7 months, we have detected 3 HBV NAT yield cases with a rate $\mathrm{HBV}$ yield at 1:4,261.In an earlier study, data presented for the first 18 months of NAT, the HBV NAT yield reported was 1: 3355 , the HCV yield was $1: 20,131$ and the HIV-1 yield was 1: 10,065. We now observe that these rates based on cumulative data on the MPX v1 test seem to have decreased. Viral load testing performed on the HBV NAT cases showed that the 13 of the 17 cases had very low level titres of $<20 \mathrm{IU} / \mathrm{ml}$, three with viral titres of $27-33 \mathrm{IU} / \mathrm{ml}$ and only one with a viral load of $4541 \mathrm{IU} / \mathrm{ml}$.

Conclusion: The implementation of NAT at our blood bank in addition to screening with serology continues to circumventthe risk of infected blood units being transfused. We will continue to monitor the rate of the HBV NAT yield cases to understand the effect of the increased sensitivity with the MPX v2 test, and the corresponding slight increase of HBV NAT yield rate. In general, the use of the MPX v2 test with real-time viral discrimination allows for faster identification of the reactive donation and prompt notification of the donors for counselling.

\section{P-108}

\section{THE IMPORTANCE OF NUCLEIC ACID TEST FOR IMPROVING} BLOOD SAFETY IN JAKARTA BLOOD TRANSFUSION UNIT

N Nurdiyati, U Bachrussani, NK Ritchie and S Salim

Jakarta Blood Transfusion Unit, DKI Jakarta, Indonesia

Background: Blood transfusion safety is the responsibility of all stakeholders (Indonesian Red Cross, Government, Blood Transfusion Units, Donors and Hospitals involved in the blood journey from donor until transfused in patients. Blood screening is one of the aspects determining the safety of blood. The screening of transfusion transmitted infections (TTIs) could prevent the patients contracting infections due to transfusion. But there is no zero risk in transfusion. There is a gap time between the time when someone was infected and the antigen or antibody could be detected in his blood, known as window period. Since 2007, NAT was introduced in Indonesia. NAT method could significantly shorten the window period but the cost was still tremendously expensive for patients because patients 'pay' for the blood in Indonesia. The government already aware but still counting for the budget and prioritize the multi-transfusion patients. If we presented the risk to the government, we hope they would cover the NAT cost for the safety of all the patients.

Aims: To know the risk of a patient being infected from blood transfusion.

Methods: We select the blood sample randomly from May 2012 until December 2014. We run Chemiluminescence method (Architect ${ }^{\circledR}$ ) and NAT method (Ultrio ${ }^{\circledR}$ ) in parallel. The reactive results either from NAT or chemiluminescence were discarded after re-tested in duplo.

Results: We have tested 171.232 samples from total 852.686 blood donors in Jakarta Blood Transfusion Unit from May 2012 until December 2014. We found 110 of 171.232 samples $0.06 \%$ ) reactive with NAT method but non-reactive in chemiluminescence method. If we only produced whole blood, 6 patients in 10.000 transfu-

(C) 2015 The Author Vox Sanguinis (C) 2015 International Society of Blood Transfusion Vox Sanguinis (2015) 109 (Suppl. 2), 1-96 
sion had risk of getting infection but if we produced blood components more patients had risk.

Summary/conclusions: The risk of contracted TTI could beprevented by implementation of $100 \%$ NAT method in TTI screening.

P-109

NAT EXPERIENCE AT CHARITABLE BLOOD BANK IN PUNE, WESTERN PART OF INDIA

\section{A Kulkarni}

Janakalyan Blood Bank, Pune, India

Background: Replacement donors still contribute a significant portion of the collected blood donations in India. Our centre, Janakalyan Raktapedhi, Pune, and many others across the country have been strictly promoting voluntary donations for the past several years. With increasing awareness on voluntary donation there is also an increased need to ensure safety and superior quality of blood supplied.

Aims: Our study was aimed to observe the seroprevalence of TTIs among the donors of Pune western parts of Maharashtra and to understand the utility of advanced screening techniques such as nucleic acid testing (NAT) over the traditional serology screening.

Materials and Methods: A total of 52,967 units were screened over a period of 32 months. Serology testing using the ELISA method (Benshera kit from Aventor) was used for detection of HBsAg, anti-HIV, and anti-HCV in donor serum. 20,148 units which were negative on ELISA were further subjected to NAT for HBV, HCV, and HIV-1 \& 2 using the cobas TaqScreen MPX assay on the fully automated cobas s201 platform (Roche Diagnostics).

Results: Our sero-reactive rates were at 0.79\% for HBV, 0.25\% for HCV and 0.06\% for HIV-1 \& 2. HBV was the predominant infective agent in these donations screened. Both the TaqScreen MPX and the TaqScreen MPX v2.0 assays were used during this study period for NAT screening. Of the 20,148 units that were screened using NAT, 11 NAT yield cases were detected; 8 for HBV, 2 for HCV and 1 for HIV-1. The combined NAT yield for HIV, HBV and HCV was 1:1832 donations. Each blood unit is further divided into 2.4 components resulting in much higher risk and in a NAT yield as high as 1:763 components.

Conclusion: In countries like India with high prevalence of infections that pose a huge risk of TTIs, a significant number of lives can be saved by eliminating window period donations which can be identified by NAT screening. Our centre now screens $70 \%$ of all seronegative donor samples by NAT. We will continue to follow up the NAT reactive donors to check their seroconversion status and soon screen 100\% donations by NAT.

\section{$\mathrm{P}-110$}

\section{IMPROVING BLOOD SCREENING IN THE REPUBLIC OF}

\section{KAZAKHSTAN}

T Savchuk $^{1}$, Z Burkitbayev $^{1}$ and E Zhiburt $^{2}$

${ }^{1}$ Scientific and production center of transfusiology, Astana, Kazakhstan ${ }^{2}$ Pirogov National medical surgical center, Moscow, Russian Federation

Background: Currently, donated blood is screened for transfusion transmissible infections (TTI) including HIV-1, HIV-2, hepatitis B and C and syphilis. In Kazakhstan, this is carried out in two stages, using two essentially different methods. The first involves an immunoassay and the second NAT-testing 2000SR (ABBOTT, USA) for immunoassays and the COBAS S-201 System TagScreen MPX (ROCHE, Switzerland) for NAT-testing.

Depending on the algorithm adopted, a confirmation of positive samples obtained from the first stage needs to be carried out using test methods by other manufacturers. In this regard, blood centers are also equipped with the automated immunoassay machine Freedom EVOLyzer 150/8 (TECAN, Austria), EVOLIS (BIO-RAD, France) and automated system for immunoblotting ProfiBlot 48 (TECAN, Austria).

The machines used to screen donated blood for TTI have an international CE certificate which is a mandatory requirement for the testing of donated blood in accordance with European Commission Directive (98/79/ EC).

Aim: To analyse the screening programme for donated blood in Kazakhstan between 2008 and 2014 years.

Methods and Materials: Qualitative and quantitative analysis by using 2000SR (ABBOTT, USA) for immunoassays and the COBAS S-201 System TagScreen MPX (ROCHE, Switzerland) for NAT-testing.

Results: From 2008 to 2014, the absolute donor numbers who showed positive during the primary screening for TTI markers by using the immunochemiluminometric

\section{(C) 2015 The Author}

Vox Sanguinis (C) 2015 International Society of Blood Transfusion

Vox Sanguinis (2015) 109 (Suppl. 2), 1-96 assay (ICLA) was reported as 11777 in 2014, which is 1.3 times lower than in 2008 As shown in Table 1, further calculations on relative index of primarily screened donors, reported an increase by $1.6 \%$.

Table 1 also reports the results obtained at the end of the secondary screening with ICLA. Number of positive results rose from $69.7 \%$ to $70.5 \%$ during the last 7 years. Introducing and using NAT-testing from 2012 have prevented using blood components collected from 653 donors with identified TTI (Table 2).

Therefore, NAT-testing has made it possible to prevent delivery of blood that contained TTI causative agents. As shown in Table 2, number of positive results during NAT-testing increased by $7.9 \%$ and $8.9 \%$, in 2012 and 2014 respectively.

Conclusions: Automation of the screening process of donated blood provided significant medical and economic benefits due to improving the detection of TTI markers and thereby reducing false-positive results during the primary screening and detecting the false-negatives during the NAT-testing.

Table 1. Results of the primary and secondary ICLA screening of donated blood samples for TTI markers and confirmed positive results during 2008-2014.

\begin{tabular}{|c|c|c|c|c|c|c|}
\hline & \multirow{2}{*}{$\begin{array}{c}\text { Number } \\
\text { Years }\end{array}$} & \multicolumn{2}{|l|}{ Positive TTI result } & \multicolumn{3}{|c|}{$\begin{array}{c}\text { Confirmation of positive TTI } \\
\text { results using different analyzers }\end{array}$} \\
\cline { 5 - 8 } examined & $\begin{array}{c}\text { Absolute } \\
\text { number } \\
\text { of donors }\end{array}$ & $\%$ & $\begin{array}{c}\text { Absolute } \\
\text { number of } \\
\text { donors }\end{array}$ & $\%$ & $\begin{array}{c}\text { Confirmed } \\
\text { primary positive } \\
\text { results (\%) }\end{array}$ \\
\hline 2008 & 257850 & 15774 & 6,1 & 10996 & 4,3 & 69,7 \\
\hline 2009 & 280862 & 16079 & 5,7 & 11522 & 4,1 & 71,6 \\
\hline 2010 & 271922 & 14670 & 5,4 & 10410 & 3,8 & 70,9 \\
\hline 2011 & 276468 & 13062 & 4,7 & 9973 & 3,6 & 76,3 \\
\hline 2012 & 302163 & 12558 & 4,2 & 9437 & 3,1 & 75,1 \\
\hline 2013 & 303561 & 11264 & 3,7 & 8294 & 2,9 & 73,6 \\
\hline 2014 & 312510 & 11777 & 3,8 & 8308 & 2,6 & 70,5 \\
\hline
\end{tabular}

Table 2. NAT-test results in blood samples during the period from 2012 to 2014 .

\begin{tabular}{|l|c|c|}
\hline $\begin{array}{l}\text { Indicator/ } \\
\text { years }\end{array}$ & $\begin{array}{c}\text { Number of } \\
\text { donors examined }\end{array}$ & $\begin{array}{c}\text { Number of NAT-positive } \\
\text { samples }\end{array}$ \\
\hline 2012 & 233499 & 185 \\
\hline 2013 & 284799 & 201 \\
\hline 2014 & 301319 & 267 \\
\hline Total & $\mathbf{8 1 9 6 1 7}$ & $\mathbf{6 5 3}$ \\
\hline
\end{tabular}

P-111

PERFORMANCE AND CLINICAL USEFULLNESS OF NANOPOROUS SOL-GEL BASED MICROARRAYS AS SCREENING TESTS OF BLOOD DONORS FOR PREVENTION OF TRANSFUSION-TRANSMITTED INFECTION WITH HBV, HCV AND HIV

\section{JY Kim}

Korea University Medical Center, Seoul, South-Korea

Background: Risk of transfusion-transmitted infections persists despite screening blood donations with advanced technologies and improved donor screening. HBV HCV and HIV have proved major risk factors for transfusion transmitted infection. Despite screening tests for these viruses, major risks of donations were given in the infectious window period. Microarrays enable high throughput screening of diseaserelated molecules, including important signaling proteins and small molecules in low quantity. The sol-gel based microarray technology meets high sensitivity, selectivity, and low signal to noise ratio.

Aims: We evaluated nanoporous sol-gel based protein microarrays as screening tests of blood donors for prevention of transfusion-transmitted infection with HBV, HCV and HIV.

Methods: Blood samples were collected at the Korea University Guro Hospital between May 2009 and October 2010. All samples were analyzed by sol-gel based microarray compared to commercially available chemiluminescent microparticle immunoassays such as Architect HBs Ag, Architect anti-HCV immunoassay and 
Architect HIV Ag/Ab Combo (Abbott Laboratories, Abbott Park, IL, USA). There were $419 \mathrm{HBs}$ Ag positive samples, $102 \mathrm{HIV}$ Ab positive samples, $431 \mathrm{HCV}$ Ab positive samples and 4048 all negative samples.

Results: Compared to Architect HBs Ag, Architect anti-HCV immunoassay and Architect HIV Ag/Ab Combo, sensitivities of sol-gel based microarray for detection of HBV, HCV and HIV were 99.76\%, 100\% and 100\%, respectively. The specificities of sol-gel based microarray for detection of HBV, HCV and HIV were 99.88\%, 99.77\% and 99.96, respectively. There is no cross reactivity between HIV and HCV. Conclusions: We concluded novel sol-gel based microarrays were able to utilize as a diagnostic tool for blood bank screening. We suggest that this technology will significantly advance sensitivity and specificity for prevention of transfusion-transmitted infection in high-throughput blood bank screening field.

\section{P-112 \\ CURRENT STATE OF NUCLEIC ACID AMPLIFICATION TESTING INCONCLUSIVE DONORS WHO ARE REACTIVE AT DISCRIMINATORY HEPATITIS B VIRUS NUCLEIC ACID AMPLIFICATION TESTING IN THE LATER DONATION IN KOREA}

HJ Jo, KE Jeong, SR Choi, YS Choi and JN Kim

Korea Centers for Disease Control and prevention, Chungcheongbuk-do Cheongju-si, South-Korea

Backgrounds: In Korea, in 2012, the nucleic acid amplification testing (NAT) system for blood donor screening tests was changed from minipool NAT (MP-NAT) which had been adopted in 2005 for detecting HCV RNA and HIV RNA to individual donation NAT (ID-NAT) which can also detect hepatitis B virus (HBV) as well as HCV and HIV.

Before 2012, for the donor screening test of HBV, only chemiluminescent immunoassay (ChLIA) targeting hepatitis B surface antigen (HBsAg) had been used. It contributes to advance of blood safety in Korea that ID-NAT for HBV DNA was added as required donor screening test since 2012 .

Aims: Analyzing the results of ID-NAT operation since 2012, the NAT inconclusive cases, which multiplex assay for HBV, HCV and HIV was reactive while each discriminatory assay for HBV, HCV and HIV was nonreactive, were reported with the annual average of 1580 cases. Recently, some studies have reported that some of these donors were converted to be reactive at discriminatory assay for HBV (dHBVNAT) in the later donation. Therefore, this study investigated the current state of the cases of NAT inconclusive in Korea.

Method: From the adopt time of ID-NAT detecting HBV, HCV and HIV in 2012 until June 30th in 2015, the data about the donors who had been NAT inconclusive and have become dHBV-NAT reactive in later donation from blood centers (Korean red cross, Hanmaeum, Chung-Ang Univ. medical center) were reviewed and analyzed. The system for ID-NAT was Procleix TIGRIS System (Grifols, USA) and Procleix Ultrio plus assay (Grifols, USA).

Results: From the adopt time of ID-NAT detecting HBV, HCV and HIV in 2012 until June 30th in 2015, there were 4741 cases that were NAT inconclusive and nonreactive at donor screening HBsAg assay. Among them, 384 donors (8.1\%) were dHBVNAT reactive in the later donation. 77 of these donors had blood donation history during the period between their past donation with NAT inconclusive and their last donation which dHBV-NAT was reactive. The blood components from 56 donors among them were used for transfusion, since for these cases, both of multiplex NAT and discriminatory NAT had been nonreactive during the period between their past NAT inconclusive case and their last donation which dHBV-NAT was reactive. Followed up the archived samples of these blood components, 18 samples were dHBVNAT reactive. However, performed the look-back process, there has been no transfusion-transmitted infections from these cases.

Conclusion: In Korea, while the blood components from NAT inconclusive cases are still discarded, these donors are not registered in Donor Deferral Registry (DDR) unlike the cases with reactive discriminatory NAT result. In this study, it has been confirmed that some donors of NAT inconclusive cases might be reactive for dHBVNAT in the later donation. Some studies have reported that some of the donors of NAT inconclusive have low HBV DNA viral load and high anti-HBc prevalence. Therefore, for the donors of NAT inconclusive, the further management should be considered, for instance, registering the donors who are reactive at anti-HBc test among NAT inconclusive cases in the DDR for HBV.
$\mathrm{P}-113$

USEFULNESS OF SIGNAL TO CUTOFF RATIO OF HEPATITIS C VIRUS ANTIBODY ASSAY IN PREDICTING HEPATITIS C VIRUS INFECTION AMONG BLOOD DONORS

SC $\mathrm{Ng}^{1}$, MH Chen ${ }^{2}$ and Y.S. Hung ${ }^{2}$

${ }^{1}$ Taipei Blood Center, Taiwan Blood Service Foundation, Taipei, Taiwan ${ }^{2}$ Taipei Blood Center, Taiwan Blood Services Foundation, Taipei, Taiwan

Background: Enzyme immunoassays (EIAs) are commonly used serological screening tests for hepatitis $\mathrm{C}$ virus (HCV) antibody detection in blood centers. EIA detects the presence of HCV antibody based on the signal to cutoff $(\mathrm{S} / \mathrm{CO})$ ratios. The diagnosis of HCV infection can be confirmed by either Western blot (WB) assay or nucleic acid test (NAT).

Aims: This study aimed to assess relationship between S/CO ratios and HCV confirmatory testing results to identify true HCV infection.

Methods: A total of 903 anti-HCV positive blood donor samples were included in this study. Diasorin Murex EIA was performed to screen for anti-HCV, and Grifols Procleix Ultrio Plus assay was used to detect HCV RNA. Donor samples that were positive for HCV antibody, but negative for HCV RNA were subjected to MP Diagnostics WB assay. The positive HCV RNA results were assumed to be WB positive. The positive results confirmed by either HCV RNA or WB were interpreted as true anti-HCV positive in this study. Receiver operating characteristic (ROC) curve was analyzed to determine the optimal S/CO ratio to predict HCV infection.

Results: The true anti-HCV positivity obtained in this study was $34.4 \%$ (311/903). Among 311 true positive samples, 218 (70.1\%) were confirmed as HCV viremic subjects. The S/CO ratio ranged from 1.01 to 8.20 . The true positive and viremic subjects had a higher S/CO ratio than the false positive and non-viremic subjects respectively $(\mathrm{P}<0.001)$. By ROC curve analysis, the EIA results with $\mathrm{S} / \mathrm{CO}$ ratio $\geq 4.24$ well predict HCV infection, giving the positive predictive value of $96.0 \%$ compared with WB results, and 77.7\% compared with NAT results.

Conclusions: This study showed that anti-HCV S/CO ratio is a useful indicator in predicting true anti-HCV results. In cases with $\mathrm{S} / \mathrm{CO}$ ratio $\geq 4.24$, the WB results will be positive in most samples, so WB assay is not recommended, but NAT should be performed to determine HCV viremia status. To confirm HCV exposure, samples with an S/CO ratio between 1 and 4.24 should be tested with WB assay, and those with positive WB results should be futher tested by NAT.

\section{$\mathrm{P}-114$}

\section{COMPARISON OF TWO ALGORITHMS TO CONFIRM AND DISCRIMINATE SAMPLES INITIALLY REACTIVE FOR NUCLEIC ACID AMPLIFICATION TESTS}

AK Tiwari, RC Dara, D Arora, G. Singh, G Aggarwal and DP Acharya

Medanta- The Medicity, Gurgaon, India

Background: Blood centers in India have published Nucleic Acid Testing (ID-NAT) data based on algorithm (Algorithm A) where serologically negative, NAT reactive sample was subsequently tested with discriminatory NAT (d-NAT) and on the basis of d-NAT initial reactive samples were classified 'NAT yield' or inconclusive. We followed Algorithm B based on replicate testing and Ultrio Plus assay and compared the results with Algorithm A

Aim: We aimed to analyze the effect of this change and compared it with the earlier algorithm (algorithm A) in blood donors.

Material and methods: Results of NAT using two algorithms were analysed. Concordant positives and negatives, seroyield and NAT yield were compared.

Table 1: Nucleic-Acid Amplification Tests (NAT) test results comparison of two algorithms.

\begin{tabular}{|l|l|l|l|l|l|l|}
\hline & $\begin{array}{l}\text { Total } \\
\text { samples } \\
\text { tested }\end{array}$ & $\begin{array}{l}\text { Serology } \\
\text { and NAT } \\
\text { Reactive }\end{array}$ & NAT-IR & DY & Inconclusive & NDY \\
\hline Total & 88583 & 698 & 229 & 25 & 200 & 4 \\
\hline Algorithm A & 31844 & 252 & 147 & 7 & 140 & NA \\
\hline Algorithm B & 56739 & 446 & 82 & 18 & 60 & 4 \\
\hline P value & NA & 0.001 & 0.001 & 0.002 & 0.001 & NA \\
\hline
\end{tabular}


Results: 88,583 (31,844 with algorithm A and 56,739 with algorithm B) samples were tested. Among 1037 reactive donations, number of concordant samples -252 with algorithm A went up to 446 with algorithm $B(P=0.0001)$. Among serology non-reactive donations, NAT inconclusive results came down from 95.2\% in algorithm $\mathrm{A}$ to $73.1 \%$ in algorithm $\mathrm{B}(\mathrm{P}=0.0001)$. Discriminated yield (DY) rate went up from $4.7 \%$ in algorithm $A$ to $21.9 \%$ in algorithm $B(P=0.001)$. Conclusion

Study data suggests replicate testing strategy and Ultrio Plus enhances the concordance between serology and NAT results, increases DY and eliminates unnecessary discriminatory tests.

\section{P-115}

\section{ABILITY OF HCV ANTIGEN AND ANTIBODY SCREENING ASSAY TO IMPROVE BLOOD SAFETY \\ P.R. Samsur}

Jakarta BTS, Jakarta, Indonesia

Background: Blood donor's screening for anti-HCV may reduce the risk of transfusion transmission of hepatitis C virus (HCV) infection. To make blood safe for transfusion, the Chemiluminescen Immuno Assay (CMIA) anti-HCV and individual multiplex Nucleic Acid Testing (NAT) has been run in Jakarta Blood Centre. However, the use of NAT results in increased cost of blood. Recently, a combo HCV Enzyme Immuno Assay (EIA) that is able to detect HCV antibody and or antigen has been developed to increase the safety of blood with less cost than using individual NAT because the HCV antigen can be detected earlier than HCV antibody. Currently, Indonesia has no evidence on its effectiveness and efficiency in detecting HCV infected blood compared to individual HCV NAT

Aims: To compare the effectiveness and efficiency of combo HCV EIA assay with CMIA anti-HCV using individual HCV NAT assays as a gold standard.

Methods: 35 HCV NAT positive samples and 100 HCV NAT negative samples by Procleix Ultrio assay were evaluated for detection of HCV antibody and antigen using CMIA anti-HCV Architect and Combo HCV EIA Biorad assays. Samples with HCV NAT negative but repeated reactive by CMIA anti-HCV and/or Combo HCV EIA assays will be tested by HCV Blot 3.0 MP Diagnostics to confirm the existence of HCV antibody.

Results: The CMIA anti-HCV assay showed reactive results on all HCV NAT positive samples, but only positive on 11 of 100 HCV NAT negative samples. From this result the sensitivity and specificity of the CMIA anti-HCV assay was calculated to be $100 \%$ and $89 \%$ respectively with $76 \%$ of positive and $100 \%$ of negative predictive value. Meanwhile, the Combo HCV EIA assay showed reactive results on all HCV NAT positive samples, but only positive on 3 of 100 HCV NAT negative samples. This result lead to the calculation of sensitivity and specificity of the Combo HCV EIA assay to be $100 \%$ and $97 \%$ respectively with $92 \%$ of positive and $100 \%$ of negative predictive value. Moreover, the HCV Blot testing on 11 samples with anti-HCV positive resulted in positive, weak positive, indeterminate and negative on four, two, one and four samples respectively.

Conclusion: Using the individual HCV NAT as a gold standard, the Combo HCV EIA assay was more effective and efficient than the CMIA anti-HCV assay.

\section{$\mathrm{P}-116$}

\section{COMPARISON OF FOUR COMMERCIAL SCREENING ASSAYS FOR DIAGNOSIS OF HEPATITIS C}

M Moghtadaei, S. prof Amini Kafi Abad, A Soleimani Ferizhandy, S Raman, N Amirhakimi, H Gholizadeh and M Abedini

'Iranian Blood Transfusion Organization Research Center, Tehran, Iran

Background: Highly sensitive hepatitis C (HCV) antibody screening assays are needed to efficiently avoid transfusion-related HCV infections associated with blood products. Also a high level specificity is required to reduce time and cost expenses .It is very important to avoid unnecessary rejection of blood donors due to falsepositive results.

Aims: This study has been designed to evaluate the performance of four different commercially available screening ELISA kits; Murex, anti_HCV (version 4.0) Diasorin and Hepanostika HCV ultra (Beijing Biomedical Co), EIAgen HCV Ab V.4 (ADALTIS), and Enzygnost Anti-HCV 4.0 (SEIMENSE) for their performance in diagnosis of Hepatitis C infection.

Methods: This study was performed at QC department of IBTO headquarter. The kits were evaluated with blood donor specimens; 2005 samples in 2013 \& 2014 .The repeatedly reactive results with ELISA kit were analyzed by HCV RIBA for confirmation. Specificity of screening kits was calculated. The relative sensitivity of kits was

\section{(C) 2015 The Author}

Vox Sanguinis (C) 2015 International Society of Blood Transfusion

Vox Sanguinis (2015) 109 (Suppl. 2), 1-96 calculated based on WHO recommendations by BBI panels produced by Boston Biomedica Inc (BBI) for evaluation of quality kits. Also 100 true positive samples were used to evaluate of these kit

Results: Initial \&t final specificity was $99.92 \%$ \&t $100 \%$ respectively for Murex, anti_HCV (version 4.0) Diasorin kit. Initial \& final specificity were 99.92\% \& 99.96\% respectively for Hepanostika HCV ultra (Beijing Biomedical Co) kit; Initial \& fina specificity for EIAgen HCV Ab V.4 (ADALTIS) was 99.8\% \& $99.85 \%$ respectively and Initial \& final specificity was $99.8 \%$ \&t $99.95 \%$ respectively for Enzygnost Anti-HCV 4.0 (SEIMENSE). All of four kits had accepted results with seroconversion panel including PHV 906, PHV908, PHV910, PHV908, PHV914, PHV916, PHV919, and PHV920, performance panels ((PHV105, PHV106, PHV205 and WWHV301).Also these kits were able to detect HCV in 100 true positive samples

Conclusion: To support the continuous safe supply of blood units and to avoid the waste of precious blood units, the assay with high sensitivity and specificity is necessary to be used. These results demonstrated that four of kits are acceptable and can be used for screening of blood donors for HCV.

\section{P-117}

This abstract has been withdrawn.

\section{P-118}

THE PREVALENCE OF TRANSFUSION-TRANSMITTED

INFECTION AMONG BLOOD DONORS IN THE LOWER NORTHEASTERN REGION OF THAILAND FROM 2005 TO 2014

S Sukpong $^{1}$, S Pheakkhuntod ${ }^{1}$, T Khanongmak ${ }^{1}$, T Sakuldamrongpanich ${ }^{2}$ and S.A. Phikulsod $^{2}$

${ }^{1}$ Nakhon Ratchasima Regional Blood Center V, Thai Red Cross Society, Nakhon Ratchasima, Thailand ${ }^{2}$ National Blood Center, Thai Red Cross Society, Bangkok, Thailand

Background: The most seriously encountered complication of blood transfusion is transfusion-transmitted infection (TTIs). For safety blood transfusions, routine blood donations have been screened and tested for transfusion-transmitted infections. The results covered sero-prevalence rates of the Lower Northeastern region of Thailand from 4 provinces including Nakhon Ratchasima, Surin, Buriram and Chaiyuphum. Objectives: To assess the seroprevalence of markers of TTIs which are syphilis, hepatitis B surface antigen (HBsAg), hepatitis C virus antibody (anti-HCV) and human immune deficiency virus antigen/antibody (HIVAg/Ab) in 10 years and to define data of this region, retrospectively.

Methods: A total of 825,643 blood donors, aged between 17-60 years, applied to Nakhon Ratchasima Regional Blood Center V, Thai Red Cross Society, Thailand from January 2005 to December 2014, were screened and tested for the seroprevalence of markers of TTIs.

Table 1: The prevalence of transfusion-transmitted infection among blood donors in the Nakhon Ratchasima Regional Blood Center V, Thai Red Cross Society.

Table 1: The prevalence of transfusion-transmitted infection among blood donors in the Nakhon Ratchasima Regional Blood Center V, Thai Red Cross Society, Thailand from 2005 to 2014

\begin{tabular}{|c|c|c|c|c|c|c|c|c|c|c|c|}
\hline \multirow{2}{*}{$\begin{array}{l}\text { years/Inferction } \\
\text { markers }\end{array}$} & \multirow{2}{*}{ donor } & \multicolumn{2}{|c|}{ Syphilis } & \multicolumn{2}{|c|}{ HBsAg } & \multicolumn{2}{|c|}{ Anti-HCV } & \multicolumn{2}{|c|}{$\mathrm{HIV} \mathrm{Ag} / \mathrm{Ab}$} & \multicolumn{2}{|c|}{ Total } \\
\hline & & Positive & $\%$ & Positive & $\%$ & Positive & $\%$ & Positive & $\%$ & Positive & $\%$ \\
\hline 2005 & 59,480 & 474 & 0.80 & 1,300 & 2.19 & 348 & 0.59 & 94 & 0.16 & 2,216 & 3.73 \\
\hline 2006 & 63,981 & 649 & 1.01 & 1,111 & 2.21 & 329 & 0.51 & 85 & 0.13 & 2,174 & 3.87 \\
\hline 2007 & 70,893 & 707 & 1.00 & 1,698 & 2.40 & 359 & 0.51 & 110 & 0.16 & 2,874 & 4.05 \\
\hline 2008 & 70.438 & 560 & 0.80 & 1,609 & 2.28 & 338 & 0.48 & 108 & 0.15 & 2,615 & 3.71 \\
\hline 2009 & 81.147 & 993 & 1.23 & 1.524 & 1.88 & 353 & 0.44 & 220 & 0.27 & 3,092 & 3.81 \\
\hline 2010 & 79,007 & 482 & 0.61 & 1,278 & 1.62 & 288 & 0.36 & 69 & 0.09 & 2,117 & 2.68 \\
\hline 2011 & 79,987 & 381 & 0.48 & 1,153 & 1.44 & 236 & 0.30 & 67 & 0.08 & 1,837 & 2.30 \\
\hline 2012 & 90,116 & 291 & 0.32 & 794 & 0.88 & 188 & 0.21 & 73 & 0.08 & 1,346 & 1.49 \\
\hline 2013 & 92,242 & 326 & 0.35 & 1,054 & 1.14 & 626 & 0.68 & 155 & 0.17 & 2,161 & 2.34 \\
\hline 2014 & 138,352 & 1041 & 0.75 & 1,817 & 1.31 & 1098 & 0.79 & 435 & 0.31 & 4,391 & 3.17 \\
\hline Total & 825,643 & 5,906 & 0.72 & 13,638 & 1.65 & 4,163 & 0.50 & 1,416 & 0.17 & 25,123 & 3.04 \\
\hline
\end{tabular}


Results: From a total of 825,643 blood donors from 2005 to 2014, 25,123 donors $(3.04 \%)$ were infected with at least one pathogen. The most prevalent marker was $\mathrm{HBsAg}(1.65 \%)$, followed by syphilis $(0.72 \%)$, anti-HCV $(0.50 \%)$ and HIVAg/Ab $(0.17 \%)$. HBsAg was significantly different between seroprevalence groups $(\mathrm{P}<0.01)$. Conclusions: The seroprevalence rate of HBsAg was higher than other markers, but it seemed to be reduced in 5 latest years. While anti-HCV and HIVAg/Ab still showed high rate in recent years.

\section{$\mathrm{P}-119$}

\section{COMPARISON OF THE RESULTS OF THE CONFIRMATORY} ASSAY FOR THE ANTI-HTLV POSITIVE DONORS

JW Kang ${ }^{1}$, KW Youn ${ }^{1}, \mathrm{JH}_{\text {Yang }}{ }^{1}, \mathrm{SY}_{\mathrm{Kwon}}{ }^{2}$ and DJ $\mathrm{Oh}^{1}$

${ }^{1}$ Korean Red Cross/Blood Transfusion Research Institute, Wonju, South-Korea ${ }^{2}$ Korean Red Cross/Seoul Nambu blood center, Seoul, South-Korea

Background: In Korean Red Cross, anti-HTLV-1/2 screening assay for the blood donations except plasmaphersis has been performed since April 15th 2009. For the anti-HTLV-1/2 positive donors, both Western blot (WB) and Nucleic acid amplification test (NAT) are done as confirmatory assays.

Aims: As a question was raised regarding the efficiency of the current confirmatory assay scheme adopting two kinds of method for anti-HTLV-1/2 positive donors, we suggest a confirmatory assay scheme using one kind of method through the analysis of the results of the confirmatory assay for the past six years.

Methods: We analyzed the results of WB and NAT in the HTLV confirmatory assay from April 15th 2009 to April 14th 2015 using Blood Information Management System of Korean Red Cross.

Results: During the study period, 12,923,854 donations were tested for anti-HTLV1/2. Among them, 3483 donations $(0.027 \%)$ showed positive results in anti-HTLV-1/ 2. 499 donations of the 3483 donations (14.3\%) showed positive results in either WB, NAT or both. The number of positive cases in both tests was 461 ; therefore, the concordance rate in the positive results of the confirmatory assay was 92.4\%. 24 cases and 14 cases showed positive results only in WB and NAT respectively. In the cases of positive results only in NAT, the WB results were all indeterminate (ID). The number of negative cases in both test was 2,337/3,483 donations (67.1\%), and 647/ 3,483 donations (18.6\%) showed ID in WB and negative in NAT.

Summary/Conclusions: With the results of the confirmatory assay for positive cases in the anti-HTLV-1/2 screening assay, it was considered that the accomplishment of WB for the confirmatory assay may be sufficient because the concordance rate in the positive results of the confirmatory assay was shown to be high, and there was no case of simultaneously positive in NAT and negative in WB. However, for the case of WB ID result, it may be better to perform NAT as a supplement test.

\section{$\mathrm{P}-120$}

\section{COMPARISON OF FOUR COMMERICAL SCREENING ASSAYS FOR DIAGNOSIS OF HUMAN T_CELL LYMPHOTROPIC VIRUS TYPES 1AND 2}

S Raman, S Amini Kafi Abad, M Moghtadaei, M Abedini, A Soleimani Ferizhandy, N Amirhakimi and HR Gholizadeh

Iranian Blood Transfusion Organization, Tehran, Iran

Background: Human T lymph cytotropic virus HTLV is a virus from retroviridae family, and more than 20 million people are infected with this virus worldwide .It can cause T_cell leukemia/lymphoma in adults, tropical spastic paraparesis and HTLV associated myelopathy (HAM/TSP). It can be transmitted by blood products and via blood transfusion.

Aims: The aim of this study was comparison sensitivity and specificity of the four ELISA commercial assays; HTLV 1/2 4.0 (MP Diagnosis), Murex HTLV 1/2 (Diasorin), EIAgen HTLV Ab kit (ADALTIS), and HTLV $1 / 2$ Ab (Dia.pro) for HTLV $1 \frac{1}{2}$ detection. Methods: This study was carried out at quality control department of Iranian blood transfusion organization headquarter. The kits were evaluated with 2023 blood donors' specimens in 2013. The repeatedly reactive results with ELISA kits were analyzed by HTLV $1 / 2$ W.Blot as a confirmatory test. The specificity of screening kits was calculated .The relative sensitivity of kits was calculated on WHO recommendation by BBI panels produced by Boston Biomedical Inc.(BBI) for evaluation of quality kits. Also 50 true positive samples were used to evaluation of these kits .Initial and final specificity was 99.95\% \& 99.95\% respectively for EIAgen HTLV Ab kit, 99.75\% At 100\% for Murex HTLV 1/2 (Diasorin) kit, $99.77 \%$ \& $99.88 \%$ for HTLV $1 / 2$ Ab (Dia.pro) kit, 99.5\% \& $99.8 \%$ for HTLV $1 / 24.0$ (MP Diagnostic) kit.All of four kits had same results in PRP 207 performance panel according BBI panel sheets and were able to detect 50 true positive samples.

Conclusion: Regarding importance of blood safety, the assay with high sensitivity and specificity is necessary to be used. These results demonstrated that all of four kits are acceptable and can be used for screening of donors for HTLV.

P-121

\section{PERFORMANCE CHARACTERISTICS OF EIA AND NAT FOR HBV/HCV/HIV AND MAIN RISK OF TTID IN SOUTHERN CHINA $\mathrm{X}$ Rong and H Wang}

Guangzhou Blood Center, Guangzhou, China

Background: Blood safety are one of the most concerned issues. Now the risk of transfusion transmitted infectious disease by transfusion have been greatly reduced by serological screening for ALT, HBV, HCV, HIV and Syphilis in China. However, because of the long window period of EIA reagents, nucleic acid test (NAT) was put into trial use in 15 blood centers from 2010 to improve the transfusion safety. Aims: (i) To compare the performance characteristics of EIA and NAT for HBV/ HCV/HIV screening and evaluate the effectiveness of two methods for blood screening. (ii) To investigate the main risk of TTID in Southern China.

Methods: We test 1,127,189 blood donations from 2011-2014. Grifols Procleix TIGRIS automatic nucleic acid test and analysis system and PROCLEIX ULTRIO assay were performed to jointly detect HBV, HCV and HIV-1. Moreove, identification test was performed to identify HBV/HCV/HIV nucleic acid positive samples. Meanwhile, HBsAg, anti-HCV, anti-HIV were test by serological reagents of all donations.

Results: (i) Of 1,127,189 blood donations, 18,833 (1.67\%) were EIA positive and $10,761(0.93 \%)$ were NAT positive. There was a significant difference between EIA positive and NAT positive $(\mathrm{P}<0.01$ ). (ii) Of 18,833 EIA positive blood donations, $8153(48.29 \%)$ were NAT positive and 10,680 (56.71\%) were NAT negative. For EIA negative samples, $0.24 \% \quad(2,608 / 1,108,356)$ were NAT positive and $99.76 \%$ $(1,105,748 / 1,108,356)$ were NAT negative. Of 2608 NAT+/EIA- blood donations, 782 (29.98\%) were positive by identification nucleic acid test, including $777 \mathrm{HBV}$, 3HCV and 2 HIV.

Conclusions: (i) EIA and NAT are complementary blood screening methods while NAT are more advanced and sensitive which will help to shorten the window period of blood transfusion infection and further improve blood safety. (ii) The present risk of transfusion transmitted infectious disease mainly was HBV (Most OBI and little WP) infection.

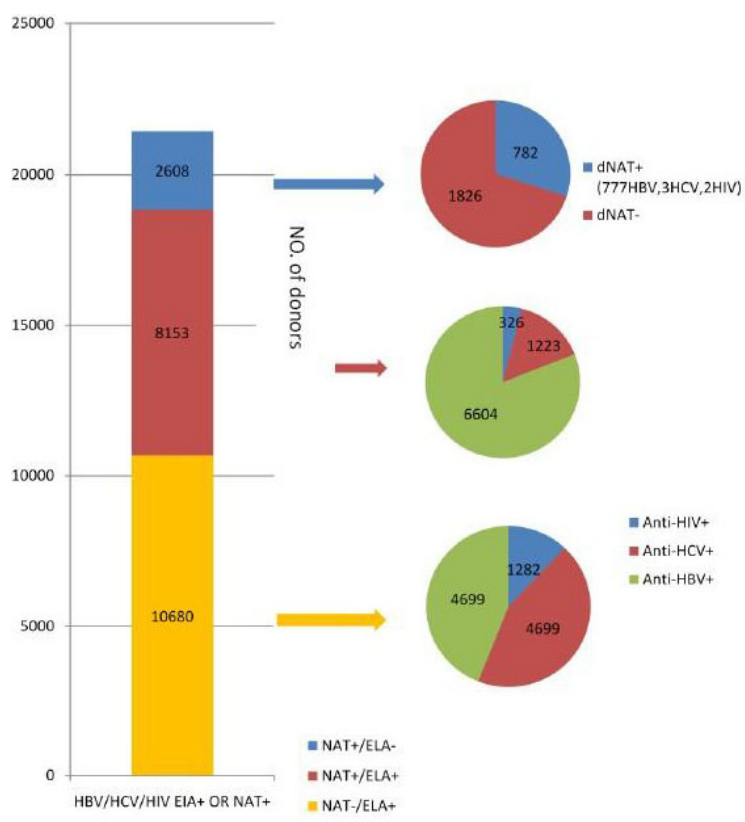

Figure 1 Composition analysis of HBV/HCV/HIV EIA positive or NAT positive

Figure 1: HBV/HCV/HIV positive rate of EIA and NAT from 2011-2014.

(C) 2015 The Author

Vox Sanguinis (C) 2015 International Society of Blood Transfusion Vox Sanguinis (2015) 109 (Suppl. 2), 1-96 
Table $1 \mathrm{HBV} / \mathrm{HCV} / \mathrm{HIV}$ positive rate of EIA and NAT from 2011-2014

\begin{tabular}{ccccc}
\hline HBV/HCV/HIV & Positive & Negative & Total & $\chi^{2}(P)$ \\
\hline EIA & 18833 & 1108356 & 1127189 & 2230.989 \\
& $(1.67 \%)$ & $(98.33 \%)$ & $(100.00 \%)$ & \\
\multirow{2}{*}{ NAT } & 10761 & 1116428 & 1127189 & P<0.01 \\
& $(0.93 \%)$ & $(99.07 \%)$ & $(100.00 \%)$ & \\
\hline
\end{tabular}

Table 2: Composition analysis of HBV/HCV/HIV EIA positive or NAT positive.

Table 2 comparison of blood screening results of EIA and NAT

\begin{tabular}{cccc}
\multicolumn{5}{c}{ Table 2 comparison of blood screening results of EIA and NAT } \\
& NAT+ & NAT- & Total \\
\hline ElA + & 8153 & 10680 & 18833 \\
& $(43.29 \%)$ & $(56.71 \%)$ & $(100.00 \%)$ \\
ElA- & 2608 & 1105748 & 1108356 \\
& $(0.24 \%)$ & $(99.76 \%)$ & $(100.00 \%)$ \\
Total & 10761 & 1116428 & 1127189
\end{tabular}

P-122

VALIDATION OF IMMUNOASSAYS FOR THE USE WITH

CADAVERIC SPECIMENS FROM TISSUE DONORS

L Pallavicini, A Cucchietti, B Jansson and E Piccin

Diasorin, Saluggia, Italy

Background: Deceased donors are the main source of organs and tissues for transplantation, but this introduces the risk of transmission of donor-derived infections, for the recipients, and thus the need for analyses. Very few CE-marked assays are specifically validated for the use with cadaveric specimens.

Aims: The aim of this study is to provide a mechanism for the qualification of standard serological blood screening LIAISON ${ }^{\circledR}$ assays for markers of infectious diseases, for use with post-mortem blood samples from potential tissue donors.

Methods: The validation study is based on an available international guideline recommended by Paul Ehrlich Institute (PEI), Germany ('Proposal for the validation of anti-HIV-1/2 or HIV Ag/Ab combination assays, anti-HCV assays, HBsAg and anti$\mathrm{HBc}$ assays for use with cadaveric samples', PEI 08/05/2014). LIAISON ${ }^{\circledR}$ immunoassays for the determination of antibodies against CMV, Chagas, Treponema, Hepatitis and Retroviruses are used in the study. Collection and preparation of cadaveric specimens have to strictly follow procedures and limitations, since hemolysis and other changes occur in blood specimens after death, which might lead to false results either positive or negative in serological testing. In order to determine if changes interfere with the assays, accuracy is evaluated by assessing cadaveric samples, unspiked and spiked at 2 different levels of reactivity (low and medium/high positive), compared to same number of human sera from living donors. Specimens are collected within $24 \mathrm{~h}$ after last heartbeat. The same procedure is used for sera from living donors, then tested in parallel as reference. The results are calculated as the ratio between mean of doses for living donors and for post-mortem subjects, at each reactivity level. Accuracy testing is considered successful if the results are comparable within the range of precision and do not differ by more than $25 \%$. Precision is evaluated by testing a cadaveric specimen with reactivity close to the cutoff, in 6 replicates. Precision testing is considered successful if the CV is $<15 \%$.

Results: In the accuracy testing, a ratio of below 5\% between mean of living donors and post-mortem is obtained, for all of the kits at all evaluated reactivity levels. Precision testing shows CV below 6\% for all kits under analysis.

Conclusions: No significant differences are observed when testing cadaveric specimens vs living donors. LIAISON ${ }^{\circledR}$ assays ensure reliable data, showing suitable performance in the determination of specific infectious disease antibodies in serum or plasma cadaveric specimens.

The qualification of testing with cadaveric specimens improves the potential of the LIAISON $^{\circledR}$ fully automated solution for the detection of infectious disease markers including Hepatitis and Retroviruses.

(C) 2015 The Author

Vox Sanguinis (C) 2015 International Society of Blood Transfusion

Vox Sanguinis (2015) 109 (Suppl. 2), 1-96

\subsection{Hepatitis B (HBV)}

P-123 HEPATITIS B VIRUS PREVALENCE IN FIRST-TIME U.S. BLOOD DONORS, 2004-2014

E Murphy $^{1}$ and Z Kaidarova ${ }^{2}$

${ }^{1}$ UCSF/BSRI, San Francisco, United States of America ${ }^{2}$ BSRI, San Francisco, United States of America

Background: Hepatitis B virus infection (HBV) is the most common blood-borne pathogen worldwide and can cause chronic hepatitis, cirrhosis and hepatocellular carcinoma. HBV vaccination has been instituted in all children in the USA during the 1990's, but its effects upon blood safety are unknown. Tracking HBV prevalence in blood donors is also important for identification of high risk groups and may be useful for public health surveillance of vaccination coverage.

Aims: To analyze trends in HBV prevalence among first-time US blood donors over more than a decade.

Methods: This was a cross-sectional seroprevalence study of all non-autologous, first-time blood donors at Blood Systems Incorporated blood centers in calendar years 2004 through 2014. Chronic HBV infection was defined as reactive HBsAg EIA and reactive neutralization. Past exposure to HBV was defined by anti-HBcore seropositivity. Secular trends in HBV prevalence were explored and demographic risk factors were analyzed using multivariable logistic regression.

Results: Among 1,852,188 first time donors who presented during 2004-2014, 1128 $(0.06 \%)$ had HBsAg and 19,844 (1.07\%) had anti-HBcore. There were significant downward trends in both HBsAg and anti-HBcore from 2004 to 2014 ( $\mathrm{p}_{\text {tren }}$ $<0.0001$; see Figure). In multivariable logistic regression, HBsAg was associated with older age (OR $=1.84,95 \%$ CI $1.51-2.24$ for age $60+$ vs age under 20 years), male sex $(\mathrm{OR}=1.14,95 \%$ CI 1.05-1.25), Asian (OR =7.72, 95\% CI 6.87-8.68), Black $(\mathrm{OR}=3.21,95 \%$ CI $2.79-3.69)$ and Hispanic $(\mathrm{OR}=1.29,95 \%$ CI $1.14-1.45)$ race/ethnicity. Anti-HBcore prevalence showed generally similar demographic associations. Conclusions: The prevalences of both chronic and past HBV infection among firs time blood donors in the USA have declined by about half over the past decade, likely due to universal vaccination of newborns and a birth cohort effect as older higher prevalence persons are less likely to become first time donors. Our results confirmed demographic associations between HBV infection and older age and Asian and Black race/ethnicity.
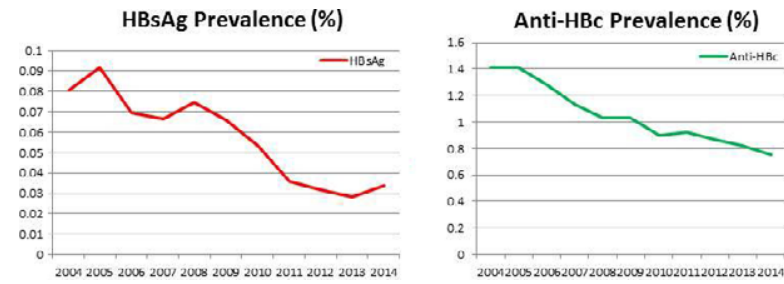

Figure 1: HBV Figures.

$\mathrm{P}-124$

RESULTS OF SCREENING FOR OCCULT HEPATITIS B VIRUS INFECTION AMONG BLOOD DONORS IN MONGOLIA

TD Magvansuren $^{1}$ and $\mathrm{E}$ Namjil ${ }^{2}$

${ }^{1}$ National Center for Transfusion Medicine Mongolia, Ulaanbaatar, Mongolia

${ }^{2}$ National Center for Transfusion Medicine, Ulaanbaatar, Mongolia

Background: Hepatitis B virus (HBV) remains a major public health problem worldwide. Implementation of hepatitis B surface antigen (HBsAg) in routine screening of blood donors in the early 1970s has greatly enhanced transfusion safety. The incidence of transfusion-transmitted hepatitis B has been steadily reduced over the last four decades. However, it was demonstrated that HBV transmission by blood components negative for HBsAg can still occur and HBV transmission remains the most frequent transfusion-transmitted viral infection; thus, the term occult hepatitis B virus infection (OBI) was introduced. OBI is simply defined as serologically undetectable hepatitis B surface antigen (HBsAg-ve), despite the presence of circulating HBV DNA with or without the presence of HBV antibodies

Aims: To determine of occult hepatitis B virus infection among blood donors and evaluate the presence of HBV DNA in HBsAg negative plasma samples.

Materials and Methods: It includes 16,700 samples which donated in NCTM in Ulaanbaatar in 2013. We used to 'triplex' PCR assay that included the detect of hep- 
atitis B virus HBV-DNA in addition HCV-RNA and HIV1/2-RNA for whom with absence of serological markers of infection. The studies used molecular biology methods were performed with the help of equipment (ROCHE COBAS S 201) and technology based on Real Time PCR (pool size: 6 donation) Then we choose HBsAg negative, DNA positive samples and determined, anti-HBc and anti-HBs by serological methods, of ELISA Wantai HBc and HBs 3.0 tests.

Results: The 14,948 samples were detected serological negative in the total of 16,700 samples. PCR test results show $35(0.23 \%)$ positive by HBV-DNA. $29(82.9 \%)$ of the 35 DNA positive blood donors were alone anti-HBc positive and $3(8.6 \%)$ were anti-HBs, anti-HBc positive. 7 (17.1\%) were seronegative. Of the 35 OBI cases, 28 $(80 \%)$ were detected the first time they were screened for HBV DNA while $7(20 \%)$ gave one more HBV PCR-nonreactive results before detection. Callback studies we determined 2 cases were pre-HBsAg window period.

Conclusion: The prevalence of HBV DNA positive in HBsAg negative blood donors is found $0.2 \%$. Its $29(82.9 \%)$ were seropositive, 7 (17.1\%) were seronegative. HBV NAT needs either extreme sensitivity or to be performed on individual donations to eliminate HBV DNA-containing units.

\section{P-125}

\section{ASSESSMENT OF AN HBSAG COMMERCIAL ASSAY WITH A PANEL OF WELL CARACHTERIZED MUTANTS SAMPLES}

RS Tedder, B Haywood and S Ijaz

Blood Borne Virus Unit, Virus Reference Department, Centre for Infections, Health Prot, London, United Kingdom

Background: HBsAg of hepatitis B virus (HBV) detection is crucial both for diagnosis of infection and for screening of blood derivatives and pregnancy. HBV harboring mutations in its sequence may challenge the ability of detection for laboratory assays, leading to false negative HBsAg results, and still today HBV vaccine and diagnostic escape mutants are a growing concern.

Aims: Testing the detection ability of a commercial assay, LIAISON XL murex HBsAg Quant (DiaSorin S.p.A., Italy), using a set of native mutant HBsAg samples. Methods: A panel of 30 plasma samples diluted to between 1 and $10 \mathrm{IU} / \mathrm{ml} \mathrm{HBsAg}$ containing native surface antigen bearing single or multiple mutations in the a determinant based on consensus genotype sequencing, have been used to assess the capability of the DiaSorin HBsAg assay to detect them. Mutation pattern of the predominant HBsAg sequence in samples has been determined by means of sequencing analysis, as well as the genotype.

Phenotyping of HBsAg has been undertaken with a set of four mAbs (P2D3 aa 121129; D2H5 aa 143-147; HB04 aa 137-147, H3F5 aa 131-142). All samples were previously detected by the Murex HBsAg Version 3, GE34/36, ELISA assay.

LIAISON XL murex HBsAg Quant is a chemiluminescent immunoassay for the quantitative determination of HBV surface antigen. The test uses monoclonal antibodies directed against linear epitopes of the external and highly conserved internal and transmembrane regions of HBsAg with a balanced reactivity against subtypes ad and ay. All testing have been performed according to the manufacturing guidelines.

Results: All samples detected as positive by the LIAISON XL murex HBsAg Quant, with a range of values between $0.050 \mathrm{mIU} / \mathrm{ml}$ and $3.90 \mathrm{mIU} / \mathrm{ml}$.

Summary/Conclusions: Occurrence of false negative HBsAg results could impact diagnostic and blood safety especially in HBV high prevalence countries where immunization programmes may facilitate the evolution of escape variants. For the blood donor screening single mutations are the most frequently described. It is therefore important to assess sensitivity performance of commercially available assays, with the use of well characterized samples covering both the more frequent substitutions and also the ones having more impact in HBsAg detection. LIAISON XL murex HBsAg Quant has been able to detect the range of variants examined.

\section{P-126}

\section{COMPARISON STUDY WITH ENZYME IMMUNOASSAY AND CHEMILUMINESCENCE IMMUNOASSAY FOR HEPATITIS B VIRUS SURFACE ANTIGEN DETECTION}

A Soleimany ferizhandy, S Amini Kafi-Abad, M Moghtadaei, H Gholizadeh, M Abedini, S Raman and N Amirhakimi

Iranian Blood Transfusion Research Center, High Institute for Research and Educa, Tehran, Iran

Background: The serological detection of the surface antigen (HBsAg) of hepatitis B virus (HBV) is the basis of detection of HBV infections in blood donors and patients with hepatitis. The aim of this study was to compare the performance of $\mathrm{HBsAg}$ enzyme-linked immunosorbent assay (ELISA) and HBsAg chemiluminescence immunoassay evaluated in Iran.

Methods: We compared Two assays: HBsAg Qualitative, Architect i2000 (Abbott Laboratories, USA), Enzygnost HBsAg (seimens 6.0, Germany). We evaluated the sensitivity and specificity of each assay by testing HBsAg, four commercial seroconversion panels BBI (Boston Biomedica Inc), PHM933, PHM912, PHM935A, PHM908, and one sensitivity panel BBI (PHA808). For specificity, randomly selected serum samples were collected from volunteer blood donors at 5 blood-donation centers in Iran. The assigned values over the 4 seroconversion series were averaged to determine a mean relative seroconversion sensitivity index for each assay and the 95\% confidence limits were determined.

Results: The lowest HBsAg level detected by each assay using reference material (BBI panel) was variable from 0.02 to $0.67 \mathrm{IU} / \mathrm{ml}$ (for sub type; ad, ay). When testing 21 seroconversion panels' members, the total number of positive samples was 20 and 22 by Enzygnost HBsAg seimens 6.0 and HBsAg Qualitative, Architect i2000 samples tested. Good correlation $\rho=0.93(\mathrm{P}<0.001)$, was found between the Architect i2000, seimens 6.0 assays with narrow 95\% limits of agreement. The specificity of HBsAg tests was found to be $99.95 \%$ and $99.91 \%$ for HBsAg Qualitative, Architect i2000 and by Enzygnost HBsAg seimens 6.0 methods, respectively. The HBsAg Qualitative, Architect i2000 assays showed a higher sensitivity for subtype ad and ay for the BBI sensitivity panel 808 than did the Enzygnost HBsAg seimens 6.0 assays. Three samples with low HBsAg concentrations (0.01 to $0.05 \mathrm{IU} / \mathrm{ml})$ tested negative by the Enzygnost HBsAg seimens 6.0 assays.

Conclusions: The ARCHITECT HBsAg Qualitative is designed to show sensitivity better than a commercially available HBsAg assay. The ARCHITECT HBsAg Qualitative is designed to show seroconversion better than a commercially available $\mathrm{HBs} \mathrm{Ag}$ assay.Analytical sensitivity detecting ability among $\mathrm{HBsAg}$ assays were variable. We suggest that each laboratory should select an HBsAg assay based on analytical performance, test throughput, and the applicability of full automation. The presented data show that the new ARCHITECT HBsAg Qualitative screening assay exhibits a low initial reactive rate, while displaying sensitivity and a specificity than

\section{$\mathrm{P}-127$}

This abstract has been withdrawn.

\subsection{HIV}

$\mathrm{P}-128$ EFFECT OF THE SAMPLES SENSITIVITY ANTI-HIV SCREENING

F Farida Faridatin Nasriyah

Blood Transfution Centre Tangerang City Red Cross, Tangerang, Indonesia

Background: For standard blood tests healthy and safe Ministry of Health issued a policy that the examination was screened using a 4 parameters, one of which is HIV testing. HIV (Human Imunodeviciency Virus) is a virus that causes the immune seseoran declined and over time the virus will be AIDS, the emergence of a set of diseases caused by the human immunodeficiency. For screening examination Tangerang City Blood Centre using screening tools ARCHITECT with CMIA method and with a sensitivity which reached $99.5 \%$.

Aim: To know the results of the sample of screenings $3000 \mathrm{rpm}$ and $5000 \mathrm{rpm}$ 2 min 5 min to the inspection of Anti-HIV screening test.

Methods: This method uses 10 samples taken from volunteer blood donors with no. Different bags that have been through the handover of samples from quarantine lab to lab screening. Sample in a centrifuge rotating at $5000 \mathrm{rpm}$ with a 5-min playback and in red blood cell sedimentation get perfect, the samples in running the instrument and reagents ARCHITECT and see the results, stelah playback results carried out on the same sample in different tubes with round $3000 \mathrm{rpm} 2$ min. Results of the first round and the second round later in the compare and concluded.

Result: Examination of Anti-HIV screening of 10 samples with no. Different pockets and with different playback is $5000 \mathrm{rpm}$ to $3000 \mathrm{rpm} 5 \mathrm{~min} 2 \mathrm{~min} 1$ sample found to Contain samples infected with HIV as a result of screening samples at $3000 \mathrm{rpm} 2 \mathrm{~min}$. Conclusions: There is a 10\% change in sample playback results from $3000 \mathrm{rpm}$ to $5000 \mathrm{rpm} 2 \mathrm{~min} 5 \mathrm{~min}$ with different results. Evidenced by the sample No. 634 I2006A bag with a screening of $3000 \mathrm{rpm} 2 \mathrm{~min}$ at OD 1.36 can result REACTIVE against Anti-HIV, and from the same sample with the screening of $5000 \mathrm{rpm}$ 5 min there is a change results OD 0.13 NON-REACTIVE terhadapAnti HIV. Changes in the results that occur in the sample playback speed of $3000 \mathrm{rpm} 2 \mathrm{~min}$ is likely to occur as a result of deposition of cells is less than perfect. It can be concluded that the use of screening samples for the screening of IMLTD would be better if you use the playback speed of $5000 \mathrm{rpm}$ and a $5 \mathrm{~min}$

(C) 2015 The Author

Vox Sanguinis (C) 2015 International Society of Blood Transfusion Vox Sanguinis (2015) 109 (Suppl. 2), 1-96 


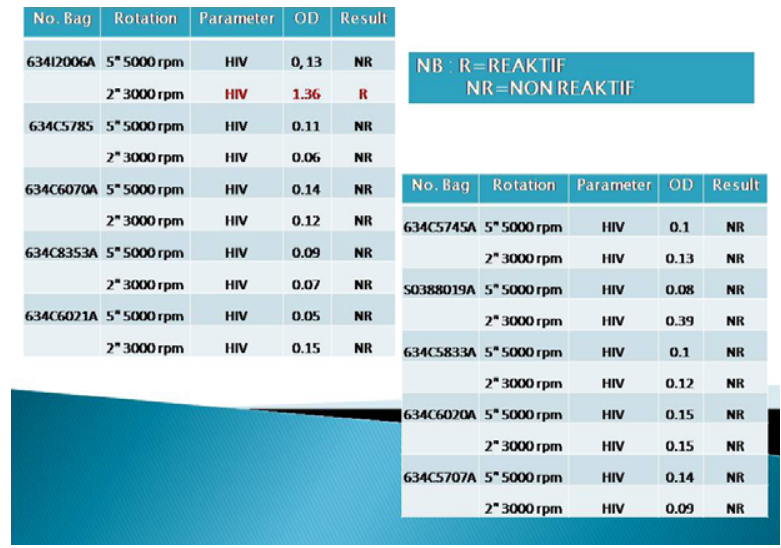

\subsection{Bacteria}

P-129

EVALUATION OF THE SENSITIVITY AND SPECIFICITY OF USE OF GLUCOSE AND PH FOR BACTERIAL SCREENING OF PLATELET CONCENTRATES COMPARED TO THE BACT/ALERT

F Razjou, A Dabir Mogaddam and H Timori Naghadeh

Iranian Blood Transfusion Research Center, High institute for Research and Education, Tehran, Iran

Background and Objectives: Bacterial contamination of blood component is the major infectious risk in transfusion medicine.Since platelets should be stored at room temperature that makes them an excellent growth medium for bacteria; it is mentioned as a major problem in transfusion medicine. Regarding that the transfusion risk of a bacterial contaminated platelet concentrate is 100 to 1000 time highe than viral pathogen such as HIV, HBV, HCV and HTLV. For reducing this risk and developing platelet safety, attempt on detection of contaminating agents affecting platelets during preparation procedure have important role.

Material and methods: 1332 platelet concentrates were screened by Bact/Alert system for aerobic and anaerobic bacterial contamination. Meanwhile bacterial contamination was also evaluated by using urine reagent strips (Multistix10 SG Bayer) and culture methods. PH screening with a pH meter (Metrohm 744 Swiss) was also used for detection of bacterial contamination.

Results: The rate of bacterial contamination detected by Bact/Alert system in platelet concentrates was 25 in $1332(1.9 \%)$. It contained 15 (1.1\%) for aerobic bacteria and $10(0.8 \%)$ for anaerobic bacteria. 226 of 1332 were considered as containing bacteria by using urine reagent strips. Six of the 226 units were also positive by Bact/Alert system. 3 of those units were culture positive for aerobic bacteria and 3 for anaerobic.The result of platelet concentrates that underwent $\mathrm{pH}$ screening by use of $\mathrm{pH}$ meter and $\mathrm{pH}$ portion of urine reagent strips was the same. The sensitivity and specificity of considering glucose alone for detection of bacterial contamination were $12 \%$ and $98 \%$ respectively .For pH alone, these were 24\% and $83 \%$.For glucose and/or $\mathrm{pH}$, these were $24 \%$ and $83 \%$; and for combination of glucose and $\mathrm{pH}$, these were $12 \%$ and $98 \%$.

Conclusion: Although measurement of $\mathrm{pH}$ and glucose are not sufficiently sensitive, in the interim, widespread use of it would improve the safety of blood products and should be encouraged.

\subsection{Parasites}

P-130 SCREENING OF BLOOD DONORS WITH MICROSCOPY, ANTIGEN RAPID TESTS AND ANTIBODY RAPID TESTS, POLYMERASE CHAIN REACTION AGAINST MALARIA PARASITE IN AMBON CITY, INDONESIA

JN Louhenapessy ${ }^{1}$, I Sutanto ${ }^{2}$, REG Syafitri ${ }^{3}$, Y Soedarmono ${ }^{4}$ and S Rahayu ${ }^{5}$ ${ }^{1}$ Hospital, Jakarta, Indonesia ${ }^{2}$ Departement of Parasitology, Faculty of Medicine, University Indonesia, Jakarta, Indonesia ${ }^{3} 2$ Central Blood Transfusion Services, Jakarta, Indonesia ${ }^{4}$ Directorate general of Health Care, Ministry of Health, Jakarta, Indonesia ${ }^{5}$ Integrated Laboratory Faculty of Medicine, University of Indonesia, Jakarta, Indonesia

Backgrounds and objectives: Screening of blood donors in Indonesia against malaria with laboratory tests have not been done. Possible risk of malaria transmission through donated blood may occur and endanger the life of recipients. This study aims to determine the most suitable laboratory test using in an endemic malaria area of Indonesia.

Materials and methods: Study was conducted in Ambon city, Maluku province from 8 March 2013 until 15 Mei 2014. Total of 550 blood sample from donors at Red Cross blood transfusion unit Ambon, was testified with various laboratory examinations: microscopy, rapid test antigen (PfHRP2-aldolase or PfHRP2-LDH) and rapid test antibody. In addition, 248 samples were also assessed for malaria DNA with 18S rRNA marker using polymerase chain reaction technique.

Results: The results showed none positive by microscopy examination and antigen rapid tests neither PfHRP2-aldolase nor PfHRP2-LDH. However, twenty-two samples $(4 \%)$ contained antibody against P. falciparum, and another five samples $(2 \%)$ showed positive reaction by PCR.

Conclusions: This study showed that the potency of malaria transmission by blood donors was $4.9 \%$ in the island of Ambon.

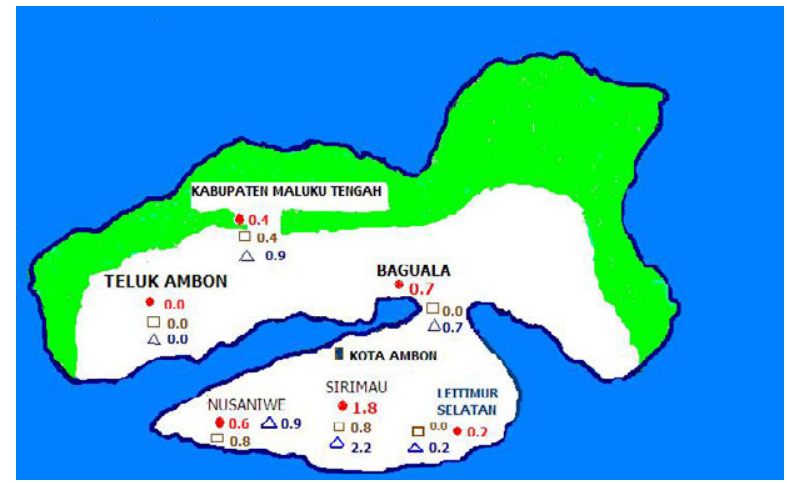




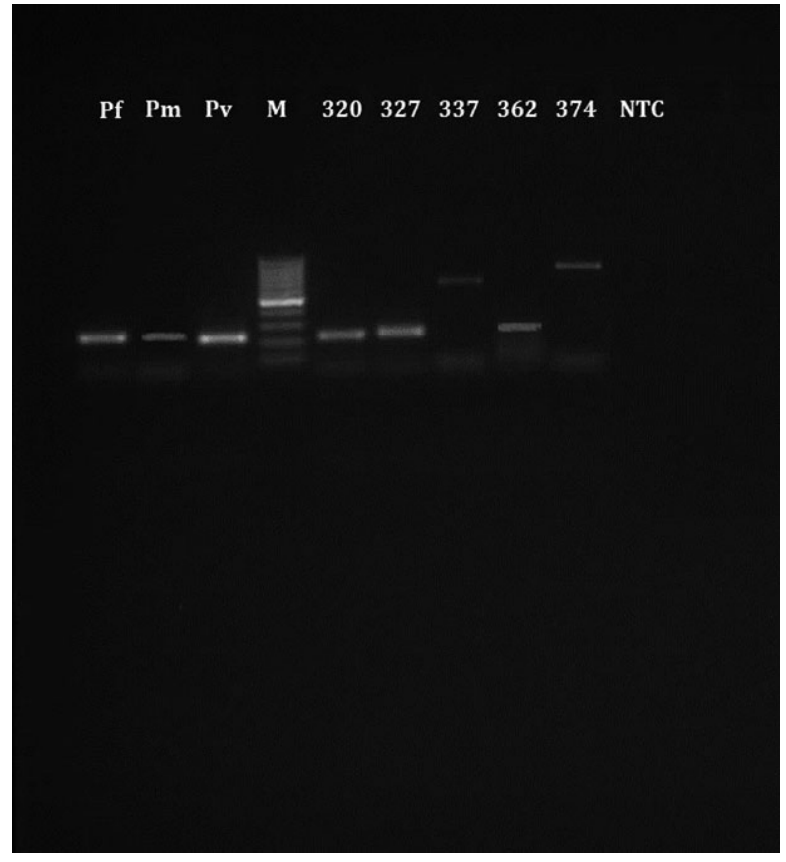

\subsection{Newly Emerging Pathogens and other Transfusion Related Pathogens}

P-131

COBASR CHIKV/DENV INVESTIGATIONAL ASSAY: A NOVEL HIGHLY SENSITIVE AND REPRODUCIBLE ASSAY FOR BLOOD SCREENING ON THE COBAS $®$ 6800/8800 SYSTEM

P Albrecht ${ }^{1}$, A Kuehler ${ }^{1}$, N Dyer ${ }^{2}$, C Roordink ${ }^{1}$, S Sudershana $^{2}$ and J Niemiec ${ }^{2}$ ${ }^{1}$ Roche Diagnostics International Ltd, Rotkreuz, Switzerland ${ }^{2}$ Roche Molecular Systems, Inc., Pleasanton, CA, United States of America

Background: The cobas ${ }^{\circledR}$ Chikungunya/Dengue test (CHIKV/DENV) for use on the cobas $^{(} 6800 / 8800$ Systems is currently in development. This automated real time PCR qualitative blood screening test is designed to both detect and discriminate CHIKV/DENV with high sensitivity and specificity.

Like other infectious diseases for which blood donations are screened, blood donations must be screened with a sensitive assay to detect chikungunya virus (CHIKV) and dengue virus (DENV) RNA so that infected units may be interdicted and discarded. The cobas ${ }^{\circledR}$ CHIKV/DENV investigational assay will offer novel capability to detect RNA from these viruses and thereby provide heightened protection from transfusion-transmitted CHIKV and DENV infection for recipients of donated blood components or products and will further improve the safety of the blood supply. Aims: To develop and evaluate the performance of a novel real-time PCR based CHIKV/DENV investigational test for blood screening utilizing the new cobas ${ }^{\circledR} 6800 /$ 8800 fully automated system.

Methods: Automated, nucleic acid extraction was used for isolation of viral RNA which amplified and detected by automated, real-time PCR. Analytical sensitivity was determined using Roche Secondary Standards of CHIKV/DENV. Clinical specificity was assessed by testing 1000 individual specimens from healthy donors.

Results: The 95\% limit of detection by Probit analysis was 0.18 PDU/ml for CHIKV and $71.8 \mathrm{PDU} / \mathrm{ml}$ for DENV. Clinical specificity was $100 \%$. The test detected genotypes Asia and ECSA for CHIKV and all four serotypes for DENV. No interference was observed in samples with albumin (60 g/l), bilirubin $(0.2 \mathrm{~g} / \mathrm{l})$, hemoglobin $(2 \mathrm{~g} /$ $1)$, triglycerides $(33 \mathrm{~g} / \mathrm{l})$, human DNA $(0.5 \mathrm{mg} / \mathrm{l})$. No cross reactivity was observed with 27 cultured microorganisms at $1.00 \mathrm{E}+06$ particles, copies, or PFU $/ \mathrm{ml}$; including 20 viral isolates, 6 bacterial strains, and 1 yeast isolate.

This study demonstrated consistent performance of the cobas ${ }^{\circledR}$ CHIKV/DENV test across kit lot and instrument-operators on different days at $\sim 0.5 \mathrm{x}$ and $\sim 1 \mathrm{x}$ and $\sim 2 \mathrm{x}$ LoD. Blood collected in EDTA anticoagulant, CPD and PPT could be stored for up to 5 days at $2-8^{\circ} \mathrm{C}$, of which $48 \mathrm{~h}$ may be at $2-25^{\circ} \mathrm{C}$ and $24 \mathrm{~h}$ at $2-30^{\circ} \mathrm{C}$ without affecting the performance of the test. For storage longer than 5 days, plasma was shown to be stored at $<-18^{\circ} \mathrm{C}$ for up to 15 days without affecting performance, including up to three freeze-thaw cycles.

Conclusions: The high specificity and sensitivity and genotype inclusivity of the novel cobas ${ }^{\circledR}$ CHIKV/DENV assay confirm that this assay is appropriate for blood and plasma screening for investigational use.

\section{$\mathrm{P}-132$ \\ THE SEARCH FOR DENGUE VIRUS IN AUSTRALIAN BLOOD DONATIONS DURING LOCAL OUTBREAKS \\ HM Faddy $^{1}$, JJ Fryk ${ }^{1}$, CR Seed ${ }^{2}$, C Hyland ${ }^{1}$, JA Holmberg ${ }^{3}$ and R Flower ${ }^{1}$ \\ ${ }^{1}$ Australian Red Cross Blood Service, Brisbane, Australia ${ }^{2}$ Medical Services, Australian Red Cross Blood Service, Perth, Australia ${ }^{3}$ Grifols, Emeryville, United States of America}

Background: Dengue virus (DENV) poses a risk to the safe transfusion of blood, with confirmed reports of transfusion-transmission from Hong Kong, Singapore, Puerto Rico and Brazil. In Australia, episodic outbreaks occur on a seasonal basis in north eastern Australia. One of the largest epidemics in over 50 years took place in the summer of 2008/2009, with over 1000 confirmed cases. Another sizeable outbreak occurred in the summer of 2012/2013. To manage this risk, fresh components are not manufactured from donors who live in or travel to areas experiencing dengue outbreaks, including countries where dengue is endemic. Dengue outbreaks pose a risk to the safety of Australia's blood supply, which justifies relatively-high cost risk mitigation strategies. An alternative approach for managing this risk could be the use of an appropriate donor screening test.

Aims: The aim of this study was to determine the rate of asymptomatic donor viraemia during local dengue outbreaks by testing plasma samples for the presence of DENV RNA using transcription mediated amplification (TMA).

Methods: Samples $(n=6182)$ from donors in North Queensland 'at-risk' of exposure to dengue were collected, including donations from two separate periods: the 2008/2009 epidemic (donors in areas of Cairns that recorded more than 20 confirmed dengue cases) and the 2012/2013 outbreak (all affected donations). Additional samples were sourced from a control region in southern Australia, where DENV transmission does not occur $(n=1601)$. All samples were tested for DENV RNA by DENV TMA (95\% detection at $<30$ copies/ml for DENV types $1-4$ ) as per the manufacturer's instructions. Exact confidence intervals (CI) were calculated for individual proportions using a standard method.

Results: None of the samples collected during the 2008/2009 epidemic (0\%, 95\% CI: $0-0.55 \%$ ) were positive for DENV RNA, despite them all being collected from 'higher-risk' areas. Likewise, none of the samples collected during the 2012/2013 outbreak (0\%, 95\% CI: 0-0.07\%) were positive for DENV RNA. All of the control samples were also negative for DENV RNA (0\%, 95\% CI: 0-0.23\%).

Summary/Conclusions: None of the donations tested from donors residing in areas experiencing dengue outbreaks had detectable DENV RNA. This was concordant with earlier studies during previous outbreaks, and is consistent with our modelling estimate of 1 in 7147 (95\% CI: 1 in 2218 to 1 in 50,021) for the risk of collecting a viraemic donation during an outbreak. The rate of DENV viraemia in blood donors residing in areas of Australia that experience episodic dengue outbreaks was lower than that in donors from Puerto Rico, where dengue is endemic, likely reflecting differences in viral epidemiology between endemic and non-endemic regions. While this study is insufficiently powered to draw definitive conclusions, the absence of DENV RNA positive donations, despite the higher number of reported cases during these outbreaks, suggests fresh component restriction for 'at risk' donors during local outbreaks is an effective strategy for minimising the risk of transfusion transmitted DENV in non-endemic regions.

\section{P-133}

\section{HEPATITIS E VIRUS INFECTIONS IN TRAVELLERS: A THREAT}

IN AUSTRALIA?

AC Shrestha ${ }^{1}$, RLP Flower ${ }^{1}$, CR Seed ${ }^{1}$, AJ Keller ${ }^{1}$, V Hoad ${ }^{1}$, R Harley $^{1}$, R Leader $^{2}$, B Polkinghorne $^{2}$, C Furlong ${ }^{3}$ and HM Faddy

${ }^{1}$ Australian Red Cross Blood Service, Queensland, Australia ${ }^{2}$ Australian Government Department of Health, Australian Capital Territory, Australia ${ }^{3}$ New South Wales Department of Health, New South Wales, Australia

Background: In developed countries such as Australia, hepatitis E virus (HEV) infections are predominantly seen in travellers returning from countries endemic for HEV. HEV can be transfusion transmissible and viral RNA has been detected in asymptomatic blood donors, demonstrating this virus is a potential threat to blood safety. To minimise the risk of HEV transfusion transmission in Australia, individuals diagnosed with HEV infection are deferred from donating for 12 months from

(C) 2015 The Author

Vox Sanguinis (C) 2015 International Society of Blood Transfusion Vox Sanguinis (2015) 109 (Suppl. 2), 1-96 
the date of recovery. Donors who have recently travelled to the majority of developing countries endemic for HEV are restricted from donating fresh components for four months after return due to risk of malaria infection.

Aims: To describe HEV cases in Australia acquired overseas in order to determine whether infection in travellers poses a risk to Australian blood supply safety.

Methods: Details of all notified cases of HEV in Australia from 2002 to 2013 were accessed from the National Notifiable Diseases Surveillance System. Countries with HEV risk were identified. The estimated HEV importation rate was determined based on the number of people in Australia who had recently (within 1 year) travelled to HEV risk countries. These data were obtained from the Australian Department of Immigration and Border Protection and include Australian travellers (short term resident returns) and visitors (short term visitor arrivals). Visitor arrivals were included in this analysis as notification data are not restricted to residents of Australia, and, providing all other donation requirements are met, visitors to Australia are able to donate in Australia, although this group likely makes a small proportion of total donations. These countries were then compared to those where donations are restricted for travel due to malaria-risk.

Results: A total of $325 \mathrm{HEV}$ cases were acquired overseas during the study period. Travel to India $(149 ; 46 \%)$ accounted for the most infections, followed by Bangladesh (39; $12 \%)$ and Nepal $(21 ; 6 \%)$. However, based on the number of travellers arriving in Australia from these countries, the importation rate was highest from Nepal (22.03 per 10,000 travellers), followed by Bangladesh (18.01 per 10,000 travellers), Sudan (16.09 per 10,000 travellers), Pakistan (5.66 per 10,000 travellers) then India ( 3.20 per 10,000 travellers). Countries where donations are restricted following travel due to malaria-risk accounted for 95\% (308) of overseas acquired HEV cases in Australia.

Conclusions: The majority of overseas acquired HEV infections were in travellers returning from South Asia, particularly countries where HEV is endemic and where large water-borne outbreaks occur. The majority of HEV cases were acquired from countries where donation-related travel restrictions for malaria exist. Specifically, such donors are precluded from donating fresh components for four months. Given that HEV viremia persists for four to six weeks in most individuals, existing travel deferral policies assist with minimising the risk to fresh components from imported HEV infections. We are currently undertaking a comprehensive risk assessment of the risk HEV may pose to transfusion safety in Australia.

\section{P-134 \\ DETECTION OF HEPATITIS E VIRUS IN AUSTRALIAN BLOOD DONATIONS}

AC Shrestha ${ }^{1}$, RLP Flower ${ }^{1}$, CR Seed ${ }^{1}$, AJ Keller ${ }^{1}$, R Harley $^{1}$, HT Chan ${ }^{1}$, V Hoad $^{1}$, D Warrilow $^{2}$, JA Holmberg ${ }^{3}$ and HM Faddy ${ }^{1}$

${ }^{1}$ Australian Red Cross Blood Service, Queensland, Australia ${ }^{2}$ Queensland Government, Queensland, Australia ${ }^{3}$ Grifols, CA, United States of America

Background: Hepatitis E virus (HEV) is known to be transfusion-transmissible. HEV has been the subject of much recent debate in transfusion medicine with the findings that asymptomatic viraemia in European blood donors is much higher than expected based on notification rates, and the acknowledgement that HEV is a disease of importance in developed countries. In such countries, sporadic cases of zoonotic transmission associated with genotypes 3 and 4 are increasingly being reported, which is in contrast to the water-borne outbreaks in developing countries that are associated with genotypes 1 and 2. HEV is detectable in blood 2-3 weeks after infection and persists for 4-6 weeks in most individuals, but can be detectable for more than 3 months in chronic infections. Australian notification data suggests HEV infections are rare, with locally acquired cases predominantly being due to genotype 3 .

Aims: To measure the rate of HEV infection in Australian blood donations.

Methods: A total of 14,799 samples from blood donations were collected from around Australia in September and October 2014. All samples were tested for HEV RNA by transcription mediated amplification (TMA). All HEV TMA reactive samples were tested in duplicate with a commercially available HEV RT-PCR, and a sample was only considered HEV RNA positive if reactive by both TMA and RT-PCR. HEV RT-PCR targeting the methyltransferase region was also performed using in-house primers, and the PCR product was sequenced. HEV RNA positive samples were tested for HEV antigen, HEV IgG and HEV IgM with commercially available ELISAs.

Results: One $(0.0068 \%$, 95\% CI: $0.0002-0.0376 \%)$ sample was confirmed positive for HEV RNA, resulting in 1 out of 14,799 Australian blood donations (95\% CI: 1 in 584,530 to 1 in 2657) being positive for HEV RNA. Phylogenetic analysis indicates the HEV RNA positive sample was most closely related to genotype 3; however, additional analyses are required to confirm this. This sample was also HEV antigen positive, but negative for HEV IgG and IgM.

Conclusions: Our finding of 1 in 14,799 donations being HEV positive is lower than the rate of HEV infection in blood donors in other developed countries, including

\section{(C) 2015 The Author}

Vox Sanguinis (C) 2015 International Society of Blood Transfusion

Vox Sanguinis (2015) 109 (Suppl. 2), 1-96 those from the United Kingdom, Spain and the Netherlands. This reflects HEV seroprevalence in these countries and is consistent with the considerably lower HEV case notification data in Australia. It is possible that the one positive sample in this study could have been from a donor who acquired HEV overseas, however, as this positive sample is provisionally genotype 3 , it seems more likely the infection was acquired through zoonotic transmission, either within Australia or overseas in a developed nation. A risk assessment is underway to determine the significance of these findings to transfusion safety in in Australia.

\section{$\mathrm{P}-135$ COMPARISON OF THE ANTI-HEV SCREENING TEST RESULTS FOR KOREAN BLOOD DONORS}

KW Youn

Korean Red Cross/Blood Transfusion Research Institute, Wonju, South-Korea

Background: Hepatitis E infection is an acute viral hepatitis infection and was recognized as pandemic mainly in the developing countries. But recently, the HEV infection has been detected in the developed countries such as Japan, and some cases resulted from blood transfusion. Blood donation of asymptomatic HEV infection can cause infectious disease in the pregnant women or immunocompressed patient. Therefore, the safety strategies related with HEV infection has to be considered.

Aims: This study was the first trial of HEV test for the Korean Blood donors. As a preliminary investigation about the HEV infection in the blood donors, we performed HEV antibody test for the Korean blood donors by using two kinds of antiHEV assay.

Methods: Anti-HEV assay was performed for 630 donors by using two kinds of anti-HEV IgG/IgM screening assay. For the anti-HEV IgG/IgM positve cases, Western blot assay as a confirmatory assay was performed. We used Wantai HEV-IgG/IgM ELISA (Wantai LTD, Beijing, China) and recomWell HEV IgG/IgM (MIKROGEN $\mathrm{GmbH}$, Neuried, Germany) for anti-HEV IgG/IgM, and recomLine HEV IgG/IgM (MIKROGEN GmbH, Neuried, Germany) for Western blot. In addition HEV RNA assay was performed for the same samples using ampliCube HEV (MIKROGEN GmbH, Neuried, Germany).

Results: Among the 630 donors, 5 donors $(0.8 \%)$ showed positive results in the two kinds of anti-HEV IgG assay, and 4 of them (0.6\%) showed positive results in Western blot. 15 donors (2.4\%) showed positive results only in one kind of assay. 6 donors (1.0\%) showed positive results only in Wantai anti-HEV IgG, 4 donors $(0.6 \%)$ showed positive results only in recomWell anti-HEV IgG, and 5 donors $(0.8 \%)$ showed positive results only in recomWell anti-HEV IgM. All of the donors showing positive results only in one kind of assay showed negative results in Western blot. There was no HEV RNA positive case among the 630 donors.

Summary/Conclusions: The frequency of anti-HEV positive samples was different according to the manufacture of the assay kit. 4 donors showing positive results in Western blot was regarded as recovery phase after infection. However, it was thought that there was some limitation in the estimation of accurate prevalence because there was no case of HEV RNA positive and the number of 630 donors which were tested was considered too small to estimate the risk among blood donors. At least, 10,000 donors are required for risk evaluation to introduce the anti-HEV screening test in Korea.

\section{P-136 PREVALENCE OF HEPATITIS E VIRUS INFECTION AMONG IRANIAN BLOOD DONORS \\ Z Sharifi and MA Jalili \\ Blood Transfusion Research Center, High Institute for Research and Education, Tehran, Iran}

Background: Hepatitis E virus (HEV) is endemic in many developing countries. HEV is transmitted by the fecal-oral route mainly. Some studies have reported HEV transmission by blood transfusions. HEV infection usually causes a self-limiting, acute illness. HEV may cause chronic infection and severe liver disease in immunocompromised patients and pregnant women.

Aims: The aim of this study was to determine the Prevalence of Hepatitis E virus infection among Iranian blood donors.

Methods: This study was carried out among 400 volunteer blood donors. Total (IgM and IgM) anti-HEV antibody was tested by ELISA method. The presence of HEVRNA was detected by RT-PCR procedure after nucleic acid extraction from plasma samples.

Results: About 4\% (16 of 400) of blood donors had total anti-HEV antibody and HEV- RNA was detected in all anti-HEV antibody positive samples. The most common genotype of HEV was genotype 1. 
Conclusion: The results of this study showed that the prevalence of anti-HEV antibody among Iranian blood donors was low. The possible of transmission of HEV especially in immunocompromised patients should be considered

\section{Immune Haematology \\ 5.1 Red Cell Immunology: Serology}

P-137

\section{MOLECULAR MODELING OF LINEAR AND CONFORMATIONAL EPITOPES OF RHD VARIANT DVII}

\section{CP Nixon ${ }^{1}$ and JD Sweeney ${ }^{2}$}

${ }^{1}$ Center for International Health Research, Providence, United States of America ${ }^{2}$ Rhode Island Hospital \& The Miriam Hospitals, Providence, United States of America

Background: The highly immunogenic and polymorphic RhD polypeptide, is the most clinically significant protein-based blood group expressed on the erythrocyte membrane and has been implicated in hemolytic disease of the newborn and hemolytic transfusion reactions. Partial D represent $\mathrm{RhD}$ variants in which one or more D antigen epitopes are missing and can result in an immune response to the missing epitope(s) upon exposure to wild type $\mathrm{Rh}(\mathrm{D})$. Such alloimmunization events however are surprisingly rare, occurring in some but not all partial Ds. Although many of these epitope alterations have been identified by monoclonal antibodies which target specific domains or exofacial loops on the erythrocyte surface, few of these variants have been visualized by structural prediction tools.

Aims: The aim was to construct a predictive model of the 3-D structure of this variant in order to visual the altered epitope.

Methods: A routine preoperative type and screen from a 73 year old male showed a positive indirect antiglobulin test with a negative direct antiglobulin test. The patient had been transfused with two units of RBC previously. The antibody panel detected anti-D alloantibodies. Passive transfer of alloantibody by plasma or derivatives was excluded. Despite the presence of anti-D alloantibodies, the patient's plasma was cross-match compatible with two units of A positive red cell units in standard gel compatibility testing, one of which was homozygous $\left(R_{1} R_{2}\right)$ and the other heterozygous $\left(R_{1} r\right)$. To elucidate the nature of the partial $D$ phenotype and the conundrum of the compatible cross-matches in the presence of anti-D alloimmunization, the patient's RHD gene was sequenced. After partial D variant detection, homology 3-D molecular modeling and structural comparison of the linear and conformational epitopes of this partial D variant vs $\mathrm{RhD}$ isoform 1 was performed with the web-based structural prediction tool ELLIPRO. The crystal structure of the ammonium transporting $\mathrm{Rh}$ glycoprotein RhCG was used as the template.

Results: Sequencing of the patient's RHD gene exons 1-10 revealed a single amino acid substitution L110P (329c > C) in the second exofacial loop of the RhD polypeptide caused by a missense mutation in $R H D$ exon 2 . This $R H D$ genotype $R H D^{*} D V I I-$ type 1, predicts a partial D phenotype. 3-D homology modeling of this partial D variant, revealed significant predicted B cell linear and conformational epitope structural differences between $\mathrm{RhD}$ isoform 1 and RhDVII in the first and second exofacial loops of the RhD polypeptide.

Conclusion: This case report documents a case of anti-D alloimmunization in a patient with $R H D$ genotype $R H D^{*} D V I I t y p e 1$ whose plasma was cross-match compatible with $\mathrm{Rh}(\mathrm{D})$ positive units. These anti-D alloantibodies are likely directed against a defined epitope appearing as a cleft between exofacial loops 1 and 2.

\section{P-138}

\section{EXTENDED RED CELL PHENOTYPE USING THE OLYMPUS PK7300 DIAGAST REAGENTS}

S Valensky and B Mitchell

Western Province Blood Transfusion Service, Cape Town, South Africa

Background: WPBTS has experienced problems finding phenotype compatible blood for Sickle Cell Disease (SCD) and other patients with multiple antibodies. The Reference lab saw an increase of 92 SCD patient cases in 18 months.

Donors are routinely typed for C, c, E, e and Kell antigens using Diagast ready-to-use reagents on the Olympus PK7300. When needed, antigen typing was performed manually, as required in the Blood Banks or Reference laboratory. A systematic approach was needed to build up a typed donor database to reduce time and costs in the Blood Banks. Aim: To find antigen negative combinations that would assist in finding compatible blood for patients with multiple antibodies. The following combinations were selected based on literature i.e. $\mathrm{Fy}^{\mathrm{a}}-\mathrm{Fy}^{\mathrm{b}}-\mathrm{Jk}^{\mathrm{b}}-\mathrm{S}-, \mathrm{Fy}^{\mathrm{a}}-\mathrm{Fy}^{\mathrm{b}}-\mathrm{S}-, \mathrm{Fy}^{\mathrm{a}}-\mathrm{Fy}^{\mathrm{b}}+\mathrm{Jk}^{\mathrm{b}}-\mathrm{S}-, \mathrm{Fy}^{\mathrm{a}}-\mathrm{Fy}^{\mathrm{b}}-$ and/or s- .

Method: A cost comparison was done between the current manual methods of randomly typing units to that of an automated method. An evaluation was performed on the Diagast extended red cell phenotype reagents $\left(\mathrm{Jk}^{\mathrm{a}}, \mathrm{Jk}^{\mathrm{b}}, \mathrm{Fy}^{\mathrm{a}}, \mathrm{Fy}^{\mathrm{b}}, \mathrm{S}\right.$ and $\mathrm{s}$ ) specifically designed for use on the Olympus PK7300 as soon as the kit was CE marked. In late 2014 a validation was performed over a 2 month period in which 858 samples were validated. Donors were selected and tested based on $\mathrm{ABO} / \mathrm{Rh}$ and race i.e. white, mixed race and black.

Results: 858 results were tested and validated of which 13 were discrepant (1.5\%). The majority of these discrepancies resulted from previous transcription or interpretation errors.

To date 1241 donors (including validation results) have been tested. 817 donor samples were from 'black' donors ( 345 Group 0 and 472 other blood groups). 423 samples with all $\mathrm{ABO} / \mathrm{Rh}$ groups were from the other race groups. The following combinations were found in 'black' donors i.e. Fy ${ }^{\mathrm{a}}-\mathrm{Fy}^{\mathrm{b}}-\mathrm{Jk}^{\mathrm{b}}-\mathrm{S}-(28 \%), \mathrm{Fy}^{\mathrm{a}}-\mathrm{Fy}^{\mathrm{b}}-\mathrm{S}-$ (9\%), Fy ${ }^{\mathrm{a}}-\mathrm{Fy}^{\mathrm{b}}+\mathrm{Jk}^{\mathrm{b}}-\mathrm{S}-(12 \%), \mathrm{Fy}^{\mathrm{a}}-\mathrm{Fy}^{\mathrm{b}}-(27 \%)$ and s- $(7 \%)$. When comparing antigen negative typing in the Western Cape population, 'whites' have a higher percentage of $\mathrm{Jk}^{\mathrm{a}}-(5.72 \%)$ whereas the 'blacks' have a higher percentage in the other 5 antigens tested i.e. Jk ${ }^{\mathrm{b}}-(45.61 \%), \mathrm{Fy}^{\mathrm{a}}-(55.76 \%), \mathrm{Fy}^{\mathrm{b}}-(48.27 \%), \mathrm{S}-(43.35 \%)$ and s- $(4.60 \%)$. Donors with the desired antigen combination were then further antigen tested using PCR in order to increase the antigen profile.

Conclusion: Automated testing on selected donors was introduced routinely in May 2015. Testing on the Olympus PK7300 using the Diagast reagents is quick and eliminates transcription errors by uploading results automatically. By targeting donors with a specific race and blood group we were able to identify samples with the preferred antigen combinations for $\mathrm{Jk}^{\mathrm{a}}, \mathrm{Jk}^{\mathrm{b}}, \mathrm{Fy}^{\mathrm{a}}, \mathrm{Fy}^{\mathrm{b}}, \mathrm{S}$ and $\mathrm{s}$, thereby increasing the WPBTS rare donor panel. Further extended antigen typing was performed by PCR on selected antigen negative donors. This study has given us a better insight to the antigen profile of the donors of the Western Cape. To date we have found a U negative, $2 \mathrm{hr}^{\mathrm{S}}$ negative, $8 \mathrm{hr}^{\mathrm{B}}$ negative and $2 \mathrm{Jo}^{\mathrm{a}}$ negative donors.

\section{P-139}

\section{SEEKING FOR POSITIVE DI ${ }^{\mathrm{A}}$ AND MI ${ }^{\mathrm{A}}$ ANTIGEN RED CELLS USING RED CELL POOLING ON COLUMN AGGLUTINATION TECHNIQUE: EXPERIENCE FROM TERTIARY CARE HOSPITAL, BANGKOK, THAILAND}

P Permpikul, T Rungroung, P Plubjuice, W Pimsamsee, K Wongchanapai and P Rojphoung

Mahidol University, Bangkok, Thailand

Background: The red cell antibodies that are frequently detected in Thailand including antibody to $\mathrm{Rh}(\mathrm{E}, \mathrm{C}, \mathrm{D}, \mathrm{c}, \mathrm{e}) \mathrm{Le}^{\mathrm{a}}, \mathrm{Le}^{\mathrm{b}}, \mathrm{Jk}^{\mathrm{a}}, \mathrm{Jk}^{\mathrm{b}}, \mathrm{Fy}^{\mathrm{a}} \mathrm{Fy}^{\mathrm{b}}, \mathrm{Mi}^{\mathrm{a}}$ and $\mathrm{Di}^{\mathrm{a}}$ antigen. The commercial screen cells produced from Good Manufacturing Practice Certification factory in Europe and America do not have $\mathrm{Mi}^{\mathrm{a}}$ and $\mathrm{Di}^{\mathrm{a}}$ antigens. From the basic knowledge of column agglutination technique which can visualized the mixed population of positive and negative reaction together .

Aims: We aim to find antigens $\mathrm{Mi}^{\mathrm{a}}$ and $\mathrm{Di}^{\mathrm{a}}$ antigens on one donor red cell in order to be used as additional screen cells for our patient's antibody detection system. The testing methods including finding the appropriate pooling size for the desired antigen detection when test with specific antisera on column agglutination.

Methods: We start from the selection of appropriate strong patient anti- $\mathrm{Di}^{\mathrm{a}}$ and test against one $\mathrm{Di}^{\mathrm{a}}$ positive mixed with various number of $\mathrm{Di}^{\mathrm{a}}$ negative red cells to find the appropriate pooling number of cells to be tested. The red cells were suspended in 'ID diluent 2' (DiaMed GmbH, Cressier, Switzerland). The column that we used in this study was BIO-RAD ID card 'LISS/Coombs' (DiaMed GmbH, Cressier, Switzerland). We then tested our donor cells for the $\mathrm{Di}^{\mathrm{a}}$ antigen in the appropriate pooling. The positive pools were tested as individual cell to identify the positive $\mathrm{Di}^{\mathrm{a}}$ antigen red cell. The positive $\mathrm{Di}^{\mathrm{a}}$ red cells were further tested for $\mathrm{Mi}^{\mathrm{a}}$ antigen using our donor anti-Mi ${ }^{\mathrm{a}}$.

Results: We found that our patient's serum contain anti- $\mathrm{Di}^{\mathrm{a}}$ strong enough to detect $\mathrm{Di}^{\mathrm{a}}$ antigen in pool of 4 . We tested 2000 samples in 500 pools (reactions). In these tested samples, 56 pools gave positive reaction and the further tests identified 58 samples of $\mathrm{Di}^{\mathrm{a}}$ positive red cells. We then tested these 58 samples for $\mathrm{Mi}^{\mathrm{a}}$ antigen using donor anti-Mi ${ }^{\mathrm{a}}$. We could identified 4 samples with positive both $\mathrm{Di}^{\mathrm{a}}$ and $\mathrm{Mi}^{\mathrm{a}}$ antigens on red cell. Overall, we tested 2000 samples for 724 reaction for $\mathrm{Di}^{\mathrm{a}}$ antigen and tested only 58 reaction for $\mathrm{Mi}^{\mathrm{a}}$ antigen. Beside saving time and money, we obtained valuable 4 red cells of $\operatorname{Di}(\mathrm{a}+)$ and $\mathrm{Mi}(\mathrm{a}+)$ which will be used as additional screen cells.

Conclusion: Our pooling of red cells tested on column agglutination technique work very well in identifying the desirable antigen combination (for example: $\mathrm{Di}^{\mathrm{a}}$ and $\mathrm{Mi}^{\mathrm{a}}$ ) which we have specific need and save our precious time and resource.

(C) 2015 The Author Vox Sanguinis (C) 2015 International Society of Blood Transfusion Vox Sanguinis (2015) 109 (Suppl. 2), 1-96 
P-140

\section{PERFORMANCE EVALUATION OF AN AUTOMATED IMMUNOHEMATOLOGIC ANALYZER FOR TESTING ABO ANTIBODY TITERS}

H Kim, YJ Hong, KU Park and KS Han

Seoul National University College of Medicine, Seoul, South-Korea

Background: Over the years, automated analyzers have become more widely used in transfusion centers. Automation in blood bank has advantages in reducing error, increased efficiency, safety, and minimizing workload. There are several instruments in the market based on a variety of different technologies, such as column agglutination, solid-phase, microplate, and red blood cell (RBC)-magnetized methods. Currently, automated systems have been developed for testing mainly $\mathrm{ABO}$ and $\mathrm{Rh}$ typing, antibody screening and identification, crossmatching, and direct antiglobulin tests. AutoVue Vision (Ortho-Clinical Diagnostics, High Wycombe, UK) is a newly introduced instrument based on column agglutination that is capable of automatically testing RBC antibody titration.

Aims: In this study, we evaluated the performance of AutoVue Vision in measuring $\mathrm{ABO}$ antibody titers and compared the results with conventional manual methods.

Methods: Plasma of five group 0 individuals were pooled and diluted with saline at a ratio of $1: 4$ to a final volume of $40 \mathrm{ml}$. The diluted plasma was divided into ten aliquots of $4 \mathrm{ml}$ and stored at $-30^{\circ} \mathrm{C}$ until testing. $\mathrm{ABO}$ antibody titration was performed twice daily for five consecutive days. Anti-A and anti-B titers were measured with AutoVue Vision using 0.8\% reagent RBCs (Affirmagen, Ortho-Clinical Diagnostics) and BioVue Reverse Diluent and AHG Anti-IgG (Ortho-Clinical Diagnostics) micro-column cassettes at room temperature (RT) and the anti-human globulin (AHG) phase, respectively. Manual comparison tests were also done using BioVue and ID-card (Bio-Rad, Cressier, Switzerland) micro-column cassettes. Titers were determined at endpoints of $1+$ and \pm reactivity, and the overall strength of hemagglutination was calculated by using the Marsh scoring system. In comparison analysis, titers that were within two standard dilutions and differences in score of $<10$ were considered as acceptable

Results: AutoVue Vision showed good correlation with the manual method using BioVue at both endpoints of $1+$ and \pm . The majority of anti-A and anti-B titers measured at both RT and AHG phase were in acceptable range (90-100\%). Manual method using ID-card at RT showed agreement rates of 70-90\%, but at AHG phase, only $0-20 \%$ were in acceptable range and many of the titer results using ID-card were two to three steps lower than AutoVue Vision. AutoVue Vision and the manual method using BioVue showed comparable results in mean values of hemagglutination scores for anti-A and anti-B at both RT and AHG phase. Mean hemagglutination scores for the manual method using ID-card were significantly lower for more than 10 compared to AutoVue Vision in all test settings. CV values for hemagglutination scores were the lowest for AutoVue Vision (anti-A, RT, 3.92\%; anti-A, AHG 3.58\%; anti-B, RT, 3.97\%; anti-B, AHG, 2.23\%) compared to other methods.

Conclusions: AutoVue Vision demonstrated good compliance in $\mathrm{ABO}$ antibody titration results with the conventional manual method using BioVue cassettes. It was more sensitive than the manual method using ID-card, and displayed good performance in terms of reproducibility. The use of automated analyzers for antibody titration in clinical laboratories can help improve consistency in test results and may contribute to reduce inter-institutional variability.

Table 1 Distribution of differences in antibody titers determined by manual methods compared to AutoVue Vision.

\begin{tabular}{|c|c|c|c|c|c|c|c|c|c|c|c|c|}
\hline & \multicolumn{6}{|c|}{ Difference in titer (endpoint 1+) } & \multicolumn{6}{|c|}{ Difference in titer (endpoint \pm ) } \\
\hline & -3 & -2 & -1 & 0 & 1 & 2 & -3 & -2 & -1 & 0 & 1 & 2 \\
\hline Anti-A, RT (BioVue) & & & & 9 & 1 & & & & & 8 & 2 & \\
\hline Anti-A, RT (ID-card) & & 1 & 9 & & & & & 3 & 7 & & & \\
\hline Anti-A, AHG (BioVue, anti-lgG) & & 1 & & 7 & 2 & & & & 1 & 8 & 1 & \\
\hline Anti-A, AHG (BioVue, anti-lgG + C3d) & & & 1 & 7 & 2 & & & & 2 & 7 & 1 & \\
\hline Anti-A, AHG (ID-card, anti-lgG + C3d) & & 8 & 2 & & & & 6 & 2 & 2 & & & \\
\hline Anti-B, RT (BioVue) & & & & 7 & 3 & & & & & 4 & 5 & 1 \\
\hline Anti-B, RT (ID-card) & & 2 & 8 & & & & & 2 & 6 & 2 & & \\
\hline Ant-B, AHG (BioVue, ant-lgG) & & & 4 & 6 & & & & & 3 & 6 & 1 & \\
\hline Anti-B, AHG (BioVue, anti-lgG + C3d) & & & 4 & 6 & & & & & 3 & 4 & 3 & \\
\hline Anti-B, AHG (ID-card, anti-lgG + C3d) & 3 & 7 & & & & & 4 & 5 & 1 & & & \\
\hline
\end{tabular}

(C) 2015 The Author

Vox Sanguinis (C) 2015 International Society of Blood Transfusion

Vox Sanguinis (2015) 109 (Suppl. 2), 1-96
Table 2 Comparison of performance for ABO antibody titration $(\mathrm{n}=10)$.

\begin{tabular}{|c|c|c|c|c|c|c|c|c|c|}
\hline & \multicolumn{3}{|c|}{ Antibody titer (endpoint $1+)^{n}$} & \multicolumn{3}{|c|}{ Antibody siter (endpoint $t)^{n}$} & \multicolumn{3}{|c|}{ Hemagglutination score } \\
\hline & Mean $\pm S D$ & $\operatorname{cv}(\%)$ & 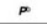 & Mean \pm SD & $\operatorname{cv}(\%)$ & $\infty$ & Mean \pm SD & cv(\%) & $p$ \\
\hline Ant-A, RT (AutoVue Vision) & $6 \cdot 0 \pm 0.0$ & 0.0 & & $6 \cdot 8 \pm 0 \cdot 4$ & $6 \cdot 2$ & & $67-0 \pm 2.62$ & 3.92 & \\
\hline Ant-A, RT (Biovue) & $6 \cdot 1 \pm 0.3$ & 5.2 & 0.317 & $7 \cdot 0 \pm 0.7$ & 95 & 0.157 & $69 \cdot 4 \pm 5 \cdot 10$ & $7 \cdot 35$ & 0.084 \\
\hline Anti-A, RT (1D-card) & $49: 0.3$ & 6.5 & 0.002 & 5.500 .5 & 96 & 0.004 & $56 \cdot 9+2.56$ & 4.50 & 0.005 \\
\hline Anti-A, AHG (Autovue Vision, anti-igG) & $7 \cdot 1 \pm 0.3$ & $4 \cdot 5$ & & $8 \cdot 3 * 0.5$ & 5.8 & & $81 \cdot 4 \pm 2.91$ & 3.58 & \\
\hline Antr-A, AHG (BioVve, ant-19G) & $7 \cdot 1 \pm 0.6$ & 8.0 & 1.000 & $8 \cdot 3+0.5$ & 5.8 & 1.000 & $81 \cdot 1+4 \cdot 98$ & $6 \cdot 14$ & 0.798 \\
\hline Anti-A, AHG (BioVue, ant-1gG + C3d) & $7 \cdot 2 \pm 0.4$ & 5.9 & 0.564 & $8 \cdot 2+0.4$ & 5.1 & 0.564 & $80 \cdot 9: 4 \cdot 38$ & 5.42 & 0.953 \\
\hline Ant-A, AHG (1D-card, ant-19G + C C3d) & $5 \cdot 3+0.5$ & $9 \cdot 1$ & 0.004 & $5.9 \pm 0.7$ & 12.5 & 0.005 & $59 \cdot 1 \pm 4 \cdot 23$ & $7 \cdot 15$ & 0.005 \\
\hline Ant-B, RT (AutoVue Vision) & $4 \cdot 9 \pm 0.3$ & 6.5 & & $5.4 \pm 0.5$ & 96 & & $542+2 \cdot 15$ & 3.97 & \\
\hline Ant-B, RT (BioVue) & $5.2+0.4$ & 8.1 & 0.102 & $6 \cdot 1+0.3$ & 5.2 & 0.020 & $609+3 \cdot 14$ & 5.16 & 0.007 \\
\hline Ant-B, RT (ID-card) & $3.7 \pm 0.5$ & 13.1 & 0.004 & $4 \cdot 4 \pm 0.5$ & 11.7 & 0.011 & $43 \cdot 1 \pm 4 \cdot 82$ & $11-18$ & 0.005 \\
\hline Ant-B, AHG (AutoVue Vision, ant-1gG) & $7 \cdot 0 \pm 0.0$ & 0.0 & & $7 \cdot 7 \pm 0.5$ & 6.3 & & $76 \cdot 3 \pm 1 \cdot 70$ & 2.23 & \\
\hline Antt-B, AHG (BioVue, ant-1gG) & $66 \pm 0.5$ & 7.8 & 0.046 & $7 \cdot 5 \pm 0.5$ & 70 & 0.317 & $75 \cdot 0 \pm 7 \cdot 29$ & 972 & 0.646 \\
\hline Ant-B, AHG (BioVue, ant-19G + C3d) & $68 \pm 0.5$ & 7.8 & 0.046 & $7 \cdot 7 \pm 0.7$ & 88 & 0.739 & $74 \cdot 4 \pm 7 \cdot 26$ & 976 & 0.314 \\
\hline Ant-B, AHG (ID-card, ant-19G + C3d) & $47 \pm 0.5$ & $10 \cdot 3$ & 0.004 & $5.4 \pm 0.7$ & $12 \cdot 9$ & 0.005 & $52 \cdot 4 \pm 5.06$ & 966 & 0.005 \\
\hline
\end{tabular}

P-141

\section{EFFECTIVENESS OF BLOOD GROUPING AUTOMATION METHOD ON ABO SUBGROUP DETECTION IN JAKARTA BLOOD TRANSFUSION UNIT}

S Sunarti, U Khasanah, U Bachrussani, NK Ritchie and S Salim

Jakarta Blood Transfusion Unit, DKI Jakarta, Indonesia

Background: $\mathrm{ABO}$ blood group testing is an important aspect to make blood safe for transfusion. The National Standard on Blood Services emphasizes that the ABO blood group testing on donor's blood should be run three times: before blood donation; at Blood Transfusion Unit (BTU's) laboratory as a confirmation test; and as part of the cross matching test. The common method used in the country for these testing are slide test at pre-donation testing and tube test at the other two occasions. Before 2013, due to lack of human resources, the ABO blood group confirmation testing in Jakarta BTU could only be run on $20 \%$ of donations consist of forward and reverse grouping using the tube method. The prevalence of confirmed ABO blood group detected in our BTU is 0 group (39\%), followed by B group (27.8\%), A group (25.8\%), and $A B$ group (7.4\%). In 2013, automation method of blood group testing was introduced with purpose to increase the accuracy of blood grouping results.

Aims: To evaluate the effectiveness of the automation method of blood group testing in Jakarta BTU.

Methods: We performed $\mathrm{ABO}$ blood grouping with the Owalys ${ }^{\circledR}$ system. The results of this testing were compared to that tested by slide test during pre-donation stage to assess human errors.

Results: During 2013-2015, 326.723 samples tested using the automation method, there were no significant different on the prevalence of each $\mathrm{ABO}$ group compared to previous data. However, surprisingly we found more donors with $\mathrm{ABO}$ sub-group with automation than with manual method; 25 of 326.723 in 2013-2015 compared to 12 of 887.157 ABO subgroups in 2010-2012 period. Interestingly, mixed-field were more often found. Currently, we found the prevalence of sub-group of $\mathrm{A} 3 \mathrm{~B}$ $(0.008 \%)$, AB3 $(0.002 \%)$, B3 $(0.004 \%)$, A3 $(0.0009 \%)$, A2 $(0.0009 \%)$, A2B $(0.0005 \%)$ and $\mathrm{A} 3 \mathrm{~B} 3(0.0005 \%)$. We also found reduction of human error while using automation method showed by decreasing of discrepancy of $\mathrm{ABO}$ blood group result in pretransfusion test.

Summary/Conclusions: Blood grouping automation method is more effective than tube method in order to decrease human error in pre-transfusion testing. Other benefit of blood grouping automation method is its ability to detect $\mathrm{ABO}$ sub-group.

\section{P-142}

DEVELOPING RED CELL ANTIGEN DATABASES OF BLOOD DONOR FOR HAVING HOMEBREW PANEL CELLS IN JAKARTA BLOOD TRANSFUSION UNIT

L Wanmuslimah, P Rini, NK Ritchie and S Salim

Jakarta Blood Transfusion Unit, DKI Jakarta, Indonesia

Background: Jakarta Blood Transfusion Unit (BTU) has a reference laboratory fo solving incompatibility cases in pre-transfusion compatibility test from blood banks around Jakarta. In the reference lab, we recheck the blood group of sample, and then we do direct anti-globulin test, antibody identification and cross match test to find 
the cause of incompatibility. To screen red cell antibody, we need 2-3 different cell suspensions derived from individual donors all with blood group 0 and 8-12 cell suspensions to identify red cell antibody. The minimum 18 of red cells antigen of screened and panel cells must have been known. At the beginning we got panel cells support for antibody detection from CSL Australia. But it is only for temporary, so we have to develop our own panel cells or buy commercial panel cells. At that time, panel cells were not available in the Indonesian market. If it was available, the expiry date would be the issue due to long bureaucracy in the custom. So, it was decided to have our own homebrew panel cells. Therefore, we started the red cell antigen phenotyping to know the characterization of red cell antigens in donor's population in Jakarta BTU.

Aims: to develop donor's red cell antigen database in order to have homebrew panel cells.

Methods: Only routine-blood group 0 donors were selected. The blood was collected in $10 \mathrm{ml}$ EDTA tube. Phenotyping was conducted using either tube method or column agglutination technique. We only typed 18 antigens as the minimum requirements for panel cells.

Results: Since 2001 until 2015, we have 1061 donors in our database. Of 1061, the most frequent antigens are D (85.8\%), C (87.6\%), e (97.9\%), k (100\%), Fy ${ }^{\mathrm{a}}(96.8 \%), \mathrm{s}$ (98.9\%), M (85.1\%) and $\mathrm{Jk}^{\mathrm{a}}(77.9 \%)$. The less frequent antigens are $\mathrm{E}(22.5 \%)$, Fyb $(24.7 \%)$, S (14.8\%), and Lea (27.1\%). The rare antigens are K (0.94\%). The most difficult types in donors are $\mathrm{Jk}^{\mathrm{a}-\mathrm{b}-}(0.38 \%)$, Fy ${ }^{\mathrm{a}-\mathrm{b}-}(0.28 \%), \mathrm{R}_{2} \mathrm{R}_{2}(1.7 \%)$ and SS (1.04\%). We have to arrange these 18 antigens from 2-3 donors into a screened panel (small panel cells). The big panel cells need 8-12 donors. Sometimes we found difficulties when combining $\mathrm{Fy}^{\mathrm{a}-\mathrm{b}+}(4.24 \%)$ or $\mathrm{Jk}^{\mathrm{a}-\mathrm{b}+}(21.87 \%)$ because of very small donors available. So, we have to expand our database to have a perfect panel. With these 18 antigens, we also found difficulties in determining other antibodies which not yet established in our panel cells.

Summary/Conclusions: We can solve the incompatibility cases using homebrew panel cells but we should screen more antigens and more donors to have a perfect panel cells.

\section{$\mathrm{P}-143$}

This abstract has been withdrawn.

$\mathrm{P}-144$

This abstract has been withdrawn.

$\mathrm{P}-145$

ALLO-ANTIBODIES MASKED BY AUTO-IMMUNE COLD ANTIBODIES IN REGULARLY TRANSFUSED EGYPTIAN

\section{PATIENTS}

M Salah, U Khasanah and E Mohammed

National blood transfusion services, Giza, Egypt

Background: Autoimmune cold antibodies are clinically non-significant but they can interfere with the pre-transfusion tests like (antibodies screening, cross-matching, etc.....) by masking the presence of other antibodies that may be clinically significant.

Aim: The aim of the study is to find out:

1. The prevalence of autoimmune cold antibodies among male and female regularly transfused Egyptian patients.

2. The prevalence of allo-antibodies masked by autoimmune cold antibodies.

Methods: The study conducted on transfusion dependent patients (2000 patients) with: Thalassemia, aplastic anemia, leukemia, renal failure and anemia of chronic diseases; presented to Egyptian NBTC for blood transfusion.

According to National Egyptian Testing Strategy, Patients were subjected to:

1. Screening for allo-antibodies against group 0 reagent RBCs (panel of 3 cells) using appropriate IAT at $37^{\circ} \mathrm{C}$ by CAT.

2. Antibody identification for positive screening samples against group 0 reagent RBCs (panel of 11 cells) using appropriate IAT at $37^{\circ} \mathrm{C}$ by CAT.

3. Screening at room temperature to detect the presence of cold antibodies.

4. Screening for allo-antibodies against group 0 reagent RBCs (panel of 3 cells) using appropriate IAT at strict $37^{\circ} \mathrm{C}$ with pre-warming of both serum \& reagents (in case of positive results of Screening at room temperature)

5. Antibody identification for positive screening samples to identify the specificity of allo-antibodies masked by autoimmune cold ones against group 0 reagent RBCs (panel of 11 cells) using appropriate IAT at strict $37^{\circ} \mathrm{C}$ with pre-warming of both serum \&t reagents.

Results: A total of 2000 patient (1000 males \& 1000 females) were examined for the presence of autoimmune cold antibodies $\&$ allo antibodies that may be masked by them

106 out of $1000(10.6 \%)$ male patients were found to have autoimmune cold antibodies.

79 out of $1000(7.9 \%)$ female patients were found to have autoimmune cold antibodies.

Out of a total of 2000 we found that 185 (9.25\%) patients had autoimmune cold antibodies and the co-incident prevalence of masked allo-antibodies was as follows:

1. Autoimmune cold antibodies only:129 out of 185 patients (69.7\%).

2. Allo-anti D: 3 out of 185 patients (1.6\%).

3. Allo-anti C: 7 out of 185 patients (3.8\%).

4. Allo-anti E: 15 out of 185 patients $(8.1 \%)$

5. Allo-antic: 13 out of 185 patients (7\%).

6. Allo-anti K: 7 out of 185 patients (3.8\%).

7. Allo-anti Fya: 1 out of 185 patients $(0.5 \%)$

8. Allo-anti Jka: 7 out of 185 patients (3.8\%).

9. Allo-anti S: 3 out of 185 patients (1.6\%).

Summary/Conclusions: Pre-warming techniques are helpful in differentiation between cold non-significant Abs and warm Abs, for proper pre-transfusion testing of the patients' samples

Table 1: co-incident prevalence of masked allo-antibodies.

\begin{tabular}{|l|c|}
\hline $\begin{array}{l}\text { Autoimmune cold } \\
\text { antibodies only }\end{array}$ & $\begin{array}{c}129 \\
(69.7 \%)\end{array}$ \\
\hline Allo-anti D & $3(1.6 \%)$ \\
\hline Allo-anti C & $7(3 \cdot 8 \%)$ \\
\hline Allo-anti E & $\begin{array}{c}15 \\
(8 \cdot 1 \%)\end{array}$ \\
\hline Allo-anti c & $13(7 \%)$ \\
\hline Allo-anti K & $7(3.8 \%)$ \\
\hline Allo-anti Fya & $1(0.5 \%)$ \\
\hline Allo-anti Jka & $7(3.8 \%)$ \\
\hline Allo-anti S & $3(1.6 \%)$ \\
\hline
\end{tabular}

$\mathrm{P}-146$

This abstract has been withdrawn.

P-147

STUDY ON RATE AND CHARACTERISTIC OF IRREGULAR

ANTIBODIES OF THALASSEMIA PATIENTS AT NATIONAL INSTITUTE OF HEMATOLOGY AND BLOOD TRANSFUSION, VIETNAM (2013-2015)

H Thi Thanh Nga, B Thi Mai An, NA Anh Tri and VD Duc Binh

National Institute of Hematology and Blood Transfusion, Hanoi, Vietnam

Background: Blood transfusion is an important treatment method for thalassemia patients. Ensuring transfusion safely and effectively for thalassemia patients is necessary. Study on result of screening and identification of irregular antibodies and selection matching blood units in order to transfuse more safely and effectively for thalassemia patients.

Aims: Study on rate and characteristic of irregular antibodies of thalassemia patients at National Institute of Hematology and Blood Transfusion (NIHBT), Vietnam from 2013 to 2015 .

Materials: 1.544 thalassemia patients who were treated in Thalassemia center, NIHBT from 01/01/2013 to 30/06/2015. Methods

Cross-sectional descriptive study. Using column agglutination technique and screening cell, panel cell which were made in NIHBT to detect irregular antibodies of patients.

(C) 2015 The Author

Vox Sanguinis (C) 2015 International Society of Blood Transfusion Vox Sanguinis (2015) 109 (Suppl. 2), 1-96 
Results: We found out 88 patients had irregular antibodies among 1.544 thalassemia patients (the rate is $5.7 \%$ ); Irregular antibodies rate in women with pregnancy higher than women without pregnancy (9.7\% and 5.3\%); Irregular antibodies rate related to blood transfusion times (no transfusion: 2.5\%, less than 5 times: 3.1\%, from 5 to 10 times: $7.2 \%$, more than 10 times: 10.7\%); There were 59 patients had single irregular antibody: anti-E (43.2\%) and anti-Mi ${ }^{\mathrm{a}}(13.6 \%)$ had the highes rate in this group; There were 29 patients had combined irregular antibodies: 2 irregular antibodies: 19 patients (21.6\%), 3 irregular antibodies: 7 patients (7.9\%), 4 irregular antibodies: 2 patients $(2.3 \%)$ and 5 irregular antibodies: 1 patient $(1.1 \%)$ the rate of anti-E combined anti-c was the highest (10.2\%); The highest irregular antibodies rate was found in Rh blood group system (anti-E: 68.2\%, anti-c: 22.7\%), follow MNS blood group system (anti-Mi ${ }^{\mathrm{a}}$ : 29.5\%), Kidd's antibodies: anti-Jk ${ }^{\mathrm{a}}$ : $6.8 \%$, anti-Jk $^{\mathrm{b}}$ : 5.7\%, Duffy's antibodies: anti-Fy ${ }^{\mathrm{b}}: 1.1 \%$, P1PK's antibodies: anti-P ${ }_{1}: 1.1 \%$. Conclusions: Irregular antibody rate in thalassemia patients was $5.7 \%$. Irregular antibody rate related to pregnancy and blood transfusion times. Irregular antibodies could appeared single (59 patients) or combination (29 patients). Anti-E (43.2\%) and anti-Mi $(13.6 \%)$ had the highest rate in single antibody group. The rate of anti-E combined anti-c was the highest (10.2\%) in combined antibodies group, we met a special patient had 5 irregular antibodies: anti-E combined anti-c, anti-Mi ${ }^{\mathrm{a}}$, anti-Jk ${ }^{\mathrm{b}}$ vá anti-Fy $(1.1 \%)$. The highest irregular antibodies rate was found in Rh blood group system (antiE: 68.2\%, anti-c: $22.7 \%$ ) and MNS blood group system (anti-Mi' ${ }^{\mathrm{a}}$ :29.5\%) was follow.

\section{$\mathrm{P}-148$}

THE IMPLEMENTATION OF ANTIBODY SCREENING IN PRETRANSFUSION TESTING FOR PATIENT SAFETY IN JAKARTA BLOOD TRANSFUSION UNIT

M Maryunis, P Rini, NK Ritchie and S Salim

Jakarta Blood Transfusion Unit, DKI Jakarta, Indonesia

Background: Pre-transfusion compatibility test aimed to know the compatibility of patients and donors or to see whether there was any antigen-antibody reactions. If it is compatible, the donor's blood expected to 'live' and patients get 'benefit' from transfusions. In other hand, if it's not compatible, we had chance to prevent transfusion reactions in patients. In general, pre-transfusion test included antibody screening beside blood group test and cross-match test. But, in Indonesia, antibody screening was excluded due to the limited of cost. There is risk of undoing antibody screening. Negative major of cross-match test was not always indicated no free antibody in patients. If 'false negative' blood was given to patients, they would have delayed transfusion reactions in the end. Therefore, Jakarta Blood Transfusion Unit (BTU) wanted to improve the blood safety in pre-transfusion test.

Aims: To know the frequency of 'false negative' of major cross test.

Methods: Patient's sample were collected in $3 \mathrm{ml}$ EDTA Blood, sent to Jakarta BTU. We screened all patient's sample using selectogen ${ }^{\circledR}, 3$ screened cells from Ortho Clinical Diagnostic ${ }^{\circledR}$. The next step, we did the major cross-match test in CAT system from Ortho Clinical Diagnostic ${ }^{\circledR}$. All test run in Autovue Innova, an automated system for pre-transfusion testing from Ortho Clinical Diagnostic ${ }^{\circledR}$

Results: We found 387 of 65,045 or 0.5\% of patient's sample from Januari 2014 until December 2014. From 387 samples contained antibody, 308 (79.6\%) samples showed negative results in major cross-match test and 79 (20.4\%) samples showed positive results in major cross-match test. If we didn't do antibody screening in 2014, among 10.000 patients, there could be 796 false negative of blood, given to patients.

Summary/Conclusions: Antibody screening in pre-transfusion testing should be implemented to prevent transfusion reactions in patients.

\section{P-149}

This abstract has been withdrawn.

\section{P-150}

DETECTION OF CIS-AB USING A MICROPLATE-BASED SYSTEMS AND TWO COLUMN AGGLUTINATION TEST IN A HOSPITAL BLOOD BANK

YJ Hong ${ }^{1}$, SH Park ${ }^{1}$, T Kim ${ }^{1}$, N Kim ${ }^{2}, \mathrm{H} \mathrm{Kim}^{2}$, KU Park ${ }^{1}$, J Song ${ }^{1}$ and KS Han ${ }^{2}$ ${ }^{1}$ Seoul National University Bundang Hospital, Seongnam, South-Korea ${ }^{2}$ Seoul National University College of Medicine, Seoul, South-Korea

Background: The ABO blood group is one of the most important antigens in transfusion medicine. Recently, a microplate-based system for ABO phenotyping has been

\section{(C) 2015 The Author}

Vox Sanguinis (C) 2015 International Society of Blood Transfusion

Vox Sanguinis (2015) 109 (Suppl. 2), 1-96 developed and is used universally in large-sized blood banks because of its effectiveness of time and cost. $\mathrm{A}$ cis- $\mathrm{AB}$, a rare and interesting $\mathrm{ABO}$ genotype, is the result of a mutated gene. Both the A glycosyltransferase and B glycosyltransferase activity are carried by the same gene which is a different from common Mendelian inheritance. This may lead to mistyping of $\mathrm{ABO}$ phenotype and adverse transfusion reactions. Aims: The evaluation of $\mathrm{ABO}$ typing using microplate-based system is required to prevent errors and to standardize protocols.

Methods: Total 28 cis-AB patients' blood samples collected from May 2011 to February 2015 were tested for ABO typing with four different methods as follows: method 1, a microplate-based system (Galileo NEO, Immucor, GA, USA); method 2, manual plate-tube method; method 3, manual column agglutination test using Ortho BioVue ABO-Rh/Reverse (Ortho, Raritan, NJ, USA), and method 4, manual column agglutination test using DG Gel ABO/Rh (2D) (Grifols, Barcelona, Spain). ABO genotyping for cis- $\mathrm{AB}$ confirmation were performed by direct DNA sequencing on exon 6 and 7 of the $A B O$ gene. $A B O$ phenotype with weak B expression in front typing and presence of weak anti-B in the back typing was defined as 'highly suspected cis-AB' is defined. We analyzed the detection rate of 'highly suspected cis-AB' as well as front/back typing discrepancies on each methods.

Results: All 28 specimens were confirmed as cis-AB: $C i s A B / 01,60.7 \%$; CisAB/O1v $32.1 \%$; Cis $A B / A 1,7.1 \%$. Using methods 1 through method 4, $4(14.3 \%), 22(78.6 \%)$, $18(64.3 \%)$, and $20(71.4 \%)$ of 28 samples were concluded as 'highly suspected cis$A B$ ', respectively. In detection rate of $\mathrm{ABO}$ discrepancies, method 1 showed 'Not Typed Determined (NTD)' in 24 (85.7\%) samples and AB in 4 (14.3\%). Twenty seven (96.4\%), $28(100.0 \%)$, and $28(100.0 \%)$ specimens resulted in front/back discrepancies in method 2, 3, and 4, respectively. One (3.6\%) was showed as phenotype AB in method 2.

Conclusions: If we perform cis- $A B$ patients' $A B O$ phenotype using only a microplate method, we can conclude wrong results (failure rate: $14.3 \%$ [4 out of 28]). Therefore, not to miss a cis- $A B$, the system must be worked together with manual methods and $\mathrm{ABO}$ genotyping, for new arrival and ambiguous patients.

\section{P-151}

PAROXYSMAL COLD HEMOGLOBINURIA: ROLE OF HOSPITAL TRANSFUSION MEDICINE \& IMMUNOHEMATOLOGY DEPARTMENT IN THE DIAGNOSIS

S Sood and S Rani

Saket City Hospital, New Delhi, India

Background: Paroxysmal cold hemoglobinuria (PCH), an autoimmune haemolytic anaemia caused due to polyclonal IgG anti-P autoantibody binding to red blood cel surface antigens and is characterized by hemoglobinuria, typically after exposure to cold temperatures.

Case Presentation: An 84-year-old female patient started developing breathing difficulty off and on with generalised weakness and loss of appetite. There was continuous fall in hemoglobin and continuous evidence of intravascular hemolysis since last 15 days. After ruling out many common diagnosis, Donath -Landsteiner Tes was done which showed Immunoglobulin G, IgG, antibodies, appearing at $4 \mathrm{c}$ and hemolysing at $37^{\circ} \mathrm{C}$ in the presence of complement. Peripheral smear showed anisopoikilocytosis and spherocytosis. Reticulocyte count was on the lower side $(0.5 \%)$.Forward and reverse blood grouping showed no group discrepancy .Direct antiglobulin test (direct Coombs test, DAT) was positive with the monoclonal C3 antisera and negative with the monoclonal anti-IgG. ICT, Indirect Coombs Test was negative. Syphilis was tested negative by Treponema pallidum hemagglutination assay (TPHA).

Results: Patient was diagnosed as a case of PCH based on the test results. Conclusions: Detecting blood antibodies, auto, allo, cold or warm, is important. The role of transfusion medicine laboratory in the diagnosis of patient PCH is emphasised. (198 Words)

\section{P-152}

PREDICTIVE VALUE OF ANTI-D SUBCLASESS IN HEMOLITIC DESEASE OF THE NEWBORN

E Velkova, V Dejanova Ilijevska, T Makarovska-Bojadzieva, L Mitevska, M Blagoevska, I Nikoloska and J Samonikov-Tosevska

Institute of Transfusion Medicine, Skopje, Macedonia

Background: Hemolytic disease of the newborn (HDN) continues to be a clinical problem in spite of the existing prophylaxis. To date, none of the available tests developed to predict the severity of HDN, has provided complete reliability. 
Aim: To evaluate the correlation of anti-D IgG1 and IgG3 subclasses with the clinical severity of HDN.

Material and methods: The IgG subclasses of anti-D antibodies were estimated in 45 pregnant women, using ID-card DAT IgG1/IgG3 (BioRad), with two dilutions of the subclasses, which indicates their concentration.

Results: IgG1 antibodies were detected in 15 samples. In 7 cases with high concentration of IgG1 (dilution 1:100) the newborns developed severe HDN.In the remaining 8 cases with low IgG1 concentration (dilution 1:1) the newborns showed symptoms of moderateto weakHDN. IgG1 and IgG3 antibodies were detected in 25 pregnant women. In 16 of them, the concentration of both subclasses (6 cases) or at least one of the subclasses (10 cases) was high with dilution of 1:100. The corresponding subclasses and their concentration were estimated in the cord blood of their newborns all of which developed severe HDN (in two cases, the pregnancy was terminated at 21 and 25 weeks of gestation because of fetus mortus). In 9 of the pregnant women, both subclasses were with low concentration and the newborns developed moderate HDN. In 5 cases with IgG3 only antibodies, their concentration was low (dilution 1:1) and the newborns developed weak or moderate HDN.

Conclusion: High concentration IgG1 antibodies was cause of sever HDN in 23 $(51 \%)$ of the cases. High concentration of IgG3 antibodies was the cause of severe HDN only in combination with IgG1 which was found in $6(13 \%)$ of the cases. When both of the subclasses IgG1 and IgG3 were present, the HDN was with stronger intensity than in the cases with only IgG1 or IgG3. These results suggest that the IgG1 and IgG3 subclasses of anti-D could be useful parameter when included in the multi-parameter protocol for evaluation of the severity of HBFN.

\section{P-153 \\ BLOOD GROUP ANTIBODY PROFILE ON ICTERIC NEONATUS IN PERSAHABATAN GENERAL HOSPITAL}

D Lesthiowati ${ }^{1}$, U Sukorini ${ }^{2}$ and T Triyono ${ }^{2}$

${ }^{1}$ Persahabatan Hospital, Jakarta Timur, Indonesia ${ }^{2}$ Medical Faculty of Gadjah Mada University, Yogyakarta, Indonesia

Background: Incidence of Hemolytic Disease in the Newborn (HDN) on neonatal hyperbilirubinemia quite much cause even death. Hemolytic state in neonates is due to the presence of maternal IgG antibodies enter the circulation of the neonate through the placenta can attack erythrocytes causing lisis of erythrocytes in neonates. The presence of maternal antibodies in the circulation which has been attached to the baby's blood can be checked with Direct AntiglobulinTest (DAT).

Objective: Knowing the proportion of Direct Antiglobulin Test (DAT) examination result and the profile of blood type antibodies in neonates with suspected jaundice Hemolytic Disease of the Newborn (HDN) in the Persahabatan General Hospital.

Method: The research design used in this study is analytical descriptive, the study subjects were infants born in the Persahabatan General Hospital with bilirubin $\geq 5 \mathrm{mg} / \mathrm{dl}$. A total of 113 subjects were included in this study examined blood type, DAT and antibody screening using a screen cell (three cells).

Result: A total of 113 neonates consisted of 55 women and 58 men with a mean age of 2.80 days with arange of 1-5 days. Of all study subjects found positive DAT as much as $23 \%(n=26)$, while on the subject of DAT positive blood group antibodies obtained as much as $84.62 \%(\mathrm{n}=22)$. Antibodies to blood group system of regular (ABO) is composed of anti-A IgG 9.09\% $(n=2)$, anti-B IgG 40.91\% ( $n=9)$, anti$A$ and $B$ IgG $45.45 \%(n=10)$. In one patient was found several possibilities for irregular antibodies, namely anti-Lea, anti-S, anti-M, anti-N, anti-Jka, anti-Fyb, anti-K, anti-c andanti-E.

Conclusion: The proportion of Direct Antiglobulin Test results of the examination in neonates born at Persahabatan General Hospital was 23\%. Profile antibodies are found in neonates HDN is a regular system of $\mathrm{ABO}$ antibodies and irregular system of non $\mathrm{ABO}$ antibodies include anti-c, anti-Fyb, anti-Jka, anti-M, anti-Lea

\section{P-154}

\section{A CASE OF RARE JR $^{\mathrm{A}}$ ANTIGEN-NEGATIVE AND ANTI-JR ${ }^{\mathrm{A}}$ ANTIBODY POSITIVE PATIENT WHO NEEDED EMERGENCY A BLOOD TRANSFUSION}

S Yoshida, H Miyano and Y Kajikawa

National hospital organization Fukuyama Medical Center, Fukuyama, Japan

A patient who sustained a fracture of the femoral neck was brought to our hospital by ambulance on December 15, 2014. She needed a blood transfusion for severe anemia and a total hip replacement operation. However, she showed negative results for $\mathrm{Jr}^{\mathrm{a}}$ antigen and positive results for anti-Jr ${ }^{\mathrm{a}}$ antibody. We report how we were able to identify irregular antibody and secure blood quickly using the Japanese Red Cross Society wide area network system.

Case: A woman about 80 years old was brought to our hospital by ambulance after femoral neck fracture. Her blood type was B RhD-positive. She was a multipara with no history of blood transfusion. Hemoglobin concentration was $5.9 \mathrm{~g} / \mathrm{dl}$. We ordered some red blood cell preparation to improve severe anemia and prepare for operation. Results: Positive results for irregular antibodies were found in screening examination for blood transfusion and all cross-matches with our stock Ir-RBC-LR were incompatible with her blood. After investigating cross-match testing thoroughly with the Hiroshima Blood Center branch of the Japanese Red Cross Society, she was revealed to be negative for $\mathrm{Jr}^{\mathrm{a}}$ antigen, and positive for anti-Jr ${ }^{\mathrm{a}}$ antibodies. We immediately ordered some suitable Ir-RBC-LR from the Japanese Red Cross Society blood center, and obtained 8 units of $\mathrm{Jr}^{\mathrm{a}}$ antigen-negative Ir-RBC-LR (Yamagata, 2 units; Kumamoto, 2 units; Osaka, 4 units), and 4 units of $\mathrm{Jr}^{\mathrm{a}}$ antigen-negative IrFTRC-LR (Kagawa). We were thus able to improve her severe anemia and perform surgery safely on December 19.

Conclusions: The Japanese Red Cross Society has built a wide area network system for blood supply beyond prefectural boundaries since April 2012. Even in urgent case like this, using the system extending beyond Hiroshima prefecture allows quick and sage performance of blood transfusions.

\section{P-155}

\section{SEVERE ALLOIMMUNIZATION, CAUSE OF DEATH IN PATIENTS WITH CHRONIC TRANSFUSION (THALASSEMIA) \\ A Azarkeivan, S Ferdowsi, M Moghadam and A Oshidar}

Iranaian Blood Transfusion Organization (IBTO), Research Center, Tehran, Iran

Chronic Transfusion is treatment of congenital anemia's such as thalassemia patients. But this treatment has some consequences. Transfusion reaction is one of these consequences. Alloimmunization against red blood cell (RBC) antigens is an immune response usually stimulated by RBC subgroups in the course of treatment with chronic blood transfusion and is one of the complications of transfusion reaction. The development of anti-red cell antibodies (alloantibodies and/or autoantibodies) can significantly complicate transfusion therapy and even may produce life threatening event. Here we describe four cases with thalassemia major and intermedia who developed post transfusion severe hemolysis because of alloimmunization. In all cases the cause of death was due to a very low hemoglobin level. Three of them were intermediate patients who had infrequent transfusion and developed severe alloantibody autoantibody after transfusion and did not respond to any therapy and finally died because of severe anemia one of them was thalassemia major who developed autoantibody after common cold episode and severe hemolysis and acute renal failure. He survived from renal failure but autoimmunization was worsened his condition gradually and he did not respond to any immunosuppressant therapy and finally he died because of severe anemia and infection .

Table 1: Serologic results of the patients.

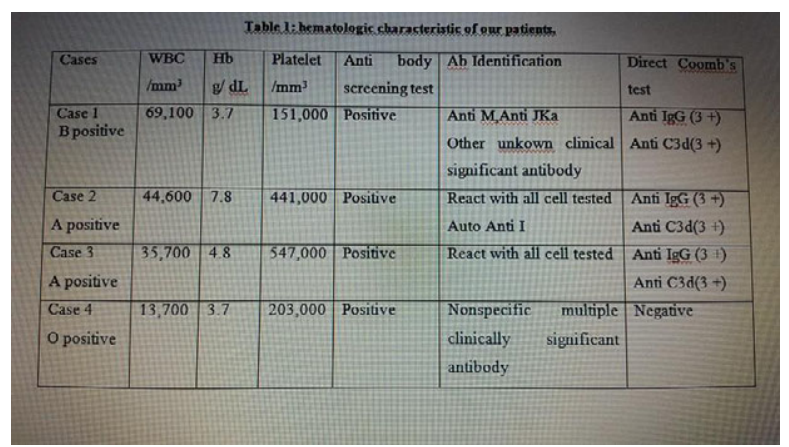

(C) 2015 The Author Vox Sanguinis (C) 2015 International Society of Blood Transfusion Vox Sanguinis (2015) 109 (Suppl. 2), 1-96 
P-156

A CASE REPORT OF PATIENTS WITH ANTI-C,D TRANSFUSED WITH RR AND 'MIA' ANTIGEN NEGATIVE PACKED RBC: INCLUDING CONSIDER TO HLA-DR TYPING

YJ Peng ${ }^{1}$, JS Chang ${ }^{2}$, YS Lin ${ }^{3}$, WLM Joyces ${ }^{3}$ and JS Sheu

${ }^{1}$ Department of Clinical Pathology, Taipei City Hospital, Ren Ai branch, Taipei, China Taiwan ${ }^{2}$ National Taiwan University Hospital, Taipei, China Taiwan ${ }^{3}$ Department of Clinical Medicine, Peking University Health Science Center, Beijing, China

The report is about a 65-Y0 male patient with chronic kidney disease and left large lung adenocarcinoma who is being transfused packed RBC with B, rr and 'Mia' antigen negative, because the patient's phenotype is rr with anti-C,D and his HLA typing results HLADR*09:13. The distribution of $\operatorname{rr}$ (ccddee) and $\mathrm{rr}^{\prime \prime}$ are $0.23 \%$ and $0.1 \%$ respectively and anti-'Mia' is the highest incidence of irregular antibody in Taiwan. Although patient with anti-C,D is compatible transfused $\mathrm{rr}$ and $\mathrm{rr}^{\prime \prime}$ red blood cel products, considered to HLA*09 presented strong association between anti-'Mia' in Taiwan and anti-E in Europe, we made the transfusion policy of transfusing Blood type B, rr and 'Mia' antigen negative packed RBC on the premise that his clinical condition isn't emergent. He has been transfused 3 times (packed RBC 6 units) with Blood type B and rr packed RBC products and no additional irregular antibodies in patient's plasma presented.

Table 1. Antibody screen, identification and phenotype.

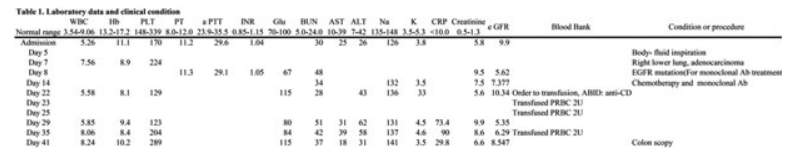

Table 2. Laboratory data and clinical condition.

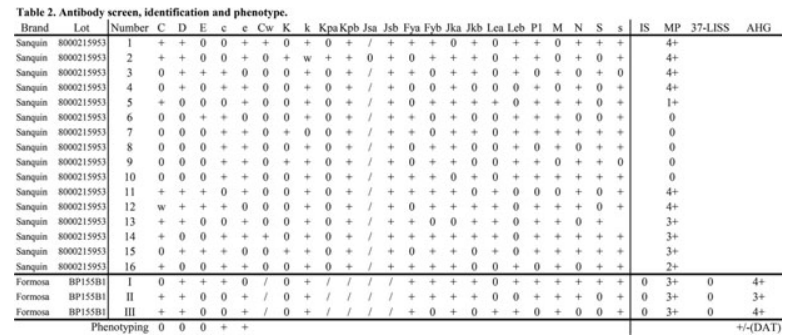

P-157

SHOULD WE DO DIRECT COOMBS TEST IN BLOOD DONOR ROUTINELY ?

D Retno, U Bachrussani, NK Ritchie and S Salim

Jakarta Blood Transfusion Unit, DKI Jakarta, Indonesia

Background: Cross-match test is part of compatibility test to see whether any antigen-antibody reaction between patient and donor. There are two method; minor and major cross-match. When we have major incompatibility major, it could be caused by free antibody in patient's serum or antibody/complement coated in donor's red blood cells (RBCs). The blood bank could return the component resulting major incompatibility to Blood Transfusion Unit (BTU) if it was suspected positive direct antiglobulin test (DAT) for further testing. In normal population, we could find positive DAT. Some BTUs abroad conduct DAT while doing antibody screening on donor's blood. Jakarta BTU had to consider the cost and benefit of testing DAT to donor's blood.

Aims: To get the prevalence of DAT in blood donor for calculating the cost and benefit of testing DAT in Jakarta BTU.

Methods: All packed red cells (PRCs) resulting major incompatibility returned to Jakarta BTU. The reference laboratory conduct blood grouping test, DAT, antibody screening, and cross-match.

Results: From Januari 2014 until July 2015, we have distributed 382.747 PRCs to all blood bank and hospitals around Jakarta. There were 72 of 382.747 or $0.019 \%$ of all distributed were returned to us due to major incompatibility. The reference lab found 51 of $72(70.8 \%)$ were only positive DAT, 16 of $72(22.2 \%)$ only positive in antibody screening and 7\% negative in both test (DAT and antibody screening). Among those samples, there were 2 samples were positive in both test. The prevalence of positive DAT among blood donors is 19 to 100.000 . If we run DAT in all donor would be costly compared to the cost of doing the cross-match.

(C) 2015 The Author

Vox Sanguinis (C) 2015 International Society of Blood Transfusion

Vox Sanguinis (2015) 109 (Suppl. 2), 1-96
Summary/Conclusions: DAT Test is not a mandatory test for blood screening for now, considering cost and benefit of DAT test.

$\mathrm{P}-158$

TNF- $\boldsymbol{\alpha}$ AND TGF- $\boldsymbol{\beta}$ LEVEL AFTER INTRAOPERATIVE ALLOGENEIC RED BLOOD CELL TRANSFUSION IN ORTHOPEDIC OPERATION PATIENTS

A Noroozi Aghideh, SR Sharifzadeh, H Hassani, MF Heydari and N Lashgari AJA University of Medical Sciences, Tehran, Iran

Background: Blood transfusion is associated with immunosuppression, referred to as transfusion-related immunomodulation (TRIM).

Aim: In this study, for the first time, changes in the concentration of TGF- $\beta$ and TNF$\alpha$ were measured postoperatively in orthopedic patients with intraoperational allogenic red blood cell transfusion. Considering the use of packed cell units with different ages, it is possible to suggest the more appropriate product for clinical applications. Methods: Two groups of 35 orthopedic surgery patients (with or without transfusion as case and control groups, respectively) were involved. Serum level of TNF- $\alpha$ and TGF- $\beta$ were measured by ELISA.

Results: Data suggested significant difference in age $(\mathrm{P}=0.0001)$, lowered hemoglobin $(\mathrm{P}=0.003)$ and hematocrit $(\mathrm{P}=0.003)$ between control and case groups. Preand post-operation levels of TNF- $\alpha$ and TGF- $\beta$ was not significantly different, however results showed a significant increase in both cytokines level after the operation $(\mathrm{P}=0.0001)$ in both groups.

Summary/Conclusions: Increased levels of TNF- $\alpha$ and TGF- $\beta$ are probably related to surgery and packed cell transfusion, respectively. Further studies using more packed cell units or other blood products, and assessment of more cytokines are needed to have better understand about this issue.

\subsection{Red Cell Immunology: Molecular}

\section{P-159}

\section{A NOVEL PROMOTER MUTATION (-76 G>C) IN THE ABO GENE IDENTIFIED FROM THREE INDIVIDUALS WITH $\mathrm{A}_{3}$ PHENOTYPE}

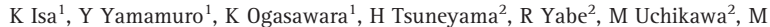
Satake $^{1}$ and $\mathrm{K}$ Tadokoro ${ }^{1}$

${ }^{1}$ Japanese Red Cross Central Blood Institute, Tokyo, Japan ${ }^{2}$ Jananese Red Cross Kanto-Koshinetsu Block Blood Center, Tokyo, Japan

Background and aims: Investigation of the molecular genetic basis of $\mathrm{ABO}$ varian phenotypes has been a continual challenge since the $A B O$ gene was identified in 1990. Several mutations in the $A B O$ gene were identified not only in coding exon and exon-intron boundaries but also in the promoter and transcription enhancer element in intron 1. We report here a novel promoter mutation identified in Japanese individuals with the $\mathrm{A}_{3}$ phenotype.

Methods: A standard serological technique was used for ABO grouping and the amounts of A antigen on RBCs were measured using a flow cytometer. Genomic DNA was extracted from whole blood and the $A B O$ gene was analyzed by PCR and sequencing. Promoter assay was performed by transient transfection into K562 cells using luciferase reporter plasmids [1].

Results and discussion: Red blood cells of three individuals showing mixed agglutination with anti-A and anti-B but not anti- $\mathrm{A}_{1}$ were observed in their plasma. Flow cytometric analysis using anti-A revealed a characteristic pattern of two main peaks of cells expressing A at a normal level compared with control group A cells, similar to that of 0 cells, with a small number of cells expressing $\mathrm{A}$ antigen ranging between the two main peaks. A transferase was detected in their plasma. From these results, the three individuals were typed as having the $A_{3}$ phenotype. Nucleotide sequence analysis of exons $1-7$ of the $A B O$ gene revealed that the status of two individuals was $A B O^{*} A 1.01 / 0$ and that of the third was $A B O^{*} A 1.02 / 0$, while al individuals had ' $76 \mathrm{G}>C$ mutation from the ATG start site of the $A$ gene. Luciferase assay revealed that $76 \mathrm{G}>\mathrm{C}$ mutation reduced the promoter activity to $23 \% \mathrm{com}-$ pared with the common $A$ allele.

Conclusion: We identified a novel $76 \mathrm{G}>\mathrm{C}$ mutation in the promoter region of the $A B O$ gene that affects the promoter activity, resulting in the $\mathrm{A}_{3}$ phenotype

Reference: 1. Takahashi Y, Isa K, Sano R, et al.: Presence of nucleotide substitutions in transcriptional regulatory elements such as the reythroid cell-specific enhancerlike element and the $\mathrm{ABO}$ promoter in individuals with phenotypes $\mathrm{A}_{3}$ and $\mathrm{B}_{3}$, respectively. Vox Sang; 2014, 107: 171-180. 
$\mathrm{P}-160$

This abstract has been withdrawn.

$\mathrm{P}-161$

A CIS-ABO4 ALLELE WITH A NONSYNONYMOUS SUBSTITUTION C796A IN EXON 7 OF ABO GENE IDENTIFIED IN A KOREAN FAMILY: THE FIRST CASE IN KOREA

JS Choi ${ }^{1}$, J Yoon ${ }^{1}$, HJ Youk ${ }^{1}$, JH Chang ${ }^{2}$, MA Jang ${ }^{1}$, JH Choi ${ }^{1}$, MH Nam ${ }^{1}$ and CS Lim ${ }^{1}$ ${ }^{1}$ Korea University College of Medicine, Seoul, South-Korea ${ }^{2}$ Korea University Ansan Hospital, Seoul, South-Korea

Background: The $A_{2} B$ phenotype is a subtype of the blood group $A B$. Some of $A_{2} B$ phenotypes may be caused by the cis- $A B$ genotype. The cis- $A B$ is a rare blood $A B O$ genotype with an unusual inheritance of both $\mathrm{A}$ and $\mathrm{B}$ genes on the same chromosome that results from a point mutation. Currently, four cis- $A B$ alleles have been identified, namely cis-ABO1, cis-ABO2, cis-ABO3, and cis-AB04. The cis-ABO1 allele has been the only one reported thus far in the Korean population and the overall frequency of the cis-AB blood group was reported to be $0.0354 \%$, which is relatively more common than the Japanese population $(0.0012 \%)$.

Aims: Recently, authors experienced a Korean family case with cis-ABO4 allele through the pedigree of two generations and report this case with a brief review of literatures.

Methods: $A B O$ serologic tests were performed on all of the family samples. The serum transferase activity levels of the proband were assayed by estimation of the degree of conversion of group 0 RBCs to A or B RBCs. ABO genotyping was performed by PCR-RFLP and DNA sequencing analysis to identify the ABO gene haploid of the proband and her family.

Results: Routine $\mathrm{ABO}$ typing of the proband's blood showed the result of phenotype $A_{2} B$ by the absence of red cell agglutination with anti- $A_{1}$ lectin. $H$ antigen was nearly undetectable and only anti-A was very weakly detected in the serum. She exhibited only strong B-transferase activity with no A-transferase activity. We determined that she harbored a cis-ABO4 allelle, having a single nucleotide substitution C796A predicting an amino acid change at residue 266 of leucine to methionine on the A102 background. Pedigree analysis revealed that the proband's twin sister, brothers, daughter, nephew and niece also had the cis-AB04 allele.

Summary/Conclusions: Our molecular studies for the first time confirm that cisABO4 allele does exist in the Korean population as well as in the previously reported Chinese population in Taiwan.

\section{$\mathrm{P}-162$}

\section{KLF1 MUTATIONS IN JAPANESE WITH IN (LU) PHENOTYPE}

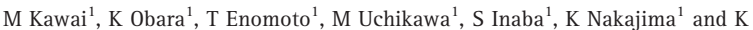
Ogasawara $^{2}$

${ }^{1}$ Japanese Red Cross Kanto-Koshinetsu Block Blood Center, Saitama, Japan

${ }^{2}$ Japanese Red Cross Central Blood Institute, Tokyo, Japan

Background: KLF1 encodes an erythroid transcription factor, KLF1 (Krüppel-like factor). Singleton et al. identified different heterozygous KLF1 mutations in most of the dominant $\mathrm{Lu}(\mathrm{a}-\mathrm{b}-)$ phenotype, which is known as $\operatorname{In}(\mathrm{Lu})$ phenotype, was likely caused by inheritance of a loss-of-function mutations in one allele of KLF1. $\operatorname{In}(\mathrm{Lu})$ is characterized by reduced expression of antigens in the Lutheran system and for CD44 glycoprotein and other blood group antigens (P1, AnWj, i, CD151 and Knops). Aims: We investigated $\operatorname{In}(\mathrm{Lu})$ Japanese to estimate the prevalence of the phenotype and to understand the molecular background.

Methods: Blood samples of $\operatorname{In}(\mathrm{Lu})$ phenotype were screened by an automated blood grouping system (PK7300; Beckman Coulter) with murine monoclonal anti-CD44 (CBC-121). The $\operatorname{In}(\mathrm{Lu})$ was confirmed by serology using anti- $\mathrm{Lu}^{\mathrm{a}}$, anti- $\mathrm{Lu}^{\mathrm{b}}$, including adsorption and elution tests. Genomic DNAs were extracted from whole blood and exons 1 to 3 of the KLF1 gene as well as exons 1 to 15 of the $L U$ gene were analyzed by polymerase chain reaction (PCR) and sequencing.

Results: One-hundred in 481,322 blood donors $(0.02 \%)$ were identified as $\operatorname{In}(\mathrm{Lu})$ phenotype. RBC samples of the 100 donors did not agglutinate with anti- $\mathrm{Lu}^{\mathrm{a}}$ and $\mathrm{Lu}^{\mathrm{b}}$ by indirect antiglobulin tests while the expression of $\mathrm{Lu}^{\mathrm{b}}$ antigen on the RBCs was confirmed by adsorption and elution tests. Nucleotide sequencing revealed that 92 in the 100 individuals had mutant $K L F 1$ allele with single nucleotide changes, heterozygote with common one, while remaining 8 individuals appeared to have common KLF1 alleles. None of the individuals had mutations in the $L U$ gene and all were $L U B / L U B$ genotype. We identified 7 known mutations and 22 new mutations in the KLF1 gene from the 92 individuals. Forty-five in the 92 individuals had one of the following mutations, c.862A $>\mathrm{G}$ (p.Lys288Glu) 19, c.947G $>$ A (p.Cys316Tyr) 15, or c.968C $>\mathrm{G}$ (p.Ser323Trp) 11, and those were major KLF1 alleles in the In(Lu) Japanese.

Conclusions: The prevalence in Japanese with $\operatorname{In}(\mathrm{Lu})$ phenotype was $0.02 \%$. We identified 7 known and 22 novel KLF1 alleles, and one of the new allele with c.862A $>\mathrm{G}$ mutation was most predominant in the $\operatorname{In}(\mathrm{Lu})$ Japanese.

P-163

\section{INVESTIGATION OF A PATIENT WITH A PAN-REACTIVE RED CELL ANTIBODY INHIBITED BY SOLUBLE CD 55: MPS REVEALS A HOMOZYGOUS NOVEL CROMER BLOOD GROUP ALLELE}

A Hyland ${ }^{1}$, E Schoeman ${ }^{1}, \mathrm{~K}$ McGrath ${ }^{1}$, B Wilson ${ }^{1}, \mathrm{HC}$ Lin $^{2}$, CW Wu ${ }^{2}$, RL Flower ${ }^{1}$, T Powley $^{1}$ and YW Liew ${ }^{1}$

${ }^{1}$ Australian Red Cross Blood Service, Brisbane, Australia ${ }^{2}$ Kaohsiung Blood Center, Kaohsiung, Taiwan

Background: The Cromer blood group system is carried on a GPI-linked glycoprotein, Decay Accelerating Factor (DAF or CD55). CD55 is also found on granulocytes, platelets and lymphocytes as well as red blood cells and in soluble forms in plasma. The ISBT Blood Group Data Base lists 10 SNP variants that are negative for high frequency antigens in this blood group system.

Antibodies to Cromer antigens are rare however there is some evidence of increased red blood cell destruction of serologically incompatible red cells, indicating the potential for transfusion reaction. We report a case where a patient presented with suspected transfusion reaction and a pan-reactive antibody. Samples were referred to the Australian Red Cross Blood Service Red Cell Reference Laboratory for further investigation.

Methods: Serological investigations were carried out using standard RCRL serological procedures. This included testing whether the antibody was inhibited by soluble CD55 (DAF).

On request, targeted DNA exomic massive parallel sequencing was performed using the TruSight One Sequencing Panel (Illumina Inc). This panel is validated for targeted genotyping including blood group analysis. Analysis targeted DNA sequences for exonic and $3^{\prime}$ untranslated regions including those for the Cromer blood group gene, CROM. DNA sequence reads were aligned against the ISBT CROM *01 gene reference sequence.

Results: Soluble CD55 (DAF) completely inhibited binding of the patient's antibody to red cells. Sequencing analysis of the CROM gene (also called CD55) did not reveal any of the known variants. A homozygous nucleotide change c.203G $>$ A was the only variation from the reference exomic sequence for $C R O M^{*} O 1$. This change predicts a p.S68N (serine to asparagine) change in the DAF protein. The c.203G $>$ A mutation is not listed among the polymorphisms on international databases nor on the ISBT blood group database.

Summary and conclusion: The serology and sequencing data are consistent with the patient being homozygous for a variant CROM allele that generates an amino acid change in the first of four CCP (complement control protein) repeat motifs. Serologically this variant is a further example of loss of a high frequency antigen. Other Cromer system antigens such as $\mathrm{Tc}^{\mathrm{b}}$ and $\mathrm{Tc}^{\mathrm{c}}$ (52Leu, 52Pro), WES (82Arg) and CROM: -6 (Ile80Asn)(also loss of a high frequency antigen) are located within 16 residues of this substitution. Further studies with synthetic peptides predicted to mimic this antigen are in progress.

The SNPs that define the CROM alleles are not represented on many SNP genotyping platforms, as antibodies to these antigens are rare. MPS provided a strategy for resolving this complex investigation by detection of a novel allele in the Cromer blood group system.

\section{P-164}

\section{PRODUCTION OF CHIMERIC MOUSE-HUMAN IGM ANTI-MUR BY RECOMBINANT TECHNIQUES}

R Tobita

Japanese Red Cross Society, Tokyo, Japan

Background: Immunoglobulin (Ig) M is a very useful Ig class for blood grouping as it directly agglutinates red blood cells (RBCs) by simple saline method or automated blood grouping equipment including PK7300 (Beckman coulter). The variable region of the heavy chain of a murine anti-Mur IgG was grafted onto the constant region of human IgM and co-expressed with light chain derived from the same antibody. GP.Mur phenotype and antibodies to GP.Mur RBC are relatively common is South-

(C) 2015 The Author

Vox Sanguinis (C) 2015 International Society of Blood Transfusion Vox Sanguinis (2015) 109 (Suppl. 2), 1-96 
East Asia. Antibodies to GP.Mur RBC have been responsible for immediate and delayed hemolytic transfusion reactions, and severe hemolytic disease of the newborn. It is recommended that a GP.Mur RBC are included in antibody screening panels in South-East Asia.

Aim: To produce chimeric mouse-human anti-Mur for use in the clinical laboratory and automated blood grouping equipment.

Methods: The full-length heavy- and light-chain domains was amplified from cDNA of a single hybridoma clone using the polymerase chain reaction (PCR) with immunoglobulin specific primers. The heavy chain variable domain was amplified by PCR, and was the expression vector ligation together human IgM constant domain fragment. Likewise, the light chain variable domain was amplified by PCR, were ligation into an expression vector with human kappa constant domain fragment. The expression vectors of the heavy- and light chain were restriction enzyme treatment to ligation as one of the expression vectors. The construct was transfected CHO cell line to express of the antibodies.

Results: The heavy and light chain variable domains from the murine anti-Mur IgG were cloned using PCR amplification and inserted into an expression vector with human IgM constant domain. The chimeric mouse-human IgM anti-Mur directly agglutinated $4+$ with the Mur+ RBCs (GP.Mur, GP.Bun and GP.Kip) in saline at room temperature, resembling the specificity of the original murine antibody. The chimeric anti-Mur also determined clearly Mur+ or Mur- RBCs by PK7300.

Conclusions: Mouse-human chimeric IgM from a murine IgG monoclonal anti-Mur has been successfully engineered for use in the clinical laboratory, and PK7300 for large number of blood samples.

\section{P-165}

\section{HIGHER LEVEL OF MUR ANTIGEN EXPRESSION ON THE RED CELLS OF GP.MUR HOMOZYGOTES THAN THAT OF HETEROZYGOTES}

L Wei, Y Ji, Z Wang, Z Shan, J Wen and G Luo

Guangzhou Blood Center, Guangzhou, China

Background: GP.Mur was one of GP (B-A-B) hybrid glycophorins in MNS blood group system. It was reported to be the most common hybrid glycophorin in Southeast Asia, with a frequency of 7.3\% in Taiwan, 8.0\% in Thailand, and 6.3\% in Hongkong. In our previous study, the frequency of heterozygous GP.Mur was about $9 \%$, and homozygous GP.Mur was very rare with an inferred frequency of about $0.2 \%$ in Guangzhou. The comparison of the level of antigens expressed on the red cells between the homozygous and heterozygous GP.Mur has not been investigated. In this study, Mur antigen expression was detected by flow cytometry in the individuals with GP.Mur homozygotes and heterzygotes to explore whether any differences exist.

Aim: To compare the level of Mur antigen expressed on the surface of the red cells with homozygous and heterozygous GP.Mur.

Methods: The whole blood samples of 528 volunteer blood donors from Guangzhou blood center were collected. Monoclonal anti-Mur was used to detect Mur antigen expressed on the red cells of each donor. Genomic DNAs of Mur positive donors were extracted, and then, GYPB pseudoexon 3 and part of adjacent intron 2 and 3 was sequenced by direct sequencing. Flow cytometry was performed to detect Mur antigen expression on the red cells of GP.Mur homozygotes and heterozygotes.

Results: In total, fifty-one Mur positive samples were identified with a frequency of $9.7 \%(51 / 528)$ by serological testing. Further sequencing of GYPB pseudoexon 3 and adjacent introns in these donors showed heterozygous GYP.Mur allele existed in 48 donors, homozygous GYP.Mur allele in 2 donors, and a novel heterozygous GYP.Bun allele in one donor. Flow cytometry results revealed higher Mur antigens expression on the red cells of GP.Mur homozygotes than heterozygotes.

Conclusions: GP.Mur was the main hybrid glycophorin in Guangzhou with a high frequency of 9.5\% (50/528). Since homozygous GP.Mur individual expressed higher level of Mur antigens, whether GP.Mur homozygotes could more easily stimulate the recipient to produce the alloantibodies than GP.Mur heterozygotes during incompatible transfusion should be further investigated.

\section{P-166}

\section{THE DISTRIBUTION OF THE RED CELLS STA (STONES)} ANTIGEN IN CHINESE BLOOD DONORS FROM GUANGZHOU GP Luo, Z Wang, YL Ji, L Wei and JZ Wen Guangzhou Blood Center, Guangzhou, China

Background: $\mathrm{St}^{\mathrm{a}}$ is a low-frequency antigen in MNS blood group system. It could be expressed by the variant hybrid glycophorins, which usually resulted from the

\section{(C) 2015 The Author}

Vox Sanguinis (C) 2015 International Society of Blood Transfusion

Vox Sanguinis (2015) 109 (Suppl. 2), 1-96 hybrid GYP (B-A) gene with unequal crossing over at the intron 3 of GYPA and GYPB. So far, six different $\mathrm{St}^{\mathrm{a}}$ related alleles (type A to F) have been identified, differing in the location of crossing-over points in the intron 3. And the frequency of $\mathrm{St}^{\mathrm{a}}$ is $1.0-5.2 \%$ in Taiwan, $6 \%$ in Japanese. But there is no report about the $\mathrm{St}^{\mathrm{a}}$ frequency of Chinese in Mainland of China.

Aim: To study the distribution of $\mathrm{St}^{\mathrm{a}}$ in Chinese blood donors from Guangzhou and clarify the molecule background

Methods: A PCR-based method was used to detect St ${ }^{\mathrm{a}}$ in 390 Chinese blood donors from Guangzhou Blood Center. Upstream primer was GYPB-specific and downstream primer was GYPA-specific. Several DNA samples with sequence-confirmed $\mathrm{St}^{\mathrm{a}}$ allele detected previously were used as positive control. Then, the samples with $\mathrm{St}^{\mathrm{a}}$ specific PCR product were analyzed by direct sequencing to clarify the allele types. The extra two presumed $\mathrm{St}^{\mathrm{a}}(+)$ samples were also included.

Results: Five donors with $\mathrm{St}^{\mathrm{a}}$ specific PCR product were identified in 390 (5/390 $1.3 \%)$ donors tested. Combined with the two extra suspected $\mathrm{St}^{\mathrm{a}}(+)$ samples, the PCR product of a total of 7 samples were analyzed by direct sequencing. Sequencing results revealed 5 of them with heterozygous $\mathrm{St}^{\mathrm{a}}$ type B allele and the other two Samples with heterozygous $\mathrm{St}^{\mathrm{a}}$ type E allele.

Conclusions: The frequency of $\mathrm{St}^{\mathrm{a}}$ in China South Han from Guangzhou is around $1.3 \%$, which is similar with Taiwanese, higher than Europeans. The distribution of anti-St ${ }^{\mathrm{a}}$ in Chinese population is still needed for further investigation in the future.

\section{P-167 DEVELOPMENT OF REAL-TIME PCR AND MELTING CURVE ANALYSIS FOR THE RAPID DETECTION OF DEL PHENOTYPE} YJ Hong ${ }^{1}, \mathrm{H} \mathrm{Kim}^{2}$, D Cho ${ }^{3}$, KU Park ${ }^{1}$, J Song ${ }^{1}$ and KS Han ${ }^{2}$

${ }^{1}$ Seoul National University Bundang Hospital, Seongnam, South-Korea ${ }^{2}$ Seoul National University College of Medicine, Seoul, South-Korea ${ }^{3}$ Samsung Medical Center, Seoul, South-Korea

Background: The Rh blood group D antigen is clinically important since it is associated with transfusion reactions in the alloimmunized individuals with RhD. DEL, a type of variant $\mathrm{RhD}$, is hard to ascertain in routine blood bank, since it expresses extremely low level of D antigens. However, it is capable of alloimmunizing a patient when transfused to RhD-negative individuals. Thus, the detection of DEL in donors with serologically $\mathrm{RhD}$-negatives is crucial in transfusion medicine.

Aims: In this study, we developed real-time PCR and melting curve analysis for the rapid detection of DEL phenotype.

Methods: Total $325 \mathrm{RhD}$-negative individuals were involved in this study. RhD negativity were confirmed with minimum two different reagents: BIOSCOT Anti-D IgM/ IgG blend (Merck Millipore, Darmstadt, Germany), Anti-D Bioclone (Ortho Clinical Diagnostics, Raritan, NJ, USA), or NOVACLONE Anti-D IgM + IgG (Immucor, Norcross, GA, USA). We designed primers and probes targeting DEL-specific single nucleotide polymorphism (SNP) in exon9 and the DEL variants were analyzed by melting temperature. The SNP at RHD $1222 \mathrm{~T}>\mathrm{C}$ and $1227 \mathrm{G}>\mathrm{A}$ were confirmed by direct DNA sequencing on exon 9 of the RHD gene.

Results: Among 325 D-negative individuals, 244 individuals (75.1\%) were true $R H D$ deletion given that amplification of exon 9 was failed in real-time PCR and melting curve analysis. Of remaining 81 individuals, 56 (17.2\%) showed distinguishing melting temperature from that of D deletion as follows: Fifty three $R H D 1227 \mathrm{G}>\mathrm{A}$ specimen had specific dual melting temperature of $55.24^{\circ} \mathrm{C}$ and $62.10^{\circ} \mathrm{C}$ in average; three RHD $1222 \mathrm{~T}>\mathrm{C}$ had single melting temperature at $61.94^{\circ} \mathrm{C}$ in average. Twenty-five specimens were revealed to have RHD genes other than DEL.

Conclusions: In this study, we developed rapid DEL typing methods by real-time PCR and melting curve analysis. The targeted SNP were relatively common in Asian. This method would be helpful in screening donors with serologically RhD-negatives and in prevention of alloimmunization of $\mathrm{RhD}$ individuals from $\mathrm{DEL}$.

\section{P-168}

DETECTION OF DEL PHENOTYPE USING ADSORPTION ELUTION TECHNIQUE AND SEQUENCE SPECIFIC PRIMERPOLYMERASE CHAIN REACTION (SSP-PCR)

S Ramli, E Kamarudin, M Bahari, NEI Maswan, D Nordin and A Che Nor MARA University of Technology (UiTM), Puncak Alam, Malaysia

Background: DEL phenotype is the most weakly expressed D antigen which only detected by Adsorption-Elution technique. It is capable to induce anti D immunization in Rhesus (Rh) negative recipient. Currently in Asia, RHD1227A is a useful genetic marker to identify DEL phenotype. 
Aims: The objective of this study was to detect DEL phenotype in Rh negative blood using Adsorption-Elution technique and Sequence Specific Primer-Polymerase Chain Reaction (SSP-PCR).

Method: A total number of 43 serologically $\mathrm{Rh}$ negative blood samples collected from National Blood Centre, Malaysia were tested for Rh phenotyping, undergo three different adsorption-elution technique which are by chloroform, freeze - thaw and heat elution technique followed by detection of RHD1227A allele by SSP-PCR

Results: Rh-phenotype identified were ccee with 79.07\%, Ccee with 13.95\%, 4.65\% of ccEe phenotype and CCee phenotype with only $2.33 \%$. Out of 43 samples, only one sample was identified as DEL phenotype carrying RHD1227A allele when tested using SSP-PCR but none was identified from Adsorption-Elution test.

Conclusion: RHD1227A analysis able to provide an accurate laboratory screening protocol for DEL detection in Rh negative population. As this study was a preliminary study, it is recommended, larger sample size and statistically the specificity and sensitivity can be determined between for RHD1227A detection as compared with the traditional adsorption/elution technique.

\section{P-169}

\section{EXTENDED RED CELL ANTIGEN TYPED REGULAR BLOOD DONORS FOR THALASSEMIC PATIENTS}

S Kulkarni, B Choudhary, H Gogri and K Ghosh

National Institute of Immunohaematology, Mumbai, India

Background: Thalassemic patients are at a risk of alloimmunization after multiple RBC transfusions because of disparity between donor and recipient antigen profile. The presence of RBC alloantibodies further complicates transfusion therapy. As a result, the two most common problems in blood bank pertain to finding the correct blood group status of multitransfused patients and issuing appropriate antigen negative blood for transfusion.

In multi-transfused patients hemagglutination fails to determine the patients phenotype due to donor derived erythrocytes from previous transfusions. DNA techniques are now being used for blood group antigen typing. Molecular genotyping for clinically significant blood group antigens in thalassemics and extended blood group phenotyping (other than $\mathrm{ABO} \mathrm{Ct} \mathrm{Rh}$ ) in regular donors, will facilitate the procurement of antigen matched blood in patients who have not produced antibodies as well as in identification of appropriate antigen negative RBCs for transfusion in alloimmunised patients.

Matching for the critical antigens of Rh, Kell, Kidd and Duffy blood group systems has been shown in literature to dramatically minimize alloimmunization. However, there is no universal agreement on the most appropriate strategy on selection of RBC units for multitransfused patients. Many transfusion services provide antigen matched donor RBCs for C, E and $\mathrm{K}$ antigens and in few countries the matching is extended to Kidd and Duffy blood group systems.

Aim: To create a database of extensively typed donors for clinically significant and common blood group antigens of Rh, Kidd, Kell, Duffy systems for multitransfused thalassemic patients.

Methods: Blood samples from seventy multitransfused beta-thalassemia patients were tested for common antigens or genes of Rh, Kell, Kidd and Duffy blood group system by hemagglutination and PCR techniques. Two hundred and forty five ' 0 ' group regular blood donors were also phenotyped for the above mentioned antigens. Results: Based on partial matching of donors and patients for Rh antigens (C, c, E, e), we identified $97 R_{1} R_{1}$ donors for 37 thalassemic patients, $66 R_{1} r$ donors for 15 patients, 25 donors $R_{1} R_{2}$ for 11 patients, five $R_{0} r$ donors for four patients and $35 \mathrm{rr}$ donors for three patients. A total of 153 regular donors matching perfectly for D, C, $\mathrm{c}, \mathrm{E}, \mathrm{e}, \mathrm{Fy}^{\mathrm{a}}, \mathrm{Fy}^{\mathrm{b}}, \mathrm{K}, \mathrm{k}, \mathrm{Jk}^{\mathrm{a}}, \mathrm{Jk}^{\mathrm{b}}$ antigens were identified for 70 thalassemic patients. For eleven alloimmunised patients 100 regular donors matching perfectly for above antigens were identified.

Conclusion: Blood group genotyping enabled determination of correct antigen profile where serology failed. Database of regular blood donors phenotyped for common antigens of Rh, Duffy, Kell, Kidd blood group systems was created which will help in providing extended antigen matched RBCs for thalassemic patients. This will thereby improve the quality and effectiveness of transfusion therapy.

\subsection{Platelet Immunology}

$\mathrm{P}-170$

This abstract has been withdrawn.

\section{P-171}

\section{EVALUATION OF THE MANAGEMENT WITH CROSSMATCH- COMPATIBLE PLATELET FOR REFRACTORY PATIENTS AND PREVALENCE OF ANTIBODIES DEVELOPED IN THESE}

\section{PATIENTS}

J Wang, J Deng, X Xu, W Xia, Y Shao, H Ding, Y Chen, J Liu, D Chen and X Ye Guangzhou Blood Center, Guangzhou, China

Background: Alloantibodies against platelets could induce platelet transfusion refractoriness in patients receiving intensive platelet transfusions. The use of crossmatch-compatible platelets improves posttransfusion corrected count increments (CCIs) in these patients.

Aims: Few studies have addressed the efficacy of crossmatch-compatible platlet transfusions and the development of the anbitibodies during the period.The aim of this study was to determine the effectiveness of the management with crossmatchcompatible platelet for refractory patients and the prevalence of the antibodies developed in these patients.

Materials and methods: Medical records of patients who were refractory to random single-donor apheresis platelet units between January 2014 and December 2014 were reviewed. Enzyme-linked immunosorbent assay (ELISA) was used to detect anti-HLA and anti-HPA antibodies in patients' serum. The platelet crossmatch assays were performed to select the compatible platelet units (PLTs) with a commercial solid-phase adherence kit.

Results: 204 patients were enrolled. Antibodies with HLA and/or HPA were detected in $125(61.27 \%)$ patients, including $83(66.4 \%)$ with only HLA antibodies, $8(6.4 \%)$ with only HPA antibodies, $34(27.2 \%)$ with both HLA and HPA antibodies. A total of 550 crossmatched PLTs were administered to those antibody-positvie patients with a mean \pm standard deviation CCI of $8000 \pm 6500$ ( $\mathrm{n}=310$ units with adequate $24-\mathrm{h}$ posttransfusion counts), a significant improvement over random PLTs $(\mathrm{P}<0.001)$ The platelet-specific antibodies were developed in 11 patients, 4 patients with antibody-negative transformed to antibody-positive, the other 7 patients had detected with HLA and/or HPA antibodies in initial ELISA assay, but appeared to demonstrate decreasing alloimmunization over successive assays. Our data do not shown any evidence for increasing alloimmunization in response to repeated transfusions of crossmatched PLTs.

Conclusions: HLA and/or HPA alloimmunization is an important factor to cause platelet transfusion refractoriness. For most patients, crossmatch-compatible PLTs offers an effective and rapid first-line approach to management of PLT transfusion refractoriness and demonstrates no trend toward progressive alloimmunization.

\section{$\mathrm{P}-172$}

\section{HUMAN PLATELET ANTIGEN (HPA) GENOTYPE AND ANTI-}

\section{PLATELET ANTIBODY DETERMINATION IN APLASTIC}

\section{ANEMIA PATIENT}

E Arwanih $^{1}$, NK Ritchie ${ }^{2}$ and SM Asmarinah

${ }^{1}$ FKUI, Jakarta, Indonesia ${ }^{2}$ Jakarta Blood Center, Jakarta, Indonesia

Background: Aplastic anemia is characterized by peripheral blood pancytopenia and bone marrow hypocellularity due to absence of a clonal hematological process. Therefore, patient needs transfusion support routinely. The incidence is approximately four per million in Asia population including Indonesia. Transfusion of HPA incompatible donor platelets may initiate the production of alloantibodies against polymorphic glycoprotein structure residing on donor's platelet, leading to failure to respond to platelet transfusion therapy (known as platelet transfusion refractoriness) in aplastic anemia patients. In contrast, the specificity of alloantibodies in aplastic anemia patient is unknown. Therefore, it is important to determine the specificity of alloantibodies and the allele of HPA in aplastic anemia patient for the management of this disease.

Aims: Determination of anti-platelet antibody and the allele of Human Platelet Antigen in aplastic anemia patients.

Methods: DNA derived from 29 aplastic anemia patients in Cipto Mangunkusumo Hospital-Jakarta was genotyped for HPA-1 to HPA-6 and HPA-15 alleles by using of polymerase chain reaction - sequence specific primer (PCR-SSP) method. Antiplatelet antibody in serum sample was screened by whole platelet ELISA method. The specificity of positive samples from whole platelet ELISA method was determined by Monoclonal Antibody Immobilization of Platelet Antigen (MAIPA) method.

Results: The gene frequencies of the rare allelic variants HPA- $1 \mathrm{~b},-2 \mathrm{~b}$, and $-6 \mathrm{~b}$ were $0.04,0.06$, and 0.04 , respectively. However, HPA- $4 \mathrm{~b}$ and $-5 \mathrm{~b}$ were not found in this cohort, indicating that the risks of alloimmunization caused by incompatibility of these HPA systems are very low. The gene frequencies of HPA-3b and $-15 b$ were 0.51 and 0.44 , this indicate that the risks of alloimmunization caused by incompati-

(C) 2015 The Author

Vox Sanguinis (C) 2015 International Society of Blood Transfusion Vox Sanguinis (2015) 109 (Suppl. 2), 1-96 
bility of these HPA systems are extremely high. Sixteen sera from 29 aplastic anemia patients have positive reaction with ELISA method. This shows that approximately 55\% of total sample had been exposed by incompatible platelet antigen from donors in platelet transfusion. Using MAIPA assay, we found 7 sera have anti- $\beta-2$ microglobulin antibody (HLA Class I), 2 sera have anti-GPIIb/IIIa antibody (HPA-3), and 1 serum has anti-CD109 antibody (HPA-15).

Conclusions: The blood center should supply HPA-genotyped platelet for multi transfusion patients such as aplastic anemia, that could result in HPA alloantibody generation. The HPA-genotyping should be focused on HPA-3 and HPA-15.

\section{P-173 HUMAN PLATELET ANTIGEN DIVERSITY IN PENINSULAR MALAYSIA}

WS Wan Ubaidillah, N Hanim Kamis, SM Manaf, Z Zulkafli and EH Atan

Universiti Sains Malaysia, Kubang Kerian, Malaysia

Background: Human genetic structure in Peninsular Malaysia is complex and comprises several groups of highly differentiated genetic lineages; e.g. Negrito, Senoi, Proto-Malays, Chinese and Indians. We have screened several genetic markers in these people including human platelet antigen (HPA) reported here.

Aims: The major aims of our study were to compile allelic data for HPA-1 to -6 and -15 systems in Malay subethnic and Orang Asli individuals in Peninsular Malaysia, use the data for studying origins and evaluate their influence in transfusion and gestation.

Methods: We have typed seven HPA loci using combination of sequence specific primer and sequence based typing techniques in 358 volunteers representing 11 subethnic/subgroups of Malays and Orang Asli currently living in Peninsular Malaysia. These study groups include five Malay subethnic groups (Banjar, Bugis, Champa, Jawa and Kelantan) and three Orang Asli groups (Negrito, Senoi and Proto-Malays). We have also included HPA data from the previous study on Malays, Chinese and Indians in our statistical analyses.

Results: Homozygous a/a was the most predominant genotype for HPA-1,-2,-4,-5 and -6 in Malays and Orang Asli. Except for Batek, Kensiu and Lanoh, the other studied population groups/subgroup were observed to have HPA 15a/a as the most common genotype. Tests for Hardy-Weinberg equilibrium were only significant in Che Wong for HPA-15. In general, HPA data collected for Malays and Orang Asli are similar to their putative ancestors (Taiwan aboriginals, Africans and Austro-Asiatic populations) and these genetic relationships were illustrated using principal coordinate plot.

Conclusion: The present study has successfully genotyped HPA-1 to -6 and -15 loci in Malay subethnic and Orang Asli individuals in Peninsular Malaysia. The results showed high genetic diversity in Peninsular Malaysia and this is related to independent waves of immigrations in pre-historic (i.e. Negrito, Senoi and Proto-Malays) and historic (Malay subethnic groups, Chinese, Indians) times into this region. The genepools of the settled Peninsular people were then further shaped by various evolutionary processes such as repeated founder effects, natural selection and admixture.

\section{$\mathrm{P}-174$}

\section{ANALYSIS OF ANTI-HPA-1A AND ANTI-NAKA ANTIBODIES ON ENDOTHELIAL FUNCTIONS: IMPACT ON THE PATHOMECHANISM OF FOETAL/NEONATAL ALLOIMMUNE THROMBOCYTOPENIA}

\section{H Wihadmadyatami ${ }^{1}, \mathrm{X} \mathrm{Xu}^{2}$, B Bayat ${ }^{3}$ and S Santoso ${ }^{3}$}

${ }^{1}$ Faculty of Veterinary Medicine Universitas Gadjah Mada, Yogyakarta, Indonesia ${ }^{2}$ Institute of Blood Transfusion, Guangzhou Blood Centre, Guangzhou, Guangdong, China ${ }^{3}$ Institut for Clinical Immunology \& Transfusion Medicine Justus Liebig University, Giessen, Germany

Background: Foetal/neonatal alloimmune thrombocytopenia (FNAIT) is bleeding disorder due to the destruction of foetal platelets by maternal alloantibodies bound to paternal human platelet antigen (HPA) expressed on foetal platelets. The majority of FNAIT cases in Caucasians are caused by anti-HPA-1a antibodies reacted with the Leu33 isoform of the b3 integrin (CD 61). In contrast, recent data indicate that anti$\mathrm{Nak}^{\mathrm{a}}$ antibodies developed in CD36 deficient mothers represent the most frequent isoantibodies responsible for FNAIT in Asian populations. The frequency of CD36 deficient individuals among Asian populations ranges between 1-7\%. Besides on platelets, CD61 is also expressed on endothelial cells, known as vitronectin receptor (avb3). CD36 is widely distributed, found in different blood cells including platelets, erythrocytes, monocytes and also on endothelial cells. However, little is known about the impact of anti-HPA-1a and anti-Nak ${ }^{\mathrm{a}}$ antibodies on endothelial function(s) Aim: In this study, we compared the capability of anti-HPA-1a and anti-Nak ${ }^{\mathrm{a}}$ antibodies derived from mothers with severe FNAIT to induce endothelial dysfunction in vitro.

Methods: The influence of antibodies on monolayer integrity, apoptosis and angiogenesis of Human Umbilical Vein Endothelial Cells (HUVEC) was evaluated by permeability assay (albumin-FITC), apoptosis assay (caspase 3/7) and angiogenesis assay (tube formation). Mammalian cell lines expressing recombinant CD61 integrin (avb3) or CD36 were produced to isolate anti-HPA-1a and anti-Nak ${ }^{\mathrm{a}}$ antibodies from the serum samples.

Results: Prior to functional analysis, binding of both antibody specificities was confirmed by antigen capture assay using HUVEC as target cells. Functional analysis showed that both anti-HPA-1a as well as anti-Nak antibodies significantly enhanced albumin-FITC penetrance through HUVEC monolayer. When anti-HPA-1 antibodies sera were tested with HPA-1a positive HUVEC, endothelial apoptosis was significantly observed. This result could be confirmed by absorption of these sera with avb3 transfected cells. After absorption, IgG antibodies remained in serum samples (absorbates) did not trigger endothelial apoptosis. Surprisingly, no apoptosis was observed when endothelial cells were treated with anti-Nak ${ }^{\mathrm{a}}$ antibodies isolated by the same approach. Similar phenomenon was observed by the angiogenesis assay. Only anti-HPA-1a antibodies, but not anti-Nak ${ }^{\mathrm{a}}$ antibodies were able to inhibit the tube formation of HUVEC.

Conclusions: Although anti-HPA-1a and anti-Nak ${ }^{\mathrm{a}}$ antibodies bound endothelial cells, both antibody specificities differ from each other in respect to their function. This result indicates that other mechanism, unlike anti-HPA-1a, may participate on the mechanism of severe bleeding and hydrops and shown by some FNAIT cases associated with anti-Nak ${ }^{\mathrm{a}}$ antibodies.

\section{P-175}

\section{THE FREQUENCY OF CD36 NEGATIVE INDIVIDUALS AMONG DIFFERENT SOUTH EAST ASIAN POPULATIONS}

$\mathrm{Y} \mathrm{Fu}^{1}, \mathrm{~N}$ Tsuno ${ }^{1}, \mathrm{Y} \mathrm{Ye}^{2}$ and S Santoso ${ }^{1}$

${ }^{1}$ ISBT Platelet Immunology Working Party (PIWP), Guangzhou, China ${ }^{2}$ Guangzhou Blood Center, Guangzhou, China

Background: CD36 is a highly glycosylated protein (known as GPIV) and is expressed widely in different cells such as platelets, monocytes, macrophages, endothelial and epithelial cells. Two types of CD36 deficiency have been identified: type I lacking CD36 surface expression on platelets and monocytes, and type II lacking CD36 on platelets only. Several mutations underlying type I CD36 deficiency have been described. Currently, immunization against CD36 (development anti-Naka isoantibodies) associated with immune mediated thrombocyte- penia (platelet transfusion refractoriness (PTR) and fetal neonatal alloimmune thrombocytopenia) has been mainly reported in Japanese and Chinese populations, but little is known about the clinical impact of CD36 among other South East Asian populations platelet transfusion refractoriness

Aim: To determine the frequency of CD36 negative individuals among South Eas Asian populations, multinational study including different South East countries and Australia has been design by PIWP and conducted by 10 laboratories in Asia (see Table 1)

Methods: All laboratories were requested to screen platelet samples from healthy blood donors $(\mathrm{n}=200)$ for CD36 deficient using flow cytometry analysis, and were asked to analyze thereafter the molecular bases underlying the CD36 deficient by nucleotide sequencing analysis. Fluorescence labelled monoclonal antibody against CD36 and flow cytometry protocol was provided by the PIWP.

Results: Among the 10 labs, 8 labs provided data derived from more than 100 individuals. Two labs submitted data from less than 100 individuals. Most laboratories analyzed CD36 expression on platelets only (Table 1), but not on platelets and monocytes. Therefore, accuracy differentiation between type I and type cannot be concluded from this study. The frequency of CD36 negative individuals varied between 0\% (Australia) until 7.92\% (Japan) (Table 1). Overall, the frequency of CD36-negative among South East Asia populations was 2.59\% (36 out of 1391). Although nucleotide sequencing platform has been provided by the Guangzhou Blood Center, only 6 labs have provided DNA samples for mutation analysis. Seventeen samples (3 Type I, 7 Type II and 7 unknown Type). Among Type I individuals $(\mathrm{n}=3)$, two carry heterozygous mutations (exon4 C268T and exon13 1228-1239 delATTGTGCCTATT; exon5 329-330 delAC and exon8 T737A), one homozygous mutation (exon4 C268T). Among type II $(n=7)$, three exhibit point mutations (exon12 A1163T and exon12 A1163T). No mutation was found in 4 other subjects. In the unknown type cohort $(n=7)$, no mutation was found in two subjects. Five indi- 
viduals carry different mutations (exon4 C268T, exon13 1228-1239 del ATTGTGCCTATT, exon5 329-330 del AC and exon14 ins 1332-1335 TGAT.

Conclusions: Our result showed that CD36 deficient exists not only in Japanese and Chinese populations, but also in other South Asian populations. Although the frequency of immunization is not known yet, screening for anti-Nak ${ }^{\mathrm{a}}$ antibodies should be considered in suspected immune-mediated thrombocytopenia. A registry of CD36-deficient blood donors should be established to maintain PTR patients and fetus with FNAIT.

Table 1: The frequencies of CD36 negative in Asia.

\begin{tabular}{|c|c|c|c|c|}
\hline No. of Lab & Country/Area & $\begin{array}{c}\text { Number of } \\
\text { results } \\
\text { provided }\end{array}$ & $\begin{array}{c}\text { Number of } \\
\text { Negative }\end{array}$ & $\begin{array}{c}\text { Negative of } \\
\text { percentage(\%) }\end{array}$ \\
\hline 1 & China(Guangzhou) & 200 & 3 & 1.50 \\
\hline 4 & China(Changchun) & 200 & 4 & 2.00 \\
\hline 5 & Taiwan & 191 & 3 & 1.57 \\
\hline 6 & South Korea & 55 & 2 & 3.64 \\
\hline 7 & Indonesia & 41 & 1 & 2.44 \\
\hline 8 & Thailand & 162 & 1 & 0.62 \\
\hline 9 & Malaysia & 200 & 4 & 2.00 \\
\hline 11 & Japan & 202 & 16 & 7.92 \\
\hline 14 & Hong Kong & 140 & 2 & 1.43 \\
\hline 19 & Australia & 200 & 0 & 0 \\
\hline Monocytes & Hong Kong & 140 & 1 & 0.71 \\
\hline 14 & & & & \\
\hline \multicolumn{7}{r|}{}
\end{tabular}

P-176

\section{PLATELET DONOR REGISTRY FOR ALLOIMMUNIZED}

\section{PATIENTS}

A Srisuddee, C Roekchai, K Inorn, S Khanuntong, S Phiancharoen and P Kupatawintu

Thai Red Cross Society, Bangkok, Thailand

Background: $\mathrm{ABO}$-major incompatible platelet transfusions are associated with lower platelet count increments than either $\mathrm{ABO}$ identical or minor incompatible transfusions. In patients with platelet specific alloantibodies, human leukocyte antigen (HLA)- or human platelet antigen (HPA)-compatible platelets are required, the transfusion of apheresis platelet across with minor ABO incompatible cannot be avoided. Plasma replacement by platelet additive solution (PAS) in group 0 platelet donor can be managed to reduce the risk of hemolysis in non $\mathrm{ABO}$ identical platelet transfusion.

Aims: This study aim to establish the HPA-typed platelet group 0 donors for all patients.

Methods: DNA sample from group 0 male Thai blood donors, ages between 1840 years, were genotyped HPA using Simple-Probe Real-Time PCR, TIB MOLBIOL $\mathrm{GmbH}$, Berlin, Germany.While, 7308 donors were genotyped HPA-1, -2, -4, -5, and $-6,589$ donors were genotyped HPA-3, -7 to $-11,-13$ to -15 and -17 . HPA genotyped frequencies were analyzed.

Table 1: Distribution of HPA in the Thai population.

\section{Distribution of HPA in the Thai}

\begin{tabular}{|r|r|r|r|r|r|r|}
\hline \multirow{2}{*}{ HPA } & \multicolumn{3}{|c|}{ HPA genotyping } & \multicolumn{3}{c|}{$\%$ Frequencies } \\
\cline { 2 - 8 } & \multicolumn{1}{|c|}{ aa } & \multicolumn{1}{|c|}{ ab } & bb & \%aa & \%ab & \%bb \\
\hline-1 & 7029 & 275 & 4 & $96.18 \%$ & $3.76 \%$ & $0.05 \%$ \\
\hline-2 & 6630 & 659 & 19 & $90.72 \%$ & $9.02 \%$ & $0.26 \%$ \\
\hline-3 & 195 & 282 & 112 & $33.11 \%$ & $47.88 \%$ & $19.02 \%$ \\
\hline-4 & 7294 & 14 & 0 & $99.81 \%$ & $0.19 \%$ & $0.00 \%$ \\
\hline-5 & 6814 & 488 & 6 & $93.24 \%$ & $6.68 \%$ & $0.08 \%$ \\
\hline-6 & 7068 & 238 & 2 & $96.72 \%$ & $3.26 \%$ & $0.03 \%$ \\
\hline-7 & 589 & 0 & 0 & $100.00 \%$ & $0.00 \%$ & $0.00 \%$ \\
\hline-8 & 588 & 1 & 0 & $99.83 \%$ & $0.17 \%$ & $0.00 \%$ \\
\hline-9 & 589 & 0 & 0 & $100.00 \%$ & $0.00 \%$ & $0.00 \%$ \\
\hline-10 & 589 & 0 & 0 & $100.00 \%$ & $0.00 \%$ & $0.00 \%$ \\
\hline-11 & 589 & 0 & 0 & $100.00 \%$ & $0.00 \%$ & $0.00 \%$ \\
\hline-13 & 589 & 0 & 0 & $100.00 \%$ & $0.00 \%$ & $0.00 \%$ \\
\hline-14 & 589 & 0 & 0 & $100.00 \%$ & $0.00 \%$ & $0.00 \%$ \\
\hline-15 & 157 & 318 & 114 & $26.66 \%$ & $53.99 \%$ & $19.35 \%$ \\
\hline-17 & 588 & 1 & 0 & $99.83 \%$ & $0.17 \%$ & $0.00 \%$ \\
\hline
\end{tabular}

Results: Similar to previous studies, the homozygous 'aa' were commonly found in HPA-1, $-2,-4$ to $-11,-13,-14$ and -17 . The heterozygous 'ab' were commonly found in HPA-3 and -15 . Interestingly, HPA- $1 \mathrm{bb},-2 \mathrm{bb},-5 \mathrm{bb}$ and $-6 \mathrm{bb}$ were newly found in Thai population which the genotyped frequencies were $0.05 \%, 0.26 \%, 0.08 \%$ and $0.03 \%$, respectively.

Conclusions: The HPA-typed donors of our study will be useful for HLA- and HPAcompatible platelets selection which can be accessed for alloimmunized patients.

\section{$\mathrm{P}-177$}

A NOVEL GLY ${ }_{540}$ ASN MUTATION ON THE BETA3 SUBUNIT LEADS TO THE PRODUCTION OF ANTI-ALPHAIIBBETA3 AND ANTI-ALPHAVBETA3 ISOANTIBODIES IN PATIENT WITH GLANZMANN THROMBASTHENIA

H Wihadmadyatami ${ }^{1}$, L Röder ${ }^{2}$, R Fischer ${ }^{3}$, G Bein ${ }^{2}$, UJ Sachs ${ }^{2}$ and S Santoso ${ }^{2}$ ${ }^{1}$ Faculty of Veterinary Medicine, Universitas Gadjah Mada, Yogyakarta, Indonesia ${ }^{2}$ Institut for Clinical Immunology \& Transfusion Medicine, Justus Liebig University, Giessen, Germany ${ }^{3}$ Haemostasis Center University Clinic Giessen Marburg, Giessen, Germany

Background: Glanzmann thrombasthenia (GT) is an autosomal recessive bleeding disorder characterized by quantitative and qualitative defects of platelet fibrinogen receptor aIIbb3 integrin. Based on the residual aIIbb3 expression on platelet surface, GT patients are categorized in two different types; type 1 expressing $<5 \%$, and type 2 expressing $5-20 \%$ allbb3 integrin. On platelets, the b3 integrin forms a heterodimer with av subunit (avb3) and serves as vitronectin receptor

Aim: In this study we aimed to elucidate the molecular characterization of two GT patients and analysed the development of platelet antibodies against b3 integrin in these patients after platelet transfusion(s).

Methods: Flow cytometry and platelet aggregation assays were performed to analysis the copy number and function of platelet receptors. PCR amplification, nucleotide sequencing, and transfection analysis were conducted to identify point mutations. Antibody screening was done by the use of antigen capture assay (MAIPA) Results: Quantitative flow cytometry analysis showed significant reduction of surface aIIbb3 expression in both patients' platelets; 3340 molecules in patient \#1 and 1523 molecules in patient \#2 when compared to platelets from healthy blood donors $\left(44,000 \pm 12,000\right.$ molecules). Molecular genetic analysis showed a novel $\mathrm{C}_{1727} \mathrm{G}$ point mutation on ITG2B gene in patient \#1 which leads to missense $\mathrm{Cys}_{545} \mathrm{Ser}$ mutation on the allb integrin subunit. In contrast, patient \#2 revealed a point mutation $\mathrm{G}_{1697} \mathrm{~A}$ on ITGB3 gene causing $\mathrm{Gly}_{540} \mathrm{Asp}$ mutation on the b3 subunit. Transfection analysis in mammalian cells (COS-7 cells) with mutant aIIb $\left(\mathrm{S}_{545}\right)$ and mutant b3 $\left(D_{540}\right)$ expression vectors showed strong reduction of mutant aIIb $\left(S_{545}\right)$ b3 isoform and lacking of mutant allbb3 (D540) expression. These results demonstrated that these mutations are responsible for diminished aIIbb3 expression in patient \#1 (type 2 GT) and lacking of aIIbb3 expression in patient \#2 (type 1 GT).

It is known that some type $1 \mathrm{GT}$ patients can develop anti-aIIbb3 antibodies after receiving multiple platelet transfusion. Interestingly, we found in our patient \#2, not only anti-aIIbb3 antibodies but also anti-avb3 antibodies when antigen capture assay was applied with platelets and endothelial cells. Although both b3 reactive antibodies were capable to induce platelet clearance as shown by phagocytosis assay, no inhibition of platelet aggregation mediated by ADP by these antibodies was observed. In patient \#1, platelet and endothelial reactive b3 specific antibody were not detectable.

Conclusions: Taking together, our results demonstrated that type I GT type patients caused by b3-mutations can alter the synthesis of allbb3 and avb3 integrin. Consequently, platelet transfusion(s) in such patients can lead to the production anti-aIIbb3 as well as anti-avb3 isoantibodies, and subsequently to the development of platelet transfusion refractoriness.

P-178

THE STUDY OF EXPRESSION AND PLATELETS PRESERVE METHOD OF HPA-15 ANTIGEN

$\mathrm{Y} \mathrm{Shao}^{1}, \mathrm{X} \mathrm{Ye}^{1}, \mathrm{~W} \mathrm{Xia}{ }^{1}, \mathrm{X} \mathrm{Xu}^{1}$ and S Santoso ${ }^{2}$

${ }^{1}$ Guangzhou Blood Center, Guangzhou, China ${ }^{2}$ Institute for Clinical Immunology and Transfusion Medicine, Giessen, Germany

Background: HPA-15 alloantigen is located in CD109 which is a GPI linked protein. Alloantigen against CD109 is reported can cause fetal and neonatal alloimmune thrombocytopenia (FNAIT). Detection of HPA-15 antibodies in several studies used the monoclonal antibody-specific immobilization of platelet antigen (MAIPA) assay

(C) 2015 The Author Vox Sanguinis (C) 2015 International Society of Blood Transfusion Vox Sanguinis (2015) 109 (Suppl. 2), 1-96 
which related to the level of HPA-15 expression. But detection of HPA-15 antibodies is difficult because of the low and instability of CD109 expression. Analysis of the HPA-15 expression and study of the platelets preserve methods may contribute to the detection of HPA-15 antibodies.

Aims: Determination and analysis the HPA-15 antigen expression on platelet. Analyze the HPA-15 genotypes frequencies in South China donors. Find a better platelets preserving method which can sustain the CD109 expression.

Methods: A total of 109 healthy blood donors were collected in Guangzhou Blood Center, China. The HPA-15 expression was determined on the platelets by flow cytometry. The HPA-15 genotypes were also determined by PCR technology. Another 76 healthy blood donors were divided into two groups. Platelets in Group 1 were suspended in PBS-BSA and stored in $4^{\circ} \mathrm{C}$. Platelets in Group 2 were stored in an orbital shaker in $22^{\circ} \mathrm{C}$. HPA- 15 expression of the two groups was determined by flow cytometry on days $0,1,2,3,7,14$.

Results: In the 109 donors, the allele frequencies of HPA-15a and HPA-15b are 0.495 and 0.505 . The MFI of HPA-15 expression ranged from 2.44 to 20.4. In the two compared groups, the linear mixed model was used to analyse the data. According to different determination time point, the MFI of the two groups have statistical difference $(\mathrm{P}=0.001)$ during the storage. Although the HPA-15 MFI of the two groups both decline during this period, the HPA-15 expression of group 2 is better than group 1.

Conclusions: The expression of CD109 on platelet is low and varies among different individuals. The CD109 expression decreases during storage, especially in $4^{\circ} \mathrm{C}$.

\section{P-179}

THE INFLUENCE OF LEUKODEPLETION OF PLATELET CONCENTRATES ON CD62P EXPRESSION AND CORRECTED

\section{COUNT INCREMENT}

T Triyono ${ }^{1}, Z_{\text {Rofinda }}{ }^{1}$ and U Sukorini ${ }^{2}$

${ }^{1}$ Medical Faculty of Andalas University, Yogyakarta, Indonesia ${ }^{2}$ Medical Faculty of Gadjah Mada University, Yogyakarta, Indonesia

Background: Platelet concentrate (PC) is a blood component which is needed to be transfused to patient with thrombocytopenia including the patient with hematology malignancies. Platelet concentrates contain contaminant leukocytes that can lead side effects and platelet activation that can reduce the efficacy of platelet transfusion. For minimazing the contaminant leukocytes, leukodepletion by filtration might be performed. Platelet activation can be assessed by CD62P expression, whereas the efficacy of platelet transfusion can be assessed by corrected count increment (CCI). Leukodepletion was not yet applied universally in Indonesia.

Aims: To analyze the CD62P expression between non-leukodepleted and leukodepleted PC groups, and also to analyze the CCI between group of patients which were transfused with non-leukodepleted and leukodepleted PCs.

Method: The study was performed into two groups of patients with hematology malignancies in Estella Children Ward of Dr.Sardjito Hospital, Yogyakarta Indonesia i.e. 24 patients were transfused with non-leukodepleted and 24 patients were transfused with leukodepleted PRP-derived PCs. The platelet expression of CD62P in PC products was measured by flowcytometri, and the $1 \mathrm{~h}$ post-transfusion corrected count increment was counted by CCI formula. The differences of CD62P expression and CCI between the two groups were analyzed by using Mann-Whitney Test with statistical significance of $\mathrm{P}<0.05$.

Results: From PC products, it was found that the CD62P expression in non-leukodepleted groups were higher with median of $34.4 \%(16.8-94.4 \%)$ compared to the leukodepleted groups with median of $21.7 \%(6.2-34.0 \%)$.There was a statistical significantly differences of the CD62P expression betweentwo groups $(P=0.00)$. It was found that in the group of patients which were transfused with non-leukodepleted PCs, the median of CCI was $18.8 \times 10^{9} / 1\left(2.4-94.8 \times 10^{9} / 1\right)$. The median of CCI in the group of patients which was transfused with leukodepleted PCs was $14.7 \times 10^{9} / 1$ $\left(2.4-124 \times 10^{9} / 1\right)$. There was no statistically significant differences of CCI between the two groups $(\mathrm{P}=0.42)$.

Conclusion: The platelet CD62P expression in leukodepleted were statistically significant lower than in non-leukodepleted PCs. But there was no statistically significant CCI differences between the group of patients which were transfused with nonleukodepleted and with leukodepleted PCs.

\section{$\mathrm{P}-180$}

This abstract has been withdrawn.
$\mathrm{P}-181$ RELEVANT ROLE OF TISSUE FACTOR IN VON WILLEBRAND FACTOR-DEPENDENT THROMBUS FORMATION UNDER FLOW: INVOLVEMENT OF INFLAMMATORY RESPONSES BY LEUKOCYTES IN I INTRA-THROMBUS FIBRIN GENERATION

Y Matsunari, H Matsui, M Kawaguchi and M Sugimoto

Nara Medical University, Kashihara, Japan

Background: von Willebrand factor (VWF) plays a pivotal role in platelet thrombogenesis at sites of injured vessel walls under high shear flow conditions. Such platelet thrombus formation is concurrently reinforced by fibrin generation initiated by tissue factor (TF). However, little is known about the functional link between VWF and TF in flow-dependent thrombus formation.

Aims: To address this issue, we have studied the relevant role of TF on von Willebrand factor (VWF)-dependent thrombus formation by use of an in vitro perfusion chamber system.

Methods: Recombinant human TF were co-coated with VWF onto a glass plate to prepare 'surface-immobilized VWF/TF complex'. Recalcified citrated whole blood was perfused over a surface-immobilized VWF/TF complex. In some experiments, neutrophil Cathepsin G $(12 \mu \mathrm{g} / \mathrm{ml})$ was added to sample blood. Platelet adhesion/aggregation were evaluated by the surface coverage of thrombi. The mural thrombi formed on VWF-surface was double-stained with fluorescently labeled anti-fibrin and anti-fibrinogen antibodies. Fibrin generation was evaluated as a ratio of fibrin relative to fibrinogen fluorescence within mural thrombi.

Results: Surface-immobilized TF significantly augmented flow-dependent fibrin generation as a function of increasing surface density of TF under both low and high shear rate conditions. In addition, immobilized TF significantly up-regulated VWF-dependent platelet adhesion and aggregation under high shear rate conditions. Cathepsin G significantly increased intra-thrombus fibrin generation on immobilized VWF-TF complex under both high and low shear rate conditions.

Summary/Conclusions: Surface-immobilized TF and VWF could build mural thrombi in a concerted manner under high shear rate conditions. The present experimental approach revealed that Cathepsin G enhanced the TF-dependent fibrin generation, suggesting the critical involvement of inflammatory responses by leukocytes on thrombus formation under whole blood flow conditions.

\subsection{Granulocyte Immunology}

P-182 GENERATION OF CYTOKINES IN RED BLOOD CELL PRODUCTS DURING STORAGE: A COMPARISON OF NON- AND LEUKOREDUCED RED BLOOD CELL COMPONENTS

T Lee ${ }^{1}, \mathrm{H} \mathrm{Lin}^{1}$, CC Chang ${ }^{1}$, LF Chang ${ }^{2}$ and FY Chu ${ }^{1}$

${ }^{1}$ FarEastern Memorial Hospital, New Taipei City, Taiwan ${ }^{2}$ Asia-Med Medical Reference Lab, Taipei City, Taiwan

Background: Leukocyte reduction before storage effectively reduced the generation of white blood cell (WBC)-derived cytokines. However, it was still unknown whether there was accumulation of bioactive mediators including non-leukocyte-derived cytokines, such as platelet-specific proinflammatory factors, in non-leukocyte-reduced RBC components during storage.

Aims: The aim of the present study was to investigate the cytokine accumulation, including MIP- $1 \alpha$, RANTES, VEGF, TGF- $\beta 1$, TGF- $\beta 2$ and TGF- $\beta 3$, in the pre-storage leuko-reduced RBC (Pre-LPR) products and standard non-leuko-reduced RBC (S-RBC) products during a 42-day storage.

Methods: Fifteen units of pre-LPR and S-RBC were included in the study and stored at $4^{\circ} \mathrm{C}$. Samples were acquired using aseptic technique from each unit on the 4 th and 42th day of storage. Supernatant was collected by centrifugation at 10,000 $\times g$ for $10 \mathrm{~min}$ and then stored at $-70^{\circ} \mathrm{C}$ until analysis. The concentration of cytokines was determined by the bead-based Luminex Bioplex system according to the manufacturer's instructions. The accumulation of various cytokines for within-sample correlations across designated time points of storage was assessed in Pre-LPR and S-RBC. Results: The cytokine levels of RANTES, VEGF, TGF- $\beta 1$, TGF- $\beta 2$ and TGF- $\beta 3$ were markedly increased in S-RBC from the 4th day to 42th day, but not in Pre-LPR. The post-storage cytokine concentrations were significantly increased in comparison with pre-storage cytokine levels of RANTES $(\mathrm{P}<0.001)$, VEGF $(\mathrm{P}<0.001)$, TGF- $\beta 1$ $(\mathrm{P}<0.001)$, TGF- $\beta 2(\mathrm{P}=0.012)$ and TGF- $\beta 3(\mathrm{P}<0.001)$ in $\mathrm{S}-\mathrm{RBC}$, but there was no significant change during storage in Pre-LPR. MIP- $1 \alpha$ decreased significantly during storage in Pre-LPR $(\mathrm{P}<0.001)$, but not in S-RBC $(\mathrm{P}=0.76)$.

Conclusions: Our results indicated that leuko-reduction in RBC products before storage effectively prevented the cytokine generation, including RANTES, VEGF, TGF- 

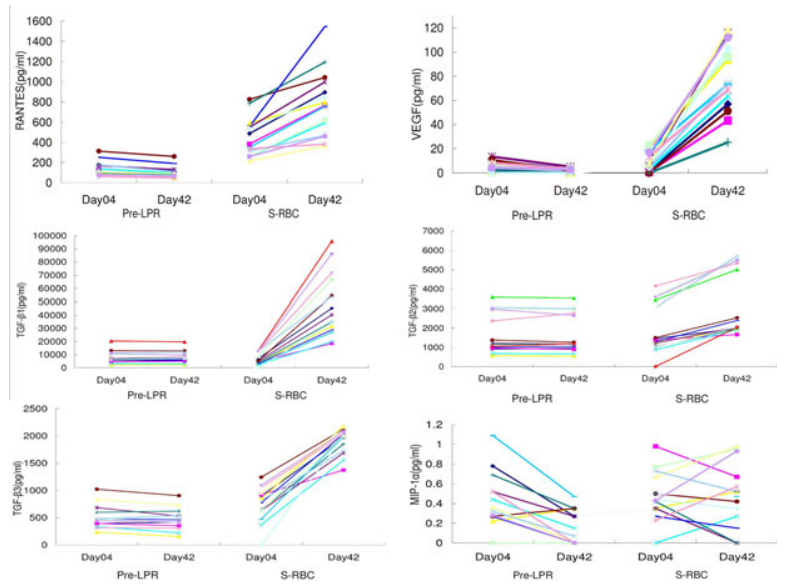

Figure 1: Comparisons of RANTES, VEGF, TGF- $\beta 1$, TGF- $\beta 2$, TGF- $\beta 3$ and MIP$1 \alpha$ levels in Pre-LPR and S-RBC at the Day 04 and Day 42 during storage of the components.

$\beta 1$, TGF- $\beta 2$ and TGF- $\beta 3$. In contrast, MIP- $1 \alpha$ level was decreased in pre-storage leuko-reduced RBC components during storage.

$\mathrm{P}-183$

\section{THE PREVALENCE OF LEUCOCYTE ALLOANTIBODIES IN BLOOD DONORS FROM SOUTH CHINA}

$\mathrm{W} \mathrm{Xia}{ }^{1}, \mathrm{X} \mathrm{Ye}^{1}, \mathrm{D} \mathrm{Chen}^{1}, \mathrm{Y} \mathrm{Fu}^{1}$ and S Santoso ${ }^{2}$

${ }^{1}$ Institute of Blood Transfusion, Guangzhou, China ${ }^{2}$ Institute for Clinical Immunology and Transfusion Medicine, Giessen, Germany

Background: Several studies had demonstrated that HLA antibodies (class I and class II) and HNA antibodies (HNA-1, -2, and -3) in the blood products are capable to induce transfusion related acute lung injury (TRALI) in recipients. Therefore, screening of HLA and HNA antibodies in blood donors may reduce the risk of TRALI. However, the prevalence of leukocyte antibodies among blood donors in China is currently not known.

Study Design and methods: Blood samples were collected from 454 male and 560 female donors (143 nulliparous and 417 multiparous female). HLA class I and II antibodies were analyzed by bead assays (Luminex). Anti-HNA-1, -2 antibodies were screened by the LABscreen Assay (One Lambda), and HNA-3 were detected by Antigen Capture Assay, and confirmed by the granulocyte agglutination assay (GAT).

Results: Screening of the total cohort showed higher prevalence of HLA antibodies in female compared with male donors $(19.64 \%$ vs $4.63 \%)$. We found antibodies against HLA class I (13.21\%) and HLA class II (11.43\%) in 560 female donors. The most frequent antibodies against HLA class I and II in parous females $(\mathrm{n}=69)$ reacted with were $\mathrm{A}^{*} 11(28.81 \%), \mathrm{B}^{*} 07(42.37 \%), \mathrm{Cw}^{*} 07(20.34 \%)$ and DRB1*04 $(40.43 \%)$ molecules.

When sera from 778 donors were screened for neutrophil specific antibodies, there were three pregnant female samples demonstrated HNA antibodies (0.39\%). Two sera showed anti-HNA-2 specificity. This result could be confirmed by flow cytometry analysis of donor serum and donor neutrophils. As shown in Figure 1, serum of donor 1 reacted with neutrophils derived from CD177 positive, but not from CD177 negative individual. Furthermore, no expression of CD177 was found on neutrophil surface of this donor. Similar results were obtained with donor 2 (data not shown). In donor 3, we found antibodies reacted with all three forms of HNA-1 $(-1 \mathrm{a},-1 \mathrm{~b}$ and -1c) and anti-HNA-2 indicating the presence of neutrophil autoreactive antibodies in this donor (Table 1). No anti-HNA-3a and anti-HNA-3b alloantibodies could be found in our cohort by screening of these sera using antigen capture assay and GAT.

Conclusions: In this study, we found alloimmunization against HLA class I, II and HNA in 4.63\%, 24.70\% and 0.39\% respectively, in our female blood donors, indicating that the use of plasma containing blood products from parous female blood donors without HLA antibodies pre-testing may increase the risk of TRALI reaction. Although alloimmunization against HNA seems to be a rare event, further observa-

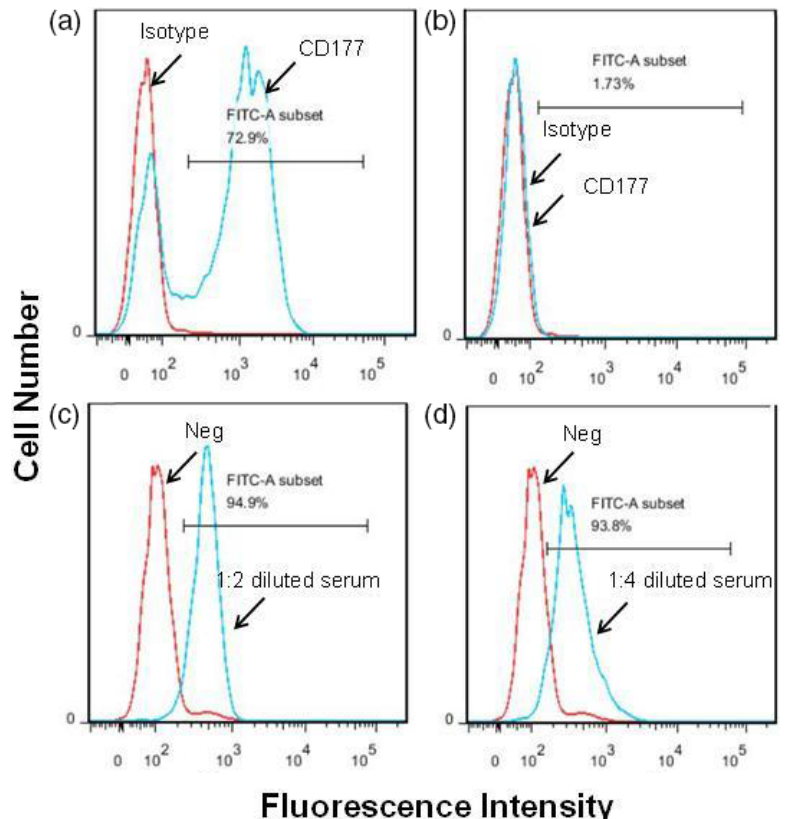

Figure 1: Analysis of CD177 expression and HNA-2 antibodies by flow cytometry.

tion is necessary to exclude the necessity of HNA antibodies screening of our blood products.

Table 1: HNA antibody specificities found in our blood donors.

\begin{tabular}{|c|c|c|c|c|c|c|}
\hline Donors & Gender & HLA Ab & HNA Ab & GIFT & HNA Genotype & Comments \\
\hline 1 & F & Neg & 2 & Pos & 1 aad, 2 Null & Possible \\
\hline 2 & F & Neg & 2 & Pos & 1 ab.2 Null & Possible \\
\hline 3 & F & Class I & 1 1a,1c,1b,2 & Pos & 1 1ab, 2aa & $\begin{array}{c}\text { Possible } \\
\text { (autoreactive) }\end{array}$ \\
\hline
\end{tabular}

$\mathrm{P}-184$

HUMAN NEUTROPHIL ANTIGEN FREQUENCIES IN MALAYS AND ORANG ASLI IN PENINSULAR MALAYSIA

\section{SM Manaf}

Universiti Sains Malaysia, Kubang Kerian, Malaysia

Background: Human neutrophil antigens (HNA) have been implicated in several hematological diseases including alloimmune neonatal neutropenia (ANN), autoimmune neutropenia (AIN) and transfusion-related acute lung injury (TRALI). This is the first study of HNA in Malays and Orang Asli and is anticipated to have benefits in population and disease studies.

Aim: To examine HNA-1, -3, -4 and -5 loci in Malays and Orang Asli in Peninsular Malaysia.

Methods: Our study involved collection of blood samples from 192 Malay subethnic groups (Banjar: $\mathrm{n}=30$, Bugis: $\mathrm{n}=37$, Champa: $\mathrm{n}=51$, Jawa: $\mathrm{n}=39$ and Kelantan: $\mathrm{n}=35$ ) and 170 blood samples from three Orang Asli groups (Negrito: Bateq; $\mathrm{n}=27$, Kensiu; $\mathrm{n}=38$ and Lanoh; $\mathrm{n}=25$, Senoi: Che Wong; $\mathrm{n}=27$ and Semai; $\mathrm{n}=42$ and Proto-Malay: Orang Kanaq; $\mathrm{n}=11$ ). A self-declared was used to assign volunteers into three generations of un-admixed ancestry. Family pedigree was then used to divide Orang Asli into smaller subset of un-admixed individuals. The HNA$1,-3$ and -4 and HNA-5 loci were genotyped by using polymerase chain reaction-sequence specific primer (PCR-SSP) and PCR-restriction fragment length polymorphism (PCR-RFLP), respectively.

Results: The most common HNA alleles in the studied population subgroups are HNA-1a (0.539-0.846), -3a (0.676-0.976), -4a (0.857-1.000) and -5a (0.529-1.000).

(C) 2015 The Author Vox Sanguinis (C) 2015 International Society of Blood Transfusion Vox Sanguinis (2015) 109 (Suppl. 2), 1-96 
Principal coordinate plot constructed using HNA-1, -3, -4 and -5 allele frequencies showed high genetic diversity in Peninsular Malaysia and the similar observation was captured using neighbor-joining analysis. Our statistical analyses showed high risk of HNA alloimmunization in transfusion and gestation between pairs or donors and recipients of different ethnicities.

Conclusion: This is the first study provides HNA allele and genotype frequencies in five Malays subethnic groups and six Orang Asli subgroups living in Peninsular Malaysia. The present study supports the significant of HNA in health and population studies.

\section{P-185}

\section{FREQUENCY OF HUMAN NEUTROPHIL ANTIGEN-2 AMONG INDIAN BLOOD DONORS}

H Gogri, S Kulkarni, K Ghosh and A Gorakshakar

National Institute of Immunohaematology, Mumbai, India

Background: Human neutophil antigen-2 (HNA-2) or NB1 is one of the antigens assigned to the HNA alloantigen systems and was first described in 1971 by Lalezari et al. It is located on a 58-64 kDa glycoprotein known as CD177 found on plasma membranes of neutrophils and secondary granules. A unique characteristic of HNA$2 \mathrm{a}$ is its heterogenous expression, i.e. a single individual has a neutrophil subpopulation which expresses HNA-2a and another that does not. CD177 deficiency on neutrophils is caused due to different off-frame insertions at RNA level. CD177 is involved in the adhesion of neutrophils to endothelial cells and their transendothelial migration by cationic-dependent interaction with the heterophilic domains of the platelet endothelial cell adhesion molecule (PECAM)-1 (CD31). The alloantibodies formed by HNA-2a-negative individuals are important in neonatal alloimmune neutropenia, transfusion related acute lung injury, autoimmune neutropenia, drug-induced neutropenia, and graft failure following bone marrow transplantation. High frequency of HNA-2 has been reported among North Americans, Europeans, Brazilians and Japanese. However, the frequency of HNA-2 is not studied among Indians. Aims: To determine the frequency of HNA-2 among Indian blood donors.

Methods: Fresh EDTA blood $(3 \mathrm{ml}$ ) was collected from 310 voluntary blood donors (79 females, 231 males). Neutrophils were phenotyped using FITC-labelled antiCD177 (Clone: MEM-166; Abcam, UK) and were analysed using BD FACSCalibu (BD Biosciences, CA). Individuals showing less than 5\% of reactive cell population were considered as HNA-2 negative.

Results: Out of 310 blood donors, five (1.6\%) donors were negative for HNA-2 antigen. Remaining donors showed two distinct populations- a positive and a negative. Among these, $44.19 \%$ showed an increased expression $(>60 \%)$ of HNA-2 on the neutrophils while $18.06 \%$ showed weak expression. All females were positive for HNA-2.

Conclusions: HNA-2 is a high frequency antigen present in $98.4 \%$ of the Indian population and has variable expression. This frequency is comparable to that of North Americans, Europeans, Brazilians and Japanese.

\subsection{Fetal-Maternal Immunology}

P-186

\section{NONINVASIVE FETAL RHD GENOTYPING BY MULTIPLEX REAL-TIME PCR}

S Kulkarni, D Parchure and K Ghosh

National Institute of Immunohaematology, Mumbai, India

Background: In large number of RhD-negative pregnant women who have not been immunized, prophylactic RhIG is recommended antenatally as a routine after potential sensitization events. Even in the absence of such events the RhIG administration at 28th and 34th week gestation has shown to reduce incidence of immunization. The identification of fetal $\mathrm{RhD}$ status in nonimmunized $\mathrm{RhD}$-negative pregnant women by invasive means is not practical. The discovery of cell free fetal DNA in maternal plasma in 1997 has opened up new possibilities for noninvasive prenatal diagnosis. With the use of real time PCR technology, circulating fetal DNA has been detected robustly in plasma of pregnant women, even early in first trimester of pregnancy. Fetal RHD genotyping by noninvasive method is safe, and numerous groups have reported fetal RHD genotyping in RhD-negative mothers with close to 100\% accuracy. If the fetal $\mathrm{RhD}$ status in these nonimmunized RhD-negative women is known by noninvasive means, antenatal prophylaxis can be avoided when fetus is RhD-negative. About 40\% of RhD-negative Caucasians women and 25\% of RhDnegative Indian women receive unnecessary prophylaxis as they are carrying $\mathrm{RhD}$ negative fetus. The precise targeting of antenatal RhIG would be of advantage from

\section{(C) 2015 The Author}

Vox Sanguinis (C) 2015 International Society of Blood Transfusion

Vox Sanguinis (2015) 109 (Suppl. 2), 1-96 clinical, economic and supply point of view. A preliminary study of noninvasive fetal RHD genotyping was carried out with the intention of routinely performing genotyping in our reference laboratory in India.

Aims: To standardize multiplex real time PCR for noninvasive fetal RHD genotyping using maternal plasma.

Methods: Noninvasive fetal RHD genotyping was performed on blood samples of thirty nonimmunized RhD-negative pregnant women (second trimester). Taqman probes were used for real time PCR to detect exon 4, 5 and 10 of the RHD gene. SRY gene and CCR5 gene probes were used as controls. When RhD-negative female fetus is predicted fetal epigenetic marker RASSF1A was used. The PCR results were compared with the RhD status of babies after delivery.

Results: Multiplex real time PCR method for noninvasive fetal RHD genotyping was standardized. There is complete concordance between results obtained by noninvasive fetal RHD typing and serological typing on cord blood sample.

Conclusion: Fetal RHD genotyping by multiplex real time PCR is being done with a high accuracy rate, as a routine clinical test in prenatal diagnostic laboratories worldwide. This method will avoid unnecessary immunoprophylaxis in RhD-negative women bearing RhD-negative fetuses and hence should become an essential part of prenatal screening in the management of $\mathrm{RhD}$ incompatible pregnancies in India. It will prove cost-effective with a substantial reduction of costs of overall immunoprophylaxis.

\section{P-187 ANTENATAL SEROLOGY ALGORITHM AND INTRAUTERINE TRANSFUSIONS IN ALLOIMMUNIZED PREGNANCY: NORTH INDIAN TERTIARY CARE CENTRE EXPERIENCE \\ A Bajpayee \\ AIIMS, Jodhpur, India}

Background: Haemolytic disease of the foetus and newborn (HDFN) is although most common due to red cell alloimmunisation against $\mathrm{Rh}(\mathrm{D})$ antigen but other red cell antigen alloimmunization are also seen. In severe fetal HDFN, intrauterine transfusions are administered to fetus to prevent or reverse hydrops fetalis and prevent fetal death. This study was conducted in the department of Transfusion Medicine in collaboration with Maternal and reproductive health department in a Tertiary care centre during a period of 2 years.

Aims: (1) To study prevalence of red cell alloimmunized pregnancies in Indian females presented with Bad Obstetrtic History. (2) To correlate mother's red cell Rh antibody titer at presentation with severity of fetal anemia. (3) To study prevalence of additional antibodies after intrauterine transfusion. (4) To study outcome of alloimmunized pregnancies treated with intrauterine transfusions.

Methods: 188 pregnant women of bad obstetric history were registered .A detailed clinical history such as patient's name, age, registration no., H/O Gravida, parity, live baby, diagnosis, transfusion history was taken. All 188 mothers were screened using commercially available red cell screening panel irrespective of gestational age. Those with negative indirect coomb tests (IAT) were followed by 4 weeks interval IAT. Those with positive indirect coombs test, the antibody screnning, identification and titration were done. If maternal antibody was confirmed and titre was $\geq 16$ or in ultrasound there are signs of hydropic changes, an invasive procedure cordocentesis was planned to monitor the fetal anemia.IUT was done to treat severe fetal anemia and to prevent intrauterine death. 7 days after each IUT antibody titre was estimated and development of additional antibody was looked. .52 IUT episodes were done over the study period. Results: Out of $188,48 \mathrm{Rh}(\mathrm{D})$ negative women and $1 \mathrm{Rh}(\mathrm{D})$ positive women was IAT positive. Overall prevalence of alloimmunization in women with bad obstetritic history was $15.42 \%(49 / 188)$. The antibody detected in $48(97.95 \%)$ was Anti-D only and $1(2 \%)$ had anti-M only. Among $48 \mathrm{Rh}(\mathrm{D})$ negative alloimmunized females only $30(62.5 \%)$ women received Anti-D immunoprophylaxis in her previous/present pregnancies. Out of 49 only 17 women showed antibody titer more than critical titre (16), titre ranged from 16-1024. Among 17 fetuses hydrops was noticed in 13 (76.4\%). In all 17 cases fetal middle cerebral artery peak systolic blood flow (MCA PSV) $>1.5 \mathrm{mom}$. On cordocentesis fetal hematocrit ranges from $8.8-33 \%$ with mean of $19+7.9$. DAT was positive in all fetuses and antibody is $\operatorname{IgG}$ and it is subtyped into IgG1 and IgG3. IgG1in 10 fetuses, IgG3in 2 fetuses and both IgG1 + IgG3 in 4 cases was seen. $3(17.6 \%)$ females developed additional antibodies C, S and Fya after IUT.Out of 17 fetuses who received IUT, 14 live births (82.4\%) outcome observed.

Summary: Despite the introduction of anti-D immunoprophylaxis $\mathrm{Rh}(\mathrm{D})$ alloimmunization is still prevalent. The red cell serologic testing is neglected in medical care and should be a part of routine antenatal testing in developing countries.In higher gravidas the severity of fetal anemia is high even at low antibody titre. Early referral of high risk cases for IUT can improve the outcome. Additional antibodies can complicate the procedure but extended phenotyping is not cost effective. 
Plan of workup in Blood Bank Immunohemtology Lab:

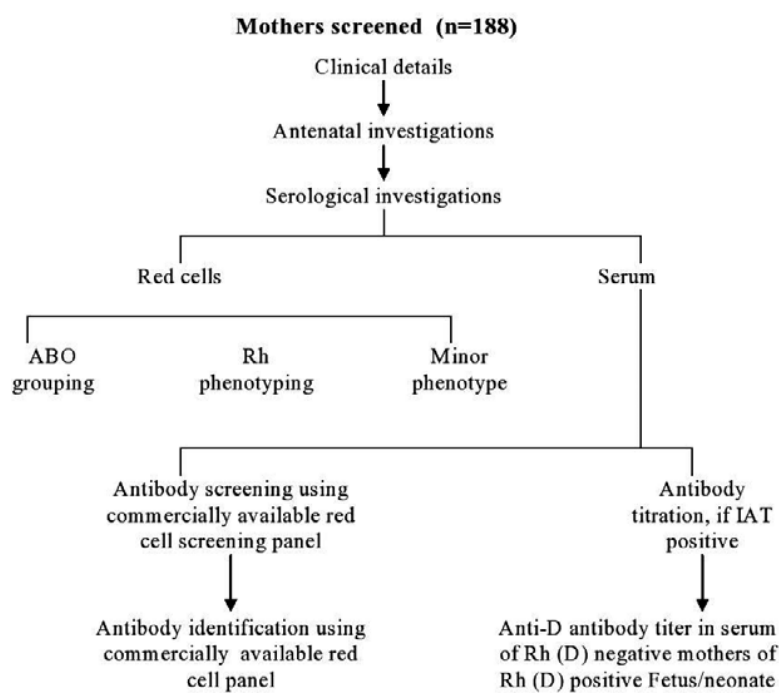

Figure 1: Immunohematologic details in mothers $(n=17)$ and fetal Hct, fetus USG finding.

\begin{tabular}{|l|l|l|l|l|l|l|l|}
\hline $\begin{array}{l}\text { S. } \\
\text { N. }\end{array}$ & $\begin{array}{l}\text { IAT } \\
\text { at } \\
\text { presen- } \\
\text { tation }\end{array}$ & $\begin{array}{l}\text { Antibody } \\
\text { Screening }\end{array}$ & $\begin{array}{l}\text { Antibody } \\
\text { Identification } \\
\text { at } \\
\text { presentation } \\
\text { and follow } \\
\text { up }\end{array}$ & $\begin{array}{l}\text { Titer of } \\
\text { anti-D }\end{array}$ & $\begin{array}{l}\text { Titer of } \\
\text { additional } \\
\text { Antibody }\end{array}$ & $\begin{array}{l}\text { Fetal } \\
\text { hydrops } \\
\text { as on } \\
\text { USG }\end{array}$ & $\begin{array}{l}\text { Fetal Hct } \\
\text { on cordo- } \\
\text { centesis } \\
\text { sample } \\
\text { before first } \\
\text { IUT }\end{array}$ \\
\hline 1 & Positive & $\begin{array}{l}\text { D,C,E,Fy, } \\
\text { Jk' S }\end{array}$ & D.C & $\begin{array}{l}1024 . \\
512,256\end{array}$ & $\begin{array}{l}\text { C-128 } \\
\text { S-8 }\end{array}$ & Present & 12.6 \\
\hline 2 & Positive & D,C,E,Fya, & D & 128 & & Absent & 21.5 \\
\hline 3 & Positive & D,C,E,Jka & D & 64 & & Present & 30.6 \\
\hline 4 & Positive & D,C,E,, & D,Fya & 128 & Fya 8 & Present & 15.8 \\
\hline 5 & Positive & D,C,E,Lea & D,C & 128,64 & C-16 & Present & 16.3 \\
\hline 6 & Positive & D,C,E,Leb, & D & 256 & & Present & 14.4 \\
\hline 7 & Positive & D,C,E,Jkb & D & 256 & & Present & 11.4 \\
\hline 8 & Positive & D,C,E,Fya, & D & 16 & & Present & 33 \\
\hline 9 & Positive & D,C,E,K,M & D & 32 & & Present & 21 \\
\hline 10 & Positive & D,C,E,Fya, & D & 64 & & Absent & 32.9 \\
\hline 11 & Positive & D,C,E,Jka & D & 128 & & Present & 21 \\
\hline 12 & Positive & D,C,E,Fyb, & D & 32 & & Present & 28 \\
\hline 13 & Positive & D,C,E,Jka & D & 16 & & Present & 18.7 \\
\hline 14 & Positive & D,C,E,Fya, & D & 128 & & Absent & 17.1 \\
\hline 15 & Positive & D,C,Jka & D & 512 & & Present & 11.2 \\
\hline 16 & Positive & D,C,E,Fya, & D.C & 128 & C-8 & Present & 9 \\
\hline 17 & Positive & D,C,E,Fya,M & M & 8 at 4 ${ }^{\circ} \mathrm{C}$ & & Absent & 17.7 \\
\hline
\end{tabular}

Figure 2: Plan of workup in Blood Bank Immunohemtology Lab.

\section{Clinical Transfusion \\ 6.1 Neonatal and Pediatric Transfusion}

$\mathrm{P}-188$

\section{NEONATAL TRANSFUSION SUPPORT FOR SAFE TRANSFUSION} PRACTICE BY TRANSFUSION SERVICE OF REGIONAL UNIVERSITY HOSPITAL

Y Fujii $^{1}$, T Kusuda ${ }^{2}$ and S Ohga ${ }^{2}$

${ }^{1}$ Yamaguchi University Hospital, Ube, Japan ${ }^{2}$ Department of Pediatrics, Yamaguchi University Graduate School of Medicine, Ube, Japan

Background: The unique requirements for neonatal transfusion affect the processing of units for neonatal transfusions, and the techniques used in those processes. Most transfusions in the neonatal intensive care unit (NICU) are administered at infusion rates so slow that they can only be given effectively through a rate-controlled syringe pump.

Aims: The aim of this study was to establish neonatal transfusion support for safe transfusion practice by the transfusion service of a regional university hospital.

Methods: Our university hospital has 20 beds in the NICU. To plan neonatal transfusion support, the transfusion service regularly discussed special needs for blood products and the precise timing needed for neonatal transfusions in the NICU. Results: For the neonatal transfusion of red blood cells (RBC) and apheresis platelets (APC), the following support was performed. The regional blood bank supplied irradiated and pre-storage leukocyte-reduced RBC. In order to avoid excess supernatant potassium, RBC units within 7 days of storage were issued for neonates at risk of hyperkalemia. A unit of RBC was divided into small-sized bags using a sterile connection device by the hospital transfusion service to avoid shortening the shelf-life. Each RBC aliquot in a small-sized bag was subsequently transferred to a syringe by NICU staff at the bedside, and simultaneously filtered with a particulate filter. This required the syringe to be labeled, in the NICU. An RBC aliquot in a small-sized bag was issued with a label for syringe and a barcode for electronic identification. The regional blood bank supplied irradiated and pre-storage leukocyte-reduced APC. For the division of APC, a transfer bag was connected with a sterile connection device to the original product bag. An APC aliquot in a transfer bag was issued just before use. This required clear communication by the clinical area to the transfusion service. APC remaining in the original bag could be used until the expiration date. Summary/Conclusion: Successful neonatal transfusion support for safe transfusion practice at a regional university hospital is feasible. However, communication between the transfusion service and NICU is essential to ensure the quality of neonatal transfusion.

P-189

\section{ANALYSIS OF RISK FACTORS ASSOCIATED WITH ALLERGIC TRANSFUSION REACTIONS AND FEBRILE NONHEMOLYTIC TRANSFUSION REACTIONS IN CHILDREN}

R Yanagisawa ${ }^{1}$, S Shimodaira ${ }^{2}, \mathrm{~K}_{\text {Sakashita }}{ }^{1}$, Y Hidaka $^{3}$, F Nishijima ${ }^{1}$, S Kojima ${ }^{2}$, E Hidaka $^{1}$, M Shiohara $^{4}$ and T Nakamura ${ }^{1}$

${ }^{1}$ Nagano Children's Hospital, Azumino, Japan ${ }^{2}$ Shinshu University Hospital, Matsumoto, Japan ${ }^{3}$ Shinshu University School of Medicine, Matsumoto, Japan ${ }^{4}$ Matsumoto Dental University School of Dentistry, Shiojiri, Japan

Background: he two major types of transfusion-related adverse reactions (TRARs) are allergic transfusion reactions (ATRs) and febrile nonhemolytic transfusion reactions (FNHTRs). Although prestorage leukocyte reduction (LR) of blood and diversion of the first aliquot of blood (diversion method; D) can reduce the incidence of FNHTR and bacterial contamination in adult blood transfusions, ATRs remain problematic. However, there is little information on TRARs in pediatric transfusion, such as underlying mechanisms of TRARs and risk factors.

Aims: To evaluate the real-world occurrence of TRARs associated with pediatric transfusions and to identify risk factors for pediatric TRARs from the recipient's background.

Methods: This retrospective study analyzed data from patients aged $\leq 18$ years who received transfusions at Nagano Children's Hospital from April 2002 to December 2014. The total number of each transfused product and patients were as follows: platelet concentrates (PCs): 6851 in 889 patients, red cell concentrates (RCCs): 11,699 in 2299 patients, and fresh-frozen plasma (FFP): 7193 in 1323 patients. Each product included LR products and LR products administered using the diversion method (LR/ D). Transfusions using secondary products such as washed PCs and RCCs that are used to prevent TRARs in patients with a history of TRARs were excluded. Analyses included TRAR frequency, both per patient and per transfusion product by product type. Possible associations between TRARs and patient background characteristics

(C) 2015 The Author Vox Sanguinis (C) 2015 International Society of Blood Transfusion Vox Sanguinis (2015) 109 (Suppl. 2), 1-96 
were evaluated using the records for all patients who were transfused with PCs (552 patients) and/or RCCs (1448 patients) separated by LR/D product. The Institutional Review Board of Nagano Children's Hospital approved this study.

Results: TRARs were primarily classified into ATRs and/or FNHTRs during pediatric transfusion of PCs and RCCs. Blood product administration after the introduction of LR significantly reduced not only FNHTRs but also ATRs during PC and RCC transfusion of each product. However, ATRs associated with PCs continued to occur, even after the introduction of LR products. Likewise, even after LR/D implementation, ATRs remained significantly more frequent in patients with primary hematologica and malignant diseases who received PCs and RCCs $(\mathrm{P}<0.01)$, in patients $\geq 1$ year old who received PCs $(P<0.01)$, and in patients who received frequent $(\geq 6)$ RCC transfusions $(\mathrm{P}<0.05)$. TRARs after FFP transfusions were extremely infrequent, and there were no significant differences.

Summary/Conclusions: These results indicate that leukocytes and factors secreted by leukocytes could explain the etiology underlying ATRs and FNHTRs associated with pediatric transfusion of PCs and RCCs. The mechanistic basis for pediatric TRARs may not be identical to that for adult transfusions, since some studies in adults indicated that LR was not effective in preventing ATRs. Furthermore, particular characteristics specific to children may also be risk factors for ATRs. Special attention may be required if children with these risk factors require PC or RCC transfusion.

\section{P-190}

\section{RED CELL ANTIBODY SCREENING IN PREGNANCY AT A TERTIARY CARE REFERAL HOSPITAL IN SOUTH INDIA: LESSONS LEARNT}

\section{S Chandrashekar and A Kantharaj}

Manipal Hospital, Bangalore, India

Background: Most international guidelines recommend antenatal antibody screening once at 10-16 weeks and next at 28 weeks to detect red cell alloimmunization and prevent fetal anemia and Hemolytic disease of fetus and newborn (HDFN).However, red cell antibody screening is seldom done routinely in many developing countries, as the problem goes unrecognized and hence its incidence is largely unknown. In the absence of this, some patients never receive Intrauterine/neonatal transfusions leading to deaths from fetal anemia.

Aims: (1) To identify $R h(D)$ negative women needing $R h(D)$ prophylaxis. (2) To identify the alloantibodies detected and assess its clinical significance. (3) To prepare for obstetrical and neonatal transfusions with antigen negative blood.

Materials and Methods: We present below Red cell antibody screening data of pregnant women, at a tertiary care referral hospital from Jan 2013-Dec14 (2 years). Screening was done by using Column agglutination (gel) technology using IgG AHG cards and a 3-cell panel comprising of R1wR1 (CwCD.ee), R2R2 (ccD.EE) and rr (ccddee) cells from BIORAD in conformance with BCSH guidelines. Screening was done at the time of booking pregnancy and all positive antibody screens were identified using extended cell panels and patients followed up where possible.

Results: A total of 2018 antenatal samples were screened for Red cell antibodies in pregnancy, out of which $116(5.75 \%)$ were antibody positive. Few Indian studies have shown a rate of $1-2 \%$. Statistical analysis by Fischer's exact test using a 2 X2 contingency table showed the results to be significant with a $\mathrm{P}$ value $<0.0001$. Higher alloimmunization rate in our study is attributed to the referred cases possibly those at higher risk. Anti-D was the most common $(\mathrm{n}=81)$ followed by Anti-E $(n=5)$, Anti-Le $(a)(n=5)$, Anti-S $(n=3)$, Anti-M $(n=2)$ and Anti-Cw $(n=1) \cdot$ Dual antibodies were found in 13 cases $(D+C, n=11),(D+K, n=1),(D+M, n=1)$, and there were 3 indeterminate cases. Another case of Anti-D was reported in a Partial D patient. Out of these 116 positive antibody screens 22 patients needed 1-4 intrauterine transfusions (IUT). Forty one IUTs were given to these 22 patients. Anti-D was the most common. In one case of IUT the patient had a dual antibody $(\mathrm{D}+\mathrm{C})$ and Le (a) antibody was found in another case. In 3 patients IUTs were carried out due to non-immune causes of fetal anemia.

Summary/Conclusion: Anti-D was the most significant with a high potential to cause HDFN.Co-existence of Anti-D and Anti-C was fairly frequent in our study Anti-E was found to be the next common Rh antibody but was not severe enough to necessitate IUTs. One case of Anti-Cw was noted but did not cause HDN. Anti-S. another clinically significant antibody was detected in 3 cases. Anti-M \&S have also been implicated in HDN. Surprisingly one case of Le(a) antibody needed IUT.Alloimmunization rates in Rh Positive women and Rh negtaive women will be discussed. Screening for RBC antibodies helped the transfusion service in its goal of identifying $\mathrm{Rh}$ (D) negative women for $\mathrm{Rh}(\mathrm{D})$ prophylaxis, identifying fetuses at risk which needed IUT, and preparing for emergency obstetrical and neonatal transfusions with antigen negative blood.

\section{(C) 2015 The Author}

Vox Sanguinis (C) 2015 International Society of Blood Transfusion

Vox Sanguinis (2015) 109 (Suppl. 2), 1-96
Table 1: Red cell antibody screening in pregnancy 2013-2014

\begin{tabular}{|l|l|l|l|}
\hline $\begin{array}{l}\text { Antenatal RBC Antibody } \\
\text { Screening }\end{array}$ & Year 2013 & Year 2014 & Total \\
\hline No Screened & 1065 & 953 & 2018 \\
\hline Positive Antibody Screens & 71 & 45 & 116 \\
\hline \% Positives & 6.66 & 4.72 & 5.75 \\
\hline Anti-D & 49 & 32 & 81 \\
\hline Anti-K & 02 & 0 & 02 \\
\hline Anti-E & 04 & 01 & 05 \\
\hline Anti-M & 02 & 0 & 02 \\
\hline AntiLe(a) & 04 & 01 & 05 \\
\hline Anti-S & 02 & 01 & 03 \\
\hline Anti-CW & 01 & 0 & 01 \\
\hline DUAL ANTIBODY & & & \\
\hline D+C & 05 & 06 & 11 \\
\hline D+K & 01 & 0 & 01 \\
\hline D+M & 01 & 0 & 01 \\
\hline Anti-D In Partial D & 01 & 0 & 01 \\
\hline Indeterminate & 0 & 03 & 03 \\
\hline $\begin{array}{l}\text { INTRAUTERINE } \\
\text { TRANSFUSIONS }\end{array}$ & 11 atients/20 & 11 Patients/21 & \\
\hline & Transfusions & Transfusions & \\
\hline
\end{tabular}

\subsection{Therapeutic Apheresis}

\section{P-191 RATIONALIZATION AND OPTIMIZATION OF THE THERAPEUTIC PLASMA EXCHANGE APPLICATION \\ Z Kirov}

Institute of Blood Transfusion Nis, Nis, Serbia

Background: Therapeutic plasma exchange (TPE) is a nonselective, nonspecific method used for the separation of circulating agents, an automated apheresis procedure with extracorporal circulation aimed at reducing the concentration or elimination of pathogen from the blood plasma, to reimburse the removed volume with suitable liquid. TPE is used in a number indications, which are currently classified in to four separate categories. The category I includes diseases in which the TPE is standard - the primary treatment option. For disorders of category II, TPE is an additional, supportive therapy, usually in combination with other treatment modalities. In the category III are pathological conditions, in which the exact role of apheresis is not stil safely defined - validity of TPE application, as well as the achieved effects are individual. The category IV includes diseases in which performing of TPE has not been proven effective.

Aim: Continuous introduction of new and more effective immunomodulatoty and other medications, the indications for performing TPE and achieved effects require continuous critical re-evaluation. For this reason, the aim of this work was analisysi of the feasibility of this type of treatment, with the categorization of indications, are essential for understanding the place and role of TPE in the treatment of these patients group.

Methods: Retrospective analysis of indications for the total of 1075 TPE during the period from 2010 to 2014, as well as categorization according to criteria AABB at the Institute for Blood Transfusion Nis, Serbia, has been done. TPEs are performed using the blood cell separator Haemonetics MCS+, according to the applicable standards and recommendations about the number and frequency of procedures, optimized time of application and the amount of extracted plasma.

Results: All of 1075 TPE procedures are analysed and categorized, and the following results were obtained, category I was represented in $42.2 \%$, 31.8\% category II, category II -III in $18.2 \%$ and category III in $7.8 \%$, while category IV was not represented. The most common indication was myasthenia gravis (MG) 55 patiens, 205 TPE, followed by Guillain - Barre syndrome with 34 patients, 141 TPE, multiple sclerosis 29 patients, 109 TPE, hiperbilirubinemia 21 patients, 72 TPE, CIDP 13 patients, 49 TPE, and thrombotic thrombocytopenic purpura (TTP) was represented in 8 patients, 35 TPE, and odher in I, II, III category.

Conclusion: The results suggest that the indications for TPE predominantly belong to category I and II, while disorders in the category IV were not presented. This can be interpreted as rationalization and optimization of the TPE application, as a special form/modality of the treatment. The positive therapeutic effect of TPE in the treatment of patients depended upon the nature of the basic disease, its stage, general condition, volume of plasma removed and additional therapy. 
P-192

\section{THERAPEUTIC PLASMA EXCHANGE: REVIEW OF THE RESULTS}

R Sood, G Aroskar, S Kumar and V Kumar

Saket City Hospital, New Delhi, India

Background: Therapeutic plasma-exchange (TPE) is used as primary as well as adjunctive therapy in treatment of several category of diseases $(1,2)$. Technological innovations and the adoption of evidence-based indications in the field of medicine have led to the establishment of guidelines on the clinical indications for the use of therapeutic apheresis (3).

Aim: Review of the outcome of the consecutive TPE procedures performed on various categories of patients.

Methods: Retrospective analysis and evaluation of the TPE procedures performed at our centre was done for a time duration of July 2013-2015 June.

Results: A total of 71 TPE procedures were performed in patients ( 2 males and 8females, with mean age of $57.2 \pm 5$ years). Three of the 10 patients had GuillainBarré syndrome (GBS), 1 patient had anti glomerular basement membrane CKD disease (Good pasteurs Syndrome), 2 patients had Wegeners Granulomatosis (Systemic Vasculitis cANCA positive), 2 had Myasthenia Gravis, 1 Chronic Inflammatory Demyelinating Polyneuropathy (CIDP) and 1 patient was given TPE treatment for SLE. Out of the total, 9 patients belonged to indication category 1 and one patient belonged to indication category 111.Fresh frozen plasma (FFP) was used as replacement fluid. Complete response (CR) was achieved on 8 patients $(80 \%)$ in of the cases. Complications were detected in none of the TPE procedures. Adverse events (AEs), were seen in 5.6\% of all procedures. None of the patients died from any complication or adverse event. AE occurred as three as Grade-I (mild -no treatment required and procedure continued) and one patient as Grade-III (severe- treatment required, procedure had to be finished). The most common $\mathrm{AE}$ were nausea/vomiting, hypotension, pruritus and abdominal pain.

Conclusions: TPE is effectively and safely carried out for various diseases. (1) MedlinePlus. 'Goodpasture syndrome'. U.S. National Library of Medicine. Retrieved 7 April 2013. (2) Jump up ^Yazdi, MF; Baghianimoghadam, M; Nazmiyeh, H; Ahmadabadi, AD; Adabi, MA (2012). 'Response to plasmapheresis in myasthenia gravis patients: 22 cases report'. Romanian journal of internal medicine $=$ Revue roumaine de medecine interne 50 (3): 245-7. PMID 23330293. (3) McLeod BC. Therapeutic apheresis: history, clinical application, and lingering uncertainties. Transfusion. 2010;50:1413-26.

\section{$\mathrm{P}-193$}

\section{A CASE SERIES OF THREE PATIENTS UNDERGOING THERAPEUTIC LEUKAPHERESIS IN A TERTIARY CARE HOSPITAL}

B Kakkar, RN Makroo, M Chowdhry, A Bhatia and UK Thakur

Indraprastha Apollo Hospitals, New Delhi, India

Introduction: Hyperleukocytosis is a condition characterised by increase in the total leukocyte count (TLC) or blast count greater than $1,00,000 /$ cumm. It is usually seen in $5-18 \%$ of patients with AML depending on the leukemic subtype. Leukostasis is characterised by abnormal intravascular leukocyte aggregation and clumping and is associated with high early mortality in these patients. As leukostasis can compromise the functioning of respiratory system and central nervous system. Leucoreduction is achieved either by leucapheresis or chemotherapy. Therapeutic leukapheresis is usually initiated as soon as clinical signs of leukostasis are seen.

Case report: We present here case reports of 3 patients who underwent therapeutic leukoapheresis at our centre. Leukapheresis was performed using Haemonetics MCS+ (USA) cell separator using central venous access. ACD was used as anticoagulant in a ratio of 1:12 with blood.

Case 1: A 25 year old male, admitted with history of sore throat and fever with chills and rigors for 1 month, petechial haemorrhages for 20 days and unexplained weight loss. On examination, patient had subconjunctival haemorrhage, petechiae, hepatosplenomegaly and bilateral submandibular and cervical lympadenopathy. After detailed laboratory workup and bone marrow biopsy along with flow cytometry, he was diagnosed as biphenotypic acute leukemia (myeloid/Tcell). On day 4 of admission, patient experienced acute blast crisis (97\%) and emergency therapeutic leukapheresis was planned with pre procedure TLC of 3,21,200/ $\mu \mathrm{l}$. A TLC fall of $51.4 \%$ was seen post procedure to $1,65,200 / \mu \mathrm{l}$. No adverse events or procedure related complications were reported. Post procedure the patient improved considerably. He was weaned of assisted ventilator support and his TLC counts stablilised. Case 2: A 28 year old male, diagnosed case of chronic myeloid leukemia on treatment since 2007. He came with relapsed disease with blast crisis (96\%). His TLC was $1,02,100 / \mu \mathrm{l})$ for which two procedures were performed on consecutive days. There was considerable fall in total leucocyte count to $78,800 / \mu \mathrm{l}$ (77\% reduction) at the end of two procedures. He experienced mild symptoms of citrate toxicity during the first procedure, which was managed effectively with calcium infusion. Marked improvement in symptoms was seen and patient went into complete remission. Case 3: This was a 69 year old diabetic, hypertensive male recently diagnosed with myeloproliferative syndrome with myelofibrosis; JAK-2 positive. His pre-procedure TLC was $87,100 / \mu l$. There was a significant fall in TLC to $54,200 / \mu l(62 \%)$ after one procedure. There were no procedure related complications. Patient improced considerably and was discharged. However, he was readmitted later with urological problems and later succumbed to his illness.

Conclusion: It is evident from the cases presented above that Therapeutic leukapapheresis is a safe and effective approach for rapidly reducing WBC counts in patients with blast crisis or any other haematological malignancies and can be a lifesaving modality in patients at risk of leukostasis.

\subsection{Evidence Based Transfusion Medicine Practice}

\section{P-194}

\section{OPTIMIZING RED CELL EXCHANGE PROCEDURES FOR SICKLE CELL PATIENTS}

E Himchak, A Bonzon-Adelson, J Uehlinger, K Mohandas, R Walsh and D Wu Montefiore Medical Center, Bronx, United States of America

In patients with sickle cell disease (SCD), transfusion therapies have been shown to reduce the risk of recurrent strokes and prevent initial strokes in patients with abnormal cerebral blood flow. Current guidelines recommend a monthly transfusion protocol using manual or automated red cell exchange (RCE) or simple transfusion. The goal of therapy in the pediatric population is to maintain the patient's HbS below $30 \%$ at the time of the next transfusion in at-risk patients. It is not clear in any given patient how much to transfuse or exchange to reach these goals.

Immediate post transfusion $\mathrm{HbS}$ (post-HbS) can be calculated with relative certainty with the number of red blood cell (RBC) units, pre-transfusion parameters like $\mathrm{HbS}$, hematocrit, and patient weight. Our goal is to find a post-HbS following which our patients will remain below $30 \% \mathrm{HbS}$ until their next transfusion.

We uploaded a cohort of 18 sickle cell patients receiving long-term RCE into our institution's patented clinical information system search tool called Clinical Looking Glass. We extracted $\mathrm{HbS}$ results from these patients and categorized them into pretransfusion $\mathrm{HbS}$ (pre-HbS), post-HbS, and follow up pre-transfusion $\mathrm{HbS}(\mathrm{f} / \mathrm{u} \mathrm{HbS}$ ) 22-40 days later (i.e. the pre-HbS at the next visit). Each set of data was considered a patient event and categorized by post-HbS of $<10 \%, 10-15 \%, 15-20 \%, 20-25 \%$, $25-30 \%$, and $30+\%$. Follow-up data was analyzed to identify if fHbS was above or below the $30 \%$ level. Linear regression analysis was used to evaluate the correlation between post-HbS and $\mathrm{HbS}$.

A total of 218 sets of data were identified from 2000-2015. Linear regression analysis revealed a weak correlation $\left(R^{2}=0.369\right)$ between post-HbS and fHbS. No particular level of post-HbS guarantees maintenance of $\mathrm{HbS}<30 \%$. In the post-HbS $<10 \%$ category $(\mathrm{n}=8), 62.5 \%$ of patients had $\mathrm{fHbS}<30 \%$ while the $10-15 \%$ category $(\mathrm{n}=16)$ had $19 \%$ of patients with $\mathrm{HbS}<30 \%$. Applying a Fischer's Exact test suggests a possible difference in these categories $(\mathrm{P}=0.06)$ which may be statistically significant with a larger cohort. Few patients with post-HbS $>15 \%$ had acceptable fHbS results ( 5 of 194 patient events). For each individual patient, monthly HbS values varied markedly over the course of years without a discernible pattern.

This project highlights the difficulty of maintaining low HbS in SCD patients especially over many years. The cutoff post-HbS level will likely lie around 10\%, in order to maintain most of the patients' $\mathrm{f} / \mathrm{u} \mathrm{HbS}<30 \%$. With several of our patients developing alloantibodies to very high frequency or multiple antigens, we try to maintain an appropriate $\mathrm{fHbS}$ while minimizing exposure to donor antigens. Further studies may be done to correlate HbS to clinically significant events like strokes and possible underlying clinical factors.

\section{P-195}

\section{A CLINICAL AUDIT OF RED CELL TRANSFUSION IN ELECTIVE TOTAL HIP REPLACEMENT SURGERY}

YG Peters ${ }^{1}$, GM Davison ${ }^{2}$, G Bellairs ${ }^{1}$ and A Bird

${ }^{1}$ Western Province Blood Transfusion Service, Cape Town, South Africa ${ }^{2}$ Cape Peninsula University of Technology, Cape Town, South Africa

Background: Previous international studies have documented a marked variation in transfusion practice for Total Hip Replacement (THR) surgery. This is despite widespread dissemination of clinical guidelines for the use of blood products. The cost

(C) 2015 The Author

Vox Sanguinis @ 2015 International Society of Blood Transfusion Vox Sanguinis (2015) 109 (Suppl. 2), 1-96 
and potential wastage of blood products as well as concerns regarding patient care and outcomes are important drivers of optimal blood management.

Aim: The aim of this study was to audit red cell product utilisation for THR surgeries at two tertiary referral hospitals in the Western Cape, South Africa.

Methods: A retrospective clinical audit was conducted at two tertiary hospitals in the Western Cape. The audit involved patient folder review and the review of computer records of the respective blood banks. The folders of two hundred and seven consecutive patients undergoing elective THR surgery from January 2013-December 2013 from each hospital were reviewed. Baseline data relating to age, gender, clinical observations, indications for surgery, pre and post operative haemoglobin values, comorbidities, length of hospital stay and transfusion history was recorded.

Data was then uploaded from individual data collection sheets onto IBM SPSS version 22 statistical package for analysis.

Results: The two institutions were coded Hospital Green (HG) and Hospital Yellow (HY). Of the two hundred and seven patients who had THR surgery, eighty seven were excluded from the analysis, leaving one hundred and twenty eligible for review, which were equally split between the two hospitals. The transfusion rate at HY (41.6\%) was significantly higher than that at HG (10\%). The mean post-operative haemoglobin $(\mathrm{Hb})$ in the transfused patients at $\mathrm{HG}$ was $8.3 \mathrm{~g} / \mathrm{dl}$, compared to HY with a mean of $9.1 \mathrm{~g} / \mathrm{dl}$. Females had a significantly higher transfusion rate $(33 \%)$ than males $(15 \%)(\mathrm{P}<0.05)$ and the mean age of transfused patients was significantly greater than untransfused patients $(\mathrm{P}<0.005)$. Although patients with comorbidities had a higher transfusion rate than those without, this did not reach statistical significance. One hundred and thirteen (94\%) patients had a blood order from the blood bank, the vast majority, one hundred and two (85\%), were group and screen requests of which twenty nine $(24 \%)$ were converted to a full crossmatch

Conclusions: The transfusion rates for the two hospitals for elective THR were significantly different although both are well within the wide ranges documented in the literature. Although there are no bench marks, several studies support a restrictive transfusion regimen strategy with a guideline 'trigger' Hb level of 7-8 g/dl. In this study HG had a transfusion rate of $10 \%$ with a mean post operative $\mathrm{Hb}$ of $8.3 \mathrm{~g} / \mathrm{dl}$ in the transfused population, compared to $9.1 \mathrm{~g} / \mathrm{dl}$ at HY which had a transfusion rate of $41.6 \%$. This suggests that a benchmark of $20 \%$ would not be unreasonable and together with rational blood ordering policies (group and screen rather than a full crossmatch) would lead to significant savings with no extra risk to the patient.

\section{P-196}

\section{RE-EVALUATION OF MAXIMUM SURGICAL BLOOD ORDER SCHEDULE (MSBOS) IN A SECONDARY CARE HOSPITAL IN KOREA}

MJ Kim and ACdr Oh

Seonam University College of Medicine, Goyang, South-Korea

Background: Effective usage of RBC product can be achieved by monitoring maximum surgical blood order schedule (MSBOS). Our previous MSBOS was set for 220 type of surgery and its range was from type and screen (TtS) to 4 units. Re-evaluation of MSBOS is essential for transfusion because surgical technique and need for transfusion can be changed. We reset MSBOS by reviewing of surgical blood order data from 2011 to 2014

Aims: We planned to reset MSBOS by reviewing of surgical blood order data from 2011 to 2014 to use RBC product effectively and feedback to surgeons.

Methods: Number of each surgery and RBC product ordered from 2011 to 2014 were reviewed. Exclusion criteria were local anesthetic surgery, emergency surgery, transfused within $24 \mathrm{~h}$ before surgery, surgery for more than 2 organs and less than 5 cases per 3 years. Mean C/T ratio, mean unit, median unit, minimum unit, maximum unit and SD of each surgery and each surgeon were calculated. MOBOS was decided as a mean of blood unit for each surgery.

Results: Total number of included surgery was 2087 (total number: 3403, excluded number: 1316) and kinds of the surgery were 51. The lesser kind of surgery and larger amount of RBC units than previous one was included in MSBOS (from TAS to 15 units). T\&S for 10 types (19.6\%), 1 unit for 10 types (19.6\%), 2 units for 12 types (23.5\%), 3 units for 12 types (23.5\%), 4 units for 2 types (3.9\%), 5 units for 1 type (2.0\%), 6 units for 1 type $(2.0 \%), 7$ units for 1 type $(2.0 \%)$, 8 units for 1 type $(2.0 \%)$ and 15 units for 1 type $(2.0 \%)$ of surgery were calculated. Mean C/T ratio, mean unit, median unit, minimum unit, maximum unit and SD of each surgeon were 1.9, $1.6,1,0,37$, and 2.8, respectively.

Conclusions: Our current MSBOS was higher than previous one and other articles reported. And amount of RBC units of each surgeon were highly variable. Regular re-evaluation of MSBOS is mandatory to reflect the progression of surgical technology. We will give surgeons information of MSBOS for each surgery and suggest the adequate number of blood component units.
P-197

This abstract has been withdrawn.

P-198

EFFICACY OF PLATELET CONCENTRATES PREPARED BY

RANDOM DONOR BUFFY COAT AND SINGLE DONOR APHERESIS IN ACUTE LEUKEMIA AND CHEMOTHERAPY INDUCED PANCYTOPENIA

AK Jindal ${ }^{1}$, A Kaur ${ }^{1}$, R Kumar ${ }^{1}$ and JS Sekhon ${ }^{2}$

${ }^{1}$ Dayanand Medical College and Hospital, Ludhiana, India ${ }^{2}$ Fortis Hospital, Ludhiana, India

Background: Since discovery of platelets, there has been continuous and accelerated progress in understanding of platelet functions and their utilization. In Oncology, platelet transfusions are indicated for prophylactically and/or therapeutically. Despite our improving knowledge about the beneficial effect of platelet concentrates, there has been limited data that can predict their therapeutic efficacy quantitatively. The need for this study arose to fill this void, to assess the therapeutic efficacy of leuko-reduced platelet concentrates made by buffy coats and single donor apheresis in thrombocytopenic patients of Acute Leukemia and Chemotherapy Induced Pancytopenia

Aims: To study the post transfusion therapeutic efficacy of Buffy Coat Random Donor Platelet Concentrates (RDP) and Single Donor Apheresis Platelet concentrates (SDP) in patients of Acute Leukemia and Chemotherapy Induced Pancytopenia.

Methods: Thrombocytopenic patients from oncology ward were included as per the inclusion criteria. Platelet count of the platelet concentrates to be transfused was done prior to transfusion. Post transfusion efficacy of platelets in the patients (in vivo efficacy) was assessed by Corrected Count Increment (CCI) [units: platelets $\mathrm{m}^{2} / \mu \mathrm{l} \times 10^{11}$, hereafter implied for the sake of clarity] and Percentage Recovery (PR) at $1 \mathrm{~h}$ and $24 \mathrm{~h}$. If the CCI at $1 \mathrm{~h}$ and $24 \mathrm{~h}$ is $<7500$ platelets $\mathrm{m}^{2} / \mu \mathrm{l}$ and $<$ 4500 platelets $\mathrm{m}^{2} / \mu \mathrm{l}$ and PPR at $1 \mathrm{~h}$ and $24 \mathrm{~h}<30 \%$ and $<20 \%$ respectively, it indicates platelet refractoriness. Data was analyzed using Student t-test on SPSS Version 21.0.

Results: Out of 61 patients, who had comparable pre-transfusion platelet count; 48 received $\mathrm{RDP}$ and 13 received SDP. In patients receiving $\mathrm{RDP}$, the mean platelet increment at $1 \mathrm{~h}$ and $24 \mathrm{~h}$ was $22784.8 \pm 1166.2 \times 10^{11}$ platelets and $16835.4 \pm 8859.9 \times 10^{11}$ platelets, respectively. The Mean CCI at 1 Hour and $24 \mathrm{~h}$ was $10727.1 \pm 5495.3$ and $7915.1 \pm 4201.1$ respectively. The Mean PR at $1 \mathrm{~h}$ and $24 \mathrm{~h}$ was $24.0 \pm 12.2 \%$ and $17.7 \pm 9.3 \%$ respectively. In patients receiving SDP, the mean platelet increment at $1 \mathrm{~h}$ and $24 \mathrm{~h}$ was $48619.0 \pm 17214.8 \times 10^{11}$ platelets and $41952.4 \pm 16758.5 \times 10^{11}$ platelets respectively. The Mean CCI at $1 \mathrm{~h}$ and $24 \mathrm{~h}$ was $18104.5 \pm 6375.5$ and $15602.6 \pm 6229.1$ respectively. The Mean PR at $1 \mathrm{~h}$ and $24 \mathrm{~h}$ was $48.4 \pm 17.2 \%$ and $41.7 \pm 16.9 \%$ respectively. Platelet refractoriness was seen in $15(24.5 \%)$ of cases out of which $13(86.6 \%)$ were seen in patients receiving RDP and $2(13.3 \%)$ in patients receiving SDP. Statistically significant $(\mathrm{P}=0.001)$ higher platelet increment, CCI and PR was observed with SDP than RDP and less refractoriness was seen with SDP transfusion.

Summary/Conclusions: Transfusing SDP is more efficient method to increase platelet count in oncologic thrombocytopenic patients and is associated with longer in vivo survival of platelets.

\section{P-199 \\ BLOOD PRODUCT USAGE IN ADULT PATIENTS WITH MASSIVE BLEEDING \\ B Zhou, A Zhou and Y Wu}

Bloodworks Northwest, Seattle, United States of America

Background: Patients with massive bleeding often require large number of blood product transfusion. These requirements often put an extra burden on the hospital transfusion service inventory as well as the local community blood resource. Understanding the usage pattern of blood products in these patients can help blood bank and blood service personnel estimate the needs and prepare for these events, which are often unexpected.

Aims: To identify usage pattern of blood products in patients with massive bleeding.

Methods: Retrospective review of transfusion data from 08/01/2013 to $07 / 31 / 2014$ was performed. Patients with massive bleeding were defined as patients who used 10 or more units of RBC over a $24 \mathrm{~h}$ period. Data included blood product usage of mul- 
tiple hospitals including two tertiary hospitals and 12 small to mid-size community hospitals. Data did not include one local children's hospital as well as a local level 1 trauma center. For platelets, the standard adult dose which is equivalent to a single donor apheresis platelet (SDP) is used. For cryoprecipitates (Cryo), the number of cryo pool with a pool size of 5 is used.

Results: Please see Table 1 for the summary of data. Of these patients, $40 \%$ were Opos, and $8 \%$ were 0 neg. More than $75 \%$ of patients with massive bleeding belong to the following four diagnosis categories: 1) Patients undergoing solid organ transplantation (liver, heart, and lung); 2) Patients undergoing cardiac surgery; 3) Patients with GI bleeding; 4) Patients with Obstetric bleeding (OB bleeding).

In addition, the occurrence of major bleeding events happened in about 25\% of days in a year. They occurred during any day of the week.

Summary/Conclusions: Major bleeding in adult patients can happen anytime. Certain diagnoses or procedures, such as solid organ transplantation, cardiac surgery, GI bleeding, Obstetric bleeding, put patients at high risk for massive bleeding and require a large number of blood products. Understanding this usage pattern can facilitate the preparedness of both the transfusion service and the blood service. Table 1.

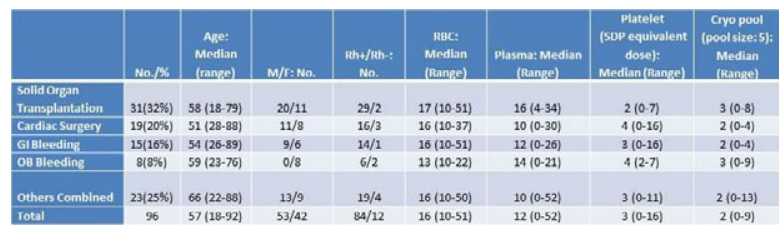

P-200

THE EFFECT OF PERI-OPERATIVE ANEMIA AND BLOOD

TRANSFUSION ON PATIENTS WITH GASTRIC CANCER

\section{RECEIVING GASTRECTOMY}

CC Chang, TC Lee and FY Chu

Far Eastern Memorial Hospital, New Taipei City, Taiwan

Background: It was reported that patients with malignancies, such as lung, breast, gastric, renal and colorectal cancers, could have shorter survival when receiving blood transfusion. Few studies had illustrated the impact of peri-operative blood transfusion on adverse outcome in patients with gastric cancer (GC)

Aims: The aim of this retrospective study was to evaluate the effect of peri-operative anemia and blood transfusion on GC patient with surgical intervention.

Methods: From Jan, 2012 to Dec, 2014, patients with GC receiving gastrectomy were enrolled. The clinical data, including patient age, gender, pathological report, TNM stage, admission day, operation (OP) day, discharge day, hemoglobin ( $\mathrm{Hb})$ levels on admission, before OP and discharge, the nadir HB from OP to discharge, the transfused units of red blood cell products from admission to OP day, during OP and after OP to discharge, were collected via electronic chart review. Risk factors associated with peri-operative anemia status and blood transfusion were estimated by calculating odds ratio (OR) with 95\% confidence interval (CI). Kaplan-Meier analysis was used for evaluation of 1-year survival rate.

Results: A total of 97 patients with GC had undergone surgical treatment. Of these, 82 were adenocarcinoma, 13 were malignant gastrointestinal stromal tumor, 1 was neuroendocrine tumor and 1 was gastric lymphoma. Patients with a pre-OP Hb level more than $10.0 \mathrm{~g} / \mathrm{dl}$ needed less blood transfusion during or after OP (OR: 0.17, 95\% CI: $0.06-0.49, \mathrm{P}<0.001)$. Besides, pre-OP transfusion was less likely to induce a nadir $\mathrm{Hb}$ level less than $8.0 \mathrm{~g} / \mathrm{dl}$ (OR: 0.21, 95\% CI: 0.05-0.94, P = 0.042). However, pre-OP transfusion increased the risk of intra- or post-OP transfusion (OR: 4.13, 95\% CI: $1.59-10.73, \mathrm{P}=0.003)$. The 1-year survival of $\mathrm{GC}$ patients with a pre-OP $\mathrm{Hb}$ level more than $10 \mathrm{~g} / \mathrm{dl}$ was lower than those less than $10 \mathrm{~g} / \mathrm{dl}$, but with no significance $(90.1 \%$ vs $96.2 \%, P=0.444)$. Similarly, there was no significant difference between those with and without pre-OP transfusion in 1-year survival (85.7\% vs 94.2\%, $\mathrm{P}=0.223$ )

Conclusions: Anemia before OP was associated with increased rate of blood transfusion intra- and post-operatively. It seemed that pre-operative blood transfusion avoided a nadir Hb level less than $8 \mathrm{~g} / \mathrm{dl}$ in GC patients receiving surgical intervention. Nevertheless, there was no evidence suggesting that pre-operative anemia or peri-operative blood transfusion affected the 1-year survival of GC patients undergoing gastrectomy.
Table 1: The odds ratio (OR) of factors associated with peri-operative anemia and blood transfusion and their 95\% confidence intervals (CI) in gastric cancer.

\begin{tabular}{ll}
\hline Variables & \\
\hline Age (year) & $68.1 \pm 11.8$ \\
Gender (M/F) & $59 / 38$ \\
Gastric cancer & \\
Adenocarcinoma & 82 \\
Malignant GIST & 13 \\
Neuroendocrine tumor & 1 \\
Gastric lymphoma & 1 \\
\hline
\end{tabular}

Table 2: The demographic characteristics of patients with gastric cancer receiving gastrectomy.

\begin{tabular}{lccc}
\hline Variables & OR & $95 \% \mathrm{Cl}$ & P value \\
\hline Pre-OP Hb level $\geqq 10.0 \mathrm{~g} / \mathrm{dL}$ & & & \\
$\begin{array}{l}\text { Intra/post-OP transfusion } \\
\text { Pre-OP transfusion } \\
\text { Nadir Hb level } \leqq 8.0 \mathrm{~g} / \mathrm{dL}\end{array}$ & 0.17 & $0.06-0.49$ & $<0.001$ \\
Intra/post-OP transfusion & 0.21 & $0.05-0.94$ & 0.042 \\
\hline
\end{tabular}

P-201

SAVING OUR BLOOD: A BLOOD MANAGEMENT APPROACH IN A REGIONAL HOSPITAL

LR Hollis $^{1}$, CA Lambooy ${ }^{1}, \mathrm{H}$ Weston ${ }^{1}$ and YL Fung ${ }^{2}$

${ }^{1}$ Sunshine Coast Hospital and Health Service, Nambour, Australia ${ }^{2}$ University of the Sunshine Coast, Sippy Downs, Australia

Background: Blood management strategies have been implemented in both Australia and overseas to reduce inappropriate blood use, exposure to blood products and indirectly reducing the number of adverse events.In 2011 the cost of blood products in Queensland was devolved from the state budget to each hospital and health service. Health services were then responsible to pay one-third of all fresh blood product cost, with the remaining two-thirds being funded by the federal government.The Sunshine Coast Hospital and Health Service (SCHHS) is a regional service with 4 public hospitals and 513 overnight beds.To address the challenges of maintaining a sustainable blood supply within the confinements of a devolved blood budget, a SCHHS blood management strategy was introduced to improve the appropriateness of transfusions practice.

Aim: To assess the effectiveness of a blood management strategy in a regional hospital for the use of fresh blood products.

Method: To improve appropriate blood use, the SCHHS Blood Management Committee in collaboration with the Queensland Blood Management Program developed and implemented an adult red cell single unit pathway and a two unit fresh frozen plasma pathway. Practice change was implemented by in-service training sessions, memos, meetings, emails and informal conversations. Compliance with the blood management strategy was monitored by blood bank laboratory scientists, the transfusion nurse and blood management committee members. Blood use, its appropriateness and transfusion related complications were tracked and reported at the SCHHS Blood Management Committee. The results for the period from July 2011 and June 2015 are reported below.

Results: Red blood cell use reduced from 5119 units in 2011 to 4047 units in 2014.Fresh Frozen Plasma use reduced from 1339 units in 2012 to 716 units in 2014.0verall, from 2011 to 2015 the total fresh blood product use has continued to decline and significant cost saving to the Health Service blood budget have been achieved. These savings amount to approximately AUD $\$ 600,000.00$.

(C) 2015 The Author Vox Sanguinis (C) 2015 International Society of Blood Transfusion Vox Sanguinis (2015) 109 (Suppl. 2), 1-96 
Transfusion related adverse events documented in the SCHHS incident reporting database (PRIME CI) have reduced significantly in alignment with the blood management strategies and reduced patient exposure to blood products. 48 adverse events were reported in 2013, 28 reported in 2014, and 21 reported in 2015, this is an overall reduction of $56 \%$.

Comparison of blood use by activity (WAU - Weighted Activity Unit) indicates that even though hospital activity is increasing, blood use has decreased by approximately $30 \%$. Current SCHHS data indicates the cost of blood by WAU was approximately $\$ 11$ in 2012/13 and has reduced to approximately $\$ 7$ in 2014/15.

Summary/Conclusion: The SCHHS data demonstrates that a blood management program including simple strategies such as single unit transfusions, complimented by promotion and education can significantly reduce blood use and transfusion related complications.

\section{$\mathrm{P}-202$}

\section{CLINICAL EFFICACY OF 'O' GROUP SINGLE DONOR} PLATELETS WITH PLATELET ADDITIVE SOLUTION

P Jain, A Tendulkar, A Gupta, J Sanal and A Navkudkar

Tata Memorial Hospital, Mumbai, India

Background: This study was conducted in a tertiary care oncology setup wherein platelet transfusions are an integral part of the supportive care. Guidelines recommend $\mathrm{ABO}$-identical platelet transfusions to patients as opposed to non-identical groups. However, maintaining an inventory of group specific platelet concentrates (PC) is challenging. Also as a prelude to this study, we performed the titres (anti A, anti B) in ' 0 ' group donors which were high $(\geq 128)$ in $57.19 \%$ of donors. Hence, we studied the feasibility of preparing group ' 0 ' Single donor platelets (SDP) with Platelet additive solution (PAS) to obtain safer low titre units and to overcome the problems related to minor incompatible platelet transfusions. This would ensure availability of group 0 units during emergency. The units produced were tested for quality parameters with respect to viability, morphology, sterility, metabolism and activation. These units were then transfused to the patients and analyzed for clinical efficacy.

Aim: To study the clinical efficacy of PAS added group 0 single donor platelets. Methods: SDP $(n=50)$ were prepared from 0 group donors on Amicus ${ }^{\circledR}$ (version 3.2. Fenwal Inc., USA) cell separator which provides 3 log leukodepletion. PAS III $\left(\mathrm{SSP}+{ }^{\mathrm{TM}}\right.$, MacoPharma, Moveaux, France) in a ratio 70:30 (PASIII: plasma) was added at source under sterile conditions (study arm). The units after being studied on day of collection (day 0) and day 4, and compared with 50 SDP's containing 100\% plasma (control arm) were transfused to the adult inpatients presenting with thrombocytopenia. The study was approved by institutional ethics committee and written informed consent from the patients was obtained prior to transfusion. The parameters studied post transfusion were Count Increment (CI), corrected count increments (CCI) and Percentage platelet recovery (PPR). The statistical analysis was performed using one way ANOVA for parametric data and Kruskal-Wallis one way ANOVA for non-parametric data.

Results: There was no statistically significant difference with respect to baseline parameters like weight, height and body surface area of the patients in two arms. The mean CI in study and control arm was $30,900 / \mu \mathrm{l}$ and $31,500 / \mu \mathrm{l}$ respectively. The $\mathrm{CCI}$ at $1 \mathrm{~h}$ and at $24 \mathrm{~h}$ amongst the two arms was not significantly different $(\mathrm{P}=0.839$, and $\mathrm{P}=0.536)$, with mean $\mathrm{CCI}$ at $1 \mathrm{~h}$ being $11,970 / \mu \mathrm{l} / \mathrm{m}^{2}$ and $13,525 / \mu \mathrm{l} /$ $\mathrm{m}^{2}$ for study and control arm respectively. The PPR for study and control arms was $33.74 \%$ and $36.6 \%$ respectively and were not significantly different $(\mathrm{P}=0.885)$. There was no adverse reaction reported in any patient in the two arms. Intra-group comparison of study arm showed that CI, CCI at 1 and $24 \mathrm{~h}$ and PPR at $1 \mathrm{~h}$ were not significantly different when group 0 SDP's with PAS were transfused to A, B and 0 group patients.

Conclusion: 0 group SDPs can be safely prepared with an additive solution and show clinical efficacy comparable to units with 100\% plasma. Patients are benefitted with these units, especially during platelet shortages which require $\mathrm{ABO}$ switchovers.

(C) 2015 The Author

Vox Sanguinis (C) 2015 International Society of Blood Transfusion

Vox Sanguinis (2015) 109 (Suppl. 2), 1-96
Table 1: Inter-Group comparison of Clinical efficacy in study arm and control arm.

\begin{tabular}{|c|c|c|c|c|c|}
\hline \multirow{2}{*}{$\begin{array}{l}\text { Patient Parameters } \\
\text { studied }\end{array}$} & \multicolumn{2}{|c|}{ Study Arm $(n=50)$} & \multicolumn{2}{|c|}{ Control Arm $(n=50)$} & \multirow{2}{*}{$\mathrm{p}$-value } \\
\hline & Mean & Median & Mean & Median & \\
\hline Weight of patients (kg.) & 62.32 & 60 & 59.30 & 58.5 & 0.258 \\
\hline Height of patients $(\mathrm{cm})$. & 158.28 & 158 & 158.44 & 158 & 0.895 \\
\hline Body surface area $\left(\mathrm{m}^{2}\right)$ & 1.6531 & 1.6202 & 1.6135 & 1.6125 & 0.271 \\
\hline Pre transfusion count $(/ \mu \mathrm{l})$ & 12560 & 10000 & 17540 & 14500 & $0.009^{\star}$ \\
\hline Platelet dose (x 1011) & 4.3 & 4.22 & 3.7 & 3.5 & $0.001^{*}$ \\
\hline $\begin{array}{c}\text { Count increment at } 1 \mathrm{Hr} \\
(/ \mu \mathrm{l})\end{array}$ & 30900 & 31500 & 31500 & 27000 & 0.368 \\
\hline $\begin{array}{c}\text { Corrected count increment } \\
\text { at } 1 \mathrm{Hr}\left(/ \mu \mathrm{l} / \mathrm{m}^{2}\right)\end{array}$ & 11970 & 11436 & 13525 & 11319 & 0.809 \\
\hline $\begin{array}{c}\text { Corrected count increment } \\
\text { at } 24 \mathrm{Hr}\left(/ \mu \mathrm{l} / \mathrm{m}^{2}\right)\end{array}$ & 6741 & 5948 & 7893 & 6165 & 0.539 \\
\hline $\begin{array}{l}\text { Percentage Platelet } \\
\text { recovery at } 1 \mathrm{Hr}(\%)\end{array}$ & 33.75 & 31.61 & 36.6 & 30.55 & 0.885 \\
\hline
\end{tabular}

* Statistically significant at $\mathrm{p}<0.05$

Table 2: Intra-group comparison of Study arm SDP's issued to A, B and 0 group patients.

\begin{tabular}{|c|c|c|c|c|}
\hline \multirow{3}{*}{ Patient Parameters studied } & \multicolumn{3}{|c|}{$\begin{array}{c}\text { Study arm SDP's }(n=50) \text { transfused } \\
\text { to }\end{array}$} & \multirow{3}{*}{$\begin{array}{l}\text { p-value } \\
\text { (ANOVA) }\end{array}$} \\
\hline & $\begin{array}{c}\text { A Group } \\
(n=17)\end{array}$ & $\begin{array}{c}\text { B Group } \\
(n=17)\end{array}$ & $\begin{array}{l}\text { O group } \\
(n=16)\end{array}$ & \\
\hline & \multicolumn{3}{|c|}{ Mean Values } & \\
\hline Height (cm.) & 155.14 & 161.35 & 159.21 & $0.024^{\star}$ \\
\hline Weight (kg.) & 55.64 & 66.64 & 66.57 & $0.001^{*}$ \\
\hline Body Surface area $\left(\mathrm{m}^{2}\right)$ & 1.55 & 1.72 & 1.71 & $0.001^{\star}$ \\
\hline Pre Transfusion count $(/ \mu \mathrm{l})$ & 15857 & 14714 & 8857 & 0.213 \\
\hline Platelet Dose (x 1011) & 4.13 & 4.15 & 4.38 & 0.570 \\
\hline Count increment at $1 \mathrm{Hr}(/ \mu \mathrm{l})$ & 29714 & 32071 & 28214 & 0.388 \\
\hline $\begin{array}{l}\text { Corrected count increment } \\
\text { at } 1 \mathrm{Hr}\left(/ \mu \mathrm{l} / \mathrm{m}^{2}\right)\end{array}$ & 11246 & 13372 & 11068 & 0.127 \\
\hline $\begin{array}{l}\text { Corrected count increment } \\
\text { at } 24 \mathrm{Hr}\left(/ \mu \mathrm{l} / \mathrm{m}^{2}\right)\end{array}$ & 6134 & 7309 & 5867 & 0.309 \\
\hline $\begin{array}{l}\text { Percentage Platelet } \\
\text { recovery at } 1 \mathrm{Hr}(\%)\end{array}$ & 30.44 & 38.3 & 32.23 & 0.089 \\
\hline
\end{tabular}

* Statistically significant at $p<0.05$

P-203

\section{ASSESSMENT OF EFFICACY PLATELET TRANSFUSION IN} ACUTE LEUKEMIA

I Frigaa, H Mahnaoui, B Rached, A Amireche and H Ouelaa

CHU Annaba /Faculty of Medecine of Annaba, Annaba, Algeria

Background: The evaluation of yield platelet transfusion is a way of testing the various factors influencing the efficiency of transfusion.The effectiveness of a platelet transfusion can be variable as a result of differences in platelet dose, platelet source, platelet donor-recipient $\mathrm{ABO}$ compatibility, duration of platelet storage and characterstic of recipient

Aims: This study was designed to determine the prevalence and assess the risk factors responsible for platelet transfusion inefficiency and fractoriness in hemato-oncological patients.

Methods: This is a prospective study, during 6 months, evaluating the transfusion efficiency of 111 blood platelet transfusionsperformed in 64 thrombocytopenic patients followed for acute leukemia admitted in the hemato-oncology unit of the Department of Hématology at our universitary hospital. Single-donor apheresis platelet concentrates (APC) were collected from our community blood center by Trima Accel system. All patients were monitored during each platelet transfusion and the response was assessed by estimating $\mathrm{CCI}$ at $1 \mathrm{~h}$ and $24 \mathrm{~h}$. The average weight of patients is $68.15 \pm 16.87 \mathrm{~kg}$ [39-125 kg].

Results: Half of the patients have an initial platelet count $<10 \times 10^{9}$ Plt $/ 1$.Bleeding evaluation comprehended petechiae, haematomas, nose and mouse bleeds, bloody 
stools and haematuria. $62.50 \%$ of transfused patients present a hemorrhagic syndrome (level 02), while $24.66 \%$ are clinically stable (no bleeding) and $79.86 \%$ of patients present a history of transfusion. The average platelet dose of APC transfused is $5.26 \pm 1.38 \times 10^{11}$ Plt $\left[2.5-8 \times 10^{11}\right]$. During the study period, $27(19,28 \%)$ patients did not show desired platelet count increment. The results found an early platelet yield effective of 80.71. The study of factors influencing on ineffective yield platelet transfusion showed that fever, antibiotic therapy, hemorrhage, insufficient dose platelet and splenomegaly are responsible for the reduction of the yield of platelet transfusion. $\mathrm{ABO}$ compatibility, and duration of storage have a modest impact on posttransfusion platelet increments.

Conclusions: The evaluation of this parameter is a very useful for the clinician and the transfusing physician to adopt better conditions for platelet transfusion. The number of platelet transfusions could be safely lowered after clinical audit.

\section{P-204}

\section{UNDER MY SKIN: PRELIMINARY OUTCOMES OF} ESTABLISHING A SUBCUTANEOUS IMMUNOGLOBULIN (SCIG) PROGRAM IN A REGIONAL HEALTH SERVICE

\section{CA Lambooy $^{1}$, LR Hollis ${ }^{1}$, YL Fung ${ }^{2}$, K Morwood ${ }^{1}$ and H Weston ${ }^{1}$}

${ }^{1}$ Sunshine Coast Hospital and Health Service, Nambour, Australia ${ }^{2}$ University of the Sunshine Coast, Sippy Downs, Australia

Background: Immunoglobulin replacement therapy (IRT) is standard of care for patients with primary or secondary immune deficiency. Intravenous immunoglobulin (IVIg) is the predominant form of IRT in Australia. In March 2013, subcutaneous immunoglobulin (SCIg) was approved for use in Australia by the National Blood Authority (NBA) for use in these patients.

Administration of immunoglobulin by the subcutaneous route in the home environment is advantageous for both the patient and health service. It reduces patient admission episodes and adverse reactions, produces more stable blood levels and thus decreases infective episodes.

The Sunshine Coast Hospital and Health Service (SCHHS) has faced a continuing challenge to provide sufficient day procedure beds for the increasing number of day infusions procedures. As patients can be trained to self-administer SCIg at home, this provided an avenue of reducing day bed requirements.

Aims: To review the process and assess the early impact of implementing a patient administered SCIg Program.

Methods: In September 2013, senior members of the immunology, haematology and transfusion teams discussed the possibility of implementing a SCIg program within SCHHS. Key stakeholders were identified and consultation took place in October 2013. A cost benefit analysis and a literature review were undertaken. A Briefing Note was submitted to the Patient Safety and Quality Committee and the Executive Leadership Team in December 2013 and subsequently endorsed implementing the program.

Results: Rollout of SCIg program:The Transfusion Clinical Nurse Consultant co-ordinated the implementation of the SCIg program in January 2014. A Training Day was undertaken in January involving 20 key medical, nursing and laboratory staff. Representatives from SCIg product manufacturers also attended.Issues and processes required for the commencement of a SCIg program were discussed, and planning was undertaken. Issues identified included: - Availability of pumps and equipment Clinic space for training, patient review and nursing support - Patient training (self infusion techniques, product knowledge) - Dispensing a blood product directly to a patien

Patient recruitment:The first patient commenced SCIg training on the 17th March 2014.In the first 12 months, 35 adult immunology and haematology patients were recruited onto the SCIg program. Although the patient competency training program was planned to include four sessions, 25 patients (71\%) gained competency in 1 to 3 sessions. 9 patients (26\%) required 4 training sessions and 1 patient (3\%) required 5 training sessions.In the first 12 months, approximately 455 day admissions were saved by patients enrolled in the SCIg program.

Summary/Conclusion: The SCHHS was the first health service in Queensland to implement a home based SCIg program which has generated valuable day bed savings. The SCHHS SCIg program has grown to be the largest cohort of adult haematology and immunology patients in Australia.Further studies are currently underway to assess the clinical, economic and patient quality of life impacts of this program.

\subsection{Adverse Events, Including TRALI}

P-206

ANALYSIS OF TRANSFUSION ADVERSE REACTIONS TO RED

CELL TRANSFUSION IN NATIONAL THALASSAEMIA CENTER TEACHING HOSPITAL KURUNEGALA SRI LANKA

BGSRRBandara

Teaching Hospital Kurunegala, Kurunegala, Sri Lanka

Back ground: Teaching Hospital Kurunegala which has a bed strength of 1991 is one of the main hospitals in Sri Lanka. It a hospital with a well equipped National Thalassaemia Center which is totally dedicated for caring the Thalassaemic patiens from all parts of the country. National Thalassaemic Center is one of the units Teaching Hospital Kurunegala, where $1032 \beta$ Thalassaemia major patients are being managed.These patients are registered since the first day of the diagnosis and are being managed whole throughout

Aim: In order to minimise the occurance of adverse reactions and FNHTR (Febrile Non Haemolytic Transfusion Reactions) a retrospective analysis of all transfusions and adverse reactions following blood transfusions were carried out with the aim of providing optimum patient care by promoting Leuco Reduced Blood and pre storage leucodepleted blood for transfusions

Method: A descriptive retrospective study was carried out by analysing all post transfusion adverse events and reaction report forms received to the Department of Transfusion Medicine of Teaching Hospital Kurunegala, over a period of six months from 01 February 2015 to 31 July 2015. A detailed collection of Haemovigilence data was gathered and analyzed.

Results: The total no transfusion episodes were 6738 . There were 31 post transfusion reactions reported, 15 were FNHTR (Febrile Non Haemolytic Transfusion Reactions). The type of reaction and their frequency of occurrence were illustrated in the table.FNHTR was the commonest with a $48.4 \%$ which is mainly due to the leucocytes in the whole blood.(Percentage of Allergic Reaction was 38.7\% may be due to under reporting of minor allergic reactions.)

Conclusion: This study clearly shows that there is an increased tendency of FNHTR (Febrile Non Haemolytic Transfusion Reactions) in Thalassaemic patients. Therefore in order to minimize the occurrence of adverse reactions and to provide best possible care the use of pre storage leucoreduced Blood and pre storage leucodepleted blood should be promoted .At Sri Lanka due to cost constraints It's difficult to provide LDB (Leuco Depletd Blood) for every patient.But we are trying our best to provide LDB at least to the thalassaemic population.

Table 1: Analysis of adverse reactions to red cell transfosions in National Thalassaemia Center Teachind Hospital Kurunegala Sri Lanka

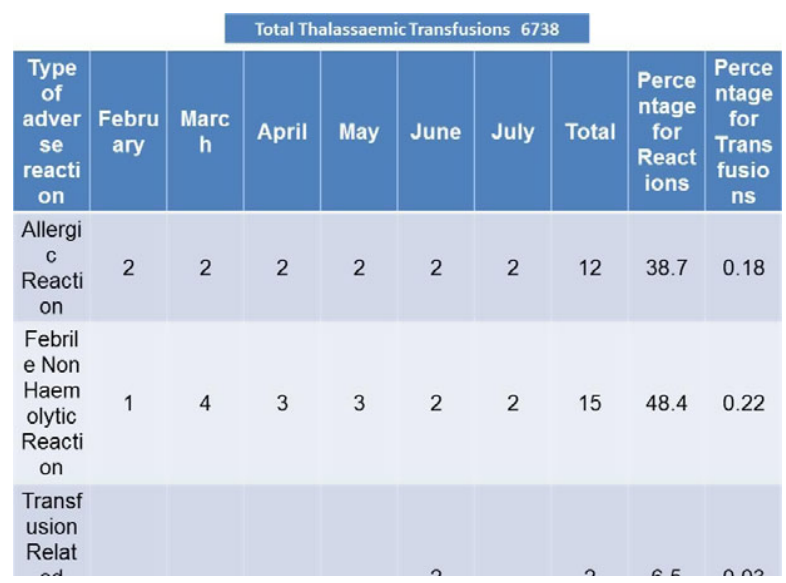

P-207

This abstract has been withdrawn. 


\subsection{Haemovigilance and Transfusion Safety}

$\mathrm{P}-208$

THE PROGRESS OF THE IMPLEMENTATION OF HOSPITAL TRANSFUSION REACTION REPORTING AND INVESTIGATION SYSTEM

FA Said

Indonesia Army Central Hospital, Jakarta Pusat, Indonesia

Background: One of the hospital blood bank duties is to report and investigate adverse event related to transfusion, as stated in the Indonesian Goverments Regulations No. 7 year 2011 concerning blood transfusion services. However, there is no established national guidelines to conduct it, such as how to assess, classify the symptoms, and standardize the reporting forms. Although there is a monthly report to the Indonesian Red Cross Society, but until now there is no institusion appointed by the local authorities to perform an audit on the transfusion reactions reports. Nevertheless, since January 2012, the hospital blood bank has developed an acute transfusion reaction reporting and investigation system to meet the function of haemovigilance. Here we reported the 2012-2014 experience of implementation.

Aim: To review the reporting and investigation system of the acute blood transfusion adverse reactions in the Indonesia Army Central Hospital- Gatot Soebroto for better health services and patient safety.

Methods: Since January 2012 we have implemented the blood transfusion related adverse effect reporting and investigating system using a paper-based reporting form with check-box format containing 11 signs and symptoms of transfusion reactions. Because of the requirement of the Joint Commission on International accreditation (JCI) to maintain the quality of patient safety by strict monitoring and evaluation during blood administration, in October 2013, the reporting form was revised and redesigned to be a blood transfusion monitoring and evaluation check-box form. Attached to it is a notification form to be completed when adverse reactions are found. Based on the above, the blood bank carried out an investigation and examination into the complete immunohematology tests.

Result: All of the transfusion reaction data from 2012-2014 were collected and analyzed. In 2012, there were 12 cases of transfusion reaction out of 17,806 blood bags $(0.067 \%)$. In 2013, the reported cases showed an increase to 33 cases out of 19,290 blood bags $(0.171 \%)$. In 2014, when transfusion monitoring and evaluation form was fully implemented, and the reported cases rose to 48 cases out of 16,791 blood bags $(0.286 \%)$. A total there were 93 cases out of 53,887 blood bags, and based on the signs and symptomps, classified accordingly: urticaria $33(0.061 \%)$, febrile reactions $42(0.078 \%)$, dyspnea $14(0.026 \%)$, hypotension $2(0.004 \%)$ and hemolytic reaction $1(0.002 \%)$. All of the cases were investigated with immunohematology confirmation examinations, It was found that there was 1 minor incompatibility and 1 mayor incompatibility, the rest was all compatible. It was discovered that there was 1 near-miss case and 1 error case because of wrong blood grouping due to blood discrepancies. There was no fatal incident in these cases.

Conclusion: The transfusion adverse reaction reporting rates vary, and may be under reported. With the better designed monitoring and evaluation form, there has been a significant increase in the reporting of suspected transfusion reaction cases. Although in this 3 years span no fatal incidents have been reported, the transfusion reaction rates especially error incidents should be minimize by practising haemovigilance program.

\section{P-209}

\section{MONITORING NEAR MISS EVENTS IN A HOSPITAL BLOOD}

BANK

MS Soomro and FK Mahar

Aga Khan University Hospital, Karachi, Pakistan

Background: Near miss errors related to sample identification are the preventable errors that may have fatal consequences if remain undetected, resulting in the determination of a wrong blood group or transfusion of an incorrect component.

Aims: This study was done with aim to identify the Near miss events in our transfusion medicine setup in order to identify the sources and prevent them from happening again.

Methods: It's a retrospective descriptive study, which was carried out at the section of hematology (Blood bank), with a purpose to identify the near miss events mainly pertaining to errors in sample identification. A total number the requests forms along with the blood sample for grouping and cross matching, from January 2013 till December 2014 were included in the study. Data was analyzed retrospectively and sample identification errors were identified on the grounds of when notified by the on call doctor/ staff nurse who has performed the sampling. when noticed by laboratory staff by delta checking with previous results.

(C) 2015 The Author

Vox Sanguinis (C) 2015 International Society of Blood Transfusion

Vox Sanguinis (2015) 109 (Suppl. 2), 1-96
Results: During these two years a total of 75,157 request slips were received in blood bank for arranging various blood products and 44 sample identification errors were identified, out of which $0.4 \%$ were in 2013 (26 in 63,670 bags) and $0.3 \%$ were in 2014 (18 in 59,690 bags) per 1000 units of various blood components issued.

Out of these 44 identification errors, 42 (95.4\%) were labeled as Wrong blood in tube (WBIT), which is further sub classified as WBIT due to miscollection (sample was labeled correctly but blood was collected from the wrong patient) which was found to be the most commonest error 26 out of 44 (59\%); and WBIT due to mislabeling of samples (sample was collected from the right patient but was labeled incorrectly with medical record number/ name of another person) was found to be 16 out of $44(36.3 \%)$. While $2(4.55 \%)$ of these errors were made by blood bank staff by issuing units on wrong blood unit releasing slips.

Most of these events, 30 out of $44(68 \%)$ happened on floor/wards. 2 out of 44 $(4.5 \%)$ were reported from ICU (intensive care unit), 8 out of $44(18 \%)$ were reported from emergency room (ER), 1 (2.27\%) of them was reported from operating room (OR), another case of misidentification happened in labor room.

All of the samples in high critical areas are taken by staff nurses which makes 12 $(27.2 \%)$ of total sample identification errors. Out of total errors 30 were reported from floor/ward, $29(65.9 \%)$ were done by residents only one $(2.27 \%)$ was carried out by trained phlebotomist.

Conclusion: In our study we observed that unlike usual most of the errors were reported from floor/ward not from high throughput areas like ER (emergency room) and ICU (intensive care unit) and most of these errors were carried out by the on cal doctors (intern/resident). Most of these errors occurred due to mal practice of labeling the samples after collection and away from patient's bed side.

\section{P-210 OVERCOMING THE CHALLENGES IN ESTABLISHING A SUCCESSFUL HAEMOVIGILANCE PROGRAMME IN A COUNTRY IN THE ASIAN REGION \\ K Gonsalkorale \\ National Blood Transfusion Service of Sri Lanka, Ratmalana, Sri Lanka}

Haemovigilance, a dire necessity to provide safe and quality transfusions was a known but a novel concept to the National Blood Transfusion Service (NBTS) of Sri Lanka in the year 2008. The need was well understood by the administration and the encouragement given to initiate the programme is praiseworthy. The unique structure of the NBTS was the greatest advantage in commencing the programme. NBTS is an island wide, centrally coordinated, non-profitable government service which comes under the ministry of health and is the sole supplier of blood and blood products to the entire patient population in the state sector and majority in the private sector. It consists of the National Blood Centre (NBC) which is the main administrative centre and 95 Hospital Based Blood Banks (HBBs) which are grouped into 17 clusters taking into consideration the proximity and the accessibility to each other. Each cluster is headed by a Consultant or a Medical officer In Charge (MOIC) who acts as the administrative officer of the said cluster.

All cluster heads were initially invited to the NBC for a familiarization programme on haemovigilance. Reporting forms, Instruction leaflets were distributed and presentations were conducted. Despite this meeting the initial reporting of adverse events was poor. The thoughts and attitudes of the Sri Lankans with their long history and the culture heritage are quite different to the western world. Yet they would understand and accept an evolving situation, probably due to the colonial rule that prevailed in the bygone era.

The next strategy was to meet the staff of each HBB during the regular Hospital Transfusion Committee (HTC) meetings and to explain the importance of the system and the blame free nature of the programme.

Fright and doubt still prevailed. Telephone number of the In-charge consultant of the haemovigilance unit was given to all $\mathrm{HBBs}$ so that they could contact her throughout the day. This was a major breakthrough. The staff would often clear their minds by phoning and gradually regular reporting became a norm.

Near miss events and delayed reactions are still under reported. The occasional hesitancy of reporting a mishap in the blood bank still prevails and refresher programmes are a must.

Lectures are now given regularly to all newly recruited medical officers and nursing officers. Undergraduates and the intern medical officers are given lectures on haemovigilance. A review programme was held this year which paved the way to clarify many doubts and queries.

The system is now a familiar concept and reporting of transfusion related adverse events (recipient haemovigilance) have become a routine procedure. The first annual haemovigilance report was published in 2010. Donor haemovigilance was commenced two years later and this was included in the annual report of 2012. With a 
lot of effort Sri Lanka has finally established a very successful haemovigilance programme.

\section{$\mathrm{P}-211$}

\section{ANALYSIS OF SELANGOR STATE HEMOVIGILANCE \\ DATABASE}

S Said Khan ${ }^{1}$, N Asidin ${ }^{2}$ and PL Lau ${ }^{2}$

${ }^{1}$ Hospital Serdang, Selangor, Malaysia ${ }^{2}$ Hospital Tengku Ampuan Rahimah Klang, Selangor, Malaysia

Background: Hemovigilance involves surveillance procedures covering the whole blood transfusion process beginning from collection of blood to following up the recipients.

Aim: We intended to analyse and report the state hemovigilance data collected for 18 months.

Methods: This is a retrospective data analysis from 10 hospitals in the state, based on the hemovigilance database template.

Results: A total of 132 reports of incidents received, of which 125 (94.7\%) are near misses while 7 (5.3\%) are transfusion errors. The main contributing factor for near misses was pre-analytical errors (85.6\%), followed by blood bank errors (14.4\%). Wrong sampling (53.3\%) constitutes the most for pre-analytical errors, while 39.3\% was due to wrong labelling and $7.4 \%$ others. There were 761 adverse transfusion reactions reported, of which $45.6 \%$ are allergic reaction, 33.4\% were febrile nonhaemolytic transfusion reaction, $6.8 \%$ anaphylactic reaction, $1.35 \%$ transfusion associated circulatory overload, $0.3 \%$ acute/delayed haemolytic transfusion reaction and $1.35 \%$ not related to transfusion.

Summary/Conclusion: The hemovigilance database analysis had illustrated the key areas which needs improvement to further improve the transfusion safety.

\section{$\mathrm{P}-212$}

\section{SETTING AN EFFECTIVE HEMOVIGILANCE SYSTEM IN A HOSPITAL}

R Sood, S Kumar, V Kumar and S RANI

Saket City Hospital, New Delhi, India

Background: Blood transfusion is often lifesaving but is not without risk.The focus has been on prevention of transfusion-transmitted infection, but there is a need to investigate other hazards related to transfusions as well. Haemovigilance is defined as 'A set of surveillance procedures covering the whole transfusion chain - from the collection of blood and its components to the follow-up of recipients, intended to collect and assess information on unexpected or undesirable effects resulting from the therapeutic use of labile blood products, and to prevent their occurrence or recurrence.' (1) Aim: To collect information on adverse incidents in donors and patients, and use it to improve patient safety.

Method: Retrospective and Prospective data of adverse donor reactions as well patient transfusion reactions and events were documented from July 2013 till May 2015and analysed.

Results: From July 2013 till May 2015; 100\% donor and transfusion reactions were reported. Out of 15 reported transfusion reactions, 9 (60\%) were febrile non-haemolytic transfusion reactions, and $6(40 \%)$ allergic reactions. Blood components that caused most of transfusion reactions were erythrocytes in $46.7 \%$, platelets in 33.3\% and fresh frozen plasma in 20\%. All except 4 patients had underwent multiple transfusions, the 4 without transfusion history and with reaction on first transfusion were either platelets or plasma. Estimates of adverse event/reactions incidence in blood donors was $0.13 \%$ adverse donor reactions. The factors correlating with the transfusion adverse effect included:

1. Individual patient characteristics-Transfusion history, parity

2. Blood component-Type

3. Equipment-Filter use

4. Concomitant medications and intravenous fluids

Conclusions: Identifying the blood donors at risk of donor reactions is important.To improve and make blood transfusion safer, it is necessary to respect all pre-transfusion procedures. The study emphasises the importance of improved education and training of the people involved in the transfusion process. Constant follow up of blood transfusion must be done and patients with diagnosed nonhaemolytic transfusion reaction should be given leukocyte reduced blood components. Proper corrective and preventive action must be taken at each step to minimize the risks associated with safety and quality in blood processing and transfusion for donors and patients.
References: De Vries RR, Faber JC, Strengers PF. Hemovigilance: An effective tool for improving transfusion practice. Vox Sang. 2011; 100:60-7.

\section{P-213 \\ BLOOD TRANSFUSION AND COMPATIBILIYTY TESTING VALIDITY \\ SKC Sim Kuan Lim, WYM Ng and M Chan \\ Health Sciences Authority, Singapore, Singapore}

Background: Prior to blood transfusion, patients undergo pre-transfusion testing which comprises of blood grouping, antibody screen and compatibility testing. Most international guidelines governing pre-transfusion testing such as AABB and BCSH recommend that antibody screen and compatibility testing results are valid for 3 days from sample drawn date if the patient has been transfused or pregnant in the preceding 3 months, or when such information is unavailable or uncertain. Compatibility testing on additional blood may be performed within this period but blood issued will have to be similarly transfused within this 3-day period. In Singapore, the Health Sciences Authority (HSA) performs pre-transfusion testing and provides compatible blood to all patients in the local hospitals and subscribe to this policy. Aims: The aim of this project is to conduct a retrospective study to review blood requests within the period of April till May 2015 to assess for blood transfused beyond the 3-day duration.

Methods: Specific dates for every unit of blood transfused were collated: sample drawn date (by transfusing facility), sample received date (by testing facility), compatibility testing date (by testing facility) and transfusion date (by transfusing facility) were analysed as contributing factors.

Results: Out of 2736 units of blood transfused in these two months, 2682 units $(98 \%)$ were appropriately transfused within the required 3-day time frame. Majority of units were transfused on day 1 (1256 units, 45.9\%), followed by day 0 (904 units, 33\%), day $2(366,13.4 \%)$ and day 3 (156 units, 5.7\%).

The remaining 54 units (2\%) were transfused beyond day 3 and stretches to as far as day 8. Two units had compatibility testing performed beyond the 3-day validity. Fifty-two units were compatibility tested and distributed within the validity time frame but were transfused beyond the 3-day recommended period.

The 54 units were transfused among 45 patients, of which 35 patients have had at least one transfusion in the preceding 3 months. Remaining 10 patients had either no prior transfusion or their transfusion history was uncertain.

Conclusion: Hospital personnel may be aware of the need to transfuse blood within 3 days from sample drawn date. However, they may still inadvertently transfuse blood beyond this duration as they may be unaware of the exact sample drawn date and the corresponding limit to the validity period. This could be due to the lack of coordination between phlebotomist and transfusion personnel. An initiative was undertaken to clearly indicate the last recommended transfusion date on the recipient label. This would provide a clear and unambiguous date for both hospital and testing personnel to deter transfusions beyond the recommended period. Other suggested initiative to minimise deviation from the 3 day policy include the possibility of extending the validity period to 7 days on a case-by-case basis when written confirmation on the lack of transfusion or pregnancy in the preceding 3 months is provided.

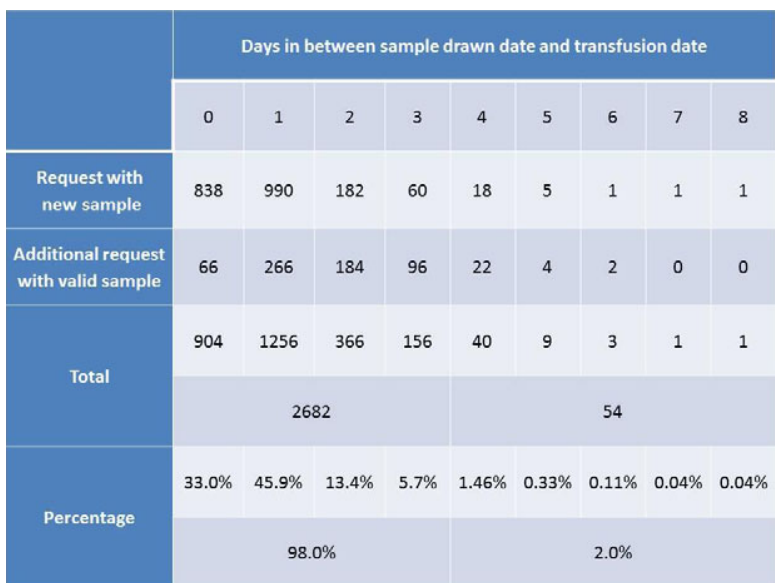

(C) 2015 The Author

Vox Sanguinis (C) 2015 International Society of Blood Transfusion Vox Sanguinis (2015) 109 (Suppl. 2), 1-96 
P-214

INCIDENCE RATE OF TRANSFUSION REACTION FOR PRBC

E Torculas, E Datu, S Saceda and M Chua

St. Luke's Medical Center-QC, Quezon City, Philippines

Background: Before 2013 no filtration was used in all blood units issued for transfusion and an increased incidence of febrile non-hemolytic transfusion reactions was observed in patients receiving unfiltered blood components. The Blood Bank then tried to introduce the use of bedside filters for packed red cells, upon requests by their attending physician for some patients. However the incidence of non-hemolytic febrile transfusion reactions (NHFTR) remained until Blood Bank and Transfusion Service (BBTS) decided to use the in-line (pre-storage) filters on all packed red blood units in addition to bedside filtration in the laboratory.

Aim: To reduce the incidence of febrile non-hemolytic transfusion reaction amongs the patients at St. Luke's Medical Center Quezon City by the use of in line filter to leuko-reduced all packed red blood cell units, thus increased patient safety and make the efficacy more feasible.

Methods: Sterile DockerTriple bag w/side pouch and In-line filter (Top and Bottom $450 \mathrm{ml} \mathrm{Bag}$ ) Automated Blood Component Separator

Collect $450 \mathrm{ml}$ blood using quadruple blood bag with in-line filter.

Stand the quadruple blood bag with freshly collected blood for $2-4 \mathrm{~h}$. This is to allow the leukocytes to perform its purpose before leukoreduction.

RESULTS

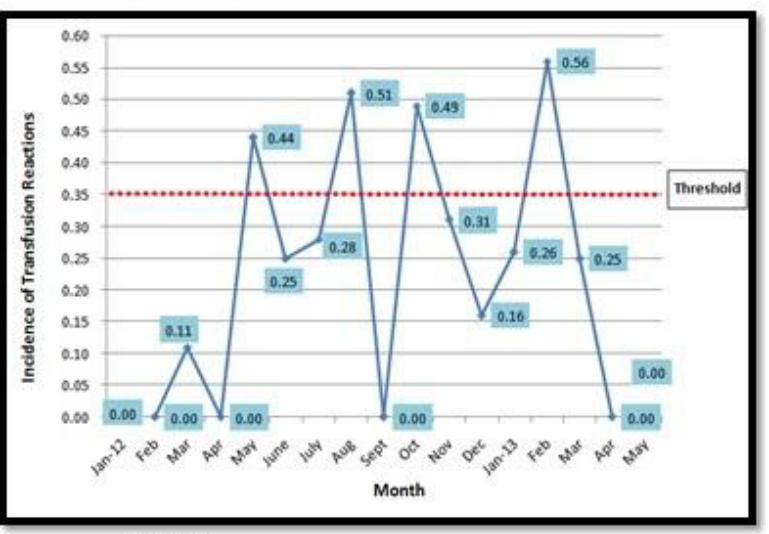

Figure 1

Incidence of Transfusion Reactions for PRBC,

St. Luke's Medical Center, Quezon City

January 2012-May 2013

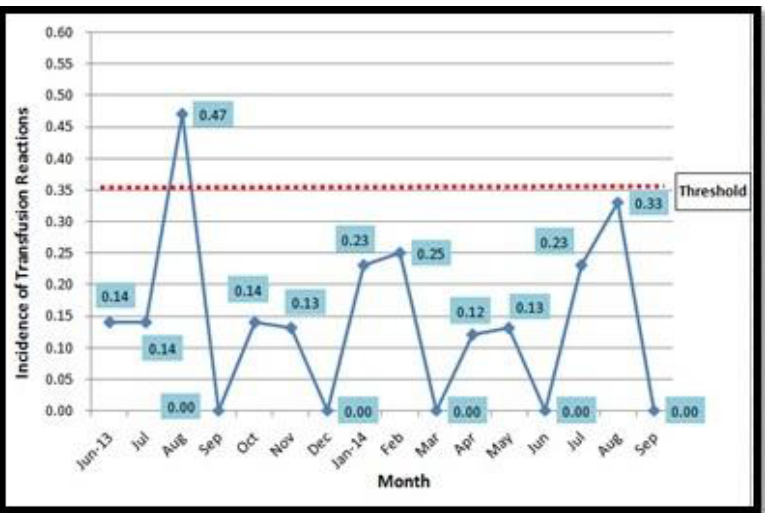

Figure 2

Incidence of Transfusion Reactions for PRBC,

St. Luke's Medical Center, Quezon City

June 2-13 - September 2014

(c) 2015 The Author

Vox Sanguinis (C) 2015 International Society of Blood Transfusion

Vox Sanguinis (2015) 109 (Suppl. 2), 1-96
Centrifuge quadruple blood bag with collected blood in a 'hard' spin with a temperature of $4^{\circ} \mathrm{C}, 4000 \mathrm{rpm}$ for $10 \mathrm{~min}$.

Switch on the Power to the automated blood component extractor. (Please see the manual of machine for proper handling of the instrument.)

Once the machine finished the separation of the components get the WBC post count for the PRBC. The value range of the Leukoreduced red blood cell unit is less than or equal to $1 \times 10^{6}$.

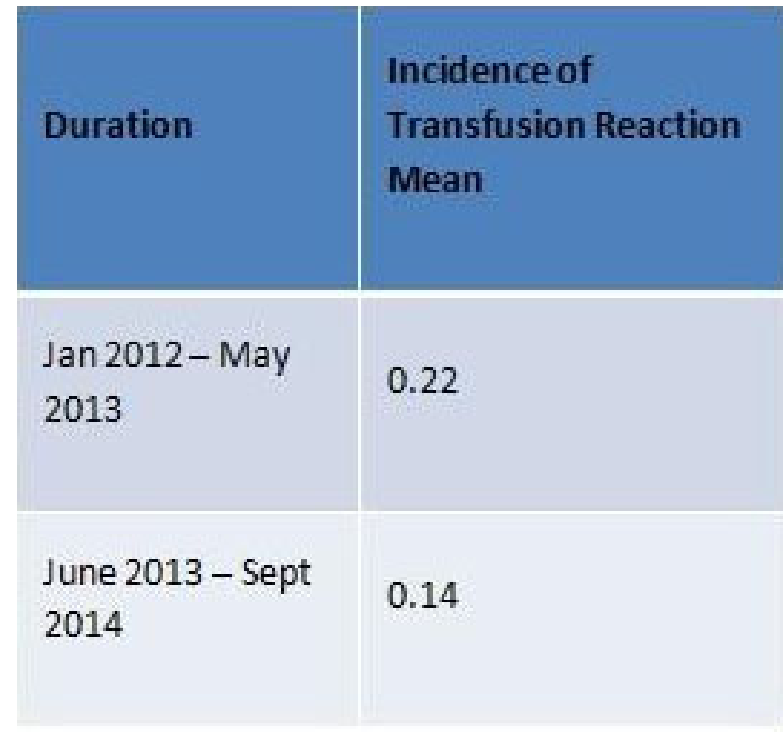

Table 1

Mean Incidence Rate of Transfusion

Reactions for PRBC, St. Luke's Medical

Quezon City, January 2012 - September

2014

\begin{tabular}{|l|l|l|}
\hline $\begin{array}{l}\text { Types of } \\
\text { Reactions }\end{array}$ & $\begin{array}{l}\text { Yale-New } \\
\text { Haven } \\
\text { HospitalUSA } \\
(1995-2002)^{1}\end{array}$ & $\begin{array}{l}\text { St. Luke's } \\
\text { Medical } \\
\text { Center-QC }\end{array}$ \\
\hline $\begin{array}{l}\text { Allergic } \\
\text { reactions }\end{array}$ & 0.09 & 0.10 \\
\hline $\begin{array}{l}\text { Febrile non- } \\
\text { hemolytic } \\
\text { reactions (FNH }\end{array}$ & 0.18 & 0.05 \\
\hline
\end{tabular}

Table 2

Mean Incidence Rate of Transfusion

Reactions after Leukoreduction,

Benchmark vs SLMC QC 
Results (See Uploaded Images): Conclusion: The monthly incidence of transfusion reactions for packed red blood cells (PRBC) is summarized in Figures 1 and 2. From January 2012 until May 2013, four (4) monthly incidence rates exceeded the set $0.35 \%$ threshold as opposed to only one (1) observed from June 2013 to September 2014.

The decrease in the mean incidence rate of PRBC transfusion reaction (Table 1) was primarily due to the use of in-line filters for leukocytes of the said blood product prior to transfusion.

Allergic type and febrile non-hemolytic type reactions were the only transfusion reactions noted. The mean incidence rate per type of transfusion reaction is comparable to international results (Table 2). No life-threatening hemolytic transfusion reactions were noted.

The decreased incidence of transfusion reaction also meant savings in the patient's hospital bill and a shorter hospital stay compared to patients who had transfusion reactions.

\section{Cellular Therapies \\ 7.1 Stem Cell and Tissue Banking, including Cord Blood}

P-215

THE IMMUNOMODULATORY EFFECT OF BONE MARROW MESENCHYMAL STEM CELLS ON EXPRESSION OF TLR3 AND TLR9 IN MICE DENDRITIC CELLS

L Kasraian $^{1}$, MH Karimi ${ }^{2}$ and L Sadeghi ${ }^{2}$

${ }^{1}$ Blood Transfusion Research Centre, High Institute for Research and Education, Shiraz, Iran ${ }^{2}$ Transplantation Reaserch Center of Shiraz Medical University, Shiraz, Iran

Background: Mesenchymal stem cells (MSCs) are multipotent cells with immunomodulatory effect on immune cells including dendritic cells (DCs). DCs are most potent antigen presenting cells. Because of immunomodulatory properties of MSCs, they can affect the function and differentiation of DCs. DCs express a kind of surface receptors called Toll-like receptors play a key role in maturation process of DCs.

Aims: In present study, we evaluated expression of two receptors (TLR3 and TLR9) in DCs after exposure to MSCs's supernatant.

Methods: MSCs and DCs were extracted from adult Balb/c mouse bone marrow and spleen, respectively. MSCs supernatant were collected 24, 48 and $72 \mathrm{~h}$ (h) after trypsinization. Isolated DCs were co-cultured with MSCs supernatant and incubated for $24 \mathrm{~h}$ and $48 \mathrm{~h}$. TLR3 and TLR9 gene expression was evaluated using real time technique.

Results: The results showed $48 \mathrm{~h}$ and $72 \mathrm{~h}$ MSCs supernatant down-regulation expression of TLR3 in DCs after both 24 and $48 \mathrm{~h}$ incubation in comparison with untreated group $(\mathrm{P}<0.01)$. DCs when treated with 24,48 and $72 \mathrm{~h}$ MSCs supernatant for $48 \mathrm{~h}$, the TLR9 mRNA level was decreased $(\mathrm{P}<0.05)$.

Conclusion: DCs were matured and activated with TLR3 and TLR9. MSCs supernatant has an immunomodulatory effect on the expression of these TLRs in DCs. Therefore, MSCs supernatant treated DCs, were not matured and could not present antigens to T cells. Generally, the findings of this study supported the immunomodulatory effect of MSCs supernatant on DCs maturation and function depends on low expression of TLR3 and TLR9

\section{P-216}

\section{IMMUNOMODULATORY EFFECTS OF MICE MESENCHYMAL} STEM CELLS ON MATURATION AND ACTIVATION OF DENDRITIC CELLS

\section{Kasraian $^{1}$, MH Karimi ${ }^{2}$ and L Sadeghi ${ }^{2}$}

${ }^{1}$ Blood Transfusion Research Centre, High Institute for Research and Education, Shiraz, Iran ${ }^{2}$ Transplantation Reaserch Center of Shiraz Medical University, Shiraz, Iran

Background: Mesenchymal stem cells (MSCs) possess a wide range of immunomodulatory functions mostly in immune cells including dendritic cells (DCs). DCs are the key cells in the immune response and play an important role in initiating cell-mediated immunity.

Objective: To evaluate the immunomodulatory effects of MSCs supernatant on maturation and function of DCs.

Methods: Bone marrow derived mice MSCs were isolated and cultured. Twenty-four, forty-eight and seventy-two hours after passage 6, supernatants were collected and
MSCs were assessed by cytometric analysis for the expression of CD34, CD44, CD45 and SCA-1. Splenic DCs were isolated using MACS and then co-cultured with MSCs supernatant. Expression of CD86, CD40 and MHC-II on DCs were also evaluated by cytometry. H3-thymidine incorporation by proliferating $\mathrm{T}$ cells was determined in two separate MLR assay settings. In one setting, DCs were co-cultured with T cells in the presence of MSCs supernatant, and in the other setting DCs were treated with MSCs supernatant and then were co-cultured with T cells. Production of IL-12, IL-6 and IL-10 cytokines was measured in the supernatant of DCs treated with MSCs supernatant. We also measured IFN- $\gamma$ and IL-4 levels in MLR supernatant.

Results: The results showed that $72 \mathrm{~h}$ MSCs supernatant could decrease the expression of MHC-II and CD86. The T cell proliferation was inhibited in the presence of MSCs supernatant and MSCs supernatant treated DCs as demonstrated by MLR assay. A significant increase in IL-4 level and a non significant decrease in IFN- $\gamma$ level in MLR supernatant were observed. However, IL-6, IL-10 and IL-12 production did not change significantly.

Conclusion: MSCs supernatant has a time dependent effect on the maturation of DCs. Also, it could alter cytokine production from responding $\mathrm{T}$ cells toward Th2. Generally, the findings of this study supported the immunomodulatory effect of MSCs supernatant on DCs maturation and function

\section{$\mathrm{P}-217$}

\section{A REVIEW ON THE EXCLUSION STATUS OF UMBILICAL CORD BLOOD COLLECTIONS IN HONG KONG RED CROSS CATHERINE CHOW CORD BLOOD BANK}

KY Lee, NK Chan, CK Wong and WC Tsoi

Hong Kong Red Cross Blood Transfusion Service, Hong Kong, China

Background: Hong Kong Red Cross Catherine Chow Cord Blood Bank (CBB) was established to provide ready-to-use umbilical cord blood units (UCB) for clinical applications. The target inventory of UCB was set at 10,000 units in consideration of cost-effectiveness analysis for a regional CBB.

Aims: To review the exclusion status and investigate the reasons of exclusion of UCB in attempt to minimise unnecessary wastage of collected UCB.

Methods: UCB units are collected from eligible donors (Table 1), which are subjected to laboratory processing procedure, including volume reduction by Sepax-2 (Biosafe, Eysins, Switzerland) and cryopreservation by BioArchive (Thermogenesis, CA, USA) if the criteria listed in Table 1 are fulfilled. Maternal blood and UCB residues are screened for mandatory infectious markers and microbial surveillance respectively. Babies' health status is surveyed shortly and 6 months after birth. The data of UCB exclusion from banking during 2007-2014 were analysed. Exclusion reasons were classified into three groups: (1) before processing; (2) during processing; and (3) after cryopreservation. Whether yearly changes in the number of excluded UCB units occurred significantly or merely due to random variation was analysed by Chi-square test ( $\mathrm{P}$-value $<0.05$ was regarded as significant).

Results: A total of 13,325 UCB units were collected in the study period and 5786 (43.4\%) units were banked. Mean yearly exclusion rate was 56.6\% (range: 54.1\% to $58.7 \%$ ). Of all the excluded UCB, 6882 units (95.9\%) were rejected before processing, 13 units (0.2\%) during processing and 281 units (3.9\%) after cryopreservation. The leading cause for exclusion before processing was insufficient volume (4852 units, 70.5\%), followed by low CD34 + cell dose (1064 units, 15.5\%), low total nucleated cell count (TNC) (802 units, 11.7\%), failure in donor health screening (129 units, 1.9\%), post-delivery complications (29 units, $0.4 \%$ ) and presence of large blood clots ( 6 units, $0.1 \%$ ). Thirteen UCB units were rejected during processing (procedure failure: 4 units, 30.7\%; blood bag leakage: 9 units, 69.3\%). Out of 281 units found unsuitable for clinical application after cryopreservation, 144 units $(51.2 \%)$ were resulted from low birth weight or abnormal Apgar score, postpartum transfusion or newborn health problems; 15 units (5.3\%) were excluded due to absence of maternal samples; 9 units $(3.2 \%)$ and 112 unit $(39.9 \%)$ were rejected due to positive infectious disease screening results and bacterial contamination respectively. One unit $(0.4 \%)$ was excluded because of improper storage. Significant yearly increment of UCB units with insufficient volume, low CD34 + cells and low TNC dose being excluded was observed over the study period $(\mathrm{P}<0.05$ all groups).

Conclusion: In this review, we were unable to identify any definitive and persistent adverse factors related to flaws in workflow design or processing procedures that could render the collected UCB ineligible for banking and clinical use as most UCB which failed qualification for banking were due to low collection volume and/or sub-standard cell dosage present. To maximise utilization of the precious collected, but failed, UCB, 473 of such excluded units were reserved for quality control purpose while suitable units were directed for research and development use.

(C) 2015 The Author Vox Sanguinis (C) 2015 International Society of Blood Transfusion Vox Sanguinis (2015) 109 (Suppl. 2), 1-96 
Table 1: Acceptance criteria for UCB to be collected/processed/cryopreserved.

\begin{tabular}{|c|c|c|}
\hline 1. Donor/newborn & $\begin{array}{l}\text { A. } \\
\text { B. }\end{array}$ & $\begin{array}{l}\text { - -34 weeks of gestation } \\
\text {-free from past history of genetic, hematologic, } \\
\text { malignant, obstetric or communicable viral diseases } \\
\text {-free from obstetric complications, maternal perinatal } \\
\text { complications, post-delivery complications, fetal } \\
\text { complications or congenital abnormalities } \\
\text {-with complete donor consent }\end{array}$ \\
\hline 2. UCB/maternal blood & A. & $\begin{array}{l}\text {-UCB: transport to } \mathrm{CBB} \text { laboratory }<36 \mathrm{hrs} \\
\text { (store at } 10-24^{\circ} \mathrm{C} \text { ) } \\
\text {-maternal blood: transport to } \mathrm{CBB} \text { laboratory }<66 \mathrm{hrs} \\
\text { (store at } 2-24^{\circ} \mathrm{C} \text { ) }\end{array}$ \\
\hline 3. UCB units & $\begin{array}{l}\text { A. } \\
\text { B. } \\
\text { C. } \\
\text { D. }\end{array}$ & $\begin{array}{l}-240 \mathrm{ml} \\
\text {-total nucleated cell dose } \geq 5 \times 10^{8} \text { cell } \\
- \text { CD } 34+\text { cell dose } \geq 1.25 \times 10^{8} \text { cell } \\
\text {-no large blood clots }\end{array}$ \\
\hline
\end{tabular}

P-218

DNA METHYL TRANSFERASE 1 EXPRESSION DURING EX VIVO EXPANSION OF UMBILICAL CORD BLOOD-DERIVED HEMATOPOIETIC STEM CELLS

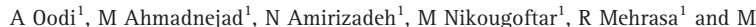
Khorshidfar $^{1}$

${ }^{1}$ Blood Transfusion Research Center, High Institute for Research and Education, Tehran, Iran

Background: HSCs reside in the ex-vivo expansion by their self-renewal capacity and also they differentiate into a myriad of cell types, as well as the regeneration of stem cells. Whereas the DNA methyltransferase1 is thought to be principally involved in the maintenance of pre-existing methylation, the role of Dnmt1 in primitive stem cells pool preservation also is not known.

Aims: In this study, mRNA expression of Dnmt1 was evaluated during CB-HSC ex vivo expansion with and without mesenchymal stem cells (MSCs).

Methods: Ex vivo cultures of CB-HSCs were performed in three culture conditions: cytokine, cytokine with MSCs and only MSCs for 7 days. Dnmt1 mRNA expression was evaluated by real time -PCR.

Results: Maximum CB-CD $34^{+}$cells expansion was observed in day 7 of expansion in all three cultures. Ex vivo expansion of $\mathrm{CB}-\mathrm{CD} 34^{+}$cells after 7 days resulted in, significant decreased expression of Dnmt1 in cytokine culture, whereas in two coculture conditions expression of Dnmt1 was increased. Also a significant difference between $\mathrm{CD}_{3} 4^{+}$cells and $\mathrm{CD} 34^{-}$cells of co-culture system with cytokine was observed.

Conclusions: Expression of Dnmt1 that are an important regulator in normal hematopoietic regeneration was increased in $\mathrm{CD}_{3} 4^{+}$expanded cells on mesenchymal stem cells.

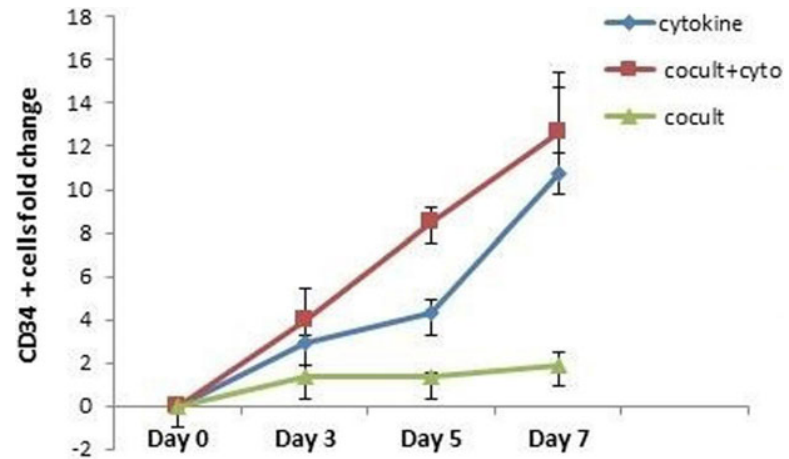

Figure 1: Dnmt1 mRNA expression fold change ratio: In the expanded cells to the fresh CD34 + cells at the three culture conditions $(P<0.05)$.

\section{(C) 2015 The Author}

Vox Sanguinis (C) 2015 International Society of Blood Transfusion

Vox Sanguinis (2015) 109 (Suppl. 2), 1-96

\section{Dnmt1 expression}

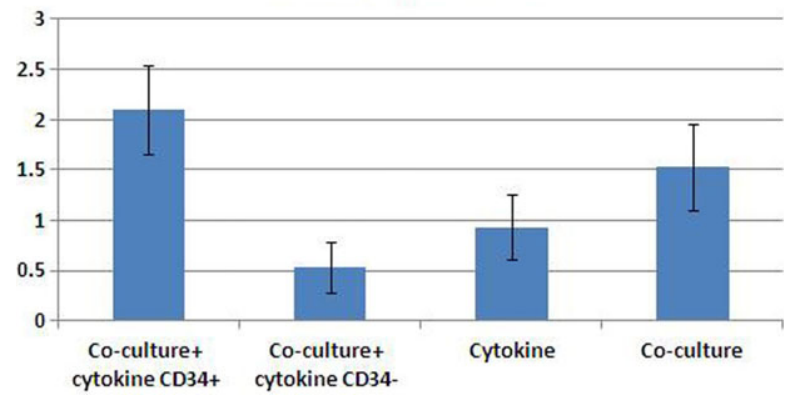

Figure 2: The fold increase of $\mathrm{CD} 34+$ cells during 7-days expansion in three culture conditions.

\subsection{Clinical Applications}

P-219

AUTOPHAGY SUPPRESSION IMPROVES REGENERATION POTENTIALITIES OF HUMAN BONE MARROW-DERIVED MESENCHYMAL STEM CELLS IN ACUTE LIVER FAILUREINDUCED MICE

F Amiri

Iranian Blood Transfusion Organization (IBTO), Tehran, Iran

Background: Mesenchymal stem cells (MSCs) are isolated and characterized from different tissues. Recently, MSCs are receiving increased attention for cell-based therapy especially in regenerative medicine. However, their low survival rate restricts their clinical applications. Interestingly, it is hypothesized that autophagy might play an important role in cellular homeostasis and survival.

Aims: The aim of this study was investigation of the regenerative capacity of autophagy-suppressed MSCs for the treatment of acute liver failure (ALF) in mice.

Methods: Authophagy was suppressed in bone marrow derived-MSCs using shRNA suppressive vectors. ALF was induced in mice by intraperitoneal injection of $1.5 \mathrm{ml} /$ $\mathrm{Kg}$ carbon tetrachloride. MSCs which are suppressed in their autophagy pathway were transplanted to afflicted mice. The mice that received normal MSCs and those that did not receive any cells (sham) were considered as control. Blood and liver samples were collected at several time intervals $(24,48$ and $72 \mathrm{hrs})$ after the transplantation of MSCs. Liver enzymes, aspartate aminotransferase (AST) and alanine aminotransferase (ALT), were evaluated with biochemical methods. Liver necrosis was also assayed using histological analysis.

Results: After $24 \mathrm{hrs}$, liver enzymes and necrosis score were significantly reduced in autophagy-suppressed MSCs-transplanted mice compared to those that transplanted with normal MSCs and sham (with no cell therapy). Normal histology without necrosis was observed in mice transplanted with autophagy-suppressed MSCs cells after $72 \mathrm{hrs}$. Interestingly, liver enzymes reached their normal levels in this period of time.

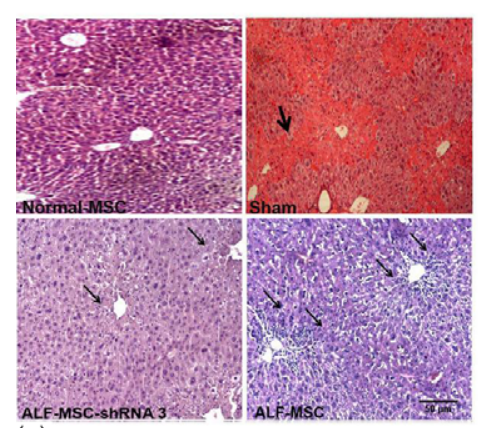

(a)

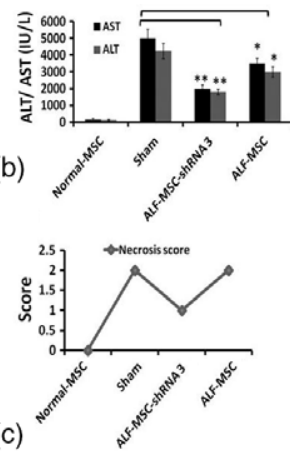

Figure 1: Therapeutic effects of different MSCs in ALF-induced mice 24 hrs after cell therapy. 


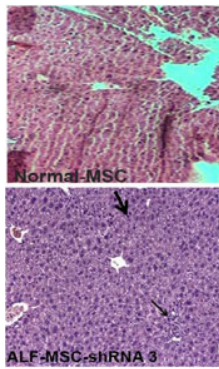

(a)

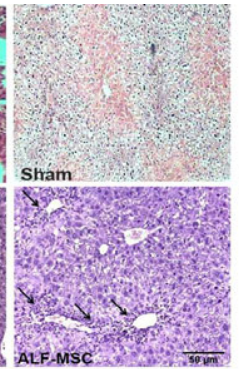

$\pi$ (b)
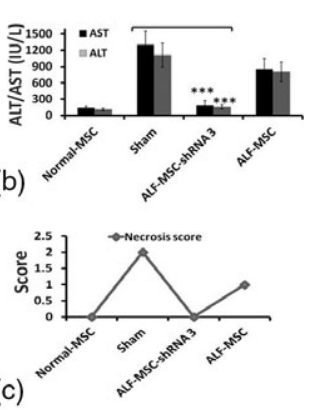

Figure 2: Therapeutic effects of different MSCs in ALF-induced mice $72 \mathrm{hrs}$ after cell therapy.

Conclusion: Autophagy suppression in MSCs might be suggested as a new strategy to improve of cell therapy in ALF. This modulation ameliorates MSCs liver regeneration potentials due to paracrine effects.

\section{P-220}

\section{CHANGE OF RBC DEFORMABILITY DURING HEMATOPOIETIC} STEM CELL TRANSPLANTATION

JM Lee, KH Suh and YT Lim

Yeungnam University Hospital, Daegu, South-Korea

Background: The red blood cell (RBC) deformability test is the measurement of the ability of the RBCs to adapt their shape to the flow conditions. The major determinants of the deformability include cell shape, composition of the cell membrane and its cytoskeleton, and internal viscosity (mean cell hemoglobin concentration). The contribution of the erythrocyte membrane to the deformability is primarily regulated by the composition and arrangement of its structural constituents. In cancer patient, chemotherapy and hematopoietic stem transplantation (HSCT) change bone marrow microenvironment, these may affect the RBC production and deformability.

Aim: We evaluate the change of RBC deformability during HSCT.

Method: Blood samples were obtained from each patients who underwent HSCT. Red cell deformability was measured with a microfluidic ektacytometer (RheoScanD, RheoMeditech, Seoul, Korea). All analyses were completed within $24 \mathrm{~h}$ after blood collection. The elongation index (EI) of erythrocytes is defined as (L-W)/(L $+W)$, where $\mathrm{L}$ and $\mathrm{W}$ are the major and minor axes of the ellipse, respectively.

Result: Eleven children ( 3 acute lymphoblastic leukemia, 5 acute myeloid leukemia, 1 aplastic anemia, 1 Ewing sarcoma, 1 retinoblastoma) were enrolled in this study. The EI of RBCs were decreased to D +5 from D-day and slowly increased from $\mathrm{D}+5$ to $\mathrm{D}+30$ after hematopoietic stem cell transplantation.

Conclusion: The deformabilites of RBCs were slowly increased during hematopoietic stem cell transplantation. RBC deformability may reflect bone marrow microenvironment of patient during HSCT. Further study about correlation with RBC deformability and prognosis of HSCT is needed.

$\mathrm{P}-221$

\section{INVESTIGATION OF ERYTHROCYTE DEFORMABILITY OF CHILDREN WITH VARIOUS HEMATOLOGIC DISEASE \\ JM Lee, YT Lim, KH Suh and YK Kim}

Yeungnam University Hospital, Daegu, South-Korea

Background: Erythrocyte deformability is expressed as the elongation index (EI) at a shear stress of $3 \mathrm{~Pa}$. The EI of erythrocyte is defined as $(\mathrm{L}-\mathrm{W}) /(\mathrm{L}+\mathrm{W})$, where $\mathrm{L}$ and $\mathrm{W}$ are the major and minor axes of the ellipse, at a shear stress of $3 \mathrm{~Pa}$. Aim: We aimed to investigate the effects of hereditary hemolytic anemia (HHA), iron deficiency anemia (IDA), immune thrombocytic purpura (ITP) and aplastic anemia (AA) on hemorrheologic parameters, especially erythrocyte deformability. Methods: Thirty-one HHA patients (age $15.3 \pm 14.7$ ), 16 IDA patients (age $9.6 \pm 5.7$ ), 16 ITP patients (age $4.8 \pm 5.4$ ) and 6 aplastic anemia patients (age $15.3 \pm 8.0$ ) and 14 healthy controls (age $11.7 \pm 12.7$ ) were enrolled. Erythrocyte deformability were estimated by ectacytometer (RheoScan-D300; Rheomeditech, Seoul, Korea). Differences between HA, IDA, ITP, AA and healthy controls were compared. Result: The EI value of IDA and AA were significantly decreased compared with normal control, but those of ITP and HHA were similar to normal control. But when shear stress were greater than $4 \mathrm{~Pa}$, EI value of HHA were significantly decreased compared with normal control.
Conclusion: This study shows different RBC deformability in various hematologic disease. Diagnostic and prognostic significance of RBC deformability is needed.

\section{$\mathrm{P}-222$ \\ A SH-SY5Y DIFFERENTIATED HUMAN NEUROBLASTOMA CELL MODEL TO EVALUATE THE POTENTIAL NEUROPROTECTIVE ROLE OF PLATELET NEUROTROPHINS CT Chang ${ }^{1}$, ML Chou $^{2}$ and T Burnouf ${ }^{1}$ \\ ${ }^{1}$ Institute of Biomedical Materials and Tissue Engineering, Taipei, Taiwan ${ }^{2}$ Institute of Medical Sciences, Taipei, Taiwan}

Background: Progressive neurodegenerative diseases, such as Alzheimer's disease (AD), Parkinson's disease (PD), or amyotrophic lateral sclerosis (ALS), are the leading cause of disability in the elderly. No therapies exist yet to delay their onset or reverse symptoms. Neurotrophins are potential drug candidates since they activate neuronal signaling pathways and modulate neurons survival. The SH-SY5Y human neuroblastoma cells have already been used to study neuroprotection induced by neurotoxins, since, using different inducers, they can be differentiated into various adult neuronal phenotypes, including dopaminergic neurons. Platelet lysates (PL) prepared from human platelet concentrates (PCs) may be a valuable physiological and balanced source of native neurotrophic growth factors (GFs) such as PDGF, BDNF, and VEGF for 'disease-modifying strategy' against PD, ALS and other neurodegenerative disorders.

Aims: The primary objectives of this study were to determine the doses of 1Methyl-4-phenyl-pyridinium ion $\left(\mathrm{MPP}^{+}\right)$neurotoxin inducing neurotoxicity in a differentiated SH-SY5Y cellular model. The second goal was to perform preliminary evaluations of the capacity of a PL to exert neuroprotective effects in this in vitro model.

Methods: SH-SY5Y neuroblastoma cells (ATCC ${ }^{\circledR}$ CRL-2266) were maintained in T75 culture flasks with a 1:1 mixture of Ham's F12 and Dulbecco Modified Eagle Medium (DMEM) supplemented with 10\% fetal bovine serum (FBS) and $1 \%$ penicillin. Cells were maintained in a humidified $5 \% \mathrm{CO}_{2}$ atmosphere at $37^{\circ} \mathrm{C}$. Differentiation was triggered by $1 \mathrm{mM}$ retinoic acid (RA) plus $0.5 \%$ of FBS during 2 and 4 days in Advanced DMEM/F12 medium or DMEM/F12 medium. On day 7, the medium was replaced by differentiation medium without FBS or RA. Viable cells $\left(2 \times 10^{4} /\right.$ well in Advanced DMEM/F12 and $5 \times 10^{4} /$ well in DMEM/F12 medium) were seeded into 24-well plates. Differentiated SH-SY5Y cells were treated with different dosage of $\mathrm{MPP}^{+}(0.01-10 \mathrm{mM})$ for $48 \mathrm{~h}$. After incubation, cell viability was determined by 3-(4,5-dimethylthiazol-2-yl)-2,5-diphenyltetrazolium bromide (MTT) assay and the absorbance was measured at $540 \mathrm{~nm}$ using a microplate spectrophotometer. PLs rich in neurotrophins were prepared from therapeutic-grade human platelet concentrates (PCs) and pre-evaluated for their neuroprotective potential.

Results: MTT assays revealed that differentiated SH-SY5Y viability was reduced to 50-60\% in Advanced DMEM/F12 medium and around 80\% in DMEM/F12 medium when using $0.3 \mathrm{mM} \mathrm{MPP}$, which was the $\mathrm{MPP}^{+}$dose selected for further experiments. Preliminary evaluation using $0.1-5 \%$ PPL to test the viability of differentiated SH-SY5Y cells exposed to $\mathrm{MPP}^{+}$, showed that PL improved cell viability. The highest level of protection (above ca. 70\%) was achieved with 1\% PPL when cells were grown in Advanced DMEM/F12 medium, and with 0.5\% PPL (ca. 90-95\%) when using DMEM/F12 medium.

Conclusions: $\mathrm{MPP}^{+}$neurotoxin can be used to induce toxicity in a differentiated SH-SY5Y human neuroblastoma cell model. This model can be of value to study the potential neuroprotective effects of platelet growth factors in neurodegenerative disorders, like PD or ALS.

\section{Clinical Immunogenetics \\ 8.1 HLA in transfusion medicine}

$\mathrm{P}-223$

\section{USING TWO SOFTWARE TOOLS TO DEFINE HLA CLASS I IMMUNOGENIC EPITOPES OF ANTIBODIES PRODUCED IN ALLOIMMUNIZED THROMBOCYTOPENIC PATIENTS} SC Pai ${ }^{1}$, JW Chen ${ }^{1}$ and SC $\mathrm{Lo}^{2}$

${ }^{1}$ Taiwan Blood Services Foundation, Taipei, Taiwan ${ }^{2}$ National Taiwan University Hospital, Taipei, Taiwan

Background: Human immune responses involve recognition of peptides presented by HLA class II molecules. In literature, the recipient HLA-DR phenotype has been sug-

(C) 2015 The Author Vox Sanguinis (C) 2015 International Society of Blood Transfusion Vox Sanguinis (2015) 109 (Suppl. 2), 1-96 
gested to play a role in determining immunogenicity of mismatched HLA class I antigens, although studies on correlation between recipient HLA-DR molecules and specific antibodies against HLA class I epitopes are limited. Antigenic proteins have functional epitopes consisting of amino acid residues which are about 3 Angstroms apart from each other. At least one of them is non-self and these patches are called eplets. HLAMatchmaker software is a computer algorithm that can determine eplet mismatches between the donor and patient's HLA antigens. NetMHCII-2.0 is a HLA class II ligand prediction tool and has been validated in vaccine research.

Aims: HLAMatchmaker and NetMHCII-2.0 software programmes were used to assess the correlation between recipient HLA DR phenotype and produced antibodies against HLA class I epitopes in HLA-alloimmunized platelet transfusion refractoriness (PTR) patients.

Methods: In this study, we combined the prediction results of two software programmes HLAMatchmaker and NetMHCII-2.0 to select platelets for HLA-alloimmunized patients. We evaluated different HLA-DRB1 alleles to determine the class II restriction for production of HLA class I epitope-specific antibodies in 20 PTR patients receiving multiple platelet transfusions.

Results: We showed that patients with HLA-DR53 could develop an epitope-specific antibody against the 65QIA eplet $(\mathrm{P}=0.004)$ on HLA-7C antigens. This could be predicted by the NetMHC II software, which revealed that the peptide TQIYKAQAQ on HLA-7C could bind with HLA-DR53 with the highest prediction score of 0.4891 . By using this prediction and epitope analysis from HLAMatchmaker, we also found a non-HLA-A2 type patient whose HLA-DR molecules did not bind with the donor's HLA-A2 epitopes and predicted to be an acceptable mismatched antigen for platelet transfusion.

Conclusions: HLA class I allopeptides containing immunogenic epitopes can be predicted using NetMHC II prediction software. These immunogenic epitopes can be determined by the presence or absence of mismatching eplets using the HLAMatchmaker software. This strategy may lead to reducing the production of HLA class specific antibodies and therefore improving the safety of platelet transfusions in the future.

\subsection{Histocompatibility in Organ Transplantation}

$\mathrm{P}-224$

EVALUATION OF HLA ANTIBODY DETECTION RATES IN PATIENTS WITH HEART TRANSPLANTATIONS

$\mathrm{J} \mathrm{Iino}^{1}$, M Matsuhashi ${ }^{2}$, M Kawabata ${ }^{1}$, S Sone ${ }^{1}$, Y Masamoto ${ }^{1}$, D Nitta ${ }^{3}$, T Imamura ${ }^{4}$, $\mathrm{M} \mathrm{Hatano}^{3}$, O Kinoshita ${ }^{5}$, K Nawata ${ }^{5}$, K Kinugawa ${ }^{4}, \mathrm{M} \mathrm{Ono}^{5}$ and H Okazaki ${ }^{1}$ ${ }^{1}$ Department of Transfusion Medicine, University of Tokyo, Tokyo, Japan ${ }^{2}$ Department of Health Sciences, Saitama Prefectural University, Saitama, Japan ${ }^{3}$ Department of Cardiovascular Medicine, Graduate School of Medicine, Univ. of Tokyo, Tokyo, Japan ${ }^{4}$ Department of Therapeutic Strategy for Heart Failure, Graduate School of Medicine, Tokyo, Japan ${ }^{5}$ Department of Cardiac Surgery, Graduate School of Medicine, University of Tokyo, Tokyo, Japan

Background: Management of patients receiving heart transplantations requires well-controlled antibody mediated rejections as well as cellular rejections. Blood transfusion associated with implanting ventricular assist devices or pregnancy causes the production of high panel reactive antibodies (PRAs) in some cases of heart transplantations in Japan because of a relatively long waiting period for transplantation. Moreover, a monitoring newly developed HLA antibody after transplantation is indispensable for a good management.

Aims: A testing of HLA antibodies has been routinely conducted in patients with heart, liver, lung, kidney and bone marrow transplantations in our institution. An aim of this study is to evaluate HLA antibodies in patients with heart transplantations. Methods: A total of 59 patients with cardiac diseases receiving heart transplantations in our institution from May 2006 to July 2015 were retrospectively analyzed. For screening and detection of anti-HLA alloantibodies, the MPHA (mixed passive hemagglutination) assay using panel kit (Beckman Coulter, Tokyo, JAPAN) and the
ELISA assay-based PakPlus platelet antibody screening kits (Immucor GTI Diagnostics, GA, USA) were used in the period between 2006 and 2009. As of then, these were examined by WAKFlow HLA antibody class I/class II (Wakunaga Pharmaceutical Co., Osaka, Japan) based on Luminex method in addition to the MPHA methods. From May 2013, the LABScreen PRA (One Lambda, CA, USA) for the determination of \%PRA and the LABScreen Single Antigen (One Lambda) for the specificity of the anti-HLA antibodies were performed.

Results: 13 out of $59(22.0 \%)$ patients receiving heart transplantations tested positive for anti-HLA antibodies. Male to female ratio is 1:0.85. Ages of patients wer between 22 and 62 (average 39) year-old. Most common underlying diseases were dilated cardiomyopathy. Among the cases where high sensitive tests for anti-HLA antibodies were performed, two patients tested positive for anti-HLA antibodies before transplantation; one case with anti-HLA class I antibody with the PRA score showing 47\% (non-DSA), another case with both class I and II with the PRA score showing 0-87\% and 94\% (DSA), respectively. These two patients received mycophenolate mofetil and are treated with plasma exchange before transplantations. Two patients newly developed anti-HLA antibodies after transplantations; one case with anti-HLA class I antibody with PRA score showing 15\% which subsequently diminished, another case with class II antibody detected persistently with PRA score showing $37-51 \%$ (DSA).

Summary/conclusions: Patients receiving heart transplantation in our institution are currently well-managed with immunosuppressant and plasma exchange even if they have anti-HLA antibody (DSA or non-DSA) before transplantation. Impact of anti-HLA antibodies on the acute graft rejection remains inconclusive because of small numbers of cases and changing methods for detecting anti-HLA antibodies. Long-term changes of cardiac function and patholophysiological features are the subjects of future investigation.

\section{$\mathrm{P}-225$ \\ CYTOKINE GENE POLYMORPHISMS IN PENINSULAR \\ MALAYSIA \\ HK Norhalifah \\ Universiti Sains Malaysia, Kubang Kerian, Malaysia}

Background: Single nucleotide polymorphisms (SNPs) in cytokine genes are uniquely distributed among populations and extensively studied due to their association with pathogenesis of diseases. Here, we report cytokine gene polymorphisms in Malay subethnic and Orang Asli groups in Peninsular Malaysia.

Aim: This study was aimed to genotype twenty-two cytokine gene polymorphisms in Malay subethnic groups and Orang Asli subgroups in Peninsular Malaysia.

Methods: Three hundred and fifteen volunteers representing the five Malay subethnic groups $($ Acheh $=34$, Kelantan $=34$, Mandailing $=31$, Minangkabau $=29$ and Patani $=22$ ) and six Orang Asli subgroups (Negrito: Batek $=25$, Lanoh $=25$ and Kensiu $=36$, Senoi: Che Wong $=26$ and Semai $=42$ and Proto-Malay: Orang Kanaq $=11$ ) of the Peninsular Malaysia were screened for twenty-two SNPs in thirteen cytokine genes $(I L-1 \alpha, I L-1 \beta, I L-1 R, I L-4 R \alpha, I L-12, I F N-y, T N F-\alpha, I L-2, I L 1-$ $R A, T G F-\beta 1, I L-4, I L-6$ and $I L-10)$ using the polymerase chain reaction-sequence specific primer (PCR-SSP).

Results: Cytokine allelic distributions are highly variable in our study groups/subgroups. For example, frequency of $I L-1 \beta-511 C$ allele is between $0.49-0.58$ in Malay subethnic groups but is relatively low in Senoi $(0.37-0.46)$ and high in Negrito (0.72-0.88) and Proto-Malays (0.73). Their genetic relationships were then mapped using principal coordinate (PCO) plot using the frequencies of the 22 cytokine SNPs. In the PCO plot, Malay subethnic groups were clustered together while other Orang Asli subgroups were displaced towards other reference populations such as those from Mainland Asia, Taiwan and Africa.

Conclusion: Our inferences using cytokine data showed population sub-structure in Peninsular Malaysia and this is associated with several ancient migration waves into this region. Their genepools were further shaped by various evolutionary pressure (founder effects and selective pressure) since they first settled. 